\title{
Investigation of the flow field in the vicinity of an axial flow fan during low flow rates
}

$$
\text { by }
$$

Francois George Louw

Dissertation approved for the degree of Doctor of Philosophy (Mechanical Engineering) in the Faculty of Engineering at Stellenbosch University

Promoters:

Prof. T W von Backstöm

Dr S J van der Spuy

December 2015 


\section{Declaration}

By submitting this dissertation electronically, I declare that the entirety of the work contained therein is my own, original work, that I am the sole author thereof (save to the extent explicitly otherwise stated), that reproduction and publication thereof by Stellenbosch University will not infringe any third party rights and that I have not previously in its entirety or in part submitted it for obtaining any qualification.

Date:

Copyright (C) 2015 Stellenbosch University All rights reserved. 


\title{
Abstract
}

\section{Investigation of the flow field in the vicinity of an axial flow fan during low flow rates}

\author{
F G Louw \\ Dissertation: PhD Eng (Mech) \\ December 2015
}

Large axial flow fans $(\approx 9 \mathrm{~m}$ in diameter) are used in air-cooled heat exchangers (ACHEs). Adverse inflow conditions occur in these systems, especially near the periphery of the air-cooled heat exchanger (ACHE) platform, affecting reduced flow rates through the fans located in these regions. The focus of the present study is to investigate the flow field in the vicinity of an axial flow fan during these low flow conditions, by means of experimentation and numerical analyses.

A 1.542 m diameter model of an axial flow fan, termed the B2a-fan, is constructed and tested in a British standard 848, type A test facility to obtain its characteristic curves. A wireless system to conduct blade surface pressure measurements (BSPMs) on both the pressure and suction sides of a fan blade is also developed and implemented. An eighth sector of the fan is modelled using the commercial CFD code, ANSYS Fluent 14.0, for a range of flow rates. Pearson correlation factors of $R_{P, \Psi_{F s}}=0.997$ and $R_{P, \eta_{F s}}=0.990$ are obtained for the respective characteristic curves of fan static pressure and static efficiency. Factors of $R_{P, B S P M} \gtrsim 0.990$ are obtained for more than $90 \%$ of the BSPMs along circumferential sections of a blade, at different radii. These correlations validate the numerical model.

Flow field visualizations show that the flow field becomes more unstable as the flow rate through the fan is decreased with considerable increases in radial velocity. Instability starts near the blade root and progressively moves towards higher blade spans with a decrease in flow rate. A deviation occurs between the numerically calculated blade sectional lift and drag characteristics and the two-dimensional NASA LS 0413 aerofoil data at low flow rates. The highest blade sectional lift coefficients occur near the hub and decrease towards higher blade spans. An Euler turbo-machinery analysis of the flow field data shows that the component of Coriolis power is close to zero at near-design flow rates, whereas the aerodynamic power component is almost equal to the total power 
applied by the fan. However, as the flow rate decreases a gradual inversion of this characteristic is observed.

Lastly, a new simplified fan model in the form of an actuator-disc model (ADM) is developed, termed the Reverse engineered empirical actuator-disc model (REEADM). This model implements the blade sectional lift, drag and radial force coefficients calculated from the CFD data. The results obtained from this model provides a fair comparison to the experimental fan characteristics at near-design flow rates, but a deviation is observed for low flow rates, although an improvement is made compared to the original ADM. 


\section{Uittreksel}

\section{Ondersoek van die vloeiveld in die omgewing van 'n aksiaalvloeiwaaier gedurende lae vloeitempo's}

("Investigation of the flow field in the vicinity of an axial flow fan during low flow rates")

F G Louw

Proefskrif: PhD Ing (Meg)

Desember 2015

Groot aksiaalvloeiwaaiers $(\approx 9 \mathrm{~m}$ in deursnee) word geïmplementeer in lugverkoelde hitteruilers (LVHRs). Ongunstige invloeipatrone, wat veral naby die rante van 'n LVHR-platform voorkom, veroorsaak 'n afname in volumevloeitempo's deur die waaiers in hierdie omgewings. Dus is die fokus van die huidige studie om die vloeiveld in die nabye omgewing van 'n aksiaalvloeiwaaier, onderhewig aan lae vloeitoestande, te ontleed deur beide eksperimentele en numeriese ondersoeke.

'n $1.542 \mathrm{~m}$ deursnee model van 'n aksiaalvloeiwaaier, genaamd die B2awaaier, is gebou en getoets in 'n toetsfasiliteit wat voldoen aan die Britse standaard 848, tipe A, om die karakteristieke waaierkrommes te bepaal. 'n Koordlose sisteem om lemoppervlak-drukmetings (LODMs) mee uit te voer, op beide die suig- en drukkant van die waaierlem, is ook ontwikkel en geïmplementeer. 'n Agtste sektor van die waaier is gemodelleer deur van die kommersiële program, ANSYS Fluent 14.0, gebruik te maak vir die reeks volumevloeie wat in ag geneem is. Pearson verwantskapsfaktore van $R_{P, \Psi_{F s}}=0.997$ en $R_{P, \eta_{F s}}=0.990$ word verkry vir die onderskeie karakteristieke krommes van waaier-statiese druk en statiese effektiwiteit. Verwanskapsfaktore van $R_{P, L O D M} \gtrsim 0.990$ word verkry vir meer as $90 \%$ van die LODMs langs omtreksnitte van die waaierlem by verskillende radiusse. Hierdie verwantskappe dien as bevestiging die numeriese model se akkuraatheid.

Visuele uitbeelding wys dat die vloeiveld toenemend onstabiel raak as die vloeitempo deur die waaier verminder. Daarbenewens word 'n aansienlike toename radiale vloei ook opgemerk. Die vloei begin onstabiel raak by die waaiernaaf en beweeg geleidelik uitwaarts na groter lemspanwydtes soos wat die vloeitempo deur die waaier verminder. 'n Afwyking tussen die numeries 
berekende hef- en sleurkarakteristieke by verskillende lemsnitte en die tweedimensionele NASA LS 0413 vleuelprofiel data, kom voor by lae vloeitempo's. Die hoogste lemsnit-hefkoëffisiënte kom by die naaf voor en verminder soos na hoër spanwydtes beweeg word. 'n Euler turbomasjienerie analise van die vloeivelddata dui aan dat die Coriolis drywingskomponent naby aan nul is vir vloeitempo's naby aan die ontwerpspunt, maar dat die aerodinamiese drywingskomponent naastenby gelyk is aan die totale drywing wat deur die waaier uitgeoefen word. 'n Gelydelike inversie van hierdie karakteristiek vind egter plaas soos die vloeitempo verminder word.

Laastens is 'n nuwe vereenvoudigde waaiermodel ontwikkel in die vorm van 'n aksie-skyf model (ASM), genaamd die Teruggewerkte empiriese aksie-skyf model (TEASM). Hierdie model implementeer die lemsnit hef-, sleur- en radiaalkragkoëffisiënte wat uit die numeriese data bereken is. Die karakteristieke, wat deur die laasgenoemde model bereken is, vergelyk goed met die eksperimentele waaierkarakteristieke by vloeitempo's soortgelyk aan die ontwerpstoestand, maar 'n afwyking kom by lae vloeitempo's voor. Die TEASM model bied egter steeds 'n verbeterde antwoord by lae vloeitempo's in vergelyking met die oorspronklike ASM. 


\section{Acknowledgements}

Firstly my acknowledgement and gratitude towards my Lord and Saviour, Jesus Christ: Here, soms kan woorde my gevoelens nie beskryf nie, maar uiteindelik staan ons nou aan die einde van een hoofstuk en aan die begin van 'n nuwe een. Dankie vir elke oomblik van karakterbou en opvoeding wat U my gegun het. Dankie vir die Pa wat $\mathrm{U}$ is. En dankie dat ons die pad verder saam kan stap. Mag ek deur U aangewend word tot in ewigheid.

To my wife, Jeanne: Dit was nogal 'n avontuur tot nou. Dankie vir al die aande wat jy saam met my gesit het in die toetsfasiliteit en die ander hulp rondom my werk. Jy is my hart se punt en ek is so bly dat ek met jou getrou het. Nou lê die volgende avontuur vir ons voor...

To my immediate family and the family-in-law: Pa Johan and Ma Irma, Pa Henry and Ma Elzabé, Zuléne and Nigel, PJ and Marike as well as Johann: Dankie vir julle bystand in hierdie tyd; Vir ma se deurlees van my proefskrif en vir al die vleis van die Vrystaat wat ons aan die gang gehou het.

To all of my friends that patiently listened while I was talking about "boring" engineering topics: Apart from the fan that I worked with in this study, I have no better group of fans than you guys. There are many, but I would like to mention, Markie and Celeste, Luan and Sammy, Johan and Avotra, Michal and Mandrie, Etienne and Salomé and also Ernie and Janneke. You are like family to me and Jeanne and we love you guys with everything in us (and more). I trust we will build on the memories we made and that our time on earth will echo into eternity.

Many thanks towards my promoters who also became my friends: Prof. Theo W. von Backström and Dr S. Johan van der Spuy, thank you for all the philosophic sessions we had during our meetings, which normally continued for at least an hour, even though only half an hour was scheduled. I enjoyed our discussions immensely. Thank you also for your contributions towards the final version of this dissertation (especially during the early morning hours). I hope your research would keep on contributing to the world. Dr van der Spuy; A personal thanks for providing me the opportunity to present at ASME Turbo in Germany and the good beer we had together. I hope we could enjoy many 
more in the future.

To the guys and girl in the post-graduate office: Eugene Joubert, Johan Basson, Johan Kotze, Kenny Allen, Christie Burger, Fine Wilms, Gareth Erfort and later also Olaf Diener. How quickly the time went by as all of our projects started and ended (and some of your's are still in progress). I will always cherish the discussions we had (as we re-engineered the world) together with a good coffee. Thank you also for the input everyone had into my project. I trust that all of you would be prosperous in your future ventures.

The late Prof Detlev G. Kröger: Thank you for the guidance that you provided me as a younger post-graduate student, before my PhD study commenced, and the interest you had in my work and me as a person all along. I suppose I would not have started on this road if it was not for you. Many thanks for the lessons I have learnt working under you and for your character which I had so much respect for.

Chris Meyer: Thank you for not hurting me when I called you Prof for the first time. I know you "despise" the title in public, but enjoy it silently. Thank you for all the inputs and discussions we could have regarding my work. Your contributions were invaluable.

Ockert Augustyn: Thank you for your help to get the simulations going and also with the initial fan tests. Your help laid a good foundation.

Jacques Muiyser: Thank you for your help regarding the wireless measuring equipment. It would have taken much longer to get started without you. May you look back onto your work with satisfaction as it also nears completion.

To the staff in the workshop: Ooms Ferdi and Cobus Zietsman, Graham and Calvin Harmse, Juliun Stanfliet, Maurisha Gallant, Jacobus Samuels, PJ Robyn, Anton van den Berg, Nathi Hlwempu and Braveboy Sotondoshe. Thank you for your interest and help as well as the many chats and laughs we had in the workshop. Specifically to oom Ferdi, Graham and Calvin, thank you for the manufacturing of my experimental setup. I appreciated all your inputs and the neat work.

Hans van der Kamp: Thank you for the perfectionism with which you constructed the blades for my experimental fan. It is a true work of art.

Lastly the funders: My gratitude goes towards the National Research Foundation (NRF) of South Africa and Paul Gauché of the Solar Thermal Energy Research Group (STERG)/Helio 100 for their financial support over the extent of my PhD. Paul, also a personal thank you for your interest in my work. 
To my wife, Jeanne, my family and friends 


\section{Contents}

$\begin{array}{ll}\text { Declaration } & \text { i }\end{array}$

$\begin{array}{ll}\text { Abstract } & \text { ii }\end{array}$

Uittreksel iv

$\begin{array}{ll}\text { Contents } & \text { ix }\end{array}$

List of Figures $\quad$ xiii

List of Tables $\quad \mathrm{xx}$

Nomenclature $\quad$ xxi

1 Introduction $\quad 1$

1.1 Background ............................. 1

1.2 Research objectives and approach ............ 3

2 Literature review $\mathbf{5}$

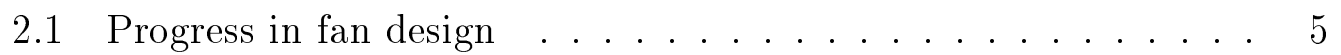

2.1.1 Vortex design and efficiency . . . . . . . . . . 5

2.1.2 Non-radial blade profile stacking . . . . . . . . . . 8

2.1.3 Arbitrary fan modifications . . . . . . . . . . . . . 10

2.1.4 Blade tip clearance effects ................... 10

2.2 Flow field investigations . . . . . . . . . . . . . . 11

2.2 .1 Inflow effects . . . . . . . . . . . . . 11

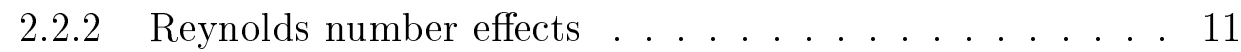

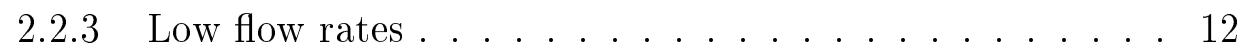

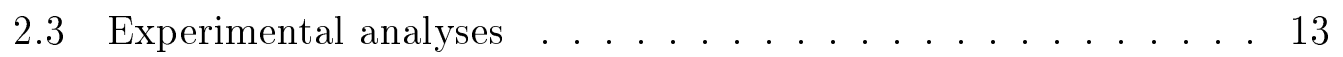

2.4 Numerical analyses . . . . . . . . . . . . . . . 15

2.4.1 Three-dimensional modelling . . . . . . . . . . 15

2.4.2 Actuator-disc modelling . . . . . . . . . . . 16

2.5 Evolution of the B2a-fan . . . . . . . . . . . . . 17

2.6 Concluding remarks . . . . . . . . . . . . . 17

$\begin{array}{lll}3 & \text { Experimental procedures } & 20\end{array}$ 
3.1 Fan performance testing .................. 21

3.1.1 British Standard 848, type A test facility . . . . . . . 21

3.1.2 Facility instruments . . . . . . . . . . . . . . 22

3.1.3 Measurement procedure . . . . . . . . . . . 23

3.2 Blade surface pressure measurement . . . . . . . . . . . . 24

3.2.1 Experimental apparatus . . . . . . . . . . . 24

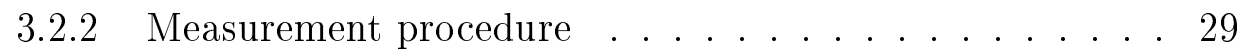

3.2 .3 Data processing ................ 30

4 Periodic three-dimensional numerical model (P3DM) and technique $\quad 32$

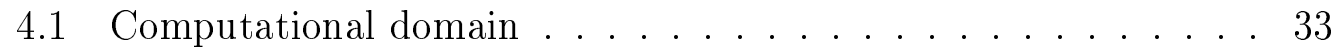

4.1.1 Rotor sub-domain construction . . . . . . . . . . 34

4.1.2 Inlet and Outlet sub-domain construction . . . . . . . 35

4.1.3 Domain assembly and boundary conditions . . . . . . . . 37

4.2 Turbulence modelling . . . . . . . . . . . . . . . . . . . 39

4.3 Solver settings . . . . . . . . . . . . . . . . . . . . 40

5 Periodic three-dimensional numerical model (P3DM) verification

5.1 Fan characteristic verification .............. 43

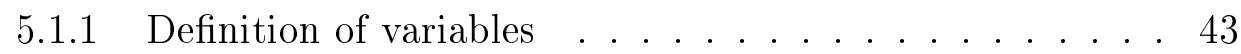

5.1 .2 Comparison of results . . . . . . . . . . . . . . . . . 44

5.2 Blade surface pressure verification . . . . . . . . . . . . . . 46

5.2 .1 Definition of variables . . . . . . . . . . 46

5.2 .2 Comparison of results . . . . . . . . . . . . . . . . 47

5.3 Concluding remarks . . . . . . . . . . . . . 53

5.3 .1 Fan characteristic comparison ................ 53

5.3 .2 BSPM comparison ............... 55

6 Spatial flow field characteristics $\quad 56$

6.1 Definition of variables . . . . . . . . . . . . . . 57

6.2 Time-averaged spanwise flow distributions . . . . . . . . . . 57

6.3 Time-dependent spanwise flow distributions . . . . . . . . . . 64

6.4 Global flow structure . . . . . . . . . . . . . . . . 65

6.5 Cascade flow structure ................... 79

6.5.1 Three-dimensional relative flow . . . . . . . . . . 79

6.5.2 Two-dimensional relative flow . . . . . . . . . . 87

6.6 Concluding remarks . . . . . . . . . . . . 87

$\begin{array}{lll}7 & \text { Blade force characteristics } & 93\end{array}$

7.1 Definition of variables . . . . . . . . . . . . . . . . 93

7.2 Time-averaged blade forces . . . . . . . . . . . . . . . . 96

7.2.1 Spanwise distributions . . . . . . . . . . . . . . 97

7.2 .2 Two-dimensional comparisons . . . . . . . . . . 99 
7.3 Time-dependent blade forces . . . . . . . . . . . . . . 106

7.4 Concluding remarks . . . . . . . . . . . . 107

8 Euler power analysis $\quad \mathbf{1 1 1}$

8.1 Definition of variables . . . . . . . . . . . . 111

8.2 Mean streamline calculation . . . . . . . . . . . . . . . . . . . . . . . . . . . . . . . .

8.3 Results . . . . . . . . . . . . . . . . 118

8.4 Concluding remarks . . . . . . . . . . . . . . 122

9 Reverse engineered empirical actuator disc model (REEADM)126

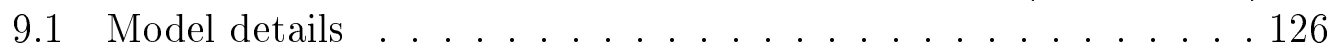

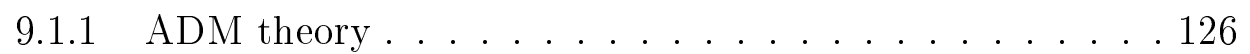

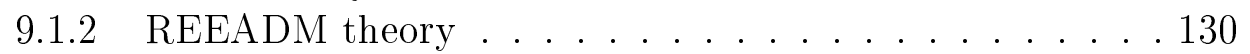

9.1.3 Computational domain . . . . . . . . . . . . 134

9.2 Results . . . . . . . . . . . . . . . . 134

9.3 Concluding remarks . . . . . . . . . . . . . 135

10 Conclusion $\quad 139$

10.1 Contributions . . . . . . . . . . . . . . . . . . . . . . . 139

10.2 Recommended future research . . . . . . . . . . . . 142

$\begin{array}{ll}\text { List of References } & 144\end{array}$

$\begin{array}{ll}\text { Appendices } & 154\end{array}$

A B2a-fan Specifications $\mathbf{1 5 5}$

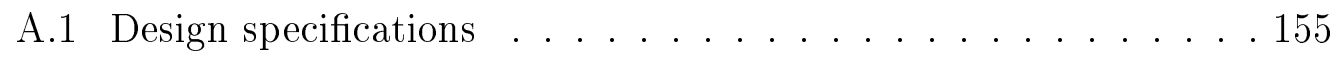

A.2 Aerofoil . . . . . . . . . . . . . . . 156

A.3 Blade shape . . . . . . . . . . . . . . . . 157

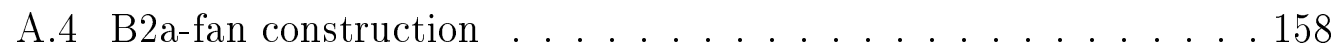

A.4.1 Fan blade construction . . . . . . . . . . . 158

A.4.2 Hub construction . . . . . . . . . . . . . . 160

A.4.3 Nose fairing construction . . . . . . . . . . . 161

$\begin{array}{ll}\text { B Summarised literature } & 162\end{array}$

$\begin{array}{lr}\text { C BSPM error and repeatability analyses } & 188\end{array}$

C.1 Error analyses . . . . . . . . . . . . . . . . . 188

C.2 Repeatability analyses .................. 189

$\begin{array}{ll}\text { D Experimental data analysis procedures } & 191\end{array}$

D.1 Fan characteristic measurement analysis . . . . . . . . . . . 191

D.2 BSPM analysis . . . . . . . . . . . . . . . . 194

D.2.1 Derivation for centrifugal pressure distribution . . . . . 195

D.2.2 Measurement corrections for blade surface pressure . . 197

D.2.3 Measured value interpolation . . . . . . . . . 199 
E P3DM sensitivity analyses 201

E.1 Rotor sub-domain grid sensitivity . . . . . . . . . . . . . 202

E.2 Boundary proximity . . . . . . . . . . . . . . 202

E.3 Computational domain grid sensitivity . . . . . . . . . . . . 203

E.4 Time-step size . . . . . . . . . . . . . . . . . . . . . 204

E.5 Simulation convergence ................. 205

F Turbulence modelling 208

F.1 Fundamental flow equations . . . . . . . . . . . . . . . . 208

F.2 Realizable $k-\varepsilon$ turbulence model . . . . . . . . . . . . . 210

G Data sampling techniques $\quad 212$

G.1 Time-averaging . . . . . . . . . . . . . . . . . . . . . . . 212

G.2 Fan performance characteristic data . . . . . . . . . . . 213

G.3 Blade pressure data . . . . . . . . . . . . . . . 214

G.4 Lift, drag and radial force data . . . . . . . . . . 215 


\section{List of Figures}

1.1 A large forced draft ACHE utilizing axial fans of approximately $10 \mathrm{~m}$ in diameter each (adapted from Louw (2011)). . . . . . . . 2

1.2 The model of the B2a-fan used in the present investigation . . . . . 3

2.1 Two-dimensional flow lines through an axial flow fan for free and controlled vortex designs ............... . . 7

2.2 Definition of various blade sweep directions (adapted from Ramakrishna and Govardhan (2009)) . . . . . . . . . . . . 8

2.3 Different shaping of the manufactured B2- and B2a-fan blades . . . 18

3.1 Stellenbosch University British standard (BS) 848, type A fan test facility (adapted from Kröger (2004)) . . . . . . . . . . . . 22

3.2 Line chart of the instrumental layout for the fan test facility . . . . 23

3.3 (a) An illustration and (b) photograph of the blade surface pressure measurement (BSPM) apparatus . . . . . . . . . . 25

3.4 (a) The suction side BSPM fan blade as well as two pressure taps and (b) the brass tubes fixed to the blade inner cavity . . . . . 26

3.5 A sectional view a) and isometric view (b) of the BSPM aluminium capsule housing seven (c) Freescale MPX 2010 pressure transducers 27

3.6 (a) LORD MictroStrain V-link and (b) LORD MictroStrain WSDABase-101........................ 28

3.7 Line schematic of the blade surface pressure measurement communication . . . . . . . . . . . . . . . . 29

4.1 Computational domain of the periodic three-dimensional model

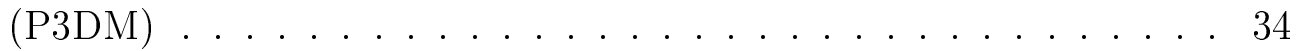

4.2 The Rotor sub-domain shape and grid structure . . . . . . . . . 35

4.3 The (a) Inlet and (b) Outlet sub-domains showing the domain shape and grid structure . . . . . . . . . . . . 36

4.4 (a) A two-dimensional depiction of the method used by Fluent to create an interpolated interface between two non-conformal grid interfaces for the transfer of flow variables and (b) the linkage between the Inlet-Rotor and Rotor-Outlet sub-domain interfaces . . . . . . . 37

4.5 Periodic boundary pairs in the computational domain (paired by

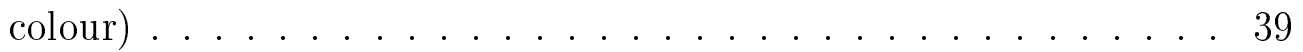


4.6 Fan static pressure and static efficiency obtained by four different methods of turbulence modelling, for steady state (SS) and timedependent (TD) analyses . . . . . . . . . . . . . . 41

5.1 The B2a-fan performance characteristic presented by the (a) fan static pressure coefficient $\left(\Psi_{F s}\right)$ and (b) fan static efficiency $\left(\eta_{F s}\right)$ as functions of flow coefficient $(\phi) \ldots \ldots . \ldots . \ldots 45$

5.2 Fan blade indicating the locations of the pressure taps at the five dimensionless spans $\left(s_{b}\right) \ldots \ldots \ldots . \ldots . \ldots 47$

5.3 Time-averaged blade surface pressure coefficients at a dimensionless blade span of $s_{b}=0.9$ for flow rates of $\phi=$ (a) 0.042 , (b) 0.074 , (c) 0.105, (d) 0.137 , (e) 0.168 , (f) $0.200 \ldots \ldots \ldots$. . . . . . 48

5.4 Time-averaged blade surface pressure coefficients at a dimensionless blade span of $s_{b}=0.7$ for flow rates of $\phi=$ (a) 0.042 , (b) 0.074 , (c) 0.105 , (d) 0.137 , (e) 0.168 , (f) $0.200 \ldots \ldots \ldots \ldots$

5.5 Time-averaged blade surface pressure coefficients at a dimensionless blade span of $s_{b}=0.5$ for flow rates of $\phi=$ (a) 0.042, (b) 0.074, (c) 0.105, (d) 0.137 , (e) 0.168 , (f) $0.200 \ldots \ldots \ldots$

5.6 Time-averaged blade surface pressure coefficients at a dimensionless blade span of $s_{b}=0.3$ for flow rates of $\phi=$ (a) 0.042, (b) 0.074, (c) 0.105 , (d) 0.137 , (e) 0.168 , (f) $0.200 \ldots \ldots \ldots$. . . . . . .

5.7 Time-averaged blade surface pressure coefficients at a dimensionless blade span of $s_{b}=0.1$ for flow rates of $\phi=$ (a) 0.042 , (b) 0.074 , (c)

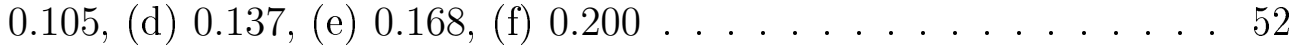

5.8 Scaled schematic of force vectors at blade sections of (a) $s_{b}=0.5$ and (b) $s_{b}=0.1$ in the $z \theta$-plane for a flow coefficient of $\phi_{D}=0.168$

5.9 Time-averaged $y^{+}$-values at different dimensionless blade spans of $s_{b}=$ (a) 0.9 , (b) 0.7 , (c) 0.5 , (d) 0.3 and (e) $0.1 \ldots \ldots . \ldots 54$

6.1 Depiction of (a) the three-dimensional velocity vectors and angles up- and downstream of the fan rotor at a specific radius, (b) the up- and downstream locations where flow variables are extracted from and (c) the velocity vectors and flow angles in the $z \theta$-plane for a single cylindrical blade section . . . . . . . . . . . 58

6.2 Circumferentially and time-averaged spanwise distributions of (a) $c_{z 1} /\left|u_{c}\right|$, (b) $c_{z 2} /\left|u_{c}\right|$, (c) $c_{\theta 1} /\left|u_{c}\right|$, (d) $c_{\theta 2} /\left|u_{c}\right|$, (e) $c_{r 1} /\left|u_{c}\right|$ and (f) $c_{r 2} /\left|u_{c}\right|$ for the range of flow rates investigated . . . . . . . . . 60

6.3 Circumferentially and time-averaged spanwise distributions of (a) $\alpha_{1}$, (b) $\alpha_{2}$, (c) $\theta_{r 1}$ and (d) $\theta_{r 2}$ for the range of flow rates investigated 61

6.4 Circumferentially and time-averaged spanwise distributions of (a) $w_{\theta 1} /\left|u_{c}\right|$, (b) $w_{\theta 2} /\left|u_{c}\right|$, (c) $\beta_{1}$ and (d) $\beta_{2}$ for the range of flow rates

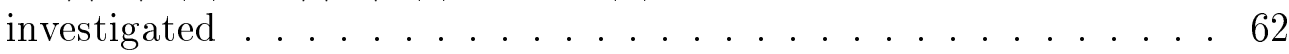

6.5 Circumferentially and time-averaged spanwise distributions of (a) $p_{s 1} / 0.5 \rho u_{c}^{2}$, (b) $p_{s 2} / 0.5 \rho u_{c}^{2}$, (c) $p_{t o 1} / 0.5 \rho u_{c}^{2}$ and (d) $p_{t o 2} / 0.5 \rho u_{c}^{2}$ for the range of flow rates investigated ........... 63 
6.6 Time-dependent spanwise axial velocity $\left(c_{z} /\left|u_{c}\right|\right)$ profiles for (a) $\phi=0.105$, upstream, (b) $\phi=0.105$, downstream, (c) $\phi=0.074$, upstream, (d) $\phi=0.074$, downstream, (e) $\phi=0.042$, upstream and (f) $\phi=0.042$, downstream of the fan . . . . . . . . . 66

6.7 Time-dependent spanwise circumferential velocity $\left(c_{\theta} /\left|u_{c}\right|\right)$ profiles for (a) $\phi=0.105$, upstream, (b) $\phi=0.105$, downstream, (c) $\phi=0.074$, upstream, (d) $\phi=0.074$, downstream, (e) $\phi=0.042$, upstream and (f) $\phi=0.042$, downstream of the fan . . . . . . . 67

6.8 Time-dependent spanwise radial velocity $\left(c_{r} /\left|u_{c}\right|\right)$ profiles for (a) $\phi=0.105$, upstream, (b) $\phi=0.105$, downstream, (c) $\phi=0.074$, upstream, (d) $\phi=0.074$, downstream, (e) $\phi=0.042$, upstream and (f) $\phi=0.042$, downstream of the fan . . . . . . . . . 68

6.9 Time-dependent spanwise absolute angle $(\alpha)$ profiles for (a) $\phi=$ 0.105, upstream, (b) $\phi=0.105$, downstream, (c) $\phi=0.074$, upstream, (d) $\phi=0.074$, downstream, (e) $\phi=0.042$, upstream and (f) $\phi=0.042$, downstream of the fan . . . . . . . . . .

6.10 Time-dependent spanwise radial flow angle $\left(\theta_{r}\right)$ profiles for $(\mathrm{a}) \phi=$ 0.105, upstream, (b) $\phi=0.105$, downstream, (c) $\phi=0.074$, upstream, (d) $\phi=0.074$, downstream, (e) $\phi=0.042$, upstream and (f) $\phi=0.042$, downstream of the fan . . . . . . . . . 70

6.11 Time-dependent spanwise relative circumferential velocity $\left(w_{\theta} /\left|u_{c}\right|\right)$ profiles for (a) $\phi=0.105$, upstream, (b) $\phi=0.105$, downstream, (c) $\phi=0.074$, upstream, (d) $\phi=0.074$, downstream, (e) $\phi=0.042$, upstream and (f) $\phi=0.042$, downstream of the fan . . . . . . 71

6.12 Time-dependent spanwise relative angle $(\beta)$ profiles for (a) $\phi=$ 0.105, upstream, (b) $\phi=0.105$, downstream, (c) $\phi=0.074$, upstream, (d) $\phi=0.074$, downstream, (e) $\phi=0.042$, upstream and (f) $\phi=0.042$, downstream of the fan . . . . . . . . . 72

6.13 Time-dependent spanwise dimensionless static pressure $\left(p_{s} / 0.5 \rho u_{c}^{2}\right)$ profiles for (a) $\phi=0.105$, upstream, (b) $\phi=0.105$, downstream, (c) $\phi=0.074$, upstream, (d) $\phi=0.074$, downstream, (e) $\phi=0.042$, upstream and (f) $\phi=0.042$, downstream of the fan $\ldots . . .73$

6.14 Time-dependent spanwise dimensionless total pressure $\left(p_{t o} / 0.5 \rho u_{c}^{2}\right)$ profiles for (a) $\phi=0.105$, upstream, (b) $\phi=0.105$, downstream, (c) $\phi=0.074$, upstream, (d) $\phi=0.074$, downstream, (e) $\phi=0.042$, upstream and (f) $\phi=0.042$, downstream of the fan . . . . . . . 74

6.15 Meridional surface streamlines for (a) $\phi=0.200$, (b) $\phi_{D}=0.168$

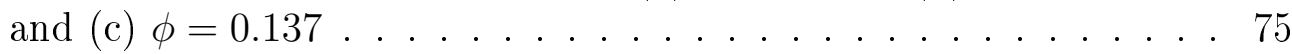

6.16 Meridional surface streamlines for $\phi=0.105$ at time instances of (a) $n_{F r}=n$, (b) $n_{F r}=n+0.475$, (c) $n_{F r}=n+0.950$ and (d) $n_{F r}=n+1.425 \ldots \ldots \ldots \ldots \ldots \ldots \ldots$. . . . . . . . . . . .

6.17 Meridional surface streamlines for $\phi=0.074$ at time instances of (a) $n_{F r}=n$, (b) $n_{F r}=n+0.700$, (c) $n_{F r}=n+1.400$ and (d)

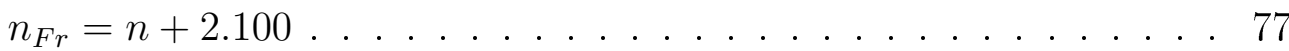


6.18 Meridional surface streamlines for $\phi=0.042$ at time instances of (a) $n_{F r}=n$, (b) $n_{F r}=n+0.825$, (c) $n_{F r}=n+1.650$ and (d) $n_{F r}=n+2.475 \ldots \ldots \ldots \ldots \ldots \ldots$. . . . . . . . . . . . .

6.19 Relative streamlines released $2 \mathrm{~mm}$ from the fan hub surface (left hand side) and relative streamlines confined to a surface $2 \mathrm{~mm}$ from the blade (right hand side) for flow coefficients of (a) $\phi=0.200$, (b) $\phi_{D}=0.168$ and (c) $\phi=0.137$ (SS: suction side, PS: pressure side) . 80

6.20 Time frames of relative streamlines released $2 \mathrm{~mm}$ from the fan hub surface for a flow coefficient of $\phi=0.105$ at time instances of (a) $n_{F r}=n$, (b) $n_{F r}=n+0.325$ (c) $n_{F r}=n+0.650$, (d) $n_{F r}=n+0.975$, (e) $n_{F r}=n+1.300$ and (f) $n_{F r}=n+1.625 \ldots 81$

6.21 Time frames of relative streamlines on a surface $2 \mathrm{~mm}$ from the fan blade for a flow coefficient of $\phi=0.105$ at time instances of (a) $n_{F r}=n$, (b) $n_{F r}=n+0.325$ (c) $n_{F r}=n+0.650$, (d) $n_{F r}=$ $n+0.975$, (e) $n_{F r}=n+1.300$ and (f) $n_{F r}=n+1.625 \ldots .$.

6.22 Time frames of relative streamlines released $2 \mathrm{~mm}$ from the fan hub surface for a flow coefficient of $\phi=0.074$ at time instances of (a) $n_{F r}=n$, (b) $n_{F r}=n+0.475$ (c) $n_{F r}=n+0.950$, (d) $n_{F r}=n+1.425$, (e) $n_{F r}=n+1.900$ and (f) $n_{F r}=n+2.375 \ldots$.

6.23 Time frames of relative streamlines on a surface $2 \mathrm{~mm}$ from the fan blade for a flow coefficient of $\phi=0.074$ at time instances of (a) $n_{F r}=n$, (b) $n_{F r}=n+0.475$ (c) $n_{F r}=n+0.950$, (d) $n_{F r}=$ $n+1.425$, (e) $n_{F r}=n+1.900$ and (f) $n_{F r}=n+2.375 \ldots .$.

6.24 Time frames of relative streamlines released $2 \mathrm{~mm}$ from the fan hub surface for a flow coefficient of $\phi=0.042$ at time instances of (a) $n_{F r}=n$, (b) $n_{F r}=n+0.550$ (c) $n_{F r}=n+1.100$, (d) $n_{F r}=n+1.650$, (e) $n_{F r}=n+2.200$ and (f) $n_{F r}=n+2.750 \ldots$.

6.25 Time frames of relative streamlines on a surface $2 \mathrm{~mm}$ from the fan blade for a flow coefficient of $\phi=0.042$ at time instances of (a) $n_{F r}=n$, (b) $n_{F r}=n+0.550$ (c) $n_{F r}=n+1.100$, (d) $n_{F r}=$ $n+1.650$, (e) $n_{F r}=n+2.200$ and (f) $n_{F r}=n+2.750 \ldots \ldots$

6.26 Relative surface streamlines and contours of relative velocity at different blade spans for flow coefficients of (a) $\phi=0.200$, (b) $\phi_{D}=$ 0.168 and (c) $\phi=0.137 \ldots \ldots \ldots \ldots \ldots$

6.27 Relative surface streamlines and contours of relative velocity at different blade spans for $\phi=0.074$ at time instances of (a) $n_{F r}=n$ , (b) $n_{F r}=n+0.475$, (c) $n_{F r}=n+0.950$ and (d) $n_{F r}=n+1.425$

6.28 Relative surface streamlines and contours of relative velocity at different blade spans for $\phi=0.074$ at time instances of (a) $n_{F r}=n$ , (b) $n_{F r}=n+0.700$, (c) $n_{F r}=n+1.400$ and (d) $n_{F r}=n+2.100$

6.29 Relative surface streamlines and contours of relative velocity at different blade spans for $\phi=0.042$ at time instances of (a) $n_{F r}=n$ , (b) $n_{F r}=n+0.825$, (c) $n_{F r}=n+1.650$ and (d) $n_{F r}=n+2.475$ 
7.1 Depiction of (a) a single blade section through the fan blade, (b) the velocity components and (c) the blade forces particular to this blade section

7.2 Circumferentially and time-averaged spanwise distributions of (a) $\alpha_{a t t}$, (b) $C_{L}$, (c) $C_{D}$ and (d) $C_{R}$ for the range of flow rates investigated 96

7.3 Blade lift $\left(C_{L}\right)$ and drag $\left(C_{D}\right)$ coefficients as a function of flow coefficient $(\phi)$ for sections of $0.9<s_{b}<1 \ldots \ldots$. . . . . 97

7.4 Blade lift $\left(C_{L}\right)$ and drag $\left(C_{D}\right)$ coefficients as a function of flow coefficient $(\phi)$ for spans of $0.1 \leq s_{b} \leq 0.9 \ldots \ldots \ldots 9$

7.5 Blade lift $\left(C_{L}\right)$ and drag $\left(C_{D}\right)$ coefficients as a function of flow coefficient $(\phi)$ for sections of $0<s_{b}<0.1 \ldots \ldots . \ldots 98$

7.6 Blade sectional lift coefficients $\left(C_{L}\right)$ as a function of angle of attack $\left(\alpha_{a t t}\right)$ at spans of (a) $0.9<s_{b}<1$ and (b) $0.6 \leq s_{b} \leq 0.9$ compared to the NASA LS 0413 aerofoil for two different Reynolds numbers obtained using XFOIL . . . . . . . . . . . . . . . 100

7.6 Blade sectional lift coefficients $\left(C_{L}\right)$ as a function of angle of attack $\left(\alpha_{a t t}\right)$ at spans of (c) $0.1 \leq s_{b} \leq 0.5$ and (d) $0<s_{b}<1$ compared to the NASA LS 0413 aerofoil for two different Reynolds numbers obtained using XFOIL . . . . . . . . . . . . . . . . . . . 101

7.7 Blade drag coefficients $\left(C_{D}\right)$ as a function of angle of attack $\left(\alpha_{a t t}\right)$ at spans of (a) $0.9<s_{b}<1$ and (b) $0.6 \leq s_{b} \leq 0.9$ compared to the NASA LS 0413 aerofoil for two different Reynolds numbers obtained using XFOIL . . . . . . . . . . . . . . . . . 102

7.7 Blade drag coefficients $\left(C_{D}\right)$ as a function of angle of attack $\left(\alpha_{a t t}\right)$ at spans of (c) $0.1 \leq s_{b} \leq 0.5$ and (d) $0<s_{b}<0.1$ compared to the NASA LS 0413 aerofoil for two different Reynolds numbers using XFOIL . . . . . . . . . . . . . . . . . . . 103

7.8 Blade radial force coefficients $\left(C_{R}\right)$ as a function of radial flow angle $\left(\theta_{r}\right)$ at spans of (a) $0.9<s_{b}<1$ and (b) $0.6 \leq s_{b} \leq 0.9 \ldots . . .104$

7.8 Blade radial force coefficients $\left(C_{R}\right)$ as a function of radial flow angle $\left(\theta_{r}\right)$ at spans of $(\mathrm{c}) 0.1 \leq s_{b} \leq 0.5$ and $(\mathrm{d}) 0<s_{b}<0.1 \ldots 105$

7.9 Time-dependent spanwise profiles of angle of attack $\left(\alpha_{\text {att }},{ }^{\circ}\right)$ and lift coefficient $\left(C_{L}\right)$ for flow coefficients of (a and b) $\phi=0.105$, (c and

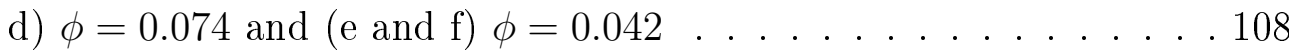

7.10 Time-dependent spanwise profiles of drag $\left(C_{D}\right)$ and radial force coefficients $\left(C_{R}\right)$ for flow coefficients of (a and b) $\phi=0.105$, (c and

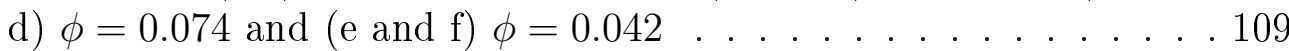

8.1 Single stream tube of fluid through a turbo-machine . . . . . . . . . 112

8.2 Sectional view of the B2a-fan depicting some terminology and the mean streamlines through the fan for flow coefficients of $\phi=$ (a)

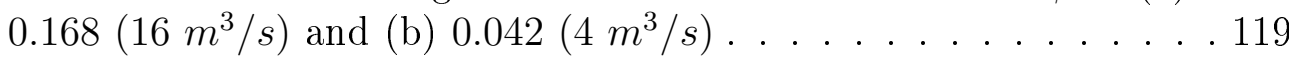

8.3 Depiction of the circumferentially and time-averaged streamlines through the B2a-fan for flow coefficients of $\phi=$ (a) 0.200, (b) 0.168, (c) 0.137, (d) 0.105, (e) 0.074 and (f) $0.042 \ldots \ldots . . . . . . .120$ 
8.4 Comparison of dimensionless fan power $\left(\Gamma_{F}\right)$ and static pressure difference $\left(\Psi_{F s}\right)$ obtained through experimental analyses, computational fluid dynamics (CFD) and Euler power calculations. . . . . . . . 122

8.5 Comparison of dimensionless effective fan work per unit mass $\left(\gamma_{F, \text { eff }}\right)$ obtained through experimental analyses, CFD and Euler power calculations. . . . . . . . . . . . . . . . . . 123

8.6 Comparison of dimensionless true fan work per unit mass $\left(\gamma_{F, \text { true }}\right)$ obtained through experimental analyses, CFD and Euler power calculations. . . . . . . . . . . . . . . . . 124

9.1 Cylindrical control volume (cell) in space . . . . . . . . . . . . 127

9.2 Comparative depiction of a three-dimensional fan model and an actuator-disc model . . . . . . . . . . . . . . . . 128

9.3 The two-dimensional force components of a two-dimensional blade section . . . . . . . . . . . . . . . . . 129

9.4 The three-dimensional force components of a two-dimensional blade

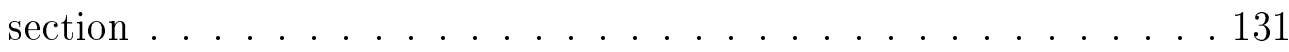

9.5 Fifth order polynomial surface fits through the (b) lift $\left(C_{L}\right)$, (b) drag $\left(C_{D}\right)$ and (c) radial force $\left(C_{R}\right)$ coefficients as a function of the applicable flow angle $\left(\alpha_{a t t}\right.$ or $\left.\theta_{r}\right)$ and blade span $\left(s_{b}\right) \ldots \ldots . \ldots 132$

9.6 Computational domain of the reverse engineered empirical actuatordisc model (REEADM) . . . . . . . . . . . . . . . 134

9.7 Comparison of the B2a-fan performance characteristics determined through different simplified numerical fan models, P3DM and the average experimental results for the (a) fan static pressure coefficient $\left(\Psi_{F s}\right)$ and (b) fan static efficiency $\left(\eta_{F s}\right)$ as functions of flow coefficient $(\phi) \ldots \ldots \ldots \ldots \ldots \ldots \ldots$

A.1 Photo and schematic of the B2a-fan . . . . . . . . . . . 155

A.2 NASA LS 0413 and 0409 aerofoil profiles (McGhee and Beasley, 1976) 156

A.3 Lift $\left(C_{L}\right)$ and Drag $\left(C_{D}\right)$ coefficients of the NASA LS 0413 aerofoil (McGhee and Beasley, 1976) profile versus angle of attack $(\alpha)$ as determined using XFOIL . . . . . . . . . . . . . . . . 157

A.4 B2a-fan spanwise chord and blade angle distribution (Louw et al., 2012) . . . . . . . . . . . . . . . . . . . . . . . . . . . . . . . . . . . . . . . .

A.5 B2a-fan blade construction . . . . . . . . . . . . . . . . . . . . . . . . . . . . . . . . . . . . . .

A.6 B2a-fan hub construction . . . . . . . . . . . . . . 160

A.7 B2a-fan nose fairing construction . . . . . . . . . . 161

C.1 Pitot tube test apparatus . . . . . . . . . . . . 188

D.1 (a) Details of the tube layout inside the fan blades and (b) a control volume of air constrained in a tube . . . . . . . . . . . 195

D.2 Schematics of the pressure lines for (a) transducer membrane zero offset measurement and (b) blade surface pressure measurement . . 197 
D.3 Measured blade surface static pressures of the pressure side at a span of $s_{b}=0.5$ (a) as a function of dimensionless chord for a range of flow rates and (b) as a function of flow rate for each pressure tap. 200

E.1 Computational domain of the numerical fan model . . . . . . . . . 201

E.2 Convergence of boundary proximity for Inlet and Outlet sub-domains $\left(\phi_{D}=0.168\left(\dot{V}_{D}=16 \mathrm{~m}^{3} / \mathrm{s}\right)\right) \ldots \ldots \ldots 2 . \ldots \ldots 3$

E.3 Simulation convergence monitors of fan static pressure and static efficiency for (a) the design flow rate, $\phi_{D}=0.168\left(\dot{V}_{D}=16 \mathrm{~m}^{3} / \mathrm{s}\right)$ and the low flow rates of (b) $\phi=0.105\left(\dot{V}=10 \mathrm{~m}^{3} / \mathrm{s}\right),(\mathrm{c}) \phi=0.074$ $\left(\dot{V}=7 \mathrm{~m}^{3} / \mathrm{s}\right)$ and $(\mathrm{d}) \phi=0.042\left(\dot{V}=4 \mathrm{~m}^{3} / \mathrm{s}\right) \ldots . . . . . .207$

G.1 Python routine for sampling and calculating fan performance characteristics . . . . . . . . . . . . . . . . 213

G.2 Python routine for sampling and calculating blade surface pressure coefficients . . . . . . . . . . . . . . . . . . 214

G.3 Python routine for sampling and calculating blade sectional lift, drag and radial force coefficients . . . . . . . . . . . . . . . 215 


\section{List of Tables}

3.1 Fan test facility and stagger angle setting instrument errors . . . . 23

4.1 Turbulence models and predicted fan static pressure for the design $\left(\dot{V}=16 \mathrm{~m}^{3} / \mathrm{s}\right)$ and the lowest $\left(\dot{V}=4 \mathrm{~m}^{3} / \mathrm{s}\right)$ flow rates . . . . . 40

4.2 Numerical solver settings . . . . . . . . . . . . . . . . . . 41

5.1 Pearson correlation $\left(R_{P}\right)$ and root mean square error (RMSE) values presenting the quality of relation between the experimental data sets and average experimental curve fits for the B2a-fan performance characteristics . . . . . . . . . . . . . . . . 44

5.2 Pearson correlation $\left(R_{P}\right)$ and RMSE values presenting the quality of relation between the numerical and average experimental data obtained for the B2a-fan performance characteristics . . . . . . 46

6.1 Cycle lengths of the flow structure in the vicinity of the fan at low flow rates . . . . . . . . . . . . . . 6 65

9.1 Fifth order surface polynomial constants for the B2a-fan blade sectional lift, drag and radial force coefficients . . . . . . . . . . 133

9.2 Comparison between P3DM and REEADM computational domain sizes and required computational resources . . . . . . . . . 135

B.1 Experimental tests on axial flow fans and comparable axial flow turbo-machinery . . . . . . . . . . . . . 163

B.2 Rotor specifications of axial flow fans and comparable axial flow turbo-machinery in literature ... . . . . . . . . . . 173

B.3 Numerical analyses on axial flow fans and comparable axial flow turbo-machinery . . . . . . . . . . . . . 178

B.4 Rotor specifications of axial flow fans and comparable axial flow turbo-machinery in literature for numerical analyses . . . . . . . 184

E.1 Rotor sub-domain grid sensitivity . . . . . . . . . . . . . 202

E.2 Computational domain grid sensitivity . . . . . . . . . . . . . 204

E.3 Computational domain time-step independence analyses . . . . . . 205

E.4 Time-steps in a single period of the converged numerical simulations at low flow rates . . . . . . . . . . . . . . 205 


\section{Nomenclature}

\begin{tabular}{|c|c|}
\hline \multicolumn{2}{|c|}{ Variables } \\
\hline$A$ & $\ldots \ldots \ldots\left[\mathrm{m}^{2}\right]$ \\
\hline$a$ & Accelaration, Constant \\
\hline$C$ & Coefficient, Constant \\
\hline$c$ & Absolute velocity \\
\hline$c_{t}$ & Tip clearance \\
\hline$c h$ & $\ldots[\mathrm{m}]$ \\
\hline$D$ & $\ldots \ldots[\mathrm{N}]$ \\
\hline$d$ & $\ldots \ldots \ldots[\mathrm{m}]$ \\
\hline$F$ & $\ldots \ldots \ldots[\mathrm{N}]$ \\
\hline$g$ & Gravitational constant . . . \\
\hline$h$ & $\ldots \ldots[\mathrm{J} / \mathrm{kg}]$ \\
\hline$k$ & Turbulence kinetic energy . \\
\hline$L$ & $\ldots \ldots \ldots[\mathrm{N}]$ \\
\hline$\dot{m}$ & $\ldots \ldots \ldots[\mathrm{kg} / \mathrm{s}]$ \\
\hline$N$ & $\ldots \ldots \ldots[\mathrm{rev} / \mathrm{mi}$ \\
\hline$n$ & $\ldots \ldots \ldots \ldots[]$ \\
\hline$P$ & $\ldots \ldots \ldots[\mathrm{W}, \mathrm{Pa}]$ \\
\hline$p$ & $\ldots \ldots \ldots[\mathrm{Pa}]$ \\
\hline$R$ & $\ldots \ldots[\mathrm{N}]$ \\
\hline$R_{P}$ & Pearson correlation factor . . . . . . . . . . . . . [ ] ] \\
\hline$R S D$ & Relative standard deviation . \\
\hline$r$ & $\ldots[\mathrm{m}]$ \\
\hline$S$ & $\ldots \ldots \ldots\left[\mathrm{s}^{-1}\right]$ \\
\hline$s$ & $\ldots \ldots \ldots\left[, \mathrm{s}^{-1}\right]$ \\
\hline$s_{p}$ & Circumferential pitch \\
\hline$T$ & Torque, Temperature \\
\hline$u$ & Velocity, Blade tangential velocity . . \\
\hline$U$ & Mean velocity \\
\hline
\end{tabular}




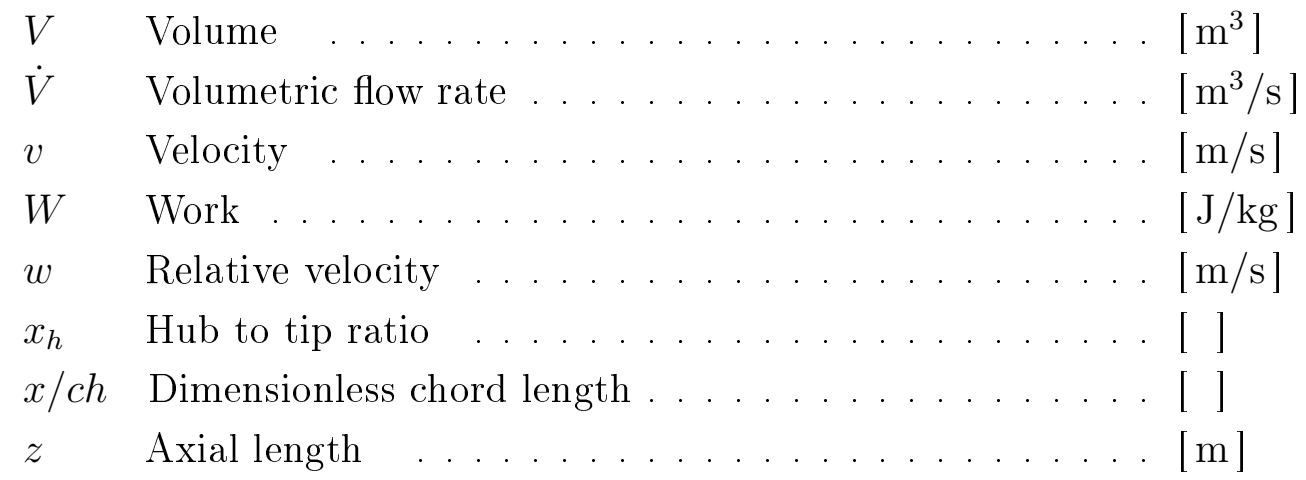

\section{Greek symbols}

\begin{tabular}{|c|c|c|}
\hline$\alpha$ & Absolute velocity angle & {$\left[{ }^{\circ}\right]$} \\
\hline$\beta$ & Relative velocity angle & {$\left[{ }^{\circ}\right]$} \\
\hline$\gamma$ & Dimensionless work & [ ] \\
\hline$\varepsilon$ & Turbulence dissipation rate, Error & {$[\mathrm{J} / \mathrm{kg} \cdot \mathrm{s}]$,} \\
\hline$\eta$ & Efficiency .... & [ ] \\
\hline$\theta$ & Angle & {$\left[{ }^{\circ}\right]$} \\
\hline$\mu$ & Dynamic viscosity & {$[\mathrm{kg} / \mathrm{m} \cdot \mathrm{s}]$} \\
\hline$\xi$ & Stagger angle . . . & {$\left[{ }^{\circ}\right]$} \\
\hline$\rho$ & Density & {$\left[\mathrm{kg} / \mathrm{m}^{3}\right]$} \\
\hline$\sigma$ & Solidity, Standard deviation & [ ] \\
\hline$\phi$ & Flow coefficient & \\
\hline$\Psi_{F s}$ & Fan static pressure coefficient & \\
\hline$\omega$ & Rotational speed & {$[\mathrm{rad} / \mathrm{s}]$} \\
\hline$\Gamma$ & Dimensionless power & \\
\hline
\end{tabular}

\section{Constants}

$g_{\text {Stel }} \quad$ Gravitational constant for Stellenbosch ....... $\left[9.796 \mathrm{~m} / \mathrm{s}^{2}\right]$

R Gas constant of air . . . . . . . . . . [ $287 \mathrm{~J} / \mathrm{kg} \mathrm{K}]$

\section{Dimensionless groups}

Re Reynolds number

\section{Subscripts}

$\begin{array}{ll}a & \text { Air } \\ \text { atm } & \text { Atmosphere }\end{array}$




$\begin{array}{ll}\text { att } & \text { Attack } \\ \text { avg } & \text { Average } \\ b & \text { Blade } \\ \text { body } & \text { Body } \\ c & \text { Casing } \\ \text { ch } & \text { Chord } \\ D & \text { Design, Drag force } \\ d & \text { Dynamic } \\ \text { dim } & \text { Dimensionless } \\ \text { Eul } & \text { Euler } \\ \text { eff } & \text { Effective } \\ \text { err } & \text { Error } \\ F & \text { Fan } \\ \text { Fr } & \text { Fan rotations } \\ F s & \text { Fan static } \\ h & \text { Hub } \\ i & \text { Inlet, Inner, Counter } \\ j & \text { counter } \\ L & \text { Lift force } \\ m & \text { Measure } \\ n & \text { Nozzle, normal } \\ o & \text { Outlet, Outer } \\ \text { orig } & \text { Original } \\ p & \text { Pressure } \\ \text { then } & \text { Plenum } \\ R & \text { Radial force } \\ \text { Res } & \text { Resultant } \\ r & \text { Tolar coordinate } r \\ r e c & \text { Recirculation } \\ s & \text { Static, Stream channel } \\ \text { sect } & \text { Sector } \\ \text { st } & \text { Static tube } \\ t & \text { Turbulence, Tip, Tube } \\ & \end{array}$




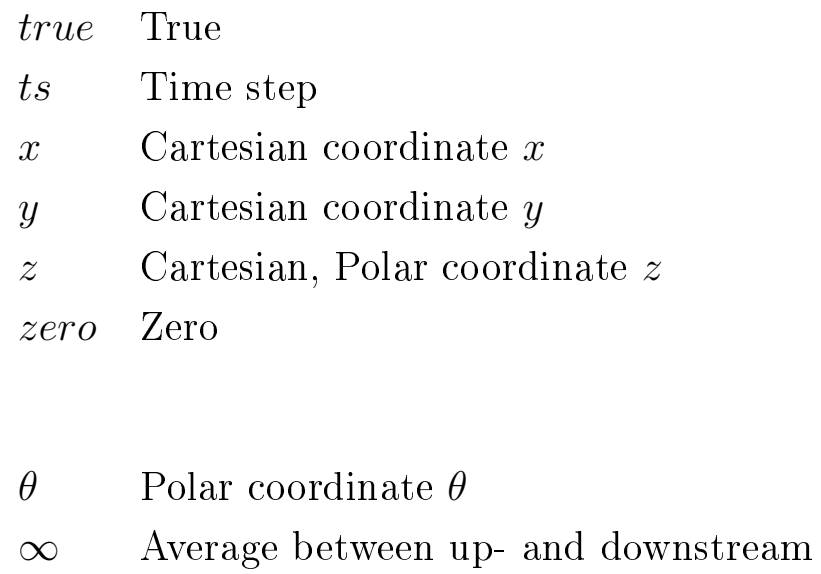

\section{Acronyms}

ACHE air-cooled heat exchanger

ADM actuator-disc model

AFF Axial flow fan

AFC Axial flow compressor

BS British standard

BSPM blade surface pressure measurement

CAD computer-aided design

CFD computational fluid dynamics

CNC computer numerical control

DES Detached eddy simulation

EADM extended actuator-disc model

HAWT horizontal axis wind turbines

hpc high-performance computer

IGES initial graphics exchange specification

IGV inlet guide vane

LDA laser Doppler anemometry

LES Large eddy simulation

OGV outlet guide vane

P3DM periodic three-dimensional model

PIV particle image velocimetry

RAM random access memory

RANS Reynolds-averaged Navier Stokes

REEADM reverse engineered empirical actuator-disc model 
RMSE root mean square error

RSM Reynolds stress model 


\section{Chapter 1}

\section{Introduction}

\subsection{Background}

Large diameter axial flow fans find application in industrial sized air-cooled heat exchangers (ACHEs) as depicted in figure 1.1. These heat exchangers are implemented in the power generation and petro-chemical industries for process cooling (Kröger, 2004). The use of ACHEs has the advantage of being water efficient compared to wet-cooling technologies (such as wet-cooling towers) and they are therefore an attractive alternative for industrial plants in arid regions. This also encourages the use of ACHEs in the renewable power industry (especially solar thermal). Despite the water efficient advantage of ACHEs, a penalty is paid in reduced overall process efficiency, since lower process temperatures of a thermodynamic power cycle can be reached with wet-cooling. This reduction in efficiency creates a need for the improvement of ACHE performance and effectiveness.

In ACHEs, the large axial flow fans (approximately 5 - $10 \mathrm{~m}$ in diameter) force a draft of atmospheric air across a number of heat exchanger bundles, arranged downstream of the fans. In this manner the atmosphere acts as a heat sink, cooling the process fluid flowing through the heat exchanger.

Large ACHEs use an array of axial flow fans ranging from thirty to nearly four hundred in number. Due to its large size, numerical analyses in the form of computational fluid dynamics (CFD) are often used to investigate effects such as wind and inflow conditions on the performance of these plants. In CFD analyses of these systems, fans are modelled using simplified models, since the computational complexity and expense of three-dimensional fan modelling would be significant.

Extensive experimental and numerical research have been conducted on ACHEs. Of this research, Bredell et al. (2006), Van Rooyen and Kröger (2008), Owen and Kröger (2010), Joubert (2010), Louw (2011) and Van der Spuy et al. (2011), through numerical simulation showed that reduced fan performance, especially along the periphery of the ACHE platform, is generally the main contributor to reduced ACHE performance. This reduction in cooling effective- 


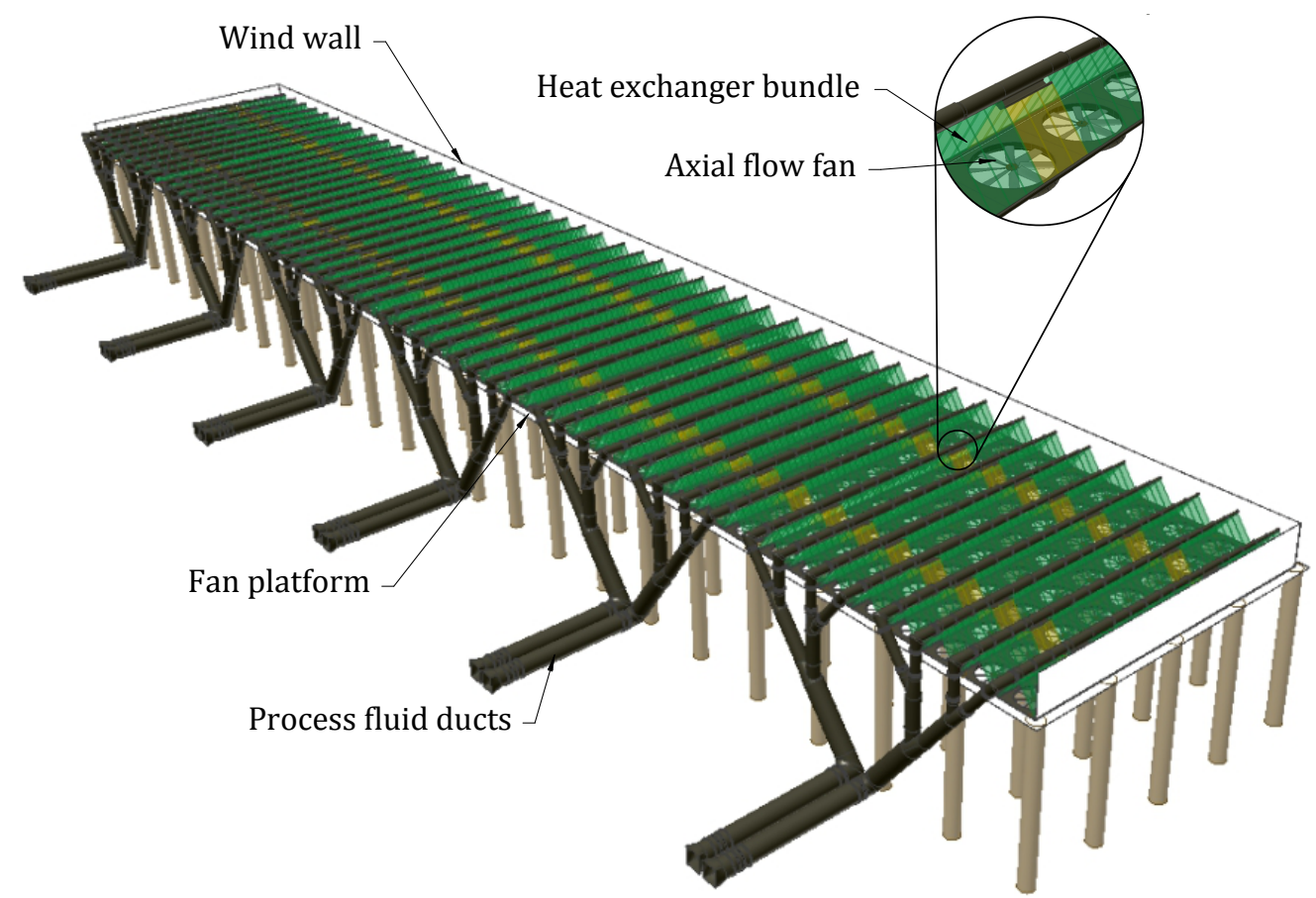

Figure 1.1: A large forced draft ACHE utilizing axial fans of approximately $10 \mathrm{~m}$ in diameter each (adapted from Louw (2011)).

ness leads to reduced plant efficiencies and potentially also unplanned turbine outages in the case of power generation as reported by Goldsschagg (1993). Van der Spuy et al. (2011) further experimentally confirmed the flow phenomena near the ACHE periphery through particle image velocimetry (PIV) measurements. The reduction in peripheral fan performance is mainly due to changes in local inlet pressure as well as distorted air inflow patterns beneath the ACHE platform. This causes some fans to operate away from their design points, resulting in reduced volumetric flow rates through these fans.

Although the influences of distorted inflow patterns and the variance in local pressure beneath the ACHE platform can to a large extent be mitigated by certain structural modifications to ACHEs, certain fans are always exposed to adverse inflow conditions, leading to lower flow rates through these fans. These conditions provide an additional challenge when large ACHE are modelled, since the simplified fan models implemented in these investigations give poor performance predictions at low flow rates as shown in the work of Van der Spuy et al. (2011). Consequently a margin of numerical inaccuracy is incurred in the modelling of ACHEs since the performance of a number of fans in the ACHE system is modelled incorrectly.

Based on this inaccuracy in large ACHE modelling, development of an improved simplified numerical fan model is required. Hence, this study is firstly directed towards understanding the flow field through and in the vicinity of an axial fan rotor, especially at low flow rates. Secondly an attempt towards the development of an improved simplified fan model is made. 


\subsection{Research objectives and approach}

Based on the background given, two main research objectives are identified:

- Investigation of the flow field that develops as a result of fan rotation. The visualization and quantification of certain flow phenomena and variables would provide valuable insight towards improved fan design and simplified numerical fan modelling, especially at low flow rates.

- Development of an improved simplified numerical fan model. Since current simplified numerical fan models give poor predictions of fan performance at low flow rates, it is attempted to design a new simplified empirical model, that would provide improvements over existing models.

An axial flow fan specifically designed for application in ACHEs, termed the B2a-fan (Louw et al., 2012) depicted in figure 1.2, is used as a test subject in the present study. Detailed specifications can be viewed in appendix A. Using this fan, the following research approach is implemented:

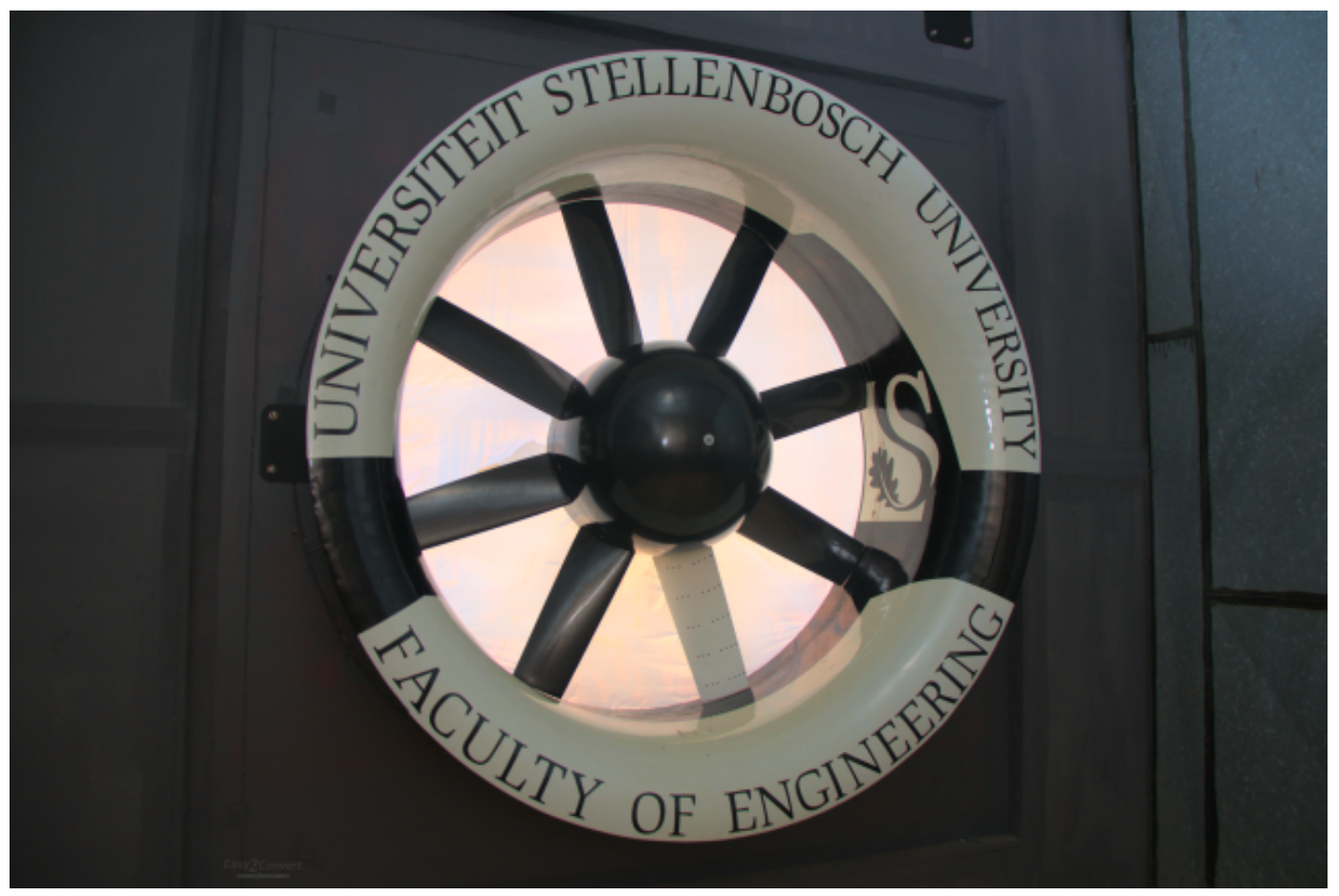

Figure 1.2: The model of the B2a-fan used in the present investigation

- Numerical modelling of the B2a-fan for a range of volumetric flow rates. Numerical simulations provide extensive amounts of data that would be difficult or expensive to measure experimentally. Hence, the three-dimensional flow patterns in close vicinity to the fan are investigated using a periodic model (termed the periodic three-dimensional 
model (P3DM)) of the B2a-fan under conditions which resemble British standard (BS) 848, type A fan testing conditions. The model saves on computational expense through the use of periodic boundaries, since only one of the eight blade passages is modelled.

- Numerical model verification. The validity of the numerical simulations may be questioned, since flow structures like rotating stall cells might occur within the fan rotor, which cannot be captured with a periodic model. The uncertainty introduced through the manner of turbulence modelling and wall conditions would also need to be quantified. The following experimental analyses are therefore conducted to verify the numerical results:

- Fan characteristic performance testing. A full-size model of the B2a-fan is constructed and tested in a BS 848, type A fan test facility in order to obtain the fan's characteristic curves.

- Fan blade surface pressure measurements (BSPMs). An additional experiment is required to verify the local flow field in close vicinity of the fan blade. As explained in chapter 3, it is decided to conduct blade surface pressure measurements similar to that of Himmelskamp (1947) and Hurault et al. (2012). A BSPM experimental system and routines for data analysis are developed and constructed.

- Flow visualisation and characterisation. Visualisation of the numerical data is required to present the three-dimensional flow patterns induced by the fan. Some of the flow variables will be characterised to present the variance in these variables for the range of flow rates investigated. These results are also necessary for the development of a new simplified numerical fan model, while also giving insights for future improvements of axial flow fan design procedures.

A survey of the relevant literature is given in chapter 2. The experimental and numerical procedures are discussed in chapters 3 and 4 , and the numerical model validated in chapter 5 . Depictions and the characterisation of the flow field and blade forces are presented in chapters 6 to 8. Finally, the development and results of a new simplified fan model, termed the reverse engineered empirical actuator-disc model (REEADM) is presented in chapter 9, followed by a concluding discussion in chapter 10 . 


\section{Chapter 2}

\section{Literature review}

A survey of literature is conducted with respect to Axial flow fans (AFFs) and other similar turbo-machinery (Axial flow compressors (AFCs) and horizontal axis wind turbiness (HAWTs)). An overview is firstly given on the progression in fan design procedures, whereafter various experimental and numerical flow field investigations are discussed. Since the aim in the present investigation is to conduct both experimental and numerical analyses, an overview is also given of the different research techniques. Hereafter, a synopsis of the B2a-fan history is presented together with concluding remarks.

\subsection{Progress in fan design}

Arguably, one of the most comprehensive manuals for the design of axial fans is the book of Wallis (1983) (first published in 1961), as well as the work published by McKenzie (1997). In general these methodologies give guidelines for the design of free-vortex fans, but a deviation from this trend is observed over time, as will be discussed hereafter. Furthermore, a discussion follows on the effects of non-radial profile stacking in fan designs, merited by the vast amount of literature on this topic. An overview is also given on other arbitrary fan modifications as well as the effect of blade tip clearance on fan performance.

\subsubsection{Vortex design and efficiency}

The use of a free-vortex swirl distribution in fan design procedures is advocated by Wallis (1983) for its simplicity with respect to the spanwise uniform axial velocity condition and the fact that no radial velocities occur through the rotor. This characteristic results in the blade design being a two-dimensional process (only designing for axial and circumferential velocity distributions). Wallis (1983) also mentions that high efficiencies can be obtained using a free-vortex design approach, which is also confirmed by Van Niekerk (1958) who investigated the theoretical (frictionless) total-to-static efficiency limits for free-vortex rotor only ducted fans. Van Niekerk (1958) found these efficiencies 
to be in excess of $\eta_{F s}=70 \%$, depending on the design parameters of the fan. However, since free-vortex designs could lead to unrealistic flow turning angles near the blade root, a set of boundaries was specified by Wallis (1983) to limit these angles such that stall would not occur at the fan design point. Van Niekerk (1958) presented a technique to calculate optimum hub-to-tip ratio's for free-vortex fans with which total-to-static efficiencies in excess of $\eta_{F s}=65 \%$ could be obtained, with the boundary conditions of Wallis (1983) and an estimation of the annular friction applied. The B2a-fan (the test subject of the present study), designed by Bruneau (1994) and also documented in Louw et al. (2012), utilized the design technique of Van Niekerk (1958) with additional relaxation of the blade stagger angle near the hub to extend the fan stall margin and obtained a maximum fan static efficiency of $\eta_{F s}=64 \%$ (total-to-total efficiency of $\eta_{F t}=90 \%$ ) after testing in a standardized test facility (British standard (BS)848, type A).

Due to the disadvantage of the free-vortex design condition (i.e. unrealistic flow turning angles near the blade root) many fan designers have resorted to controlled vortex designs, where the outlet swirl distribution is not a freevortex distribution, but still specified. According to Vad et al. (2001) a larger pressure increase can be obtained by employing controlled vortex designs, especially when new fans need to be retrofitted in existing installations, thus placing a constraint on the amount of design variables (such as rotational speed, blade number, etc.). However, as shown by Van Niekerk (1958), larger pressure differences can also be obtained through free-vortex fans by variation of the hub-tip ratio, while maintaining fan static efficiencies in excess of $\eta_{F s}=65 \%$. High efficiency fans of large hub-tip ratios is also observed by Bamberger and Carolus (2014) who investigated the performance of approximately ten thousand different fan designs through CFD trained meta-models.

In the literature acquired on fans, a total of forty-one publications presented research using controlled or arbitrary vortex fans as test subjects, compared to eight publications where free-vortex fans were used. One reason for the larger amount of controlled vortex fans in research could be due to the involvement of industry, requiring improved designs for operational fans which in many cases are of arbitrary vortex design (such as in the work of Morris et al. (1998), Xu and Amano (2002), Kergourlay et al. (2006), Hurault et al. (2010), Bianchi et al. (2012) and Hurault et al. (2012) to mention a few). Of the literature surveyed, thirty-two, twenty-five and nine fans are of controlled, unknown and free vortex type, respectively (excluding the fan in the work of Bamberger and Carolus (2014)). Of the controlled and unknown vortex fans with no stator blades, maximum and average fan static efficiencies of $\eta_{F s, c_{\max }}=62 \%$ and $\eta_{F s, c_{a v e}} \approx 55 \%$ are noted, where the maximum efficiency value was obtained for a fan with no tip clearance. The average fan static efficiency of the freevortex cases is $\eta_{F s, f_{a v e}} \approx 63 \%$, although more data are required to make the comparison more representative.

The possible reasons for lower efficiencies in the controlled vortex fans sur- 


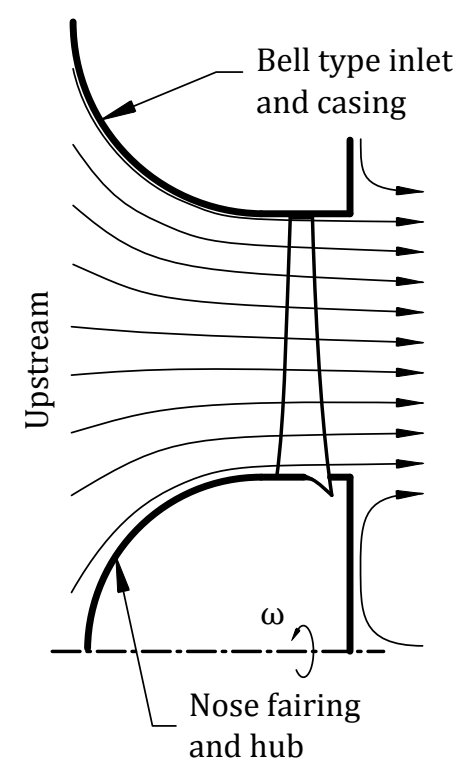

Free vortex

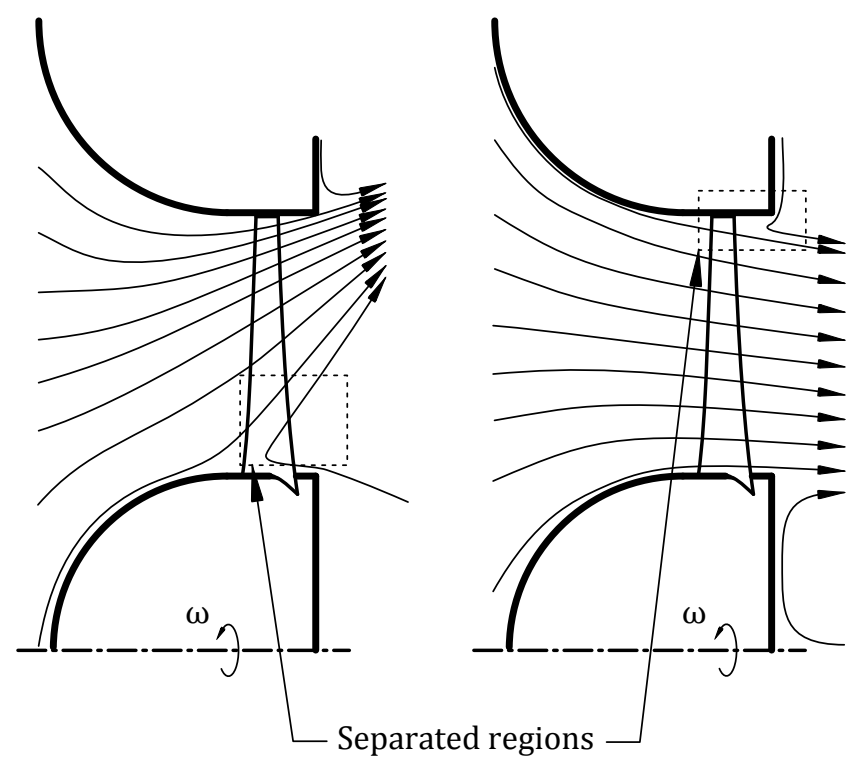

Controlled vortex

Figure 2.1: Two-dimensional flow lines through an axial flow fan for free and controlled vortex designs

veyed in literature might be attributed to a number of reasons:

- The occurrence of radial flow in controlled vortex distributions is inevitable and also separation of the flow in the meridional plane near the hub and/or tip region as depicted in figure 2.1, unless the hub and/or casing is shaped to maintain flow adhesion. Fundamentally, if a turbomachine actuates the flow in both the axial and radial directions along the meridional plane, it is normally referred to as a mixed flow machine. Therefore, it could be argued that controlled vortex fans are essentially mixed flow machines that operate away from the optimal condition due the separation effect mentioned earlier. A quantified value to present the proportion of these losses to others occurring in a fan could however not be obtained in literature.

- A fairly large component of literature involves investigations to mitigate fan noise. The fans considered are in the order of $d_{c}=0.5 \mathrm{~m}$, consuming much less power compared to large diameter fans installed in air-cooled heat exchangers (ACHEs). Subsequently, a larger priority might be placed on the demand for quieter fans, compared to more efficient fans, since a small change in efficiency would not lead to a considerable decrease in power consumption.

- A vast amount of design codes for controlled vortex fans exist compared to free vortex designs, since a free vortex is fundamentally one unique case of a controlled vortex design. Fan designers are more dependent on 


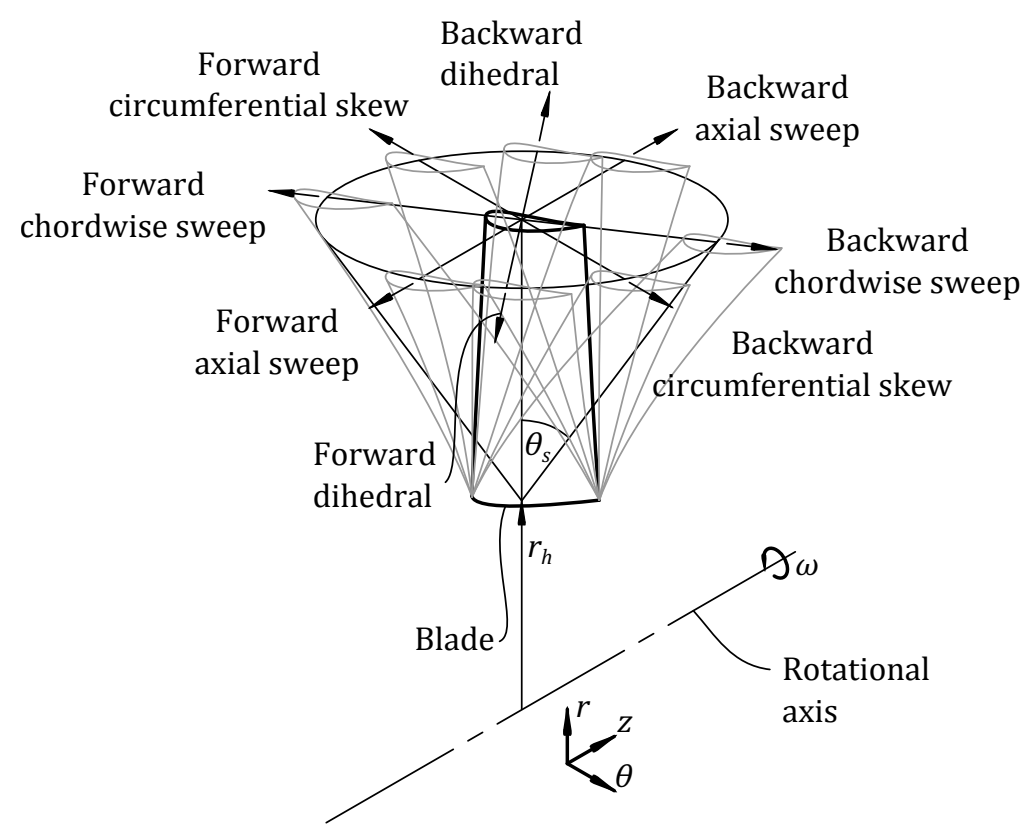

Figure 2.2: Definition of various blade sweep directions (adapted from Ramakrishna and Govardhan (2009))

empirical correlations, such as that of Downie et al. (1993) and Vad and Bencze (1998) or optimisation techniques such as Seo et al. (2008) and Yang and $\mathrm{Gu}$ (2011), causing efficient controlled vortex designs to rely much more on the designer's ability, compared to free vortex designs.

\subsubsection{Non-radial blade profile stacking}

Of the literature surveyed, more than twenty publications deal specifically with the effect of non-radial profile stacking in the design and performance of axial flow fans. Non-radial profile stacking refers to the shaping of a fan blade by stacking the sectional blade profiles in either a swept, axially swept, circumferentially skewed and dihedral manner or in a combination of these, where the definitions are discussed with reference to figure 2.2.

Normal blade sweep or chordwise sweep refers to the shaping of the blade by repositioning the individual blade sections in the direction of the leading or trailing edge along the chord line. The sweep directions are termed forward and backward sweep in the case of sweep in the directions of the leading and trailing edges, respectively. Axial sweep is the repositioning of the sectional profiles in the axial direction. Circumferential skewing of the blade is obtained by moving the blade sections in the circumferential direction and lastly, blade dihedral refers to the relocation of these sections in the direction normal to the profile chord.

The use of sweep and dihedral of turbo-machinery blades originates from its use in the aircraft industry, where the backward sweeping of wings helped to control Mach numbers and consequently shock losses. Forward sweep was 
found to be beneficial in reducing drag (Lewis and Hill, 1971). Dihedral of aircraft wings are implemented to provide more control over aircraft roll.

Smith and Yeh (1963) and Lewis and Hill (1971) are arguably among the first to publish results for the effects of blade sweep and dihedral in axial flow turbo-machinery and they present techniques to incorporate these effects in the design phase. Wright and Simmons (1990) constructed and tested axial fans with swept blades and analysed its effect on the specific performance curves and noise, finding that forward sweep reduced the average fan noise by $7 \mathrm{dBA}$. Furthermore it was also found that the performance characteristic of the swept configurations was slightly altered compared to the unswept case.

In more recent literature (of which a summary can be seen in tables B.1-B.4 of appendix B), numerous articles investigate the effect of sweep on fan noise and performance, both experimentally and numerically. The general consensus on sweep effects are summarized as follows:

- Forward sweep in axial fans is more beneficial compared to backward sweep. Forward sweep leads to unloading of the tip region and increased loading near the blade root, causing tip leakage flow to be less compared to unswept blade profiles. Subsequently, the use of forward sweep is especially advantageous in fans of controlled vortex designs, since these fans generally cause a radially outward migration of fluid, predominantly on the suction side of the blade, the side most sensitive to blade stall. (Vad et al., 2007)

- Forward sweep in fans is found to increase the stall margin of a fan for operation at lower flow rates. (Wadia et al., 1998), (Corsini and Rispoli, 2004) and (Vad, 2008).

- Forward sweep changes the radial distribution of axial velocity in controlled vortex designed fans and decreases radially outward velocities. On the contrary backward sweep increases radial velocities and causes fluid build-up near the tip, leading to higher tip leakage losses. An alteration in inflow conditions is noted for forward swept fans due to the protrusion of the tip of the blade into the upstream flow field. Consequently, flow approaching the fan is actuated by the tip of the fan first and then progressively at lower radii. (Vad et al., 2007) and (Govardhan et al., 2007).

- Findings in regard to the effect of sweep on the losses of a fan are varied. Sweep tends to either give similar or lower peak efficiency values at different operating points compared to the unswept case. Kergourlay et al. (2006), Vad et al. (2007), Hurault et al. (2010) and Ilikan and Ayder (2015) found the peak efficiencies of forward swept (and backward swept in the case of Hurault et al. (2010) and Ilikan and Ayder (2015)) fans to be less than the unswept case, whereas Corsini and Rispoli (2004) and 
Li et al. (2008) found the peak efficiencies of the forward and unswept cases to be the same.

- Finally, one of the major effects of forward and backward sweep is the reduction and increase of fan noise, respectively, as found by Wright and Simmons (1990) and later also Beiler and Carolus (1999) and Kergourlay et al. (2006) (only obtained in controlled vortex designs).

\subsubsection{Arbitrary fan modifications}

Research was conducted on arbitrary fan modifications and their effect on noise and performance. They are discussed as follows:

Longhouse (1977) investigated the noise level of three unswept fans and its mitigation by placing serrations on the leading edge and other areas of the blade suction side. It was found that laminar separation is the main contributor to noise generation for these fans. The addition of serrations to the blade along the leading edge increased the peak fan static efficiency by $3 \%$ and reduced the overall noise by $5 \mathrm{~dB}$. However, serrations added noise at low flow conditions when stall occurred.

Eberlinc et al. (2009) noted separation on the suction side, near the blade root and tip regions of a rotating fan. It was then attempted to reduce this phenomena by means of a hollow blade, where flow is entrained near the blade root on the pressure side by the centrifugation of the volume inside the blade and ejected at the trailing edge in the tip region. A similar technique to control separation and noise in fans is also reported by Bianchi et al. (2014), but not applied to a specific fan. In the case of Eberlinc et al. (2009) a small increase in both pressure difference and peak total efficiency $\left(\Delta \eta_{F t} \approx 5 \%\right)$ was noted.

Corsini et al. (2010) and Wilkinson and Van der Spuy (2015) investigated the effect of winglets, mounted on the blade tip, on fan performance, respectively. In the case of Corsini et al. (2010) a maximum static efficiency increase of $\Delta \eta_{F s} \approx 3 \%$ was obtained, depending on the shape of the winglet used. Wilkinson and Van der Spuy (2015) tested different configurations of winglets at varying tip clearances, but found reductions in both fan pressure and static efficiency, which in some instances were marginal and in other cases $\Delta \eta_{F s} \approx 2 \%$ lower.

Finally, Ye et al. (2015) investigated the effect of various forms of tip grooving on axial fan performance. A total of seven different cases were investigated of which six marginally reduced tip leakage flow and a total efficiency increase $\Delta \eta_{F t} \approx 1 \%$ was obtained. Ye et al. (2015) however, observed a considerable increase in noise with the additions of tip groovings, but did not measure these.

\subsubsection{Blade tip clearance effects}

The effect of tip clearance on axial fan performance was investigated by Venter and Kröger (1992), Downie et al. (1993) and Wilkinson and Van der Spuy 
(2015). The general finding is that a decrease in fan static pressure and efficiency can be observed with an increase in tip clearance. Venter and Kröger (1992) produced a linear correlation between tip clearance and fan static pressure difference and efficiency, but mentioned that the effects of tip clearance is specific to fan design, size and the type of installation. Downie et al. (1993) noticed a reduction of $20 \%$ in fan total pressure difference at near stall conditions for a change in tip clearance from $0.5 \%$ span to $2.5 \%$.

\subsection{Flow field investigations}

\subsubsection{Inflow effects}

Much research have been conducted on fan performance in ACHEs, especially considering the effect of inflow patterns. Of these only Stinnes and Von Backström (2002) investigated oblique inflow into a single fan experimentally, which was also numerically computed by Hotchkiss et al. (2006). Oblique inflow angles from axial to $45^{\circ}$ from the axial direction were investigated, in which it was found that fan power consumption was unaffected by the inflow angle. Furthermore, the total to total pressure rise across the fan remained unaffected up to an angle of $27^{\circ}$. However, a progressive decrement in fan total to static pressure difference was observed with an increase in oblique inflow angle, which was found to be equal to the dynamic pressure based on the cross-flow velocity component.

\subsubsection{Reynolds number effects}

Research has also been conducted on the effect of blade chord Reynolds number when fans are scaled up or down by means of the turbo-machine scaling laws. Meyer and Kröger (2004) empirically investigated the effect of Reynolds number on fan scaling using the actuator-disc model (ADM) described by Meyer and Kröger (2001). A test fan of diameter $d_{c}=1.542 \mathrm{~m}$ and an up-scaled fan of $d_{c}=9 \mathrm{~m}$ were used as subjects. Since the ADM is based on two-dimensional blade element theory to simulate the flow in the vicinity of an axial fan, the two-dimensional aerofoil lift and drag characteristics are referenced as a function of the angle of attack onto the blade. Using this approach, Meyer and Kröger (2004) referenced the lift and drag characteristics obtained at different Reynolds numbers when different fan diameters (giving different Reynolds numbers for different blade sizes) were simulated. A difference between the fan static pressure and power consumption, determined by the ADM and fan scaling laws, respectively, was obtained. The fan scaling laws predicted lower values for the characteristic total to static pressure and shaft power compared to those predicted by the ADM. However, the fan static efficiencies were found to be the same. 
Pelz (2013) developed a method to incorporate Reynolds number effects into the scaling of axial and centrifugal flow turbo-machines. It is also observed that machine efficiency increases with an increase in Reynolds number. Their method is based on a semi-empirical relation between the performance curve of the specific machine and the determination of friction factors as part of the conversion between a model and a full-scale machine. The method is validated with an axial fan example. Correlation between their modelled and experimental values varies, and the article mentions that more research is required to further improve the method.

Lastly Augustyn et al. (2015) numerically modelled two fans of the same design, but of different diameters $\left(d_{c}=1.25 \mathrm{~m}\right.$ and $\left.10.36 \mathrm{~m}\right)$. Similar to Pelz (2013), it is observed that efficiency increases with an increase in fan diameter (together with Reynolds number). In their case the total to static efficiency predicted by the fan scaling laws were $2-5 \%$ lower, and the shaft power $10 \%$ higher, compared to the numerically obtained values.

\subsubsection{Low flow rates}

Himmelskamp (1947) conducted blade surface pressure measurements on a propeller through a complex system of tubes and manometers. The propeller had two blades of constant chord length along the span. It was found that the aerofoil characteristics at various radii of the propeller blade differed considerably from that of the same two-dimensional aerofoil profile, especially at low flow rates. The most noticeable discovery was that an increase in the aerofoil stall margin as well as increased lift coefficients at high angles of attack, compared to the two-dimensional case, could be observed. This phenomenon was ascribed to the presence of a three-dimensional boundary layer and radial flow occurring close to the propeller blade.

The earliest results obtained on downstream spanwise flow profiles measured with a cobra probe for three controlled vortex AFFs are presented by Downie et al. (1993). These profiles are presented for flow rates ranging from $65 \%$ to $110 \%$ of the design flow rate. It was observed that reversed flow first started to occur near the hub with a decrease in flow rate and progressively increased to higher radii with a further reduction in flow rate. An increase in outward radial velocity with a decrease in flow rate was noted, together with a progressive shift to higher radii of the location of maximum radial velocity. Downie et al. (1993) also found a blade design utilizing aerofoil sections to be of superior performance, especially at lower flow rates, compared to a design using cambered plate sections, since a higher total pressure could be maintained at lower flow rates.

Bak et al. (2006) developed corrections of two-dimensional blade sections (essentially two-dimensional aerofoils) of a rotating wind turbine for the purpose of performance analysis using the vast amount of experimental data obtained by Hand et al. (2001) and Lindenburg (2003). A fair correlation with 
experimentally measured results was obtained. Since the flow in the vicinity of a fan at low flow rates is highly unstable and three-dimensional this model might be implemented for axial fans as well, but no such analysis could be found in the literature.

Li and Zhou (2008) attempted to investigate the flow field in the vicinity of a mine axial flow fan at low flow rates, since unwanted vibrations occurred under these conditions. The work focussed on numerical simulation and oil flow visualization, but it was concluded that the flow was too complex to analyse. This was partially because their main focus was not to understand the flow field, but develop a means to ensure stable operation during these conditions. Quoted from their publication:

"The inner-flow of an axial mine flow fan, especially under off working conditions, is too complex to be explained fully by the current imperfect theory of fluid dynamics, such as flow separation, stall and sharp vibration. To understand the intrinsic phenomenon of the inner-flow of drivers, detailed and reliable flow experiments as well as numerical simulation are urgently needed."

\subsection{Experimental analyses}

Since experimental measurements form part of the present study, a short summary is given of all axial fan experimental work surveyed in the literature. More details of these measurements and the specific fans investigated can be found in tables B.1-B.2 of appendix B. Of the measurements conducted in literature, a distinction can be made between multi-hole probe measurements, hot wire anemometry and laser techniques (such as laser Doppler anemometry (LDA) and particle image velocimetry (PIV)) to obtain spatial flow field values. The most notable experiments are highlighted

A novel investigation into the flow field in the vicinity of a rotating propeller was conducted by Himmelskamp (1947). Blade surface pressure measurements were obtained through a series of tubes located on the inside of the profile connected to holes on the surface of the blade. These tubes were each connected to a complex system of rotating seals from where pressure lines were coupled to a series of manometers. The time for a single test run was limited by the sealing capability of the rotating seals and consequently photos were taken of the manometers during the experiment. The photographic images were then used to obtain the manometer readings. Velocity measurements were also conducted up and downstream of the propeller as well as in the boundary layer of the fan blade.

Downie et al. (1993) investigated the downstream flow field of three axial flow fans by means of a Cobra, four-hole probe. Although no comparison was made to numerical results at the time, a fair correlation was obtained between the measured and theoretically expected outlet velocity profiles at the design condition, proving the feasibility of this technique. However, as 
mentioned earlier, measurements were limited to $65 \%$ of the design flow rate. The feasibility of measurements at lower flow rates remains unknown.

One of the first investigations to correlate experimentally and numerically obtained spatial flow field data was conducted by Beiler and Carolus (1999) who used a three pronged hot wire probe to measure the flow field downstream of a series of axial flow fans at the design condition. A fair comparison between the numerical and experimental results was obtained for the downstream axial flow velocities. A fair comparison does however not always exist in literature as indicated in the publications of Kergourlay et al. (2006) and Hurault et al. (2010) who investigated the downstream flow field with X-configuration hot wires. Whether this comparison is due to the modelling approach or the difficulty in obtaining measurements for the specific flow fields is unknown.

Ubaldi et al. (1994), Vad and Bencze (1998), Vad et al. (2006) and Sarraf et al. (2011) conducted detailed flow field measurements and data visualization of the flow structure downstream of fan rotors using LDA. One advantage of this technique is the ability to also measure the instantaneous velocity within the fan blade passage in a non-intrusive manner. No comparison to any analytical or numerical estimations were made, but the measured flow field depicted radial outflow at lower flow rates and vortical patterns downstream of the fan due to the controlled vortex design condition.

PIV measurements in an axial flow fan have been documented by Estevadeordal et al. (2000) who also presented detailed flow field visualization and data of flow structure within blade passage and downstream off the fan. A comparison of the flow field in the tip region of the fan was made to inhouse panel code results and a fair comparison was obtained. Similar to LDA measurements, PIV is also a non-intrusive technique, but more applicable to determine timeaveraged flow fields. Similar measurements in axial turbo-machinery were also successfully conducted by Roehle et al. (2000), Stella et al. (2000), Wernet (2000) and Yoon and Lee (2004).

A study conducted by Schreck (2007) showed how blade pressure measurements were performed using blade surface pressure taps and transducers mounted on the inside of a large HAWT. Since, these blades are large (compared to axial fans), enough space in the blade cavity was available to encapsulate the pressure transducers and other measuring equipment. The relative flow angle upstream of the blades were also measured with five-hole probes protruding from the blade leading edges. The only recent reference found in literature that refers to blade surface pressure measurements performed on AFFs were conducted by Hurault et al. (2012). Piezo-resistive pressure sensors, moulded into the tested fan blades and coupled to a slip-ring system, were utilized in their study. A very good correlation existed between their experimental and numerical results. 


\subsection{Numerical analyses}

Similar to the experimental work discussed, a fair amount of literature on the numerical modelling of AFFs is summarised in table B.3 of appendix B. More details to the fan specifications investigated in these cases are presented in B.4, if not already given in B.1.

Two methods of axial turbo-machine modelling exist in literature. The first is three-dimensional modelling, where either an axis-symmetrical (flow through a single blade passage) or a full three-dimensional model (all passages of the rotor) is used. The second approach involves flow modelling using an actuator-disc model (ADM). These two approaches are discussed.

\subsubsection{Three-dimensional modelling}

Of the literature surveyed with regards to three-dimensional fan modelling, fifteen out of the twenty-five cases (excluding the publications from the present study) modelled the flow domain by considering an annular duct only (i.e. infinitely long hub and shroud in the axial direction). The remaining ten considered open inlet to open outlet or similar cases. The tendency to model annular configurations is understandable, since the grid and solver requirements for fans in an open inlet or open outlet configuration are avoided in this manner. From the fifteen ducted cases, only Lee et al. (2003) modelled the entire fan rotor with all blades present. More recently, Liu et al. (2010) and Ye et al. (2015) also conducted a full three-dimensional study, by considering open inlet to open outlet or annular duct outlet conditions.

Thirteen out of the twenty five publications used a variant of the $k$ - $\varepsilon$ model, whereas nine used variants of the $k$ - $\Omega$ model of Menter et al. (2003). Of the remaining four publications, three utilized the Reynolds stress model. Initially the Standard $k-\varepsilon$ model of Launder and Spalding (1974) was used extensively due to it being inexpensive compared to other turbulence models. However more recently a shift is seen towards the $k-\Omega$ and Realizable $k-\varepsilon$ (Shih et al., $1994)$ models. The $k$ - $\Omega$ models are specifically tailored for aeronautical applications and consequently give good predictions for flow across aerofoils. Similarly, the Realizable $k-\varepsilon$ model gives superior performance compared to the Standard $k-\varepsilon$ model, especially in swirling and rotating flows, which occur during flow separation off solid bodies. The Reynolds stress model, although more expensive to solve, gave superior performance compared to the $k$ - $\Omega$ model in the case of Hurault et al. (2010) when compared to fluid stresses calculated from experimentally determined results.

The work of Beiler and Carolus (1999), where the Standard $k-\varepsilon$ turbulence model was used, resulted in very good correlations between the experimentally and numerically determined values for spanwise axial velocity. However, no comparison was shown regarding the fans' characteristic performances. In the publications of Corsini and Rispoli (2005), Hurault et al. (2010) and Hurault et al. (2012) fair comparisons were obtained between experimentally and nu- 
merically determined spanwise flowfield values, where the Standard $k$ - $\varepsilon$ and Reynolds stress models were used, respectively. In the case of Hurault et al. (2010) and Hurault et al. (2012) fair correlations were also obtained for fan performance, although they did not model at low flow rates. Ye et al. (2015) also obtained a fair comparison for an entire range of flow rates (even close to zero flow) using the Realizable $k-\varepsilon$ model. In their case, the fan pressure difference was generally over predicted by $5 \%$, although the average difference for fan total efficiency was smaller.

\subsubsection{Actuator-disc modelling}

Actuator-disc modelling refers to the modelling of the flow field in the vicinity of a turbo-machine rotor, without explicitly modelling the blades and blade passages. Instead the flow actuated by the fan is modelled empirically through two-dimensional blade element theory, better described in chapter 9 .

Originally, Thiart and Von Backström (1993) developed an ADM for the purpose of investigation of distorted inflow patterns to ACHEs. This work was based on a model developed by Pericleous and Patel (1987) for the modelling of fluid agitators in chemical reactors. In the case of Thiart and Von Backström (1993), the Standard $k-\varepsilon$ model was used together with an inhouse developed code. The comparisons between the numerically and experimentally measured results for spanwise velocity profiles near the fan varied. Furthermore, it is mentioned that fan power consumption is underestimated by nearly half the measured value.

Meyer and Kröger (2001) described the fundamental working of the ADM by considering and axial flow fan. The ADM is implemented in a commercial code, Flo ++ to calculate the fan performance characteristics of the B2-fan of Bruneau (1994). A fair comparison was obtained between the numerically and experimentally determined performance values, although Meyer and Kröger (2001) did not investigate the performance at low flow rates. Bredell et al. (2006) implemented the ADM as a user defined sub-routine in the commercial code, Fluent. They focussed on the implementation of their model in large ACHE modelling and thus the fundamental flow field in an AFF was not investigated explicitly.

Van der Spuy et al. (2011) continued the work of Bredell et al. (2006) and investigated the accuracy of the ADM for ACHEs. In their work, a second model was also developed termed the extended actuator-disc model (EADM). The model works on the same principle as the ADM (i.e. two-dimensional blade element theory), but with empirically altered lift and drag coefficients. Through the research of Himmelskamp (1947), which showed a difference between the lift and drag coefficients of the blade sections (essentially two-dimensional aerofoils) of a rotating propeller compared to the two-dimensional case, Van der Spuy et al. (2011) manipulated the two-dimensional characteristics of the aerofoils in their study, effectively extending the linear part of the lift characteristic 
and using these new correlations in their model. The EADM produced a fair prediction of the fan characteristics (compared to experiments) for the range of flow rates investigated in the B2a-fan, but the same level of comparison is not obtained when other fans were modelled. Generally good performance agreements exist for flow rates near the design flow condition, but the quality of correlations vary at lower flow rates. Further research is thus required to develop a model that would give improved fan performance predictions for these flow conditions.

\subsection{Evolution of the B2a-fan}

The B-fan series was originally developed by Bruneau (1994) for application in ACHEs. Two fans of $d_{c}=1.542 \mathrm{~m}$ were developed, constructed and tested in his study, termed the B1- and B2-fans. These fans were designed for exactly the same operating and vortex conditions, with the only difference being that the Clark-Y and NASA LS 0413 (McGhee and Beasley, 1976) aerofoil profiles were used for the B1- and B2-fan, respectively. Hereafter, the B2-fan was used in many other academic investigations at Stellenbosch University.

For the purpose of this study a new B2-fan was constructed, since the original fan was weathered and the specific shaping of the fan blades questioned. In the case of the B2-fan, the aerofoils used to shape the fan blade were kept "flat" and not cylindrically folded as depicted in figure 2.3. For the purpose of comparing numerically and experimentally obtained results, detailed information regarding the geometry from which the fan was manufactured is required. It was discovered that the B2-fan hub diameter specified in the thesis of Bruneau (1994) differed from the B2-fan model actually constructed, leading to large discrepancies in numerically and experimentally determined performance values by various other authors. The B2a-fan constructed in the present study is an exact model of the B2-fan described in the thesis of Bruneau (1994) with respect to hub diameter, chord length and blade twist. The blade was shaped from cylindrically fitted aerofoil sections as indicated in figure 2.3.

\subsection{Concluding remarks}

From the reviewed literature the following concluding remarks are made:

Regarding fan design techniques, investigations into the effects of blade sweep on fan performance is presently the main topic of investigation. From the literature reviewed the consensus is that forward sweep and skew are advantageous for increasing the stall margin and noise reduction. The contrary is true for backward sweep and skew. Unswept blades provide the highest nominal efficiency.

It is found that a fan of free vortex design is more efficient compared to controlled vortex designs. The designer should however carefully consider op- 


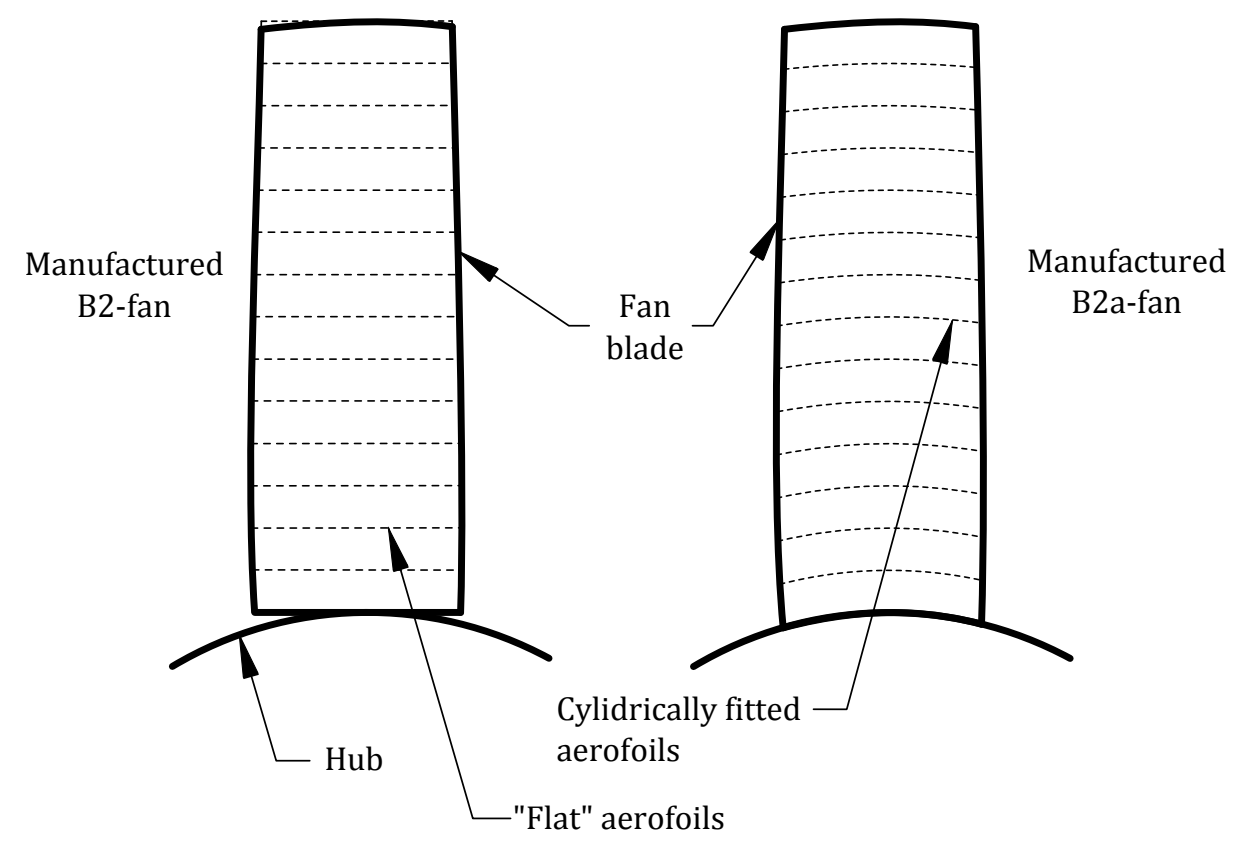

Figure 2.3: Different shaping of the manufactured B2- and B2a-fan blades

timal hub-tip ratios as well as constraints on the blade stagger angle near the root.

Of all the publications reviewed, only Hurault et al. (2012) conducted blade surface pressure measurements in an AFF. These measurements are in addition to those conducted by Himmelskamp (1947) on a propeller blade and other research in the wind turbine research field. One of the main disadvantages of these measurements is the unknown effect of centrifugal forces on the final readings given by transducers, since the transducer membranes would distort under centrifugal loading, presenting a false pressure value. The cost of commercial piezo-resistive pressure sensors, such as the ones used by Hurault et al. (2012) is significant.

Although measurements with multi-hole probes or hot wire anemometers are impressive ((Downie et al., 1993) and (Beiler and Carolus, 1999)) at flow rates as low as $65 \%$ of the design flow rate, it is suspected that more difficulties would arise when conducting such measurements at lower flow rates, which is of interest in the present investigation. These probes and anemometers are accurate within a certain reference cone of flow angle onto the probe, a restriction that might be violated since flow behaviour would become increasingly erratic near the fan with a decrease in flow rate.

The laser techniques have the advantage of being non-intrusive and allow for the instantaneous measurement of velocity, although PIV is more accurate when a time averaged flow field is measured. Since no LDA machine is available at Stellenbosch University and the simpler technique proposed in the present investigation also showed promise, the use of LDA was not investigated further. One of the disadvantages of PIV is that of inaccuracy or the inability 
to measure in the regions close to walls, where the laser sheet illuminating the measuring plane produces reflections. Hence, if the blade passage is small, only a small percentage of the measured data would be useful and only in the middle section of the passage (away from the blade and hub surfaces). Also, since a possibility exists that rotating stall might occur at low flow rates, the time averaging of PIV data sets might be erroneous, as rotating stall typically occurs at different frequencies than the fan rotational frequency. Lastly, the suitability of using PIV outdoors (as is the case for the test facility in the present study) is unknown, since a certain level of darkness is required for accurate results. For these reasons PIV measurements were not considered further in the present study, but despite these disadvantages, it might be a useful technique to consider in future investigations.

It was shown that three-dimensional numerical modelling is possible and that a fair estimation of the flow field could be obtained with less expensive turbulence formulations such as the $k-\varepsilon$ and $k$ - $\Omega$ models, compared to more complex and time-dependent models such as Large eddy simulation (LES) or Detached eddy simulation (DES). However, more research is required for open inlet to open outlet configurations (similar to BS 848, type A fan testing conditions), especially with regards to boundary proximity and mesh quality, presenting a greater challenge compared to the annular duct simulations mostly conducted in literature.

Lastly, a small amount of literature is available on the simplified numerical modelling of axial fans, where the actuated flow field is obtained through other means than the explicit modelling of the fan blades. To date, only the work of Van der Spuy et al. (2011) addresses the low flow region with varying success. Thus, more research on this topic is required.

Lastly the following questions still remain for AFFs:

- How is the flow field altered at low flow rates and to what extent can it be visualised?

- How do blade sectional lift and drag coefficients compare to the characteristics of the same two-dimensional aerofoil sections?

- What is the fundamental mechanism driving the flow at low flow rates and to what extent does radial flow play a role in this, since it is generally not considered in fan designs?

- Can improvements be made to simplified numerical fan models that would provide improved performance predictions at low flow rates?

The approach outlined in chapter 1 is taken in an attempt to answer these questions. 


\section{Chapter 3}

\section{Experimental procedures}

Experimental analyses are conducted in the present study to obtain flow field data that can be used to verify the numerical fan model. These experiments are:

Fan characteristic testing. The B2a-fan is tested in a British standard (BS) 848, type A fan test facility to obtain its performance characteristics. Originally, verification of the numerical model is assumed to be satisfactory by comparing the numerically predicted and experimentally measured characteristic fan performance data. This only gave assurance of a numerically converged global flow field and did not necessarily prove that the local flow field in the vicinity of the blade is well predicted. A second experiment is necessary to obtain flow field data in closer vicinity of the fan blades.

Blade surface pressure measurements (BSPM). After consideration of the literature discussed in chapter 2 a decision is made to conduct blade surface pressure measurements in similar fashion to the measurements of Himmelskamp (1947), using a more advanced blade manufacturing technique and wireless pressure sensing system.

The decision to settle for this specific technique is due to the following: As discussed in chapter 2, spatial flow field measurements (such as measurements with multi-hole probes and hot wire anemometry) have been conducted in turbo-machinery with relative success. The difference between many of these measurements and those required in the present investigation is the anticipated instability of the flow field. Since measurements are required at low flow rates, the induced unstable flow field complicates measurements using hot wire anemometry and multi-hole probes as discussed in the conclusion of the previous chapter (section 2.6). The challenges associated with laser Doppler anemometry (LDA) and particle image velocimetry (PIV) have also been discussed.

BSPMs are opted for in the present study since it is an alternative that would mitigate the uncertainties occurring in spatial measurements previously 
described. The pressure measured at a surface interface is static, since the flow immediately next to a wall is parallel to it. Hence, a high level of certainty of the measured values exist. The use of a wireless pressure measuring system mounted on the fan and coupled to a set of piezo-resistive transducers also means that the noise that would have been produced in slip-rings would be mitigated. The system developed in this study also has the ability to be scaled up fairly easily, to accommodate a large number of transducers.

In this chapter, the experimental performance testing of the B2a-fan, as published in Louw et al. (2014b,a), is firstly explained. Details of the fan test facility and fan testing procedures are given in section 3.1. Subsequently, the BSPM apparatus and measurement procedure, as published in Louw et al. $(2015 a)$, are discussed.

\subsection{Fan performance testing}

\subsubsection{British Standard 848, type A test facility}

Fan characteristic testing is conducted in a BS 848, type A (open inlet to open outlet) test facility at Stellenbosch University as depicted in figure 3.1. These tests produce the characteristic curves of a specific fan. The characteristic curves comprise the total to static pressure difference $\left(\Delta p_{F s}\right)$ across the fan, fan power $\left(P_{F}\right)$ and total to static efficiency $\left(\eta_{F s}\right)$ as functions of the volumetric flow rate $(\dot{V})$, respectively.

During a typical fan test, flow is drawn into the facility through a calibrated inlet at location 1 (figure 3.1). The flow through the facility is controlled by a set of louvres (2), whereafter straightening occurs by the first set of flow straighteners. An auxiliary fan located at 3 provides additional actuation of the flow to overcome the mechanical losses of the facility. The flow is then straightened through a second set of straighteners and expanded into the settling chamber. A set of guide vanes (4) at the inlet of the settling chamber ensure an even flow distribution. A set of three wire screens in the middle of the settling chamber (5) supplements the even distribution of the flow as it enters into the final part of the settling chamber (6). From here, flow is finally drawn out to the atmosphere through the test fan (7).

During a single characteristic test run, measurements are made for a range of flow rates through the facility. These measurements comprise the following for each flow rate:

1. Static pressure difference $\left(\Delta p_{s, n}\right)$ relative to atmospheric pressure at the calibrated inlet.

2. Static pressure difference $\left(\Delta p_{s, p l e n}\right)$ relative to atmospheric pressure inside the plenum chamber.

3. Shaft rotational speed $(N)$. 

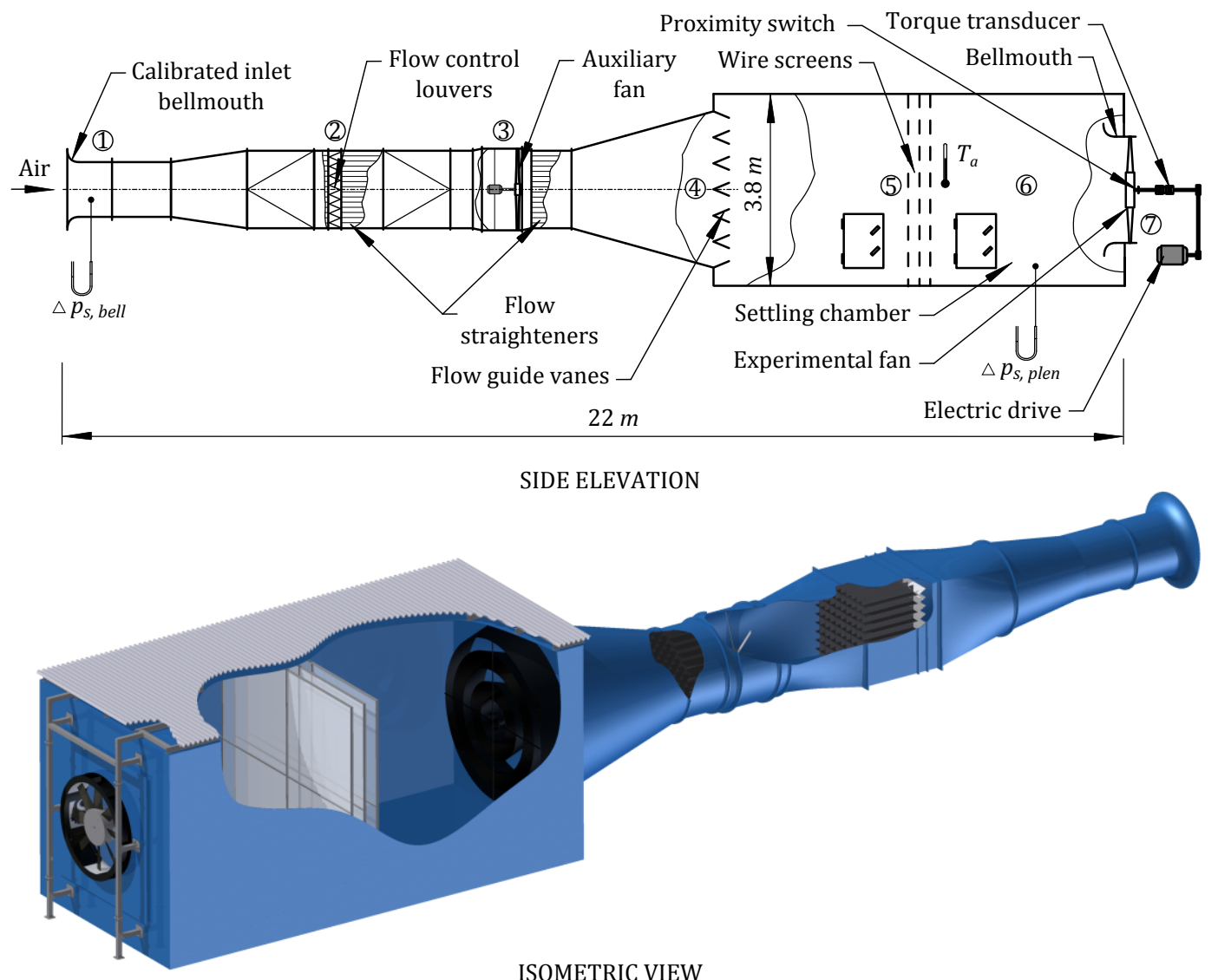

Figure 3.1: Stellenbosch University BS 848, type A fan test facility (adapted from Kröger (2004))

\section{Shaft torque $(T)$.}

Additional measurements are made that include the air temperature $\left(T_{a}\right)$ inside the test facility and the barometric pressure $\left(p_{\text {atm }}\right)$. These measurements are only made once before and after each test run. A linear interpolation is then made to obtain the $p_{a t m}$ and $T_{a}$ values for each flow rate measured. These values are subsequently used to calculate the air density $\left(\rho_{a}\right)$.

\subsubsection{Facility instruments}

The instrumental layout of the present test facility is shown in figure 3.2. Two HBM PD1 pressure transducers are used for the measurement of the static pressure inside the calibrated inlet and plenum chamber respectively. The torque is measured by an HBM T22 torque transducer fixed to the fan shaft downstream of the fan as shown in figure 3.1. Lastly the shaft rotational speed is measured by a proximity switch connected to a frequency to voltage converter. These four measuring devices are connected to a HBM PMX data acquisition system that interfaces with a desktop computer through the Catman software package. The HBM PMX provides excitation to all the instruments, amplifies 


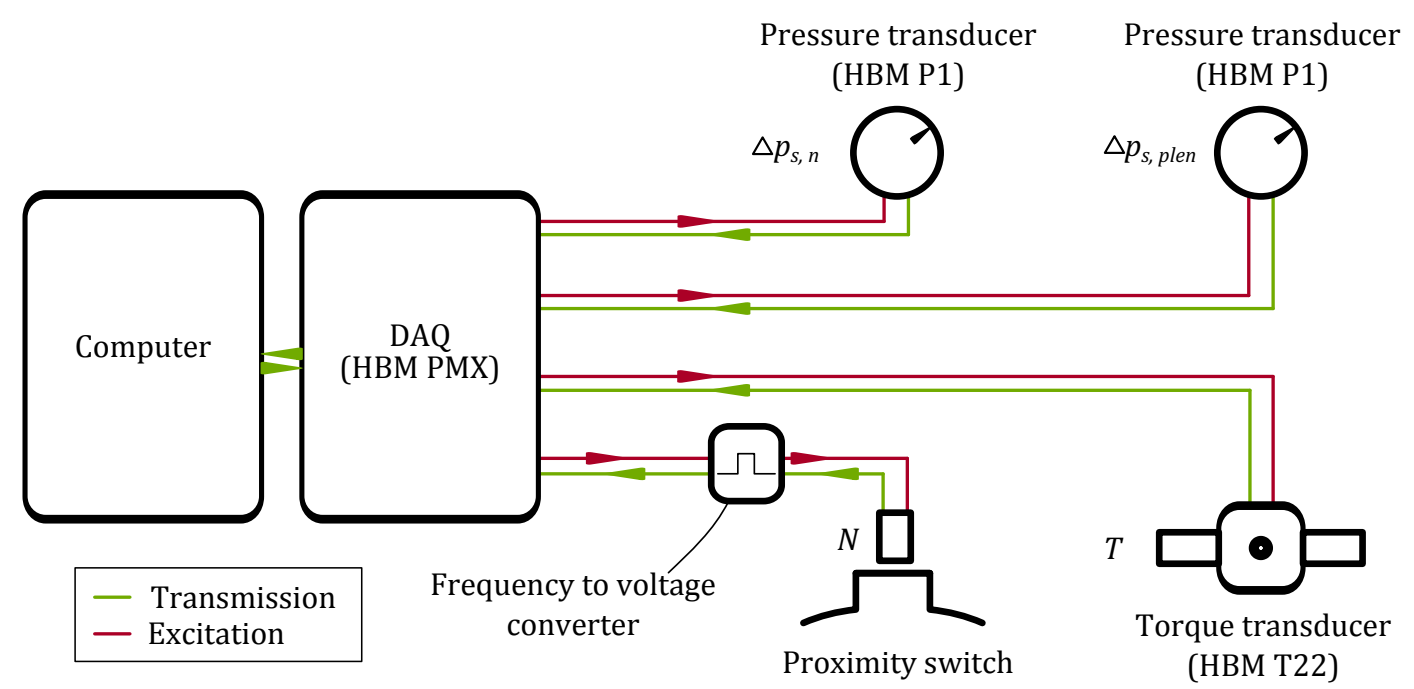

Figure 3.2: Line chart of the instrumental layout for the fan test facility

the measured signals and acts as a control interface between the computer and the sensors.

Regarding the blade stagger angle of the B2a-fan, adjustments are made using a custom manufactured jig, that is fitted to the blade root. A digital protractor is then used in conjunction with this jig to obtain the blade angle.

The measurement errors for each instrument are given in table 3.1.

Table 3.1: Fan test facility and stagger angle setting instrument errors

\begin{tabular}{lll}
\hline Instrument & Error (\%) & $\begin{array}{l}\text { Error } \\
\text { (Unit) }\end{array}$ \\
\hline Pressure transducer (HBM PD1) & $\leq \pm 1.6 \%$ & $\pm 16 \mathrm{~Pa}$ \\
Torque transducer (HBM T22) & $\leq \pm 1.1 \%$ & $\pm 2.2 \mathrm{Nm}$ \\
Frequency/Voltage converter & - & - \\
Digital protractor (SmartTool) & $\leq \pm 0.1 \%$ & $\pm 0.1^{\circ}$ \\
\hline
\end{tabular}

\subsubsection{Measurement procedure}

During a typical characteristic test the blade stagger angles of the test fan are set to the desired value $\left(\xi_{h}=59^{\circ}\right.$ in the present study), whereafter the fan is mounted at the end of the test facility (location 7, figure 3.1). The tip clearance is set to $\Delta r_{\text {tip }}=3 \mathrm{~mm}$ and the small air gap between the blade root and hub sealed with silicone to avoid air leakage in this region. 
After all connections are made to the sensors, a zero reading is firstly triggered through the HBM PMX while the fan is still static. Additionally the air temperature inside the facility and the barometric pressure is also taken. The test fan is then brought up to the design speed of $750 \mathrm{rpm}$ and the flow control louvres set to the desired setting. The auxiliary fan is switched on only for measurements during higher flow rates (typically in the order of the design flow rate and higher), but otherwise remains off. Data acquisition is then triggered through the HBM PMX for ten seconds at a rate of $100 \mathrm{~Hz}$ and stored onto a computer. Subsequently, the louvres are adjusted to obtain a new volumetric flow rate and the acquisition of data repeated. This process is then replicated until data for the desired amount of flow rates have been obtained. Finally the test fan is stopped and a second zero measurement taken in a similar fashion than before together with the barometric pressure and plenum air temperature. Hereafter the data is downloaded for analyses and processed as discussed in appendix D.1 to obtain the fan static pressure $\left(\Delta p_{F s}\right)$ and static efficiency $\left(\eta_{F s}\right)$ characteristic.

\subsection{Blade surface pressure measurement}

The BSPM apparatus is designed and constructed to measure the blade surface static pressures at five radial locations on a B2a-fan blade as indicated in figure 3.3. This apparatus includes two specially manufactured fan blades (additional to the eight existing fan blades) containing a series of pressure taps, a capsule mounted on the fan shaft enclosing a set of piezo-resistive pressure transducers and a wireless telemetry system for transducer actuation and data capturing. All of the measurements are conducted in the BS 848, type A fan test facility at Stellenbosch University. The BSPM apparatus, measurement procedure and error analyses are discussed.

\subsubsection{Experimental apparatus}

The experimental apparatus used to obtain the blade surface static pressures shown in figure 3.3, supplements the following explanation. The two carbon fibre B2a-fan blades are manufactured with the purpose of locating thirty-five pressure taps, of $1 \mathrm{~mm}$ in diameter, on either the suction or pressure side of the blades (i.e. seven taps per blade span at five different span lengths of $s_{b}=0.1,0.3,0.5,0.7$ and 0.9 ). The spacing is chosen to have at least one spanwise location near the hub, near the tip and at midspan. The remaining two locations are chosen halfway in between these three locations to give an even spanwise distribution of pressure taps. The first blade is used to measure the surface pressures on the suction side of the blade whereas the second is used for measurements on the pressure side. The white coloured gel coat on the blade surfaces (visible in figure 3.4a) ensures a smooth finish. 

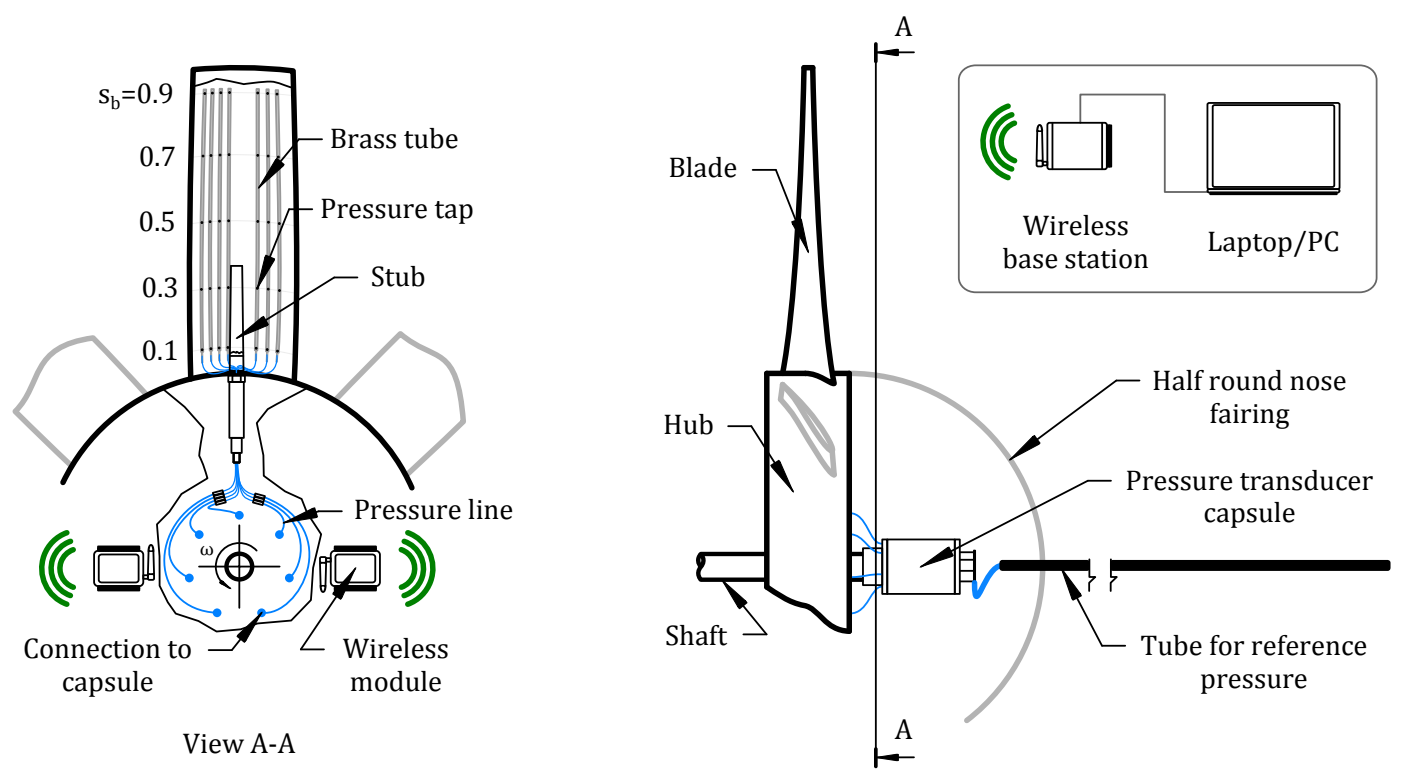

(a)

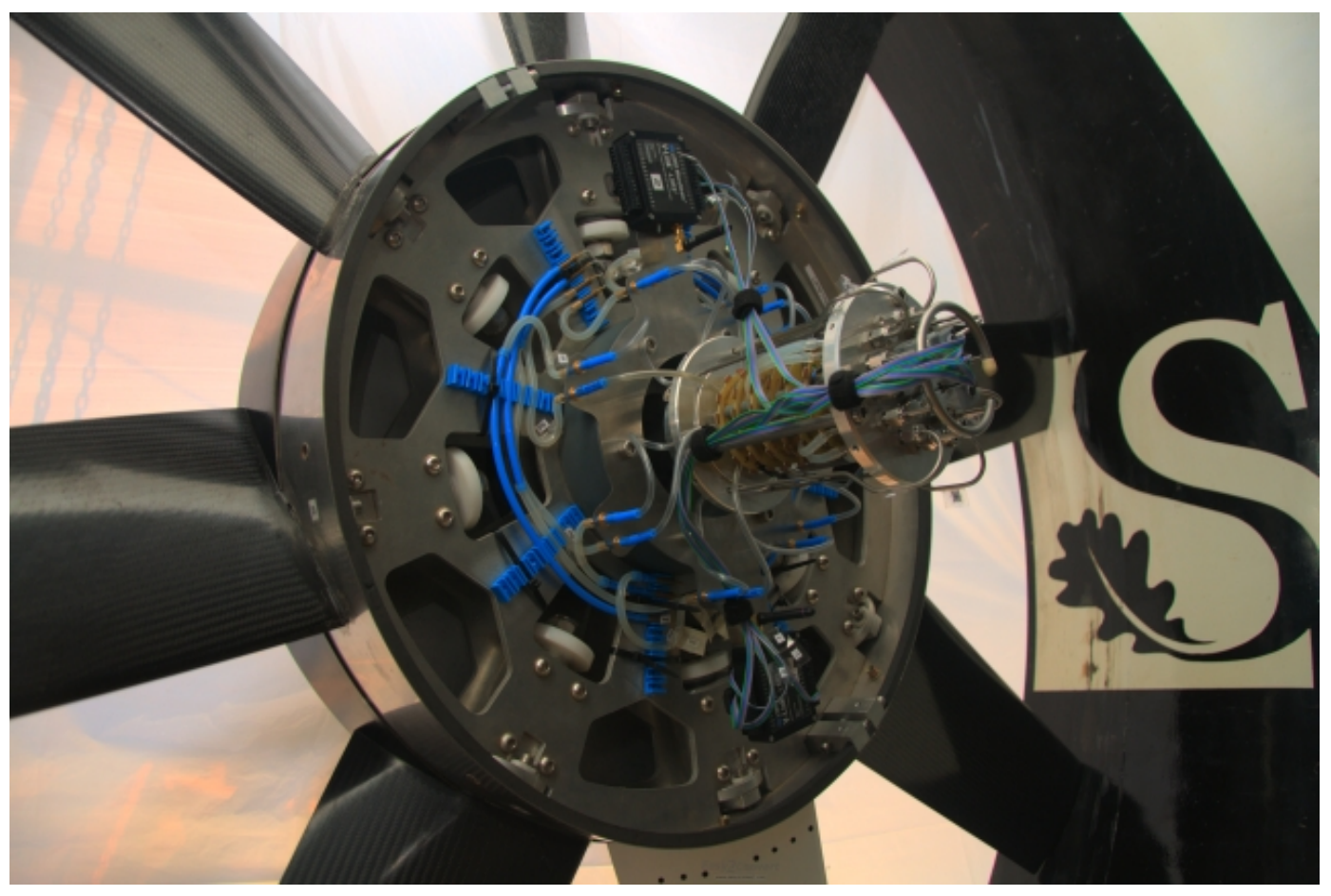

(b)

Figure 3.3: (a) An illustration and (b) photograph of the BSPM apparatus 

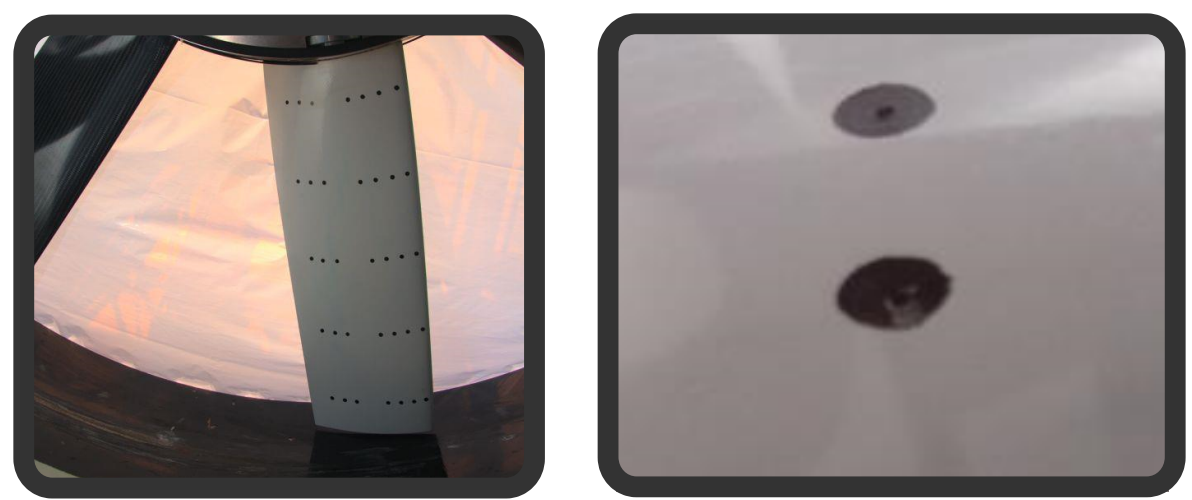

(a)

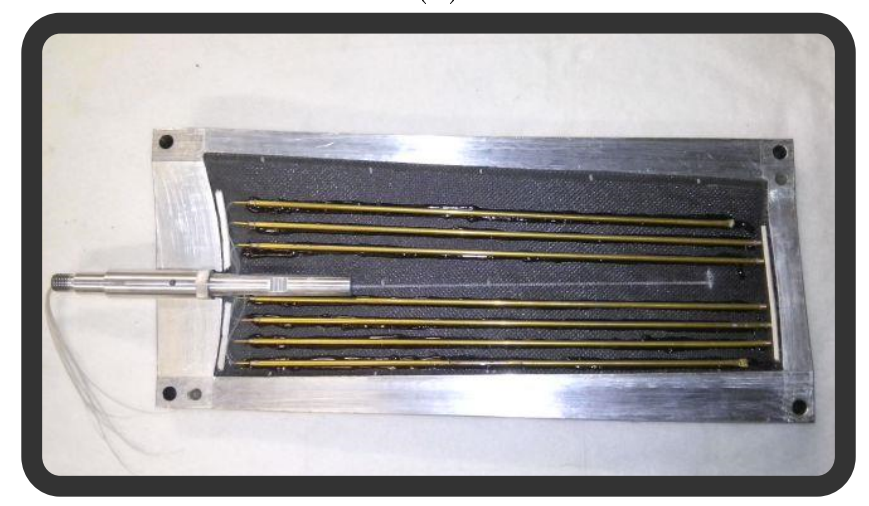

(b)

Figure 3.4: (a) The suction side BSPM fan blade as well as two pressure taps and (b) the brass tubes fixed to the blade inner cavity

The pressure taps are drilled through the blade surface into brass tubes located inside the blade cavity. Each of the two blades has seven brass tubes ( $5 \mathrm{~mm}$ in diameter) glued to the inner cavity with epoxy resin (figure $3.4 \mathrm{~b}$ ), extending from the blade root to the tip. These tubes are spaced inside the blade to locate the pressure taps at dimensionless chord lengths of $x / \mathrm{ch}=$ $0.05,0.15,0.25,0.35,0.6,0.7$ and 0.8 , where $x / c h=0$ and 1 refer to the leading and trailing edges respectively, for each of the five blade spans to be measured. The chordwise spacing is chosen to avoid interference with the stub inside the blade cavity and to obtain a fairly even distribution of taps across the chord of the blade. The placement of tubes near the trailing edge is constrained by the thickness of the blade in this region, not allowing the tubes to be placed closer to the trailing edge than $x / c h=0.8$. Seven silicone tubes of $2 \mathrm{~mm}$ in diameter are connected to each of the brass tubes via a brass reducer and led through the stub of the blade into the fan hub. These tubes are then ultimately connected to a custom manufactured aluminium capsule (figures 3.5a, b), housing seven piezo-resistive pressure transducers (Freescale MPX 2010, figure 3.5c), one for each tap to be measured.

During test measurements, the capsule is fixed to the fan shaft and also 


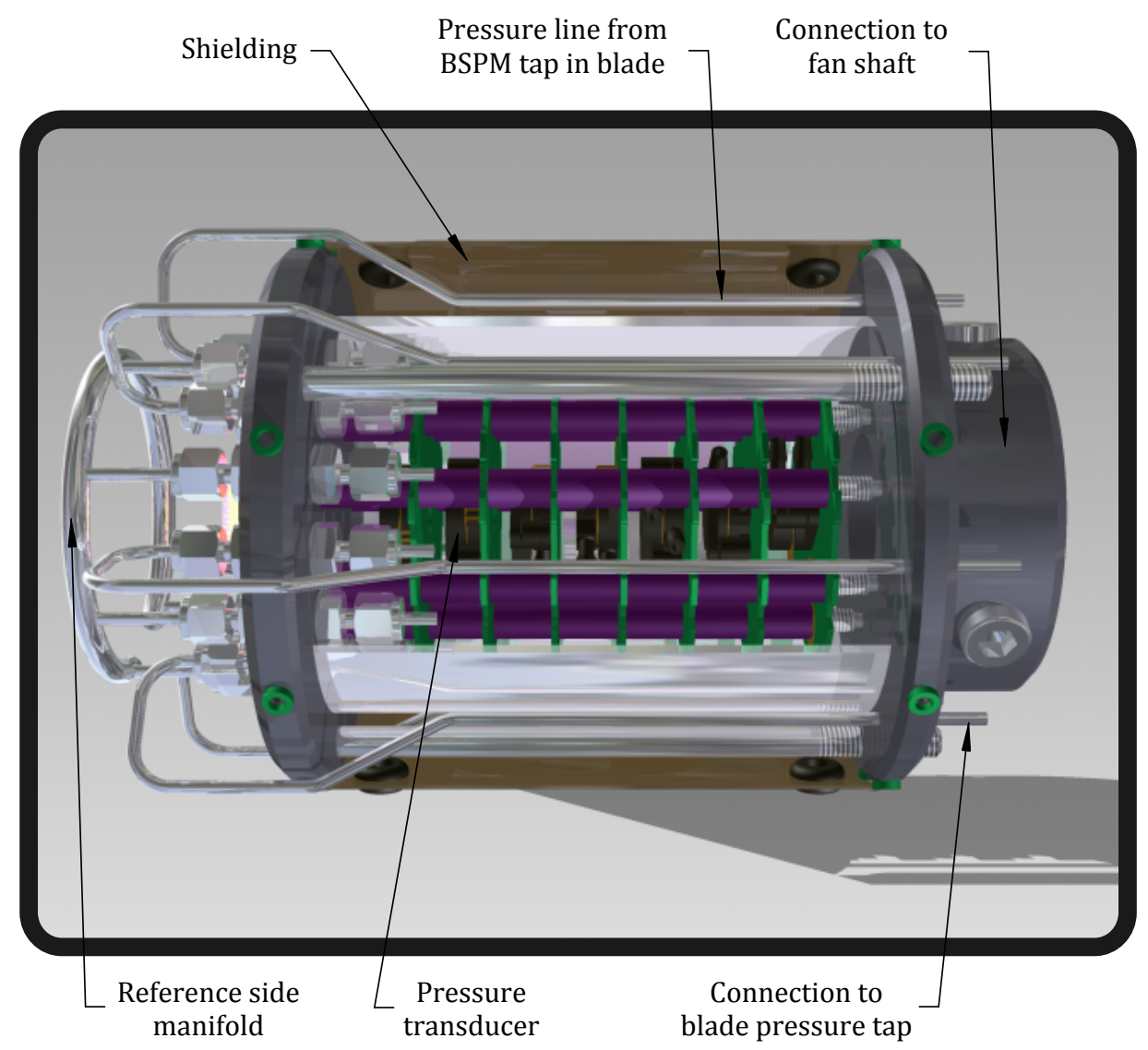

(a)

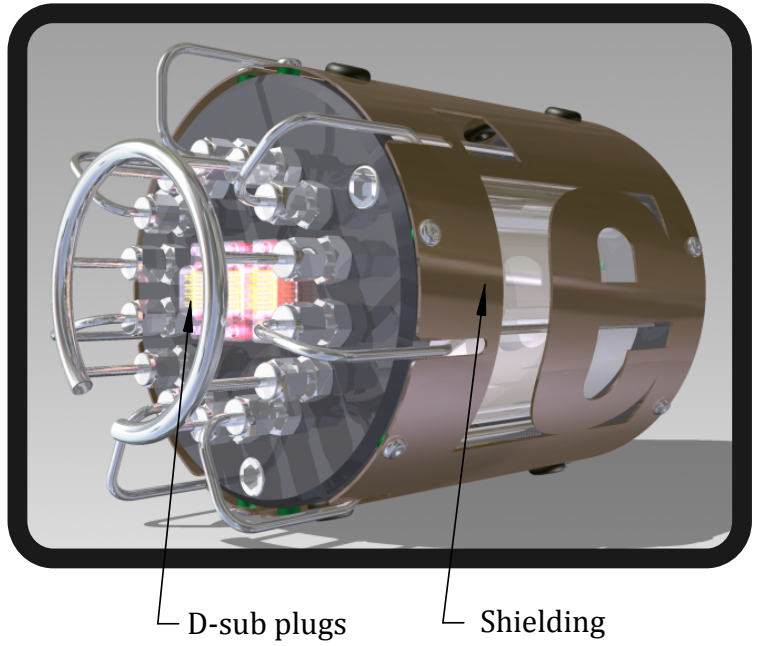

(b)

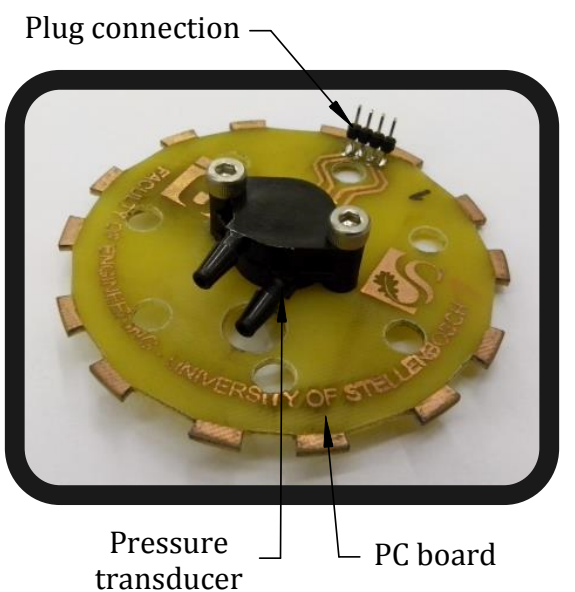

(c)

Figure 3.5: A sectional view a) and isometric view (b) of the BSPM aluminium capsule housing seven (c) Freescale MPX 2010 pressure transducers 


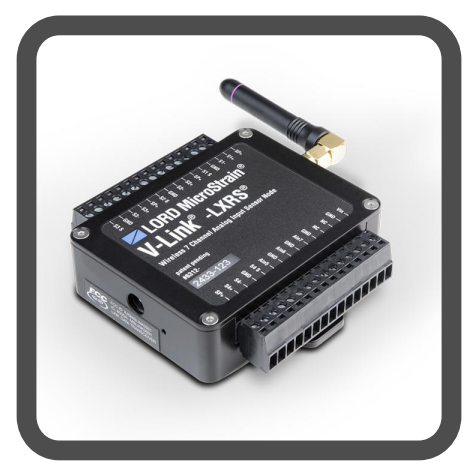

(a)

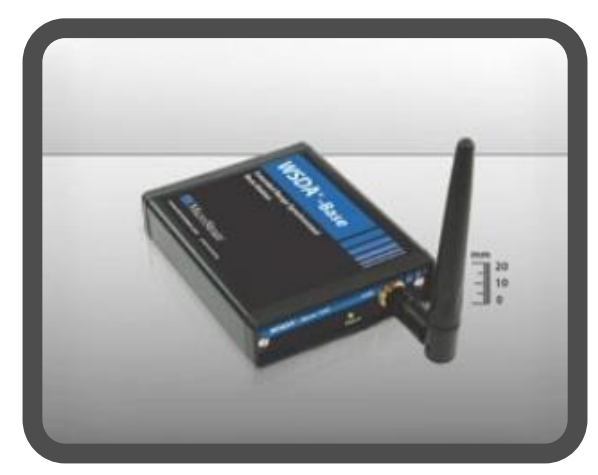

(b)

Figure 3.6: (a) LORD MictroStrain V-link and (b) LORD MictroStrain WSDA-Base-101

rotates at fan speed. Due to this rotation, the membranes of the transducers mounted inside the capsule would inevitably also be deformed by centrifugal forces, giving an error in the measured pressure if it is not accounted for. The pressure transducers are therefore mounted such that their membranes are located on and perpendicular to the axis of rotation in an attempt to minimize the offset in measured results due to centrifugal loading.

These transducers measure a differential pressure and consequently have two ports on either side of the membrane, which will be referred to as the tap and reference sides, respectively. Pressure lines leading from the blade surface pressure taps are connected to the tap side of the transducers, whereas the reference sides of each transducer is connected to a manifold on the end of the capsule. This manifold, in turn, is connected to a $2 \mathrm{~m}$ long stainless steel tube that is mounted on bearings and leads into the plenum chamber of the test facility.

To conduct wireless measurements, telemetry equipment supplied by LORD MictroStrain is used. The seven pressure transducers are connected to two modules (LORD MictroStrain V-link, figure 3.6a) fixed on the fan hub. These modules are operated wirelessly (as shown in the line schematic of figure 3.7) through a network that is set up by a transponding base station (LORD MictroStrain WSDA-Base-101, figure 3.6b) connected to a PC/Laptop and serve the following purposes: Firstly, the modules provide an excitation of $3 \mathrm{~V}$ to the pressure transducers and secondly amplify and save the measured signal from the transducers based on the calibrations spe-cified by the user. After a complete measurement run (measurements for a range of volumetric flow rates), the measured data can be downloaded via the wireless network for further analyses.

Error and repeatability analyses are also conducted for the instrumentation and measurement procedure. These can be obtained in appendix C. 


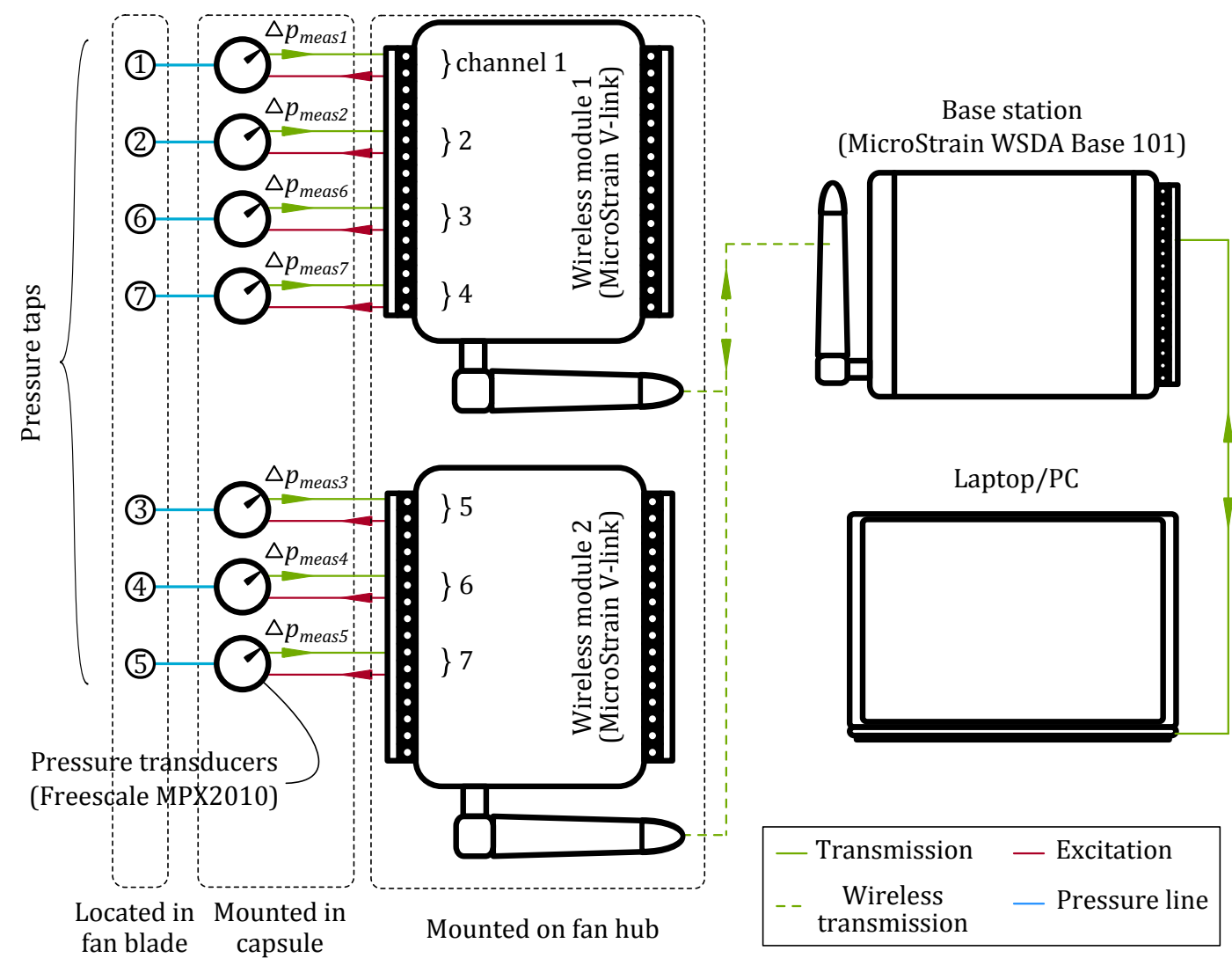

Figure 3.7: Line schematic of the blade surface pressure measurement communication

\subsubsection{Measurement procedure}

Before the measurement procedure commences, the capsule is mounted onto the fan shaft, upstream of the fan as shown in figure 3.3b. The electronics are connected to the capsule by three D-Sub plugs on the upstream end of the capsule. The system is then checked by means of live streaming from the modules to verify that all transducers are connected and responding correctly.

The measuring procedure then commences by firstly acquiring a zero pressure reading $\left(\Delta p_{z e r o}\right)$ for each transducer as described in appendix D.2.2. Subsequently the fan is stopped and connections between each transducer's tap side and the corresponding pressure taps are made. The reference sides of the transducers are connected to the stainless steel tube via the manifold, which leads into the plenum chamber of the test facility. The total pressure inside the plenum chamber $\left(p_{t o, p l e n}\right)$ therefore serves as the reference pressure for all the transducers during measurements. Finally the nose fairing is fastened to the fan hub, enclosing the capsule and all the electronic equipment.

In order to measure the pressure on one specific radius, the taps on all other radii are closed using clear adhesive tape. The fan is then once again brought to the operating speed of $750 \mathrm{rpm}$ and the test facility's flow control louvres set to the desired flow rate. For a certain flow rate, data acquisition 
of the pressure on the fan blades is wirelessly triggered by the operator from a $\mathrm{PC} /$ Laptop and stored onto the wireless modules at a sampling rate of $1 \mathrm{kHz}$ for $10 \mathrm{~s}$ (10000 data points in total for 125 fan rotations at a rate of 80 data points per rotation). Simultaneously, the four measurements pertaining to the fan characteristic test (as explained in section 3.1.1) are also made using the instrumentation of the test facility. The acquisition is then repeated for a range of flow rates comprising a test run. After each test run a second zero reading is taken in the same manner as described previously. The data on the wireless modules are then downloaded for further analysis. Subsequently the measurement procedure is repeated for a different radius on either the suction or pressure side of the measurement fan blades.

\subsubsection{Data processing}

The measured blade surface pressure data needs to be processed in order to obtain the correct static blade surface pressures $\left(p_{s, t a p}\right)$ for each tap being measured. The processing of the data addresses the following:

1. Offset in measured data due to centrifugal loading on the membrane. Although it is attempted to minimize this effect by means of transducer membrane placement and orientation as discussed in section 3.2.1, the offset in measurements is inevitable.

2. Offset in measured data due to centrifugal loading on the air enclosed in the pressure lines between the taps in the blade and the capsule. This loading leads to a radial pressure distribution inside the pressure lines and consequently additional suction occurs on the tap sides of the membranes, affecting the measured pressure.

3. Interpolation to obtain the measured values for specific volumetric flow rates.

A derivation of the radial pressure distribution in a rotating tube is shown in appendix D.2.1. Using the derived equation and taking the zero offsets in measured blade surface pressure (mentioned above) into account, the static pressure for each tap on the blade surface can be calculated from the measured values by implementation of:

$$
p_{s, t a p}=\mathrm{e}^{\omega^{2} r_{\text {tap }}^{2} / 2 R T}\left[\Delta p_{m}+p_{\text {to,plen }}+p_{\text {atm }}-\Delta p_{\text {zero }}\right]-p_{\text {atm }} \quad[\mathrm{Pa}]
$$

as derived in appendix D.2.2. In equation 3.2.1, omega is the rotational speed of the fan $(\mathrm{rad} / \mathrm{s}), r$ is the radius of the tap, $R$ the specific gas constant of air $(\mathrm{J} / \mathrm{kg} \mathrm{K})$ and $T$ the atmospheric temperature $(\mathrm{K})$. The pressures $\Delta p_{m}$, $p_{t o, p l e n}$ and $\Delta p_{\text {zero }}$ refer to the pressure measured by the transducer, the total 
pressure inside the plenum chamber of the fan test facility and the zero pressure measurement. $p_{\text {atm }}$ is the absolute atmospheric pressure.

Lastly, since the test facility only allows the measurement of set volumetric flow rates, interpolation is used to obtain values for desired volumetric flow rates. The interpolation procedure is discussed in appendix D.2.3. 


\section{Chapter 4}

\section{Periodic three-dimensional numerical model (P3DM) and technique}

Flow field data in the vicinity of the B2a-fan are required for further analyses. Such information at low flow rates, especially in the form of velocity measurements, are difficult and expensive to measure (as discussed in chapter 3), particularly due to the unstable nature of the flow field at low flow rates. Since a large amount of flow field information is more easily obtainable through numerical computation compared to experiments, this attribute is exploited to obtain the desired data.

In the present study numerical analyses of the flow field in the vicinity of the eight bladed B2a-fan is conducted to:

Prove numerical fan modelling feasibility. The characteristic performance of a fan is normally determined by means of standardized testing, requiring the construction of a physical fan model. Therefore, if an accurate prediction of the fan induced flow field could be obtained numerically, these results would also be valid to determine the specific fan's performance characteristics without experimentation.

Understand governing fan induced flow structures. The large amount of information obtained by numerical simulation is analysed to understand the existing flow structures that govern a fan's performance, of which low flow rates in the present study are of particular interest.

Develop a simplified numerical fan model. As stated in the introductory chapters, the ultimate goal is the development of a simplified fan model that would be able to accurately predict fan performance at design as well as low flow rates.

Computational fluid dynamics (CFD) simulations are conducted to obtain flow field solutions for a range of flow rates in the vicinity of the B2a-fan, but 
CHAPTER 4. PERIODIC THREE-DIMENSIONAL NUMERICAL MODEL (P3DM) AND TECHNIQUE

especially at low flow rates, where the unstable flow conditions are difficult to model. These analyses are conducted for an open-inlet to open-outlet case (simulating the BS 848, type A experimental fan performance tests).

To save on computational expenses, frozen rotor analyses are performed, considering only an $1 / 8^{\text {th }}$ segment of the fan (one blade). The model is conveniently termed the periodic three-dimensional model (P3DM) for the remainder of this document. With this technique it is assumed that the flow condition is rotationally symmetric, which is not necessarily accurate as phenomena such as rotating stall patterns could occur in the fan blade cascade near the hub, especially at low flow rates. However, its use is justified by the fair comparisons found at these flow conditions with the rotationally symmetric model, as will be presented in chapter 5 .

Using this model, it is found that steady-state analyses provide converged solutions for flow rates near and higher than the design flow rate $(13 \leq \dot{V} \leq$ $\left.19 \mathrm{~m}^{3} / \mathrm{s}\right)$. However, time-dependent analyses are required for lower flow rates of $\left(\dot{V}<13 \mathrm{~m}^{3} / \mathrm{s}\right)$.

All of the modelling and grid constructions are conducted using the ANSYS 14.0 Workbench software package. Unless otherwise stated all the software programs used in the sections that follow, are incorporated in the ANSYS 14.0 package. Simulations are run on the Rhasatsha high-performance computer (hpc) facility at Stellenbosch University using the CFD solver of Fluent. A single simulation is typically run in parallel on eight processors of 2.1-3.0 $\mathrm{GHz}$ each, using approximately $15 \mathrm{~Gb}$ of random access memory (RAM). Steady-state analyses took between one to two days to complete, whereas time-dependent analyses only converged after two to four weeks of computation.

For the sake of brevity, only a summary of the computational model and the modelling technique are presented in this chapter. Analyses for grid and time-step sensitivity and the effect of domain boundary proximity relative to the fan rotating plane are presented in appendix $\mathrm{E}$ and also published in (Louw et al., 2014a). The construction of the computational domain and grid setup is discussed in section 4.1. Thereafter, sections 4.2 and 4.3 elaborate on the specific turbulence models and solver settings used.

\subsection{Computational domain}

The computational domain for the P3DM and boundary types is shown in figure 4.1 and consists of three sub-domains, individually referred to as the Inlet, Rotor and Outlet sub-domains. Sensitivity analyses are conducted to obtain the grid density and domain size which can be viewed in appendix E. Of the three sub-domains, the Rotor's construction and grid setup is different to the other two and therefore focus will firstly be placed on this sub-domain in section 4.1.1. Subsequently, the construction of the Inlet and Outlet sub-domains 
CHAPTER 4. PERIODIC THREE-DIMENSIONAL NUMERICAL MODEL (P3DM) AND TECHNIQUE

are discussed in section 4.1.2. Finally, the assembly of the three domains and the boundary conditions are explained in section 4.1.3.

\subsubsection{Rotor sub-domain construction}

The Rotor sub-domain, presented in figure 4.2, comprises an annular section that encloses the blade profile and is located between the Inlet and Outlet subdomains. Before this sub-domain is created, it is firstly required to construct a computer-aided design (CAD) model of the blade profile. This is obtained by importing the blade design coordinates into Autodesk Inventor 13 (not part of the ANSYS 14.0 package), where a solid model of the blade is constructed and the geometry exported in a parasolid or initial graphics exchange specification (IGES) format. Subsequently the blade geometry is imported to ANSYS Design Modeller, where the annular domain (figure 4.2) is constructed around the blade using design tools made available through CFX Turbogrid.

A structured hexagonal type computational grid is used in the Rotor subdomain, constructed using CFX Turbogrid. Since CFX Turbogrid is specifically tailored to construct grids for turbo-machinery, a fairly smooth grid is obtained, where the cells are aligned with the general direction of the relative flow field around the blade. Cells on the blade surface are constructed such, to give $y^{+}$values of $30<y^{+}<300$ to accommodate using the Standard wall function (Launder and Spalding, 1974). The number of cells required in this sub-domain to provide converged flow field results is established through a sensitivity analysis presented in appendix E.1. Consequently it is determined that a cell count of approximately $1(10)^{6}$ in the Rotor sub-domain is sufficient for further analyses.

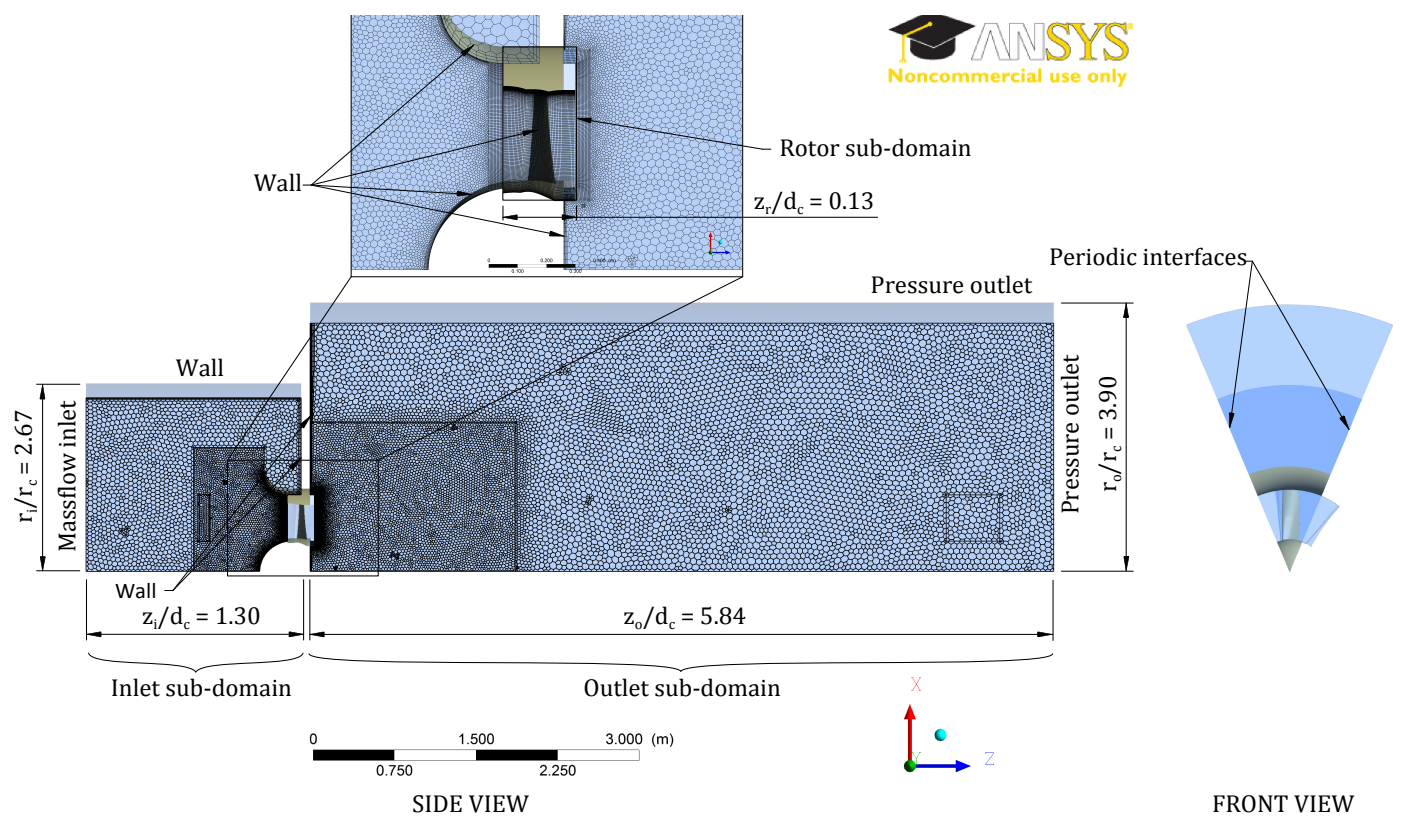

Figure 4.1: Computational domain of the P3DM 
CHAPTER 4. PERIODIC THREE-DIMENSIONAL NUMERICAL MODEL (P3DM) AND TECHNIQUE

\subsubsection{Inlet and Outlet sub-domain construction}

The Inlet and Outlet sub-domains are both constructed using ANSYS Design Modeller and Mesher. The Inlet and Outlet are both wedge shaped at an angle of $\theta_{\text {sect }}=45^{\circ}$, representing an $1 / 8^{\text {th }}$ sector of the fan. As depicted in figure 4.3, the domains are constructed with the same annulus radii where it fits onto the upstream and downstream sides of the Rotor sub-domain. The sub-domain boundary proximities are determined through sensitivity analyses as shown in section E.2. Through these analyses it is firstly determined that inlet and outlet axial respective lengths of $z_{i} \leq 1.30 d_{c}$ and $z_{o} \leq 4.54 d_{c}$ fan diameters from the fan rotation plane provide converged flow solutions for the design flow rate $\left(\dot{V}_{D}=16 \mathrm{~m}^{3} / \mathrm{s}\right)$. Hereafter grid size analyses are conducted at both the design and lowest flow rates of $\dot{V}=16 \mathrm{~m}^{3} / \mathrm{s}$ and $4 \mathrm{~m}^{3} / \mathrm{s}$ as discussed in section E.3, which showed that a computational grid size of $2.5(10)^{6}$ is sufficient to obtain converged results.

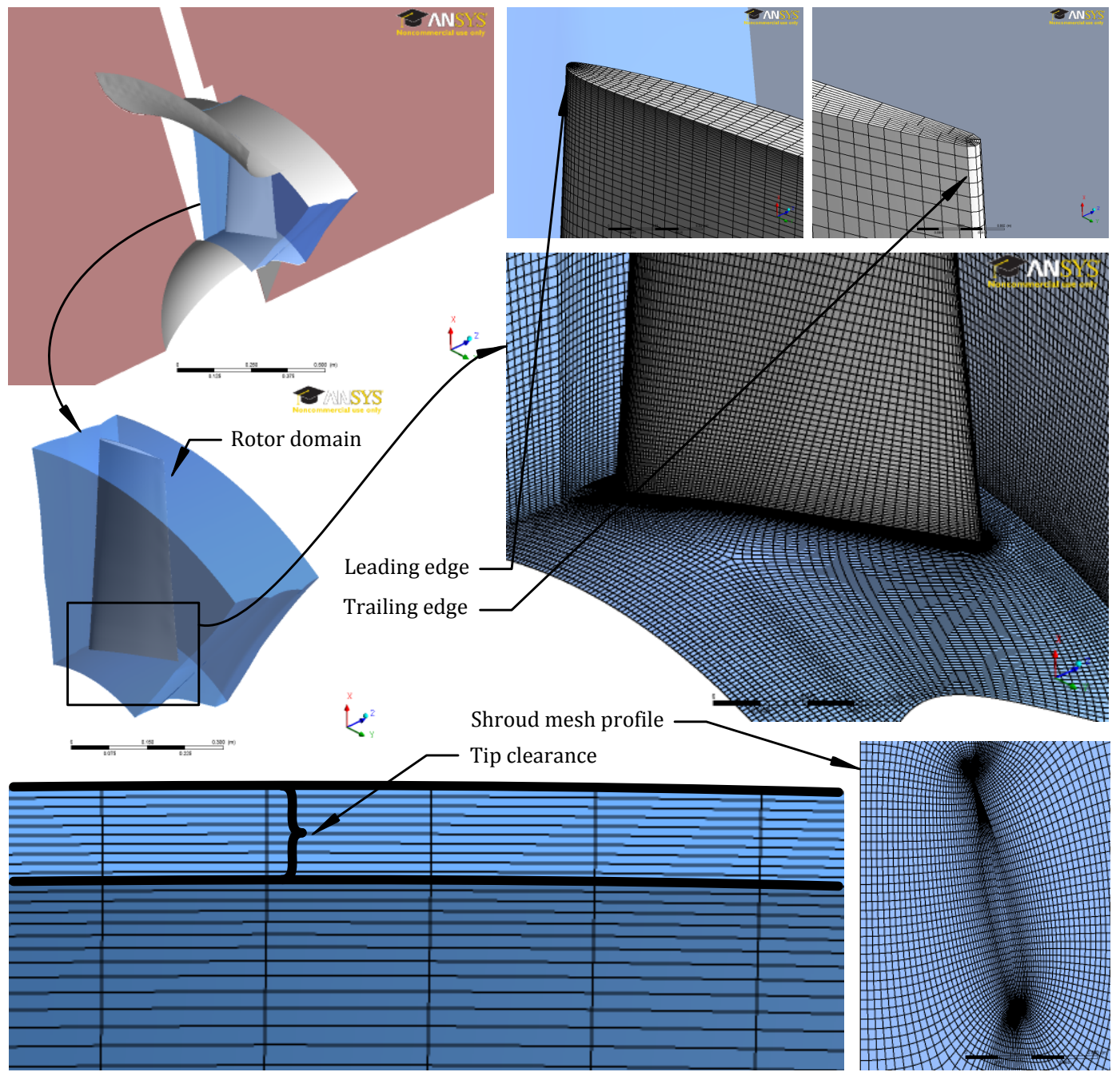

Figure 4.2: The Rotor sub-domain shape and grid structure 


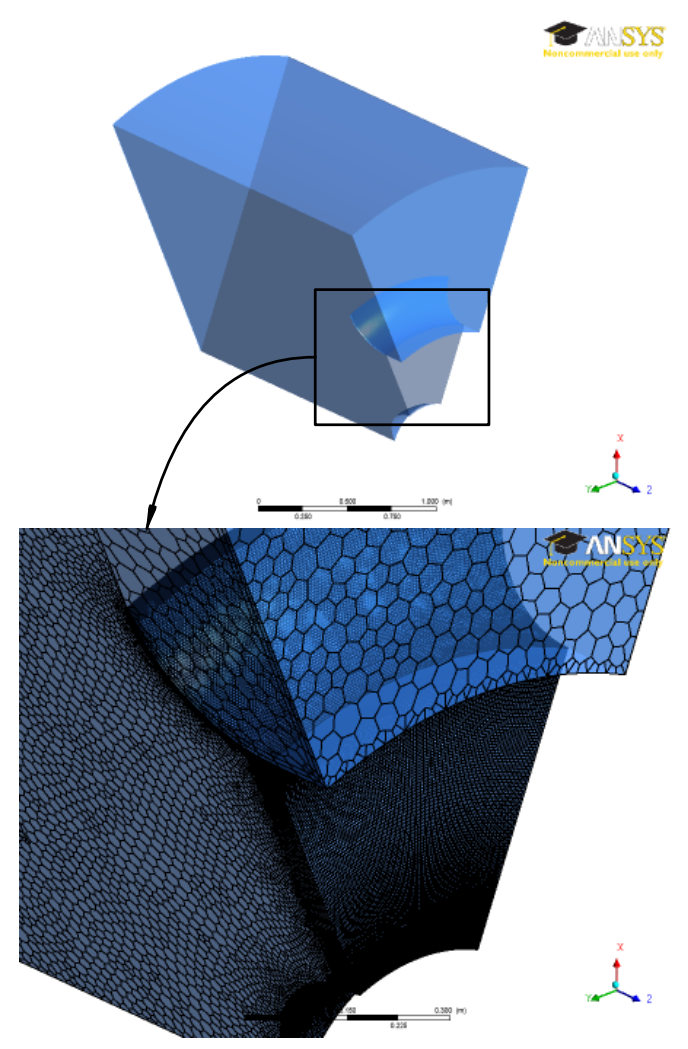

(a)

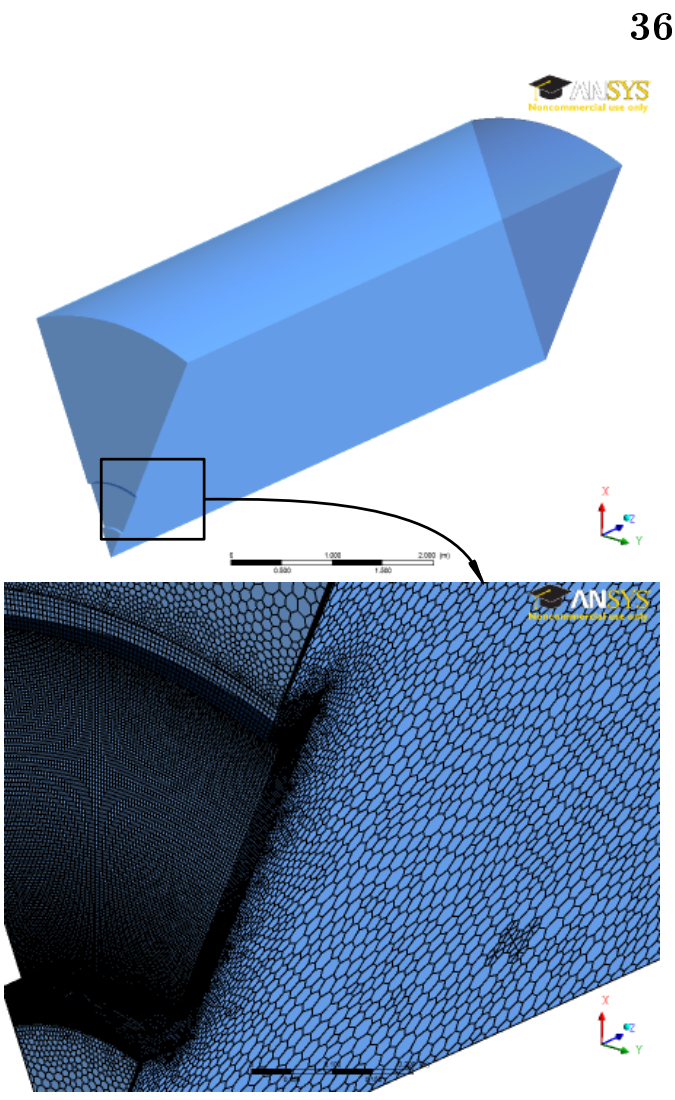

(b)

Figure 4.3: The (a) Inlet and (b) Outlet sub-domains showing the domain shape and grid structure

Unstructured polyhedral grids are used to enable a variable density of cells throughout the computational domains. The cell sizes in these sub-domains are specified to not be larger than $0.02 \mathrm{~m}$ (along the side of a specific cell) in the dense region immediately up- and downstream of the Rotor sub-domain and to be not larger than $0.04 \mathrm{~m}$ in the remainder of the sub-domains. The transition between these cells' sizes are set to "soft" in the ANSYS mesher to ensure a smooth transition between these regions. The grids are firstly set up using Design Mesher by constructing tetrahedral cells, since the option to construct polyhedral cells in this software package is not available. This option only becomes available when the computational grid is imported into Fluent, where the tetrahedral cells are converted to polygons. One of the advantages of polyhedral grids is that it provides an unstructured grid that is similar in accuracy to structured hexahedral grids, but generally leads to a considerable reduction (factor of two) in the number of cells, compared to tetrahedral cells (Lanzafame et al., 2013). Grids on the surfaces of wall boundaries, such as the nose fairing, hub and bellmouth are constructed using prism layers.

The advantage of the unstructured polyhedral grid is exploited in the present study to obtain a high density of cells in the vicinity of the interfaces between the sub-domains. The cell density then gradually reduces toward the 


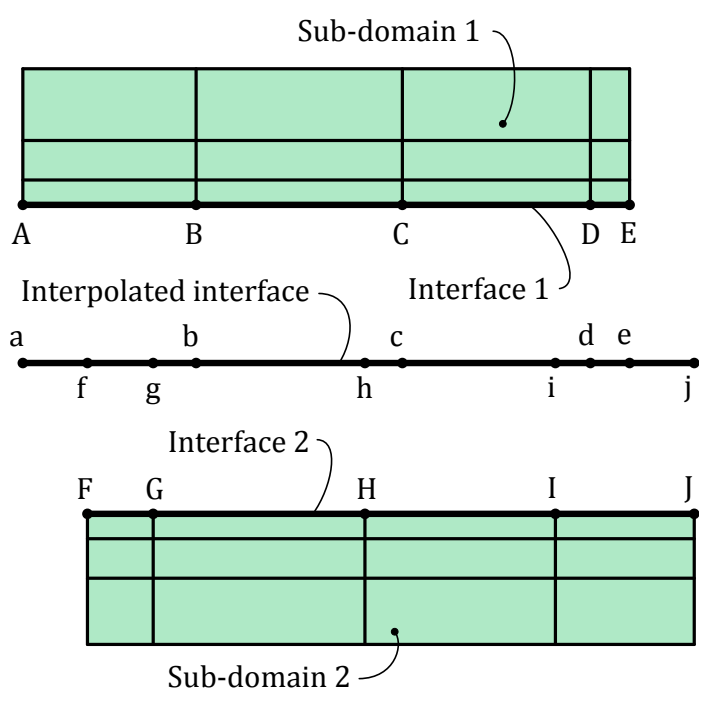

(a)
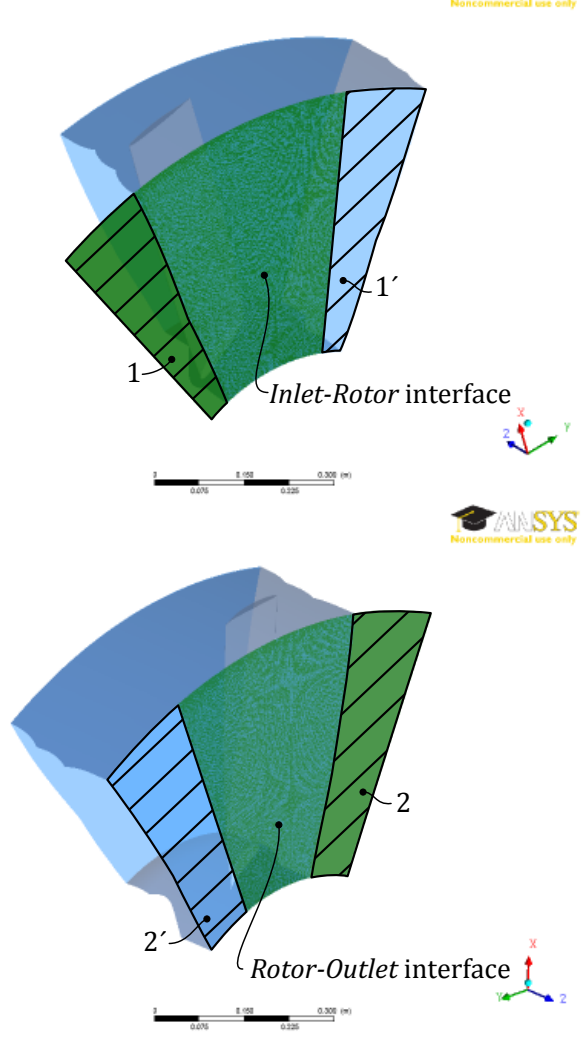

(b)

Figure 4.4: (a) A two-dimensional depiction of the method used by Fluent to create an interpolated interface between two non-conformal grid interfaces for the transfer of flow variables and (b) the linkage between the Inlet-Rotor and Rotor-Outlet sub-domain interfaces

domain outer extents, where little fluctuation in the flow merits the use of a coarser grid. To minimize averaging errors across the sub-domain interfaces, the number of cells on opposite sides are generated to be of the same amount.

\subsubsection{Domain assembly and boundary conditions}

Upon completion of the sub-domain construction, these domains are imported into Fluent and linked together to obtain the domain shown in figure 4.1. Two features of the interfaces between the sub-domains need to be addressed before continuing. These are:

Grid non-conformity. Since the grid type of the Rotor differs from the other two sub-domains, non-conformal interfaces exist at the Rotor up- and downstream boundaries. Due to the difference in the amount of grid increments on the blade suction and pressure sides, a misaligned cell interface also exists between these sides in the region of the tip clearance 
CHAPTER 4. PERIODIC THREE-DIMENSIONAL NUMERICAL MODEL (P3DM) AND TECHNIQUE

(figure 4.2). These interfaces are linked in Fluent by specification. Fluent then creates a new interface using a virtual polygon approach (as described in (ANSYS Fluent Inc, 2009)), by placing nodes on the new interface as shown in figure 4.4a. The solver ignores the individual interfaces of the two (or more) adjacent sub-domains (figure 4.4a) and uses the new interface to facilitate the flow of numerical variables from one subdomain to the next. Since averaging errors due to interpolation across the interfaces is inevitable, it is attempted to create the same amount of grid points on the interfaces of the adjacent sub-domains, essentially minimizing this error.

Rotor tangential offset. Although the Rotor also consists of an $1 / 8^{\text {th }}$ fan sector, this sub-domain is offset in the tangential direction from the other two sub-domains. This offset is inevitable since the meshing algorithm in CFX Turbogrid determines the Rotor domain shape and position. When the interfaces are linked up, flow across the overlapping parts continues through as if unobstructed, but flow across the non-overlapping interfaces is accounted for by linking these 1 to $1^{\prime}$ and 2 to $2^{\prime}$ as shown in figure $4.4 \mathrm{~b}$. For example, the flow moving out of the Inlet sub-domain at 1, enters the Rotor sub-domain at $1^{\prime}$, since the computational domain is rotationally symmetric.

The simulation is solved in the moving reference frame and therefore a rotational rate of $N=750 \mathrm{rpm}$ is assigned to the computational domain. This is done since a more stable solution is observed compared to when simulations are conducted in a static reference frame. The following are specified for the applicable boundary conditions (indicated in figure 4.1):

- A Mass flow inlet boundary introduces a uniform inlet velocity profile, based on the specified mass flow rate, inlet area and density values.

- Pressure outlet boundaries are located on the domain outlet where a static pressure of zero is specified.

- The fan blade, nose fairing and hub surfaces are modelled assuming smooth Wall boundary conditions and that no slip of the fluid occurs. A rotational speed of $N=750 \mathrm{rpm}$ is also specified relative to the rest of the computational domain.

- Wall boundary conditions are also assigned to the inlet bellmouth, and as indicated for the Inlet sub-domain as shown in figure 4.1. All of these boundaries are static relative to the rest of the computational domain, but only the bellmouth are specified with a no-slip condition. The other two boundaries are defined as slip walls, since they would not exist in reality and only provide a conduit to channel the flow into the fan. 
CHAPTER 4. PERIODIC THREE-DIMENSIONAL NUMERICAL MODEL (P3DM) AND TECHNIQUE

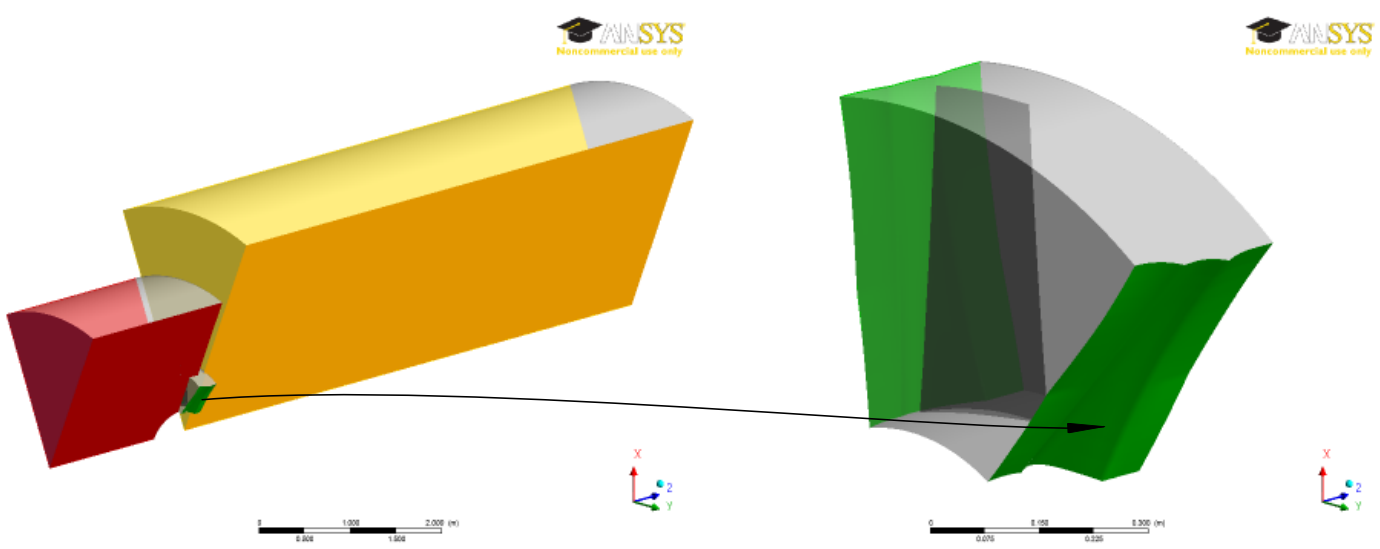

Figure 4.5: Periodic boundary pairs in the computational domain (paired by colour)

- Periodic boundaries are placed on the sides of the domain as indicated and linked to each other as shown in figure 4.5.

Turbulence intensity levels of $10 \%$ and a hydraulic diameter of $1 \mathrm{~m}$ are specified at the domain extents. Since, the turbulence levels far away from the fan (similar to the domain extents) would have a small effect on the solution near the fan rotor, the choice of turbulence levels would have a small effect on the final result. However, a turbulence intensity of $1 \%$ would have been a more appropriate choice since this is discovered to be the relevant case for the test facility at Stellenbosch University.

\subsection{Turbulence modelling}

A Reynolds-averaged Navier Stokes (RANS) modelling approach is used in the present investigation to solve the flow field. This approach is used since a direct numerical simulation is yet still computationally impractical for more complex geometries such as fans. A RANS approach also requires less computational resources compared to models such as Large eddy simulation (LES) and Detached eddy simulation (DES) (Versteeg and Malalasekera, 2007).

Four different turbulence approaches are investigated to determine which model would provide a suitable means to simulate the flow in the vicinity of the fan. These models are given below and compared in table 4.1:

- Model 1: Realizable $k$ - $\varepsilon$ turbulence model (Shih et al., 1994) with Standard wall function (Launder and Spalding, 1974) (2 equations)

- Model 2: Realizable $k$ - $\varepsilon$ turbulence model with Enhanced wall function (2 equations)

- Model 3: Transition $k$ - $k l-\omega$ turbulence model (Walters and Cokljat, 2008) (3 equations) 
CHAPTER 4. PERIODIC THREE-DIMENSIONAL NUMERICAL MODEL (P3DM) AND TECHNIQUE

- Model 4: Transition Shear Stress Transport (SST) turbulence model (Menter et al., 2003) (4 equations)

Table 4.1: Turbulence models and predicted fan static pressure for the design $\left(\dot{V}=16 \mathrm{~m}^{3} / \mathrm{s}\right)$ and the lowest $\left(\dot{V}=4 \mathrm{~m}^{3} / \mathrm{s}\right)$ flow rates

\begin{tabular}{lll}
\hline Turbulence model & $\boldsymbol{\Delta} \boldsymbol{p}_{\boldsymbol{F s}, \text { Des }(\boldsymbol{P a})}$ & $\boldsymbol{\Delta} \boldsymbol{p}_{\boldsymbol{F s}, \mathbf{L o w}(\boldsymbol{P a})}$ \\
\hline Model 1 & 184 & 382 \\
Model 2 & 185 & \\
Model 3 & 203 & 391 \\
Model 4 & 197 & \\
Experimental & 200 & 405 \\
\hline
\end{tabular}

Of these, the Realizable $k$ - $\varepsilon$ turbulence model with the Standard wall function required the least amount of computational expense and also provided fair results compared to those obtained experimentally, as presented in chapter 5 . The other three models required a finer computational grid at the walls $\left(y^{+}<5\right)$ to solve the boundary layer, almost doubling the amount of cells in the computational domain, but provided similar results compared to the Realizable $k-\varepsilon$ model as can be seen in the comparison of numerically obtained fan characteristics presented in figure 4.6. Time-dependent simulations are also conducted for some lower flow rates $\left(\dot{V}<13 \mathrm{~m}^{3} / \mathrm{s}\right)$ using the Transition $k$-kl- $\omega$ model which also produced similar time-averaged results compared to those using the Realizable $k-\varepsilon$ model.

The Realizable $k-\varepsilon$ model differs from the Standard $k-\varepsilon$ in that it has a different formulation for turbulent viscosity $\left(\mu_{t}\right)$ and the dissipation rate $(\varepsilon)$ transport equation. The Realizable $k-\varepsilon$ model more accurately predicts flow fields where adverse pressure gradients, separation and rotating flow occur (Shih et al., 1994), compared to the Standard model. The model also remains a two-equation model and therefore requires less computational power compared to turbulence models with more equations. Hence, the Realizable $k$ - $\varepsilon$ model with Standard wall function is used for the simulation of the flow field in the vicinity of the fan. The model equations, together with the RANS equations are presented in appendix $\mathrm{F}$.

\subsection{Solver settings}

Solver settings and discretization methods used are indicated in table 4.2. These settings are discussed: 
CHAPTER 4. PERIODIC THREE-DIMENSIONAL NUMERICAL MODEL (P3DM) AND TECHNIQUE

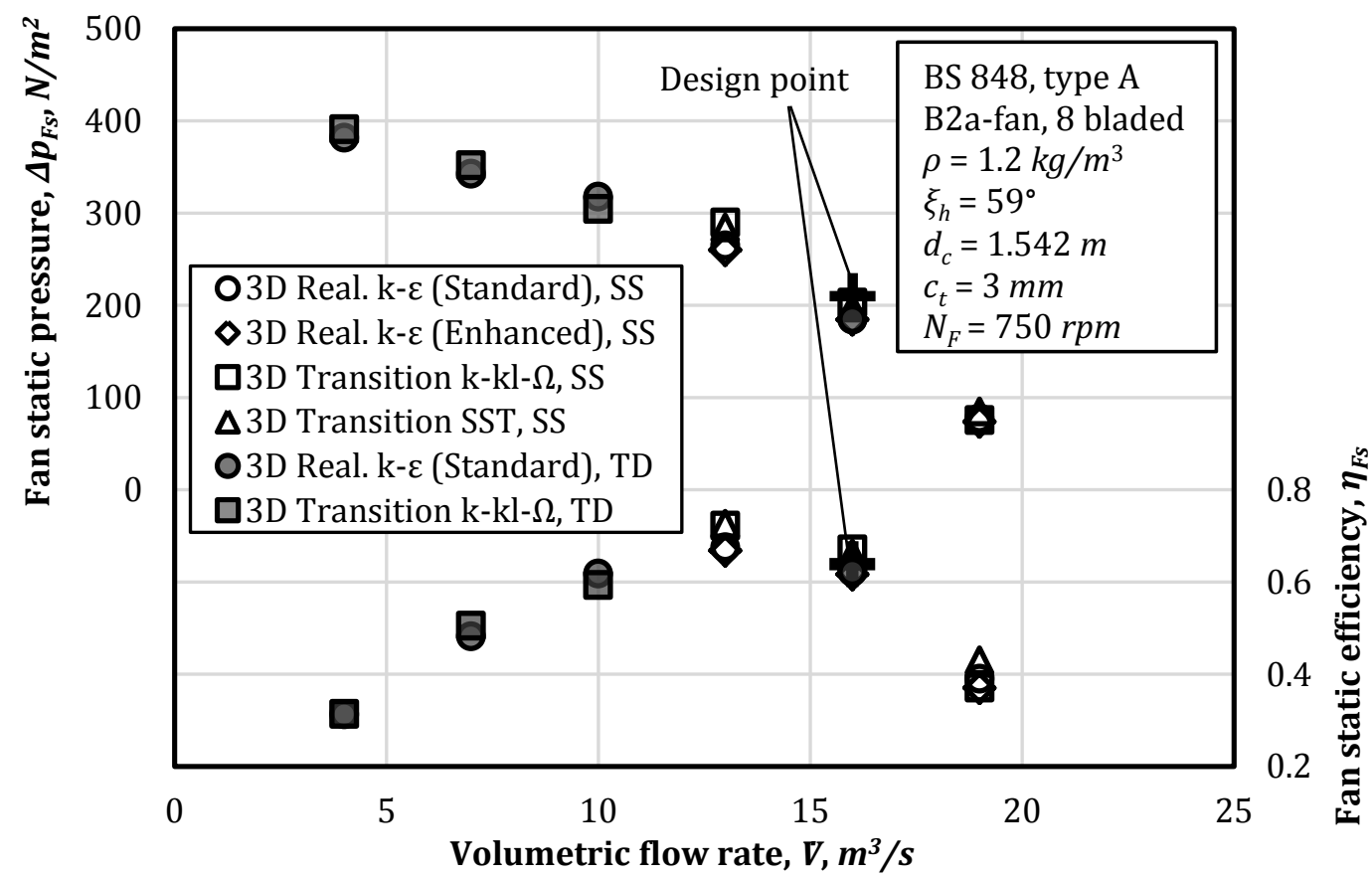

Figure 4.6: Fan static pressure and static efficiency obtained by four different methods of turbulence modelling, for steady state (SS) and time-dependent (TD) analyses

Table 4.2: Numerical solver settings

\begin{tabular}{|c|c|c|}
\hline Setting & Steady-state & Time-dependent \\
\hline $\begin{array}{l}\text { Discretization scheme } \\
\text { (Gradient) }\end{array}$ & Least squares cell based & Least squares cell based \\
\hline $\begin{array}{l}\text { Discretization scheme } \\
\text { (Pressure) }\end{array}$ & PRESTO! & PRESTO! \\
\hline $\begin{array}{l}\text { Discretization scheme } \\
\text { (Other) }\end{array}$ & $\begin{array}{l}\text { QUICK } / 2^{\text {nd }} \text { order up- } \\
\text { wind }\end{array}$ & $\begin{array}{l}\text { QUICK } / 2^{\text {nd }} \text { order up- } \\
\text { wind }\end{array}$ \\
\hline $\begin{array}{l}\text { Pressure-velocity } \\
\text { coup-ling }\end{array}$ & SIMPLE & PISO \\
\hline Convergence & $10^{-5}$ & $10^{-3}$ \\
\hline
\end{tabular}

Each simulation is started on a first-order upwind differencing scheme setting and solved until the simulation is nearly converged. A higher order accuracy is then obtained by continuing the simulations using the QUICK $/ 2^{\text {nd }}$ order upwind scheme. The QUICK scheme calculates the values of variables on the cell boundaries of hexahedral cells using a weighted average of the second order upwind scheme and the interpolation of the variable from centre of the cell. This approach results in good accuracies, especially for grids aligned 
CHAPTER 4. PERIODIC THREE-DIMENSIONAL NUMERICAL MODEL (P3DM) AND TECHNIQUE

with the flow, which is the case for the grid close to the blade profile. For the unstructured grids, Fluent resorts to the $2^{\text {nd }}$ order upwind scheme if QUICK is selected.

The least squares cell based scheme is chosen to calculate the gradients of the flow variables, since it provides a fair accuracy for unstructured grids and requires less computational power compared to the Green-Gauss cell based scheme, also available in Fluent. The pressure-velocity coupling for all steady simulations is calculated using the SIMPLE algorithm. For time-dependent simulations, PISO is used, because these algorithms provided a converged solution. PRESTO! is used for the discretization of pressure, since it is suited for flows with high pressure gradients and swirl (Versteeg and Malalasekera, 2007). 


\section{Chapter 5}

\section{Periodic three-dimensional numerical model (P3DM) verification}

The results of the experiments discussed in chapter 3 are used to validate the periodic three-dimensional model (P3DM) presented in chapter 4 and are also published in Louw et al. (2015b). To extract and analyse the necessary data from the numerical results, an automated method is required, since manual sampling of the large volume of data would be impractical. For this purpose routines written in Python are used to sample and analyse specific numerical data from ANSYS 14.0 CFD Post. Line diagrams of these routines are given in appendix $\mathrm{G}$.

In order to compare the experimental and numerical results to each other for low flow rates $\left(4 \leq \dot{V} \leq 10 \mathrm{~m}^{3} / \mathrm{s}\right)$, a time-average of the time-dependent numerical data is required. This time-averaging technique is described in appendix G. In this chapter the compared results are depicted in the two sections that follow, being:

1. The comparison of experimentally and numerically obtained fan performance characteristics (fan static pressure and static efficiency curves).

2. The comparison of experimentally and numerically obtained blade surface static pressure distributions.

Finally, a discussion of these results follow in the last section of this chapter.

\subsection{Fan characteristic verification}

\subsubsection{Definition of variables}

As discussed in section 3.1.3 fan performance characteristics are represented by the fan static pressure $\left(\Delta p_{F s}\right)$ and fan static efficiency $\left(\eta_{F s}\right)$ as a function 
CHAPTER 5. PERIODIC THREE-DIMENSIONAL NUMERICAL MODEL (P3DM) VERIFICATION

of volumetric flow rate $(\dot{V})$. For the remainder of this dissertation the variables of fan static pressure difference and volumetric flow rate are presented in dimensionless form, expressed as fan static pressure and flow coefficients $\left(\Psi_{F s}\right.$ and $\phi$ ), respectively. These coefficients are calculated by:

$$
\begin{gathered}
\Psi_{F s}=\frac{\Delta p_{F s}}{0.5 \rho u_{c}^{2}} \\
\phi=c_{z, a v g} / u_{c}
\end{gathered}
$$

where $u_{c}$ is the circumferential speed of the blade tip and $c_{z, \text { avg }}$ the axial flow velocity through the fan annulus area $\left(A_{F}\right)$ calculated as:

$$
c_{z, a v g}=\frac{\dot{V}}{A_{F}} \quad[\mathrm{~m} / \mathrm{s}]
$$

\subsubsection{Comparison of results}

The experimentally and numerically obtained B2a-fan performance characteristics are presented in figures $5.1 \mathrm{a}$ and $\mathrm{b}$. In these figures, the lines representing the experimental average are third and fifth degree polynomials fitted through thirty seven sets of experimental data for fan static pressure and static efficiency, respectively. One experimental data set represents the data obtained in a single characteristic fan test run. The Pearson correlation $\left(R_{P}\right)$ and root mean square error (RMSE) between the experimental averages and all data sets are given in table 5.1.

Table 5.1: Pearson correlation $\left(R_{P}\right)$ and RMSE values presenting the quality of relation between the experimental data sets and average experimental curve fits for the B2a-fan performance characteristics

\begin{tabular}{lll}
\hline Variables & Pearson $\left(\boldsymbol{R}_{\boldsymbol{P}}\right)$ & RMSE \\
\hline$\Psi_{F s}$ & 0.997 & $3.375(10)^{-3}$ \\
$\eta_{F s}$ & 0.990 & $1.785(10)^{-2}$ \\
\hline
\end{tabular}

The numerical data shown in figures $5.1 \mathrm{a}$ and b consists of two groups. The first group, indicated in clear circles, is the data obtained through steadystate analyses. The second group, indicated in grey circles, is that obtained through time-dependent analyses, since steady-state data was not obtainable for these flow rates (except for $\phi_{D}=0.168$ where both transient and steady analyses are conducted). Apart from the time-dependent simulations at low 
CHAPTER 5. PERIODIC THREE-DIMENSIONAL NUMERICAL MODEL (P3DM) VERIFICATION

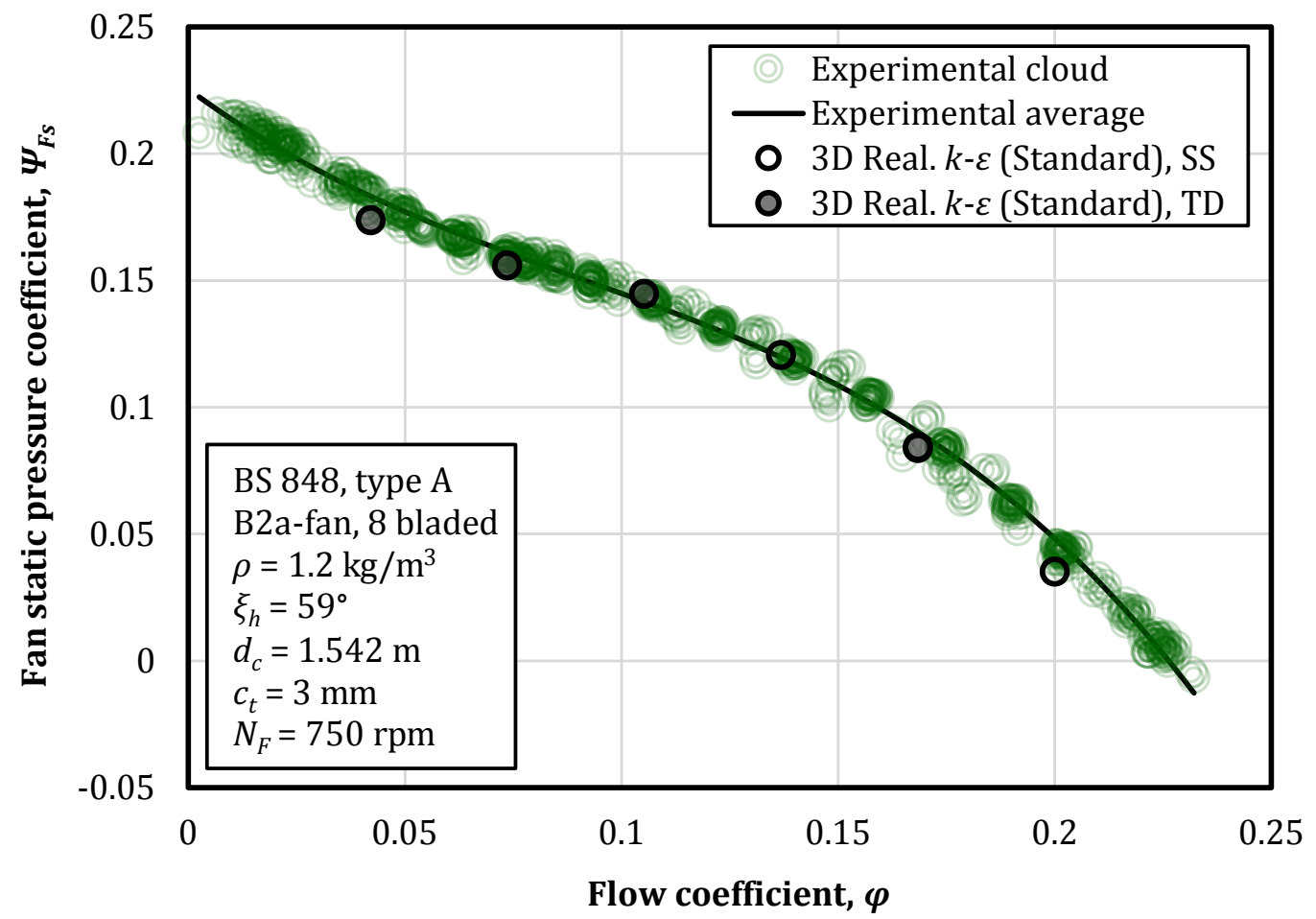

(a)

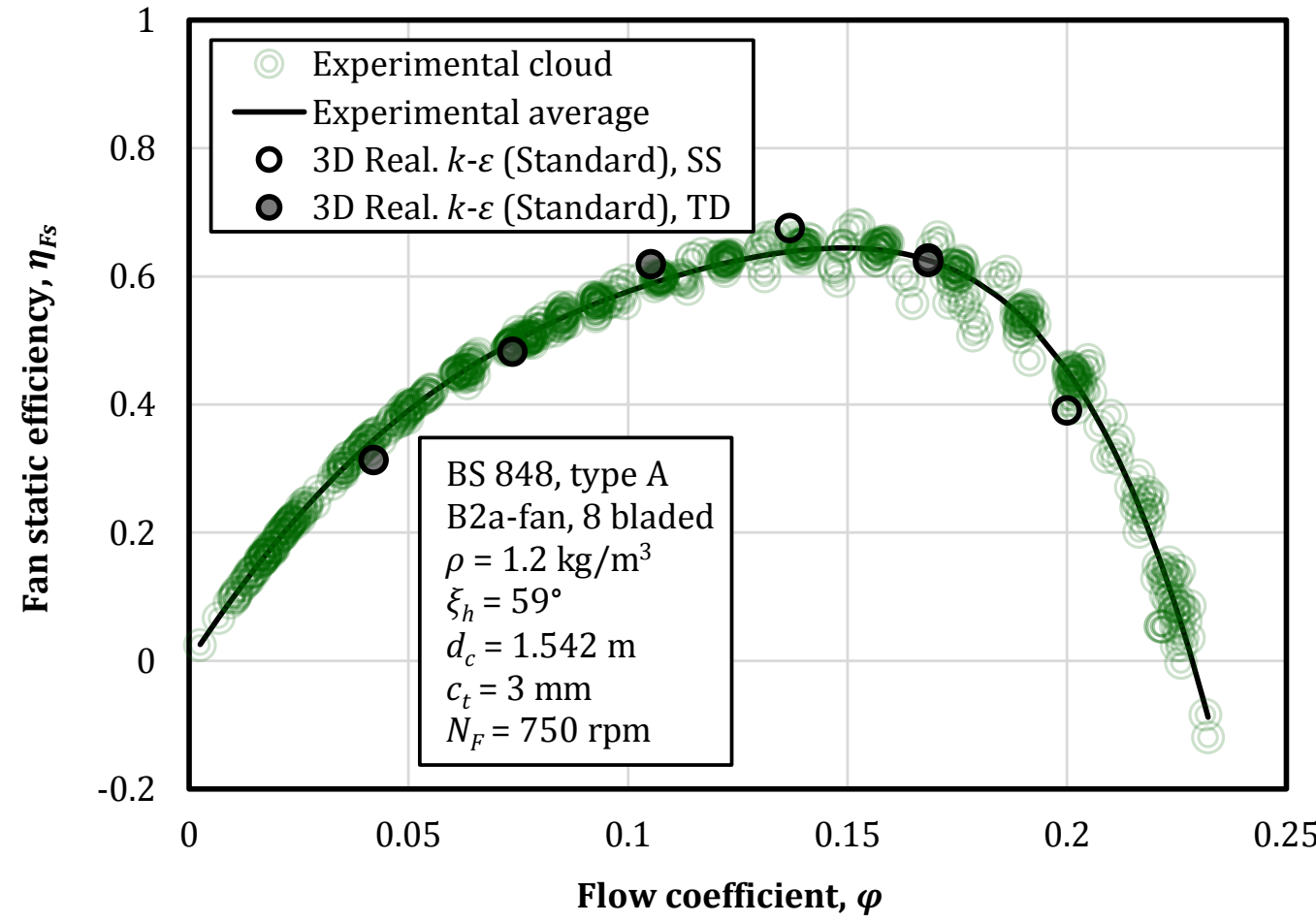

(b)

Figure 5.1: The B2a-fan performance characteristic presented by the (a) fan static pressure coefficient $\left(\Psi_{F s}\right)$ and (b) fan static efficiency $\left(\eta_{F s}\right)$ as functions of flow coefficient $(\phi)$ 
CHAPTER 5. PERIODIC THREE-DIMENSIONAL NUMERICAL MODEL (P3DM) VERIFICATION

flow rates, another time-dependent simulation is conducted for $\phi_{D}=0.168$ alongside the steady simulation to verify if a similar result would be obtained. The flow solution obtained through the time-dependent analyses converge to a state where the flow field in the vicinity of the fan becomes periodic as presented in figures E.3b-d in appendix E.4. The numerically obtained fan characteristic values at low flow rates are therefore time-averaged for the last period simulated, discussed in appendix G.1. Lastly, the Pearson correlation and RMSE values for the relation between the numerical and experimental average can be calculated in a similar fashion to the experimental comparison done previously. These values are given in table 5.2.

Table 5.2: Pearson correlation $\left(R_{P}\right)$ and RMSE values presenting the quality of relation between the numerical and average experimental data obtained for the B2a-fan performance characteristics

\begin{tabular}{lll}
\hline Variables & Pearson $\left(\boldsymbol{R}_{\boldsymbol{P}}\right)$ & RMSE \\
\hline$\Psi_{F s}$ & 0.994 & $7.425(10)^{-3}$ \\
$\eta_{F s}$ & 0.971 & $4.222(10)^{-2}$ \\
\hline
\end{tabular}

\subsection{Blade surface pressure verification}

\subsubsection{Definition of variables}

The experimentally and numerically obtained B2a-fan blade surface static pressure $\left(p_{s}\right)$ distributions are compared at five cylindrical sections along the blade span $\left(s_{b}=0.9,0.7,0.5,0.3\right.$ and 0.1 , figure 5.2$)$, where blade span is defined by:

$$
s_{b}=\frac{r-r_{h}}{r_{c}-r_{h}}
$$

with $r$ the radius under consideration and $r_{h}$ and $r_{c}$ the hub and casing radii, respectively. Apparent from figure 5.2 is that a single cylindrical section of the blade is fundamentally a two-dimensional aerofoil in the polar $\theta z$-plane, at a specific radius. For presentation purposes, the blade surface pressure values are converted to a non-dimensional static pressure coefficient $\left(C_{p}\right)$ defined as:

$$
C_{p}=\frac{p_{s}}{\frac{1}{2} \rho u^{2}}
$$




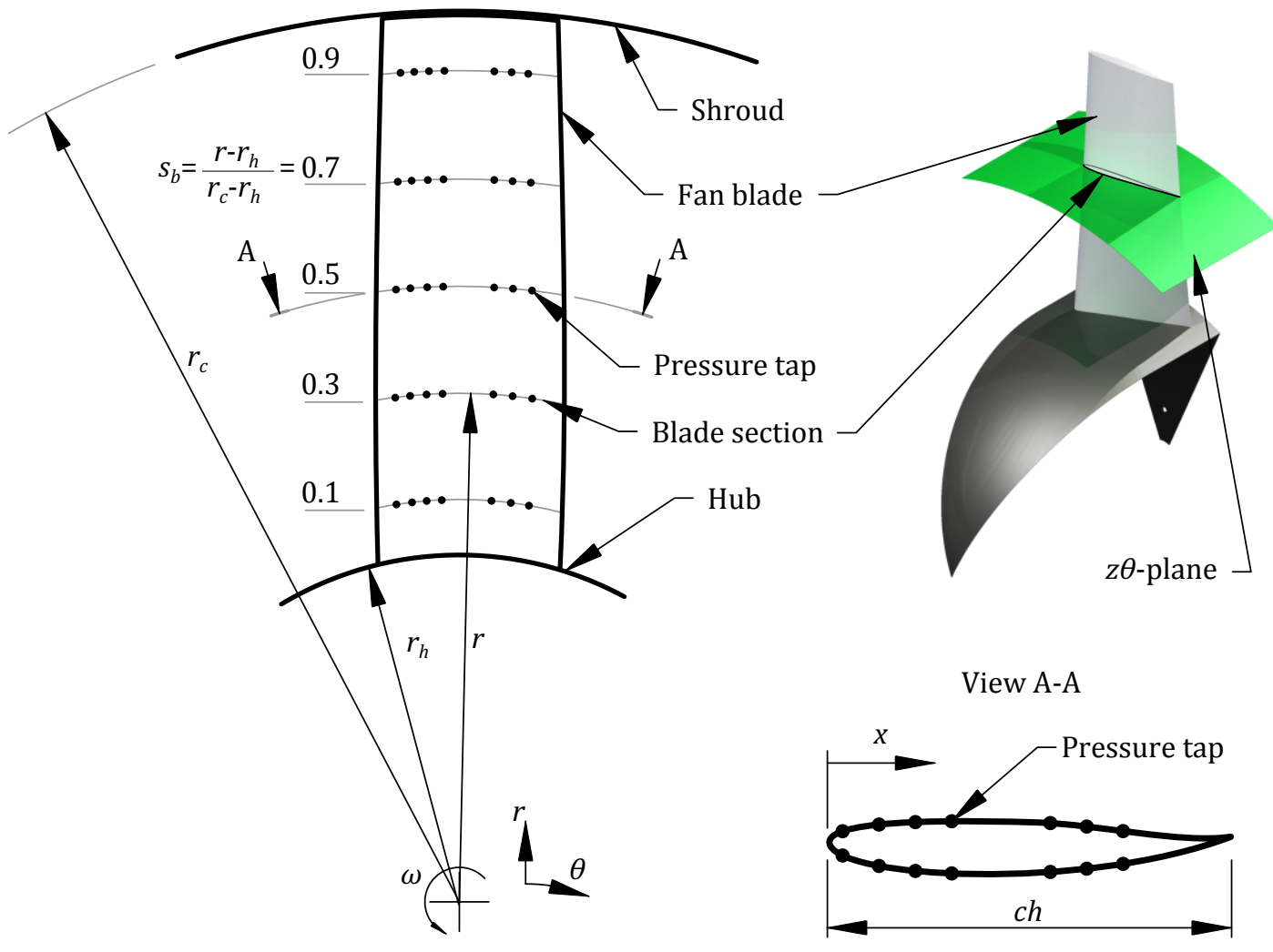

Figure 5.2: Fan blade indicating the locations of the pressure taps at the five dimensionless spans $\left(s_{b}\right)$

where $\rho$ is the atmospheric density, and $u$ the tangential blade speed. Lastly, the blade sectional pressure coefficients are presented as a function of dimensionless chord $(x / c h)$ where values of 0 and 1 represent the blade leading and trailing edges, respectively.

\subsubsection{Comparison of results}

A comparison between the experimentally and numerically obtained blade surface pressures are presented in figures 5.3 to 5.7 for the five different blade spans considered. The six sub-figures per blade span display the pressure coefficients for each of the simulated volumetric flow coefficients $(0.042 \leq \phi \leq 0.200)$. The numerically obtained blade surface pressures for the time-dependent simulations are time-averaged as discussed in section G.1. The quality of relation is once again calculated by means of Pearson correlation factors $\left(R_{P}\right)$ and the RMSEs. These values for each of the respective spans and flow coefficients are indicated on each figure. 
CHAPTER 5. PERIODIC THREE-DIMENSIONAL NUMERICAL MODEL (P3DM) VERIFICATION

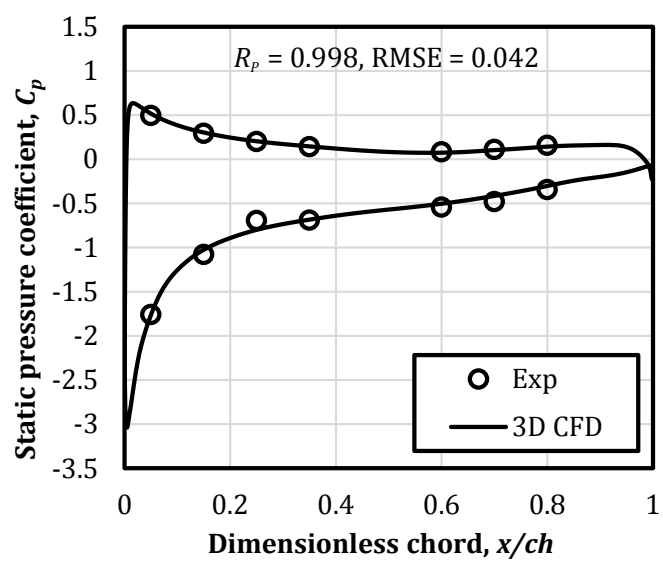

(a)

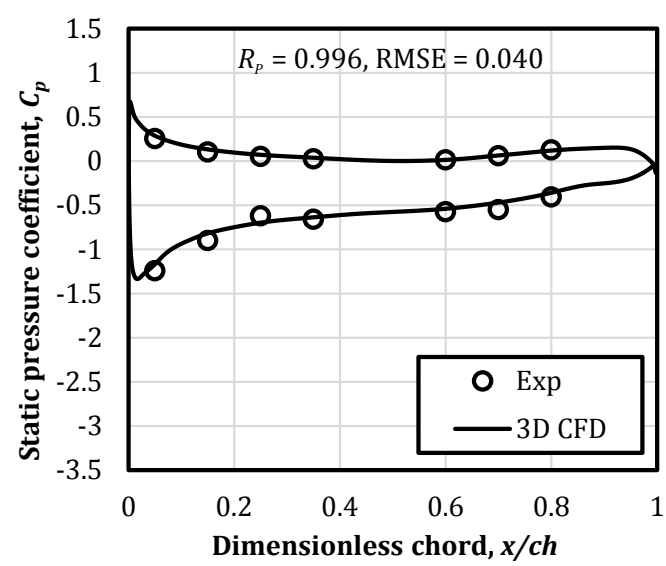

(c)

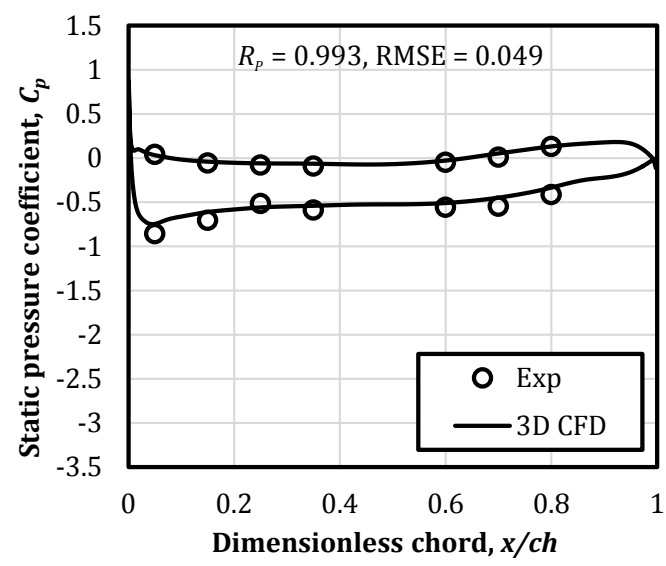

(e)

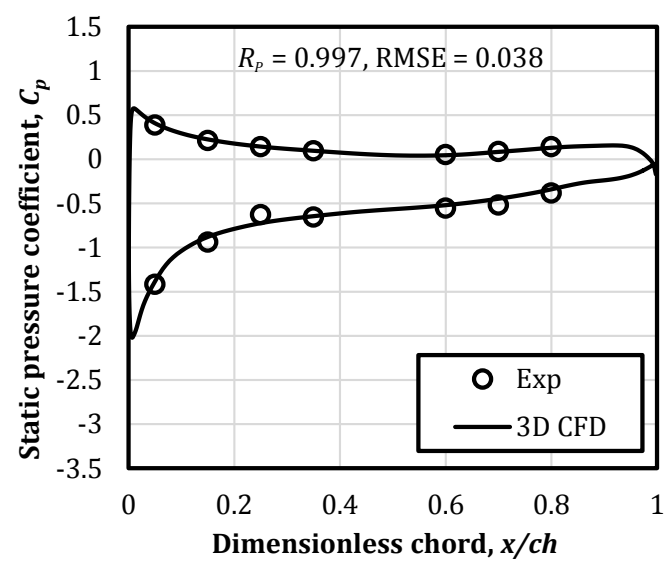

(b)

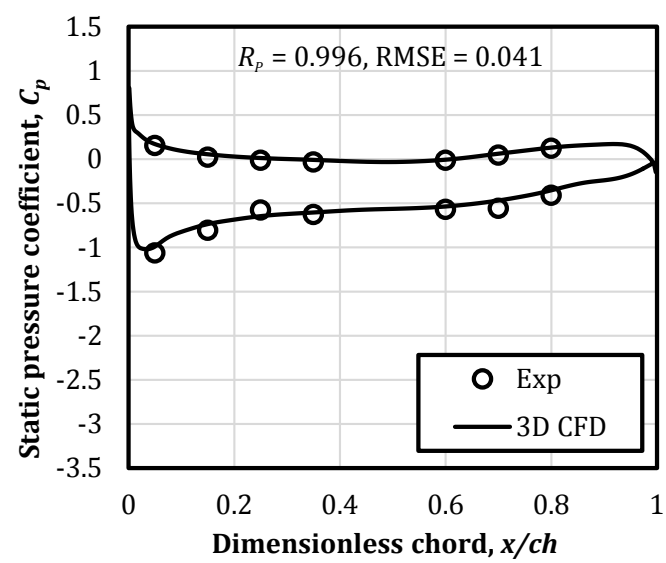

(d)

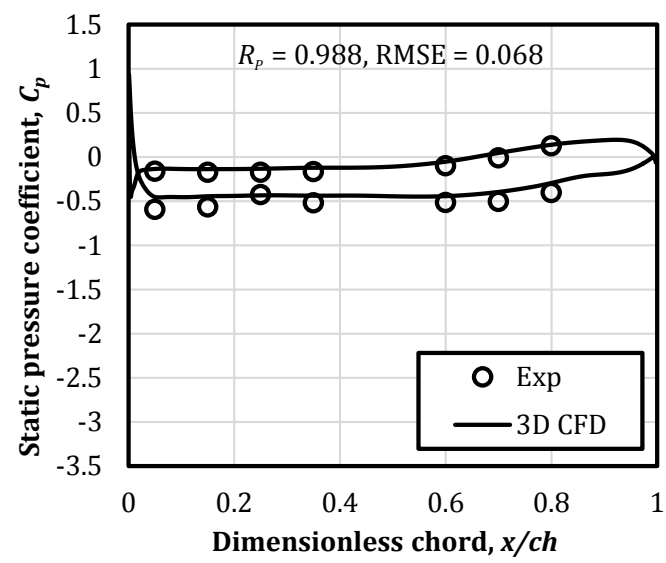

(f)

Figure 5.3: Time-averaged blade surface pressure coefficients at a dimensionless blade span of $s_{b}=0.9$ for flow rates of $\phi=$ (a) 0.042, (b) 0.074, (c) 0.105, (d) 0.137 , (e) 0.168, (f) 0.200 
CHAPTER 5. PERIODIC THREE-DIMENSIONAL NUMERICAL MODEL (P3DM) VERIFICATION

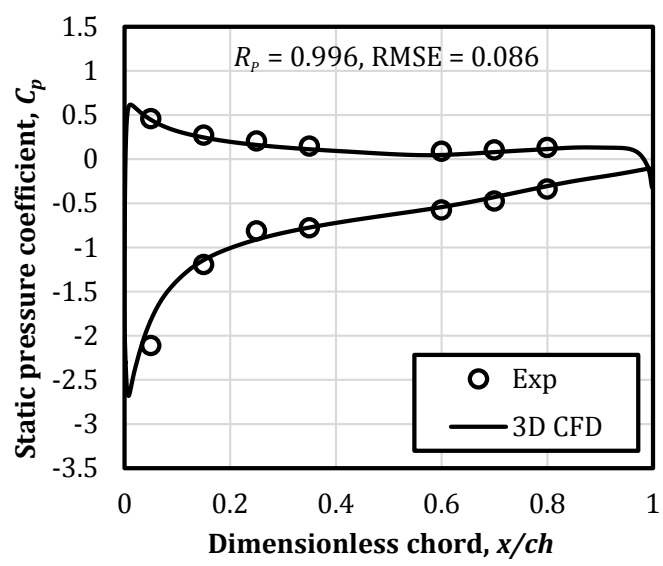

(a)

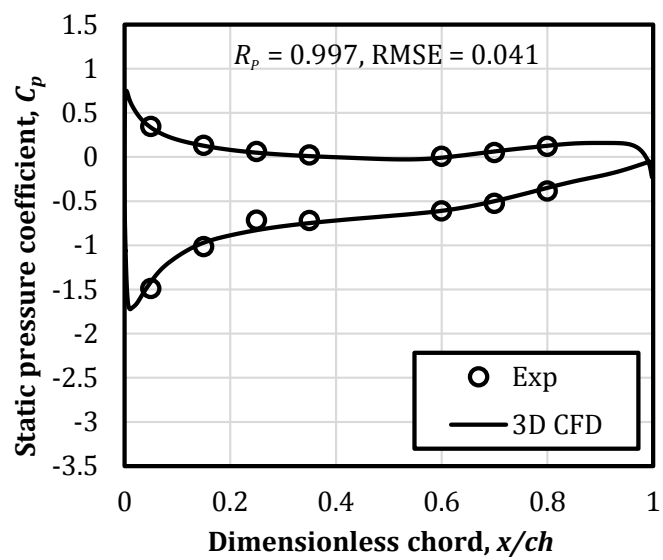

(c)

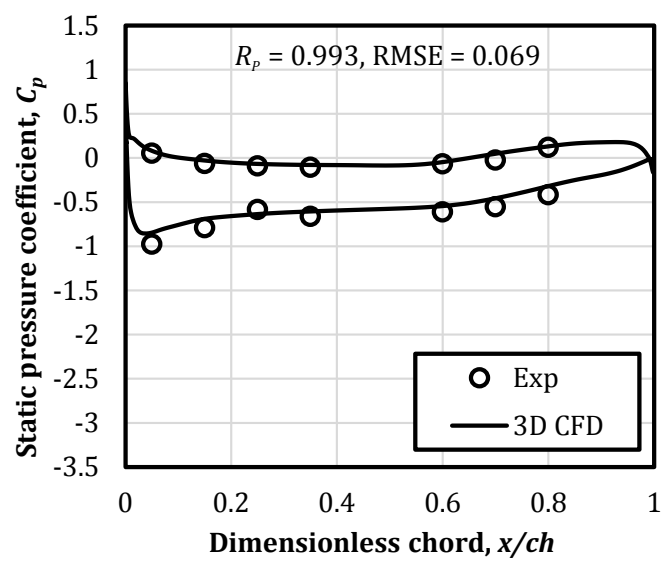

(e)

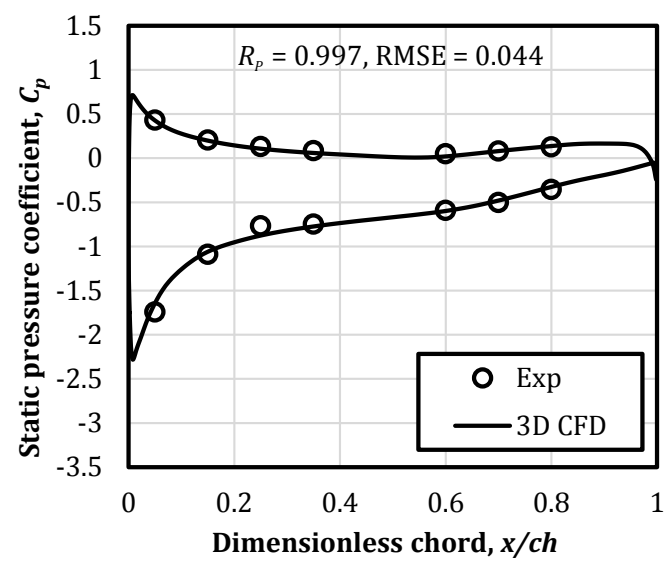

(b)

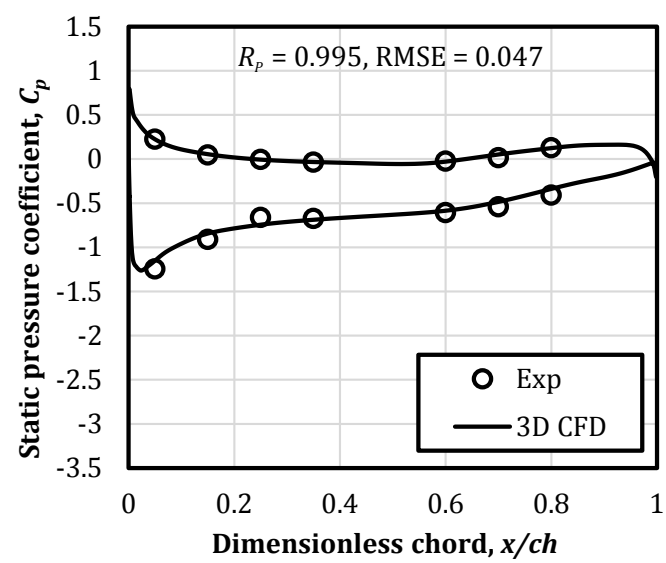

(d)

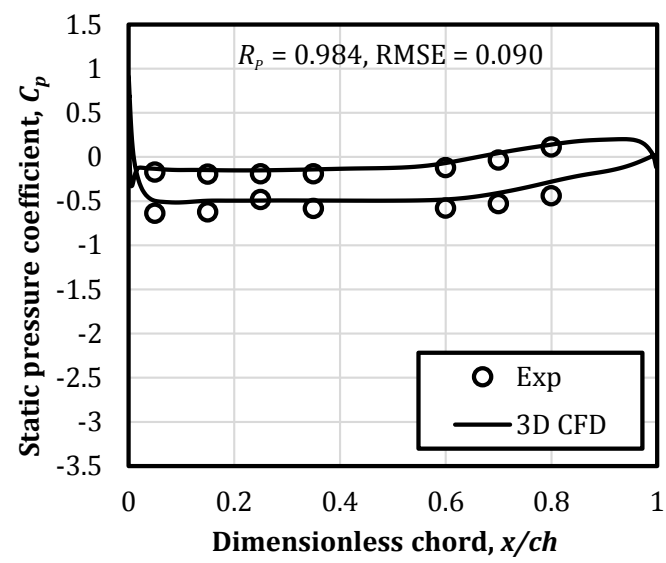

(f)

Figure 5.4: Time-averaged blade surface pressure coefficients at a dimensionless blade span of $s_{b}=0.7$ for flow rates of $\phi=$ (a) 0.042, (b) 0.074, (c) 0.105, (d) 0.137 , (e) 0.168 , (f) 0.200 
CHAPTER 5. PERIODIC THREE-DIMENSIONAL NUMERICAL MODEL (P3DM) VERIFICATION

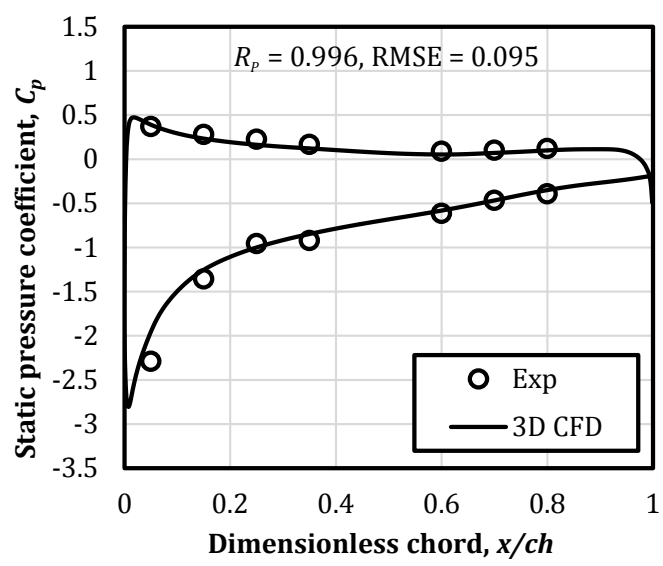

(a)

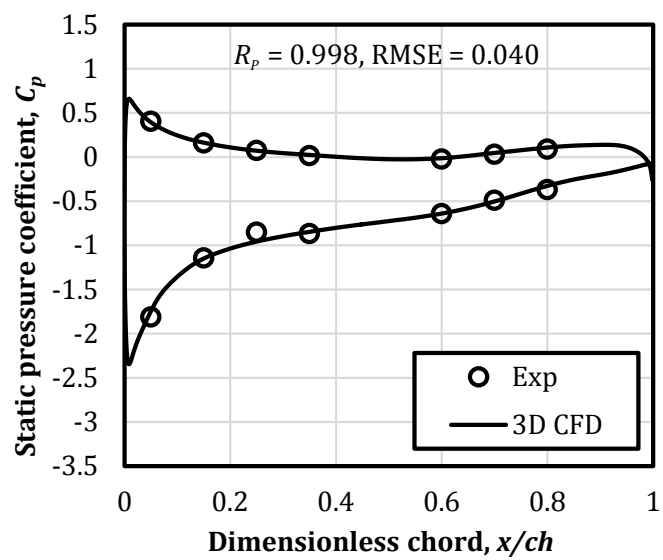

(c)

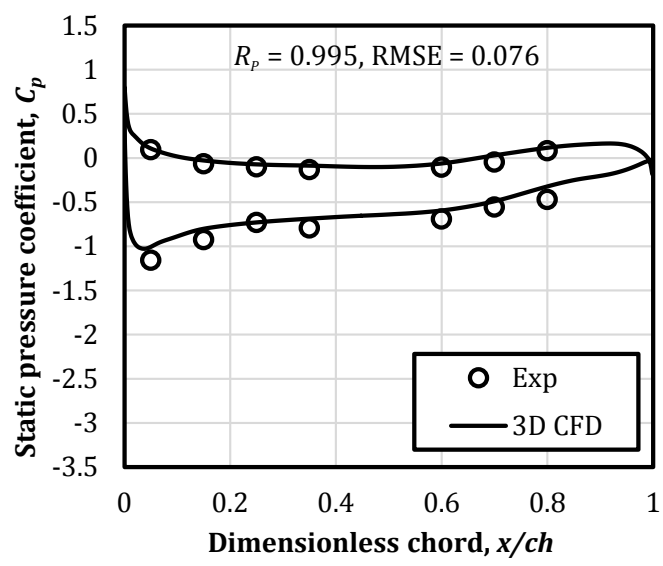

(e)

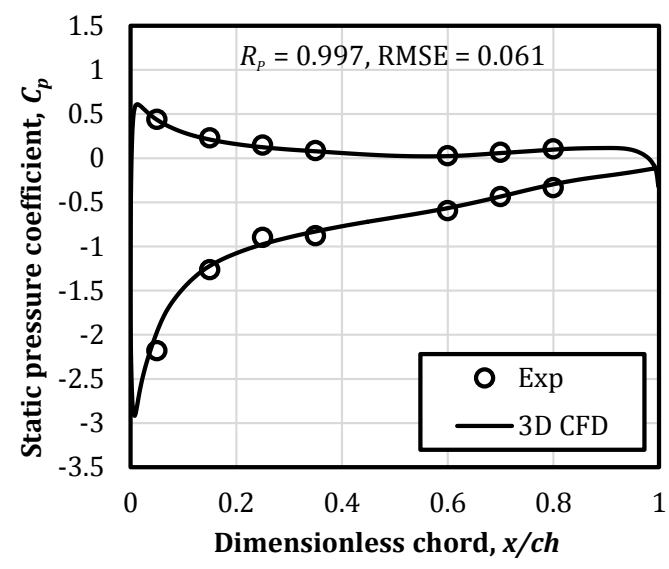

(b)

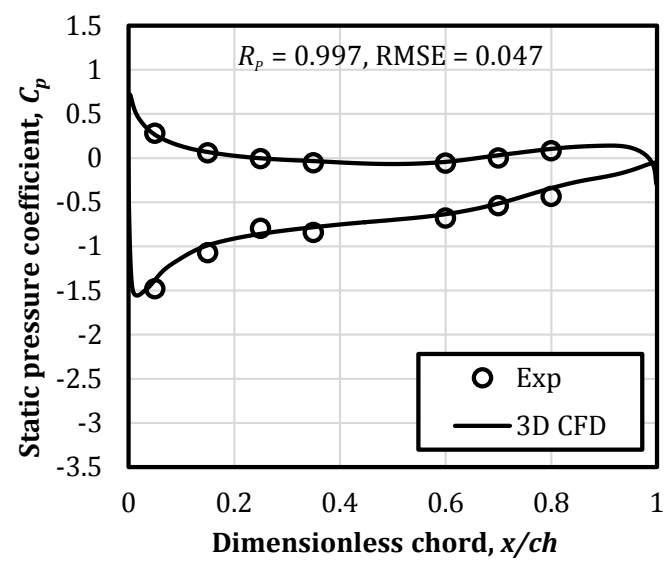

(d)

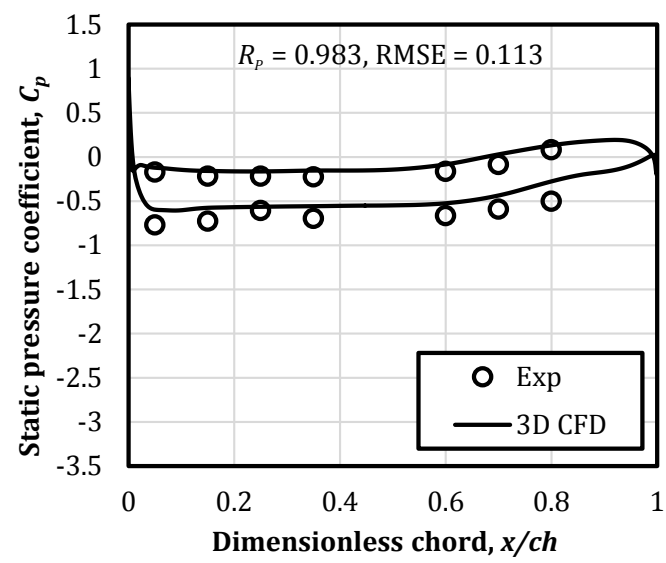

(f)

Figure 5.5: Time-averaged blade surface pressure coefficients at a dimensionless blade span of $s_{b}=0.5$ for flow rates of $\phi=$ (a) 0.042, (b) 0.074, (c) 0.105, (d) 0.137 , (e) 0.168 , (f) 0.200 
CHAPTER 5. PERIODIC THREE-DIMENSIONAL NUMERICAL MODEL (P3DM) VERIFICATION

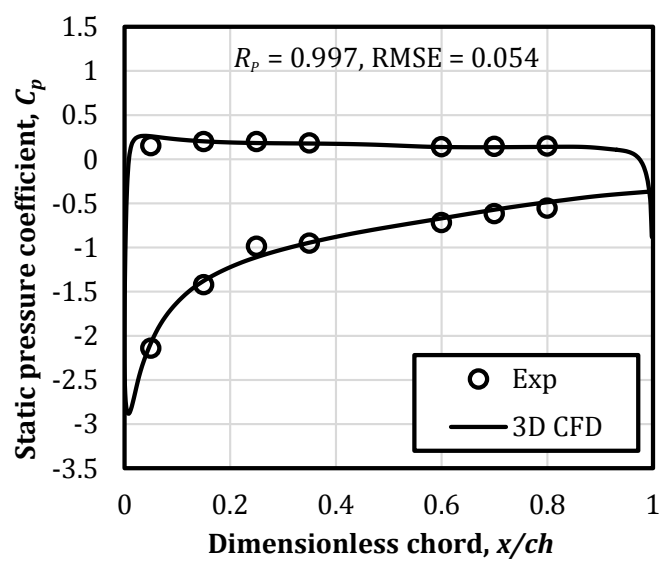

(a)

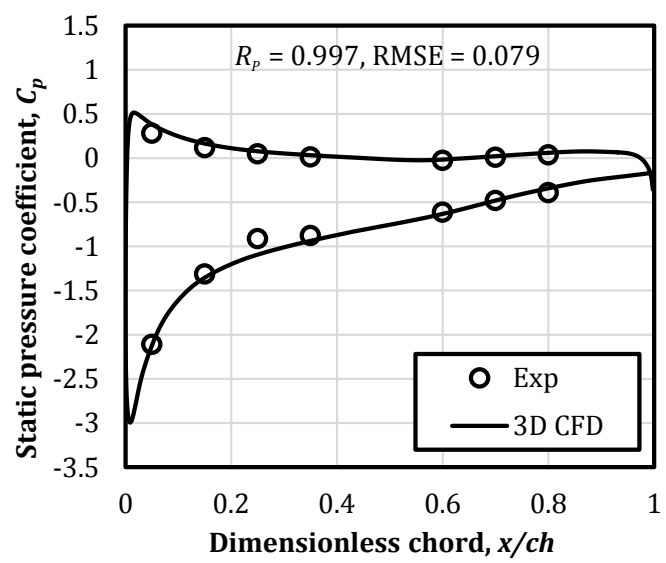

(c)

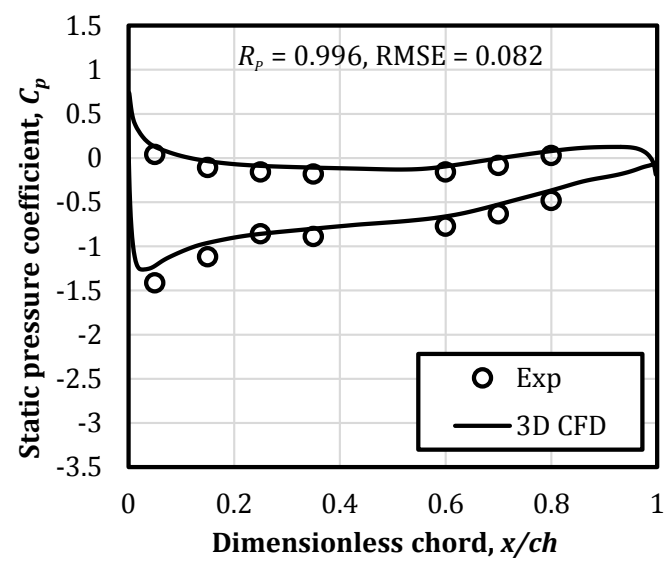

(e)

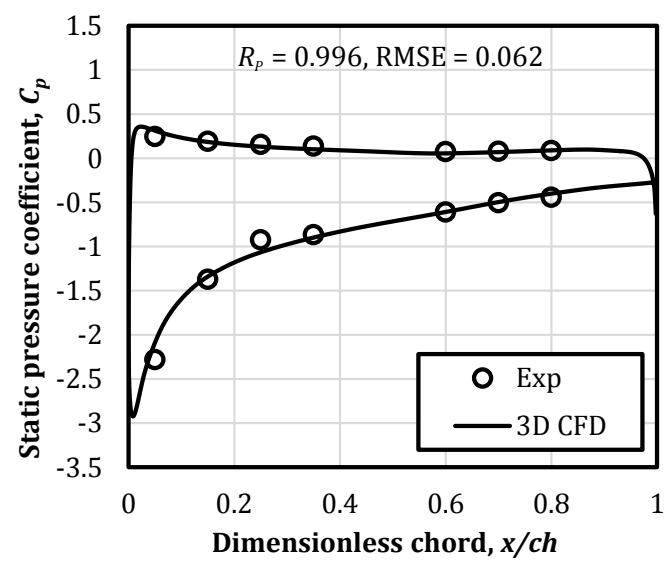

(b)

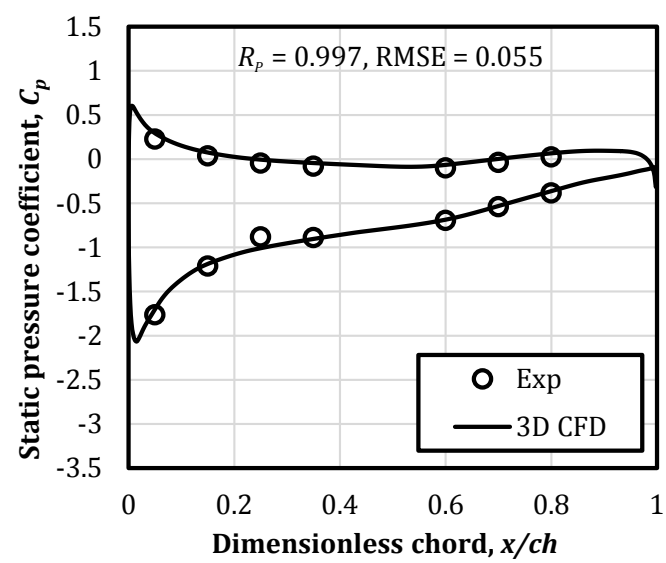

(d)

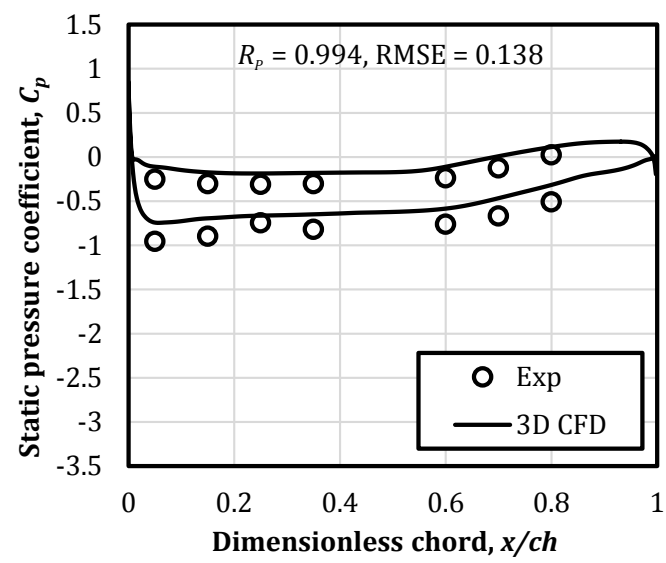

(f)

Figure 5.6: Time-averaged blade surface pressure coefficients at a dimensionless blade span of $s_{b}=0.3$ for flow rates of $\phi=$ (a) 0.042, (b) 0.074, (c) 0.105, (d) 0.137 , (e) 0.168, (f) 0.200 
CHAPTER 5. PERIODIC THREE-DIMENSIONAL NUMERICAL MODEL (P3DM) VERIFICATION

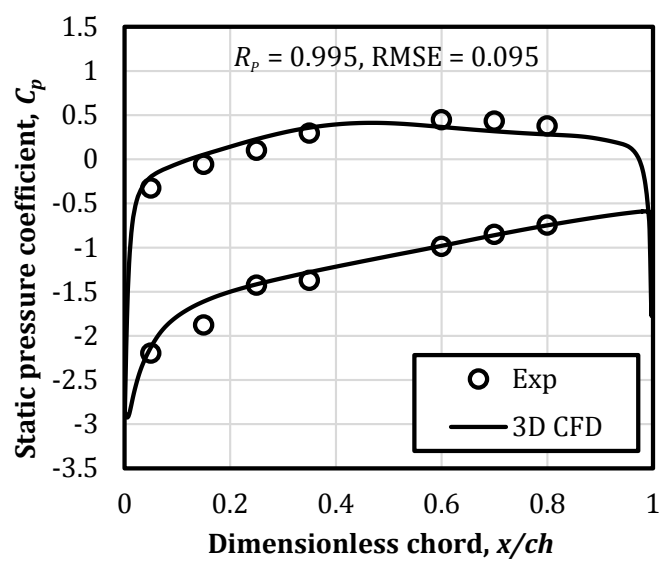

(a)

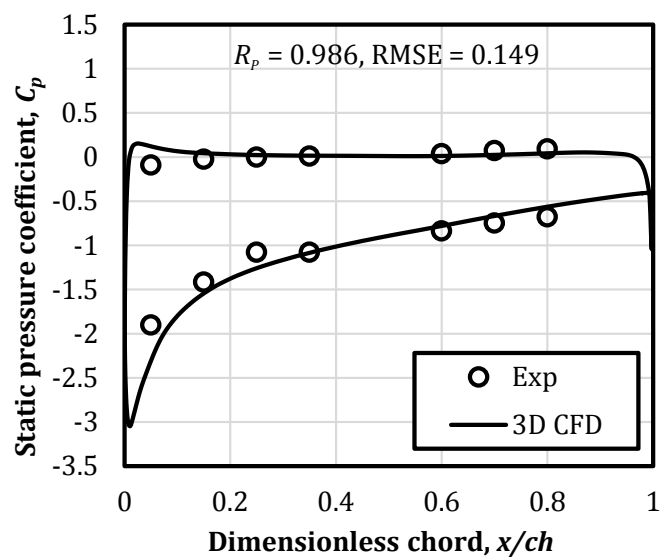

(c)

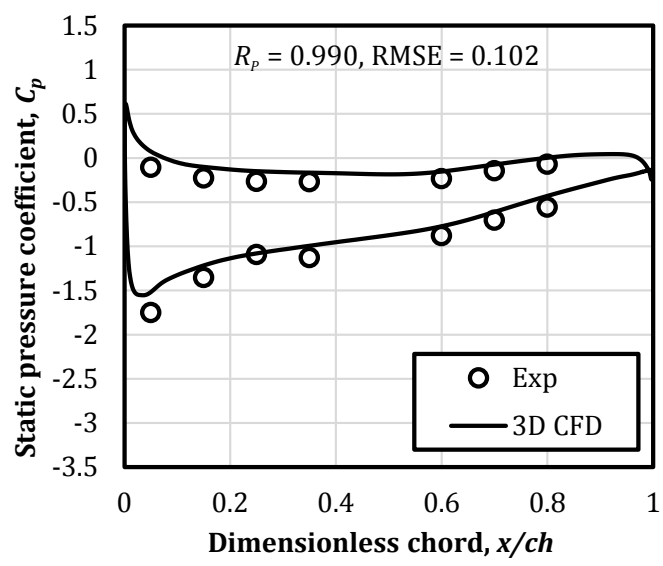

(e)

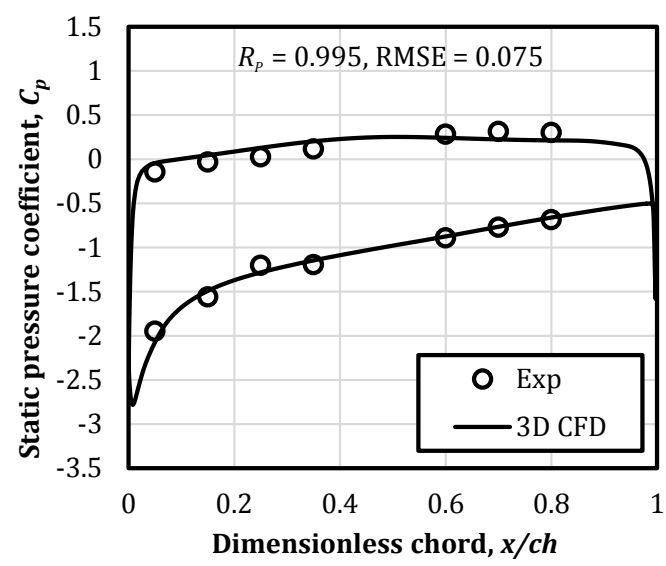

(b)

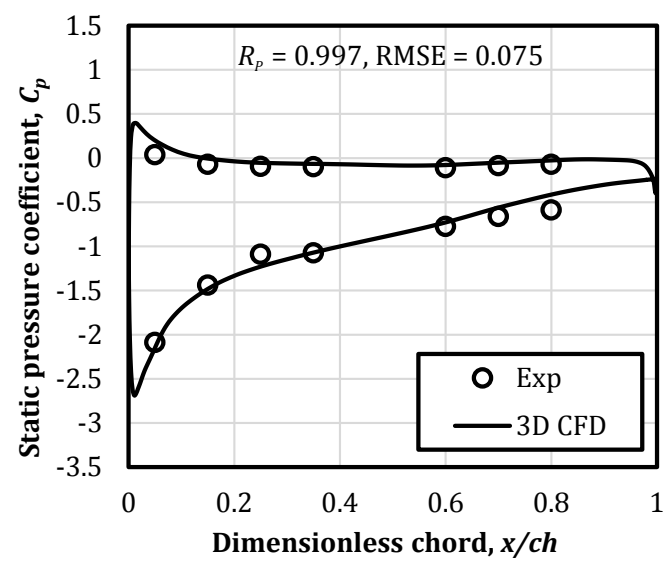

(d)

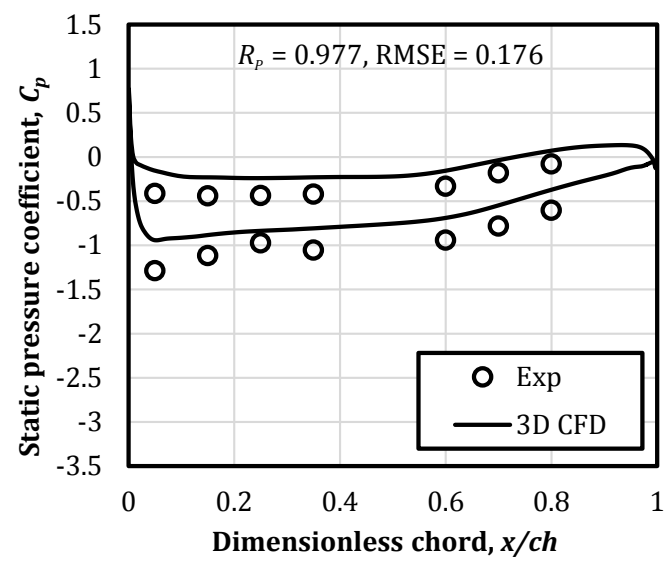

(f)

Figure 5.7: Time-averaged blade surface pressure coefficients at a dimensionless blade span of $s_{b}=0.1$ for flow rates of $\phi=$ (a) 0.042 , (b) 0.074, (c) 0.105, (d) 0.137 , (e) 0.168, (f) 0.200 


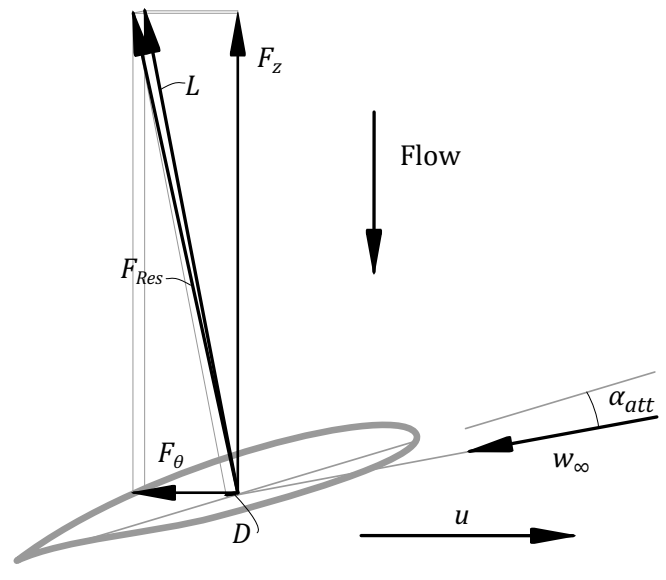

(a)

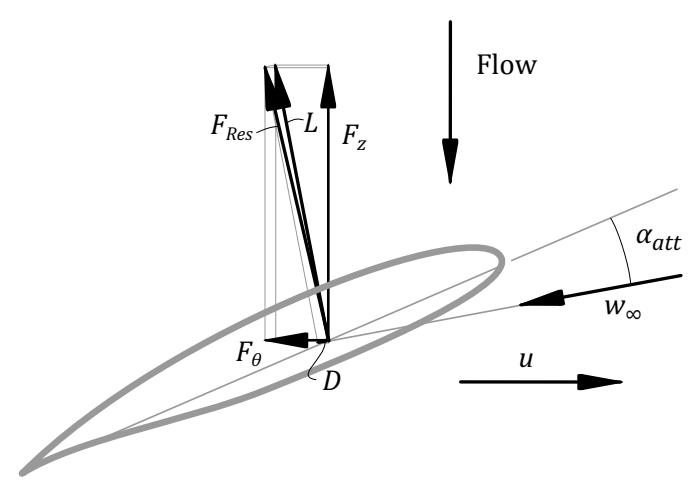

(b)

Figure 5.8: Scaled schematic of force vectors at blade sections of (a) $s_{b}=0.5$ and (b) $s_{b}=0.1$ in the $z \theta$-plane for a flow coefficient of $\phi_{D}=0.168$

\subsection{Concluding remarks}

\subsubsection{Fan characteristic comparison}

With regards to the verification of the numerical model through comparison of the numerical and experimental fan performance characteristics, a good correlation can be observed, as presented in figures 5.1a-b. Most numerical values are within the experimental cloud. According to the values in table 5.2 a good correlation exists between the fan static pressure $\left(R_{P, \Psi_{F s}}=0.994\right)$ in contrast to a slightly poorer correlation for fan static efficiency $\left(R_{P, \eta_{F s}}=\right.$ 0.971 ), due to the underestimation of fan power. This is more prominent at higher flow rates.

The cause of this phenomenon is explained by considering the nature of the forces acting on the blade, as shown for blade sections at $s_{b}=0.5$ and at $s_{b}=0.1$, for the design flow coefficient of $\phi_{D}=0.168$ in figures $5.8 \mathrm{a}-\mathrm{b}$. These figures represent a typical case for flow conditions near the design point, where $u$ is the tangential blade speed and $w_{\text {inf }}$ the averaged relative velocity between the up- and downstream relative velocities. $\alpha_{\text {att }}$ is the angle of attack between the averaged relative velocity and the blade section chord.

As can be observed in figures $5.8 \mathrm{a}$ and $\mathrm{b}$, the blade sectional lift force $(L)$ constitutes the largest part of the resultant force $\left(F_{\text {Res }}\right)$ for higher flow rates. On the contrary the sectional drag force $(D)$, barely visible in figure $5.8 \mathrm{a}$ and $\mathrm{b}$, is generally two orders of magnitude smaller than the lift. Hence, the lift force is the main contributor towards the axial $\left(F_{z}\right)$ and tangential $\left(F_{\theta}\right)$ force components, whereas the drag force only make a small contribution at near-design and higher flow rates. Of these force components, the axial force 
CHAPTER 5. PERIODIC THREE-DIMENSIONAL NUMERICAL MODEL

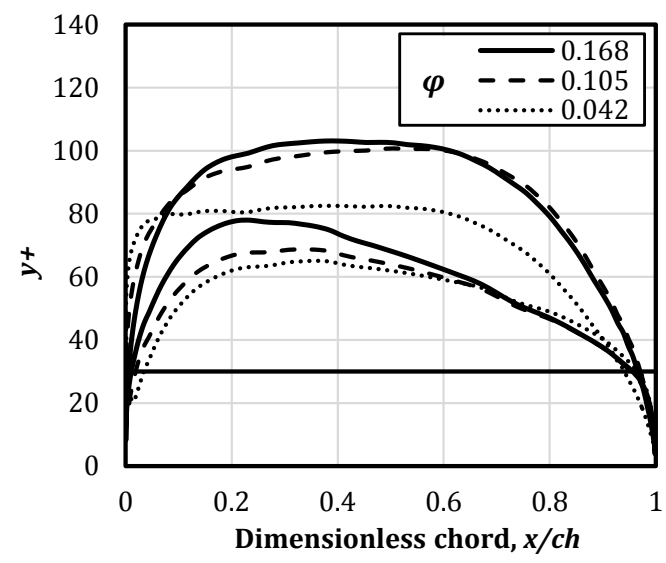

(a)

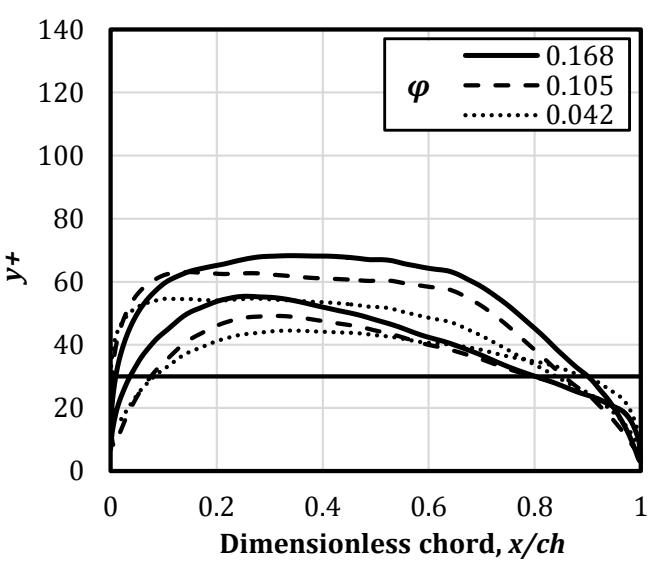

(c)

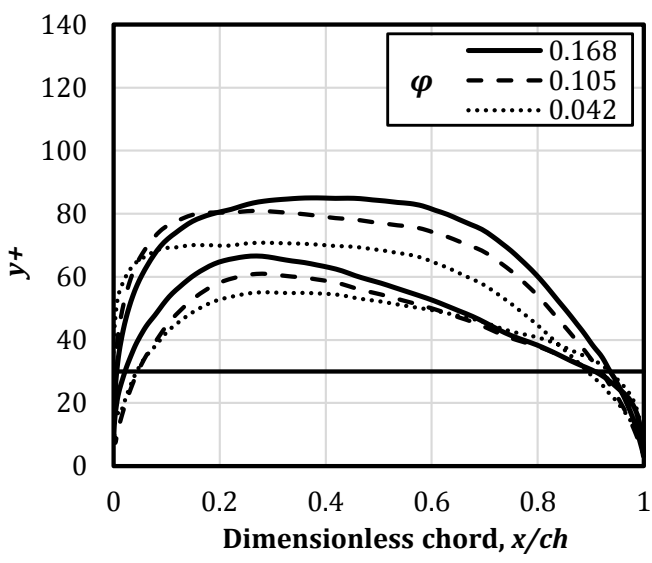

(b)

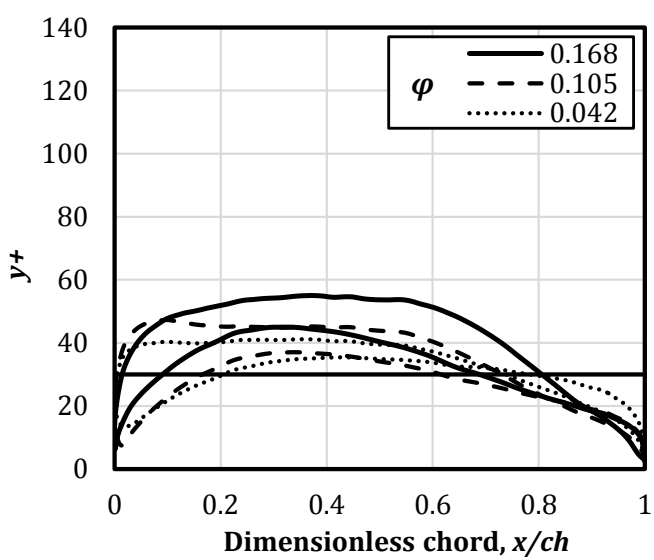

(d)

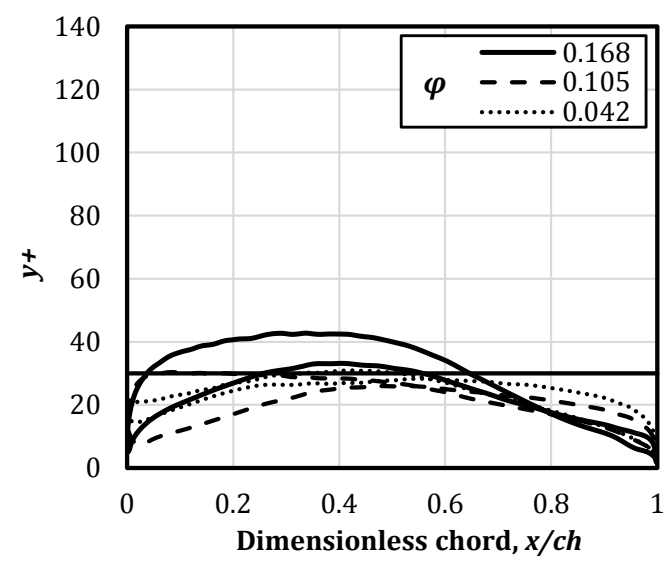

(e)

Figure 5.9: Time-averaged $y^{+}$-values at different dimensionless blade spans of $s_{b}=$ (a) 0.9 , (b) 0.7, (c) 0.5, (d) 0.3 and (e) 0.1 
CHAPTER 5. PERIODIC THREE-DIMENSIONAL NUMERICAL MODEL (P3DM) VERIFICATION

contributes to the pressure difference across the fan and the tangential force to the shaft torque. The tangential force is therefore proportional to the fan power consumption.

The error calculated in blade lift for higher flow rates (where the discrepancy between the experimental and numerical data is the highest) inevitably has a considerable effect on the tangential force component and subsequently the difference in fan power consumption, especially at higher flow rates. It follows that a more realistic numerical wall boundary condition near the blade surface would lead to an improved approximation of blade forces and subsequently also the fan power. This remains a subject for further investigation. However, since the correlation obtained in the present study remains fair, it is decided to use the numerical results obtained for further analyses.

\subsubsection{BSPM comparison}

A good comparison exists between the experimental and numerical blade surface static pressure trends presented in figures 5.3-5.7. A slight deviation from the trend for the third pressure tap from the leading edge on the suction side is evident. This deviation might have been due to a small leak in the pressure line, but since it is small, the values were included. The RMSE values of these comparisons are generally below 0.1 with the largest RMSE being 0.176 for a flow coefficient of $\phi=0.2$ at $s_{b}=0.1$ (figure 5.7f). The comparisons are fair for lower flow rates, but become poorer toward higher flow rates. The poorest comparisons typically occur near the blade root with progressive improvements towards the tip of the blade.

The discrepancy in results could once again be attributed to the boundary layer condition at the blade surface in the numerical model. Since the Standard wall function (Launder and Spalding, 1974) is used to model the shear stress in the wall boundary layer, $y^{+}$-values are recommended to be in the range of $30<y^{+}<300$. This criterion is met for approximately $90 \%$ of the blade surface as presented in figures $5.9 \mathrm{a}$-e where the $y^{+}$-values for the flow coefficients of $\phi=0.168,0.105$ and 0.042 at the aforementioned cylindrical blade sections are indicated. The dark horizontal line in these figures indicate the $y^{+}=30$ mark. $y^{+}$-values are below 30 near the leading and trailing edges of the blade as well as near the hub of the fan $\left(0 \leq s_{b} \leq 0.1\right)$, possibly explaining the reason for the discrepancy in numerically and experimentally obtained blade static pressure coefficient values in this region of the blades.

Despite the computational error made due to the boundary layer criteria not being perfectly met in some zones of the blade surfaces, fair comparisons are still obtained between the numerical and experimental data for both the fan performance characteristics and the blade surface pressure distribution. These results, together with the compared fan performance characteristics, affirmed the use of the numerically obtained results for further analyses of the flow field near the axial flow fan. 


\section{Chapter 6}

\section{Spatial flow field characteristics}

Fan design procedures firstly rely on deciding upon a desired flow field (design condition) and secondly shaping a blade profile to induce this flow field. Hence, each design condition theoretically requires a unique set of blades that would induce the desired flow field in the most efficient manner. This is not always practically obtainable, especially for systems requiring or which are subject to variable flow rates. It would lead to investigations on the effects of various fan blade design alterations on fan performance, such as controlled vortex designs or the unloading of the blade root. Since it is also desired to develop a simplified numerical fan model in the present study, visualisation of the flow structures in close vicinity to the fan rotor would be beneficial to:

- comprehend the existing flow mechanisms occurring for the range of flow rates investigated.

- understand to what extent the unloaded blade root in the present fan design keeps the blade from stalling at lower flow rates.

- obtain flow field values to be utilised in the development of a simplified fan model.

For this purpose the flow field data obtained using the periodic threedimensional model (P3DM) are analysed to present the spatial flow structures that occur in close vicinity to the B2a-fan rotor. Definitions of the various flow variables are described in the first section. In the following section, the twodimensional, circumferentially-averaged spanwise flow profiles are displayed for the range of modelled flow rates. These results give an indication of the change in flow distribution as a function of flow rate. Subsequently the time-dependent nature of these two-dimensional spanwise flow profiles are also presented for low flow rates, emphasizing the unsteady nature of the flow field in the hub region of the fan. Finally, the three-dimensional nature of the flow occurring near and inside the fan rotor cascade is visualised numerically for the range of flow rates simulated. 


\subsection{Definition of variables}

The spanwise flow profiles presented in sections 6.2 and 6.3 , are shown at the locations indicated in figure $6.1 \mathrm{~b}$. These locations are placed at 0.46 of the hub chord length $\left(0.46 \mathrm{ch}_{h}\right)$ in the axial direction, up- and downstream of the fan rotation plane. All three-dimensional flow vectors and components are indicated in figure 6.1 for a specific radius and are defined as follows:

The absolute and relative velocity vectors are denoted as $c$ and $w$, respectively. The tangential blade speed is given as $u$. Three flow angles are presented, namely the absolute, relative and radial flow angles denoted as $\alpha$, $\beta$ and $\theta_{r}$, respectively. The subscripts $r, \theta$ and $z$ signify the directions in cylindrical coordinates and the numbers 1 and 2 refer to the up- and downstream locations at which the flow is considered. Also visible in figure 6.1 is the direction of fan rotation, being negative according to the right hand rule. Similarly, the values of all vector components in the $\theta$-direction also comply with the right hand rule.

All spatial velocity components presented in the following sections are made dimensionless as follows:

$$
v_{x, \operatorname{dim}}=\frac{v_{x}}{u_{c}}
$$

where $v_{x, d i m}$ and $v_{x}$ are generic variables for dimensionless velocity and the specific velocity component, respectively. $u_{c}$ is the tangential blade velocity at the radius of the fan casing.

\subsection{Time-averaged spanwise flow distributions}

As a first indication of the flow structure in the vicinity of the fan rotor, the circumferentially averaged flow variables are calculated at the defined up- and downstream locations. 146 data points along the circumference of the fan sector in the numerical model are sampled to calculate an average value, for each variable, at each spanwise location (as well as a single time-step if applicable). Hence, a data point is taken approximately every $0.3^{\circ}$ along the fan circumference, which is the maximum obtainable number of points, since it is constrained by the number of cells in this direction. For the time-dependent results at low flow rates $(0.042 \leq \phi \leq 0.105)$ these variables are also time-averaged. The variables are presented as functions of the blade span in figures 6.2-6.4.

The absolute axial velocity $\left(c_{z} /\left|u_{c}\right|\right)$ profiles (figures $6.2 \mathrm{a}$ and $\mathrm{b}$ ) show a near-uniform trend for flow rates near the design condition up- and downstream, across the largest span of the fan. This observation confirms the free-vortex flow field that the B2a-fan is designed to induce (with the exception of the region near the blade root) since a uniform axial velocity profile through the rotor is indicative of a free-vortex condition. However, a deviation 


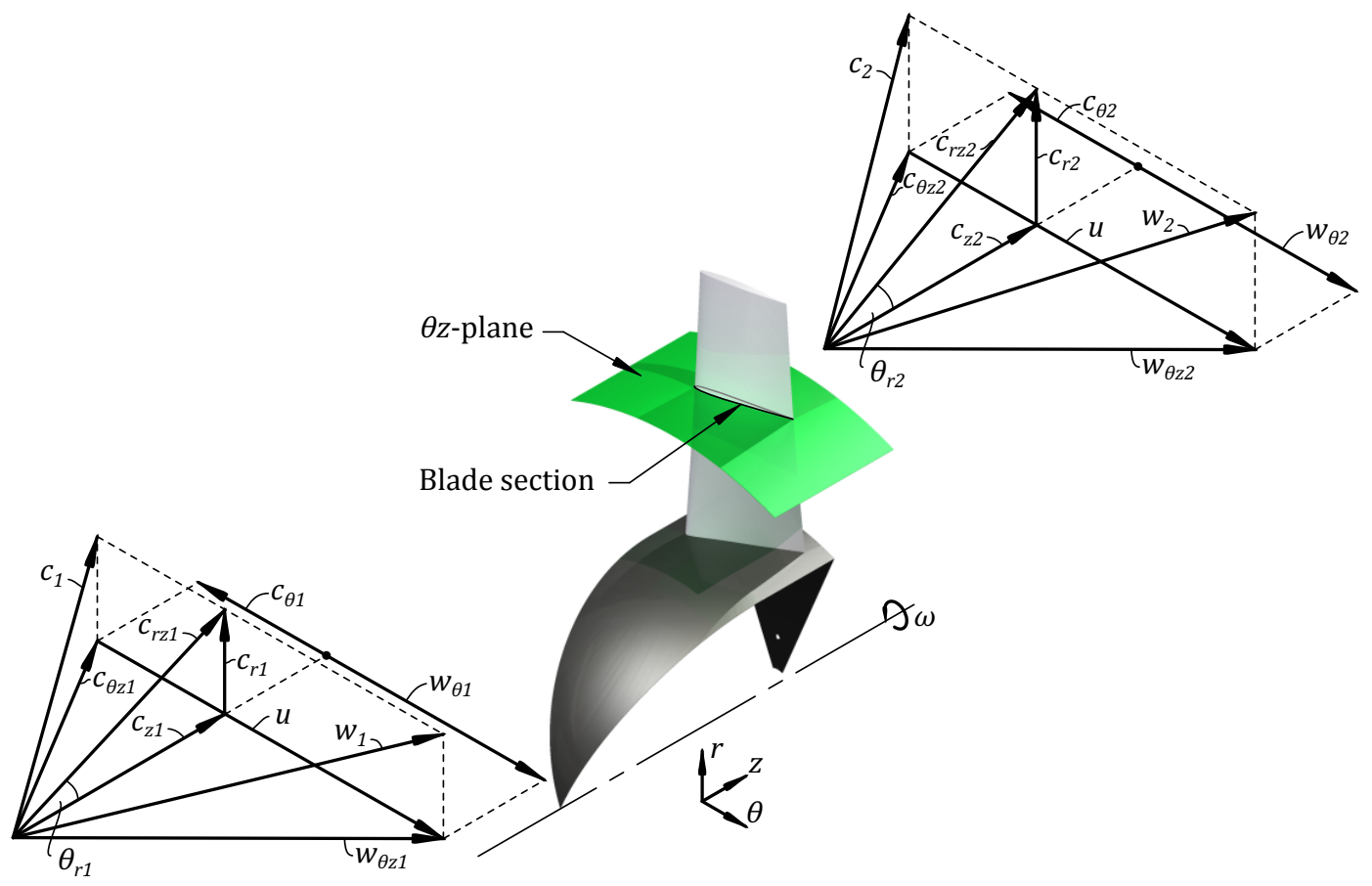

(a)

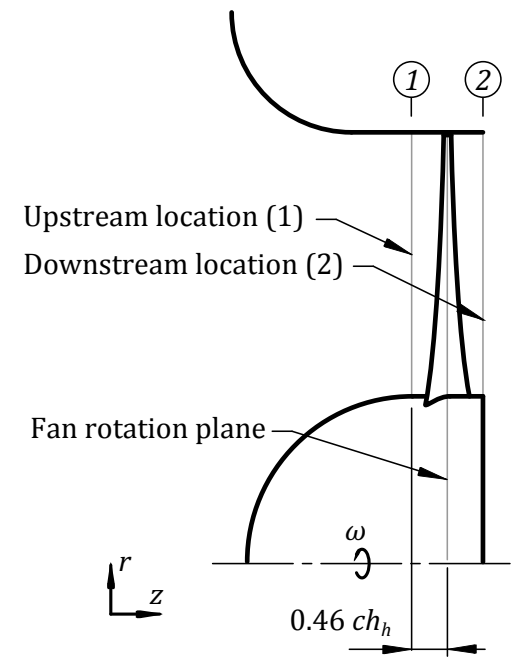

(b)
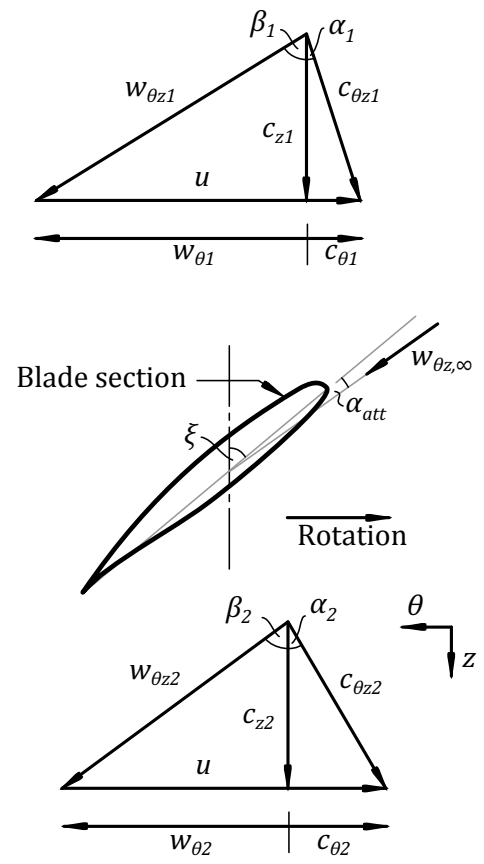

(c)

Figure 6.1: Depiction of (a) the three-dimensional velocity vectors and angles up- and downstream of the fan rotor at a specific radius, (b) the up- and downstream locations where flow variables are extracted from and (c) the velocity vectors and flow angles in the $z \theta$-plane for a single cylindrical blade section 
from the uniform profile can be observed as the flow rate through the fan is decreased. For flow coefficients of $\phi=0.074$ and 0.042 the increasing flow leakage from the downstream to the upstream side at the blade tip increases to the extent that flow is entrained further upstream forming a ring vortex in this region as will be shown in a later section. Reversed flow is also visible in these velocity profiles, occurring near the hub region on the downstream side of the fan, which is a trend also noticed in measurements by Venter (1990) as well as Downie et al. (1993). The reversed flow in the present investigation does however not move entirely through the rotor to the upstream side as the upstream flow profiles near the hub indicate flow in the positive axial direction only. Hence, the flow in the hub region approaches the fan from both the upand downstream sides at low flow rates from where it moves radially outward.

Considering the absolute tangential (swirl) velocity $\left(c_{\theta} /\left|u_{c}\right|\right)$ components (figures $6.2 \mathrm{c}$ and $\mathrm{d}$ ), a value of zero can be observed upstream of the fan for flow coefficients of $0.105<\phi<0.2$ over the largest portion of the blade span. This is expected since no inlet guide vanes (IGVs) or other obstructions exist to impart pre-swirl to the flow before it reaches the fan rotor. Small deviations from zero near the hub and tip are also visible. Near the hub, these deviations are due to the shear effect of the rotating hub on the flow. Near the tip, the occurrence of tip leakage is the cause for the small difference from zero. Once again, as the flow coefficient is reduced from $\phi=0.105$ through to 0.042 , a strong deviation from the higher flow rates can be observed upstream of the fan as larger span sections exist with pre-swirl. Near the tip, this is partially attributed to the existing ring vortex and near the hub, to three-dimensional flow effects discussed in a later section. On the downstream side of the fan, the tangential velocity is nearly linear (theoretically hyperbolic, but the difference is difficult to observe for the applicable radii) across the largest span of the fan for flow coefficients of $0.168 \leq \phi \leq 0.2$, whereas at $\phi=0.137$ a slight bump in the swirl profile is visible near the hub. This point of maximum swirl (excluding effects at the tip) gradually moves to higher radii as the flow through the fan is further decreased. However the value of this maximum swirl remains near constant just below $c_{\theta 2} /\left|u_{c}\right|=-0.2$. Once again deviations from the spanwise trend is visible near the fan tip and hub, due to three-dimensional and boundary flow effects.

With regards to the radial velocity components $\left(c_{r} /\left|u_{c}\right|\right.$, figures $6.2 \mathrm{e}$ and $\mathrm{f})$, the inward convergence of the flow towards the fan face from higher and lower radii further upstream leads to the radial components not being zero in the observed profiles immediately upstream of the fan for flow rates close to the design point. However, as the flow rate through the fan is decreased, a deviation from this trend is once again apparent, especially at the tip, caused by the vortical pattern in the tip region. On the downstream side of the fan, the radial velocity profiles are close to zero for the largest section of blade span for flow rates near the design point. This, once again affirmed the correct behaviour of the fan at these flow rates, since zero radial flow is also one of the 


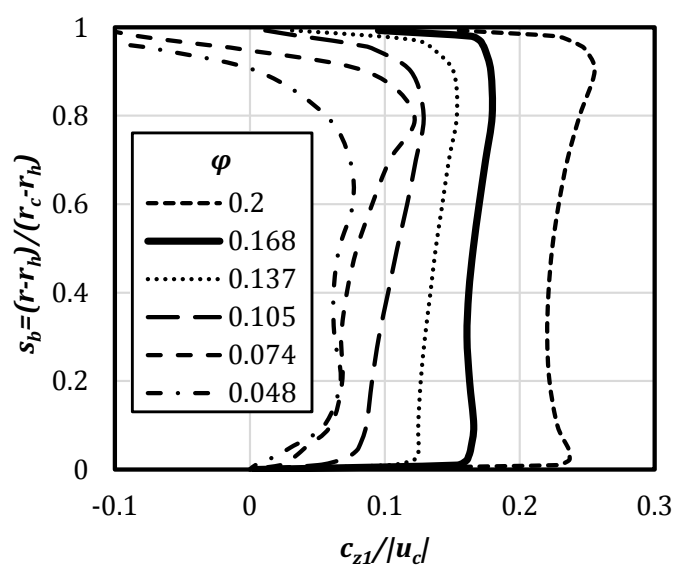

(a)

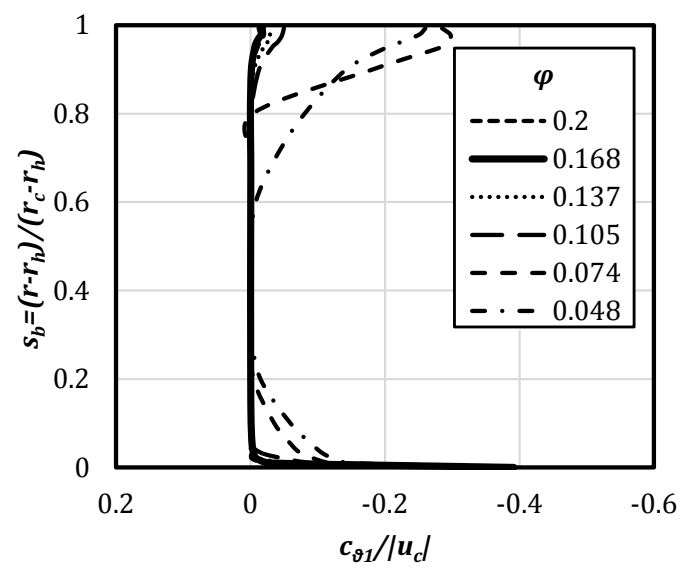

(c)

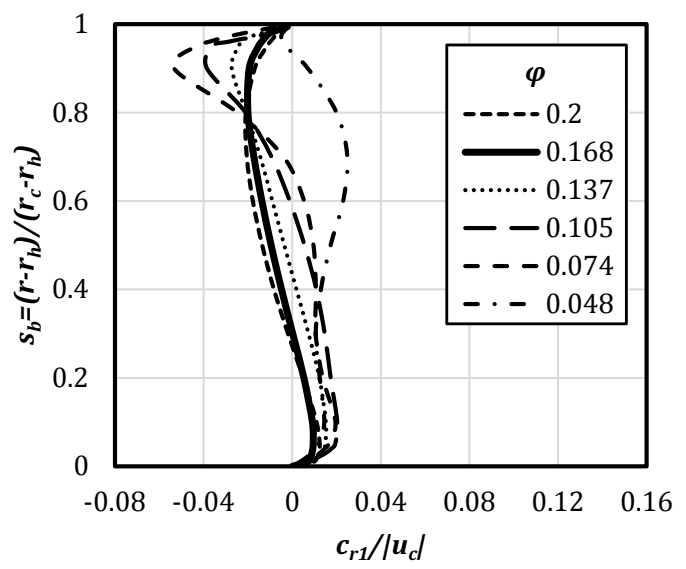

(e)

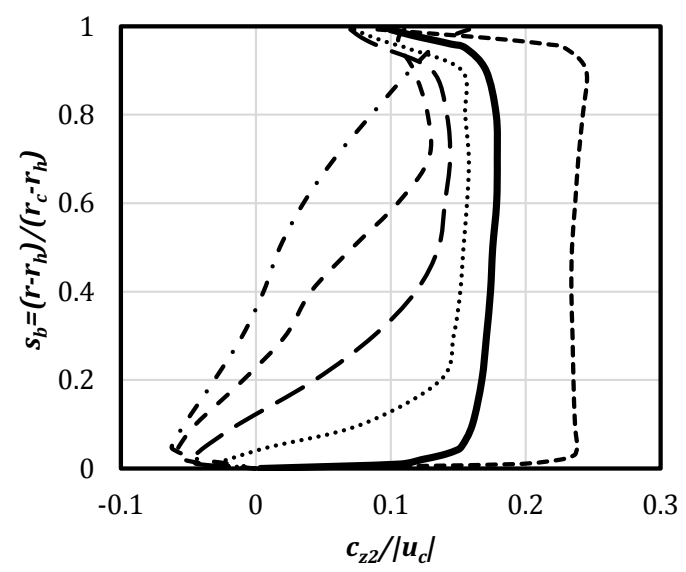

(b)

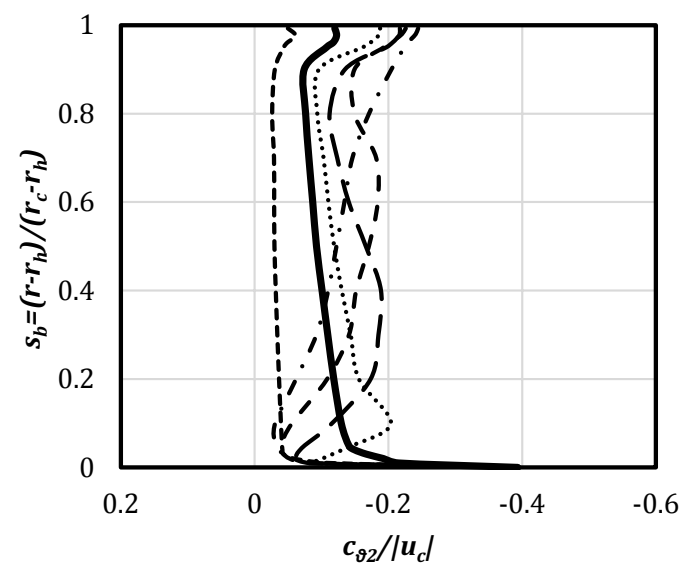

(d)

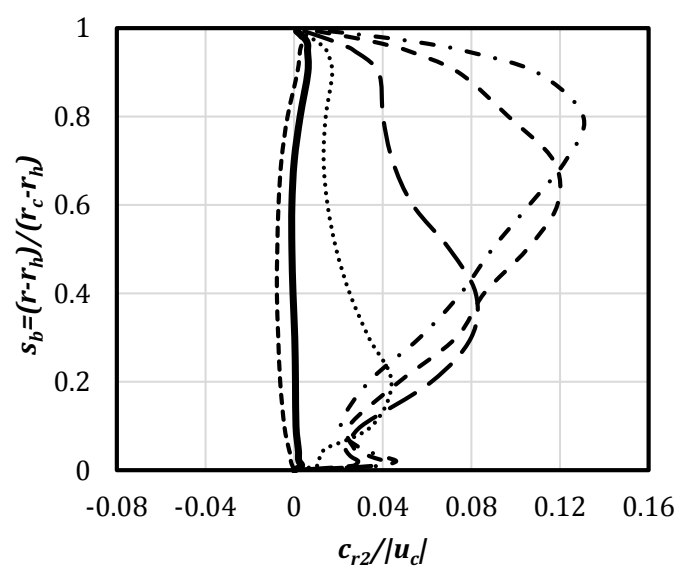

(f)

Figure 6.2: Circumferentially and time-averaged spanwise distributions of (a) $c_{z 1} /\left|u_{c}\right|$, (b) $c_{z 2} /\left|u_{c}\right|$, (c) $c_{\theta 1} /\left|u_{c}\right|$, (d) $c_{\theta 2} /\left|u_{c}\right|$, (e) $c_{r 1} /\left|u_{c}\right|$ and (f) $c_{r 2} /\left|u_{c}\right|$ for the range of flow rates investigated 


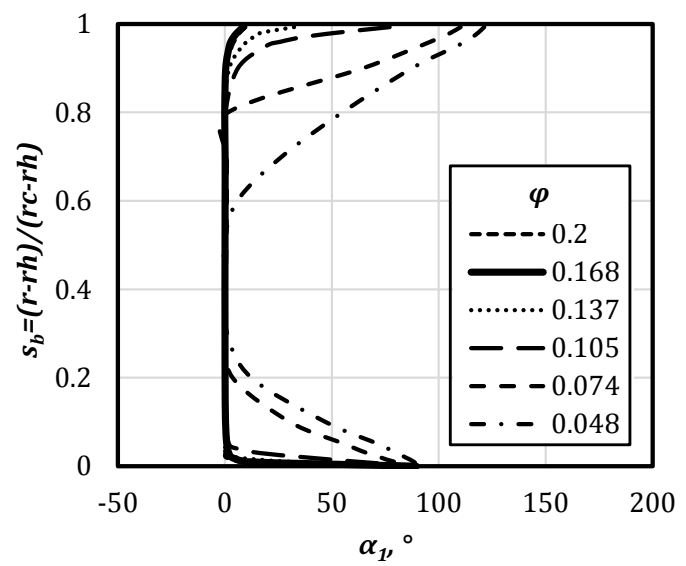

(a)

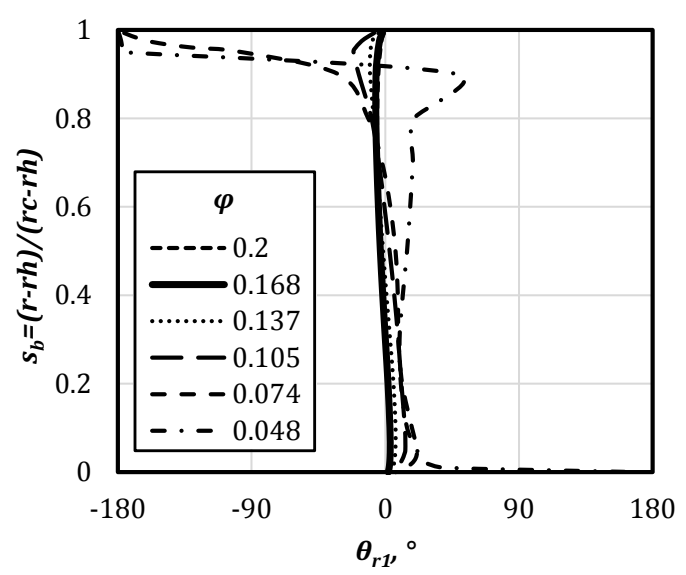

(c)

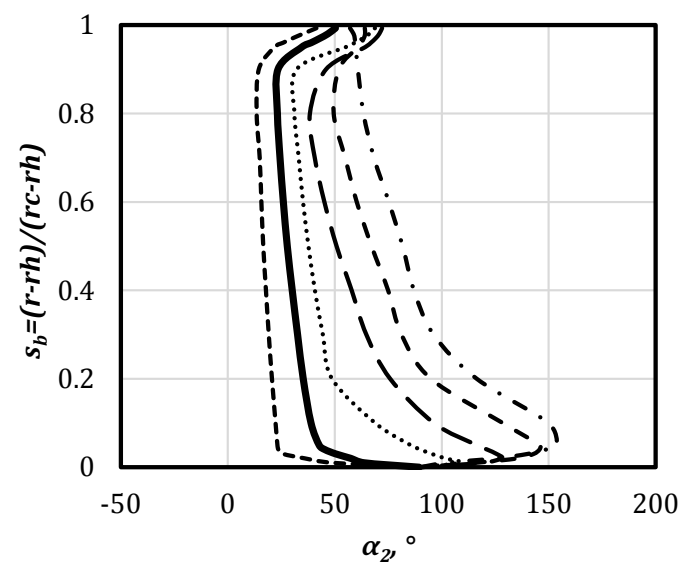

(b)

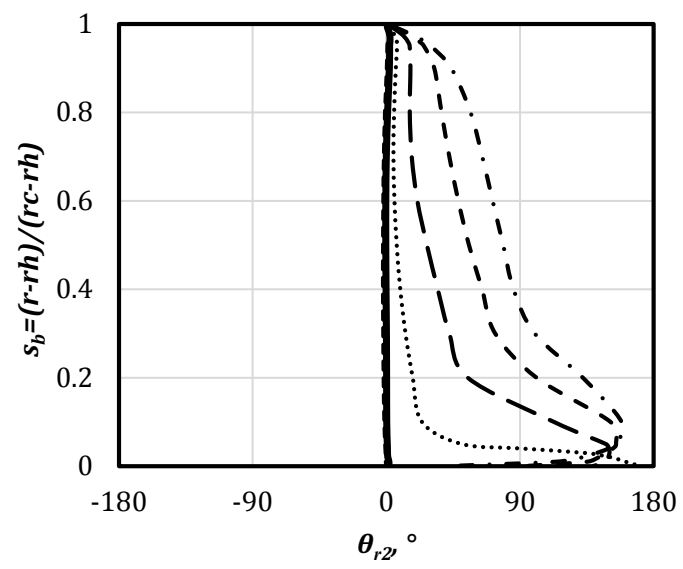

(d)

Figure 6.3: Circumferentially and time-averaged spanwise distributions of (a) $\alpha_{1}$, (b) $\alpha_{2}$, (c) $\theta_{r 1}$ and (d) $\theta_{r 2}$ for the range of flow rates investigated

characteristics of a free-vortex design. As the flow rate is decreased, an increase in radial velocity can be observed. Considering the low flow coefficient of $\phi=$ 0.137 , the maximum radial velocity component is visible near the hub. This magnitude increases with a decrease in flow rate, but the spanwise location of maximum radial velocity also moves in the radially outward direction. Once again this phenomenon can also be observed in the measurements of Downie et al. (1993). It follows that the downstream jet of the fan gradually changes from an axial direction at design flow conditions, towards a more diagonally outward direction as the flow rate through the fan decreases.

The absolute angle $(\alpha)$ characteristics (figures $6.3 \mathrm{a}$ and $\mathrm{b}$ ), representing the relationship between the absolute velocity components in the $\theta z$-plane, further establish the flow directions approaching and leaving the fan on the up- and downstream sides. The value of zero at the upstream location for the larger part of the blade span at higher flow rates are due to no swirl being present in the flow. However, the decrease in flow rate causes a change in this char- 


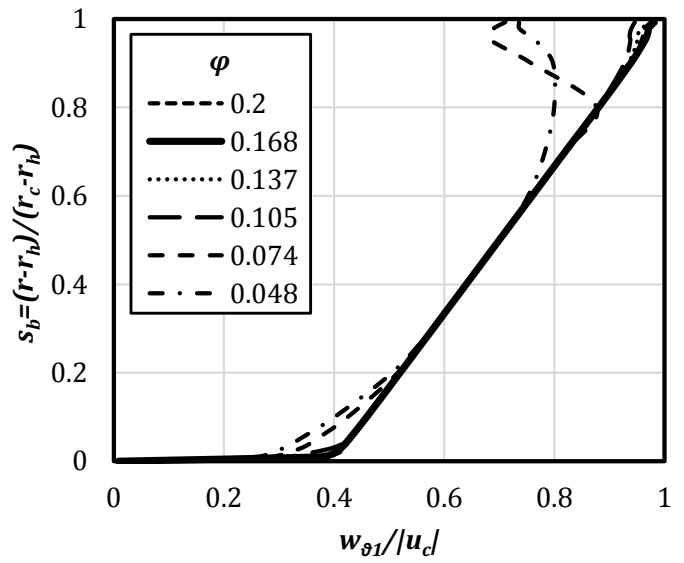

(a)

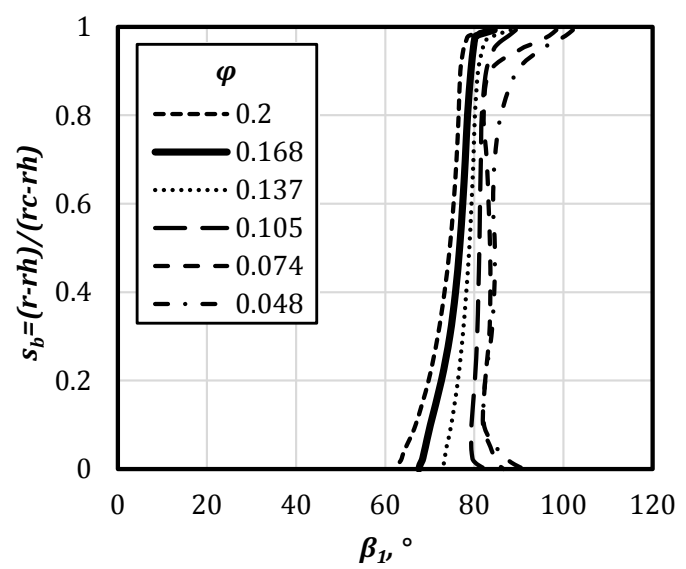

(c)

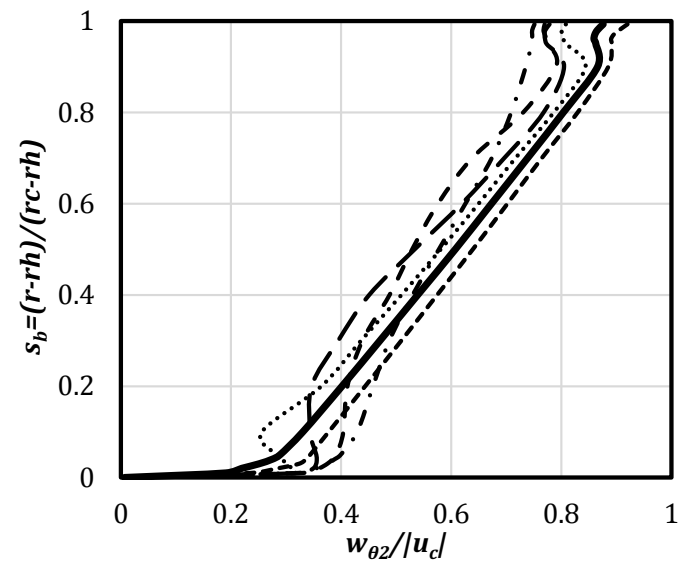

(b)

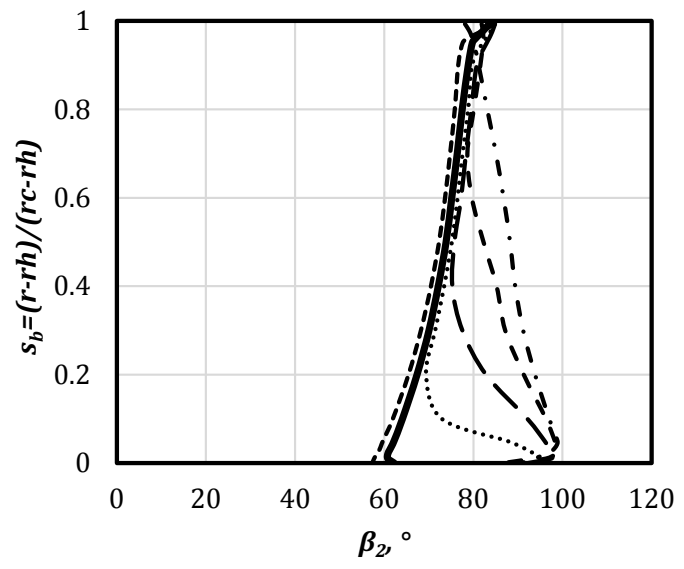

(d)

Figure 6.4: Circumferentially and time-averaged spanwise distributions of (a) $w_{\theta 1} /\left|u_{c}\right|$, (b) $w_{\theta 2} /\left|u_{c}\right|$, (c) $\beta_{1}$ and (d) $\beta_{2}$ for the range of flow rates investigated

acteristic to the extent where the flow is fully tangential $\left(\alpha=90^{\circ}\right)$ in certain spanwise locations and also starts reversing from the downstream towards the upstream side. The downstream profile shows the spanwise increase in reversed flow near the hub $\left(\alpha>90^{\circ}\right)$ as the flow rate through the fan decreases. The observation is also confirmed by the shape of radial flow angle distributions $\left(\theta_{r}\right.$, figures $6.3 \mathrm{c}$ and $\left.\mathrm{d}\right)$ starting in the hub region, but gradually covering a larger section of the blade span towards the tip, with a reduction in flow rate.

A linear trend in the tangential relative velocity $\left(w_{\theta}\right)$ component (figures $6.4 \mathrm{a}$ and b) can be observed on the up- and downstream sides of the fan for the larger section of blade span. Once again, deviations from this trend are observed at reduced flow rates. The relative flow angles ( $\beta$, figures $6.3 \mathrm{c}$ and d) in the $\theta z$-plane also confirm the drastic change in flow angles, starting near the hub and gradually moving radially outwards as the flow rate is decreased.

With regards to the pressure profiles (figures $6.5 \mathrm{a}-\mathrm{d}$ ) a near uniform (Sshaped) distribution is noted upstream of the fan at near-design flow rates, 


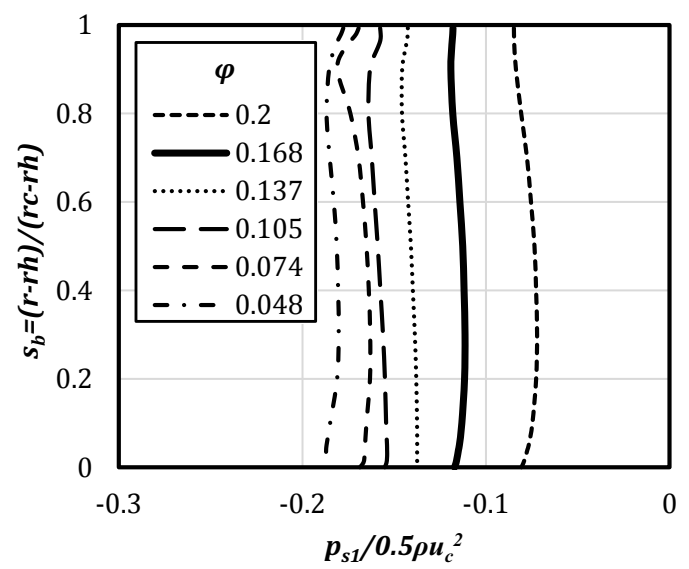

(a)

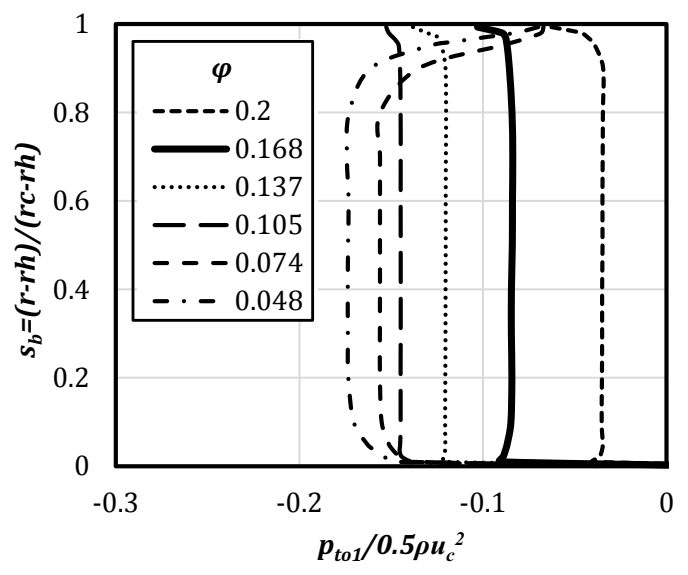

(c)

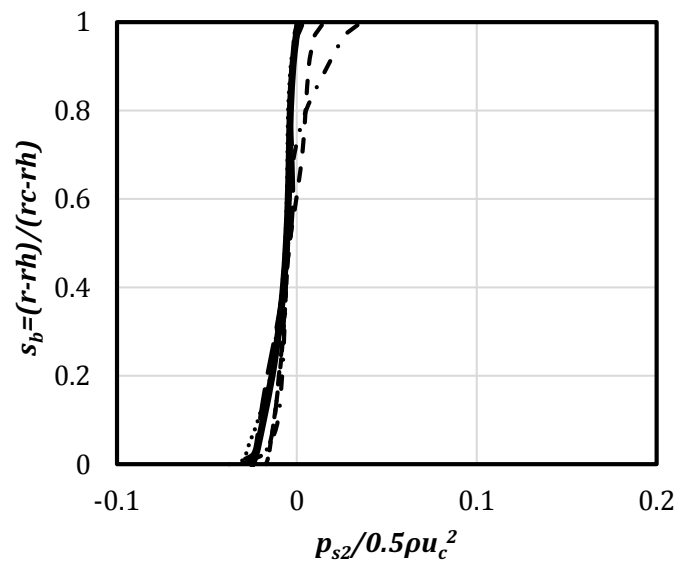

(b)

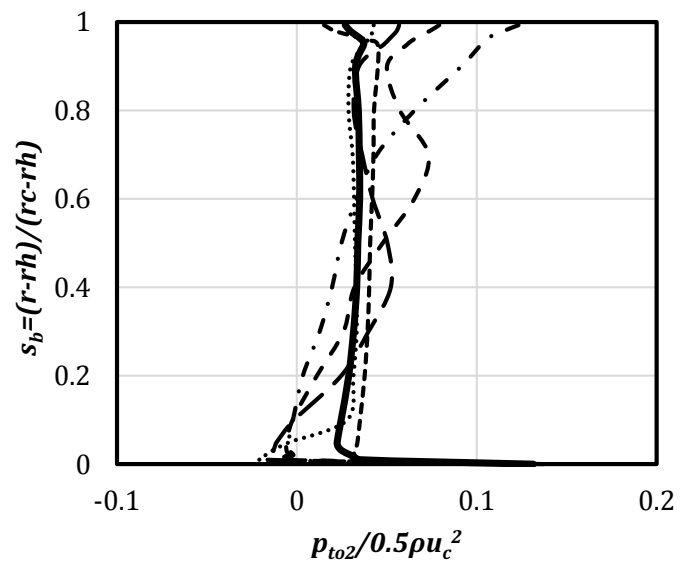

(d)

Figure 6.5: Circumferentially and time-averaged spanwise distributions of (a) $p_{s 1} / 0.5 \rho u_{c}^{2}$, (b) $p_{s 2} / 0.5 \rho u_{c}^{2}$, (c) $p_{t o 1} / 0.5 \rho u_{c}^{2}$ and (d) $p_{t o 2} / 0.5 \rho u_{c}^{2}$ for the range of flow rates investigated

but deviates from this trend at lower flow rates. The static pressure distribution on the downstream side is also slightly S-shaped and near zero as expected, since a reference static pressure value of zero is specified on the pressure outlet boundaries of the computational domain. Static pressure values higher than zero are observed near the tip for lower flow rates and could be attributed to the induced pre-swirl as well as the radial outflow in this region. A uniform total pressure distribution is observed upstream of the fan at higher flow rates, which is also expected since little energy has been added to the fluid at this stage. Once again a deviation from this trend near the tip can be observed at lower flow rates due to reversed flow propagating upstream of the fan. On the downstream side, a uniform distribution of total pressure is also noted for near-design flow rates. However, as the flow rate is reduced, a local maximum in the profile near the hub becomes visible and moves radially outwards with a further decrease in flow, similar in trend to the downstream circumferential 
and radial velocity profiles.

\subsection{Time-dependent spanwise flow distributions}

Additional insight in the flow field at low flow rates $(0.042 \leq \phi \leq 0.105)$ is obtained by visualizing the circumferentially averaged flow profiles, discussed in the previous section (6.2), with respect to time. These instantaneous flow profiles are presented in figures 6.6 to 6.12 for three cycles of the time-wise periodic flow field, where time is expressed in terms of fan rotations $\left(n_{F r}\right)$ and one fan rotation takes $80 \mathrm{~ms}$ to complete. In these figures the left- and right-hand sides represent the up- and downstream locations, respectively.

Considering the time-dependent axial velocity profiles (figures $6.6 \mathrm{a}$ to f), a near-steady profile can be observed at the upstream location for the largest section of the blade span for a flow coefficient of $\phi=0.105$. However, a timedependent flow structure occurs in the region of the hub. On the downstream side the flow structure in the hub region is amplified and pulsating reversed flow is visible. An increase in the flow instability is observed as the flow rate is decreased. In addition, the progressive redistribution of fluid can also be observed to shift to higher radial locations as the flow rate is decreased.

Examining the time-dependent circumferential velocity profiles (figures 6.7a to f), a steady nature of the flow at $\phi=0.105$ for the largest span, upstream of the blade, is noticed. Thus, no upstream swirl is induced by the rotor, except in the hub and tip regions. Again, the instability in upstream tangential velocity gradually spreads over a larger section of the span as the flow rate decreases. At the downstream location a shift in maximum tangential velocity from the hub towards the tip is observed, with a reduction in flow rate. A secondary flow pattern is also visible as the location of maximum tangential velocity shifts up and down the span with time, for a single flow rate.

With regards to the transient radial velocity profiles (figures $6.8 \mathrm{a}$ to $\mathrm{f}$ ) radial pulses can be observed near the hub and spreads to higher radii at lower flow rates. The redistribution and pulsating nature of the maximum radial velocity on the downstream side of the fan can also be observed, with a second smaller pulse per period visible for $\phi=0.042$.

The above observations are summarized when the absolute and radial angle spanwise distributions (figures $6.9 \mathrm{a}$ to $\mathrm{f}$ and $6.10 \mathrm{a}$ to $\mathrm{f}$ ) are considered. Considerable time-dependent changes in flow direction are visible near the hub region on the downstream side and become more pronounced and occur over larger portions of the blade span as the the flow rate decreases. These figures also indicate the reversed flow near the hub region, which is subsequently directed radially outward. These observations are confirmed by the relative circumferential velocity and angle profiles (figures 6.11a to f and 6.12a to f). 
The static and total pressure distributions (figures 6.13a to f and 6.14a to f) emphasize the pulsating nature of the flow through the fan at low flow rates. Similar to the time-averaged pressure distributions, near-uniform inlet profiles can be observed on the upstream side of the fan, except for the tip region. The downstream total pressure distribution also shows a similar trend to the circumferential and radial profiles as the location of maximum total pressure shifts to higher radii with a decrease in flow rate, presenting the locations of the bulk fluid flow through the fan.

Table 6.1: Cycle lengths of the flow structure in the vicinity of the fan at low flow rates

\begin{tabular}{ll}
\hline $\boldsymbol{\phi}\left(\dot{\boldsymbol{V}}, \mathrm{m}^{3} / \mathrm{s}\right)$ & $\boldsymbol{n}_{\boldsymbol{F r}}$ \\
\hline $0.105(10)$ & 1.950 \\
$0.074(7)$ & 2.775 \\
$0.042(4)$ & 3.225 \\
\hline
\end{tabular}

To conclude this section, one more observation is emphasised. Visible from the majority of time-dependent contour plots (especially at the downstream location) are the time wise diagonal "valleys" of the flow variables. This indicates that a flow structure starts developing in the hub region and then propagates to the tip of the fan with time, whereafter the periodic nature of the flow field repeats itself. The time for a single flow period also increases with a decrease in flow rate as listed in table 6.1.

\subsection{Global flow structure}

The global flow structure induced by the fan is depicted in this section by twodimensional streamlines on a meridional plane through the fan, for the range of flow rates investigated. For simulations at near-design flow rates $(0.137 \leq$ $\phi \leq 0.200$ ), single instances of the flow field are depicted in figure $6.15 \mathrm{a}$ to $\mathrm{c}$, since steady state results are obtained. However, four time frames of the time-dependent flow field for each of the low flow rates $(0.042 \leq \phi \leq 0.105)$ are depicted in figures 6.16 to 6.18 .

Regarding the steady near-design $(0.137 \leq \phi \leq 0.200)$ global flow fields depicted in figure $6.15 \mathrm{a}$ to $\mathrm{c}$, it is evident that the flow through the fan is generally in the axial direction. The radial flow also increases with a decrease in flow rate.

Considering the section between the fan centre line and the hub radius on the downstream side, it can be observed that a reversed flow pattern is always present, although originally small at the highest flow rate $(\phi=0.200)$ and 


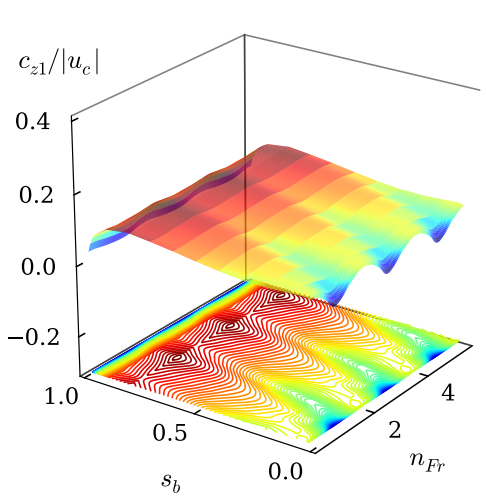

(a)

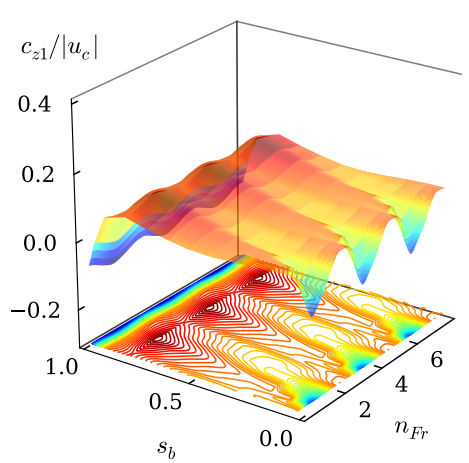

(c)

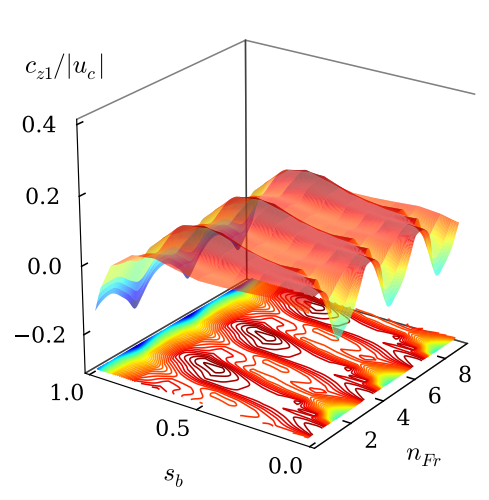

(e)
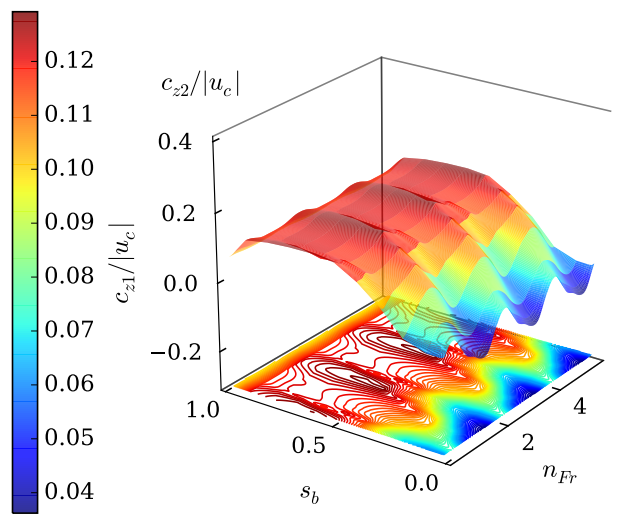

(b)
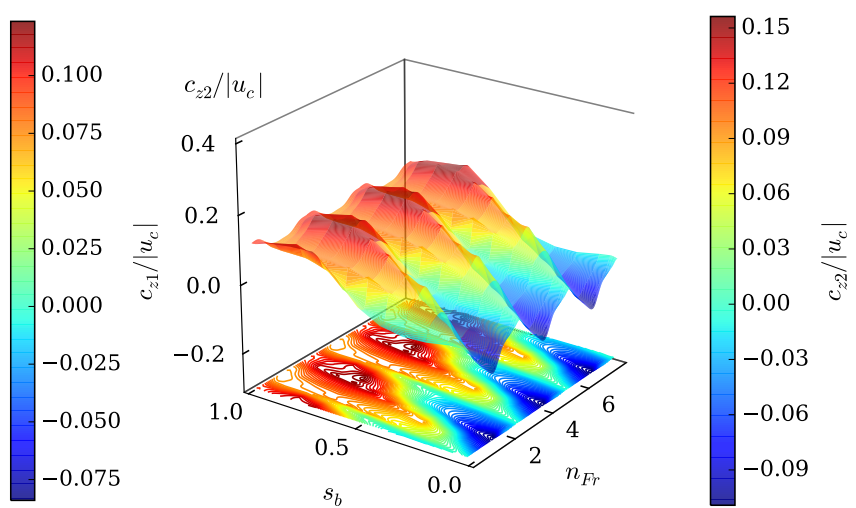

(d)
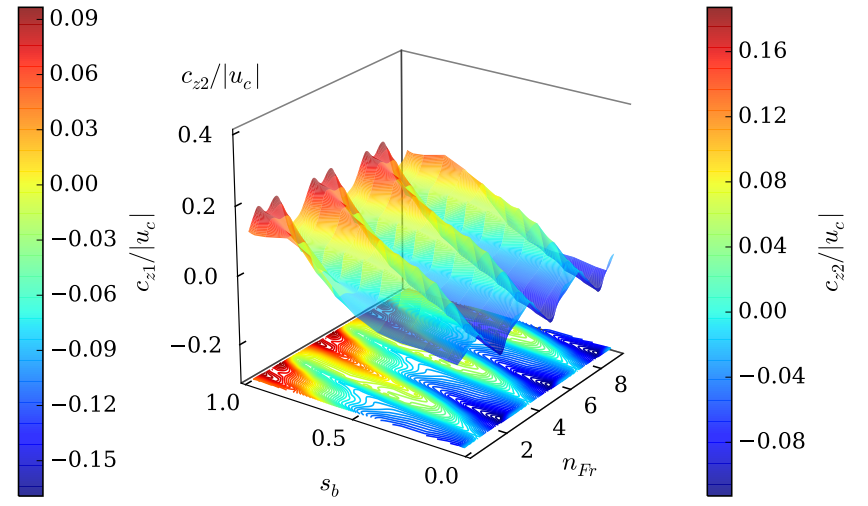

(f)

Figure 6.6: Time-dependent spanwise axial velocity $\left(c_{z} /\left|u_{c}\right|\right)$ profiles for (a) $\phi=0.105$, upstream, (b) $\phi=0.105$, downstream, (c) $\phi=0.074$, upstream, (d) $\phi=0.074$, downstream, (e) $\phi=0.042$, upstream and (f) $\phi=0.042$, downstream of the fan 


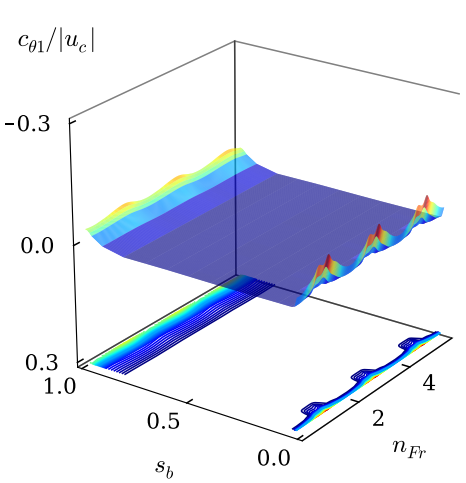

(a)

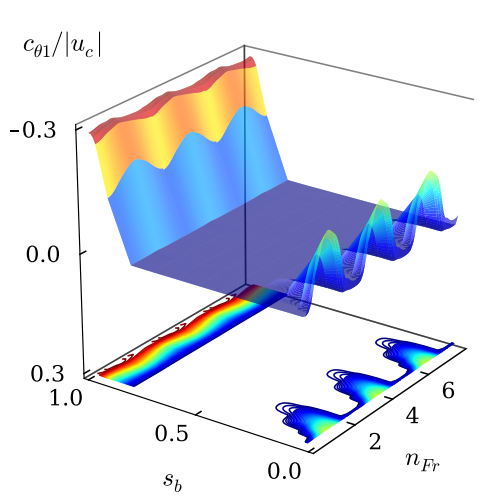

(c)

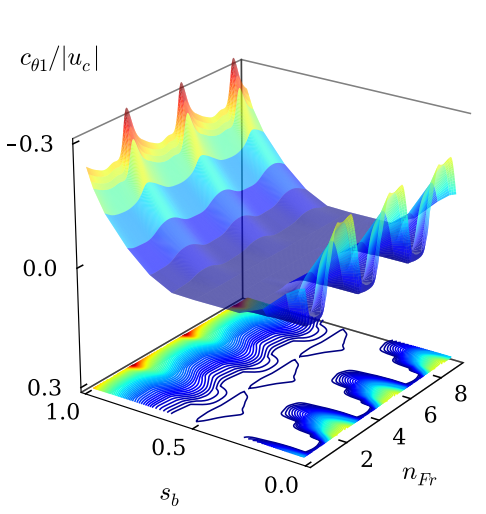

(e)
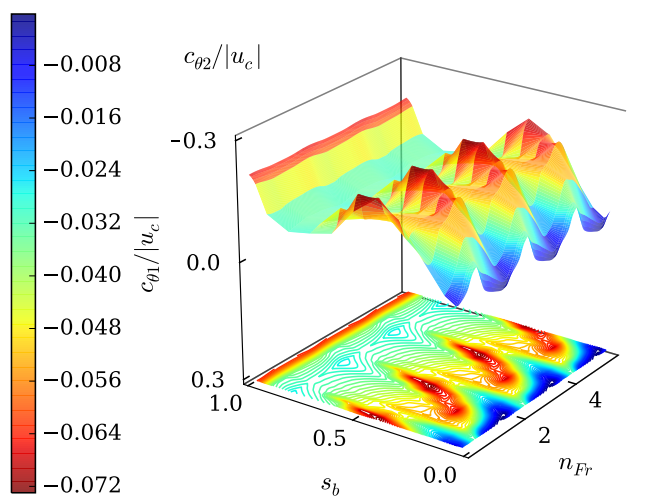

(b)
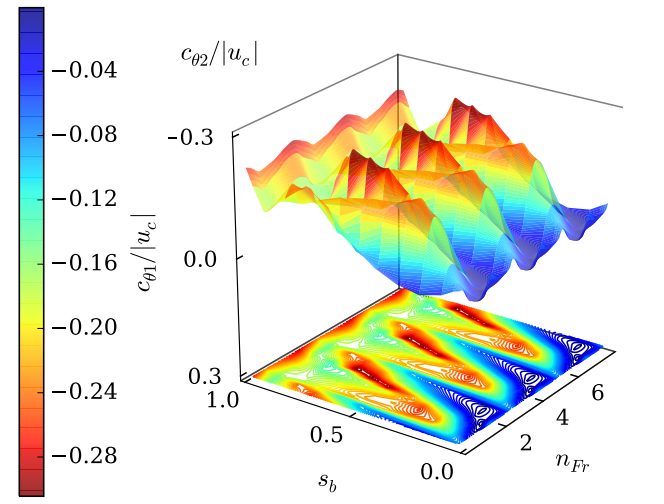

(d)
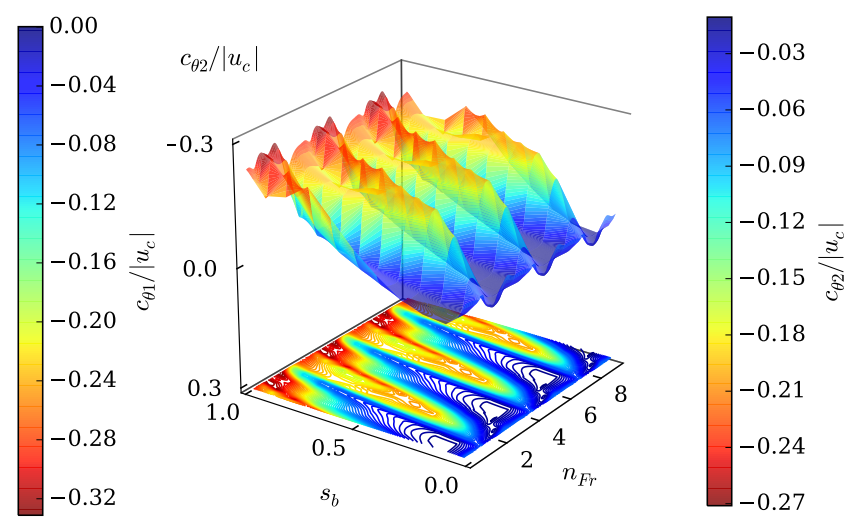

(f)

Figure 6.7: Time-dependent spanwise circumferential velocity $\left(c_{\theta} /\left|u_{c}\right|\right)$ profiles for (a) $\phi=0.105$, upstream, (b) $\phi=0.105$, downstream, (c) $\phi=0.074$, upstream, (d) $\phi=0.074$, downstream, (e) $\phi=0.042$, upstream and (f) $\phi=$ 0.042 , downstream of the fan 


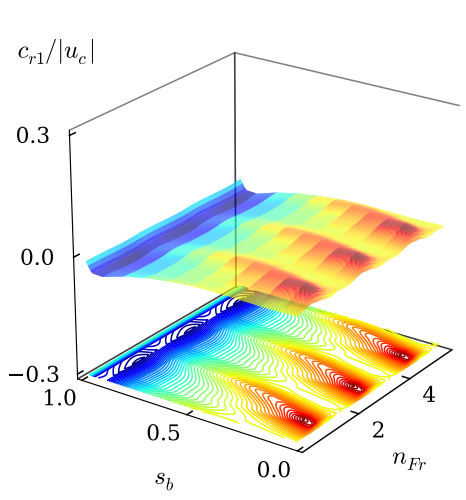

(a)

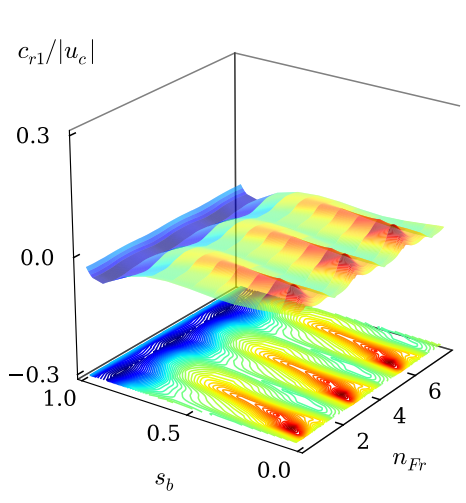

(c)

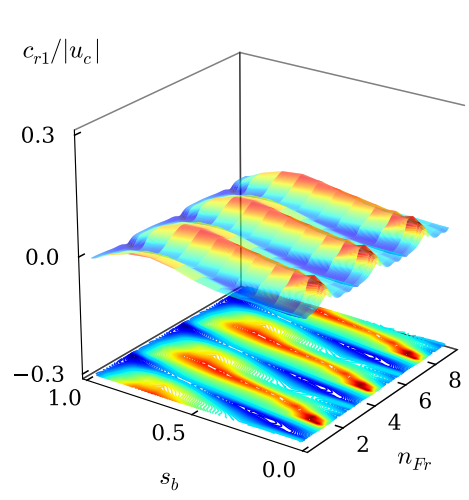

(e)
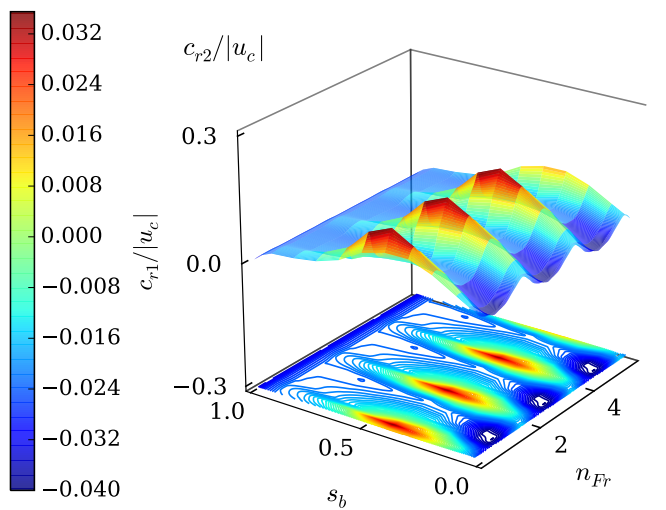

(b)
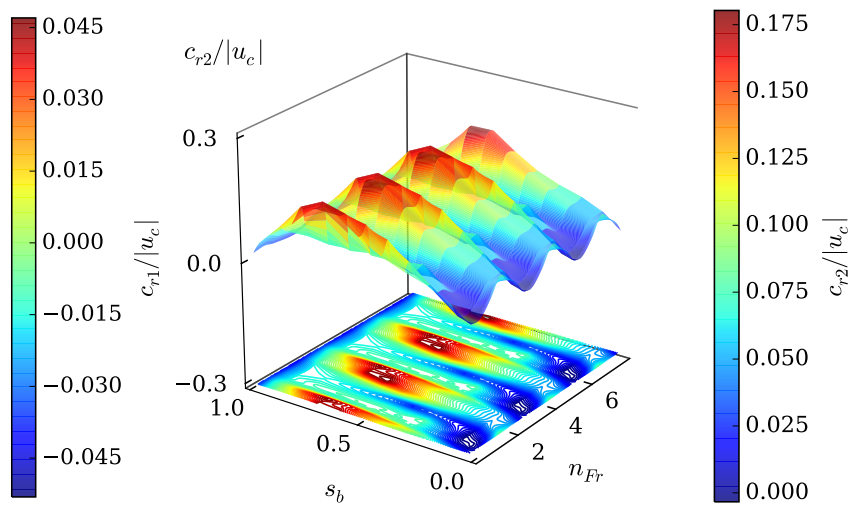

(d)
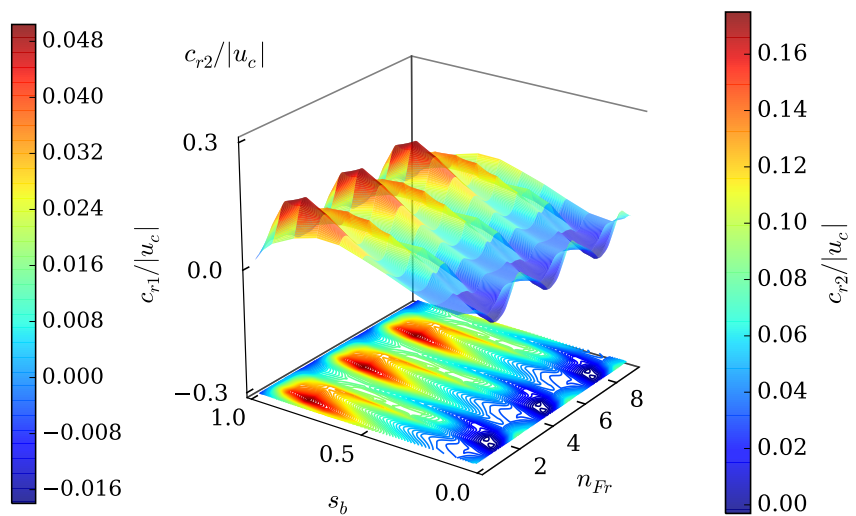

(f)

Figure 6.8: Time-dependent spanwise radial velocity $\left(c_{r} /\left|u_{c}\right|\right)$ profiles for (a) $\phi=0.105$, upstream, (b) $\phi=0.105$, downstream, (c) $\phi=0.074$, upstream, (d) $\phi=0.074$, downstream, (e) $\phi=0.042$, upstream and (f) $\phi=0.042$, downstream of the fan 


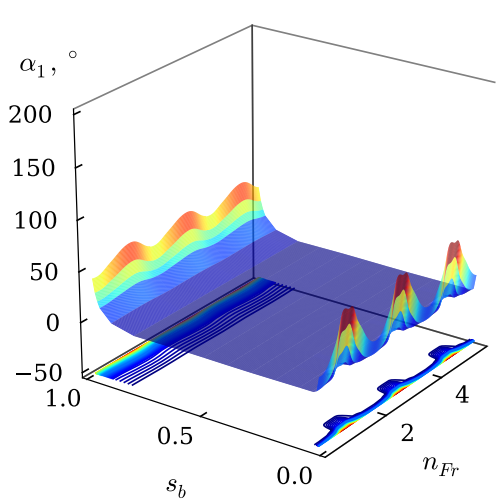

(a)

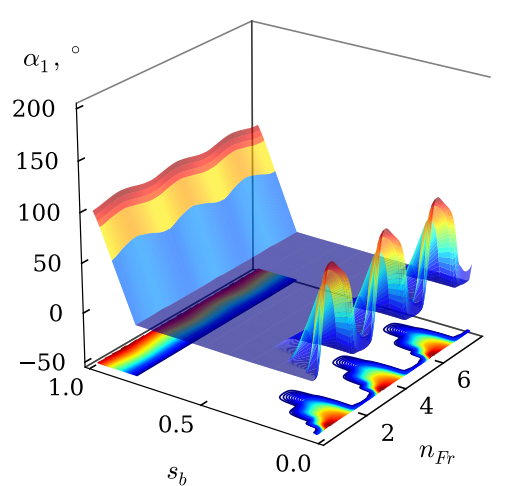

(c)

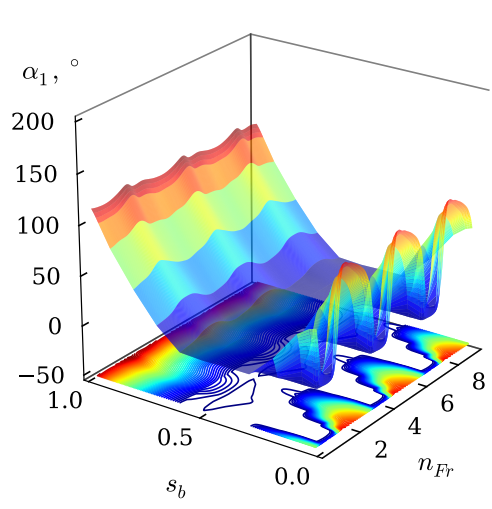

(e)
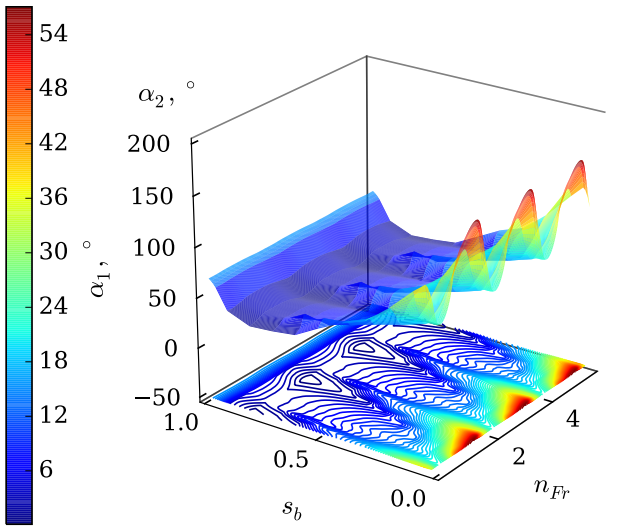

(b)
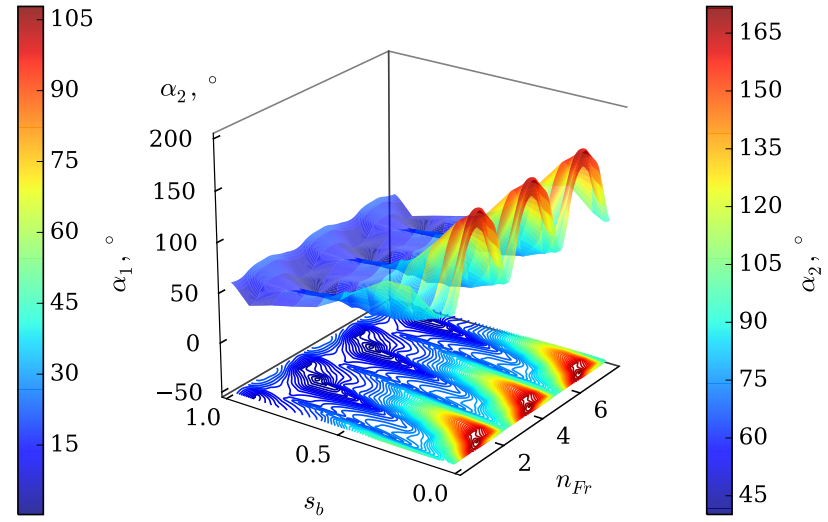

(d)
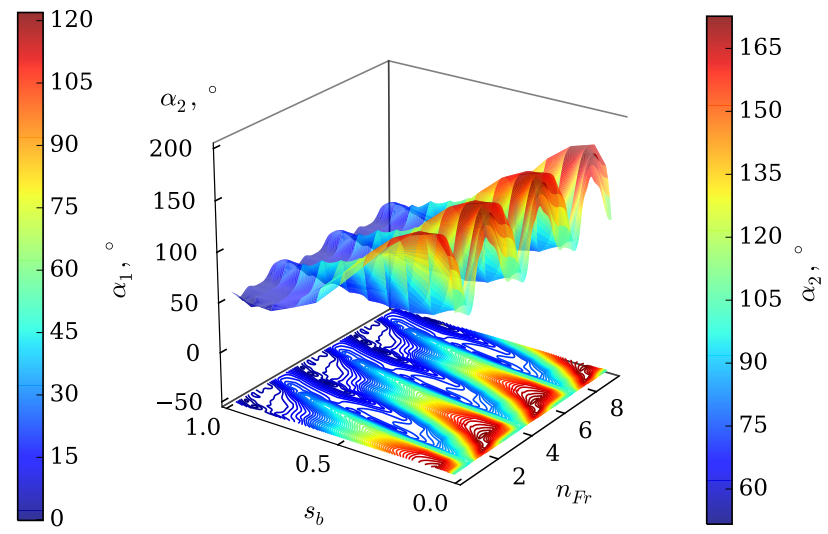

(f)

Figure 6.9: Time-dependent spanwise absolute angle $(\alpha)$ profiles for (a) $\phi=$ 0.105, upstream, (b) $\phi=0.105$, downstream, (c) $\phi=0.074$, upstream, (d) $\phi=$ 0.074 , downstream, (e) $\phi=0.042$, upstream and (f) $\phi=0.042$, downstream of the fan 


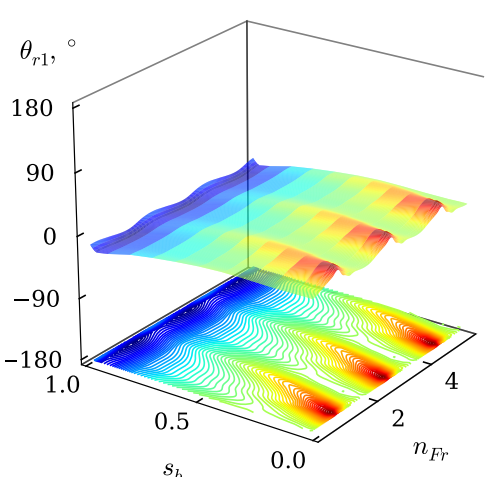

(a)

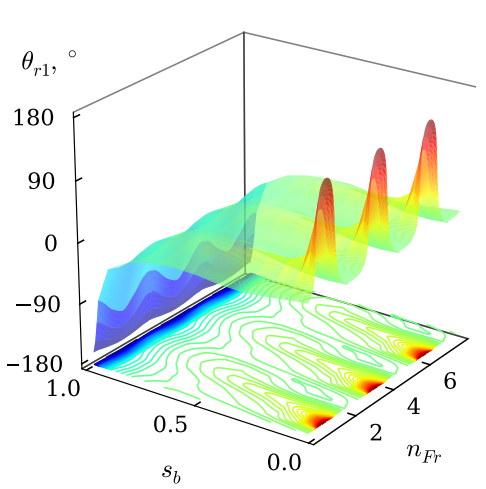

(c)

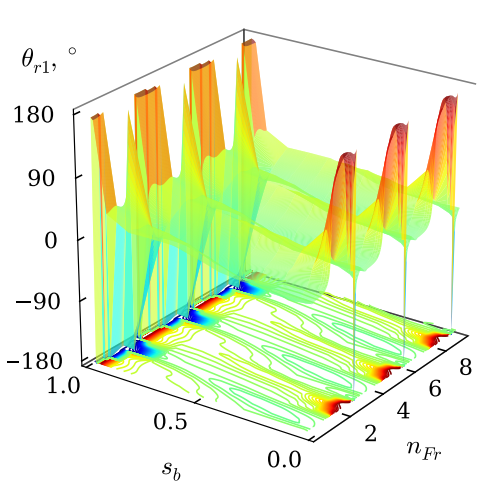

(e)
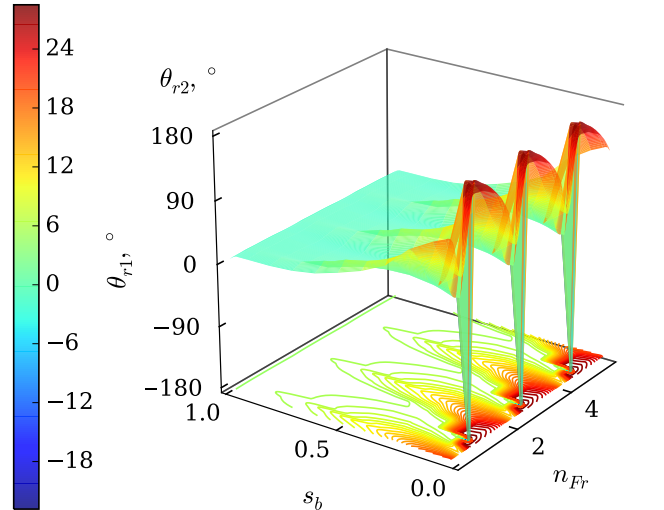

(b)
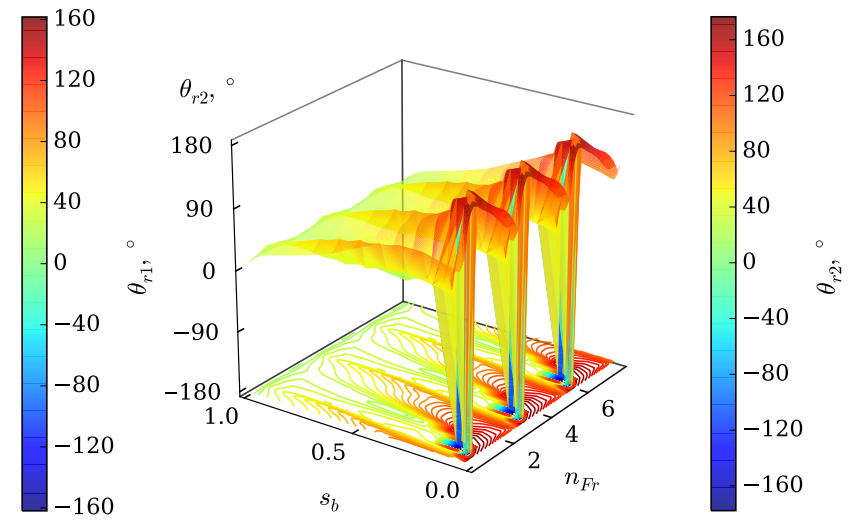

(d)
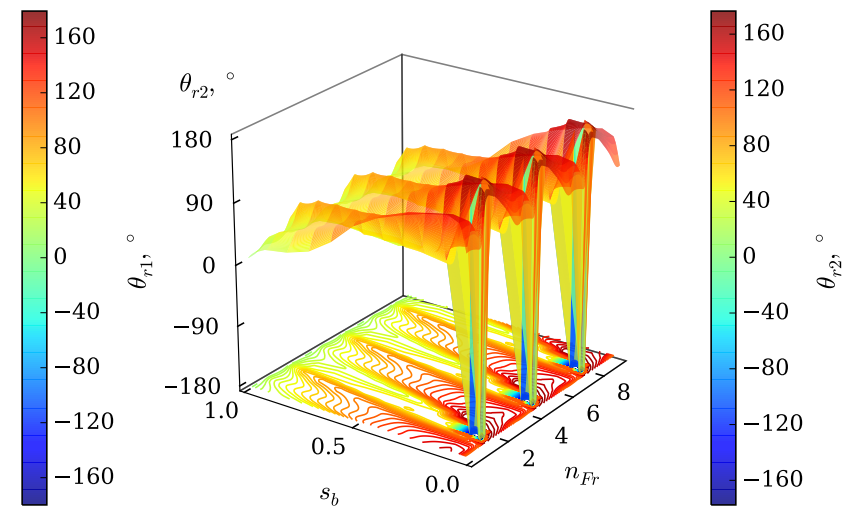

(f)

Figure 6.10: Time-dependent spanwise radial flow angle $\left(\theta_{r}\right)$ profiles for (a) $\phi=0.105$, upstream, (b) $\phi=0.105$, downstream, (c) $\phi=0.074$, upstream, (d) $\phi=0.074$, downstream, (e) $\phi=0.042$, upstream and (f) $\phi=0.042$, downstream of the fan 


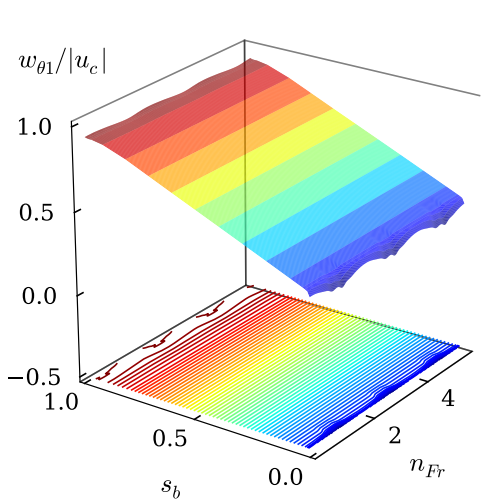

(a)

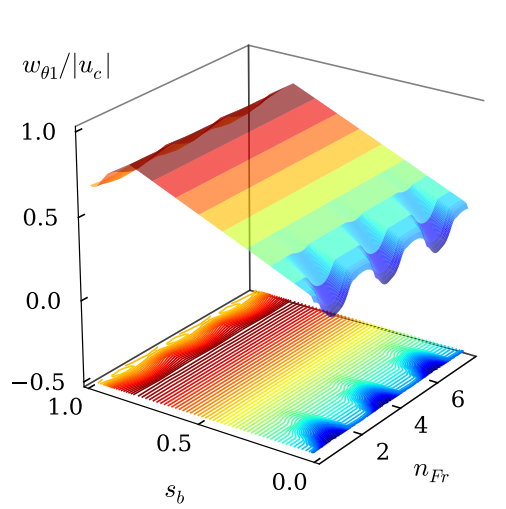

(c)

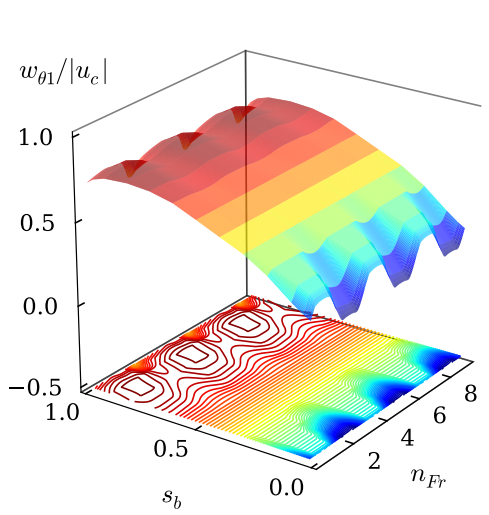

(e)

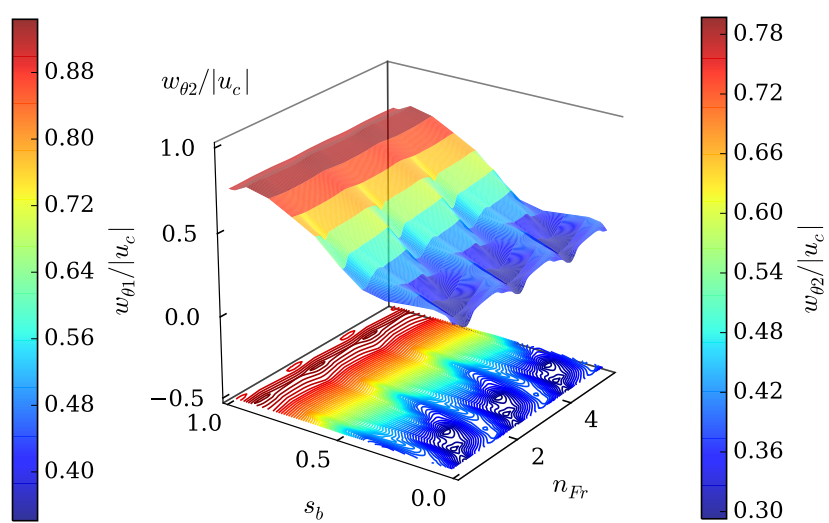

(b)
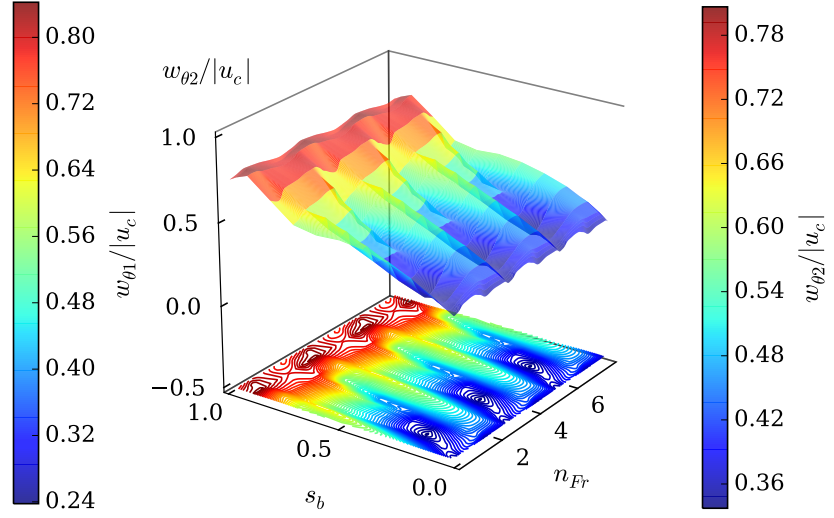

(d)
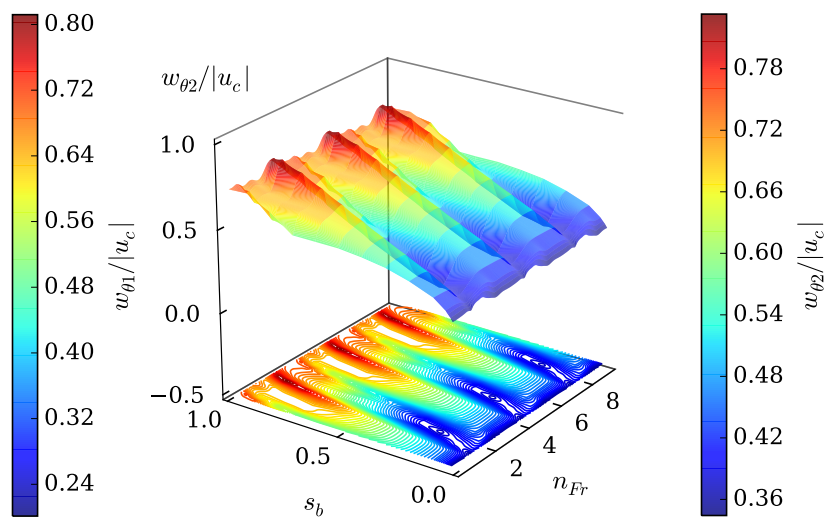

(f)

Figure 6.11: Time-dependent spanwise relative circumferential velocity $\left(w_{\theta} /\left|u_{c}\right|\right)$ profiles for (a) $\phi=0.105$, upstream, (b) $\phi=0.105$, downstream, (c) $\phi=0.074$, upstream, (d) $\phi=0.074$, downstream, (e) $\phi=0.042$, upstream and (f) $\phi=0.042$, downstream of the fan 


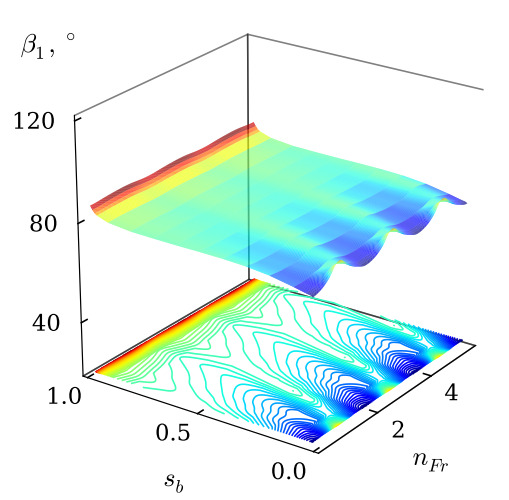

(a)

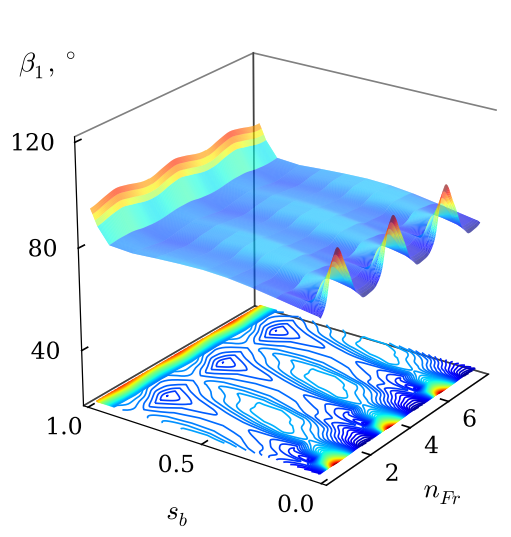

(c)

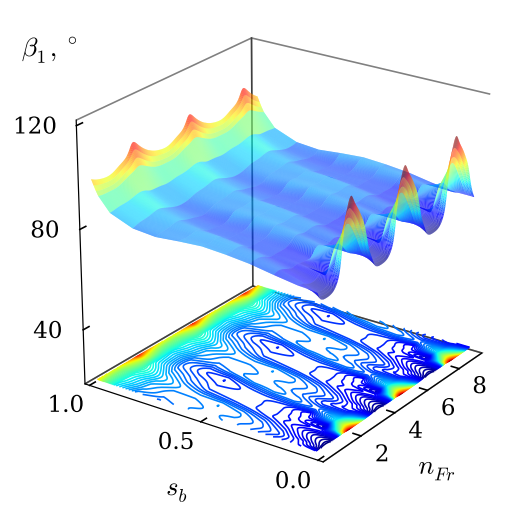

(e)
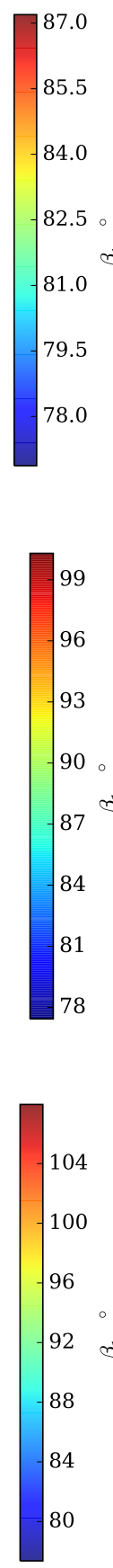

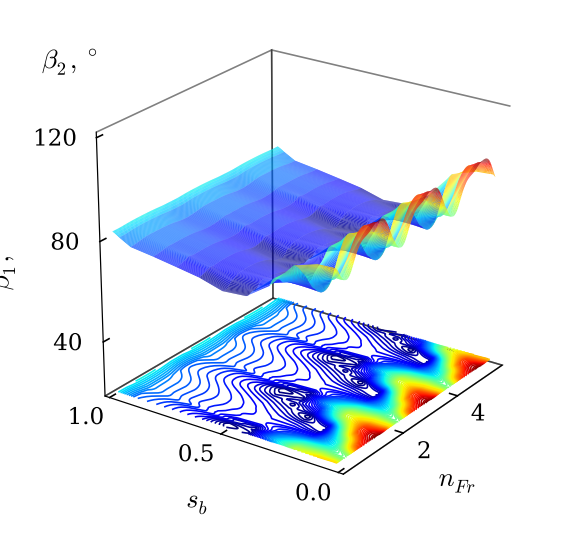

(b)

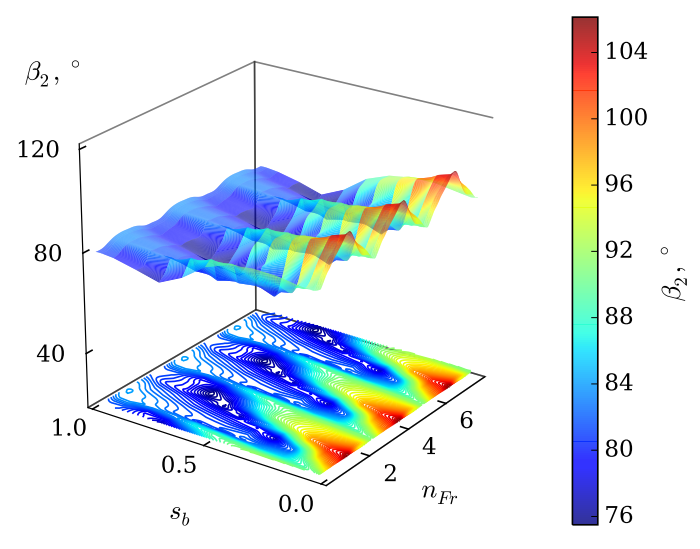

(d)

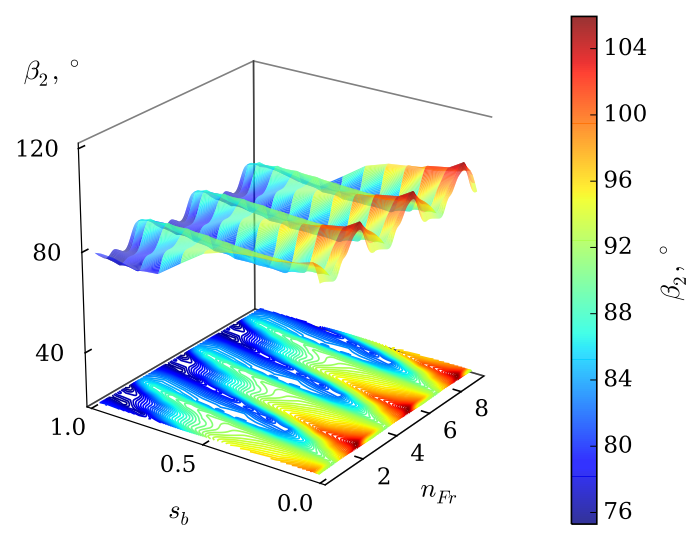

(f)

Figure 6.12: Time-dependent spanwise relative angle $(\beta)$ profiles for (a) $\phi=$ 0.105, upstream, (b) $\phi=0.105$, downstream, (c) $\phi=0.074$, upstream, (d) $\phi=$ 0.074 , downstream, (e) $\phi=0.042$, upstream and (f) $\phi=0.042$, downstream of the fan 


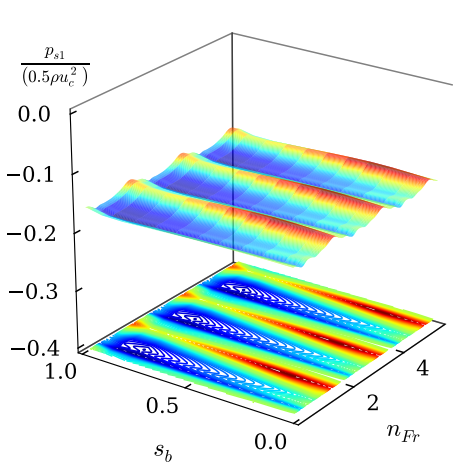

(a)

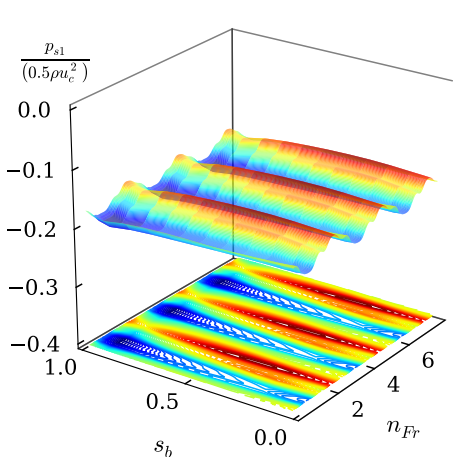

(c)

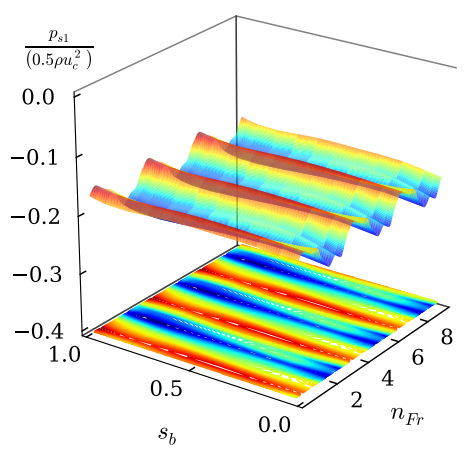

(e)
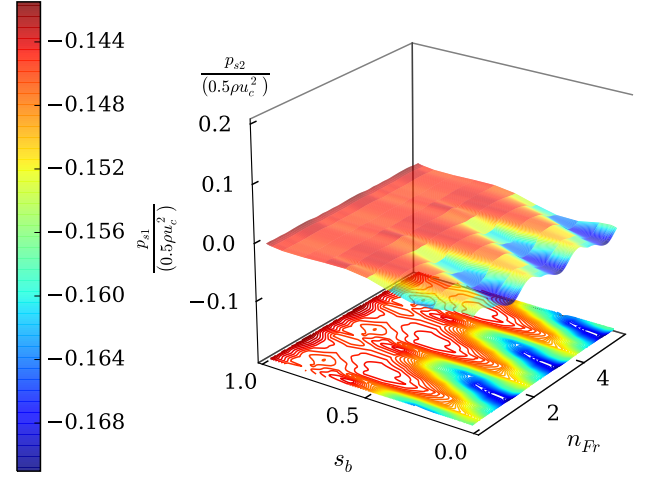

(b)
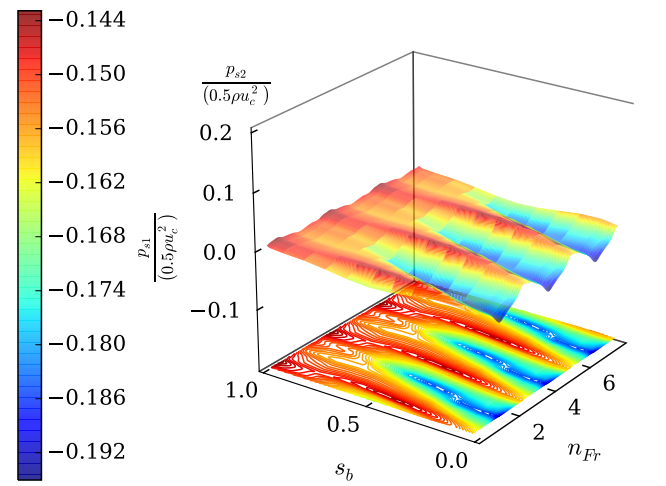

(d)
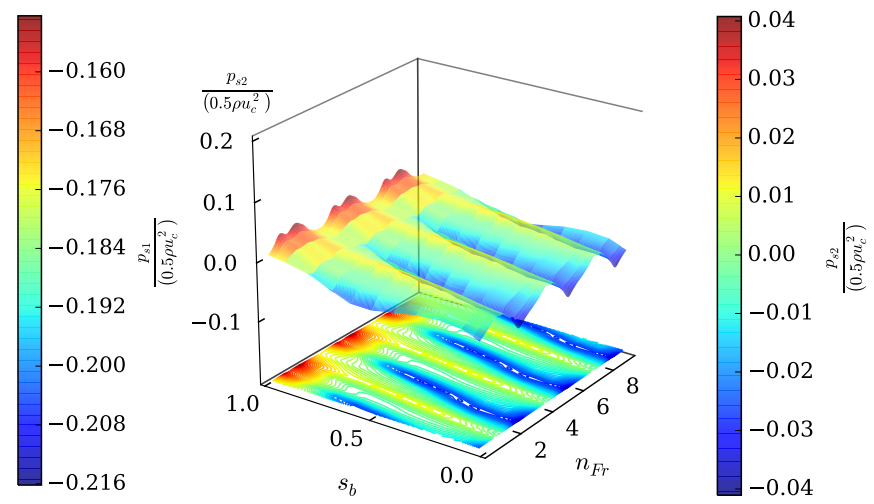

(f)

Figure 6.13: Time-dependent spanwise dimensionless static pressure $\left(p_{s} / 0.5 \rho u_{c}^{2}\right)$ profiles for (a) $\phi=0.105$, upstream, (b) $\phi=0.105$, downstream, (c) $\phi=0.074$, upstream, (d) $\phi=0.074$, downstream, (e) $\phi=0.042$, upstream and (f) $\phi=0.042$, downstream of the fan 


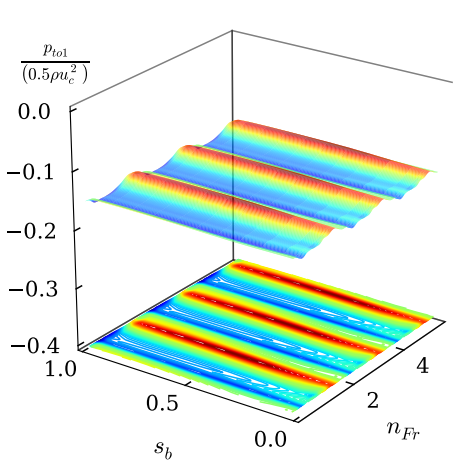

(a)

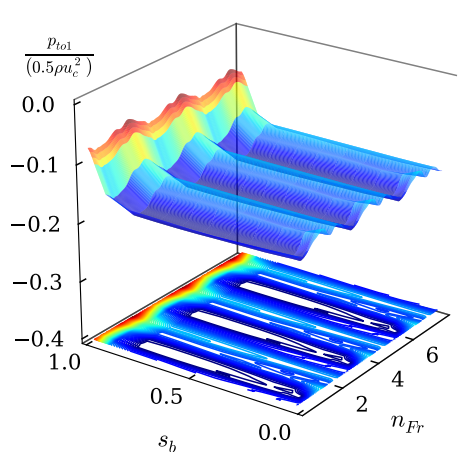

(c)

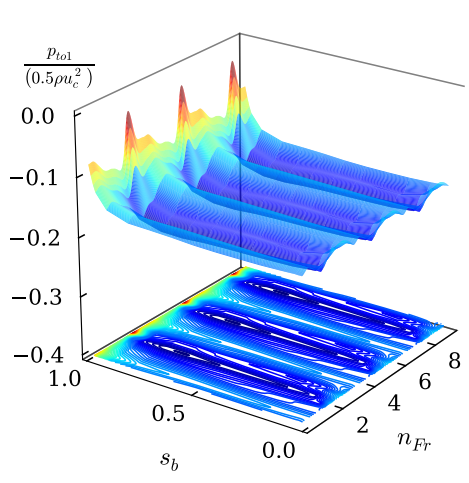

(e)

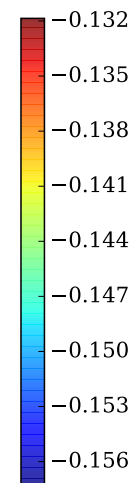

年

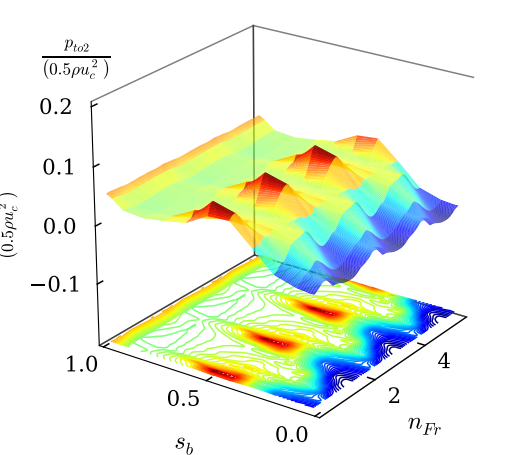

(b)
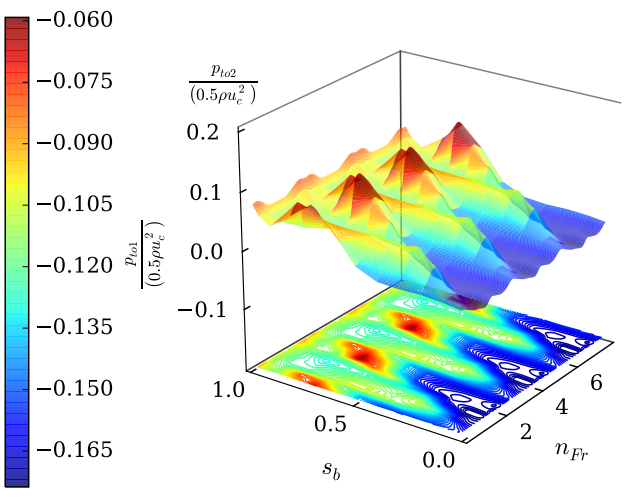

(d)
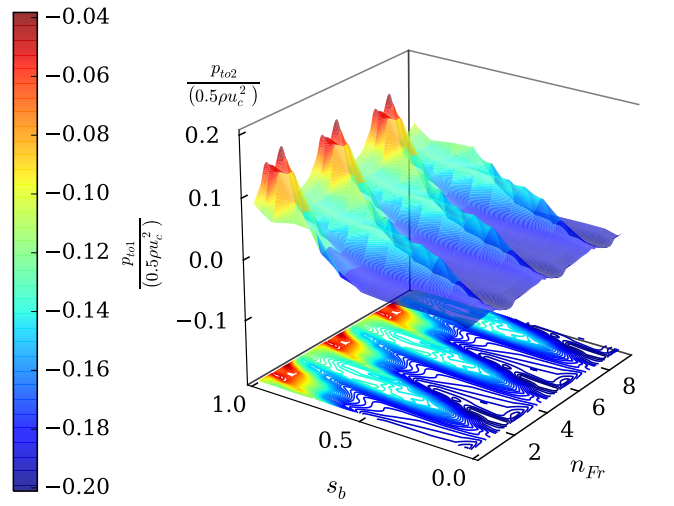

(f)

Figure 6.14: Time-dependent spanwise dimensionless total pressure $\left(p_{t o} / 0.5 \rho u_{c}^{2}\right)$ profiles for (a) $\phi=0.105$, upstream, (b) $\phi=0.105$, downstream, (c) $\phi=0.074$, upstream, (d) $\phi=0.074$, downstream, (e) $\phi=0.042$, upstream and (f) $\phi=0.042$, downstream of the fan 


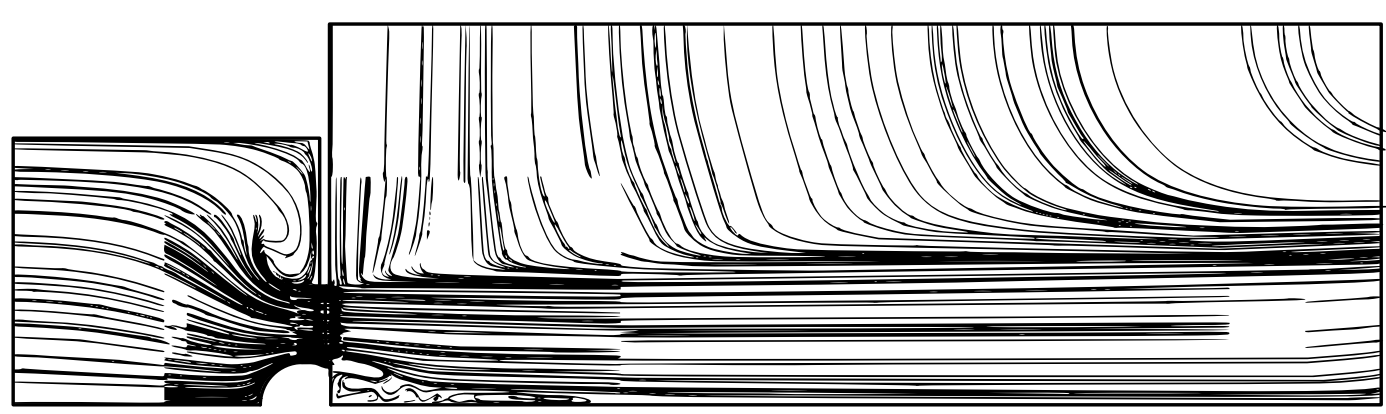

(a)

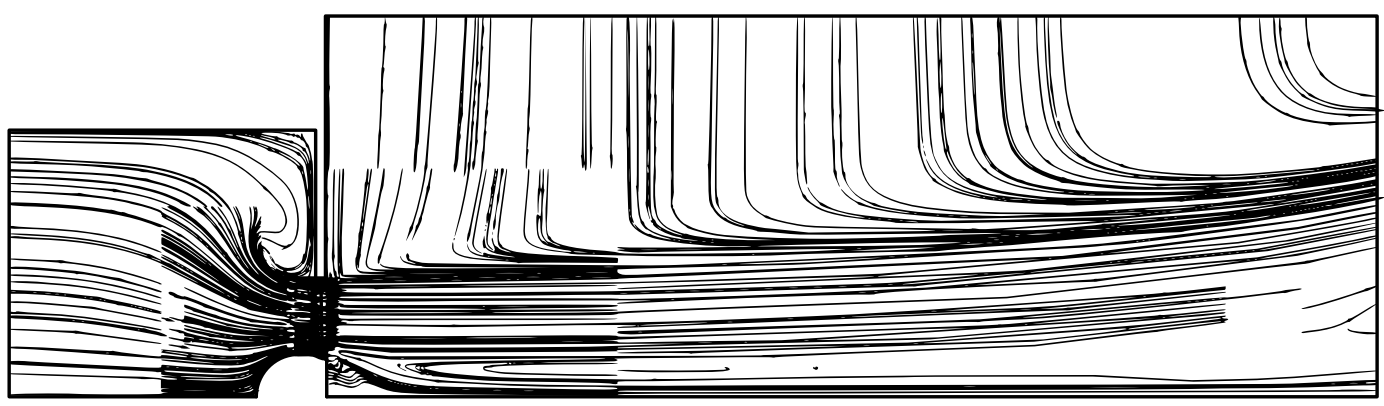

(b)

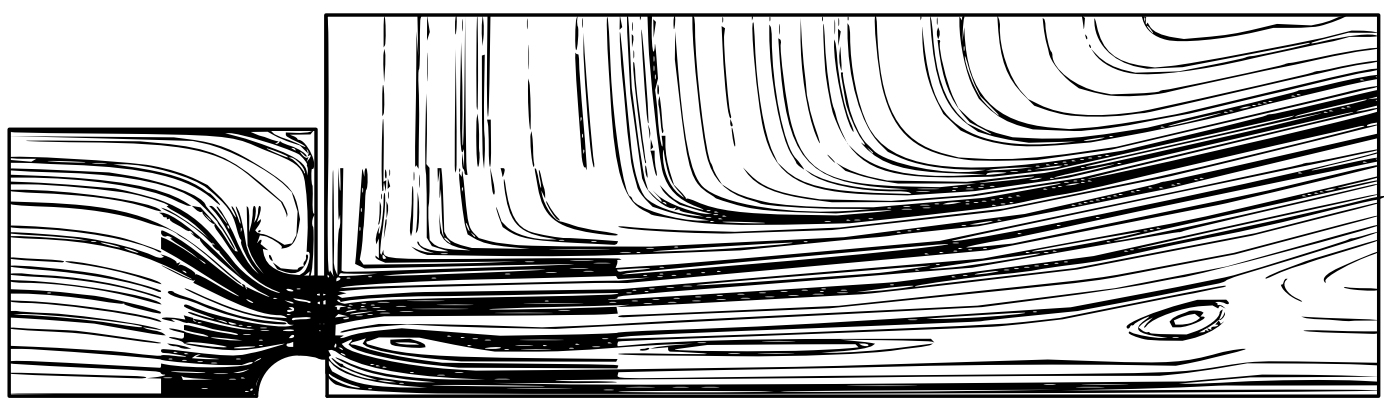

(c)

Figure 6.15: Meridional surface streamlines for (a) $\phi=0.200$, (b) $\phi_{D}=0.168$ and (c) $\phi=0.137$

confined only to this region. However, this pattern grows larger with a further decrease in flow rate, extending to higher radii.

Apparent from the time-dependent flow field depictions for low flow rates $(0.042 \leq \phi \leq 0.105)$ is that the downstream reversed flow pattern increases to a state where a standing ring vortex is present downstream of the fan. Hence, a component of flow through the fan is entrained in the standing vortex and flows back towards the fan near the hub. Also, changes in the global flow field are small with respect to time, with the largest variations occurring near the fan.

For flow rates of $\phi \leq 0.074$ a second ring vortex exists on the upstream side of the fan. This vortex, originating from the fan blade tip, leads to altered 


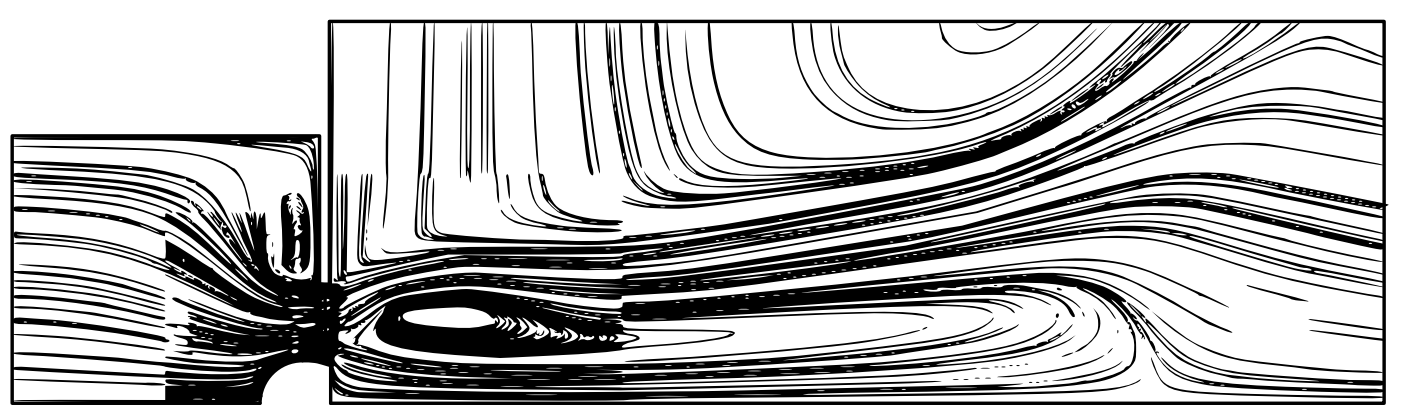

(a)

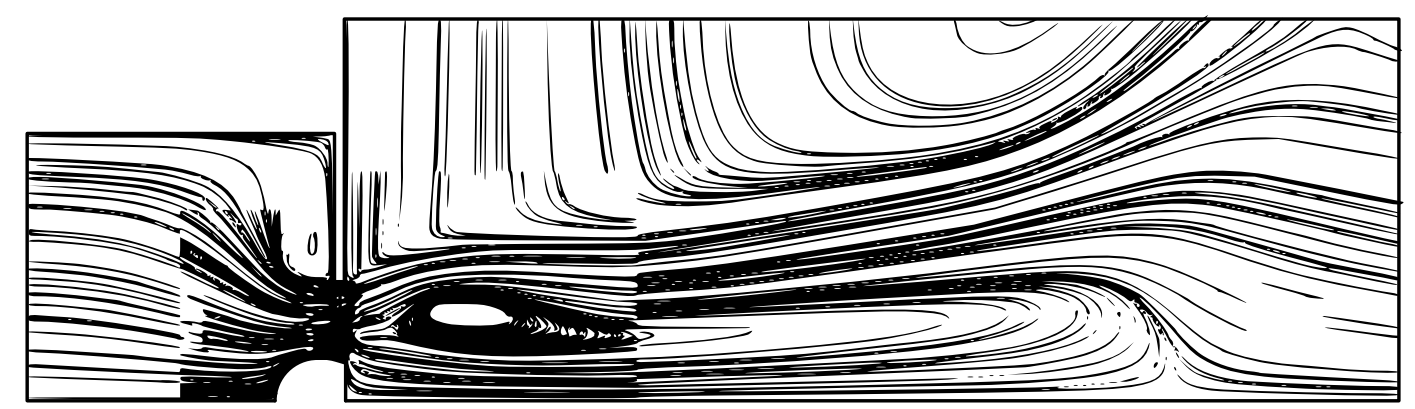

(b)

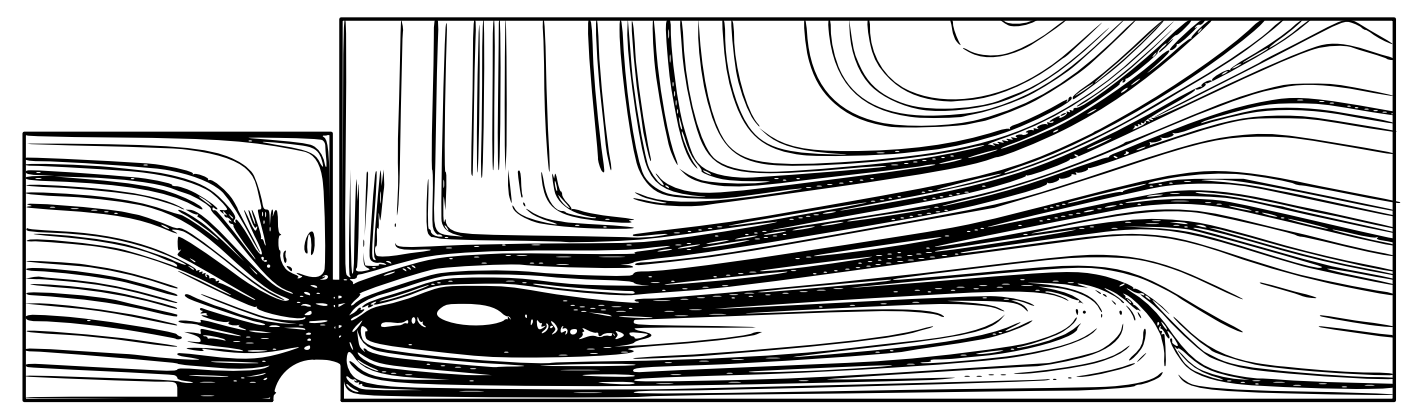

(c)

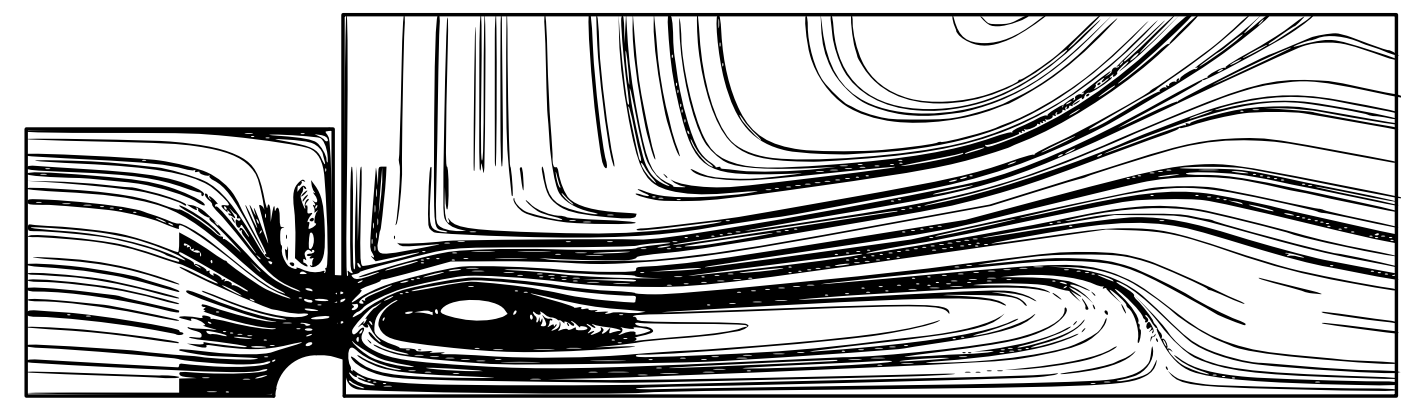

(d)

Figure 6.16: Meridional surface streamlines for $\phi=0.105$ at time instances of (a) $n_{F r}=n$, (b) $n_{F r}=n+0.475$, (c) $n_{F r}=n+0.950$ and (d) $n_{F r}=n+1.425$ 


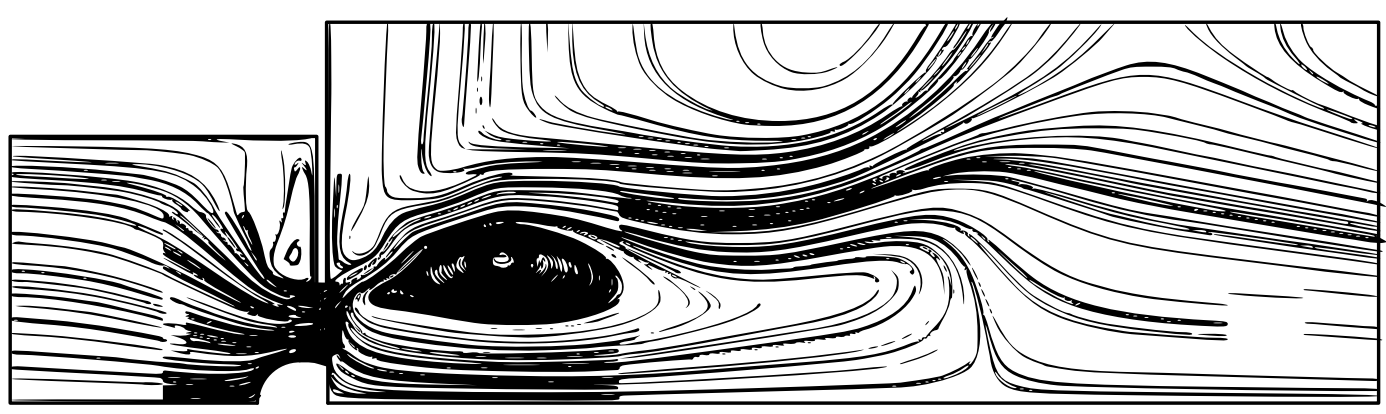

(a)

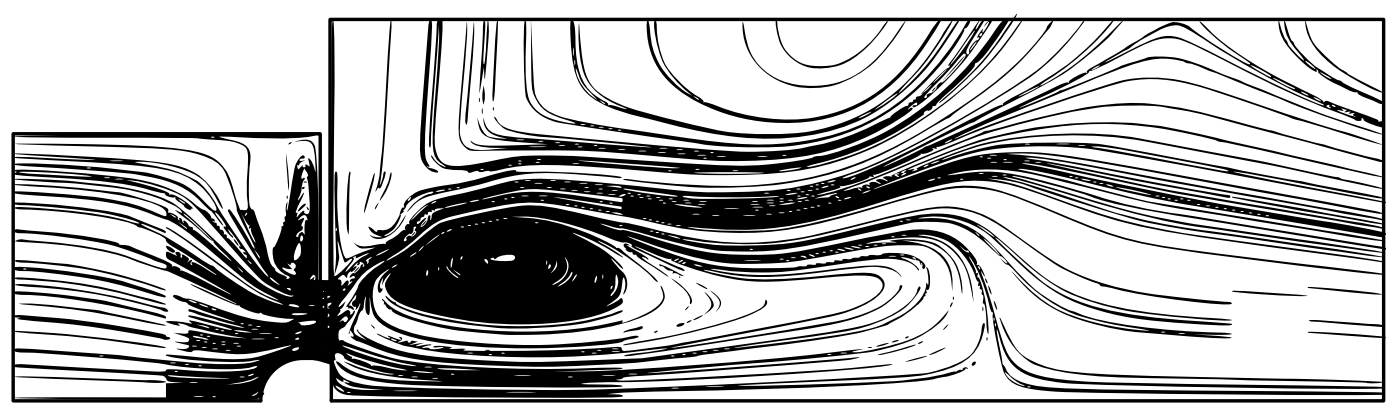

(b)

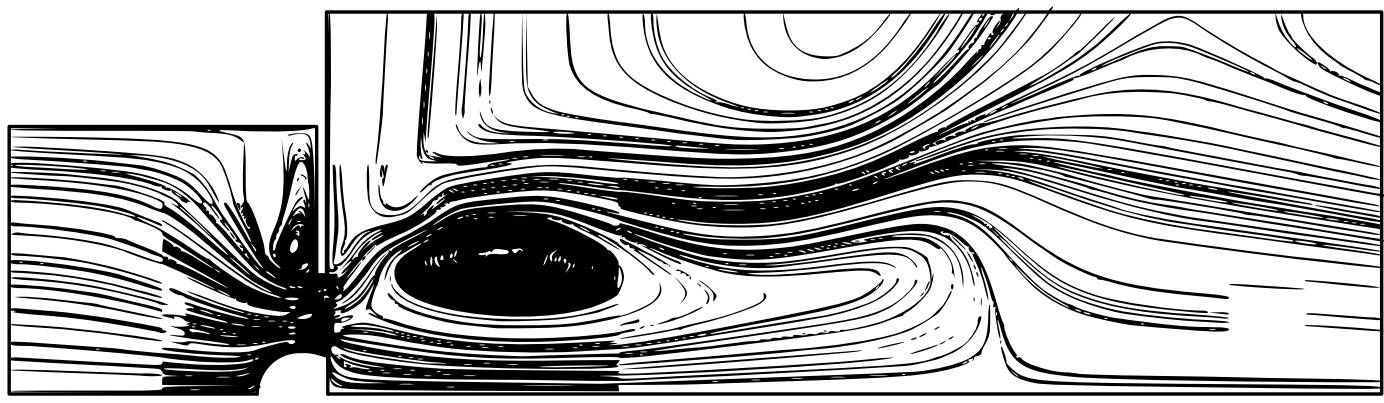

(c)

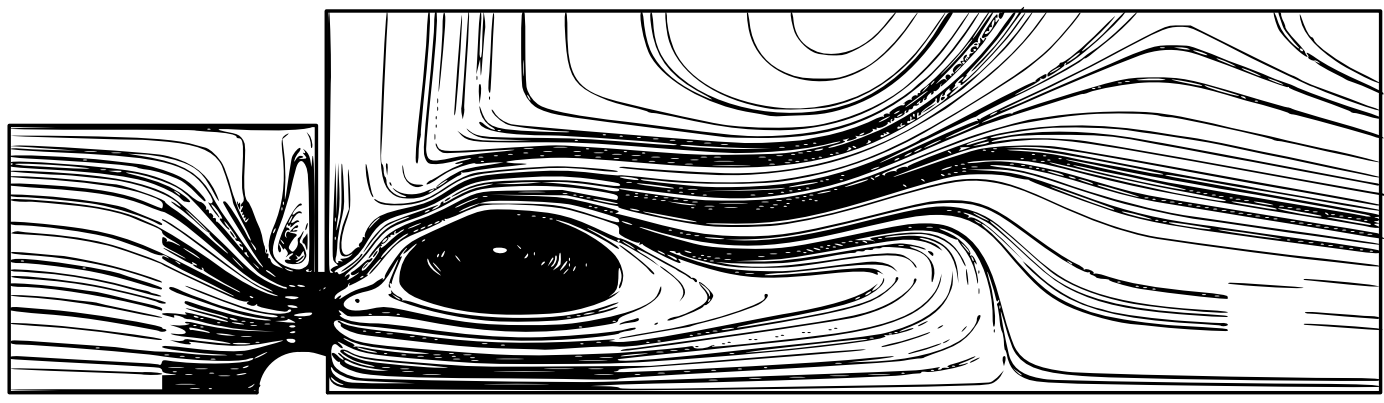

(d)

Figure 6.17: Meridional surface streamlines for $\phi=0.074$ at time instances of (a) $n_{F r}=n$, (b) $n_{F r}=n+0.700$, (c) $n_{F r}=n+1.400$ and (d) $n_{F r}=n+2.100$ 


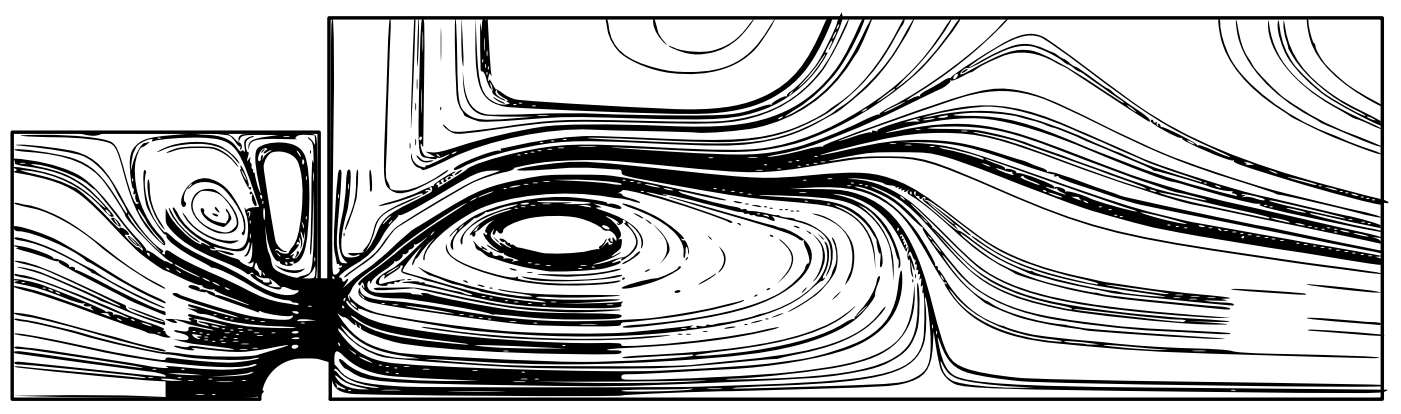

(a)

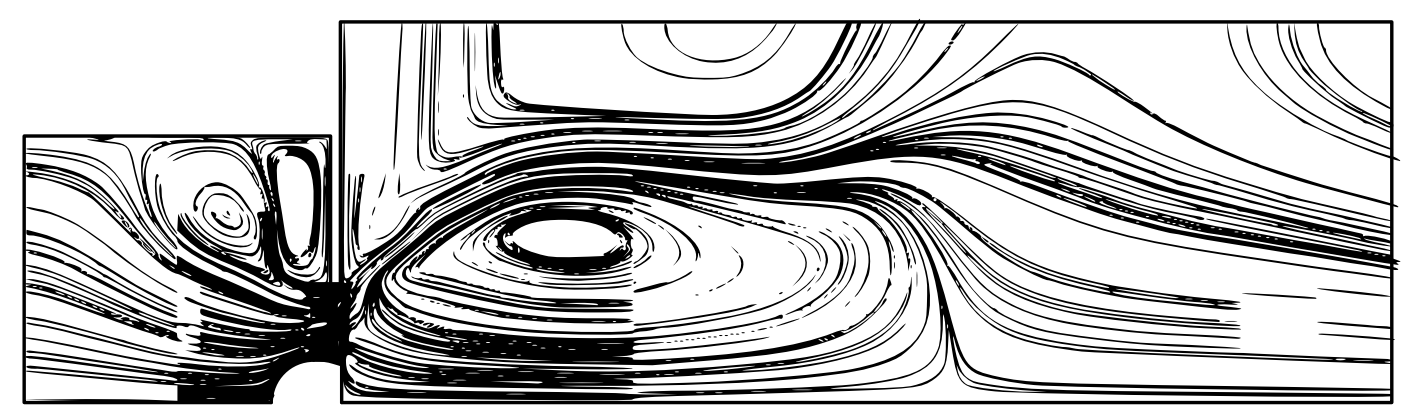

(b)

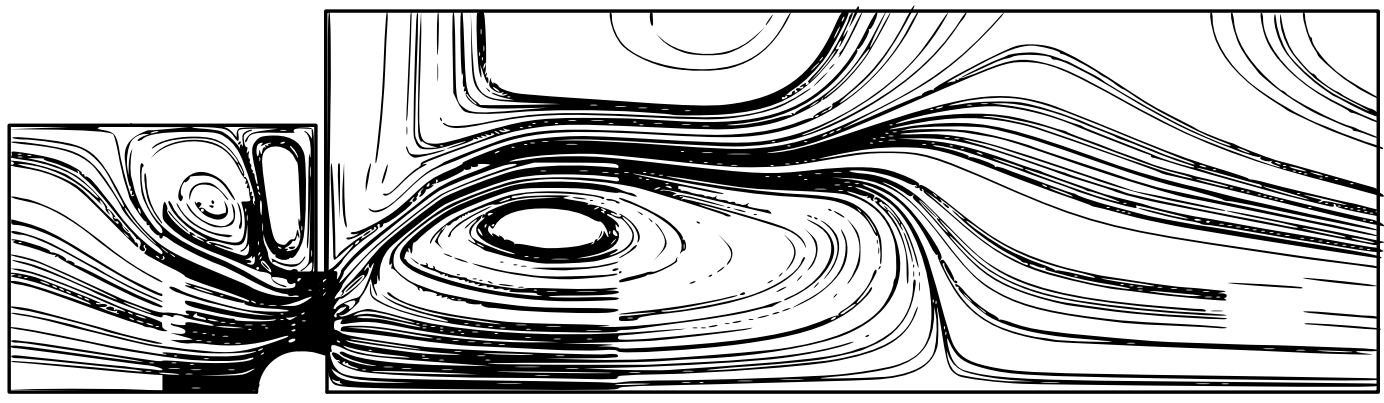

(c)

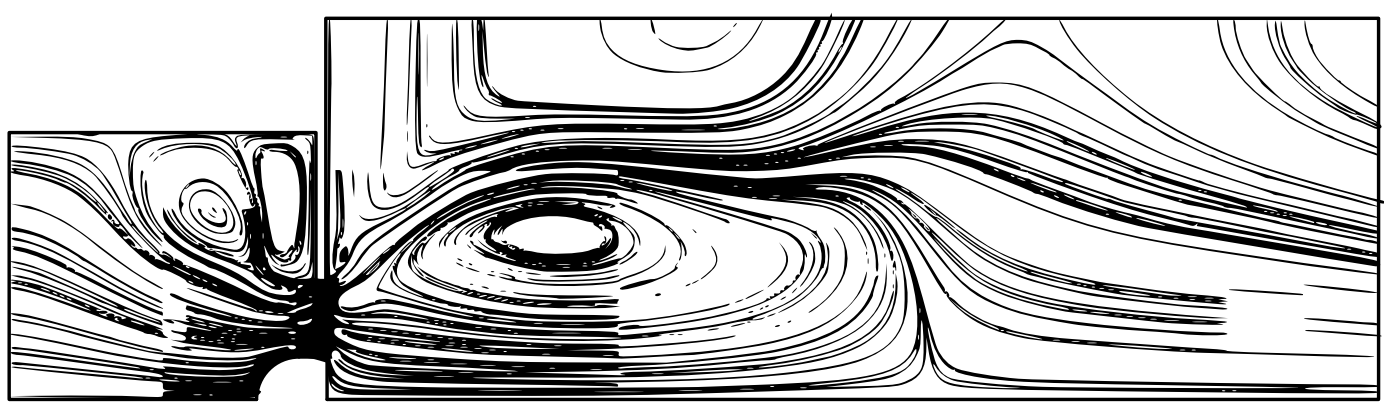

(d)

Figure 6.18: Meridional surface streamlines for $\phi=0.042$ at time instances of (a) $n_{F r}=n$, (b) $n_{F r}=n+0.825$, (c) $n_{F r}=n+1.650$ and (d) $n_{F r}=n+2.475$ 
inflow angles as depicted in figure 6.3.

\subsection{Cascade flow structure}

Although the averaged up- and downstream flow structure induced by the fan is important for the purpose of fan design and simplified fan modelling, some interesting flow patterns in the blade cascade also deserve some attention. Blade cascade flow is visualised through relative streamlines and surface contour plots in the following subsections.

\subsubsection{Three-dimensional relative flow}

The three-dimensional relative flow structure is visualised through streamlines released $2 \mathrm{~mm}$ from the hub surface. For near-design flow conditions $(0.137 \leq \phi \leq 0.200)$ depicted in figures 6.19 a to $\mathrm{c}$ the flow remains axial and circumferential with a small radial component. This is confirmed by plots of streamlines confined to a surface created $2 \mathrm{~mm}$ from the blade surface in the same figures. Hence, flow through the fan is near two-dimensional confirming the free-vortex flow field induced by the fan. Significant radial flow only appears at $0.137 \leq \phi \leq 0.168$.

Also visible in figure $6.19 \mathrm{c}$ is the small amount of reversed flow near the hub, effectively causing flow blockage and forcing flow to form a vortex near the hub. In this figure, the dark blue indicates that the fluid velocity relative to the fan rotor is nearly zero and is therefore entrained together with the fan rotational motion near the hub. Only a small amount of radial flow occurs near the hub, whereas the flow through the remaining blade span is two-dimensional.

Visualisation of the passage and blade surface flow for lower flow rates $(\phi=0.105,0.074$ and 0.042$)$ are indicated in figures 6.20 to 6.25 . Since the flow pattern is periodic, six time instances of a single period for each flow rate are presented.

The flow pattern is generally similar for low flow rates although the behaviour becomes increasingly erratic with a decrease in flow rate. Also, the length of one period changes from $n_{F r}=1.85$ at $\phi=0.105$ to $n_{F r}=3.25$ at $\phi=0.042$.

During a single period, the flow through the passage initially resembles attached flow across the largest span. Hereafter reversed flow near the hub causes a blockage of flow in the passage and a gradual build-up of fluid upstream of the fan rotor near the hub. This forces a component of flow to move radially outward. Reversed flow also enters the rotor in one passage from the downstream side near the hub and flows in a counter-rotating direction towards the adjacent passage along the upstream side of the rotor.

Flow along the blade suction side near the hub seems to remain attached for the largest part of a single period, although the flow is not two-dimensional (axial and circumferential), but have an increased radial component. The flow 


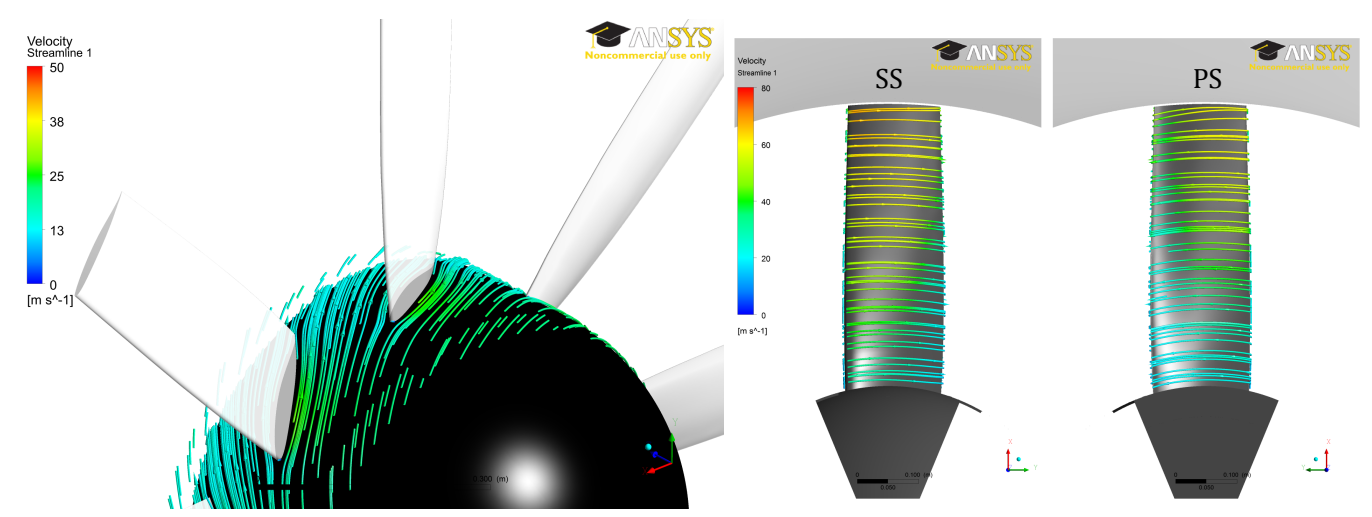

(a)

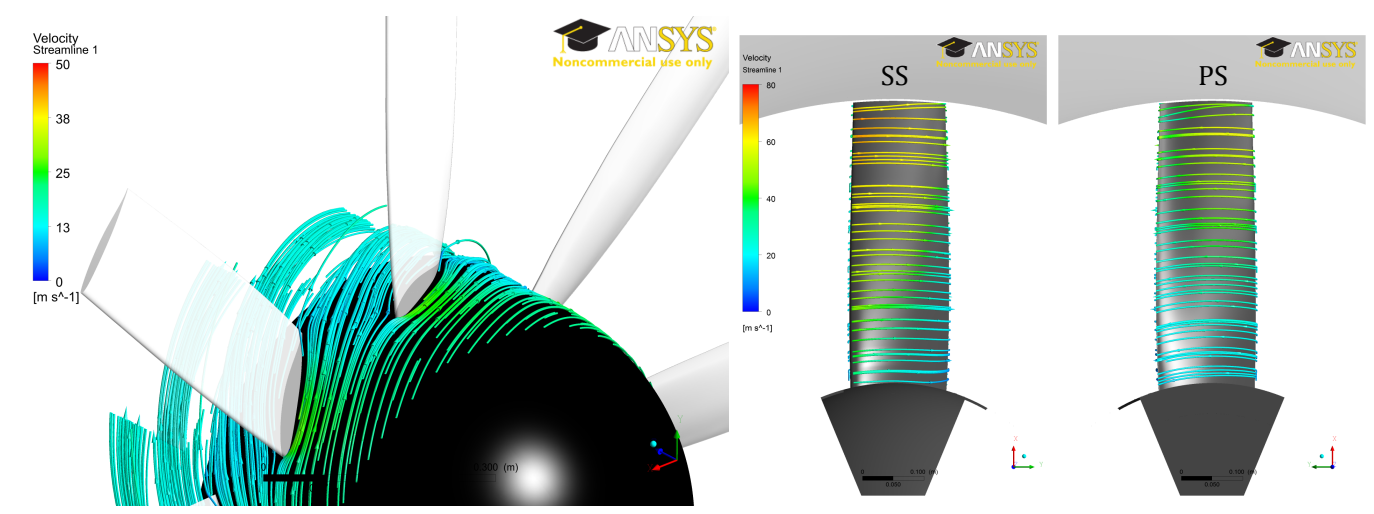

(b)

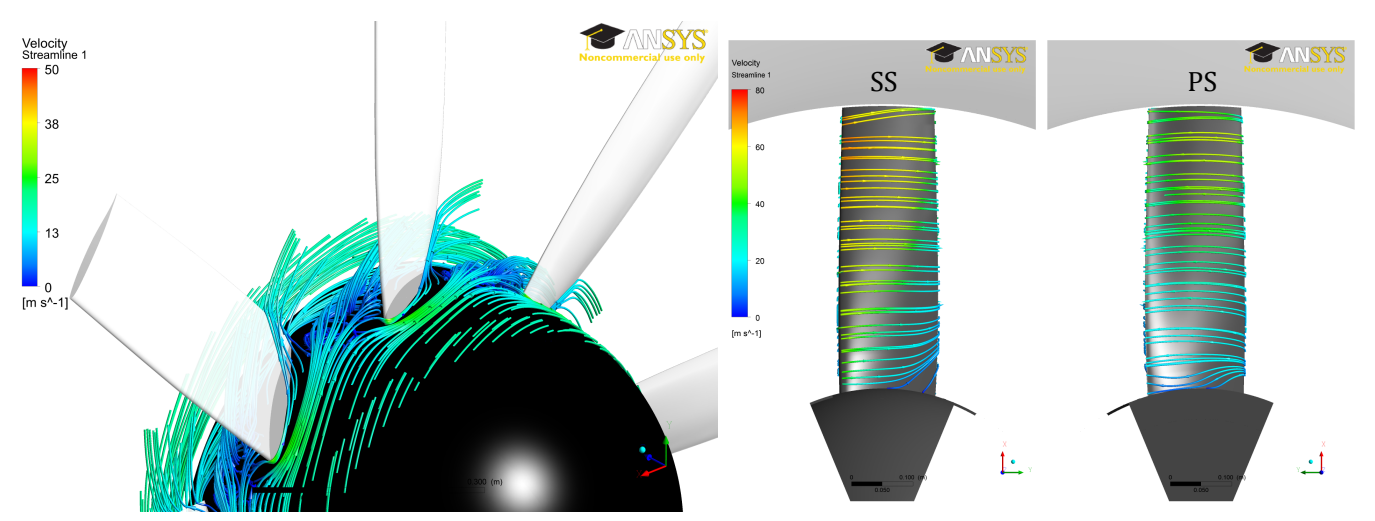

(c)

Figure 6.19: Relative streamlines released $2 \mathrm{~mm}$ from the fan hub surface (left hand side) and relative streamlines confined to a surface $2 \mathrm{~mm}$ from the blade (right hand side) for flow coefficients of (a) $\phi=0.200$, (b) $\phi_{D}=0.168$ and (c) $\phi=0.137$ (SS: suction side, PS: pressure side) 


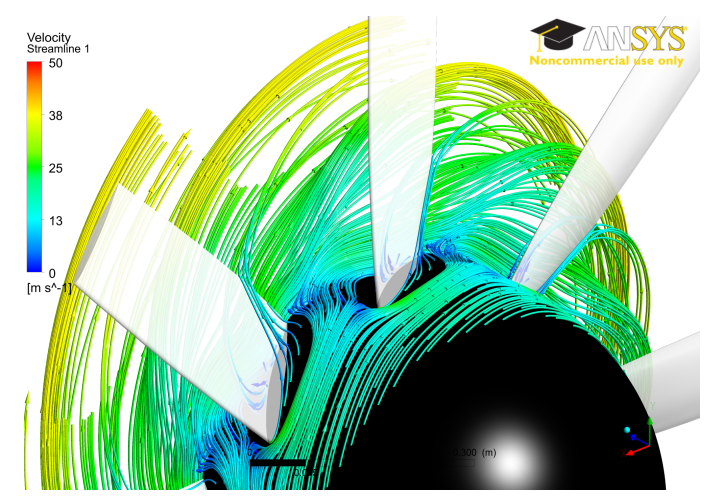

(a)

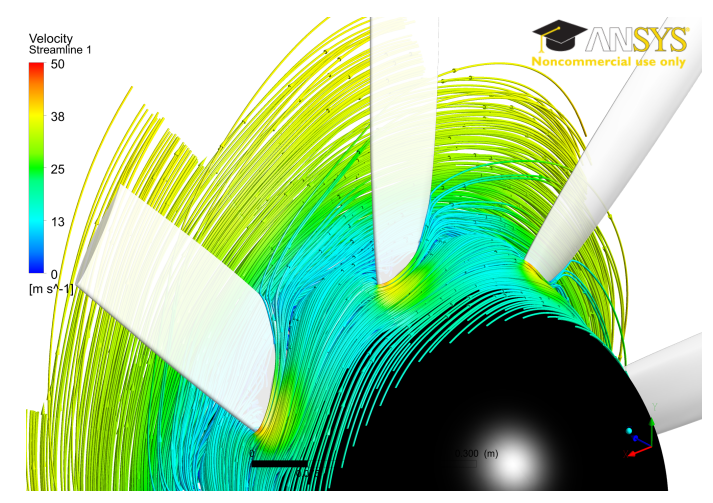

(c)

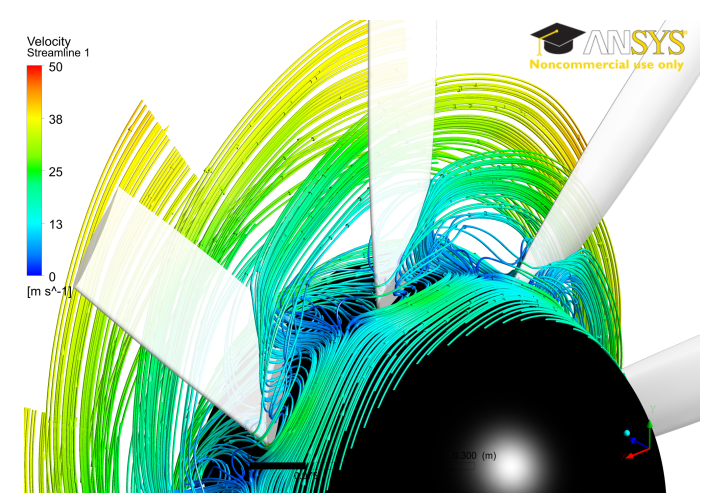

(e)

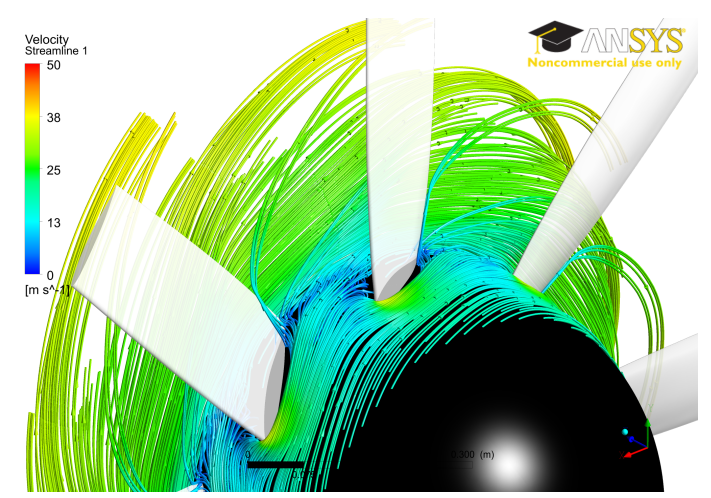

(b)

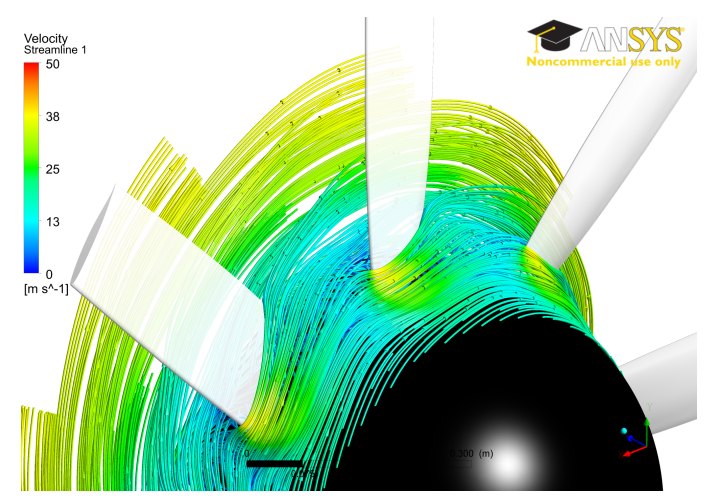

(d)

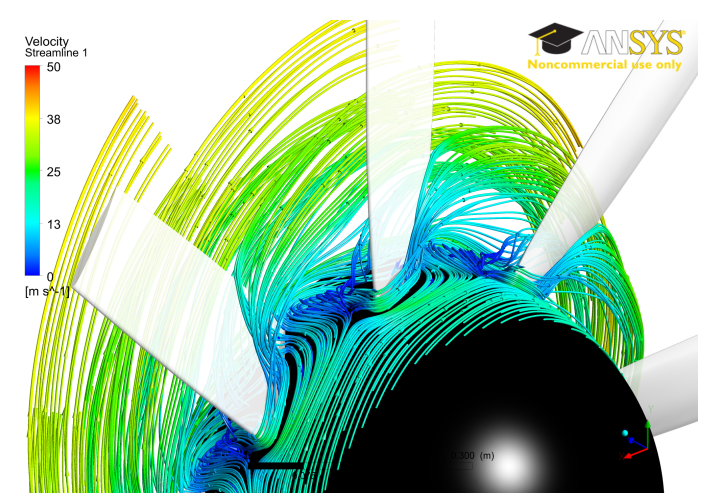

(f)

Figure 6.20: Time frames of relative streamlines released $2 \mathrm{~mm}$ from the fan hub surface for a flow coefficient of $\phi=0.105$ at time instances of (a) $n_{F r}=n$, (b) $n_{F r}=n+0.325$ (c) $n_{F r}=n+0.650$, (d) $n_{F r}=n+0.975$, (e) $n_{F r}=n+1.300$ and (f) $n_{F r}=n+1.625$ 


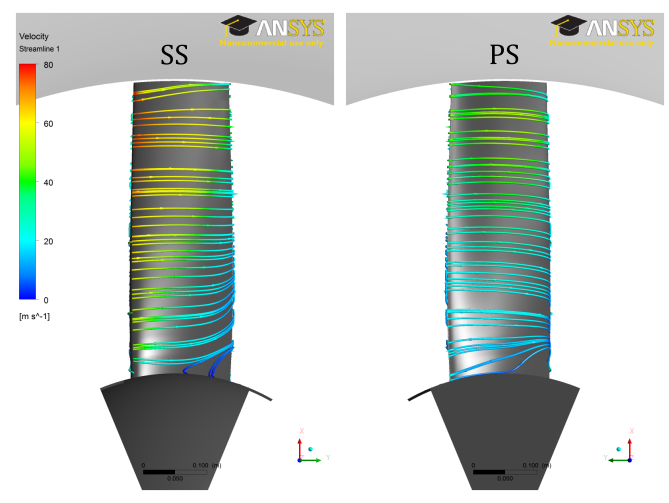

(a)

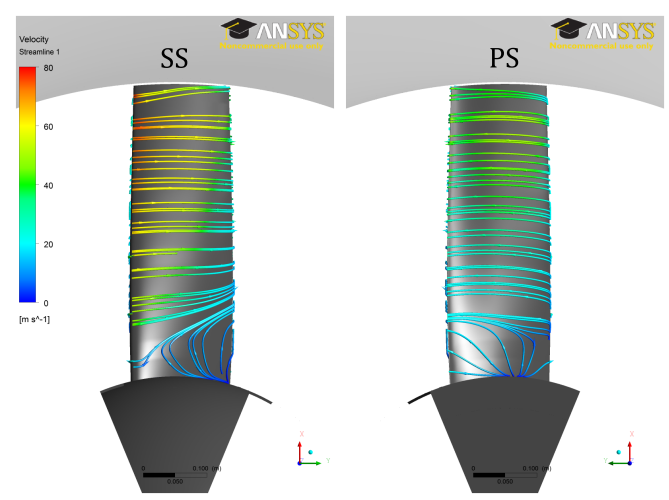

(c)

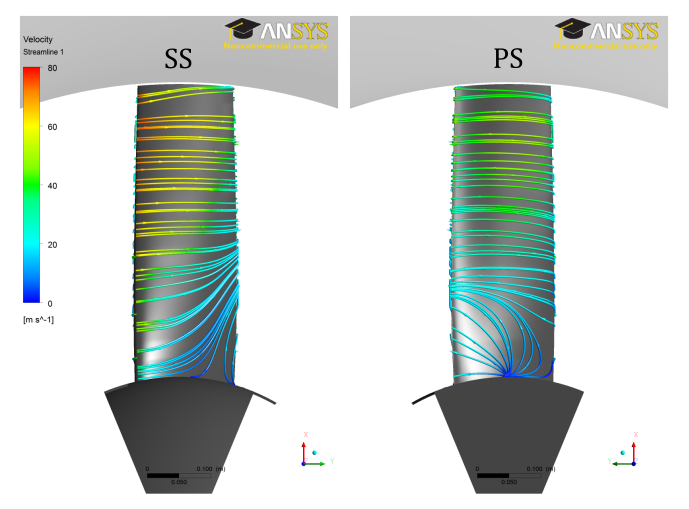

(e)

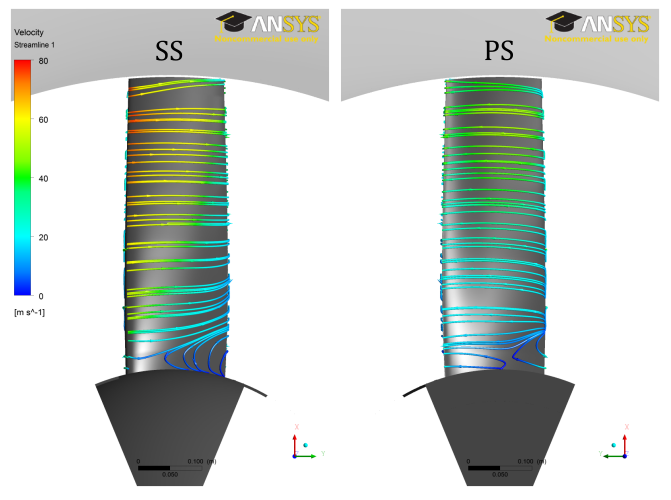

(b)

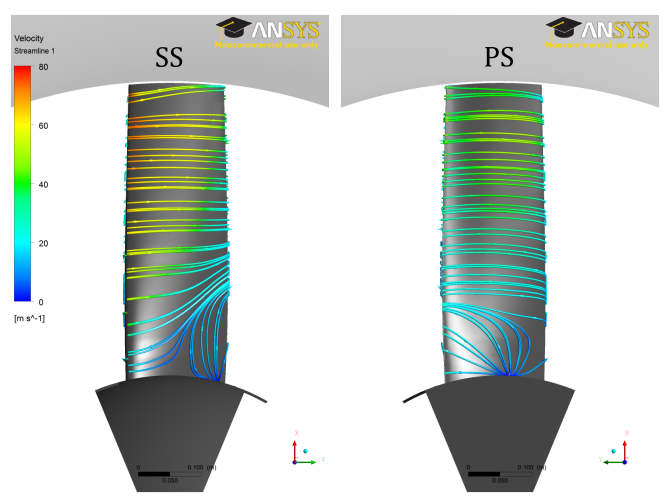

(d)

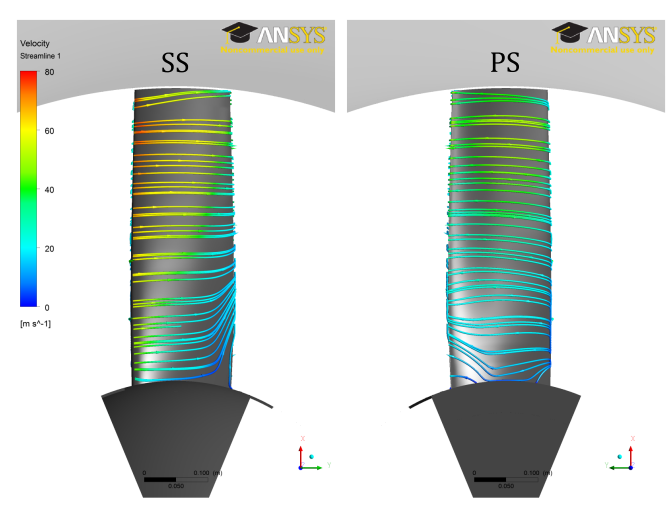

(f)

Figure 6.21: Time frames of relative streamlines on a surface $2 \mathrm{~mm}$ from the fan blade for a flow coefficient of $\phi=0.105$ at time instances of (a) $n_{F r}=n$, (b) $n_{F r}=n+0.325$ (c) $n_{F r}=n+0.650$, (d) $n_{F r}=n+0.975$, (e) $n_{F r}=n+1.300$ and (f) $n_{F r}=n+1.625$ 


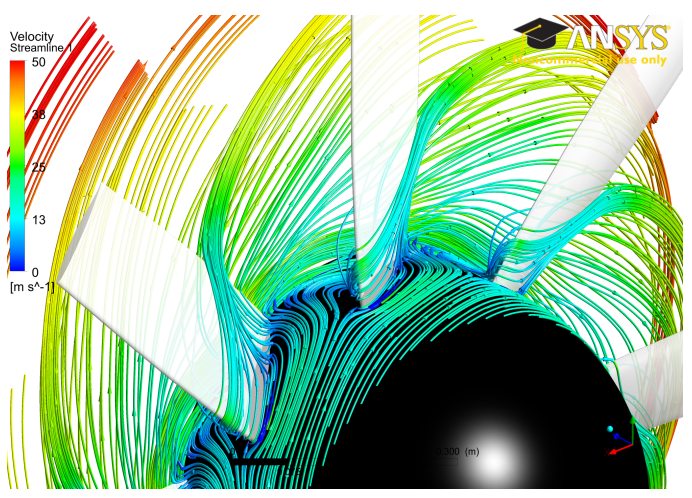

(a)

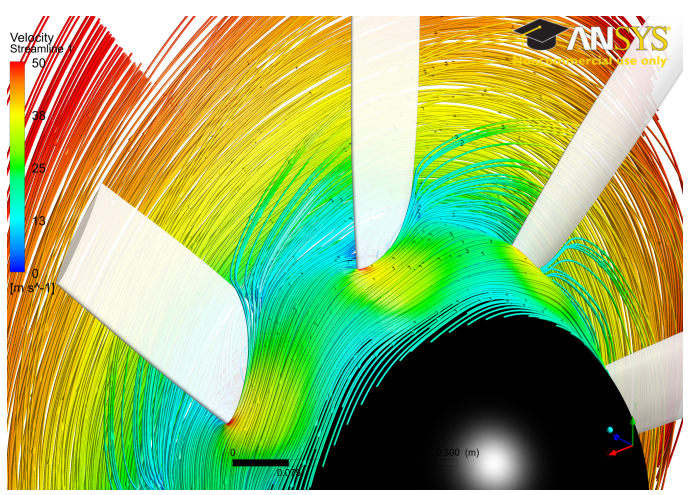

(c)

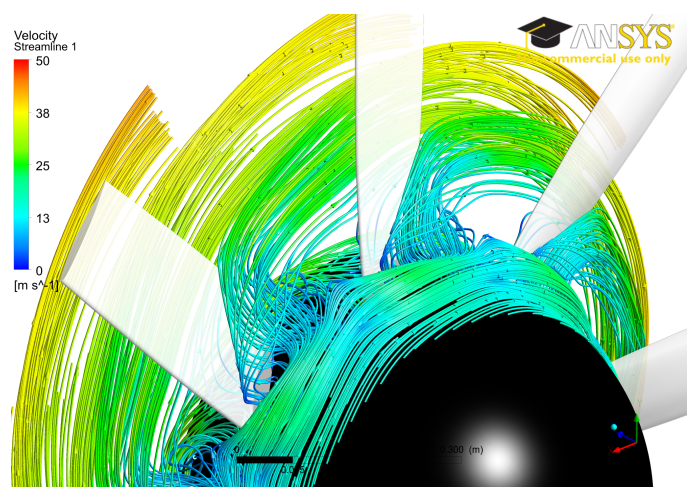

(e)

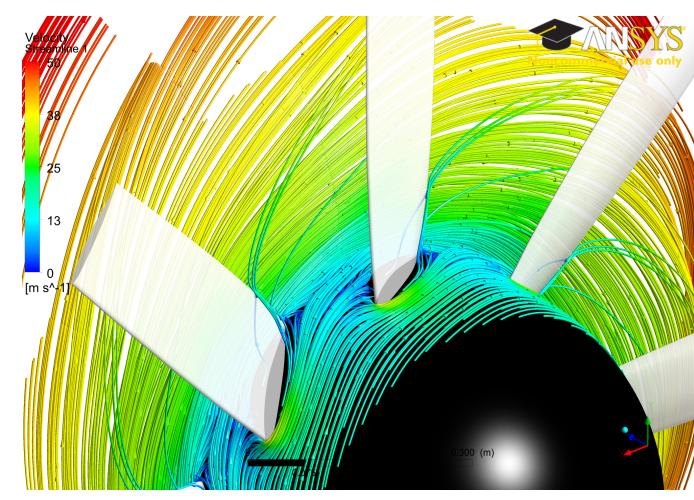

(b)

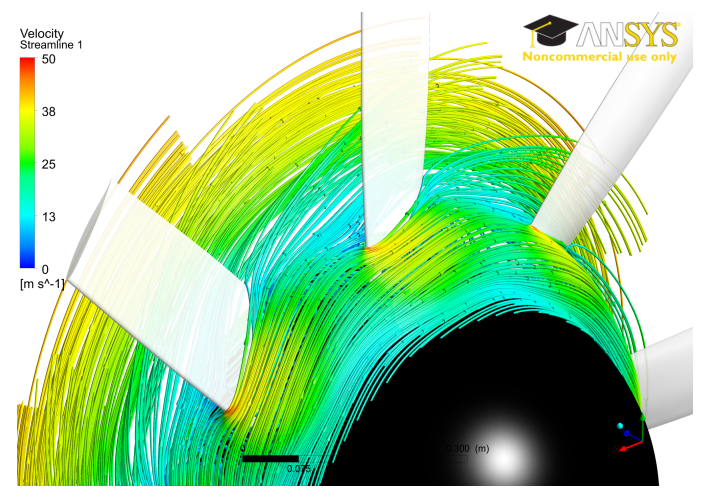

(d)

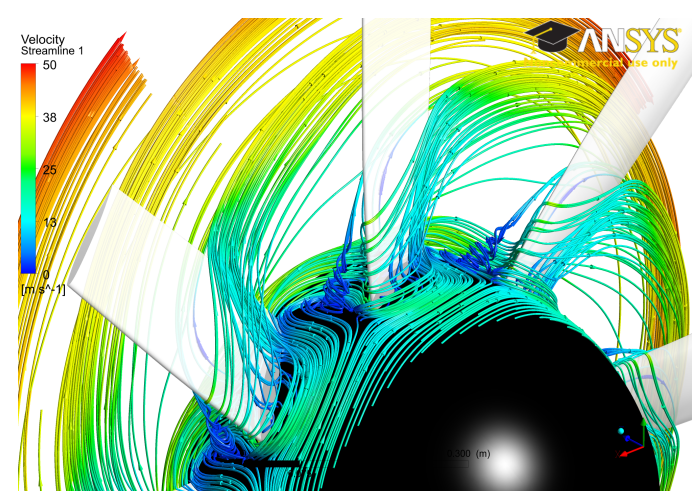

(f)

Figure 6.22: Time frames of relative streamlines released $2 \mathrm{~mm}$ from the fan hub surface for a flow coefficient of $\phi=0.074$ at time instances of (a) $n_{F r}=n$, (b) $n_{F r}=n+0.475$ (c) $n_{F r}=n+0.950$, (d) $n_{F r}=n+1.425$, (e) $n_{F r}=n+1.900$ and (f) $n_{F r}=n+2.375$ 


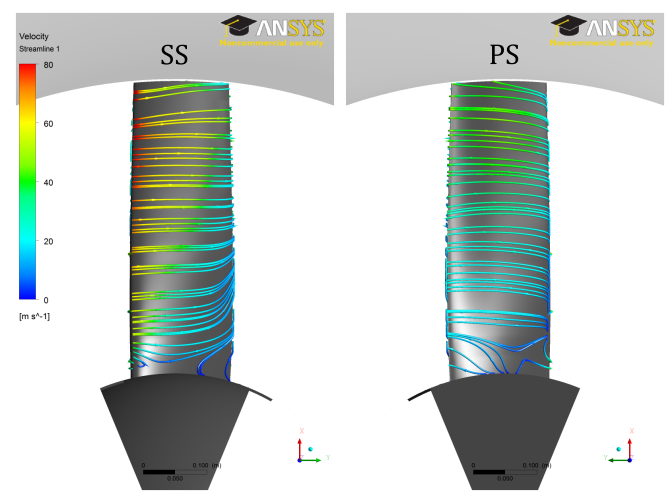

(a)

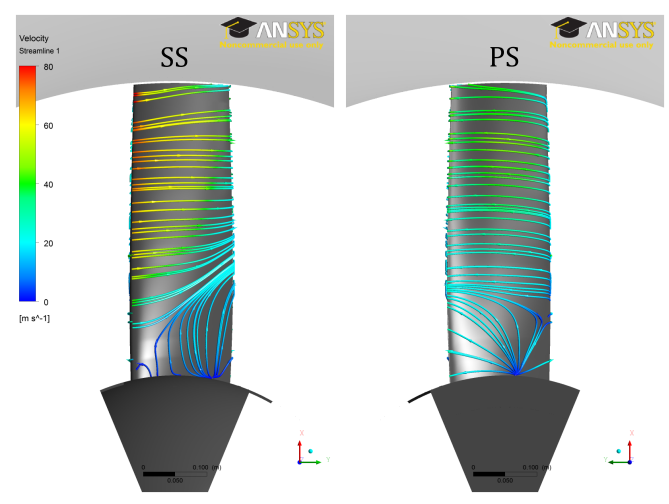

(c)

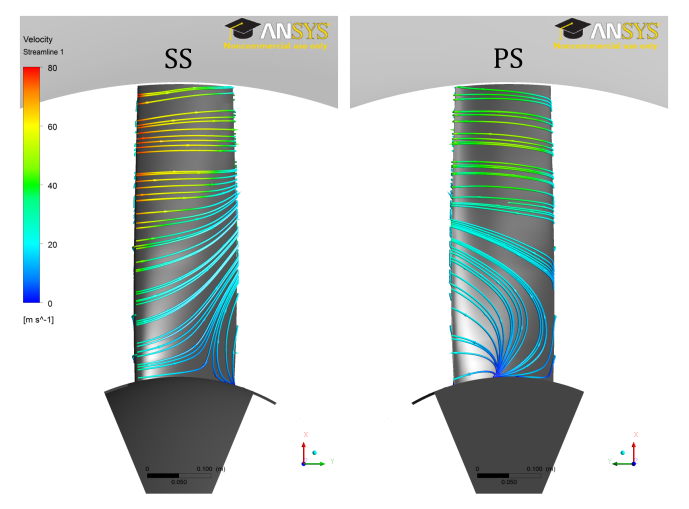

(e)

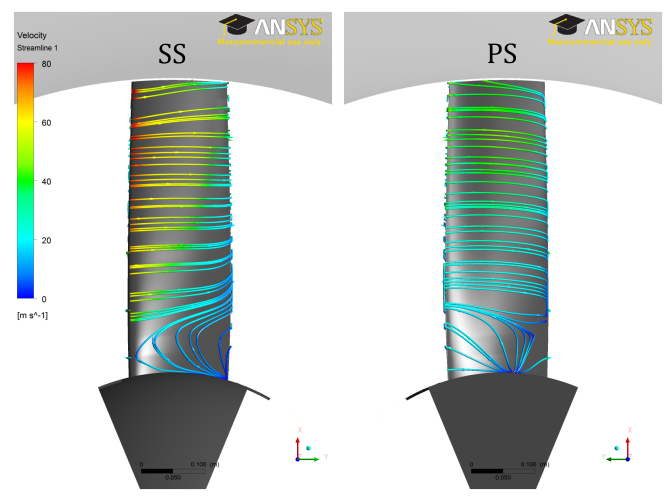

(b)

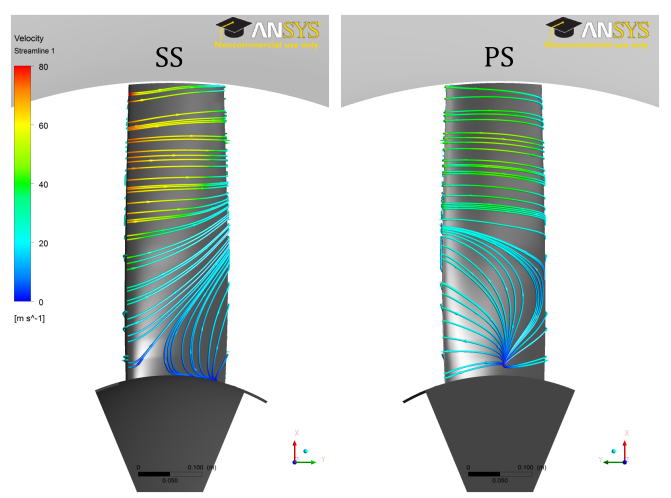

(d)

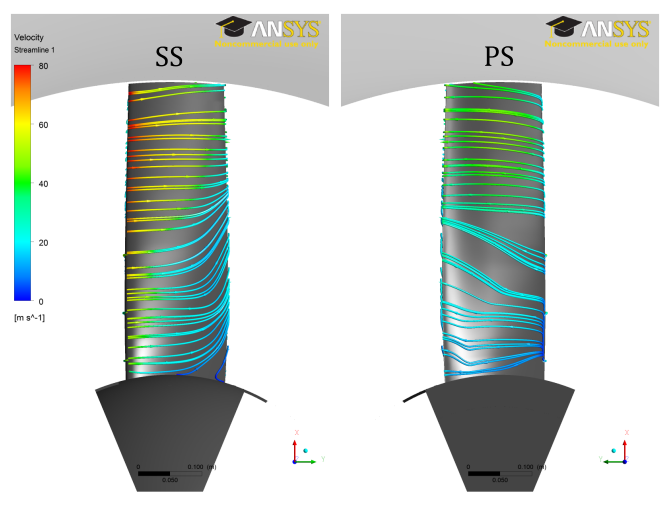

(f)

Figure 6.23: Time frames of relative streamlines on a surface $2 \mathrm{~mm}$ from the fan blade for a flow coefficient of $\phi=0.074$ at time instances of (a) $n_{F r}=n$, (b) $n_{F r}=n+0.475$ (c) $n_{F r}=n+0.950$, (d) $n_{F r}=n+1.425$, (e) $n_{F r}=n+1.900$ and (f) $n_{F r}=n+2.375$ 


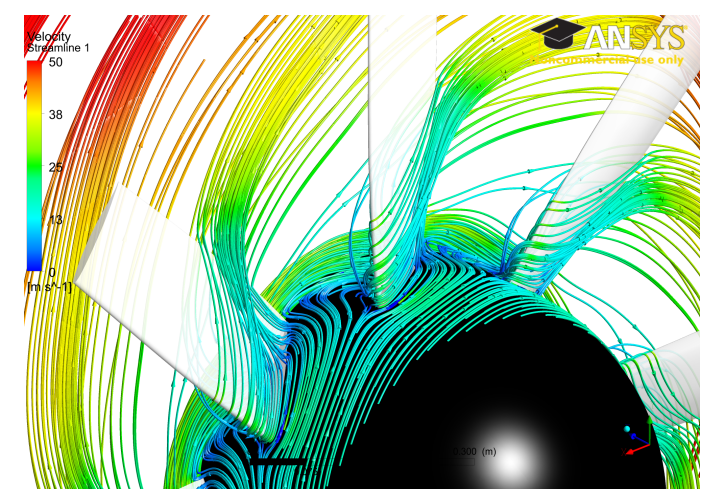

(a)

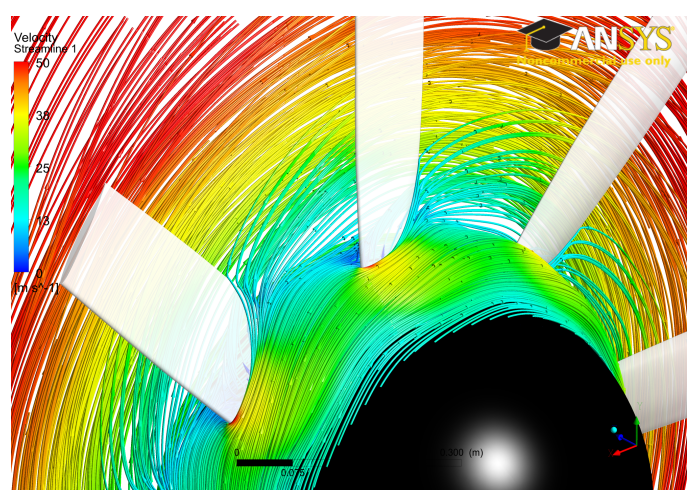

(c)

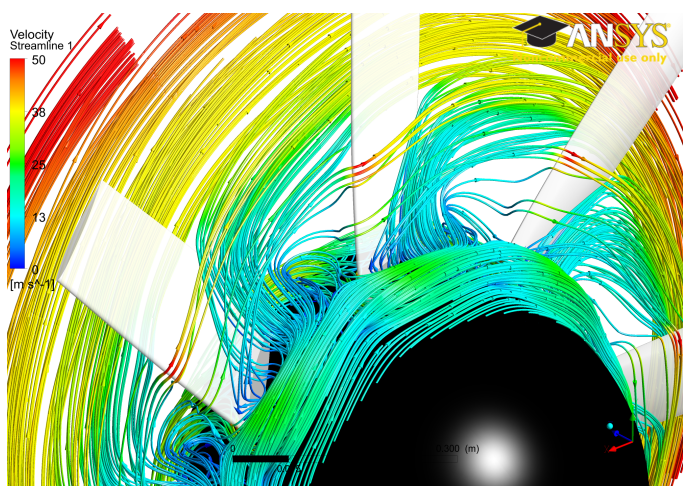

(e)

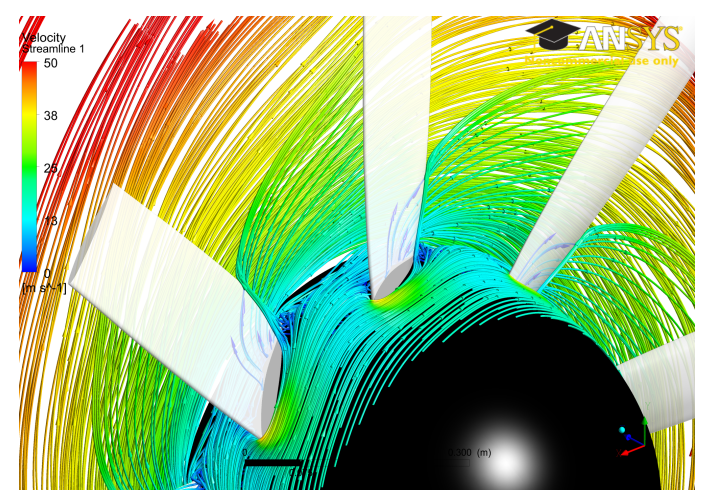

(b)

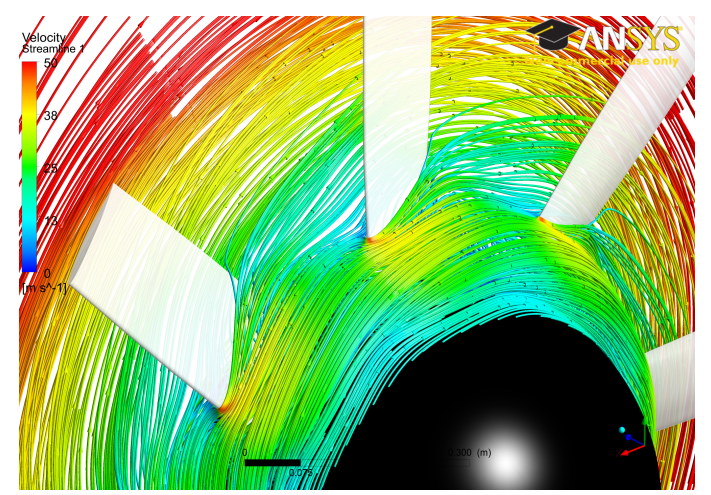

(d)

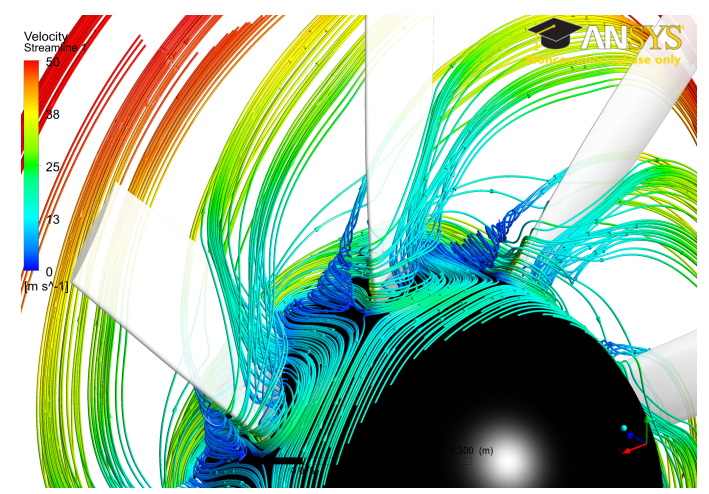

(f)

Figure 6.24: Time frames of relative streamlines released $2 \mathrm{~mm}$ from the fan hub surface for a flow coefficient of $\phi=0.042$ at time instances of (a) $n_{F r}=n$, (b) $n_{F r}=n+0.550$ (c) $n_{F r}=n+1.100$, (d) $n_{F r}=n+1.650$, (e) $n_{F r}=n+2.200$ and (f) $n_{F r}=n+2.750$ 


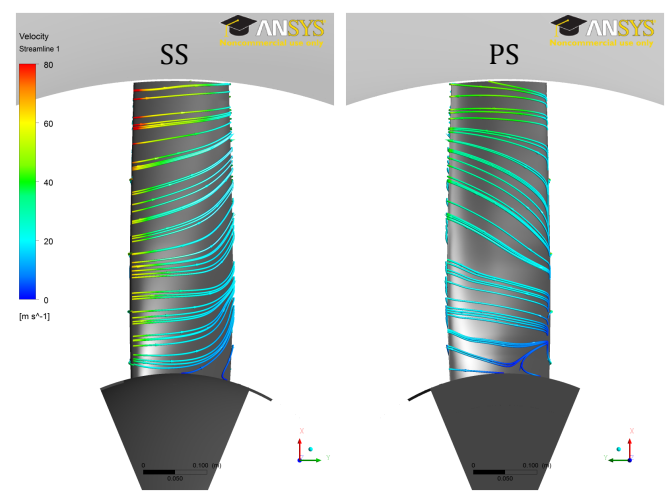

(a)

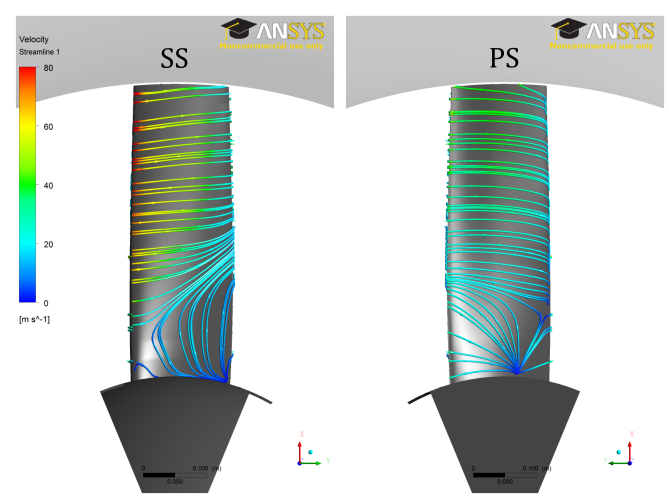

(c)

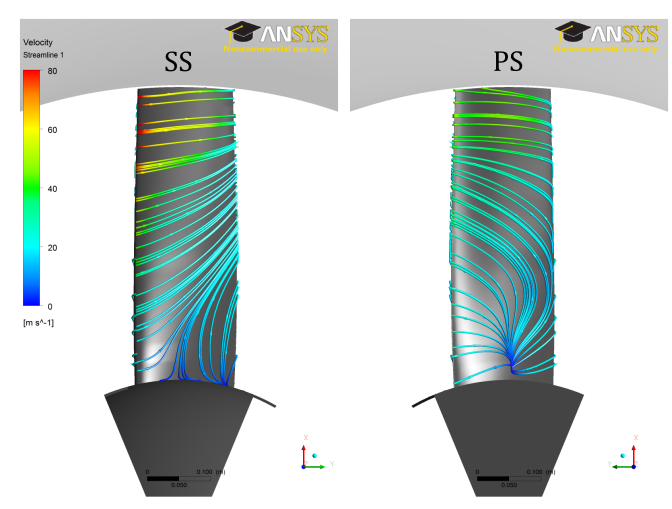

(e)

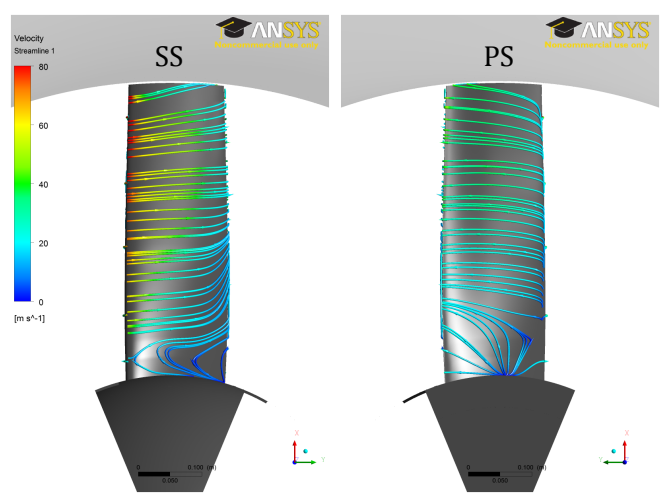

(b)

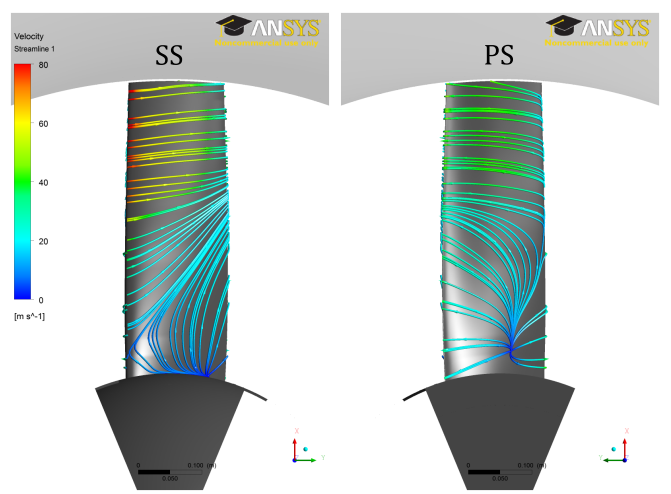

(d)

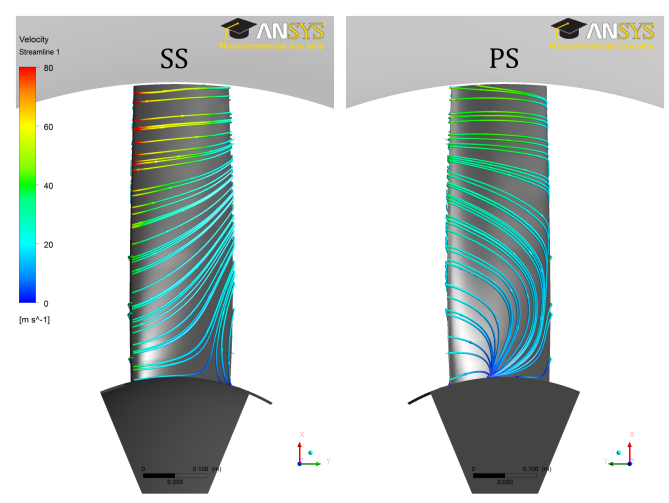

(f)

Figure 6.25: Time frames of relative streamlines on a surface $2 \mathrm{~mm}$ from the fan blade for a flow coefficient of $\phi=0.042$ at time instances of (a) $n_{F r}=n$, (b) $n_{F r}=n+0.550$ (c) $n_{F r}=n+1.100$, (d) $n_{F r}=n+1.650$, (e) $n_{F r}=n+2.200$ and (f) $n_{F r}=n+2.750$ 
pattern occurring in the passage forces the flow towards the blade near the trailing edge on the suction side (frame e, f and a of figures 6.20, 6.22 and 6.24 ), whereafter it flows radially outward. A vortical structure appears inside the blade passage (frame f of figures 6.20, 6.22 and 6.24), drawing flow out in the radial direction.

Considering the streamlines confined to a surface adjacent to the blade (figures $6.21,6.23$ and 6.25) it can be observed that flow across the blade at higher blade spans $\left(s_{b} \approx 0.5\right.$ to 1$)$ is nearly two-dimensional over time. However, strong periodic flow patterns are visible at lower blade spans. An impingement point becomes visible near the hub on the pressure side of the blade, where this impingement is also a function of periodicity. From this impingement a component of flow leaks towards the upstream side of the fan blade, whereas another component flows radially outward.

\subsubsection{Two-dimensional relative flow}

The two-dimensional relative flow structures are depicted by means of relative streamlines and velocity contour plots at five different blade sections (i.e. $s_{b}=0.1,0.3,0.5,0.7$ and 0.9$)$ in figures 6.26 a to $\mathrm{c}$ for the near-design flow coefficients of $p h i=0.200,0.168$ and 0.137 and figures 6.27 to 6.29 ) for low flow coefficients $(\phi=0.105,0.074$ and 0.042$)$. For these low flow rate cases, four time frames of the flow field for a single period are depicted.

At near design flow rates the flow remains attached along the blade for all sections depicted, although a small amount of flow separation occurs near the trailing edge on the suction side of the fan blade for $\phi=0.137$ for $s_{b}=$ 0.1 (figure 6.26c). As the flow rate decreases, periodic blade stall keeps on increasing near the hub and progresses towards higher radii as depicted in figures 6.27 to 6.29 . Although flow separates along the leading edge of the fan blade near the hub, reattachment of the flow near the trailing edge can also be noticed. Lastly it is emphasised how the flow remains attached at higher blade spans $\left(0.5<s_{b}\right)$ for the range of flow rates investigated.

\subsection{Concluding remarks}

The analysed numerical results depicted in the present chapter give some insight to the governing flow structures in close vicinity to the fan. These results are presented through circumferentially averaged flow profiles, up- and downstream of the fan as well as numerical flow visualisation.

At near-design flow rates the flow through the fan is representative of a free-vortex flow profile (i.e. the axial velocity profile through the fan is uniform and little radial flow is present), which agrees with the original design criteria of the B2a-fan. However, at low flow rates the flow structure in the vicinity of the fan becomes time-dependent relative to the rotation of the fan and follows a periodic trend. This time-dependent behaviour becomes more erratic with 
a decrease in flow rate. At low flow rates fluid is partially blocked in the passages at lower radii by reversed flow near the hub, effectively causing a radially outward deflection of the flow. The majority of the massflow moves through the fan at higher radii, keeping the outer span of the blade out of stall, since the decrease in the effective fan annulus area causes an increase in axial velocity at the tip and subsequently leads to a decrease in angle of attack. The flow is more stable in the tip region, whereas partial blade stall occurs in

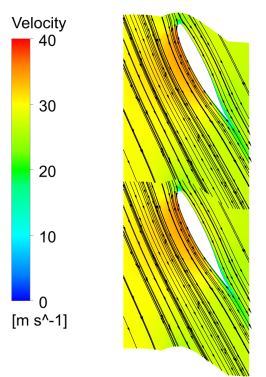

$\mathrm{s}_{\mathrm{b}}=0.1$

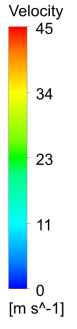

$\mathrm{s}_{\mathrm{b}}=0.3$

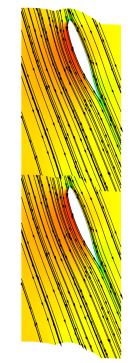

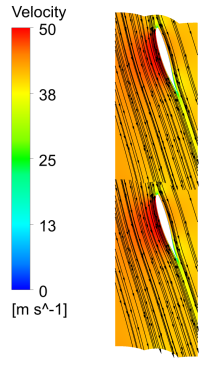

$\mathrm{s}_{\mathrm{b}}=0.5$

(a)

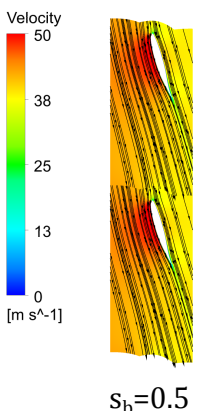

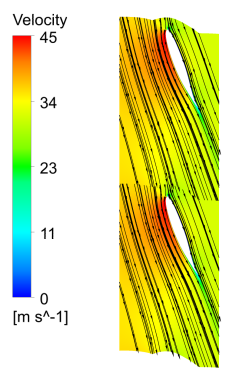

$\mathrm{s}_{\mathrm{b}}=0.3$

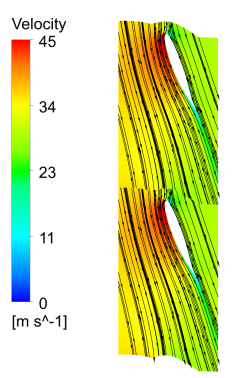

$\mathrm{s}_{\mathrm{b}}=0.3$

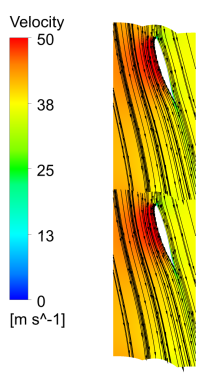

$\mathrm{s}_{\mathrm{b}}=0.5$ (b)

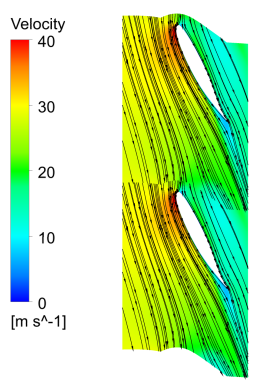

$\mathrm{s}_{\mathrm{b}}=0.1$

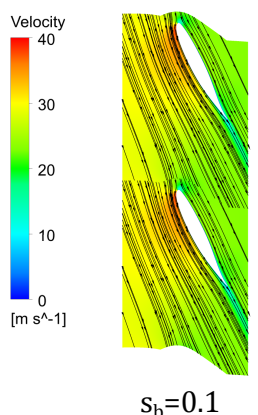

(c)

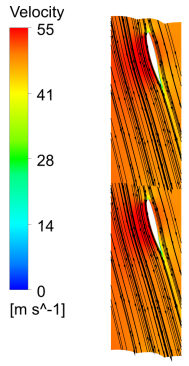

$\mathrm{s}_{\mathrm{b}}=0.7$

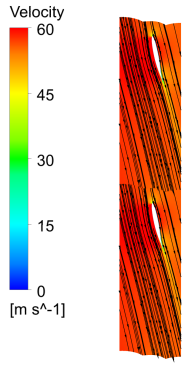

$\mathrm{s}_{\mathrm{b}}=0.9$
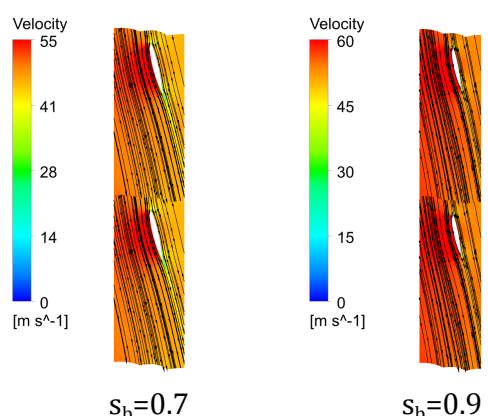

$\mathrm{s}_{\mathrm{b}}=0.9$
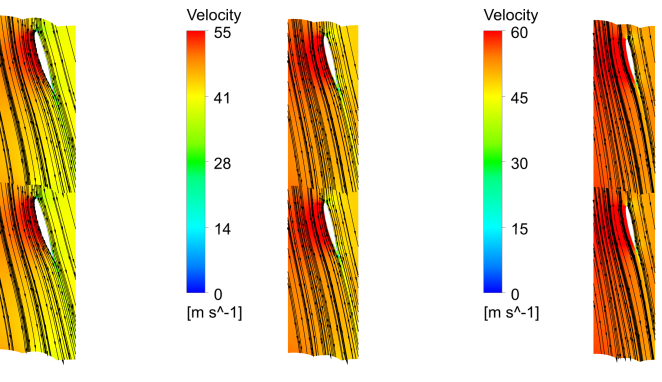

$\mathrm{s}_{\mathrm{b}}=0.7$

$\mathrm{s}_{\mathrm{b}}=0.9$

Figure 6.26: Relative surface streamlines and contours of relative velocity at different blade spans for flow coefficients of (a) $\phi=0.200$, (b) $\phi_{D}=0.168$ and (c) $\phi=0.137$ 

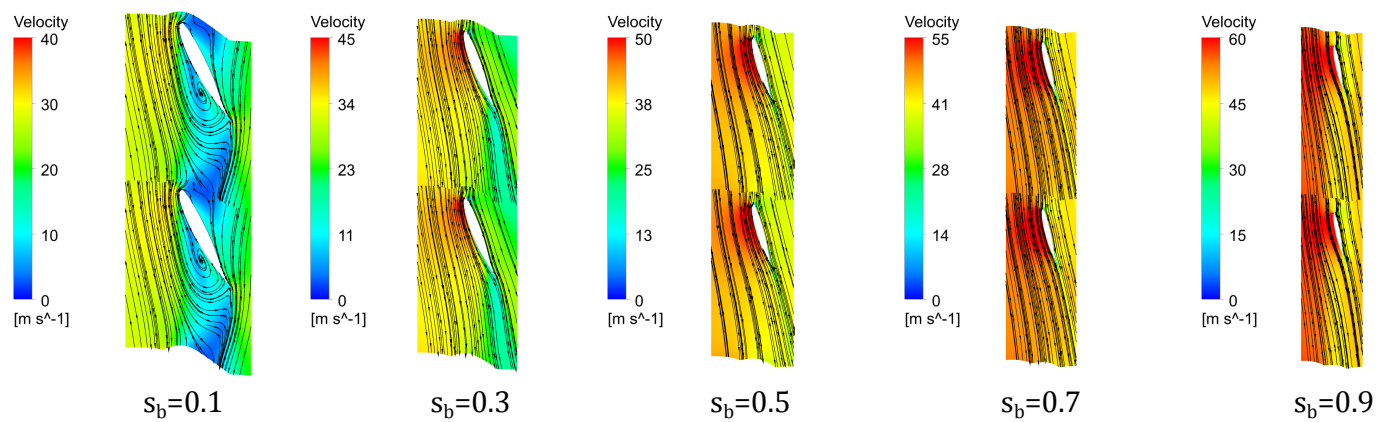

(a)
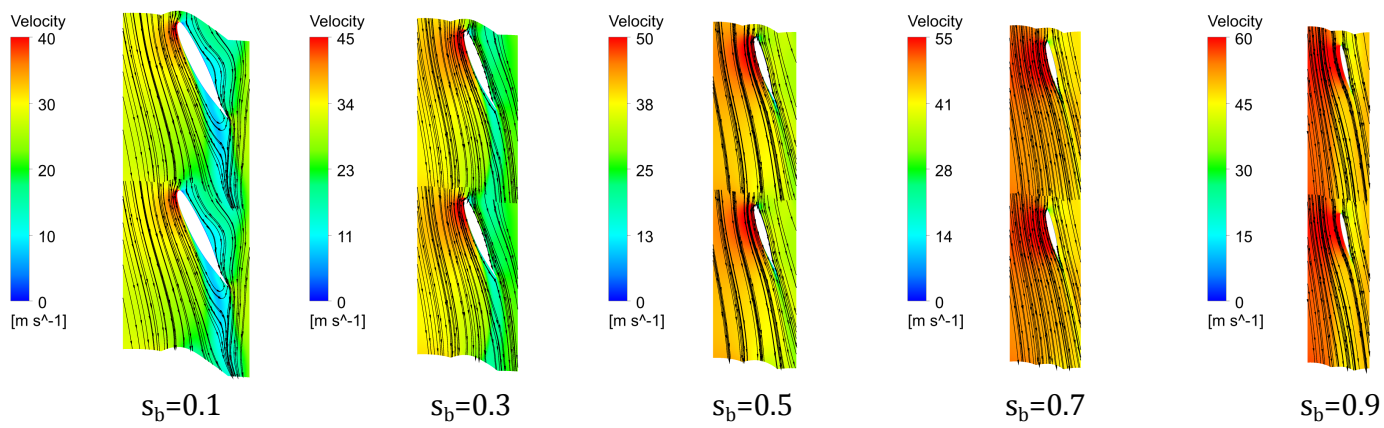

(b)
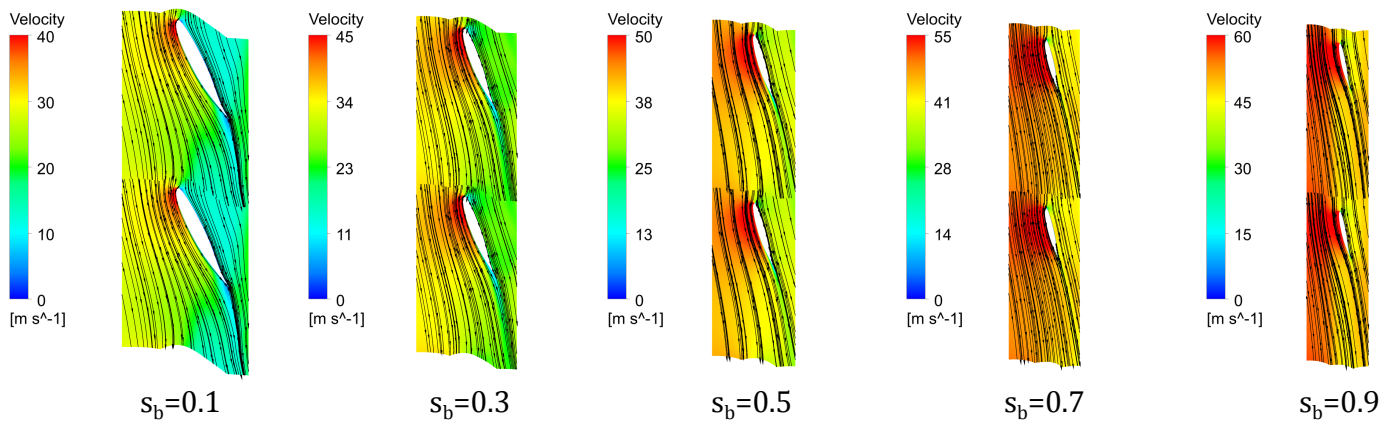

(c)
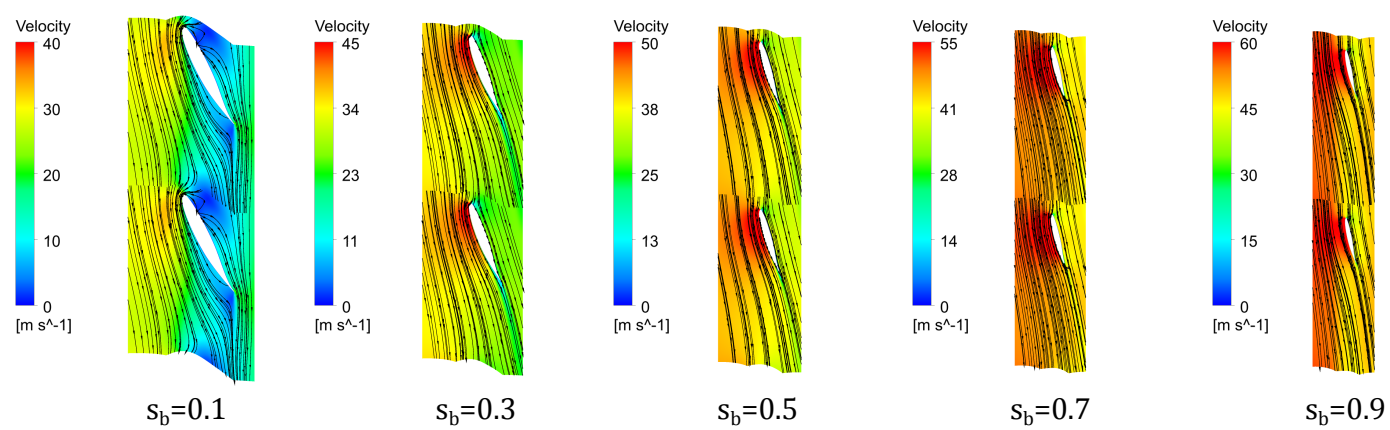

(d)

Figure 6.27: Relative surface streamlines and contours of relative velocity at different blade spans for $\phi=0.074$ at time instances of (a) $n_{F r}=n$, (b) $n_{F r}=n+0.475$, (c) $n_{F r}=n+0.950$ and (d) $n_{F r}=n+1.425$ 


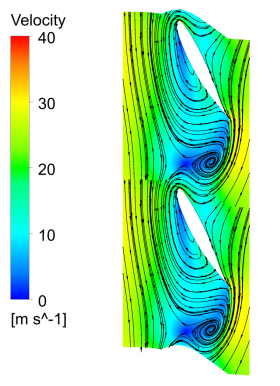

$\mathrm{s}_{\mathrm{b}}=0.1$

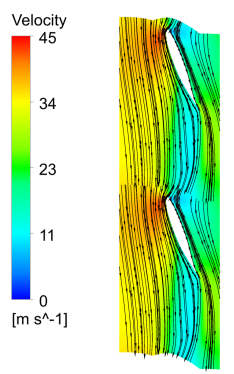

$\mathrm{s}_{\mathrm{b}}=0.3$

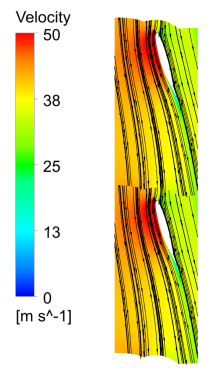

$\mathrm{s}_{\mathrm{b}}=0.5$

(a)

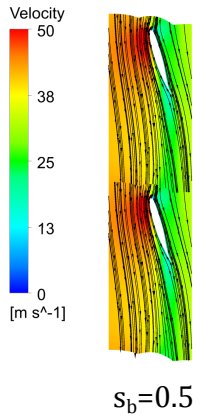

(b)
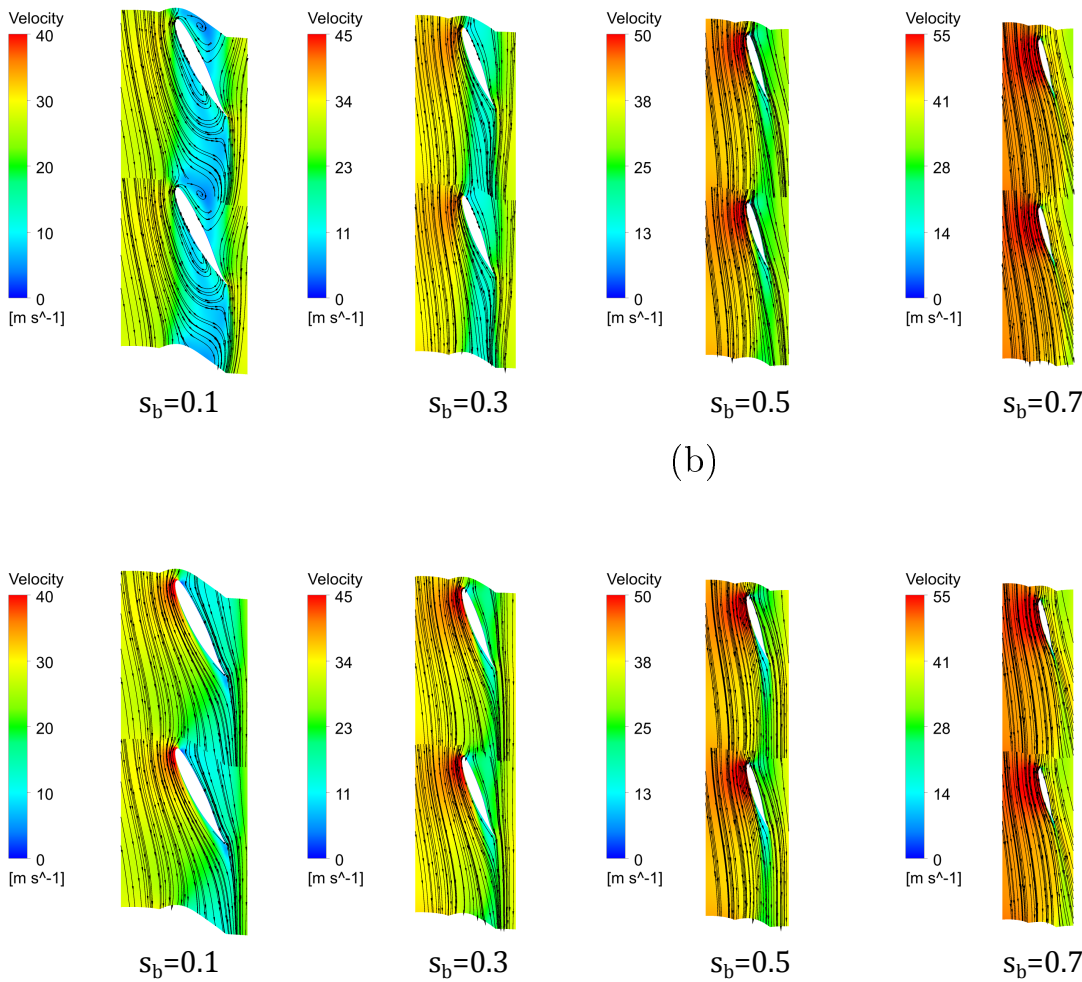

$\mathrm{s}_{\mathrm{b}}=0.7$

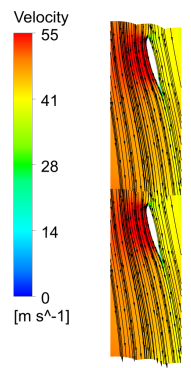

$\mathrm{s}_{\mathrm{b}}=0.7$

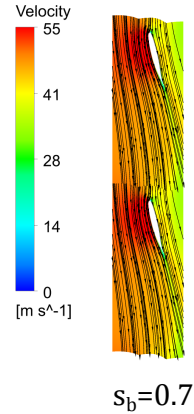

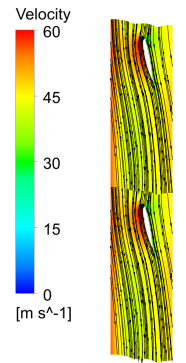

$\mathrm{s}_{\mathrm{b}}=0.9$

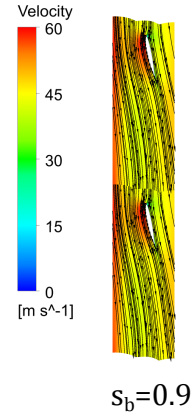

(c)
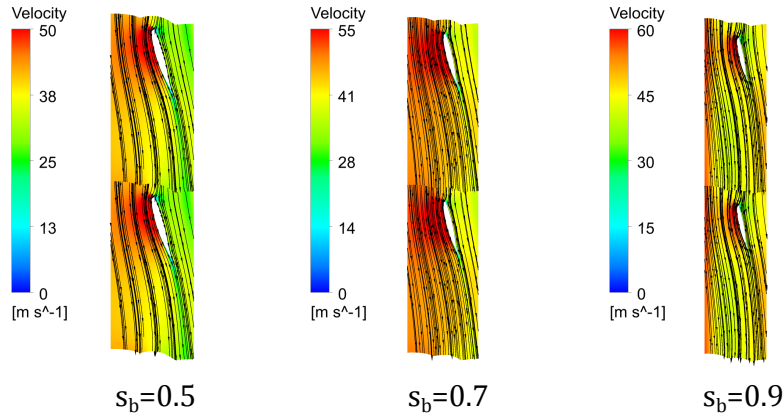

(d)

Figure 6.28: Relative surface streamlines and contours of relative velocity at different blade spans for $\phi=0.074$ at time instances of (a) $n_{F r}=n$, (b) $n_{F r}=n+0.700$, (c) $n_{F r}=n+1.400$ and (d) $n_{F r}=n+2.100$ 


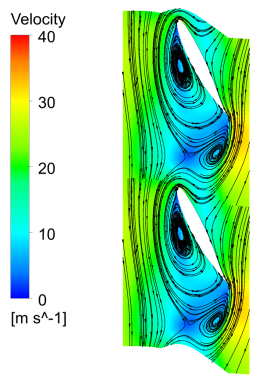

$\mathrm{s}_{\mathrm{b}}=0.1$

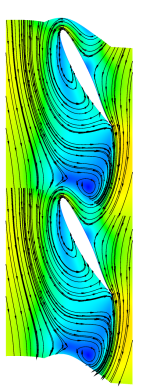

$\mathrm{s}_{\mathrm{b}}=0.1$

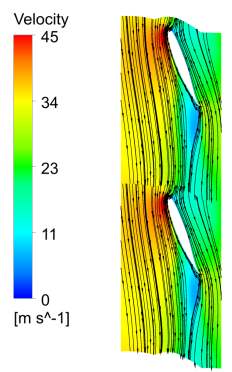

$s_{b}=0.3$

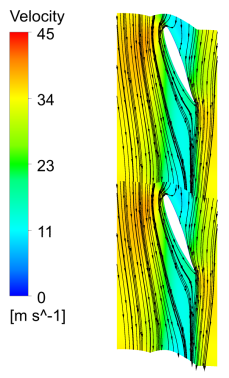

$\mathrm{s}_{\mathrm{b}}=0.3$

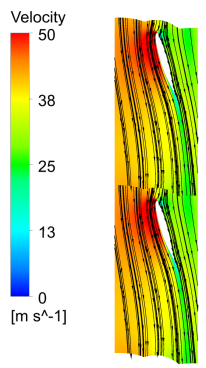

$\mathrm{s}_{\mathrm{b}}=0.5$

(a)

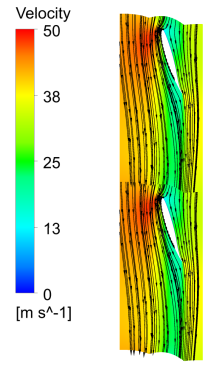

$\mathrm{s}_{\mathrm{b}}=0.5$

(b)
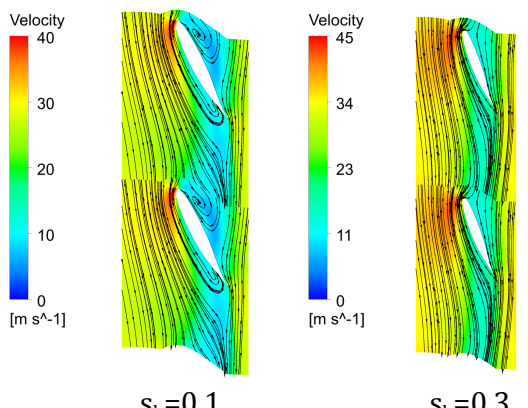

$s_{b}=0.3$
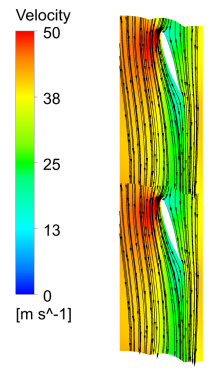

$\mathrm{s}_{\mathrm{b}}=0.5$

(c)

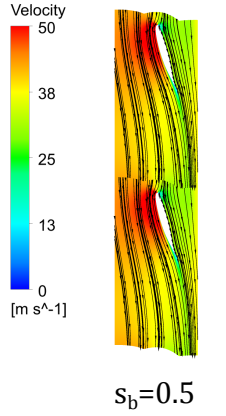

(d)
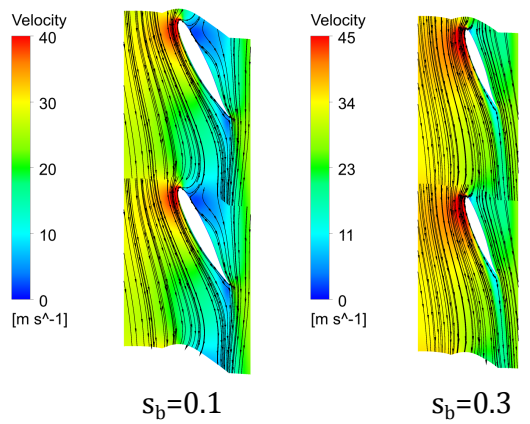

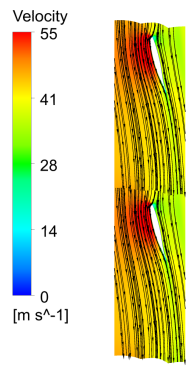

$\mathrm{s}_{\mathrm{b}}=0.7$

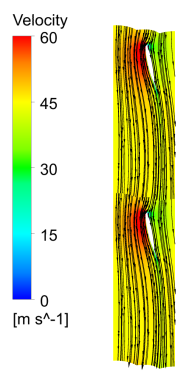

$\mathrm{s}_{\mathrm{b}}=0.9$
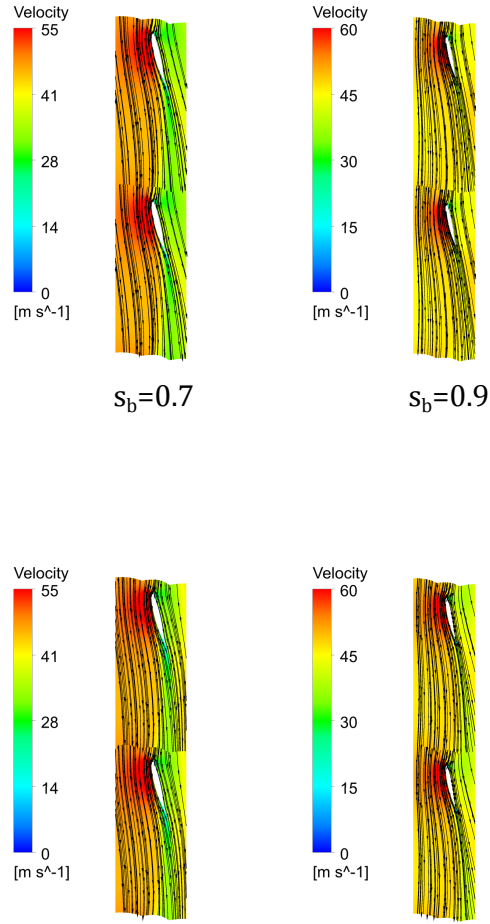

$\mathrm{s}_{\mathrm{b}}=0.7$

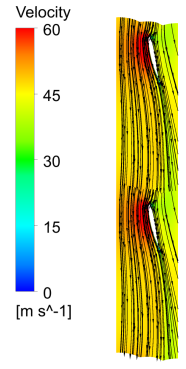

$\mathrm{s}_{\mathrm{b}}=0.9$
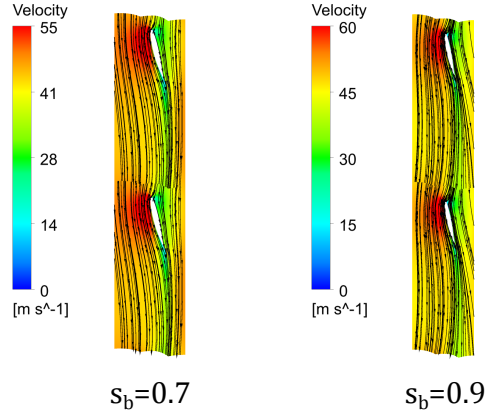

Figure 6.29: Relative surface streamlines and contours of relative velocity at different blade spans for $\phi=0.042$ at time instances of (a) $n_{F r}=n$, (b) $n_{F r}=n+0.825$, (c) $n_{F r}=n+1.650$ and (d) $n_{F r}=n+2.475$ 
the hub region at first and progressively increases to higher blade spans with decreases in flow rate from the design condition.

Research conducted by (Downie et al., 1993) also observed an increase in radial flow with a decrease in flow rate and similarly noticed reversed flow near the hub. (Downie et al., 1993) subsequently recommended the use of altered free-vortex designs at the root to alleviate the critical loading conditions of the inner span of the blade, a technique applied in the design of the B2a-fan of the present study. Unloading of the blade at the hub should give an increase in the fan stall margin while having a small effect on overall fan performance, since three quarters of the work imparted by the fan is induced by the outer half of the blade span in the case of a free-vortex design. The flow visualization however shows that, despite the effort to alleviate blade stall near the hub of the fan in the present study, stall is already present at flow rates of $\phi=0.137$, which is relatively close $\left(\phi / \phi_{D}=0.82\right)$ to the design condition of $\phi_{D}=0.168$. Unloading of the blade root would indeed have different contributions to a fan's performance depending on the specific fan's design (i.e. number of blades, lift and drag characteristics of blade sections as well as the spanwise chord length distributions). Hence, although unloading of the blade root has a small effect on the overall fan performance (as estimated by (Downie et al., 1993)) in the present study, further research to present root unloading design criteria would be valuable. 


\section{Chapter 7}

\section{Blade force characteristics}

Considering the intention to develop a simplified numerical fan model for the prediction of fan performance at low flow rates, knowledge of the forces exerted by the fan blade is required, in addition to the flow field characteristics presented in chapter 6 . Hence, the numerically obtained results are analysed to display the blade force characteristics for the range of modelled flow rates.

A discussion firstly follows on the presentation of results, whereafter the spanwise distributions of the time-averaged blade forces are given. Subsequently, the time-dependence of these forces at low flow rates are presented and some concluding remarks given. The content of this chapter is also partially presented in (Louw et al., 2015b).

\subsection{Definition of variables}

Considering a cylindrical blade section as presented in figure 7.1, the induced resultant force of the blade acting on the flow is calculated by:

$$
F_{\text {Res }}=\sqrt{\sum_{j=1}^{3} F_{j}^{2}}[\mathrm{~N} / \mathrm{m}]
$$

where

$$
F_{j}=\sum_{i=1}^{n} F_{j i} \quad[\mathrm{~N} / \mathrm{m}]
$$

and $j$ refers to the specific cylindrical direction $(r, \theta, z), i$ to a single node and $n$ the number of nodes (computational cells in the CFD model) on the peripheral surface of a single blade section.

Also shown in figure 7.1 is the vector-averaged velocity triangles between the up- and downstream velocities and the lift $(L)$, drag $(D)$ and radial $(R)$ 


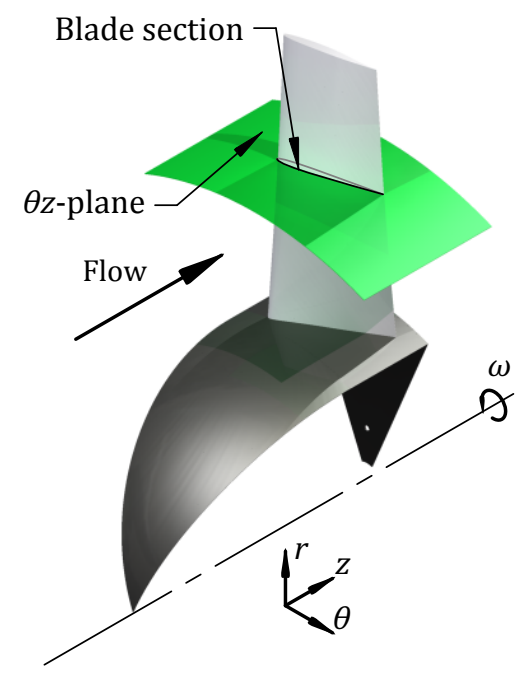

(a)
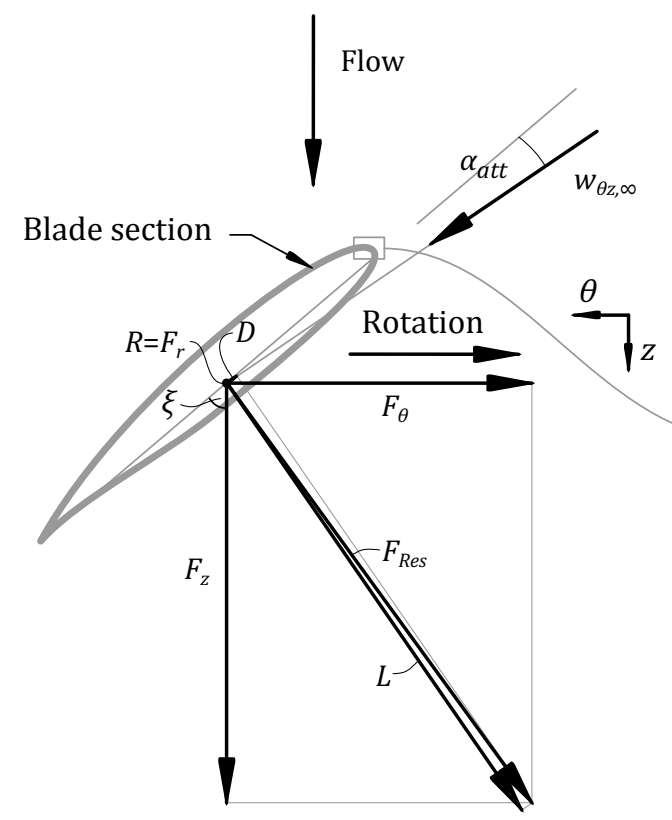

(c)
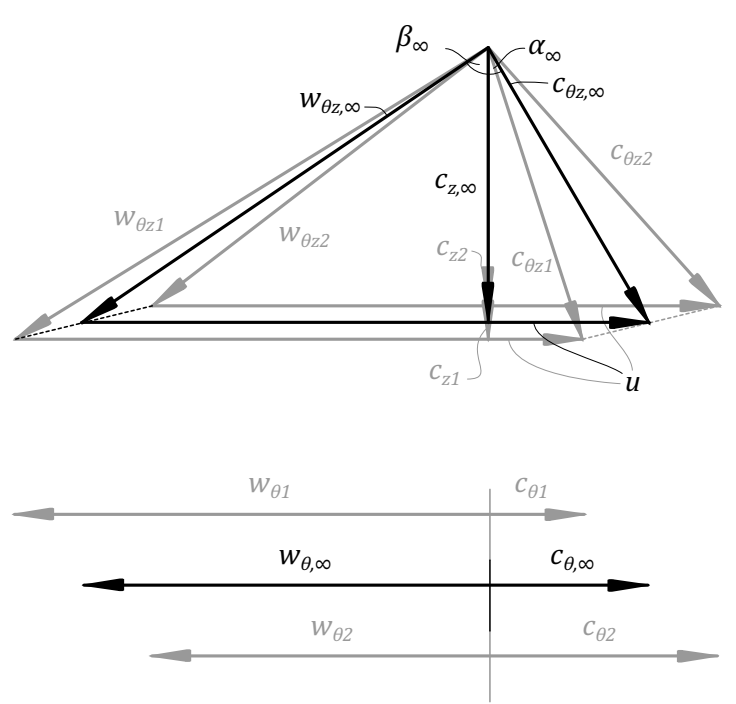

(b)

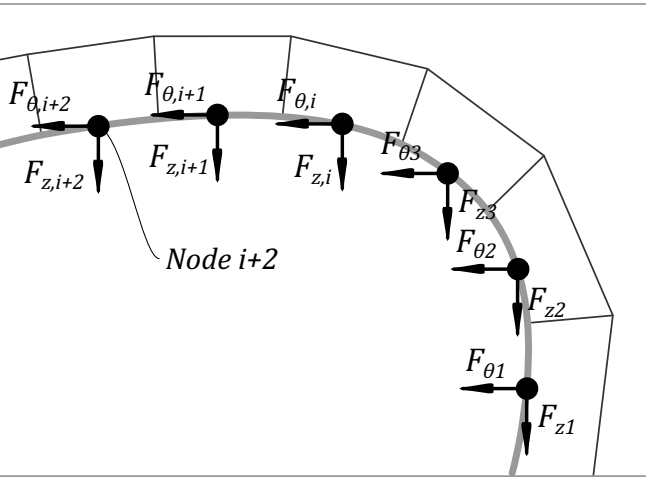

Figure 7.1: Depiction of (a) a single blade section through the fan blade, (b) the velocity components and (c) the blade forces particular to this blade section

force components in relation to the relative flow direction onto the blade section. This angle between the vector-averaged relative velocity and the blade chord, known as the angle of attack $\left(\alpha_{a t t}\right)$, is calculated as follows:

$$
\alpha_{\text {att }}=\beta_{\infty}-\xi
$$


Remaining with the convention of the indicated cylindrical coordinate system, the lift, drag and radial force components are calculated by:

$$
\begin{aligned}
& L=-F_{z} \sin \beta_{\infty}+F_{\theta} \cos \beta_{\infty} \quad[\mathrm{N} / \mathrm{m}] \\
& D=F_{z} \cos \beta_{\infty}+F_{\theta} \sin \beta_{\infty} \quad[\mathrm{N} / \mathrm{m}] \\
& R=F_{r} \quad[\mathrm{~N} / \mathrm{m}]
\end{aligned}
$$

where $\beta_{\infty}$ is the vector-averaged relative flow angle and $\xi$ the blade section stagger angle.

The low value of the drag force compared to the lift force is typical for a blade section at higher flow rates $(0.137 \leq \phi \leq 0.200)$ and higher blade spans. The radial force component, indicated as perpendicular to the page, is typically also in the same order or smaller than the drag force. Since each section is infinitely thin, the magnitude of the force vectors are given in Newton per unit length $(\mathrm{N} / \mathrm{m})$. Subsequently the force components $(L, D$ and $R$ ) are made dimensionless into force coefficients as follows:

$$
\begin{aligned}
C_{L} & =\frac{L}{0.5 \rho w_{\theta z, \infty}^{2} c h} \\
C_{D} & =\frac{D}{0.5 \rho w_{\theta z, \infty}^{2} c h}
\end{aligned}
$$

and

$$
C_{R}=\frac{R}{0.5 \rho w_{\infty}^{2} c h}
$$

where $C_{L}, C_{D}$ and $C_{R}$ are the lift, drag and radial force coefficients, respectively. Additionally $w_{\theta z, \infty}$ is the vector-averaged relative velocity in the $\theta z$-plane and $w_{\infty}$ the vector averaged total relative velocity, between the upstream and downstream locations of the fan. $\rho=1.2 \mathrm{~kg} / \mathrm{m}^{3}$ is the density of air and $c h$ the blade chord at the particular span being considered.

The reason for using $w_{\theta z, \infty}$ (instead of $w_{\infty}$ ) in the calculation of $C_{L}$ and $C_{D}$ is to make these coefficients comparable to a two-dimensional case of an isolated aerofoil. However, $C_{R}$ is calculated using $w_{\infty}$ to make it a function of the radial velocity in addition to the axial and circumferential velocities. 


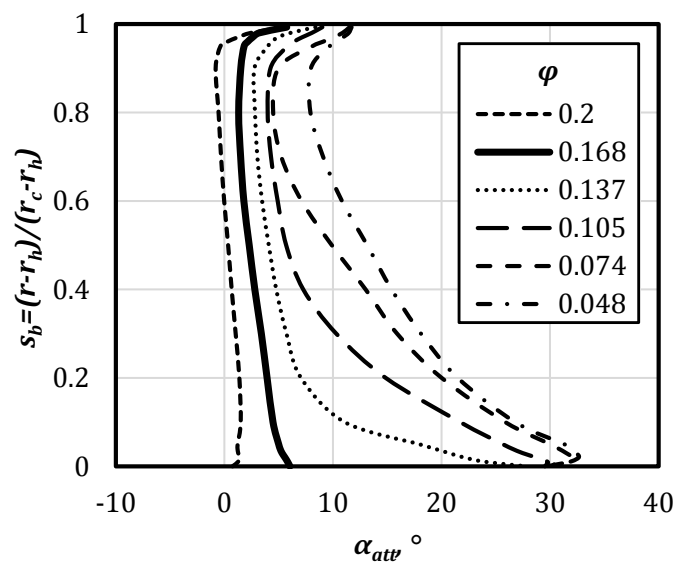

(a)

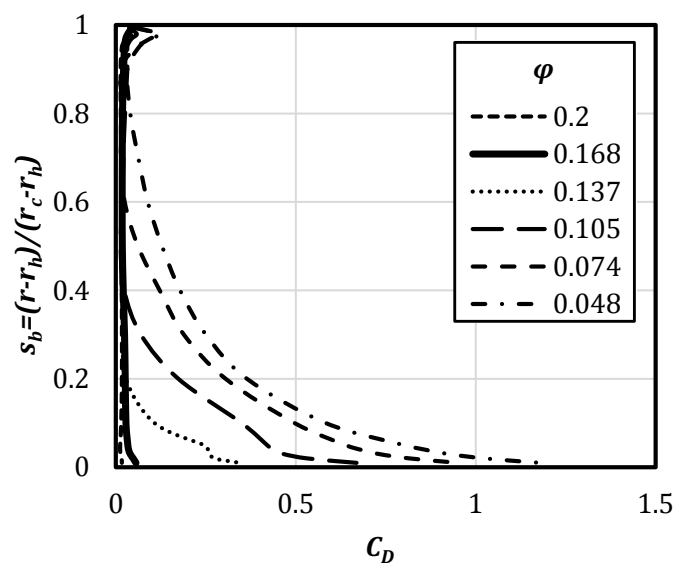

(c)

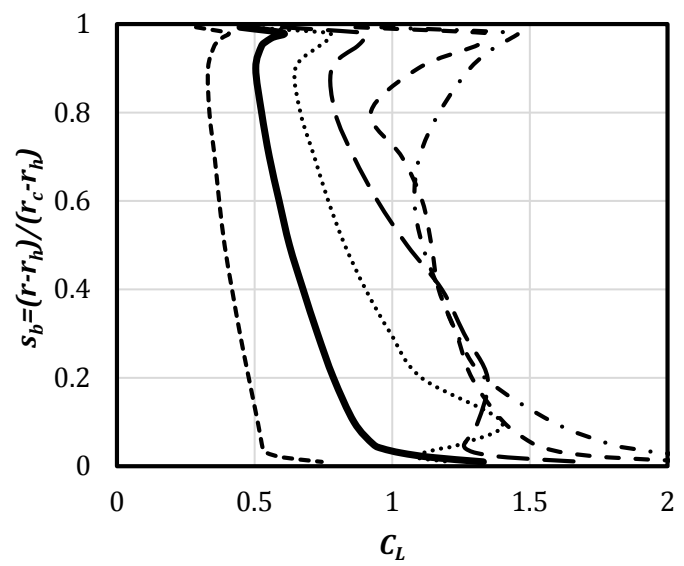

(b)

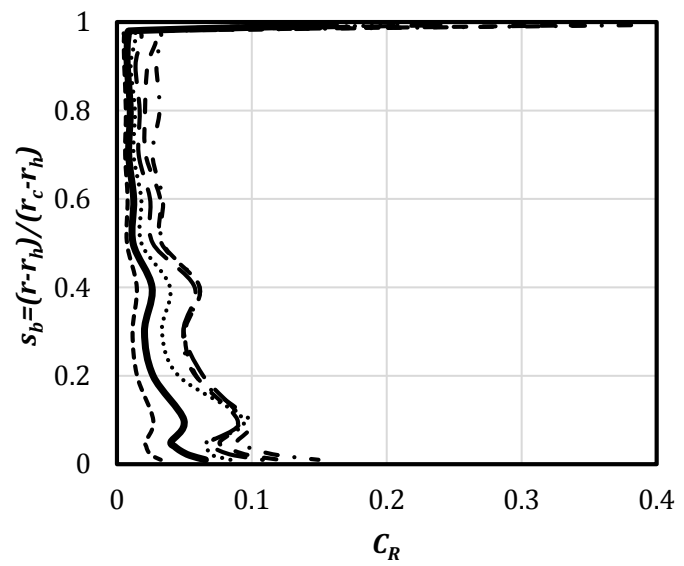

(d)

Figure 7.2: Circumferentially and time-averaged spanwise distributions of (a) $\alpha_{a t t}$, (b) $C_{L}$, (c) $C_{D}$ and (d) $C_{R}$ for the range of flow rates investigated

\subsection{Time-averaged blade forces}

The time-averaged spanwise force coefficients are firstly presented giving an indication of the change in these values for the range of flow rates that are simulated. Hereafter, these coefficients are also presented as a function of angle of attack and compared to the lift and drag characteristics of a two-dimensional NASA LS 0413 aerofoil (McGhee and Beasley, 1976) as obtained using XFOIL. Furthermore, the radial force coefficient is also presented as a function of the radial flow angle $\left(\theta_{r}\right)$ defined in chapter 6 . For all of these coefficients, the main $\left(0.1 \leq s_{b} \leq 0.9\right)$, root $\left(0<s_{b}<0.1\right)$ and tip spans $\left(0.9<s_{b}<1\right)$ of the fan blade are considered individually. 


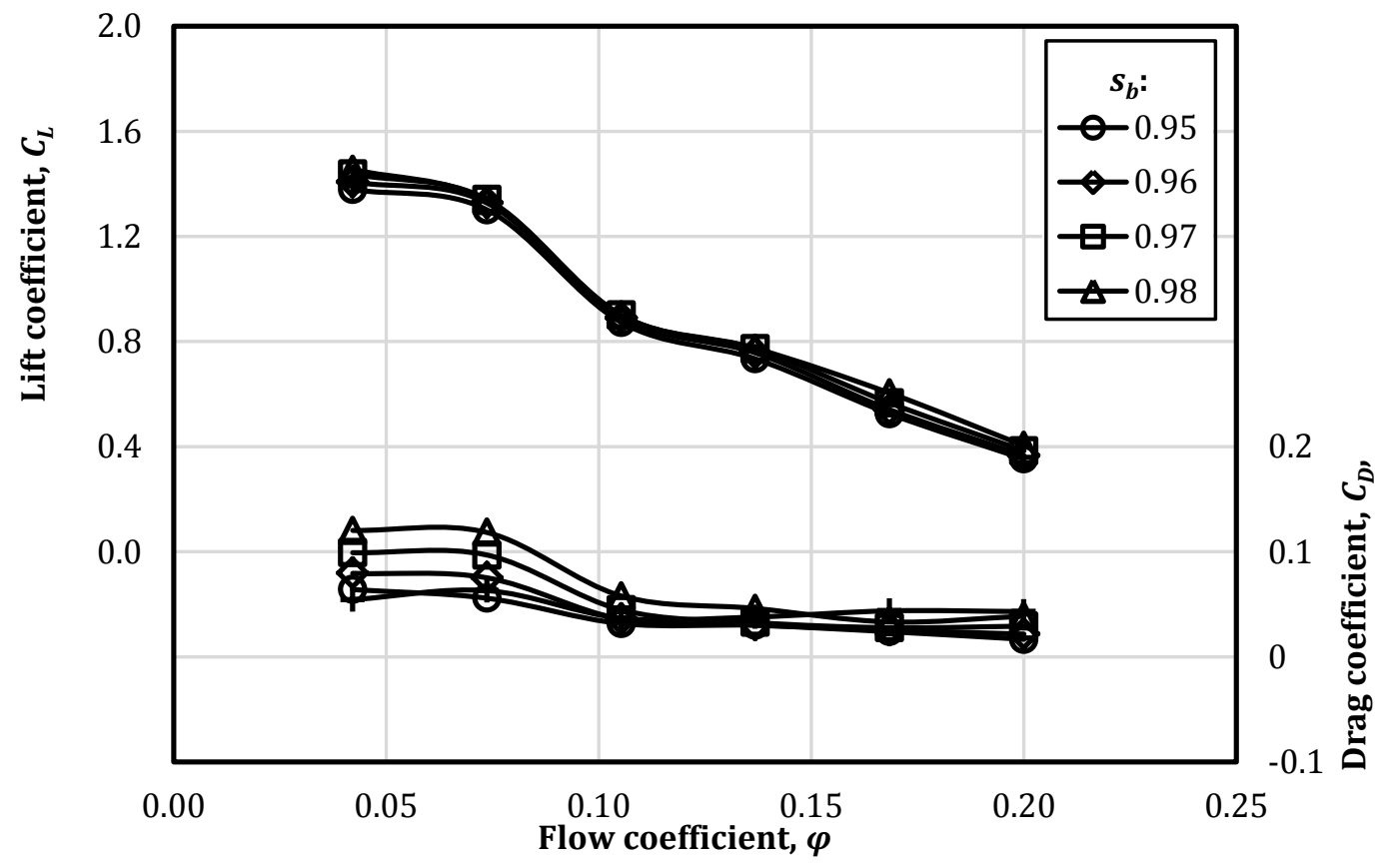

Figure 7.3: Blade lift $\left(C_{L}\right)$ and drag $\left(C_{D}\right)$ coefficients as a function of flow coefficient $(\phi)$ for sections of $0.9<s_{b}<1$

\subsubsection{Spanwise distributions}

The time-averaged spanwise angles of attack and force coefficients are presented in figures 7.2a-d. For design and higher flow rates $(\phi \geq 0.168)$, the angle of attack remains near-linear in the spanwise direction except for the tip region, which is influenced by the leakage flow across the tip. As the flow through the fan is reduced, a deviation from this trend can be observed as a large increase in angle of attack appears, starting in the hub region, where local maxima occur, and progressively increases towards higher blade spans as the flow rate is reduced. The lift coefficient is the highest in the hub region and shows an increase with a decrease in flow rate, due to the higher angles of attack onto the blade. However, a change in the lift coefficient profile shape can be observed for lower flow rates, especially near the hub. A sharp increase in lift coefficient near the tip can also be observed again due to the increased angle of attack induced by the flow structure occurring in the tip region. The drag coefficient is in the order of $1 / 100^{\text {th }}$ of the lift coefficient for near-design flow rates, but a progressive increase in drag magnitude can be observed with a decrease in flow rate. Moreover, a trend in the drag magnitude is visible that starts in the hub region and progressively moves to higher radii. Although a radial force is present, its magnitude is smaller or in the same order than the drag force, although this component also increases marginally with a decrease in flow rate and is at a maximum in the hub region.

Considering the blade sectional lift coefficients as functions of flow rate in figures 7.3-7.5 an almost linear increase can be observed for $0.1 \leq s_{b} \leq 0.9$ 


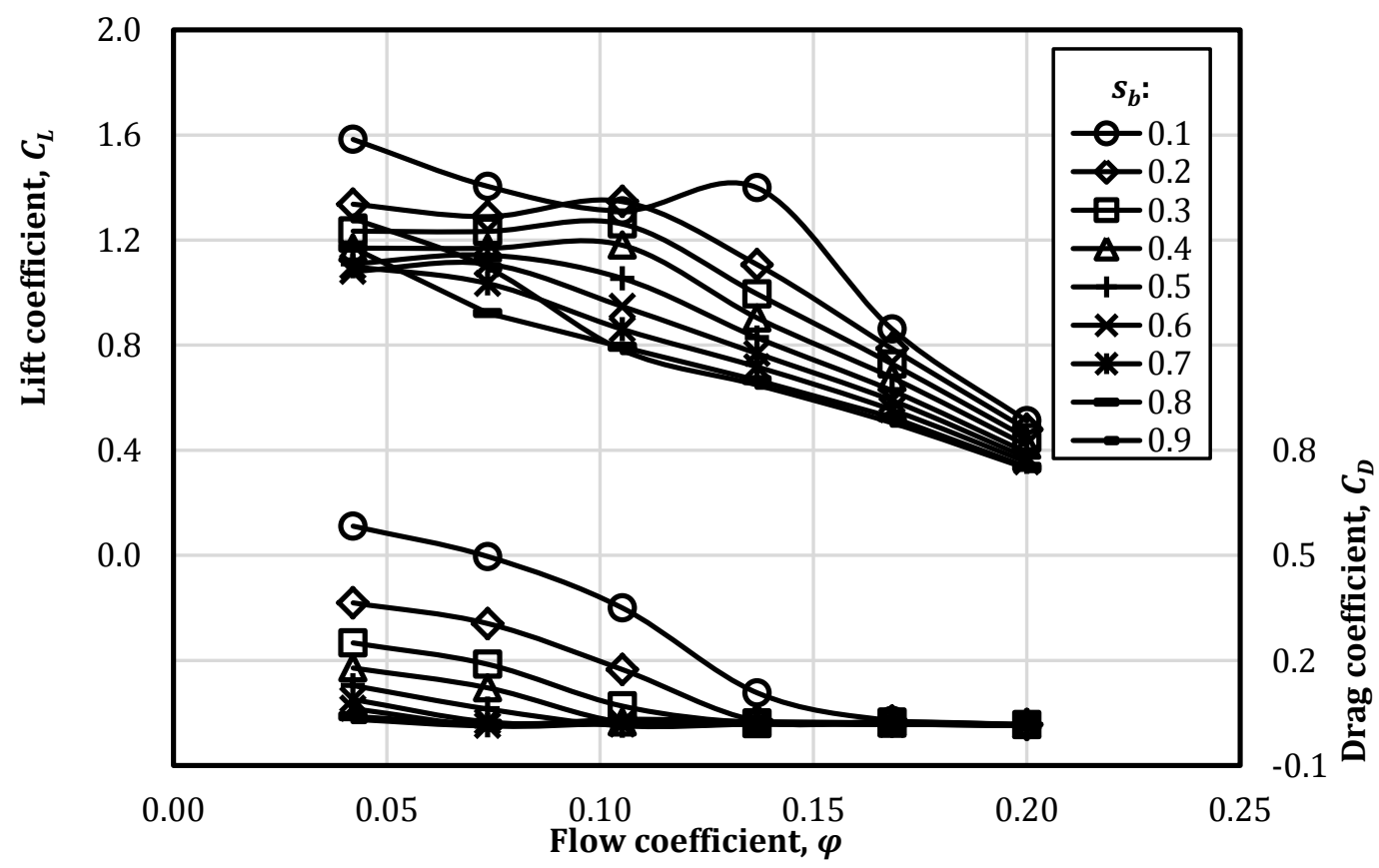

Figure 7.4: Blade lift $\left(C_{L}\right)$ and drag $\left(C_{D}\right)$ coefficients as a function of flow coefficient $(\phi)$ for spans of $0.1 \leq s_{b} \leq 0.9$

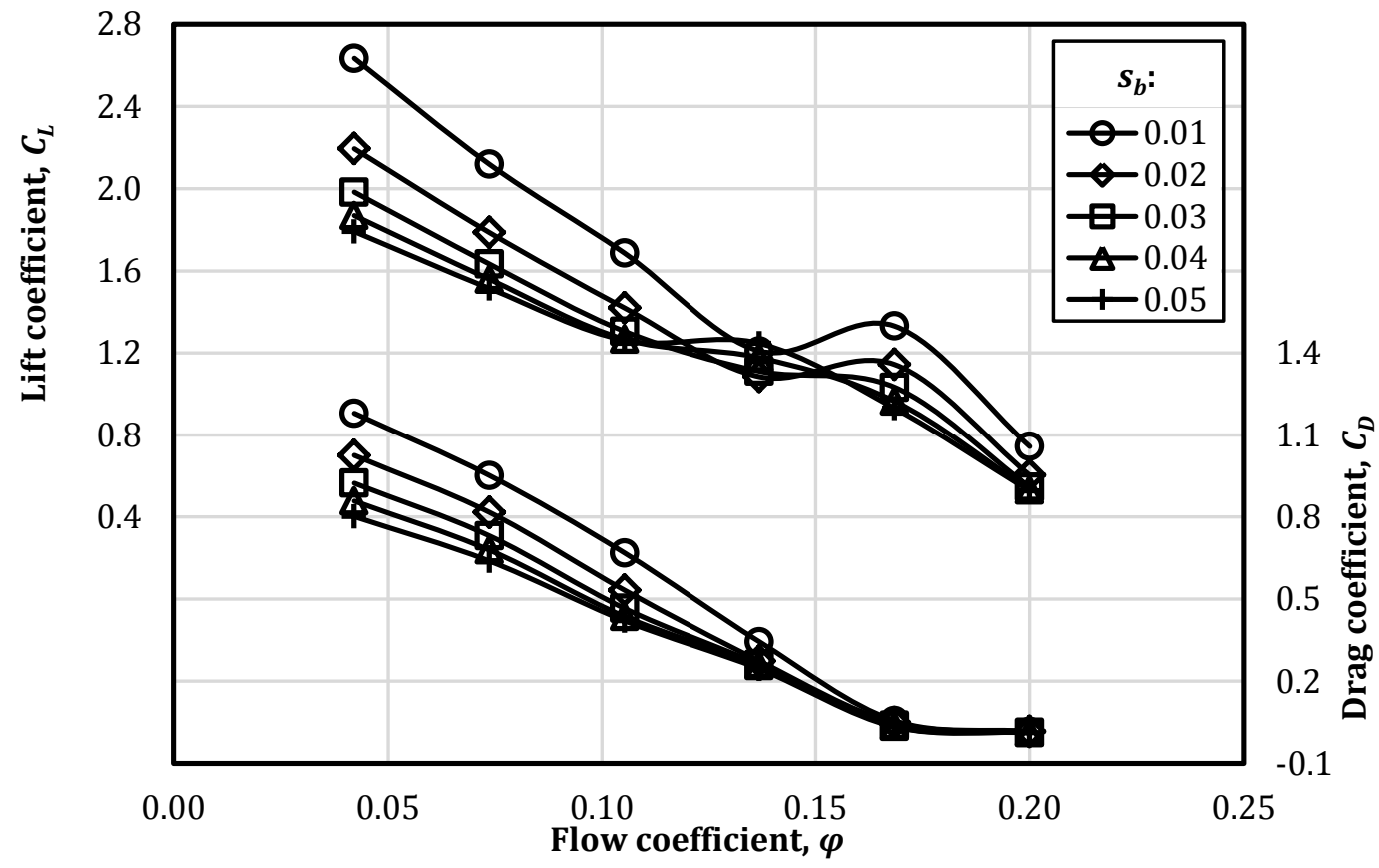

Figure 7.5: Blade lift $\left(C_{L}\right)$ and drag $\left(C_{D}\right)$ coefficients as a function of flow coefficient $(\phi)$ for sections of $0<s_{b}<0.1$ 
with a decrease in flow rate. However, the sudden increase in drag near the hub is once again accentuated as blade stall starts occurring in this region at $\phi<0.168$, and progresses to higher radii with a decrease in flow rate. At the lowest flow rate the drag coefficients in the hub region are in the order of $1 / 3^{\text {rd }}$ of the lift coefficient compared to $1 / 100^{\text {th }}$ for near-design flow rates. The lift coefficients for each blade section reaches a plateau for flow rates of $\phi<0.105$ along the main part of the blade span, but deviations from this trend are observed in the hub and tip regions due to the three-dimensional flow effects.

\subsubsection{Two-dimensional comparisons}

A comparison is made between the lift and drag characteristics obtained at the various blade sections already presented and the two-dimensional case of the NASA LS 0413 aerofoil profile used in the initial design of the fan blade. For this purpose the lift and drag characteristics of the NASA LS 0413 profile at Reynolds numbers of $2(10)^{5}$ and $5(10)^{5}$ are used for comparison. These represent the upper and lower bounds of Reynolds numbers for the B2a-fan over the range of flow rates investigated, where the highest is at the blade tip and the lowest at the blade root. Additionally, the radial force coefficients as a function of radial angle are also presented. These comparisons are depicted in figures 7.6-7.8 and discussed.

A slight offset in lift characteristics (figure 7.6) between the two-dimensional NASA-LS 0413 reference profile and the blade sectional profiles can be observed at low angles of attack $\left(\alpha_{\text {att }}<5^{\circ}\right)$ for the largest span of the fan blade, but both data sets have approximately the same gradient of inclination. In the tip region $\left(0.95<s_{b}<1\right)$ this offset is more significant, due to the three-dimensional flow structures in this region. A strong deviation in lift characteristics exists for $\alpha_{\text {att }}>5^{\circ}$ at lower blade spans as these coefficients reach a plateau for $5^{\circ} \lesssim \alpha_{a t t} \lesssim 20^{\circ}$. For $\alpha_{a t t}>5^{\circ}$, a trend can be observed where the lift characteristic near the hub is the highest and gradually decreases towards the tip, with the exception of $s_{b}=0.8$ and 0.9 . The blade sectional lift coefficients are generally always lower than that of the NASA-LS 0413 reference profile, although a sharp increase in lift can be observed for $20^{\circ}<\alpha_{\text {att }}<30^{\circ}$. For the highest angles of attack along the blade span, the lift coefficients near the hub are almost twice the value obtained compared to the tip region. The highest lift coefficient obtained at the hub is $C_{L}=2.63$ for $\alpha_{a t t}=32^{\circ}$. 


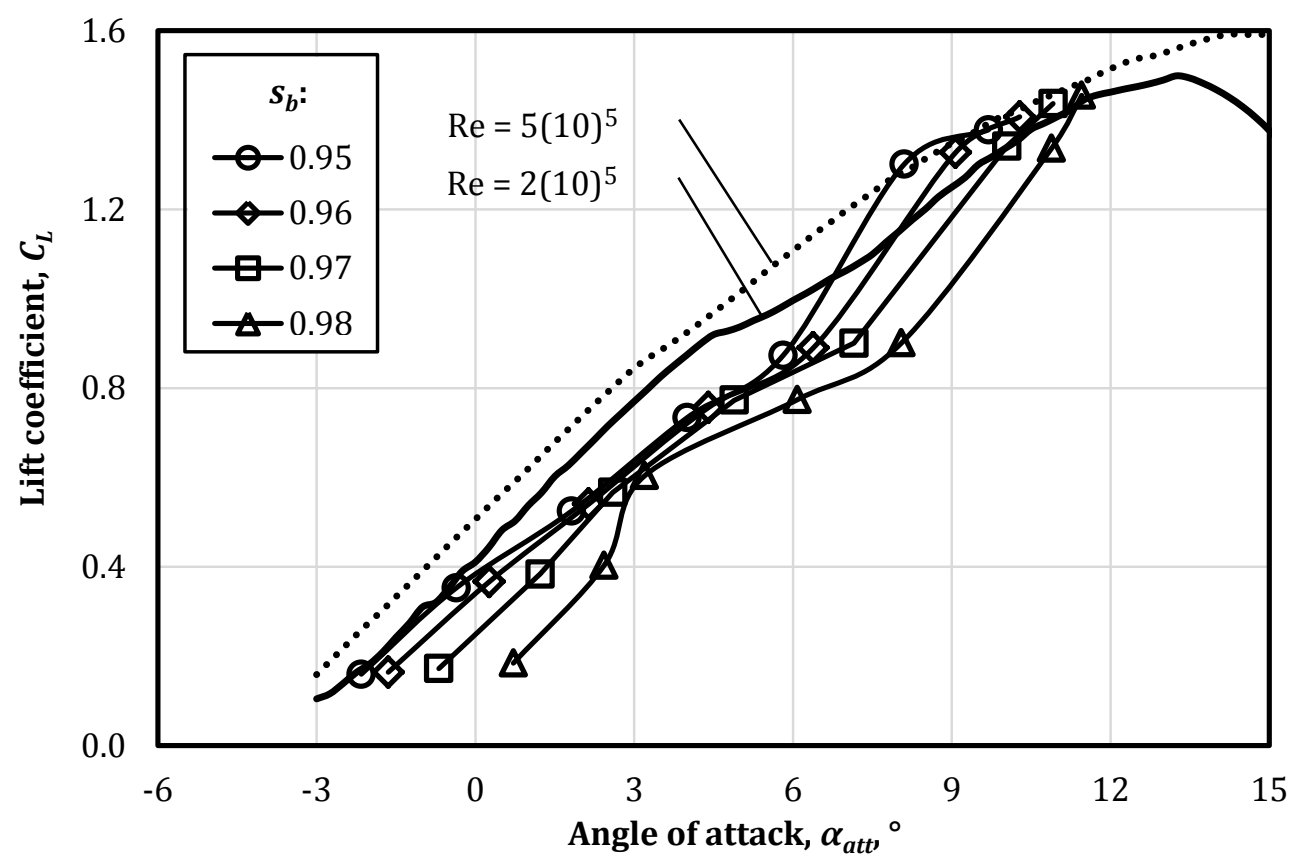

(a)

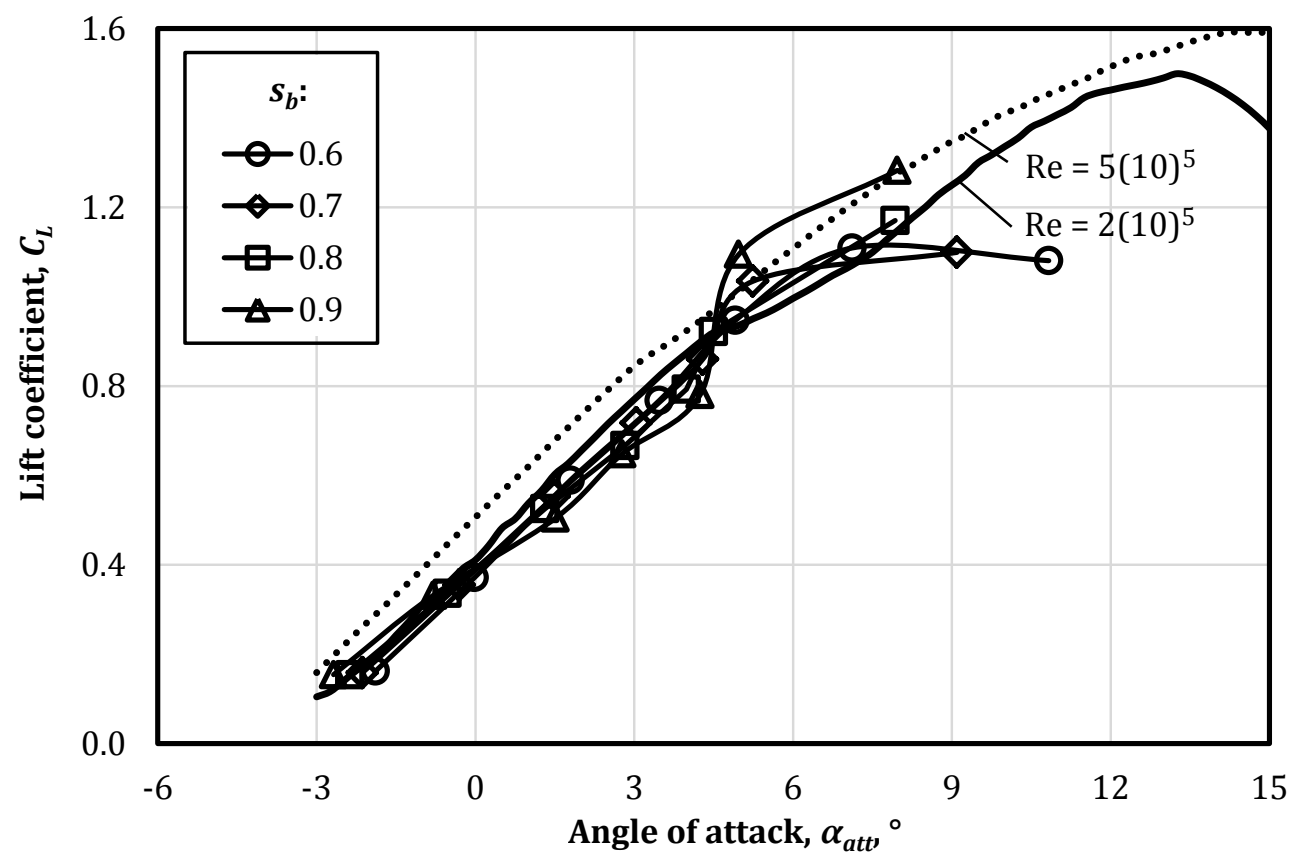

(b)

Figure 7.6: Blade sectional lift coefficients $\left(C_{L}\right)$ as a function of angle of attack $\left(\alpha_{a t t}\right)$ at spans of (a) $0.9<s_{b}<1$ and (b) $0.6 \leq s_{b} \leq 0.9$ compared to the NASA LS 0413 aerofoil for two different Reynolds numbers obtained using XFOIL 


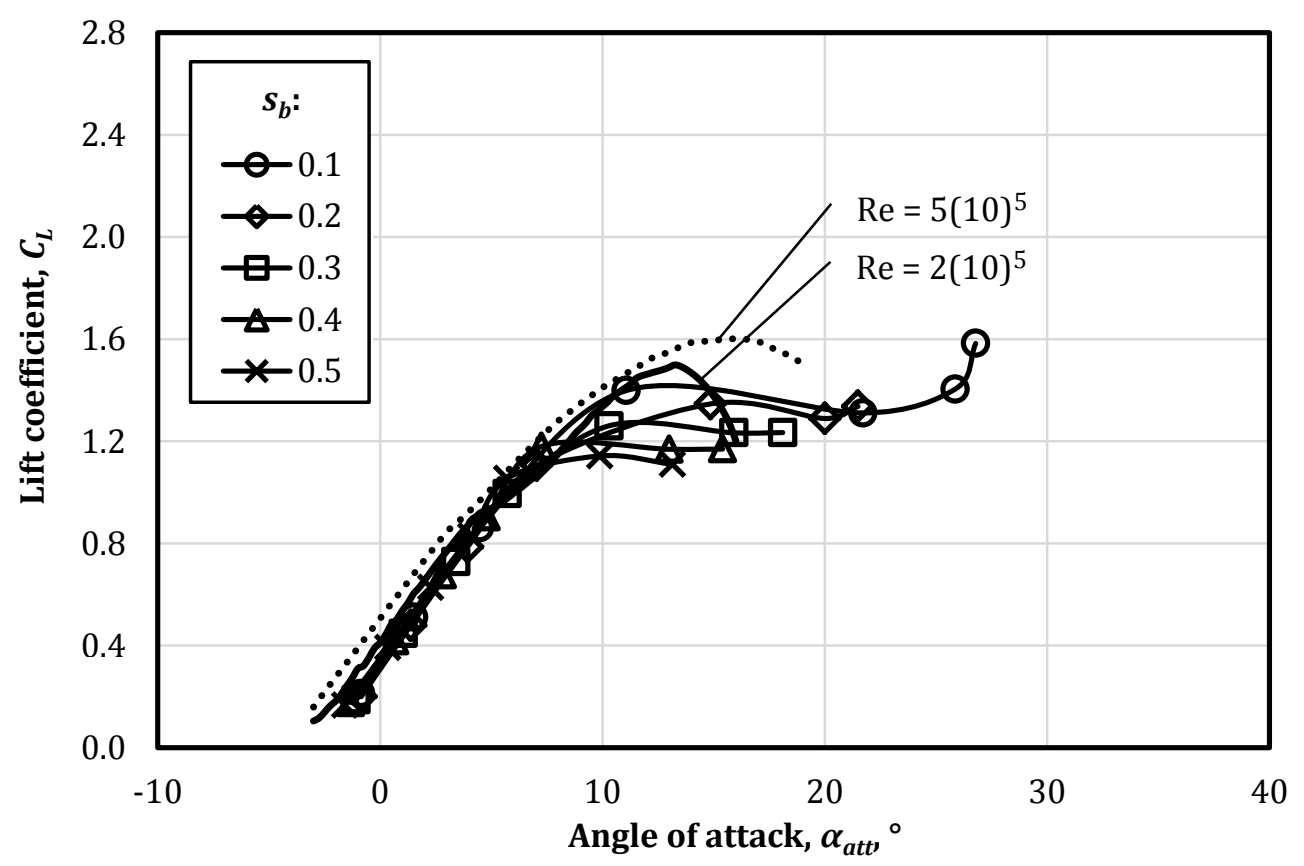

(c)

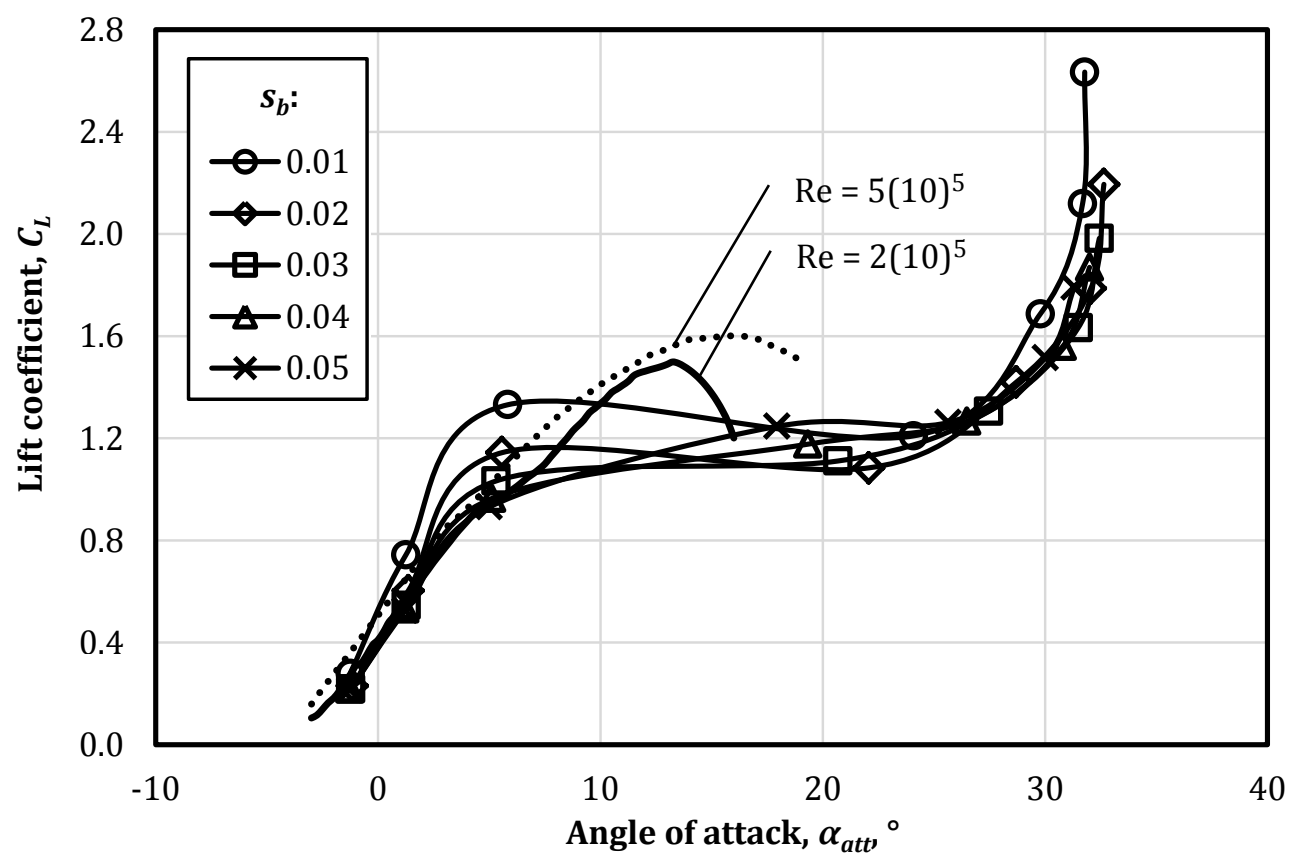

(d)

Figure 7.6: Blade sectional lift coefficients $\left(C_{L}\right)$ as a function of angle of attack $\left(\alpha_{a t t}\right)$ at spans of (c) $0.1 \leq s_{b} \leq 0.5$ and (d) $0<s_{b}<1$ compared to the NASA LS 0413 aerofoil for two different Reynolds numbers obtained using XFOIL 


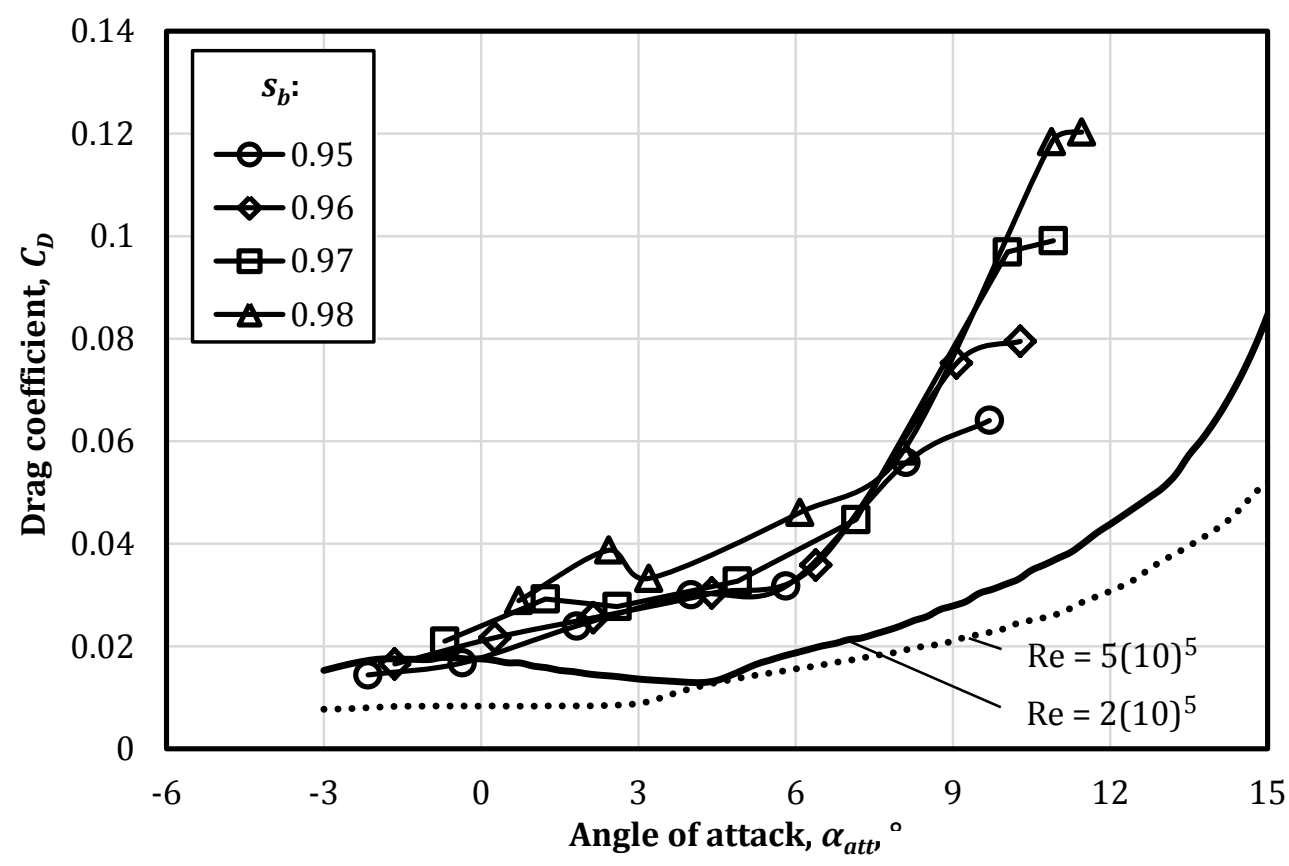

(a)

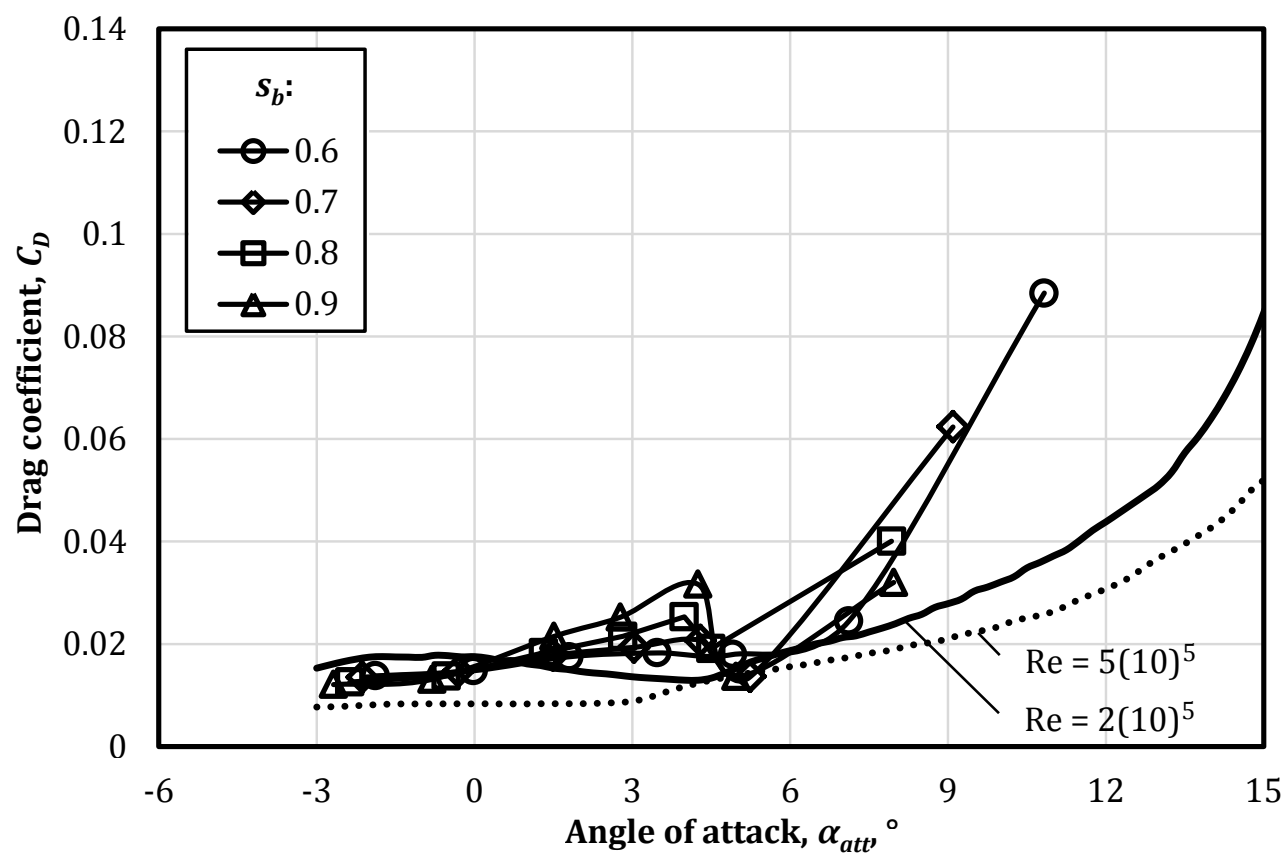

(b)

Figure 7.7: Blade drag coefficients $\left(C_{D}\right)$ as a function of angle of attack $\left(\alpha_{\text {att }}\right)$ at spans of (a) $0.9<s_{b}<1$ and (b) $0.6 \leq s_{b} \leq 0.9$ compared to the NASA LS 0413 aerofoil for two different Reynolds numbers obtained using XFOIL 


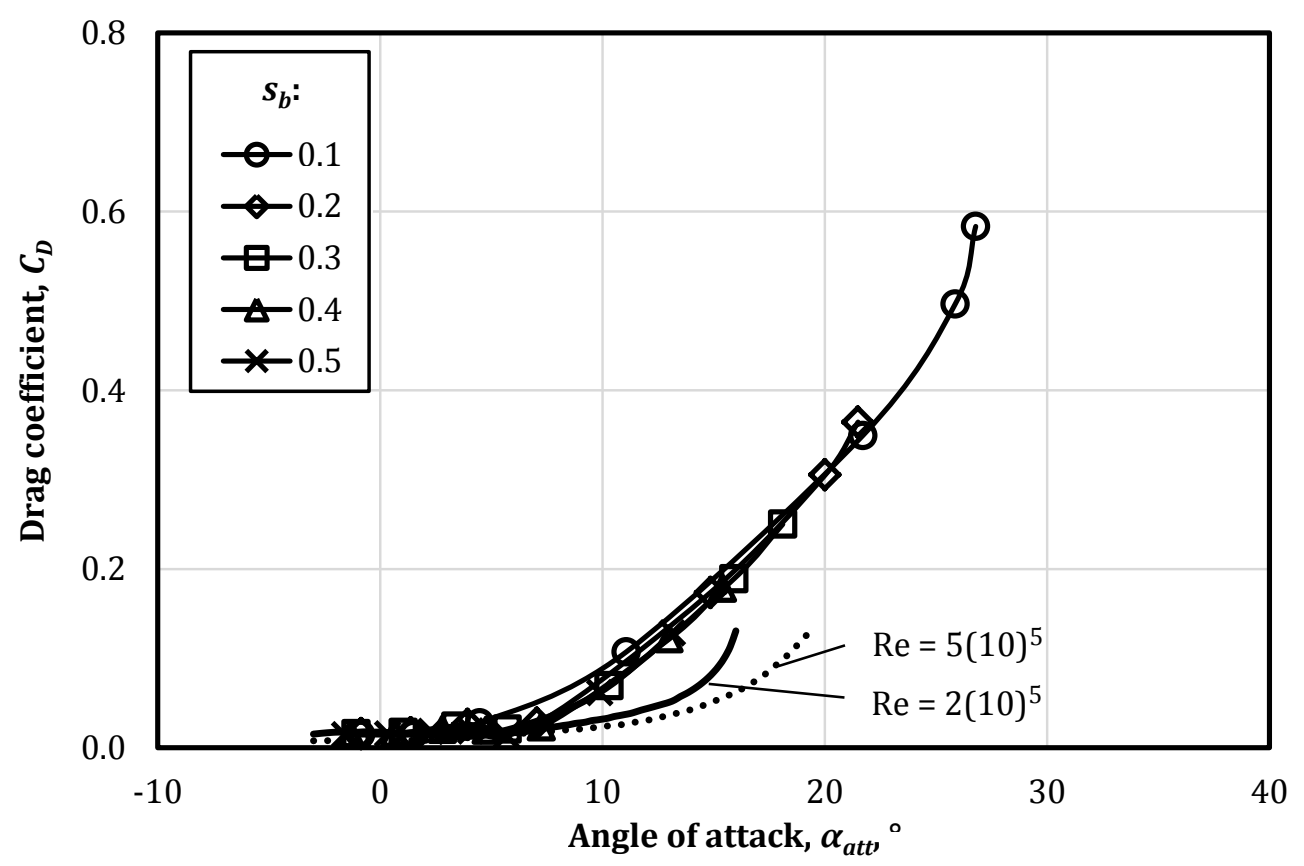

(c)

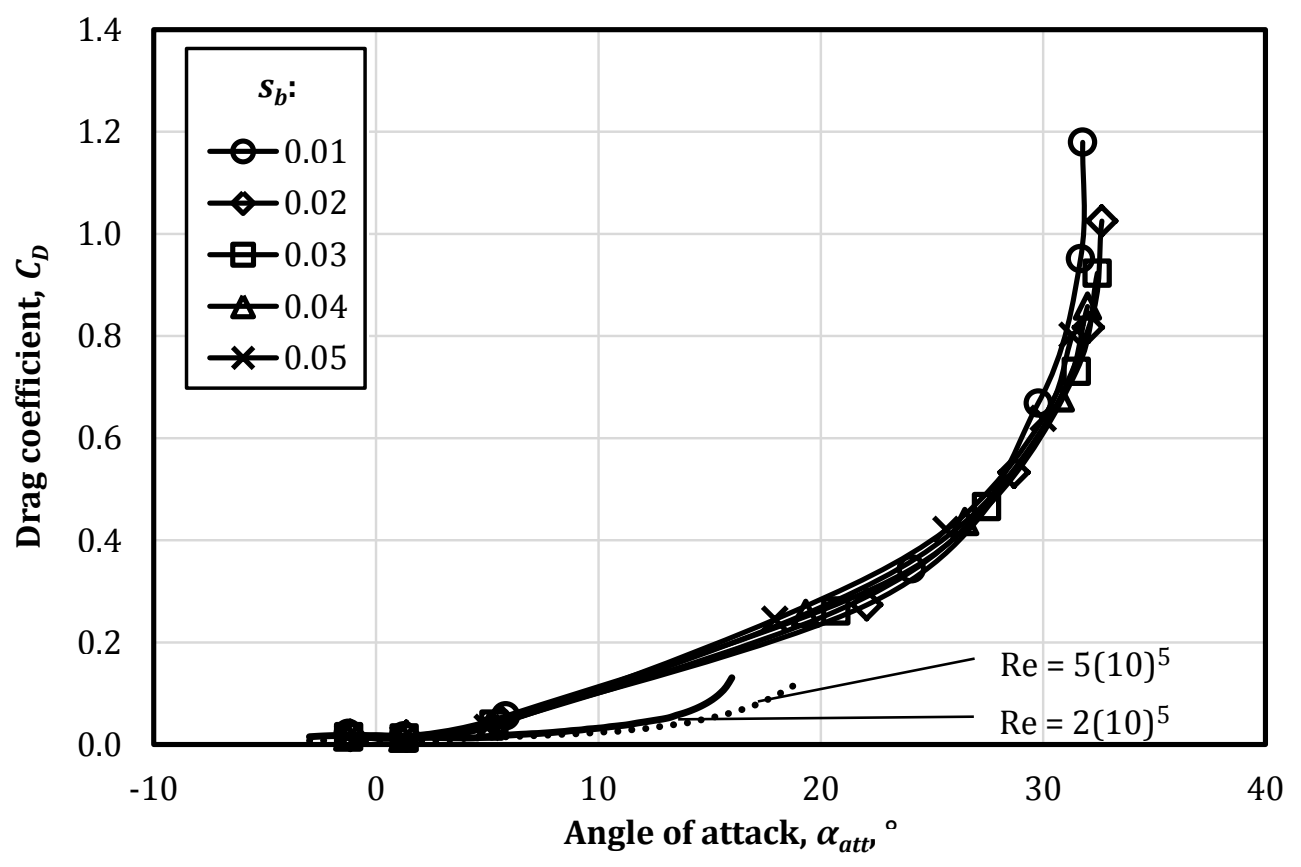

(d)

Figure 7.7: Blade drag coefficients $\left(C_{D}\right)$ as a function of angle of attack $\left(\alpha_{\text {att }}\right)$ at spans of (c) $0.1 \leq s_{b} \leq 0.5$ and (d) $0<s_{b}<0.1$ compared to the NASA LS 0413 aerofoil for two different Reynolds numbers using XFOIL 


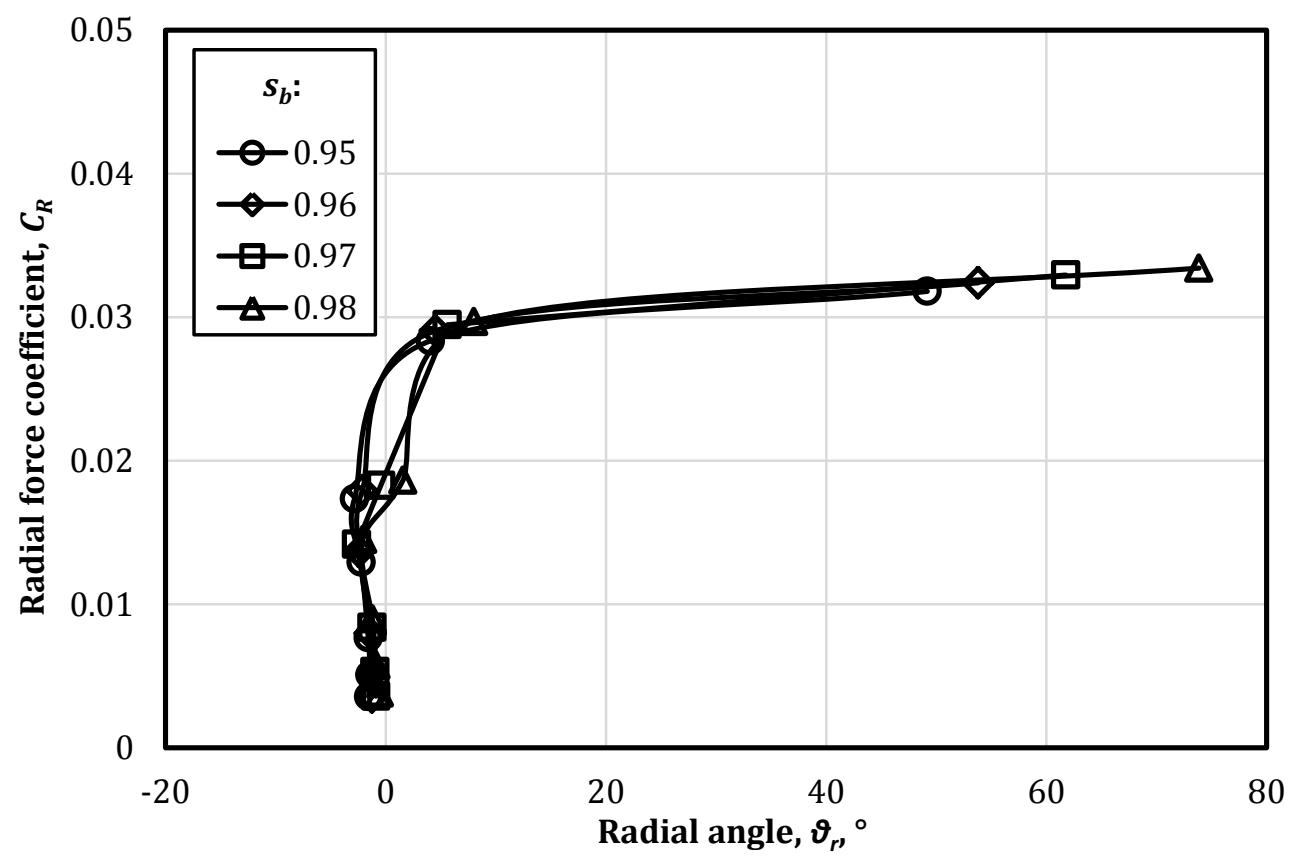

(a)

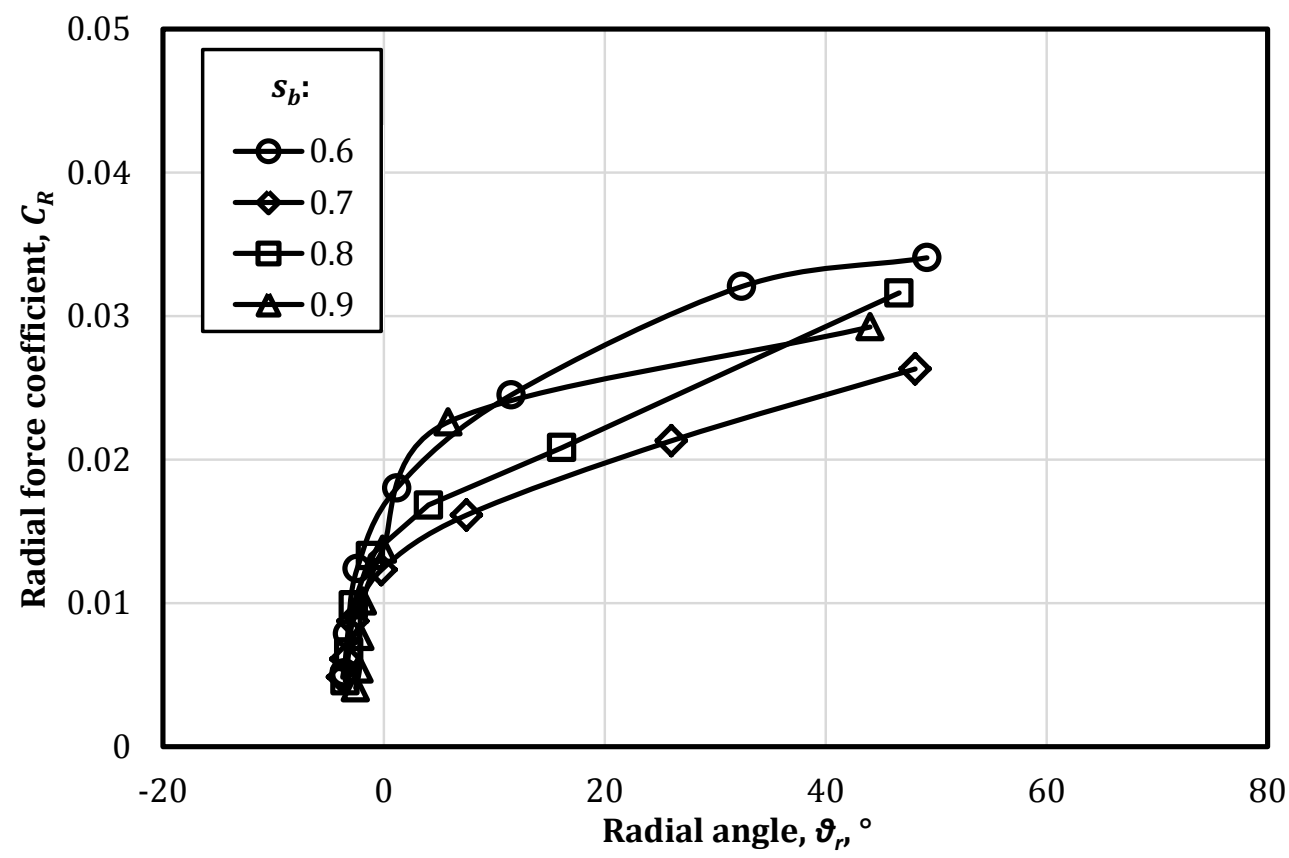

(b)

Figure 7.8: Blade radial force coefficients $\left(C_{R}\right)$ as a function of radial flow angle $\left(\theta_{r}\right)$ at spans of (a) $0.9<s_{b}<1$ and (b) $0.6 \leq s_{b} \leq 0.9$ 


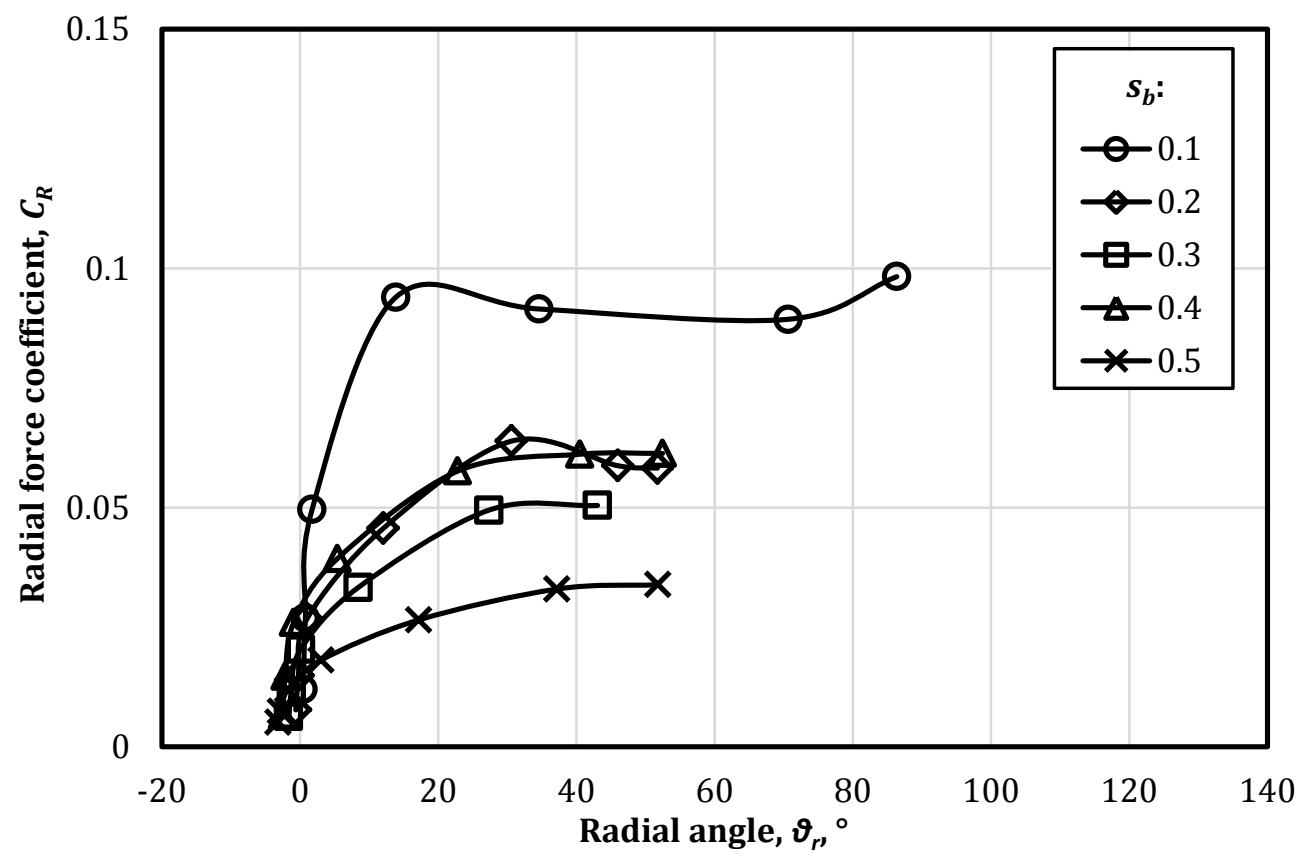

(c)

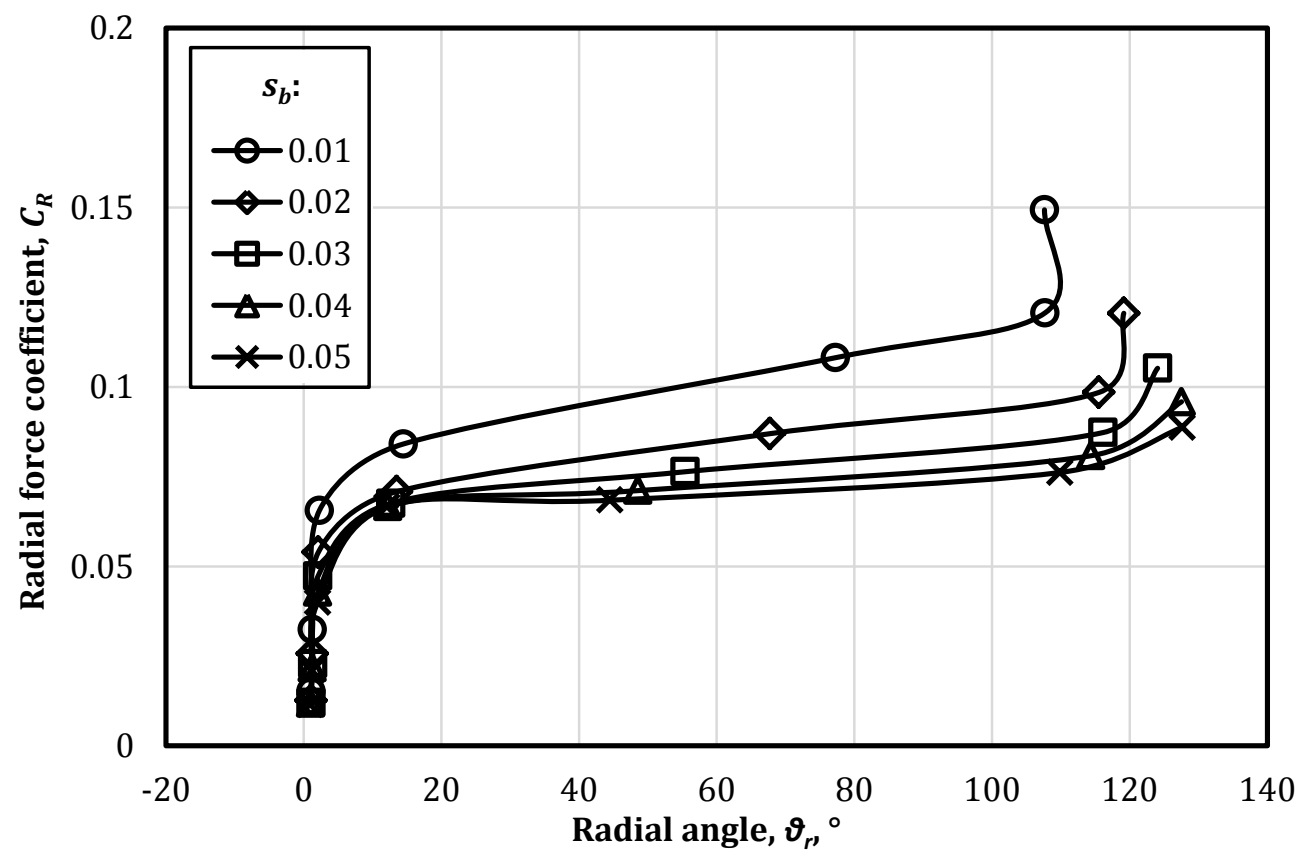

(d)

Figure 7.8: Blade radial force coefficients $\left(C_{R}\right)$ as a function of radial flow angle $\left(\theta_{r}\right)$ at spans of (c) $0.1 \leq s_{b} \leq 0.5$ and (d) $0<s_{b}<0.1$ 
The blade sectional drag coefficients are generally always higher than the NASA LS 0413 profile drag (figure 7.7). The closest comparison can be observed for $\alpha_{a t t}<5^{\circ}$. The largest discrepancy between these results occurs in the tip region similar to the lift characteristics. As the flow rate decreases through the fan (and consequently the angle of attack increases), a sudden deviation in drag coefficient is noted at $\alpha_{a t t} \approx 5^{\circ}$, where the gradient of $C_{D}$ gradually increases with an increase in angle of attack compared to the NASA LS 0413 profile drag. The highest drag occurs near the hub and decreases towards the tip, similar to the lift. In the hub region, the maximum drag coefficient obtained is $C_{D}=1.18$ for $\alpha_{\text {att }}=32^{\circ}$, which is of similar order across the largest span of the blade compared to the lift coefficient.

The radial force coefficients (figure 7.8), though small compared to the drag and lift coefficients, present a sharp increase for small radial angles (high flow rates), but gradually decrease in gradient at lower flow rates as the radial flow angle increases significantly through the fan. This coefficient is generally the highest in the hub region and decreases progressively towards the tip. A maximum radial flow coefficient of $C_{R}=0.15$ at a radial angle of $\theta_{r}=110^{\circ}$ can be observed at the hub, where the angle of $\theta_{r}=110^{\circ}$ indicates reverse flow through the fan.

\subsection{Time-dependent blade forces}

For low flow rates through the fan $(0.042 \leq \phi \leq 0.105)$, the angles of attack and blade force coefficients can also be visualized with reference to time. Hence, these spanwise characteristics are presented in figures 7.9-7.10 as a function of time and discussed.

The spanwise angle of attack (figures 7.9a, c and e) further confirms the unstable nature of the flow near the hub and its more stable attribute towards the tip. The flow also becomes more unstable with a decrease in flow rate, emphasized by the increase in valleys and troughs in these figures, implying an increase in chaotic behaviour of the flow especially near the hub where the variance in angle of attack for the lowest flow rate is in the order of $\Delta \alpha_{a t t} \approx 30^{\circ}$. The latter number indicates the significant variation in maldistributed flow that the blade near the hub is subjected to, since the stable operating region (before stall occurs) of the blade is within the boundaries of $-3^{\circ} \lesssim \alpha_{a t t} \lesssim 8^{\circ}$ (as indicated in figure 7.6).

The spanwise variations in lift, drag and radial force coefficients (indicated in figures $7.9 \mathrm{~b}, \mathrm{~d}$ and $\mathrm{f}, 7.10 \mathrm{a}, \mathrm{c}$ and e and $7.10 \mathrm{~b}, \mathrm{~d}$ and $\mathrm{f}$, respectively) are synchronized with the variation in angle of attack, i.e. the maximum values for $C_{L}, C_{D}$ and $C_{R}$ occur when the angle of attack is at a maximum for a specific blade section. $C_{L}$ is generally two orders of magnitude larger than $C_{D}$ and $C_{R}$ for the largest span of the blade with the exception of the $C_{D}$ values near the hub. The maximum variations in $C_{L}, C_{D}$ and $C_{R}$ once again occur in the 
hub region at the lowest flow rate $(\phi=0.042)$ and have values of $\Delta C_{L} \approx 2$, $\Delta C_{D} \approx 1.3$ and $\Delta C_{R} \approx 0.2$.

\subsection{Concluding remarks}

The present chapter focusses on the numerical calculation of the blade sectional lift and drag coefficients of the B2a-fan, where a blade section is essentially similar to the two-dimensional NASA LS 0413 aerofoil used in the initial design of the fan blade. These results are compared with the two-dimensional NASA LS 0413 aerofoil profile data obtained experimentally by McGhee and Beasley (1976). Additionally the radial force coefficients for these sections are also presented.

Similar comparisons pertaining to blade sectional lift and drag were first conducted experimentally by Himmelskamp (1947) in a two-bladed propeller. Himmelskamp (1947) found that blade sectional lift and drag characteristics differed from those obtained for the two-dimensional aerofoil used in the initial propeller design, where the lift at the hub was the highest and progressively became smaller for blade sections towards the tip, similar to the observations in the present study. However, the results of Himmelskamp (1947) show that the lift coefficients obtained were always higher, compared to the two-dimensional case, which is opposite to those calculated for the B2a-fan blade in the present study, where blade sectional lift coefficients are lower compared to the twodimensional aerofoil characteristics, with exceptions at certain blade spans and flow rates. Furthermore, Himmelskamp (1947) also observed a significant increase in blade sectional drag characteristics, similar to that of the present study.

The only other research the author could find on blade sectional lift and drag characteristics in turbo-machines was in the field of horizontal axis wind turbiness (HAWTs) as presented in the work of Tangler (2004) and Johansen and Sorensen (2004) and more recently Yu et al. (2011). Although the fundamental mechanism of HAWTs is opposite to that of a fan, these researchers also found similar trends compared to the present investigation, i.e. that the lift characteristics are generally the highest at the hub and decrease towards the tip. However, higher lift coefficients were also observed in these studies compared to the two-dimensional aerofoil characteristics used in their turbine designs, with some exceptions. Regarding characteristics in drag coefficients, the blade sectional drag coefficients are generally significantly higher compared to the two-dimensional case and increase with a decrease in flow rate through the turbines, similar to the results obtained in the present study.

Another observation of the trend in time-averaged blade sectional lift characteristics obtained in the present study suggests that the stall margins of these sections are extended, especially near the hub, since these lift coefficients reach a plateau for $5^{\circ} \lesssim \alpha_{a t t} \lesssim 20^{\circ}$ and then increase again for $\alpha_{a t t} \gtrsim 20^{\circ}$, whereas the NASA LS 0413 lift decreases for $\alpha_{a t t} \gtrsim 16^{\circ}$. The drag also increases con- 


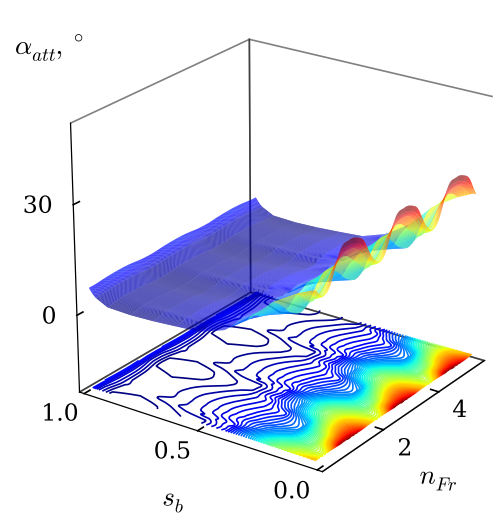

(a)

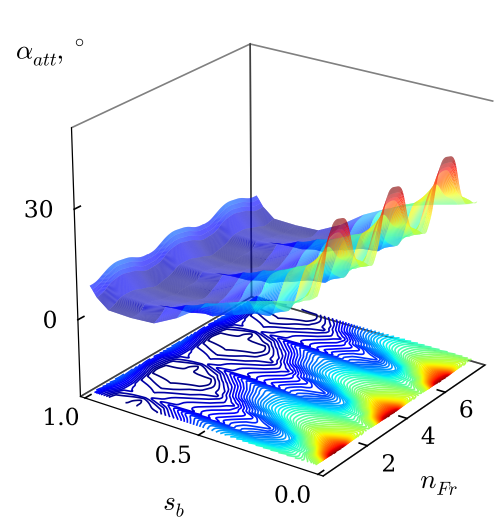

(c)

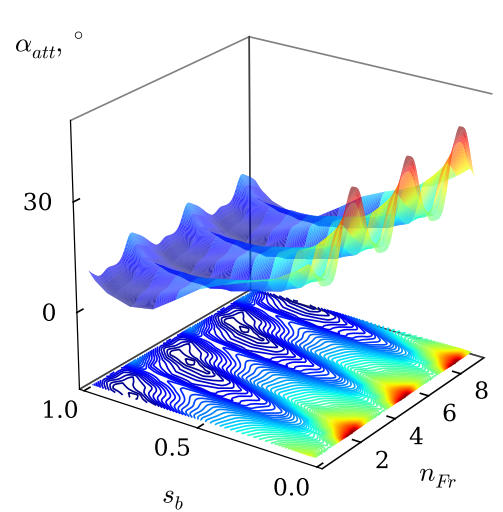

(e)
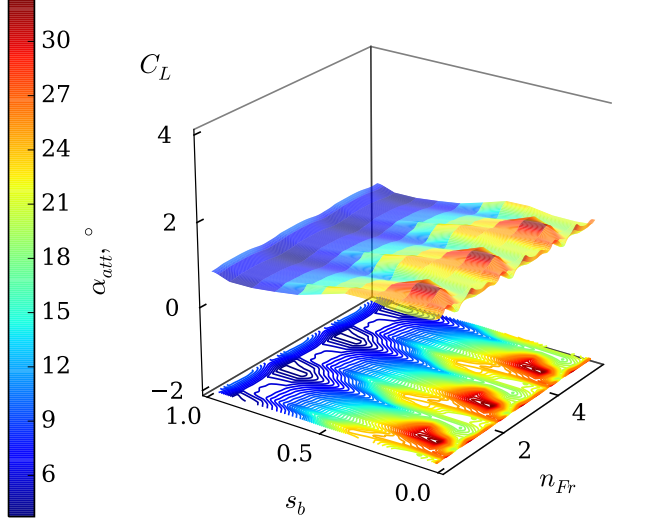

(b)
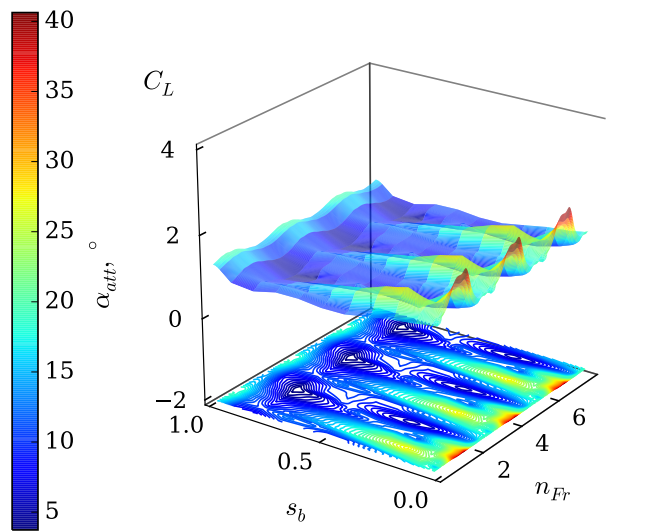

(d)
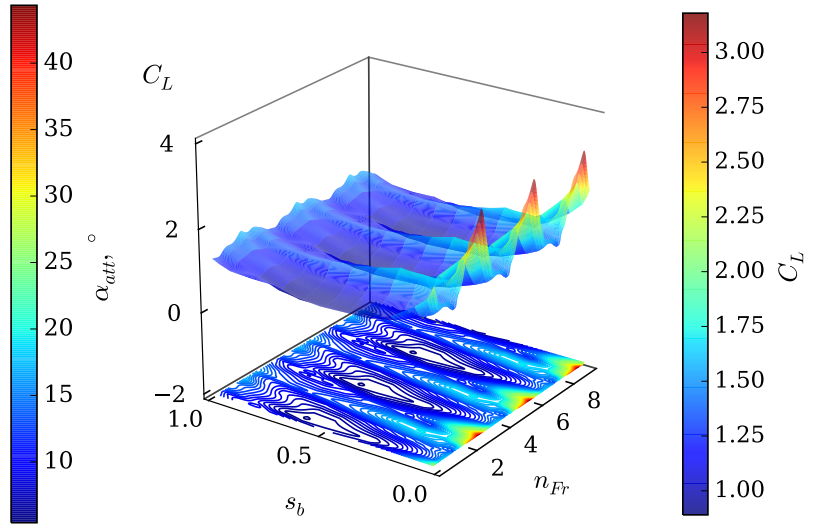

(f)

Figure 7.9: Time-dependent spanwise profiles of angle of attack $\left(\alpha_{a t t},{ }^{\circ}\right)$ and lift coefficient $\left(C_{L}\right)$ for flow coefficients of (a and b) $\phi=0.105$, (c and d) $\phi=0.074$ and (e and f) $\phi=0.042$ 


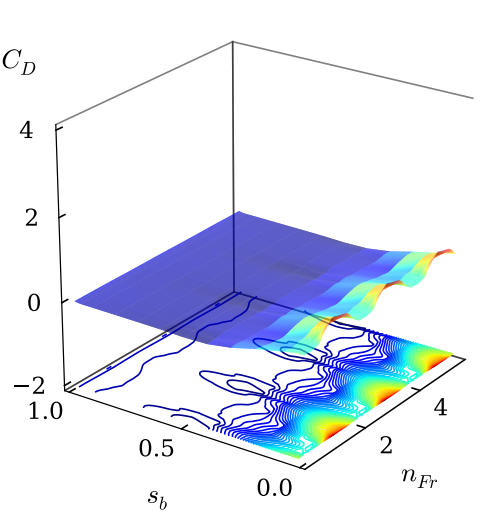

(a)

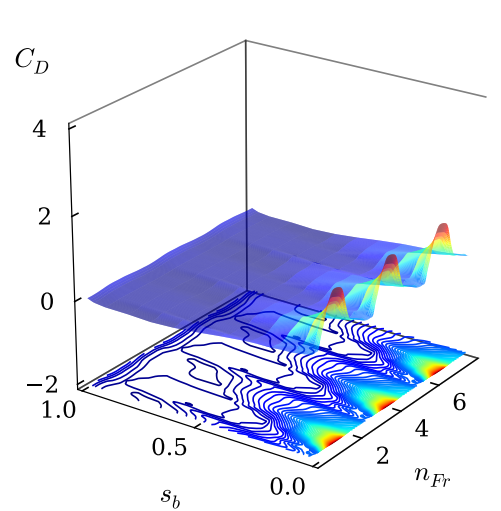

(c)

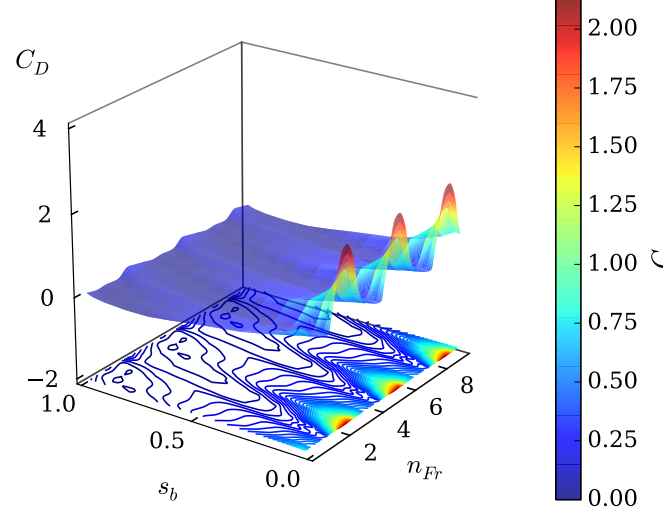

(e)

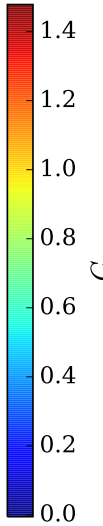

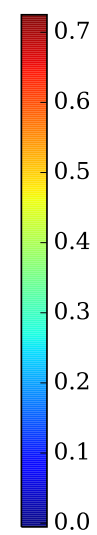
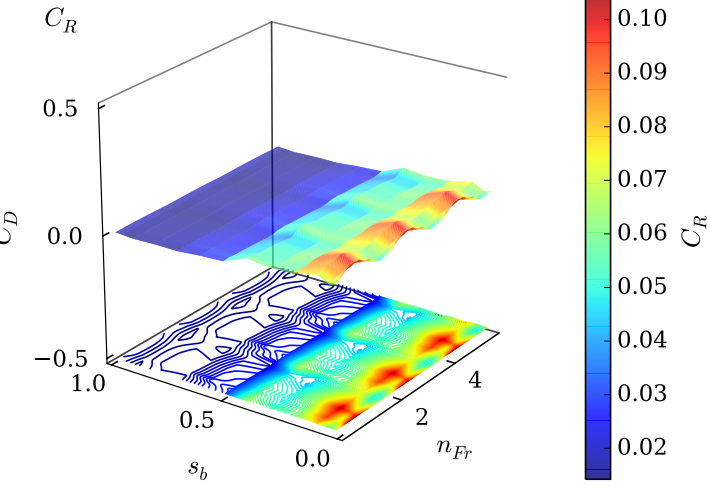

(b)

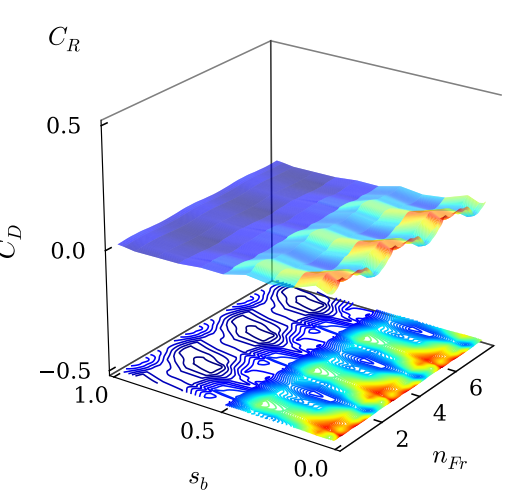

(d)

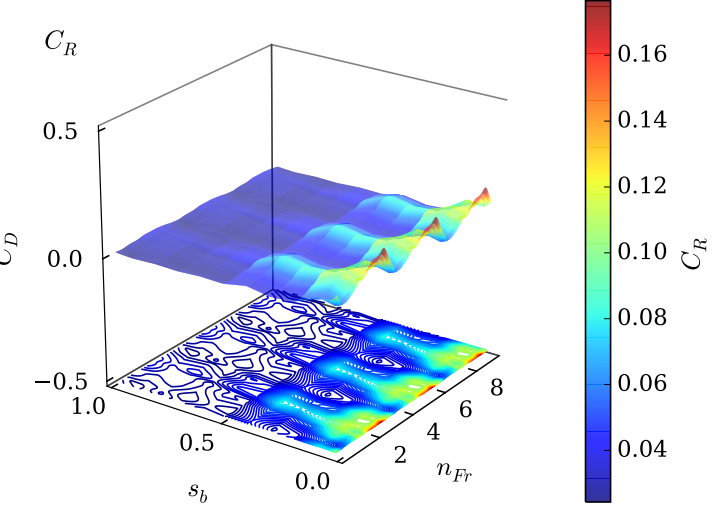

(f)

Figure 7.10: Time-dependent spanwise profiles of drag $\left(C_{D}\right)$ and radial force coefficients $\left(C_{R}\right)$ for flow coefficients of ( $\mathrm{a}$ and b) $\phi=0.105$, (c and d) $\phi=0.074$ and (e and f) $\phi=0.042$ 
siderably compared to the characteristic of the NASA LS 0413 aerofoil. The time-dependent lift and drag characteristics also suggest that the fan blade near the hub region operates within and out of stall in a periodic fashion, which is also confirmed by the two-dimensional streamline visualizations (figures 6.26 to 6.29) in chapter 6 .

Although interesting to observe, the presentation of the time-averaged blade sectional lift and drag characteristics is not truly comparable to the characteristics of the NASA LS 0413 aerofoil, since the flow across the fan blade is not purely two-dimensional, especially at low flow rates. Hence, the flow condition experienced by a blade section is different compared to a twodimensional case due to factors such as reversed flow near the hub, leakage flow near the tip and radial flow across the blade. However, the presentation of these results still show that the functioning of a blade section (essentially similar to a two-dimensional NASA LS 0413 aerofoil) as part of a fan blade is similar to the functioning of a purely two-dimensional aerofoil at near design flow rates, since a fair comparison between the blade sectional lift and drag characteristics and those of McGhee and Beasley (1976) is obtained for these conditions. However, deviation of the blade sectional lift and drag characteristics from those of the two-dimensional NASA LS 0413 aerofoil is observed for decreased flow rates through the fan. These blade sectional lift and drag characteristics are used in the development of a simplified empirical fan model similar to the actuator-disc model (ADM) as presented in chapter 9.

Lastly, the radial force coefficient, also presented in the present study is in the order of the drag coefficient at high flow rates and increases with a decrease in flow rate, but remains an order (or two orders in some instances) lower compared to the lift and drag coefficients. Similar to these coefficients the radial coefficients are the highest near the hub and decrease toward the tip. Since the radial coefficients and therefore the radial forces induced by the fan blade is small compared to its two other counterparts, the considerable increase in radial flow through the fan as the flow rate decreases, is therefore also attributable to other phenomena occurring in the flow field. These phenomena would include changes in the spanwise static pressure distribution in balance with the centrifugal and coriolis forces, drawing air from lower radii to higher radii. 


\section{Chapter 8}

\section{Euler power analysis}

Near its design condition the flow through the B2a-fan has a negligible radial component. However, as presented in chapter 6, an increase in radial flow through the fan can be observed with a decrease in flow rate. The result of this is that the functioning of the B2a-fan shifts from an effective axial-flow to an ineffective mixed-flow machine.

In an attempt to quantify the effect of this change in turbo-machine functioning, an analysis is conducted considering the Euler pump equation in terms of relative coordinates as presented in Lewis (1996). The Euler pump equation contains two types of contribution to the overall work imparted to the flow by the fan rotor, being the contributions due to aerodynamic and Coriolis forces in the fluid, respectively. For this analysis the circumferentially- and time-averaged flow field data are used. Hence, only the time-averaged flow condition is considered.

The Euler turbo-machinery equation is firstly presented from Lewis (1996) and the variables defined. This is followed by a description of the mean streamline calculation procedure, since they are necessary for the Euler power analysis that follows. Finally, these streamlines together with certain circumferentiallyand time-averaged flow variables extracted from the numerical results are used to calculate the two terms in the Euler turbo-machinery equation and these are subsequently compared to another. The chapter is concluded with a short discussion.

\subsection{Definition of variables}

The Euler work per unit mass $\left(W_{E u l, s}(r, \theta)\right)$ and power $\left(P_{E u l, s}(r, \theta)\right)$ exerted on the flow along a single stream tube $(s t)$ as shown in figure 8.1 are fundamentally defined as 


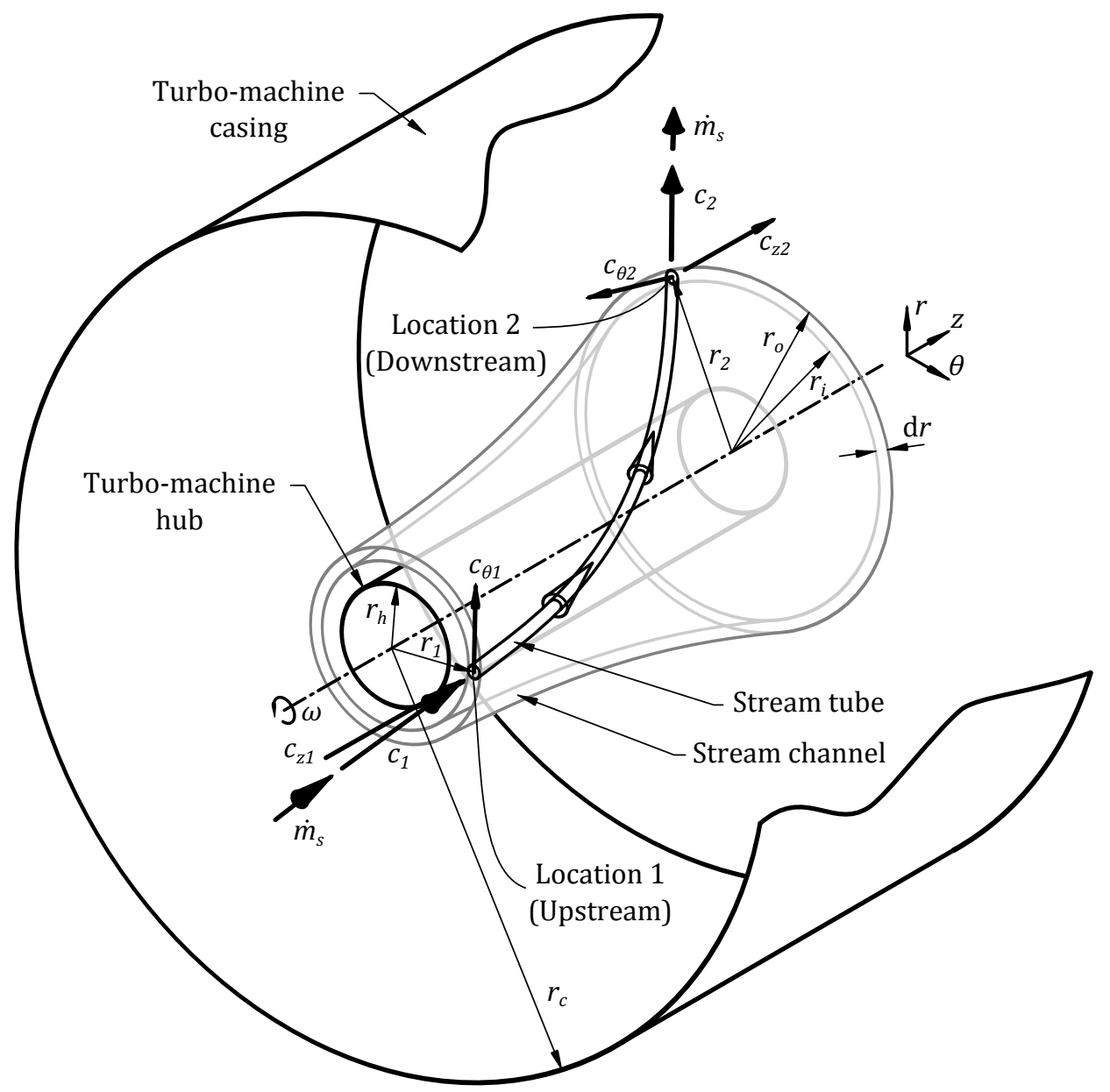

Figure 8.1: Single stream tube of fluid through a turbo-machine

$$
\begin{aligned}
W_{E u l, s t}(r, \theta) & =h_{t o 2, s t}(r, \theta)-h_{t o 1, s t}(r, \theta) \\
& =u_{2}(r) c_{\theta 2}(r, \theta)-u_{1}(r) c_{\theta 1}(r, \theta) \\
& =\omega\left[r_{2} c_{\theta 2}(r, \theta)-r_{1} c_{\theta 1}(r, \theta)\right] \quad[\mathrm{J} / \mathrm{kg}]
\end{aligned}
$$

and

$$
\begin{aligned}
P_{E u l, s t}(r, \theta) & =\dot{m}_{s t}(r, \theta) W_{\text {Euler }, s t}(r, \theta) \\
& =\dot{m}_{s t}(r, \theta) \omega\left[r_{2} c_{\theta 2}(r, \theta)-r_{1} c_{\theta 1}(r, \theta)\right] \quad[\mathrm{W}]
\end{aligned}
$$

These are functions of the radial $(r)$ and circumferential $(\theta)$ locations between the start (1) and end (2) of the stream tube considered. In equation 8.1.1 $h_{t o, s t}(r, \theta)$ is the total enthalpy at the specific upstream (1) or downstream (2) location and the velocity components $\left(c_{\theta}(r, \theta)\right)$ and radii $(r)$ are as indicated in figure 8.1. Furthermore $u(r)$ is the tangential blade speed. 
Since the circumferential average of all flow variables is used in the present Euler work analysis, annular stream channels ( $s$, figure 8.1 ) will be considered, rather than a collection of stream tubes originating from the same radius along the circumference. Subsequently, equations 8.1.1 and 8.1.2 become functions of radius alone as follows:

$$
\begin{aligned}
W_{E u l, s}(r) & =h_{t o 2, s}(r)-h_{t o 1, s}(r) \\
& =u_{2}(r) c_{\theta 2}(r)-u_{1}(r) c_{\theta 1}(r) \\
& =\omega\left[r_{2} c_{\theta 2}(r)-r_{1} c_{\theta 1}(r)\right] \quad[\mathrm{J} / \mathrm{kg}]
\end{aligned}
$$

and

$$
\begin{aligned}
P_{\text {Eul }, s}(r) & =\dot{m}_{s}(r) W_{\text {Euler }, s}(r) \\
& =\dot{m}_{s}(r) \omega\left[r_{2} c_{\theta 2}(r)-r_{1} c_{\theta 1}(r)\right]
\end{aligned}
$$

where the mass flow $\left(\dot{m}_{s}(r)\right)$ is calculated by:

$$
\dot{m}_{s}(r)=\rho c_{z}(r) 2 \pi r \mathrm{~d} r \quad[\mathrm{~kg} / \mathrm{s}]
$$

and $c_{z}(r)$ is the axial velocity at the applicable radius.

In equations 8.1.3 and 8.1.4 the absolute tangential velocity $\left(c_{\theta}(r)\right)$ can be written as

$$
c_{\theta}(r)=u-w_{\theta}(r)=\omega r-w_{\theta}(r) \quad[\mathrm{m} / \mathrm{s}]
$$

Substituting equation 8.1.6 into 8.1.3 and 8.1.4 and rearranging produces:

$$
\begin{aligned}
W_{\text {Eul,s }}(r) & =\omega\left[r_{2} w_{\theta 2}(r)-r_{1} w_{\theta 1}(r)\right]+\omega^{2}\left(r_{2}^{2}-r_{1}^{2}\right) \\
& =W_{E u l, s \text { Aero }}(r)+W_{\text {Eul }, s \text { Cor }}(r) \quad[\mathrm{J} / \mathrm{kg}]
\end{aligned}
$$

and

$$
\begin{aligned}
P_{\text {Eul }, s}(r) & =\dot{m}_{s}(r) \omega\left[r_{2} w_{\theta 2}(r)-r_{1} w_{\theta 1}(r)\right]+\dot{m}_{s}(r) \omega^{2}\left(r_{2}^{2}-r_{1}^{2}\right) \\
& =P_{\text {Eul }, s \text { Aero }}(r)+P_{\text {Eul }, s \text { Cor }}(r) \quad[\mathrm{W}]
\end{aligned}
$$

Considering equation 8.1.7, the first term $\left(W_{E u l, s \text { Aero }}(r)\right)$ represents the work performed on a stream channel by a turbo-machine purely due to aerodynamic forces. The second term $\left(W_{E u l, s \text { Cor }}(r)\right)$ is the component of work contributed by Coriolis forces as detailed in Lewis (1996). For axial flow turbomachines, where the radial flow component is zero or negligibly small through 
the rotor (and therefore the fluid experiences no change in radius), the Coriolis term becomes zero, since $r_{1}=r_{2}$. In this case, all the work input would be a function of aerodynamic forces alone. However, when flow contains a radial component, as is the case with low flow rates in the present study, the Coriolis term's contribution to the overall work performed becomes significant, as shown in the sections that follow.

The Euler work per unit mass $\left(W_{E u l}\right)$ and total power $\left(P_{E u l}\right)$ applied to the flow by the turbo-machine can be calculated by integrating across the span between the hub and casing radii $\left(r_{h}\right.$ and $\left.r_{c}\right)$ :

$$
\begin{aligned}
W_{E u l} & =\frac{\int_{r_{h}}^{r_{c}} 2 \pi r W_{E u l, s}(r) \mathrm{d} r}{\int_{r_{h}}^{r_{c}} 2 \pi r \mathrm{~d} r} \quad[\mathrm{~J} / \mathrm{kg}] \\
P_{E u l} & =\int_{r_{h}}^{r_{c}} P_{E u l, s}(r) \quad[\mathrm{W}]
\end{aligned}
$$

The total pressure difference $\left(\Delta p_{t o, s}(r)\right)$ resulting from the work applied along a stream channel can also be calculated by manipulation of equation 8.1.3:

$$
\begin{aligned}
h_{t o 2, s}(r)-h_{t o 1, s}(r) & =W_{E u l, s}(r) \\
p_{t o 2, s}(r)-p_{t o 1, s}(r) & =\rho W_{E u l, s}(r) \\
\Delta p_{t o, s}(r) & =\rho W_{E u l, s}(r) \quad[\mathrm{Pa}]
\end{aligned}
$$

where $\rho$ is the density of the fluid passing through the fan.

The total pressure $\left(p_{t o, s}(r)\right)$ can also be written as the sum of the static and dynamic components:

$$
\begin{aligned}
p_{t o, s}(r) & =p_{s, s}(r)+p_{d, s}(r) \\
& =p_{s, s}(r)+\frac{1}{2} \rho c(r)^{2} \quad[\mathrm{~Pa}]
\end{aligned}
$$

where $c$ is the absolute velocity at the location under consideration. By substitution of equation 8.1.12 into 8.1.11 the total-to-static pressure difference $\left(\Delta p_{t s, s}(r)\right)$ along a stream channel can be calculated by:

$$
\begin{aligned}
{\left[p_{s 2, s}(r)+\frac{1}{2} \rho c_{2}(r)^{2}\right]-p_{t o 1, s}(r) } & =\rho W_{E u l, s}(r) \\
p_{s 2, s}(r)-p_{t o 1, s}(r) & =\rho W_{E u l, s}(r)-\frac{1}{2} \rho c_{2}(r)^{2} \\
\Delta p_{t s, s}(r) & =\rho W_{E u l, s}(r)-\frac{1}{2} \rho c_{2}(r)^{2}
\end{aligned}
$$


The mass-weighted average of the total-to-total and total-to-static pressure differences $\left(\Delta p_{F t o}\right.$ and $\Delta p_{F s}$, respectively) across the fan is calculated by:

$$
\begin{aligned}
\Delta p_{F t o} & =p_{t o 2}-p_{t o 1} \\
& =\frac{\int_{r_{h}}^{r_{c}} \dot{m}_{s}(r)\left(p_{t o 2, s}(r)-p_{t o 1, s}(r)\right)}{\dot{m}_{t o t}} \quad[\mathrm{~Pa}]
\end{aligned}
$$

and

$$
\begin{aligned}
\Delta p_{F s} & =p_{s 2}-p_{t o 1} \\
& =\frac{\int_{r_{h}}^{r_{c}} \dot{m}_{s}(r)\left(p_{s 2, s}(r)-p_{t o 1, s}(r)\right)}{\dot{m}_{t o t}} \quad[\mathrm{~Pa}]
\end{aligned}
$$

where the total mass flow $\left(\dot{m}_{t o t}\right)$ is calculated by:

$$
\dot{m}_{t o t}=\int_{r_{h}}^{r_{c}} \dot{m}_{s}(r) \quad[\mathrm{kg} / \mathrm{s}]
$$

Since the numerically obtained upstream and downstream flow field values are used in the present Euler analysis, the given integral equations are solved numerically. Thus, equations 8.1.3, 8.1.4 8.1.14 and 8.1.15 are discretized as follow:

$$
\begin{aligned}
W_{\text {Eul }} & =\sum_{s=1}^{n_{s}} \frac{W_{E u l, s}}{n_{s}} \\
& =\sum_{s=1}^{n_{s}} \frac{\omega\left(r_{2} w_{\theta 2, s}-r_{1} w_{\theta 1, s}\right)}{n_{s}}+\sum_{s=1}^{n_{s}} \frac{\omega^{2}\left(r_{2}^{2}-r_{1}^{2}\right)}{n_{s}} \\
& =\sum_{s=1}^{n_{s}} \frac{W_{E u l, s \text { Aero }}}{n_{s}}+\sum_{s=1}^{n_{s}} \frac{W_{E u l, s \text { Cor }}}{n_{s}}[\mathrm{~J} / \mathrm{kg}] \\
P_{E u l} & =\sum_{s=1}^{n_{s}} P_{E u l, s}+\sum_{s=1}^{n_{s}} \dot{m}_{s} \omega^{2}\left(r_{2}^{2}-r_{1}^{2}\right) \\
& =\sum_{s=1}^{n_{s}} \dot{m}_{s} \omega\left(r_{2} w_{\theta 2, s}-r_{1} w_{\theta 1, s}\right)+[\mathrm{W}] \\
& =\sum_{s=1}^{n_{s}} P_{E u l, s \text { Aero }}+\sum_{s=1}^{n_{s}} P_{E u l, s} \text { Cor }
\end{aligned}
$$




$$
\begin{aligned}
\Delta p_{\text {Fto }} & =p_{t o 2}-p_{t o 1} \\
& =\sum_{s=1}^{n_{s}} \frac{\dot{m}_{s}}{\dot{m}_{t o t}}\left(p_{t o 2, s}-p_{t o 1, s}\right) \quad[\mathrm{Pa}]
\end{aligned}
$$

and

$$
\begin{aligned}
\Delta p_{F s} & =p_{s 2}-p_{t o 1} \\
& =\sum_{s=1}^{n_{s}} \frac{\dot{m}_{s}}{\dot{m}_{t o t}}\left(p_{s 2, s}-p_{t o 1, s}\right) \quad[\mathrm{Pa}]
\end{aligned}
$$

where $n_{s}$ is the number of stream channels considered through the fan and $w_{\theta}$ the relative circumferential velocity. Values for $w_{\theta}$ are obtained from the numerical data. The radii $(r)$ in these equations are obtained by conducting a mass flow balance between the up- and downstream locations of the fan rotor, a procedure which is discussed in the following section. The incremental and total mass flows $\left(\dot{m}_{s}\right.$ and $\left.\dot{m}_{t o t}\right)$ are calculated by:

$$
\begin{aligned}
& \dot{m}_{s}=\sum_{s=1}^{n_{s}} \rho c_{z, s} \pi\left(r_{o}^{2}-r_{i}^{2}\right) \quad[\mathrm{kg} / \mathrm{s}] \\
& \dot{m}_{t o t}=\sum_{s=1}^{n_{s}} \dot{m}_{s} \quad[\mathrm{~kg} / \mathrm{s}]
\end{aligned}
$$

The Euler work per unit mass $\left(W_{E u l}\right)$, Euler power $\left(P_{E u l}\right)$ and pressure $(p)$ already described are made dimensionless for the purpose of presentation. A dimensionless pressure coefficient $(\Psi)$ is already defined in chapter 5 by equation 5.1.1. The dimensionless work per unit mass $(\gamma)$ and power $(\Gamma)$ are defined as:

$$
\begin{aligned}
\gamma & =\frac{W_{E u l}}{u_{c}^{2} / 2} \\
& =\frac{P_{E u l}}{\dot{m}_{\text {tot }}^{*} u_{c}^{2} / 2}
\end{aligned}
$$

and

$$
\Gamma=\frac{P_{E u l}}{\dot{m}_{t o t, D} u_{c}^{2} / 2}
$$


where $\dot{m}_{t o t, D}$ is the design mass flow rate through the fan.

As can be observed in equation 8.1.23 the value of $\gamma$ is, amongst other, a function of the mass flow $\dot{m}_{\text {tot }}^{*}$. This is significant, since the dimensionless work per unit mass $(\gamma)$, could be based on either the total amount of mass flow actuated by the fan $\left(\dot{m}_{t o t}\right)$ or the effective amount of mass flow through the fan $\left(\dot{m}_{t o t, e f f}\right)$. For this purpose, let:

$$
\begin{gathered}
\gamma_{F, \text { true }}=\frac{P_{E u l}}{\dot{m}_{t o t} u_{c}^{2} / 2} \\
\gamma_{F, \text { eff }}=\frac{P_{E u l}}{\dot{m}_{\text {tot }, \text { eff }} u_{c}^{2} / 2}
\end{gathered}
$$

The difference between the effective and total mass flow rate $\left(\dot{m}_{t o t}\right.$ and $\left.\dot{m}_{t o t, e f f}\right)$ is as follows. The effective mass flow rate is the mass flow that moves from the upstream location of the fan through to the downstream location, whereas the total mass flow rate is the effective mass flow through the fan plus any additional recirculating mass flow in the vicinity of the fan. Hence, the effective and total mass flow rates would be equal, should no recirculation patterns occur at the up- and/or downstream boundaries defined in figure 8.1, similar to the flow indicated in figure 8.2a. In the present investigation this is true for flow coefficients of $\phi>0.168\left(\dot{V}>16 \mathrm{~m}^{3} / \mathrm{s}\right)$. However, as indicated in section 8.2, recirculation patterns occur in the vicinity of the hub and tip regions of the fan (figure 8.2b), for flow rates below the design point $(\phi<$ $\left.0.168\left(\dot{V}<16 \mathrm{~m}^{3} / \mathrm{s}\right)\right)$. Mathematically this is given as:

$$
\begin{aligned}
\dot{m}_{t o t} & =\sum_{s=1}^{n_{s}} \dot{m}_{s} \\
& =\dot{m}_{t o t, \text { eff }}+\dot{m}_{t o t, r e c} \quad[\mathrm{~kg} / \mathrm{s}]
\end{aligned}
$$

where

$$
\dot{m}_{\text {tot }, \text { rec }} \begin{cases}=0, & \phi>0.168 \\ >0, & \phi<0.168\end{cases}
$$

and $\dot{m}_{t o t, r e c}$ the amount of mass flow kept in recirculation in the vicinity of the fan hub and tip.

\subsection{Mean streamline calculation}

In order to calculate the mean meridional streamlines through the fan, a mass balance is conducted between the up- and downstream sides of the fan rotor, 
using the time and circumferentially averaged axial velocity profiles obtained through the numerical analyses. This gives the meridional streamlines that would occur through the fan on average over time for a specific flow rate. The secondary flow patterns occurring in the fan are therefore not regarded. This calculation procedure is discussed with reference to the sectional view of the fan rotor in figure 8.2.

Three zones in the fan rotor are considered individually and termed the Hub, Tip and Main zones. For near design flow rates, consideration of only the Main zone is sufficient, since no or small recirculation zones are present in the hub and tip regions of the fan. However, as the flow rate through the fan decreases, the meridional streamlines due to recirculation are incorporated by first considering the $H u b$ and Tip zones individually, before calculating the streamlines through the Main zone.

When only the Main zone is considered (near-design flow rates) the mass balance calculation commences by first seeding the upstream side (location 1 , figure $8.2 \mathrm{a}$ ) of the fan rotor with fifteen locations in the radial direction with equal radial increments. These locations serve as starting points for each stream channel. The mass flow through each of the seeded locations is then calculated by equation 8.1.21 using the time and circumferentially averaged upstream axial velocity $\left(c_{z 1}(r)\right)$ profile as input. Next, starting from the fan casing towards the hub, the corresponding flow areas on the downstream side (location 2, figure 8.2a) of the fan rotor are calculated, this time utilizing the downstream average axial velocity profile $\left(c_{z 2}(r)\right)$. This procedure ultimately provides the downstream radial locations for each of the stream channels seeded from the upstream side of the rotor.

For lower flow rates through the fan, the streamlines through the $H u b$ and Tip zones are firstly calculated to obtain the boundaries of the Main zone in addition to the mean streamlines of these zones. Hereafter the mean streamlines through the Main zone are calculated in the same manner as described in the previous paragraph. For the Hub and Tip zones, the up- and downstream radial locations where the axial velocity is zero (termed the zero mark, figure $8.2 \mathrm{~b}$ ) are identified. This would typically be the location where the axial velocity changes direction and hence flow recirculation occurs. Considering the $H u b$ zone, the region between the fan hub and zero mark is seeded with five points. Hereafter the mass flow through these points (figure 8.2b, location 2) are calculated as described previously and the corresponding radial locations between the zero mark and the fan casing calculated, assuming that the recirculation pattern is similar to that indicated in figure 8.2b. A similar approach is followed to obtain the streamlines for the Tip zone.

\subsection{Results}

The mean meridional streamlines calculated using the mass balance procedure described above are graphically depicted in figure 8.3a-f for flow coefficients 


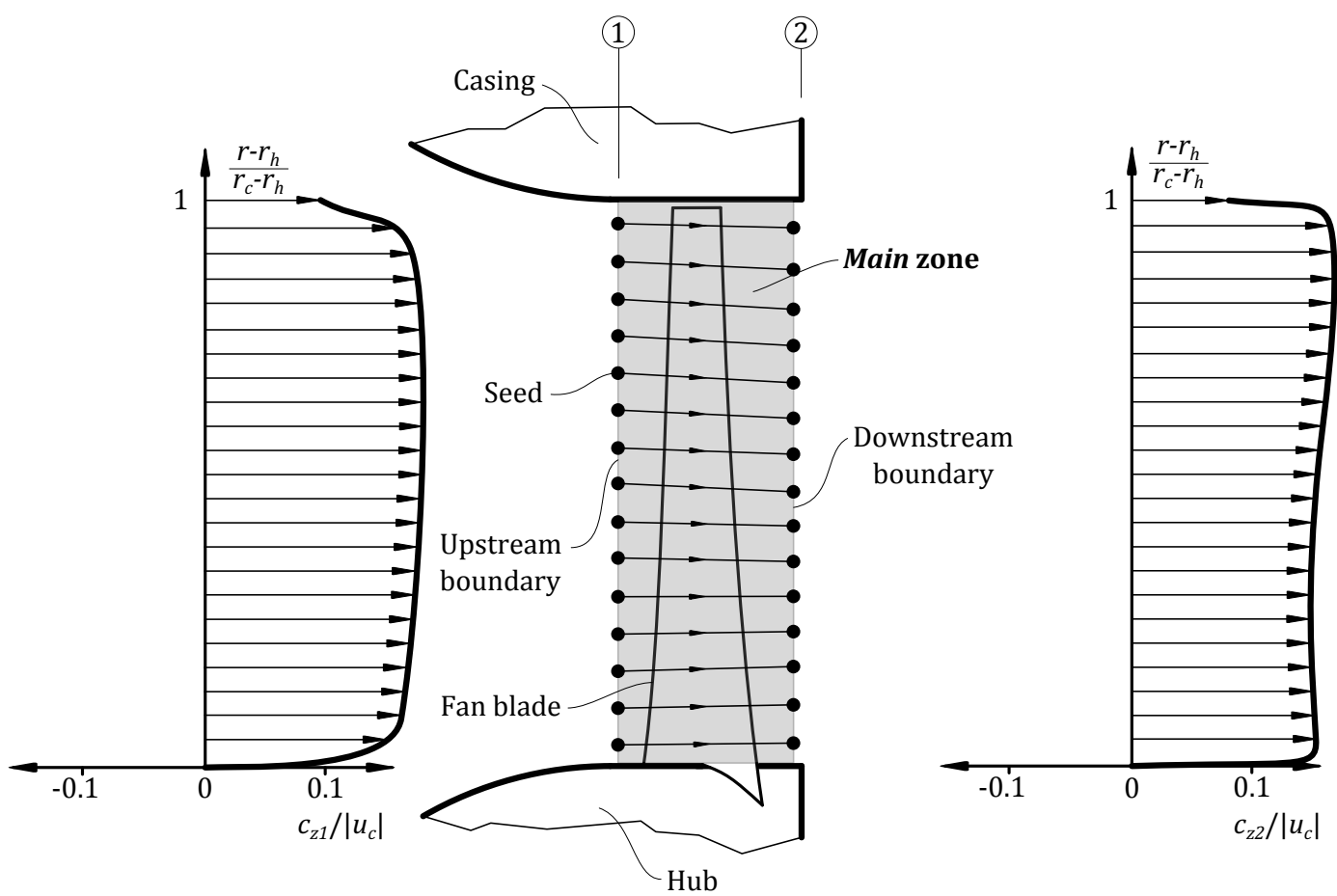

(a)

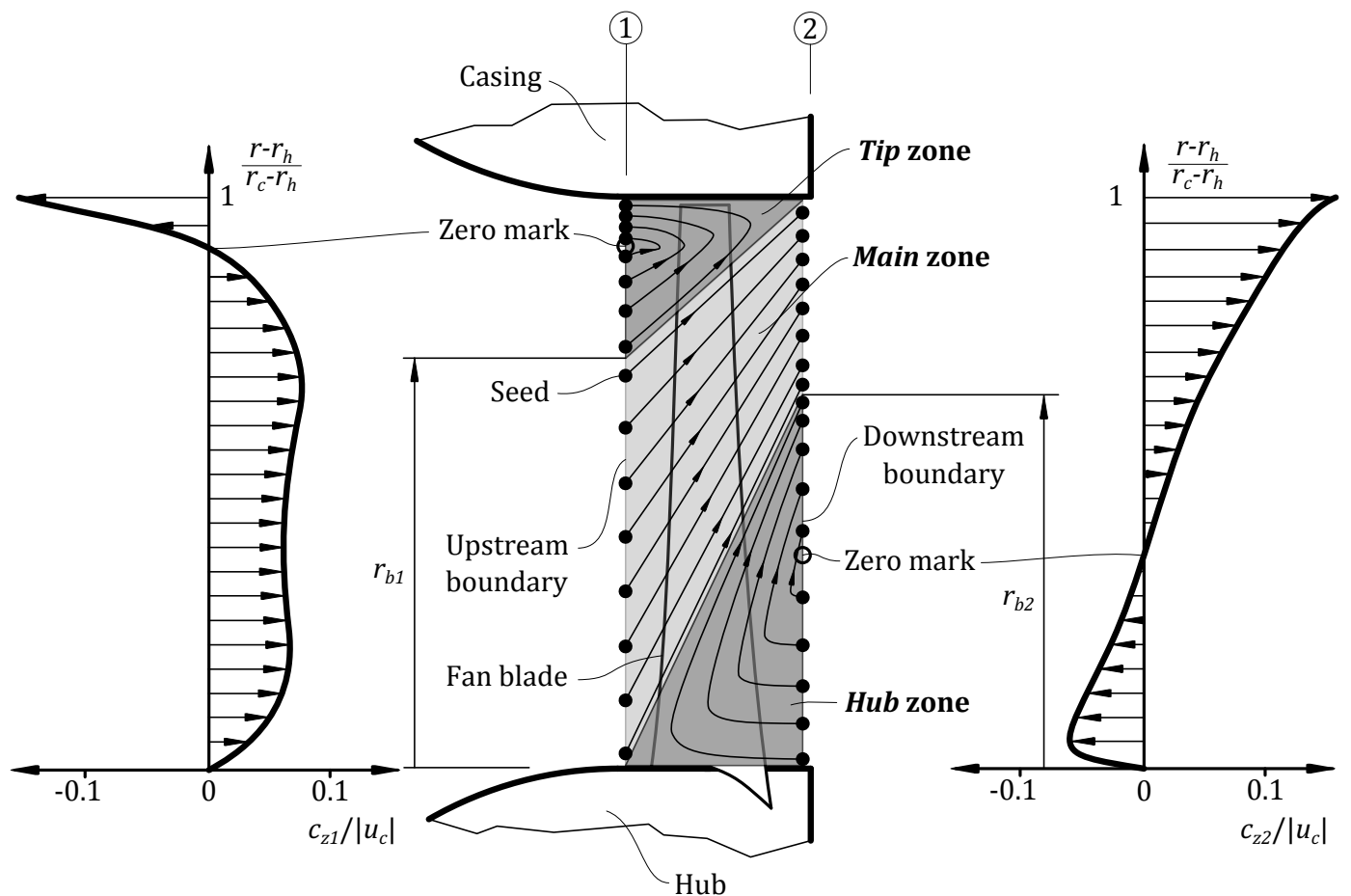

(b)

Figure 8.2: Sectional view of the B2a-fan depicting some terminology and the mean streamlines through the fan for flow coefficients of $\phi=$ (a) $0.168\left(16 \mathrm{~m}^{3} / \mathrm{s}\right)$ and (b) $0.042\left(4 \mathrm{~m}^{3} / \mathrm{s}\right)$ 


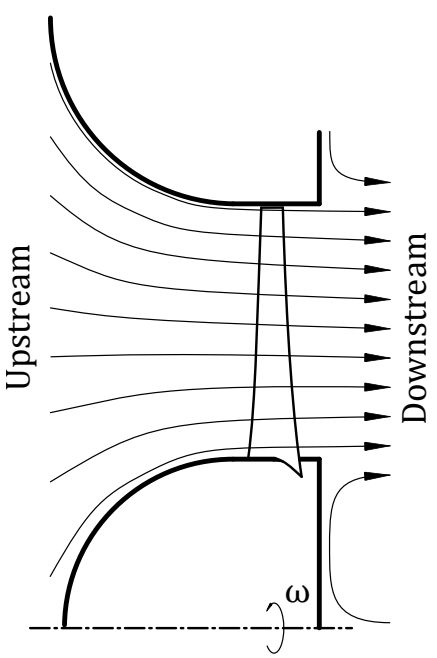

(a)

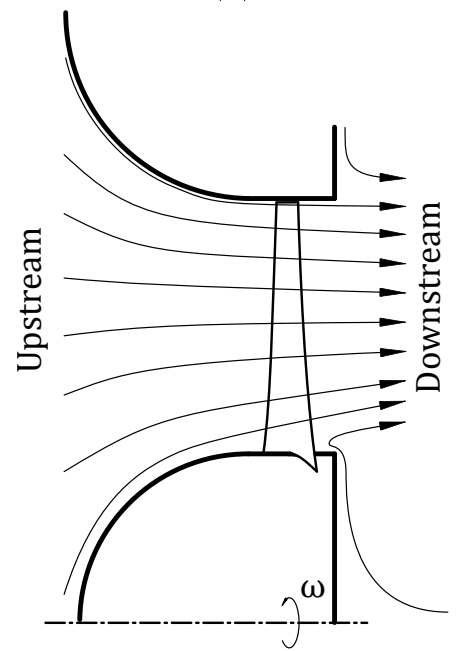

(c)

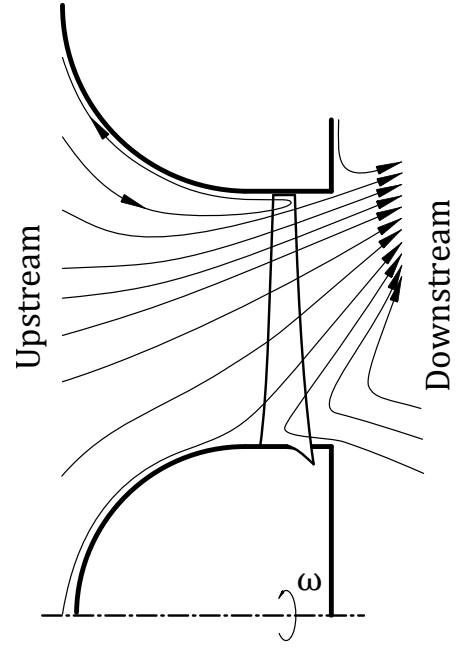

(e)

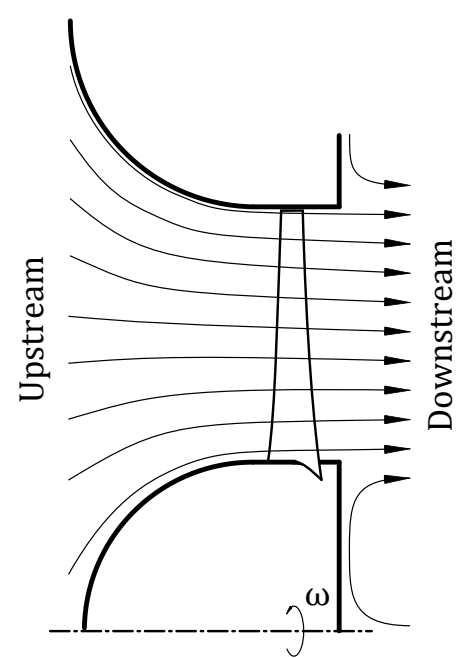

(b)

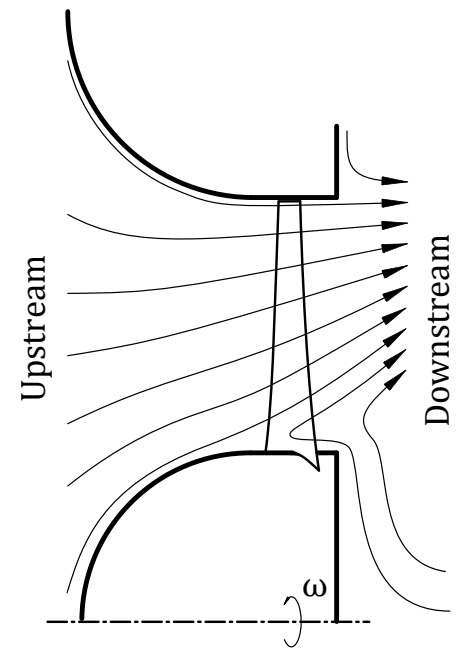

(d)

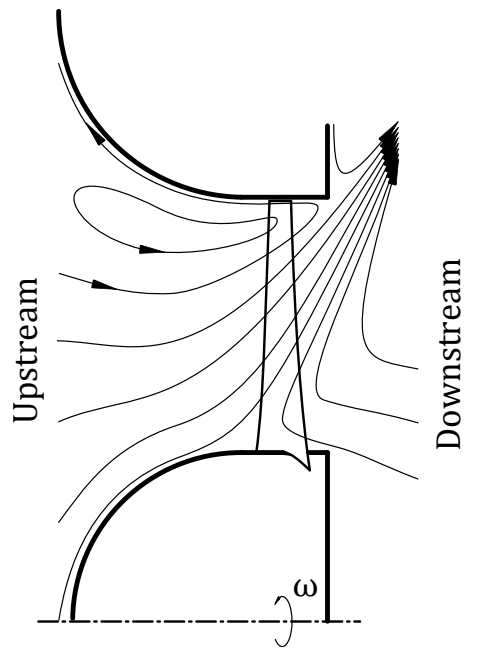

(f)

Figure 8.3: Depiction of the circumferentially and time-averaged streamlines through the B2a-fan for flow coefficients of $\phi=$ (a) 0.200, (b) 0.168, (c) 0.137, (d) 0.105 , (e) 0.074 and (f) 0.042 
of $0.042 \leq \phi \leq<0.200\left(4 \leq \dot{V} \leq 19 \mathrm{~m}^{3} / \mathrm{s}\right)$. Apparent in these figures is the significant increase in radial outflow with a decrease in flow rate through the fan. The occurrence of reversed flow patterns near the hub and tip can also be observed. Reversed flow on the downstream side of the fan near the hub starts occurring if the flow is decreased below $\phi<0.168\left(\dot{V}<16 \mathrm{~m}^{3} / \mathrm{s}\right)$. A second reversed flow pattern appears on the upstream side of the fan in the tip region along with the reversed flow near the hub on the downstream side if the flow rate is further decreased below $\phi<0.105\left(\dot{V}<10 \mathrm{~m}^{3} / \mathrm{s}\right)$.

Considering equation 8.1.18, that presents the Euler power exerted by the fan as a function of the individual aerodynamic and Coriolis components, the significant changes in radial outflow through the fan with reduced flow rate would inevitably have an effect on the relationship between these two terms. Hence, the Euler power is shown in figure 8.4 in dimensionless form as a function of flow coefficient.

For high and near-design flow rates through the fan, it is evident that the fan power is almost equal to the aerodynamic power component, whereas the Coriolis power component is negative and close to zero. This is due to the small decrease in radius of the majority of streamlines between the upstream and downstream sides of the fan, upon which the Coriolis component is solely dependent(refer equation 8.1.18). As the flow through the fan is decreased, the aerodynamic component decreases, whereas the Coriolis component increases to where these intersect at $\phi \approx 0.105\left(\dot{V} \approx 10 \mathrm{~m}^{3} / \mathrm{s}\right)$. From here the aerodynamic component decreases to a negative value for $\phi \lesssim 0.084\left(\dot{V} \lesssim 8 \mathrm{~m}^{3} / \mathrm{s}\right)$. A similar trend for the aerodynamic and Coriolis static pressure components can also be observed in figure 8.4.

Since the CFD data is used to conduct the Euler power analysis, it is appropriate to compare these results with each other (figure 8.4), although the experimental results are also indicated for completeness. A fair correlation exists between these results for higher flow rates $\phi>0.137\left(\dot{V}>13 \mathrm{~m}^{3} / \mathrm{s}\right)$, but the correlation becomes poor at low flow rates. This is due to the initial assumption that streamlines through the fan could be solved by a simple two-dimensional time-averaged mass balance between the up- and downstream locations of the fan. An additional error might be attributed to the assumptions regarding the shape of the hub and tip reversed flow patterns. In reality, the time dependent nature of the flow patterns in the vicinity of the fan blade as well as the vortical patterns in the blade passages underline the complexity of the flow field. The collection of pathlines through the fan in these instances would ultimately present different and more realistic values for the Euler power equation than presently obtained with the more simple two-dimensional approach. However, the two-dimensional approach in the present investigation still presents a fair approximation to the general trend that would occur.

The fan work per unit mass is displayed in figures 8.5 and 8.6. The two variables displayed are the true and effective work per unit mass $\left(\gamma_{F, \text { true }}\right.$ and $\left.\gamma_{F, \text { eff }}\right)$ as described by equations 8.1.25 and 8.1.26, respectively. A straight 


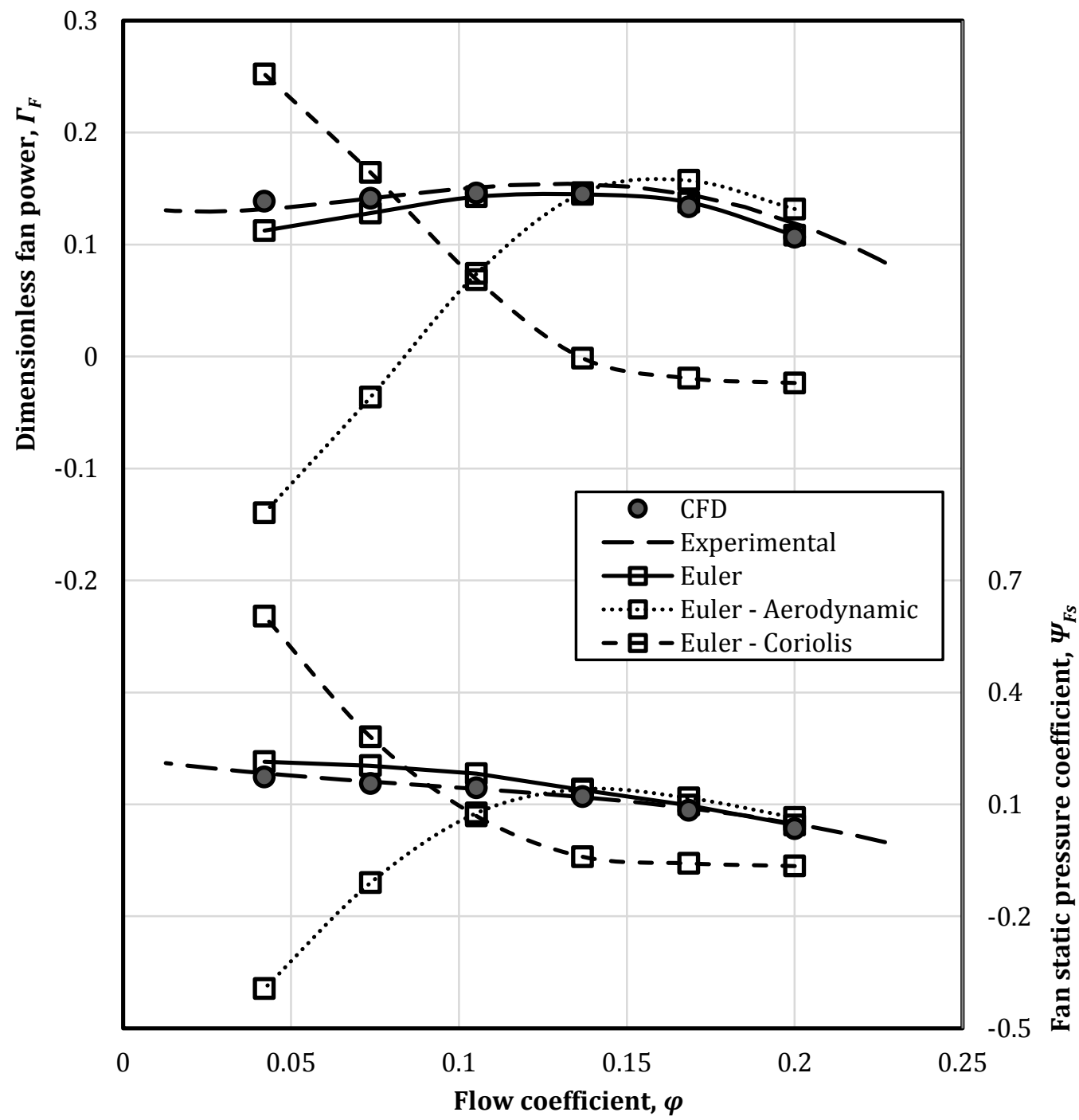

Figure 8.4: Comparison of dimensionless fan power $\left(\Gamma_{F}\right)$ and static pressure difference $\left(\Psi_{F s}\right)$ obtained through experimental analyses, CFD and Euler power calculations.

line can be observed for $\gamma_{F, \text { eff }}$ at near design flow conditions. However, with a further decrease in flow rate the effective energy per unit mass increases exponentially. Contrarily the true energy input per unit mass $\gamma_{F, \text { true }}$ is near linear for the range of flow rates examined in this study.

\subsection{Concluding remarks}

From the results presented in this chapter a fair comparison can be observed between the Euler and CFD calculated fan power. However, since the Euler pump equation is fundamentally only applicable to inviscid flow, this might be one of the reasons for the discrepancy in the compared results, especially at low 


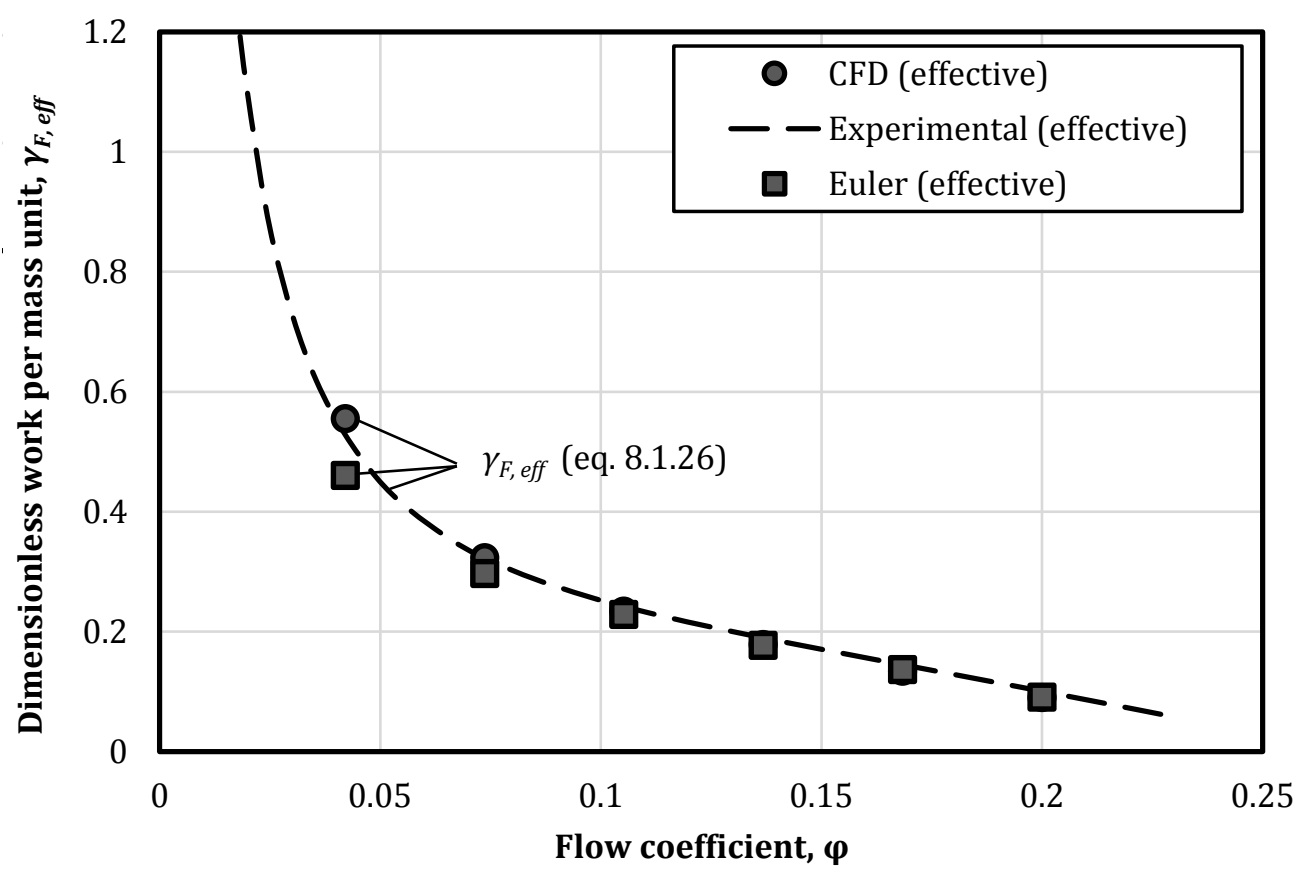

Figure 8.5: Comparison of dimensionless effective fan work per unit mass $\left(\gamma_{F, \text { eff }}\right)$ obtained through experimental analyses, CFD and Euler power calculations.

flow rates. A model to include the effects of viscosity should be incorporated in future research.

It is evident that aerodynamic forces are the main contributors to the functioning of the axial B2a-fan at near design flow rates, but they reduce significantly with a decrease in flow rate. The Coriolis force contribution is small at near-design flow rates, since little flow is displaced in the radial direction during these conditions. Yet, the Coriolis component becomes the more significant contributor as the flow rate through the fan decreases, whereas the aerodynamic component becomes negative for very low flow rates. The latter could imply that part of the fan functions as a turbine aerodynamically, but its contribution is significantly smaller than the positive Coriolis component.

With regards to the energy per unit mass that the fan exerts on the air, the linear trend in $\gamma_{F \text {, true }}$ is initially interesting and the reason not fully understood. From the Euler turbo equation (equation 8.1.7) and the velocity diagrams (figure 6.1) it can be shown that the theoretical Euler energy for a single stream channel is linear, with certain assumptions as described below:

The Euler turbo equation is once again given as:

$$
W_{E u l, s}=\omega\left(r_{2} w_{\theta 2}-r_{1} w_{\theta 1}\right)+\omega^{2}\left(r_{2}^{2}-r_{1}^{2}\right) \quad[\mathrm{J} / \mathrm{kg}]
$$

However the relative tangential velocity can also be given by: 


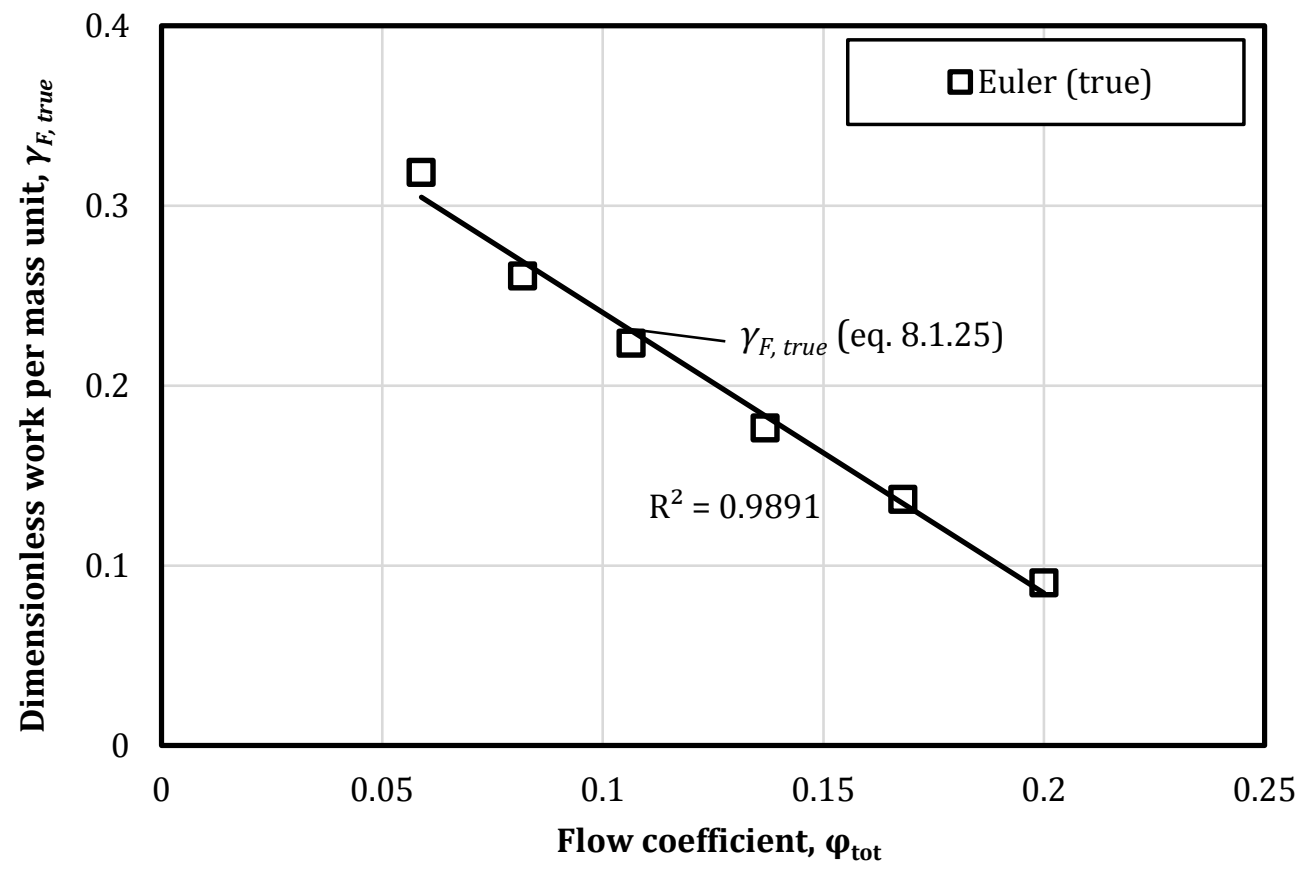

Figure 8.6: Comparison of dimensionless true fan work per unit mass $\left(\gamma_{F}\right.$, true $)$ obtained through experimental analyses, CFD and Euler power calculations.

$$
w_{\theta}=c_{z} \tan \beta \quad[\mathrm{m} / \mathrm{s}]
$$

Substituting 8.4.2 into 8.4.1 gives:

$$
W_{E u l, s}=\omega\left(r_{2} c_{z 2} \tan \beta_{2}-r_{1} c_{z 1} \tan \beta_{1}\right)+\omega^{2}\left(r_{2}^{2}-r_{1}^{2}\right) \quad[\mathrm{J} / \mathrm{kg}]
$$

The axial velocity $\left(c_{\theta}\right)$ can also be written as:

$$
c_{z}=\dot{V}_{s} / A_{s}
$$

where $\dot{V}_{s}$ is the volumetric flow rate and $A_{s}$ is the annular through flow area of the stream channel considered. Substituting 8.4.4 into 8.4.3 gives:

$$
\begin{aligned}
W_{E u l, s} & =\omega\left(r_{2} \frac{\dot{V}_{s}}{A_{s 2}} \tan \beta_{2}-r_{1} \frac{\dot{V}_{s}}{A_{s 1}} \tan \beta_{1}\right)+\omega^{2}\left(r_{2}^{2}-r_{1}^{2}\right) \\
& =\omega\left(\frac{r_{2} \tan \beta_{2}}{A_{s 2}}-\frac{r_{1} \tan \beta_{1}}{A_{s 1}}\right) \dot{V}_{s}+\omega^{2}\left(r_{2}^{2}-r_{1}^{2}\right) \quad[\mathrm{J} / \mathrm{kg}]
\end{aligned}
$$

If it is assumed that the relative angles $\left(\beta_{1}\right.$ and $\left.\beta_{2}\right)$, the areas $\left(A_{s 1}\right.$ and $\left.A_{s 2}\right)$ and the radii $\left(r_{1}\right.$ and $\left.r_{2}\right)$ remain constant with variable volumetric flow rate, then a linear relationship can be observed for the Euler turbo equation (8.4.5): 


$$
W_{E u l, s}=C_{1}+C_{2} \dot{V} \quad[\mathrm{~J} / \mathrm{kg}]
$$

where

$$
\begin{aligned}
& C_{1}=\omega^{2}\left(r_{2}^{2}-r_{1}^{2}\right) \\
& C_{2}=\omega\left(\frac{r_{2} \tan \beta_{2}}{A_{s 2}}-\frac{r_{1} \tan \beta_{1}}{A_{s 1}}\right)
\end{aligned}
$$

However, as presented in chapter 6 , the relative angles $(\beta)$ are not constant with a variation in flow rate. Additionally, as indicated in the present chapter, the stream channel radii $(r)$ and therefore also the annular stream channel areas $\left(A_{s}\right)$ up- and downstream of the fan, change with variation in flow rate. Hence, the fact that the power per unit mass is still linear in figure 8.6 implies that the relationship between $\beta, A_{s}$ and $r$ is always such that the power per unit mass as a function of flow remains linear, at least for the B2a-fan in the present study. Whether this is a fundamental characteristic observable for all non-compressible flow turbo-machinery remains subject to future research.

Lastly, the exponential trend in effective work per unit mass $\left(\gamma_{F, \text { eff }}\right)$ for low flow rates, is described by equations 8.1.26 and 8.1.27. As the effective flow through the fan decreases, an increase in recirculating flow develops. If the effective flow through the fan becomes zero, the recirculating flow would be at a maximum. All the energy that the fan imparts would be exerted on the recirculating flow and hence $\gamma_{F, \text { eff }}$ would strive to infinity, since a division of zero would occur in equation 8.1.26. This variable $\left(\gamma_{F, \text { eff }}\right)$ is however only a measure of the fan effectiveness, but does not exist in reality. 


\section{Chapter 9}

\section{Reverse engineered empirical actuator disc model (REEADM)}

The ultimate goal of the present study is the development of a new simplified numerical fan model. Such a model would be used to represent an axial flow fan in the numerical modelling of large fan systems. Due to the advantages inherent in the computational requirements of the actuator-disc model (ADM), a similar model is developed termed the reverse engineered empirical actuatordisc model (REEADM). This model works on the same principle as the ADM, but with some alterations.

ADM theory is firstly discussed, explaining the difference between the original ADM and REEADM. Hereafter, details are given regarding the computational domain used to solve the REEADM, including a comparison between the computational resources required for the periodic three-dimensional model (P3DM) and the REEADM. Lastly, results obtained in determining the fan characteristic curves with the REEADM are presented and discussed.

\subsection{Model details}

\subsubsection{ADM theory}

The three-dimensional momentum equations in cylindrical coordinates for a single control volume (figure 9.1) are given as:

$$
\begin{aligned}
& \sum F_{r, b o d y} / \mathrm{d} V-\frac{\partial p}{\partial r}+\mu\left[\frac{1}{r} \frac{\partial}{\partial r}\left(r \frac{\partial u_{r}}{\partial r}\right)+\frac{1}{r^{2}} \frac{\partial^{2} u_{r}}{\partial \theta^{2}}+\frac{\partial^{2} u_{r}}{\partial z^{2}}-\frac{2}{r^{2}} \frac{\partial u_{\theta}}{\partial \theta}-\frac{u_{r}}{r^{2}}\right] \\
& =\rho\left(\frac{\partial u_{r}}{\partial t}+u_{r} \frac{\partial u_{r}}{\partial r}+\frac{u_{\theta}}{r} \frac{\partial u_{r}}{\partial \theta}+u_{z} \frac{\partial u_{r}}{\partial z}-\frac{u_{\theta}^{2}}{r}\right)
\end{aligned}
$$




$$
\begin{aligned}
& \sum F_{\theta, b o d y} / \mathrm{d} V-\frac{\partial p}{\partial r}+\mu\left[\frac{1}{r} \frac{\partial}{\partial r}\left(r \frac{\partial u_{\theta}}{\partial r}\right)-\frac{u_{\theta}}{r^{2}}+\frac{2}{r^{2}} \frac{\partial u_{r}}{\partial \theta}+\frac{1}{r^{2}} \frac{\partial^{2} u_{\theta}}{\partial \theta^{2}}+\frac{\partial^{2} u_{\theta}}{\partial z^{2}}\right] \\
& =\rho\left(\frac{\partial u_{\theta}}{\partial t}+u_{r} \frac{\partial u_{\theta}}{\partial r}+\frac{u_{\theta}}{r} \frac{\partial u_{\theta}}{\partial \theta}+u_{z} \frac{\partial u_{\theta}}{\partial z}+\frac{u_{r} u_{\theta}}{r}\right) \\
& \sum F_{z, b o d y} / \mathrm{d} V-\frac{\partial p}{\partial z}+\mu\left[\frac{1}{r} \frac{\partial}{\partial r}\left(r \frac{\partial u_{z}}{\partial r}\right)+\frac{\partial^{2} u_{z}}{\partial r^{2}}+\frac{1}{r^{2}} \frac{\partial^{2} u_{z}}{\partial \theta^{2}}+\frac{\partial^{2} u_{z}}{\partial z^{2}}\right] \\
& =\rho\left(\frac{\partial u_{z}}{\partial t}+u_{r} \frac{\partial u_{z}}{\partial r}+\frac{u_{\theta}}{r} \frac{\partial u_{z}}{\partial \theta}+u_{z} \frac{\partial u_{z}}{\partial z}\right)
\end{aligned}
$$

where $\mathrm{d} V$ is the volume and $p$ the absolute pressure at the control volume.

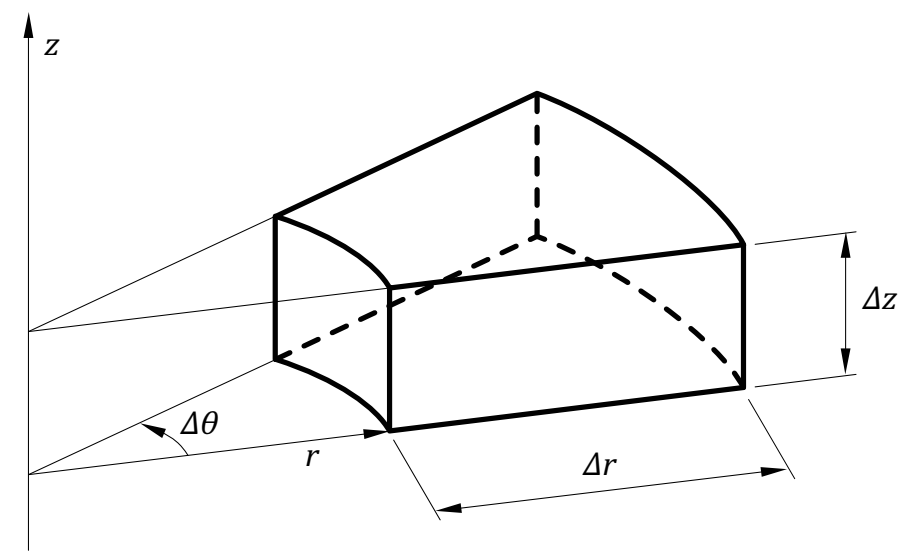

Figure 9.1: Cylindrical control volume (cell) in space

In these equations $\sum F_{r, b o d y} / d V, \sum F_{\theta, b o d y} / d V$ and $\sum F_{z, b o d y} / d V$ are body force source terms in the respective cylindrical coordinates that can be applied to the control volume. Accurate representation of the values of these source terms could simulate the flow field that would have been induced by rotating fan blades. This is done in the following manner:

The flow in the vicinity of a fan is modelled using a discretized computational domain, where the ADM technique models the actuated flow field by replacing the actual fan blades with three annular discs of finite thickness $\Delta z$, as shown in figure 9.2. Each disc is one cell-size thick in the axial direction and consists of exactly the same radial and tangential cell distribution. A single cell in the actuator disc plane is linked with two cells in the adjacent up- and downstream discs, also shown in figure 9.2. The actuator disc coincides with the fan rotational plane and is used to actuate the modelled flow field by addition of the source terms previously mentioned, to each cell in the actuator disc. The up- and downstream discs are placed at an axial length of $z / c h_{\text {avg }}>0.5$ from the actuator disc, where $c h_{\text {avg }}$ is the average chord length of the fan blade 
(Bredell et al., 2006). The average velocity vectors are obtained from these discs. These vectors are then used to obtain the angle of attack across the fan blade from where the lift and drag coefficients at the specific radial locations of the fan blade can be determined.

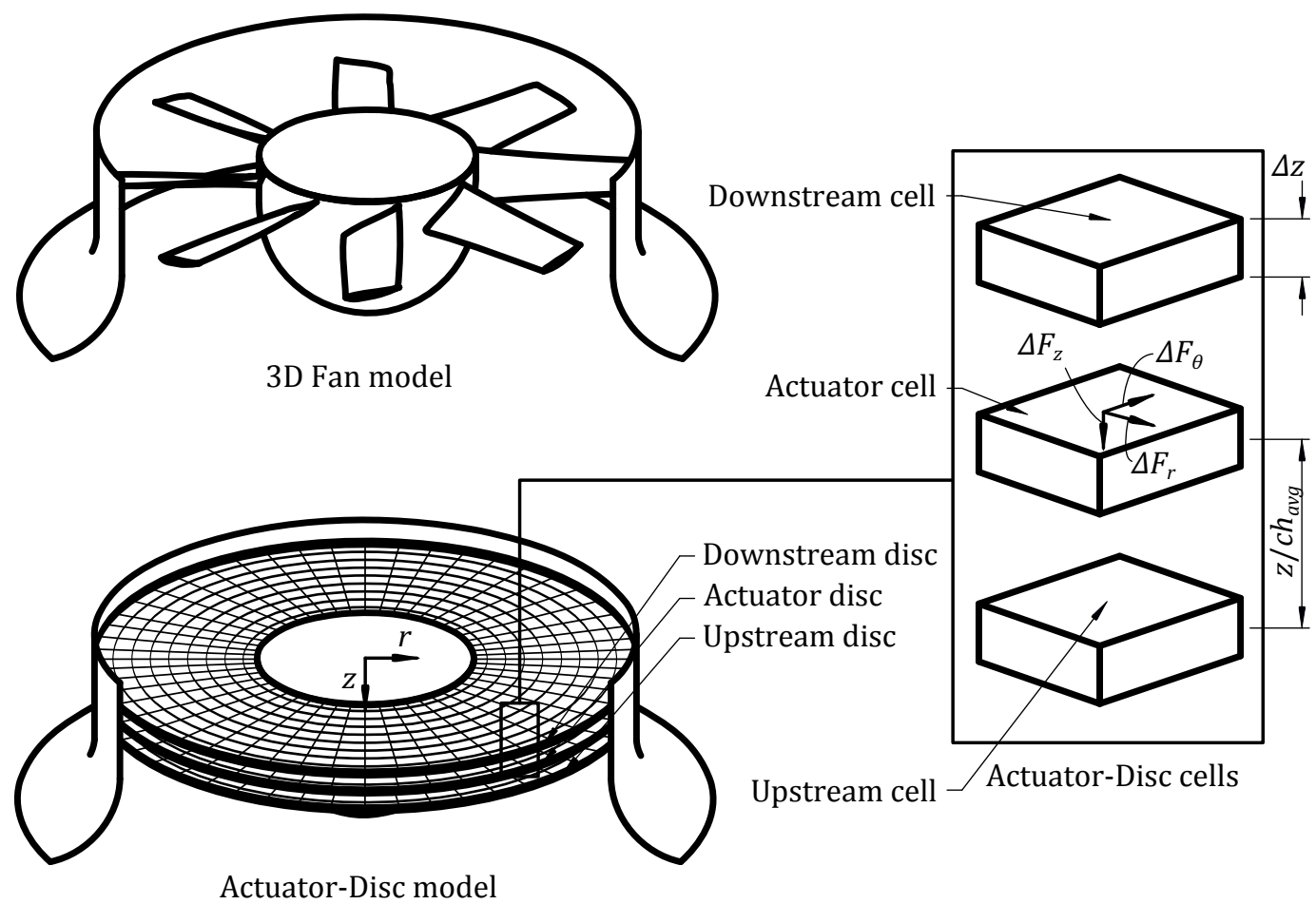

Figure 9.2: Comparative depiction of a three-dimensional fan model and an actuator-disc model

The source terms for the ADM are calculated using two-dimensional blade element theory, which is explained with reference to figure 9.3 depicting a two-dimensional blade section and the forces exerted on it. Two-dimensional blade element theory is an appropriate method to determine the forces exerted by the fan blades if the the fan solidity is below 0.7 (Bruneau, 1994). Hence, cascade theory should be used for solidities above 0.7 . The lift $(L)$ and drag $(D)$ force components are defined perpendicular and parallel to the vector averaged relative velocity $\left(w_{\theta z, \infty}\right)$ experienced by the blade element in the $\theta z$-plane. These force components are calculated by:

$$
\begin{aligned}
& L=\frac{1}{2} \rho w_{\theta z, \infty}^{2} C_{L} \times c h \times \delta r \quad[\mathrm{~N} / \mathrm{m}] \\
& D=\frac{1}{2} \rho w_{\theta z, \infty}^{2} C_{D} \times c h \times \delta r \quad[\mathrm{~N} / \mathrm{m}]
\end{aligned}
$$




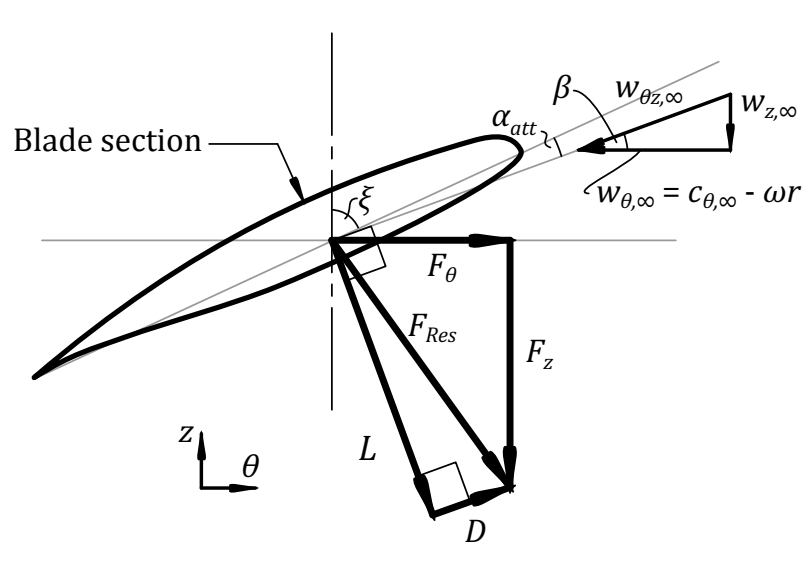

$\overline{\text { Rotation }}$

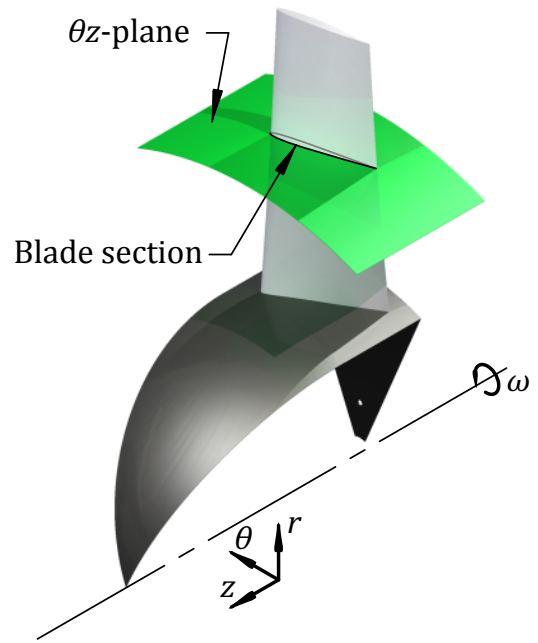

Figure 9.3: The two-dimensional force components of a two-dimensional blade section

where $\delta r$ is the finite radial thickness of the elemental blade section considered and the values of the lift and drag coefficients $\left(C_{L}\right.$ and $\left.C_{D}\right)$ are obtained from two-dimensional aerofoil data. From this figure it can also be deduced that the force components exerted by this elemental blade in the axial $\left(F_{z}\right)$ and circumferential $\left(F_{\theta}\right)$ directions are:

$$
\begin{aligned}
& F_{z}=L \cos \beta_{\infty}+D \sin \beta_{\infty} \\
& =\frac{1}{2} \rho w_{\theta z, \infty}^{2} \operatorname{ch}\left(C_{L} \cos \beta_{\infty}+C_{D} \sin \beta_{\infty}\right) \quad[\mathrm{N} / \mathrm{m}] \\
& F_{\theta}=L \sin \beta_{\infty}-D \cos \beta_{\infty} \\
& =\frac{1}{2} \rho w_{\theta z, \infty}^{2} \operatorname{ch}\left(C_{L} \sin \beta_{\infty}-C_{D} \cos \beta_{\infty}\right) \quad[\mathrm{N} / \mathrm{m}]
\end{aligned}
$$

Furthermore, the induced radial force $\left(F_{r}\right)$ is assumed to be small and is neglected (Bredell et al., 2006). Hence:

$$
F_{r}=0 \quad[\mathrm{~N} / \mathrm{m}]
$$

Since the blades are not explicitly modelled by the ADM, only the pitchaveraged induced flow is simulated. In this manner, the source terms induced into the momentum equations for each cell in the actuator disc plane is calculated on the basis of the cell volume by: 


$$
\begin{aligned}
\sum F_{z, \text { body }} / d V & =\frac{F_{z}}{s_{p} \Delta z} \\
& =\frac{1}{2} \rho w_{\theta z, \infty}^{2} \frac{\sigma}{\Delta z}\left(C_{L} \cos \beta_{\infty}-C_{D} \sin \beta_{\infty}\right) \quad\left[\mathrm{N} / \mathrm{m}^{3}\right] \\
\sum F_{\theta, b o d y} / d V & =\frac{F_{\theta}}{s_{p} \Delta z} \\
& =-\frac{1}{2} \rho w_{\theta z, \infty}^{2} \frac{\sigma}{\Delta z}\left(C_{L} \sin \beta_{\infty}+C_{D} \cos \beta_{\infty}\right) \quad\left[\mathrm{N} / \mathrm{m}^{3}\right]
\end{aligned}
$$

and

$$
\sum F_{r, b o d y} / d V=0 \quad\left[\mathrm{~N} / \mathrm{m}^{3}\right]
$$

where $s_{p}$ is the circumferential pitch between fan blades and $\sigma$ the fan solidity at a specific radius given by:

$$
\sigma=\frac{c h}{s_{p}}
$$

Finally, the ADM is incorporated into the numerical model by means of a user subroutine as discussed by Bredell et al. (2006). This subroutine calculates the source terms to be added to each cell in the actuator disc for each iteration of the numerical simulation, where the source values are based on the flow field data extracted from the up- and downstream discs and other information predefined by the user, such as the number of blades, blade angle, two-dimensional aerofoil data and other fan geometric values.

\subsubsection{REEADM theory}

The REEADM is identical to the ADM in that it models the fan induced flow field by assigning values to the momentum source terms in the actuator disc plane. However, the drag and lift coefficients used to calculate these source terms are obtained in a different manner. The radial force (indicated in figure 9.4) induced by the fan blade is also accounted for. Hence, the blade lift, drag and radial force coefficients are calculated (or reverse engineered) from the results of the P3DM (presented in chapter 7), instead of the two-dimensional aerofoil data. These coefficients are then used to calculate the source terms discussed in the previous section. Once again, the implementation of these source terms into the computational fluid dynamics (CFD) code is accomplished through the user subroutine. Hence, in developing a REEADM for a 


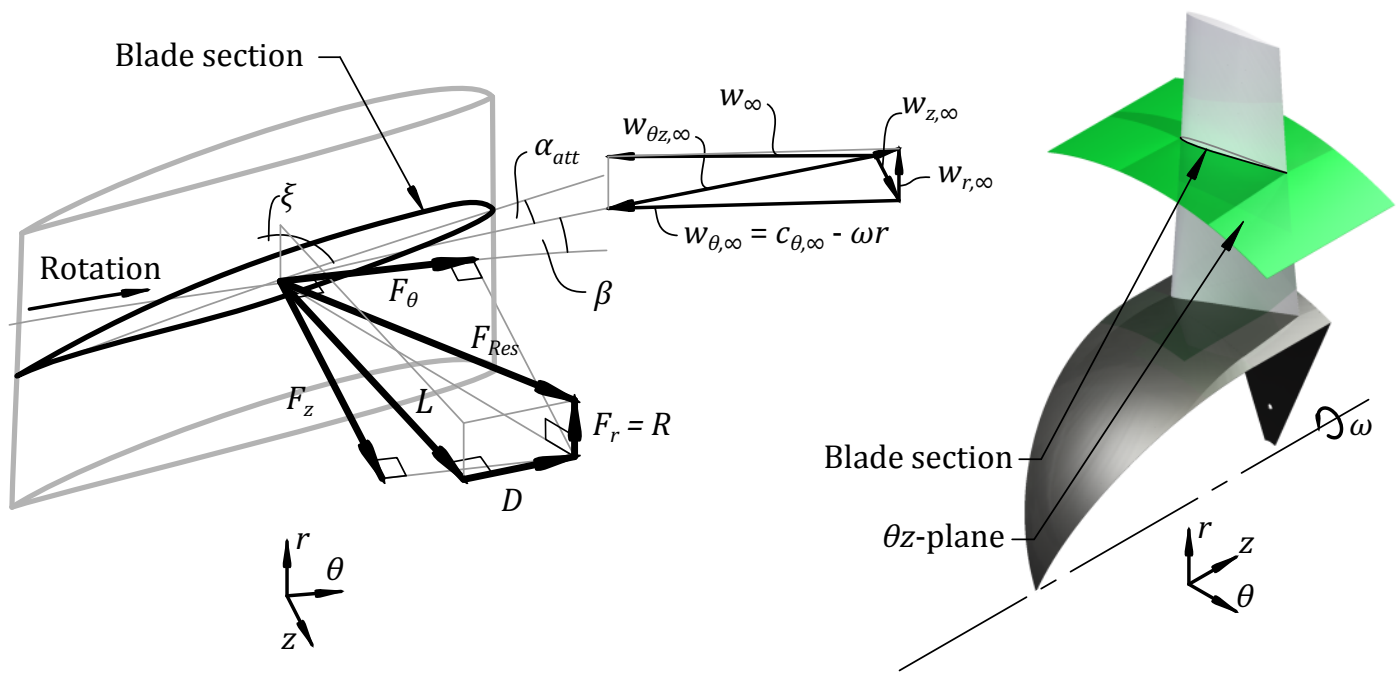

Figure 9.4: The three-dimensional force components of a two-dimensional blade section

specific fan, it is required to conduct three-dimensional CFD analyses for the operating envelope of the fan at least once.

To implement the force coefficients in the present investigation, fifth order surface polynomials are fitted through the lift, drag and radial force coefficients (shown in figures $7.6 \mathrm{~b}-\mathrm{c}, 7.7 \mathrm{~b}-\mathrm{c}$ and $7.8 \mathrm{~b}-\mathrm{c}$ ), as indicated in figures $9.5 \mathrm{a}-\mathrm{c}$ using a surface fitting tool from the commercial software code, Matlab. In this manner the coefficients are not functions of the approaching flow angle onto the blade alone (as is the case with the ADM), but also the radial location of an applicable blade section.

Due to the strong deviation of lift, drag and radial force results near the hub and tip $\left(s_{b}<0.1\right.$ and $\left.s_{b}>0.9\right)$ from the trend across the main section of the blade $\left(0.1<s_{b}<0.9\right.$, figures $\left.7.2 \mathrm{~b}-\mathrm{d}\right)$, only data from the main section is used to obtain the polynomial surface fits. Hence, force coefficient values calculated in the hub and tip regions, are extrapolations from the main section.

The general equation for a fifth order polynomial is given as:

$$
\begin{aligned}
C_{i}\left(s_{b}^{\prime}, \theta_{a}^{\prime}\right)= & a_{00}+a_{10} s_{b}^{\prime}+a_{01} \theta_{a}^{\prime}+a_{20} s_{b}^{\prime 2}+a_{11} s_{b}^{\prime} \theta_{a}^{\prime}+a_{02} \theta_{a}^{\prime 2}+a_{30} s_{b}^{\prime 3} \\
& +a_{21} s_{b}^{\prime 2} \theta_{a}^{\prime}+a_{12} s_{b}^{\prime} \theta_{a}^{\prime 2}+a_{03} \theta_{a}^{\prime 3}+a_{40} s_{b}^{\prime 4}+a_{31} s_{b}^{\prime 3} \theta_{a}^{\prime}+a_{22} s_{b}^{\prime 2} \theta_{a}^{\prime 2} \\
& +a_{13} s_{b}^{\prime} \theta_{a}^{\prime 3}+a_{04} \theta_{a}^{\prime 4}+a_{50} s_{b}^{\prime 5}+a_{41} s_{b}^{\prime 4} \theta_{a}^{\prime}+a_{32} s_{b}^{\prime 3} \theta_{a}^{\prime 2}+a_{23} s_{b}^{\prime 2} \theta_{a}^{\prime 3} \\
& +a_{14} s_{b}^{\prime} \theta_{a}^{\prime 4}+a_{05} \theta_{a}^{\prime 5}
\end{aligned}
$$

where the polynomial constants $\left(a_{s \theta}\right)$ and Pearson correlation factors $\left(R_{P}\right)$ are given in table 9.1 for the lift, drag and radial force coefficients respectively. In equation 9.1.13, $i$ refers to either the lift, drag or radial force components $(L$, $D$ or $R$ ). $s_{b}^{\prime}$ and $\theta_{a}^{\prime}$ are normalised values for the blade span and applicable angle defined as: 
CHAPTER 9. REVERSE ENGINEERED EMPIRICAL ACTUATOR DISC MODEL (REEADM)

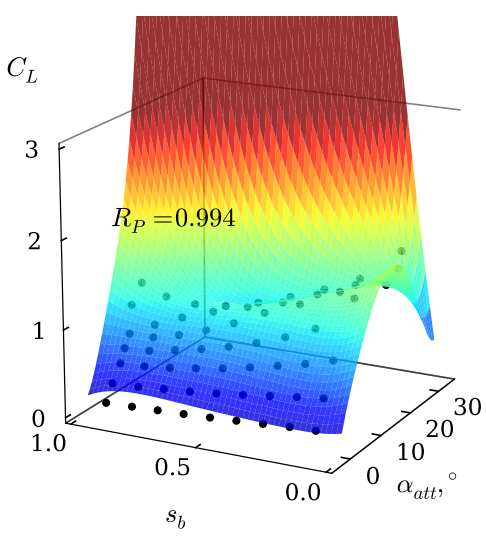

(a)

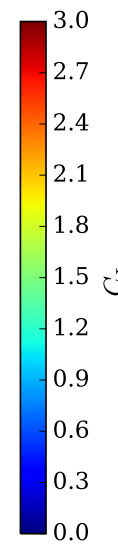

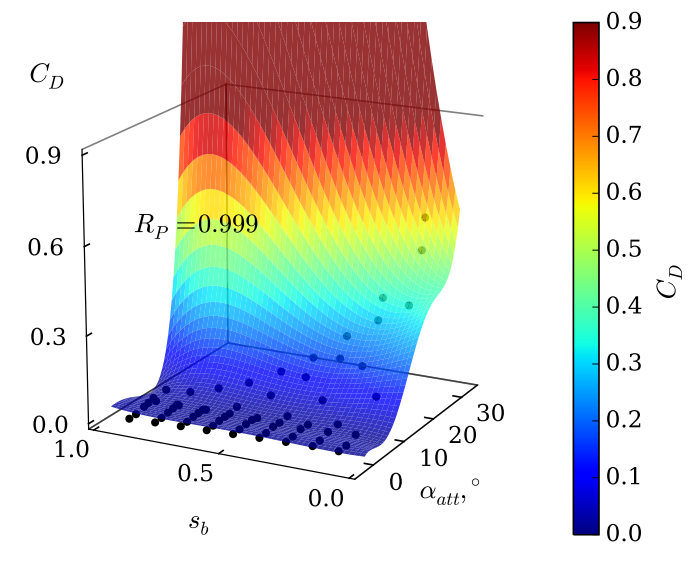

(b)

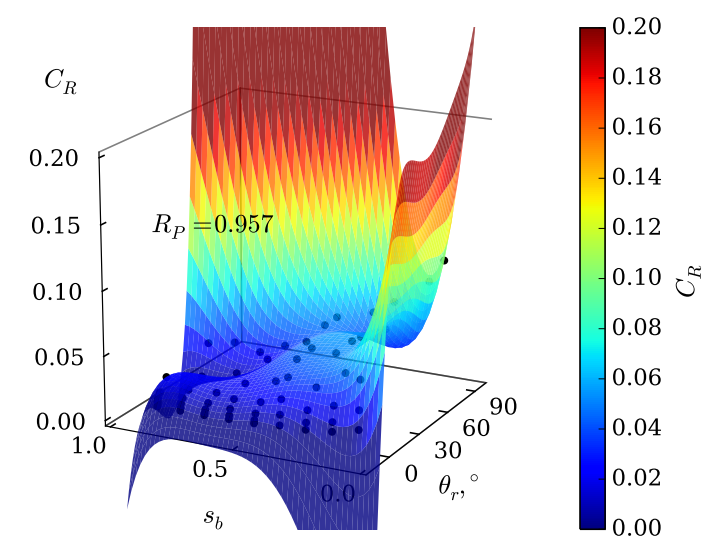

(c)

Figure 9.5: Fifth order polynomial surface fits through the (b) lift $\left(C_{L}\right)$, (b) drag $\left(C_{D}\right)$ and (c) radial force $\left(C_{R}\right)$ coefficients as a function of the applicable flow angle $\left(\alpha_{\text {att }}\right.$ or $\left.\theta_{r}\right)$ and blade span $\left(s_{b}\right)$

$$
\begin{aligned}
& s_{b}^{\prime}=\frac{s_{b, j}-s_{b, a v g}}{s_{b, s t d}} \\
& \theta_{a}^{\prime}=\frac{\theta_{a, j}-\theta_{a, a v g}}{\theta_{a, s t d}}
\end{aligned}
$$

where the subscripts $j$, avg and std refer to a specific data point, the average value and standard deviation in the data set of each force coefficient (i.e. $C_{L}$, $C_{D}$ and $C_{R}$ ), respectively. Furthermore, $\theta_{a}$ refers to the applicable angle, i.e. $\alpha_{\text {att }}$ in the case of blade lift and drag coefficients and $\theta_{r}$ (the radial flow angle defined in figure 6.1) for the radial force coefficient.

Finally, the calculation of the momentum source terms for the axial and circumferential directions described by equations 9.1.9-9.1.10 remains the same, 
CHAPTER 9. REVERSE ENGINEERED EMPIRICAL ACTUATOR DISC MODEL (REEADM)

Table 9.1: Fifth order surface polynomial constants for the B2a-fan blade sectional lift, drag and radial force coefficients

\begin{tabular}{|c|c|c|c|}
\hline & $C_{L}$ & $C_{D}$ & $C_{R}$ \\
\hline$R_{P}$ & 0.994 & 0.999 & 0.957 \\
\hline \multicolumn{4}{|c|}{ Constants } \\
\hline$a_{00}$ & 1.029250867 & 0.019603986 & 0.033077509 \\
\hline$a_{10}$ & -0.018474808 & 0.003621708 & -0.022428843 \\
\hline$a_{01}$ & 0.552201901 & 0.034378485 & 0.014980918 \\
\hline$a_{20}$ & -0.05856383 & 0.009642936 & -0.001291348 \\
\hline$a_{11}$ & -0.178100536 & 0.013250025 & -0.004245669 \\
\hline$a_{02}$ & -0.493205118 & 0.061576633 & -0.003321821 \\
\hline$a_{30}$ & 0.030043805 & -0.001747101 & 0.013658021 \\
\hline$a_{21}$ & 0.009959619 & 0.009255332 & -0.015810076 \\
\hline$a_{12}$ & -0.145181759 & -0.003878479 & -0.003847661 \\
\hline$a_{03}$ & -0.110397243 & 0.009919167 & 0.005313988 \\
\hline$a_{40}$ & 0.027003795 & -0.003049744 & 0.004834297 \\
\hline$a_{31}$ & 0.088811185 & -0.010625442 & -0.003686479 \\
\hline$a_{22}$ & 0.13552544 & -0.001133059 & -0.020552174 \\
\hline$a_{13}$ & 0.05825148 & 0.002832612 & -0.016441898 \\
\hline$a_{04}$ & 0.121338646 & -0.010983699 & -0.010928049 \\
\hline$a_{50}$ & -0.005531554 & 0.000000611 & -0.006085431 \\
\hline$a_{41}$ & 0.033004608 & -0.004575164 & 0.004077903 \\
\hline$a_{32}$ & 0.061888008 & -0.008379334 & 0.006996506 \\
\hline$a_{23}$ & 0.05503202 & 0.004377608 & 0.018576158 \\
\hline$a_{14}$ & 0.089340614 & 0.018142546 & 0.012649717 \\
\hline$a_{05}$ & 0.013372621 & 0.009463266 & 0.004222753 \\
\hline
\end{tabular}

where only the lift and drag coefficients are calculated differently. However, since the radial force is also considered (indicated in figure 9.4), the radial momentum source can be obtained through

$$
\begin{aligned}
\sum F_{r, b o d y} / d V & =\frac{F_{r}}{s_{p} \Delta z} \\
& =\frac{1}{2} \rho w_{\infty}^{2} \frac{\sigma}{\Delta z} C_{R} \quad\left[\mathrm{~N} / \mathrm{m}^{3}\right]
\end{aligned}
$$

where $C_{R}$ is the radial force coefficient defined in equation 7.1.9 and $w_{\infty}$ the relative velocity. 
CHAPTER 9. REVERSE ENGINEERED EMPIRICAL ACTUATOR DISC MODEL (REEADM)

\subsubsection{Computational domain}

Similar to the P3DM analyses, REEADM simulations are conducted using ANSYS Fluent 14.0. The computational domain is an $1 / 8^{\text {th }}$ fan sector as indicated in figure 9.6, corresponding to the P3DM in chapter 4 and conforms to the BS 848, type A fan test specification. The boundary conditions on the domain extents are the same for the REEADM and P3DM computational domains, but differ in cell count and structure.

Regarding the three discs of the REEADM, the actuator disc also coincides with the fan rotational plane, identical to the ADM. However, the up- and downstream discs are placed at the same locations where the original velocity vectors are sampled from in the P3DM, i.e. 0.46 of the hub chord $\left(0.46 c h_{h}\right)$ up- and downstream in the axial direction of the fan rotational plane. A comparison between the amount of cells required for the P3DM and REEADM is given in table 9.2

\subsection{Results}

The REEADM is used to determine the fan performance characteristics for the B2a-fan. These results are compared to the average experimental results as well as two other simplified numerical fan models (ADM and extended actuator-disc model (EADM), Van der Spuy et al. (2011)) in figure 9.7a-b. Fan characteristic values obtained using the REEADM are only valid for the range of flow coefficients modelled by the P3DM, being $0.042 \leq \phi \leq 0.2$, since the former model is developed using the latter. However, three operating points (indicated by the dotted triangles in figure 9.7a-b) outside of these boundaries

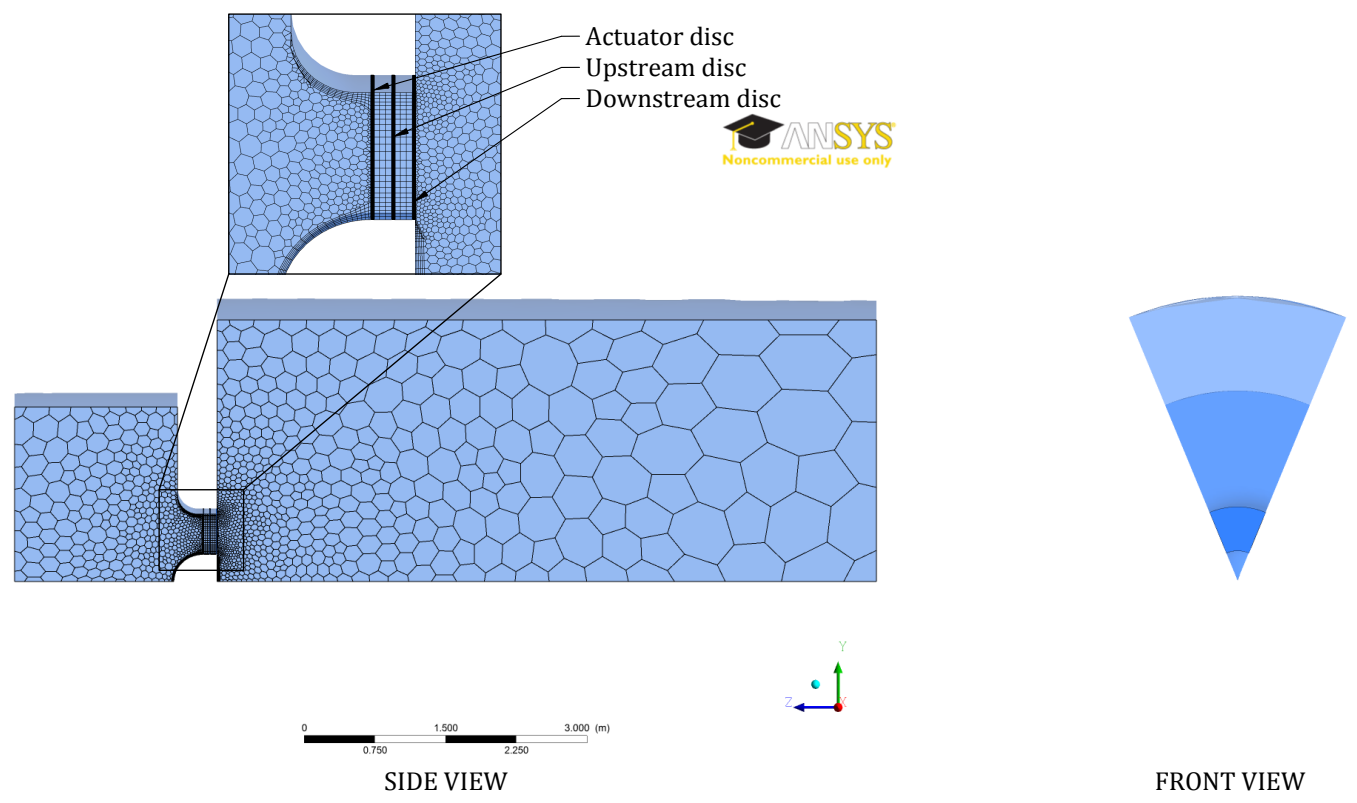

Figure 9.6: Computational domain of the REEADM 
CHAPTER 9. REVERSE ENGINEERED EMPIRICAL ACTUATOR DISC MODEL (REEADM)

Table 9.2: Comparison between P3DM and REEADM computational domain sizes and required computational resources

\begin{tabular}{|c|c|c|}
\hline & P3DM & REEADM \\
\hline \multicolumn{3}{|l|}{ Cell count } \\
\hline Rotor sub-domain & $1.5(10)^{6}(\mathrm{Hex})$ & $2.5(10)^{3}($ Hex $)$ \\
\hline Entire domain & $2.5(10)^{6}$ (Poly, Hex) & $47(10)^{3}$ (Poly, Hex) \\
\hline \multicolumn{3}{|l|}{ Computational } \\
\hline Processors & 8 CPUs $(2.1-3 \mathrm{GHz})$ & 2 CPUs $(3.4 \mathrm{GHz})$ \\
\hline $\begin{array}{l}\text { Random access me- } \\
\text { mory (RAM) }\end{array}$ & $15 \mathrm{~Gb}$ & $32 \mathrm{~Gb}$ \\
\hline Time & 2-28 days & $10-120 \mathrm{~min}$ \\
\hline
\end{tabular}

are also modelled to observe the REEADM's performance, but these values are only a result of the extrapolated lift, drag and radial force coefficients.

All of the simplified fan models give a fair prediction of the fan characteristics for near-design flow rates $(\phi>0.147)$. Yet, a deviation from the experimental results can be observed at low flow rates. Of all the models the ADM shows the furthest deviation under these flow conditions. The EADM gives a fair prediction of the fan performance at low flow rates, but Van der Spuy et al. (2011) mention that such good agreement is not obtained for all of the fans investigated in their study. The characteristic prediction of the REEADM is also poor at low flow rates, although it is an improvement upon the ADM for these flow conditions. A comparison between the REEADM and P3DM results give a Pearson correlation of $R_{\Psi_{F s}}^{2}=0.986$ and $R_{\eta_{F s}}^{2}=0.997$ for the fan static pressure coefficient and static efficiency, respectively. Possible reasons for the discrepancies occurring at low flow rates are discussed in the following section.

\subsection{Concluding remarks}

The present chapter explored the effectiveness of a simplified fan modelling technique in the prediction of fan performance characteristics. Although all the models explored give a fair approximation of these characteristics, the discrepancy at low flow rates require some consideration. The presented models are discussed individually.

The main reason for the deviation between the ADM and the experimental data might be attributed to the fan blade not experiencing two-dimensional (axial and circumferential) flow at low flow rates, but three-dimensional. Also, as shown in the previous chapters, transient flow patterns near the hub and 


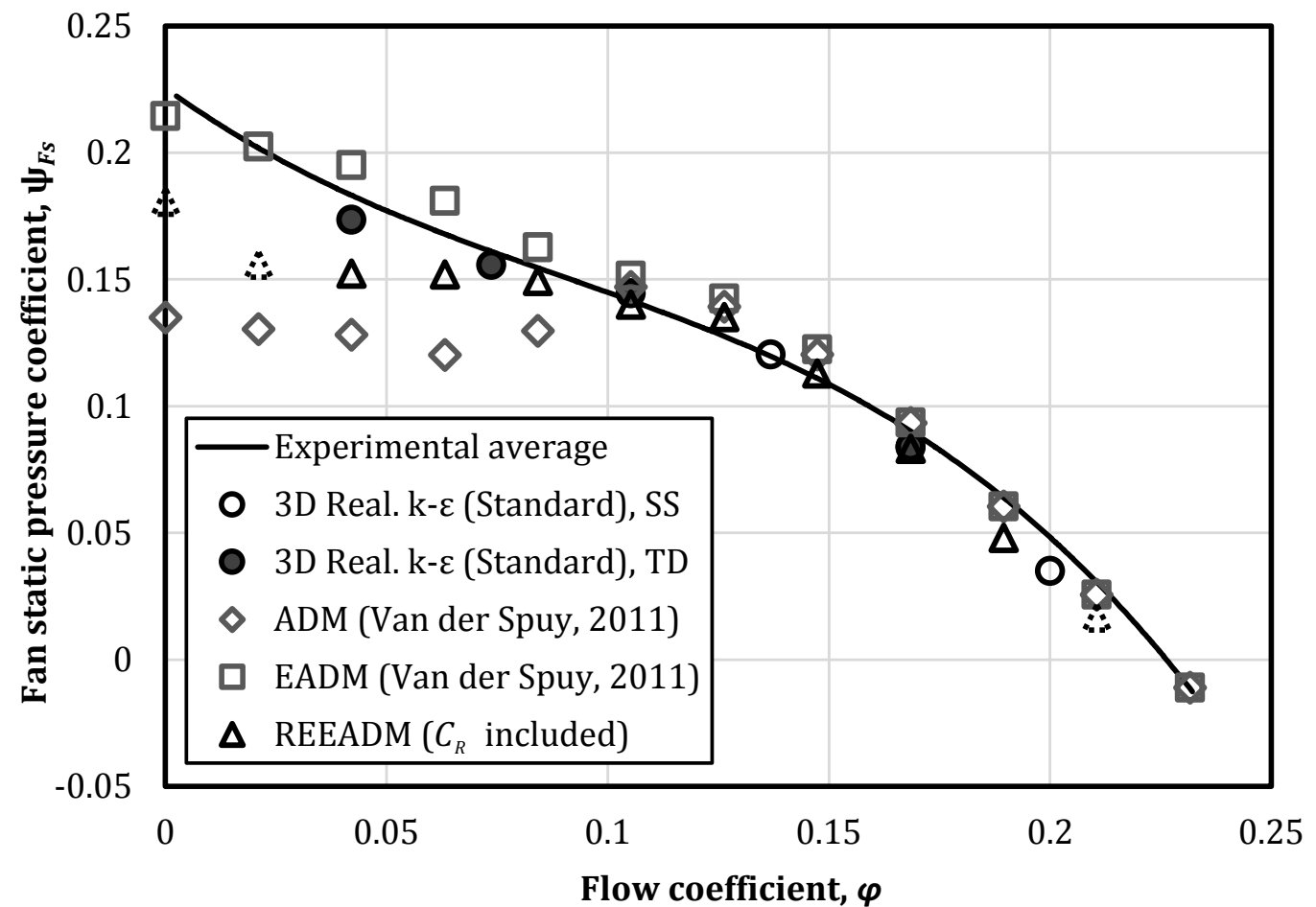

(a)

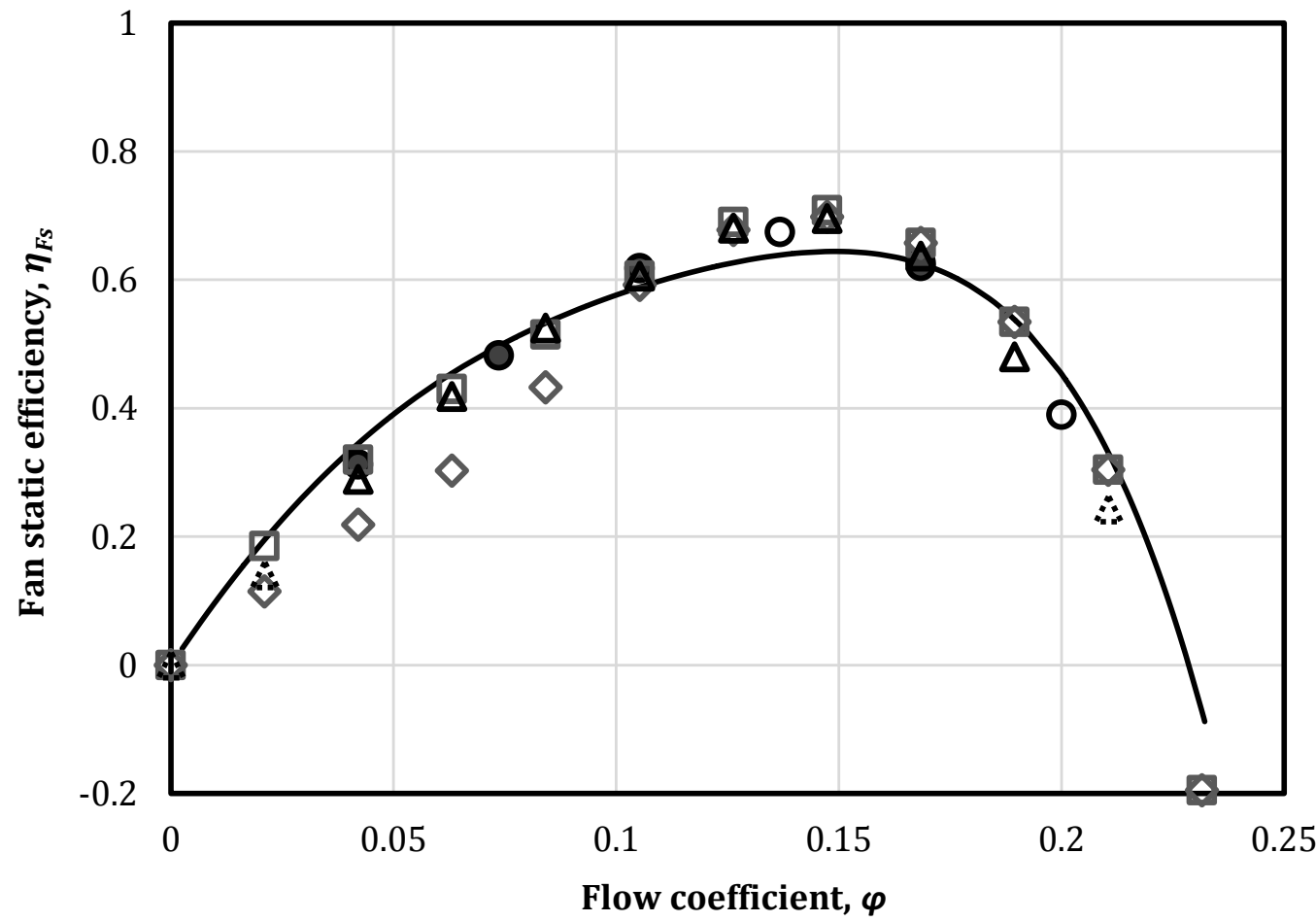

(b)

Figure 9.7: Comparison of the B2a-fan performance characteristics determined through different simplified numerical fan models, P3DM and the average experimental results for the (a) fan static pressure coefficient $\left(\Psi_{F s}\right)$ and (b) fan static efficiency $\left(\eta_{F s}\right)$ as functions of flow coefficient $(\phi)$ 
tip of the fan blade become more unstable with a decrease in flow rate, to the extent that the two-dimensional aerofoil characteristics are improbable.

The EADM, derived from lift/drag observations in a rotating propeller by Himmelskamp (1947), models the flow purely by empirically extending the two-dimensional lift curve at low angles of attack with a straight line past the stall point of the aerofoil characteristic. Hence, flow onto the blade at high angles of attack experiences a higher lift magnitude compared to an ordinary two-dimensional aerofoil that would have experienced stall. The fact that the accuracy of this model is variable, points to the unknown interaction between the fan blade and the flow field at low flow rates.

Since the lift, drag and radial force coefficients are empirically derived through analyses of the P3DM data, it is fair to compare the fan characteristics obtained through the REEADM to those obtained by the former model. Although a fair comparison is found at near-design flow rates, the poor comparison at low flow rates initially raises concern. The following reasons might explain the discrepancy in the compared data:

- Observation of the visualized flow field from the P3DM data in the vicinity of the fan (chapter 6) and inside the fan passage presents the large degree of instability at low flow rates. For the B2a-fan, non-linear vortical patterns appear and dissipate periodically over time. The time and circumferential averaging of the flow field and blade force data, to obtain the reverse engineered lift, drag and radial force coefficients for the B2afan (being a linear process), might not be the appropriate procedure to capture the time-averaged flow field.

- It could also be argued that the assumption of periodicity through the P3DM and the manner of turbulence modelling are incapable of modelling the flow in the vicinity of the fan at low flow rates. The data obtained would be erroneous as a consequence. However, a fair comparison is found between these results and those obtained for two different experiments in the present study, confirming the accuracy of the P3DM.

- Up- and downstream averaging of the numerically obtained data of the P3DM might not be the correct approach in order to quantify the average flow experienced by the fan rotor, especially during unstable flow conditions. As the flow visualizations in chapters 6 and 8 show, flow approaches the fan from both the up- and downstream locations near the hub, of which some are entrained in a stall pattern near the blade and some flow drawn radially outward. The actuation of the flow field by the blade is therefore transient. Thus, the average flow condition experienced by different spanwise locations on the blade would not necessarily be equal to the average of the up- and downstream flow conditions, upon which the actuator disc models are based.

Further investigations are therefore suggested: 
- Fundamental three-dimensional flow field analysis. If the fundamental flow structure induced by the fan could be understood, the discrepancy between the experimentally measured and actuator-disc modelled fan performance values could be explained. In chapter 8 it is attempted to understand the unsteady flow field at low flow rates by observation of the time and circumferentially averaged flow field data. These averaged results are also used in the development of the REEADM, which attempts to model the averaged flow field induced by the fan only, similar to other actuator-disc models. This approach disregards the three-dimensional and time-dependent flow structures. A full threedimensional model of the fan (all of the 8 blades included) would give more insight and also distinguish the effect of periodic boundaries in the P3DM.

- Actuator-line type model. A more complex actuator-line type model could be considered, such as the one proposed for wind turbines in Hansen et al. (2006). This model might provide a better approximation of the fan performance characteristic, since a three-dimensional flow structure is allowed to develop. However, this model would be solved in a time-dependent manner and would therefore require more computational resources compared to the actuator-disc models. 


\section{Chapter 10}

\section{Conclusion}

The present study focussed on the investigation of the flow field in the vicinity of an axial fan for a range of flow rates, mainly to obtain an understanding of the flow field at low flow rates. A near free-vortex fan designed for implementation in large air-cooled heat exchangers (ACHEs), termed the B2a-fan (Louw et al. (2012) and Louw et al. (2014a)) is used as a test subject to investigated these effects. The results obtained proved to be valuable in quantifying certain flow phenomena occurring at low flow rates, which were used in the development of an improved simplified numerical fan model.

The first section of the concluding chapter to this study lists the contributions and findings made. Thereafter, the final section elaborates on potential future research on this topic.

\subsection{Contributions}

From the literature surveyed on axial fans it is identified that many publications are related to the effects of sweep on fan performance. In these cases the consensus is that forward sweep and skew are advantageous for increasing stall margin and noise reduction. The contrary is observed for backward sweep and skew. The highest nominal efficiency is found for fans with unswept blades. Concerning the choice of vortex design, it is found that free vortex designed fans are generally more efficient compared to controlled vortex designs, at least for the publications compared in the present literature review. Careful consideration should however be given to optimal hub-tip ratios and constraints on the blade stagger angle near the root.

In the present investigation a numerical model is developed to simulate the flow field in the vicinity of the B2a-fan according to the British standard (BS)848, type A (open inlet to open outlet). This model is published in Louw et al. (2014a). The effect of turbulence is modelled using the Realizable $k-\varepsilon$ model together with a Standard wall function for boundary layer modelling near wall surfaces. The model, termed the periodic three-dimensional model (P3DM), simulates the flow for an $1 / 8^{\text {th }}$ sector of the fan representing 
a single blade passage. Hence, rotational symmetry is assumed. It is found that the difference in fan characteristic values are negligible for inlet and outlet boundaries placed further away than approximately 1.5 and 4.5 fan diameters in the axial direction, up- and downstream of the fan rotor, respectively. A computational grid larger than $2.5(10)^{6}$ cells, where $1(10)^{6}$ cells are located in close vicinity to the rotor, also provided negligible differences in fan characteristic predictions. Lastly, steady simulations proved to be sufficient for flow coefficients of $\phi \geq 0.137$, whereas time-dependent analyses are required for lower flow coefficients.

A $d_{c}=1.542 \mathrm{~m}$ experimental model of the B2a-fan is constructed and tested in a BS848, type A fan test facility. The characteristic results of the numerical model show fair comparison to these results with Pearson correlation factors of $R_{P, \Psi_{F s}}=0.997$ and $R_{P, \eta_{F s}}=0.990$, for fan static pressure coefficient and static efficiency. An experimental technique is developed to conduct time-averaged blade surface pressure measurements (BSPMs) and published in Louw et al. $(2015 b)$. These results are used to verify the numerical model, by comparing the measured data to the numerically obtained static blade surface pressures for five different spanwise locations on the pressure and suction sides of a B2a-fan blade. As a result the flow conditions occurring in the vicinity of the fan, especially at low flow rates, are confirmed experimentally. Comparisons between the experimentally and numerically obtained blade surface pressures are fair for all flow rates and blade spans, where the poorest comparison is found near the blade root. The reason might be attributed to the boundary layer not being calculated correctly by the numerical simulations, since the $y^{+}$-values for a portion of cells in this region are below the prescribed value of $y^{+}=30$. However, the comparison in trend is still fair in this region and a Pearson correlation of $R_{P} \gtrsim 0.99$ is found for $90 \%$ of the blade span.

A large number of flow visualisations drawn from the numerical results is presented. With regards to the time-averaged spanwise velocity profiles a near-uniform axial velocity profile is found for near-design design flow rates, confirming the free-vortex functioning of the fan. As the flow rate is decreased, the axial velocity profile gradually changes such that a maximum velocity near the tip is clear, but a gradual decrease in this velocity occurs along the span of the fan towards the hub. A reverse flow pattern starts occurring near the hub on the downstream side of the fan at a flow coefficient of $\phi=$ 0.137 , which is $\phi / \phi_{D}=0.82$. This reversed flow pattern increases with a further decrease in flow rate. A negligible amount of radial flow occurs at near-design flow coefficients. However, an increase in radial flow through the fan is observed with decreasing flow coefficients. The location of maximum radial velocity also shifts along the blade span from the hub towards the tip. A ring vortical structure is present on the upstream side near the tip of the fan at flow coefficients of $\phi \lesssim 0.074$. Similar behaviour was noted by Venter (1990) and also measured by Downie et al. (1993).

It is visually shown that the time-dependent nature of the flow becomes 
more erratic with a decrease in flow rates, starting near the hub, but hereafter extending to higher spans. In these cases a periodic trend is noticed at low flow rates $(\phi \lesssim 0.105)$ ranging from $n_{F r} \approx 2$ fan rotations per period for $\phi=0.105$ to $n_{F r} \approx 2.5$ for $\phi=0.042$.

Blade force characteristics are also analysed and published in Louw et al. $(2015 a)$. The lift, drag and radial force coefficients for blade sections at various span lengths are presented. In these cases a blade section represents an aerofoil similar to the NASA LS 0413 profile (McGhee and Beasley (1976)), used in the initial calculation of the B2a-fan blade shape. It is found that the blade lift characteristics agree with the NASA LS 0413 lift characteristic at low angles of attack (near-design flow conditions). However, a slight offset of these values occurs. At higher angles of attack (low flow conditions), the blade sectional lift coefficients deviate strongly from that of the NASA LS 0413 profile. A trend is noticed where these lift coefficients are always the highest near the hub and decrease towards the tip for a given flow rate. The maximum lift coefficient occurs near the fan hub, but remains lower than the NASA LS 0413 lift characteristic, except at very high angles of attack where a sharp increase in the gradient of the blade sectional lift characteristic can be observed. The value of the blade sectional drag also remains of the same order compared to that of the NASA LS 0413 profile at low angles of attack for all blade sections although a slightly different trend in the characteristic is noticed. A sharp increase in the blade sectional drag coefficients is observed at low angles of attack, to the point where it is of the same order of magnitude of the lift coefficients. The blade sectional radial force coefficients remain small and is an order smaller compared to the drag coefficients at low angles of attack. This characteristic of the radial force coefficient holds true for all flow coefficients. The large increase in radial velocity through the fan is therefore not attributable to the radial blade forces, but rather to the change in the pressure profile downstream of the fan, drawing flow from lower radii to higher radii. These force characteristics prove the difference in functioning of fan blade sectional profiles in a rotating environment compared to two-dimensional aerofoils, a trend also observed by Himmelskamp (1947) who measured blade force characteristics on a two-bladed propeller.

An analysis is conducted where the Euler turbo-machinery equation is implemented to obtain the individual aerodynamic and Coriolis power components as indicated by Lewis (1996). For this purpose, the time- and circumferentially averaged up- and downstream velocity profiles are calculated from the numerical results for all flow rates and used in the analysis. It is found that aerodynamic work is the main contributor to the actuation of flow for near-design conditions, whereas the Coriolis component is nearly zero. However, an eventual decline in aerodynamic work can be observed as the flow rate is decreased, whereas an increase in Coriolis component is visible. The rate of decline in aerodynamic work is approximately proportional to the inverse of the rate of gain in Coriolis work at flow rates of $\phi \leq 0.137$. The aerody- 
namic and Coriolis component is equal at $\phi \approx 0.105\left(\phi / \phi_{D}=0.64\right)$. At flow coefficients of $\phi \leq 0.074$ the aerodynamic work is negative. This suggests that part of the fan functions as a turbine aerodynamically. On the contrary, the Coriolis work applied is positive and almost double the aerodynamic amount. Hence, the nett amount of work applied at low flow rates consists entirely of Coriolis work.

Lastly, the development of an improved actuator-disc model (ADM) is attempted, since its use would be computationally advantageous in the modelling of large fan systems in comparison to the use of full three-dimensional fan models. This model, termed the reverse engineered empirical actuator-disc model (REEADM), implemented the reverse-engineered lift and drag force characteristics instead of the two-dimensional lift and drag coefficients of the NASA-LS 0413 aerofoil implemented in the ADM. The radial force coefficients obtained from the P3DM are also added to the REEADM. The results obtained through this model show a good comparison to the fan characteristic determined by the P3DM at near-design flow rates. However, a deviation from these results is observed at low flow rates $(\phi<0.065)$, although an improvement can be observed when compared to the ADM. Possible reasons for this deviation are listed in chapter 9 .

\subsection{Recommended future research}

In view of the contributions made in the present study, the following future research is proposed:

- Although the results obtained through the P3DM in the present investigation compare well to the data of two different experiments, numerical results are not obtained for flow coefficients of $\phi<0.042$. It could be speculated that part of the reason for the occurrence of these small discrepancies might be due to rotating stall present in the fan at these low flow conditions, especially near the hub. A numerical study is proposed where the flow rate through all eight fan blades are modelled by timedependent analyses.

- Since much experience is gained in the present BSPM technique, it is proposed to extend this technique to enable time-dependent blade surface pressure measurements, that could be compared to the time-dependent numerical analyses.

- A study should be conducted to investigate the discrepancy in fan characteristic values as obtained by the acP3DM and REEADM at low flow rates. A possible reason for this discrepancy might be due to the velocities and blade forces being time- and circumferentially averaged before the blade sectional lift, drag and radial force coefficients are calculated. Hence, any non-linear phenomena such as vortical patterns occurring in 
the blade passage at low flow rates (chapter 6) are disregarded. It is therefore proposed that an actuator-line model be developed and compared to the results of models presented in this study. An actuator-line model, being time-dependent and having the ability to allow rotationally asymmetric flow, would possibly provide a more accurate flow solution at low flow rates. 


\section{List of References}

ANSYS Fluent Inc (2009). ANSYS Fluent 12.0 Users Guide. April. ANSYS Inc.

Augustyn, O. (2013). Experimental and numerical analysis of axial flow fans. Ph.D. thesis, University of Stellenbosch, South Africa.

Augustyn, O., Van der Spuy, S.J. and Von Backström, T.W. (2015). Numerical and experimental investigation into the accuracy of the fan scaling laws applied to large diameter axial flow fans. In: Fan2015, pp. 1-12. Lyon, France.

Bak, C., Johansen, J. and Andersen, P. (2006). Three-dimensional corrections of airfoil characteristics based on pressure distributions. In: Proc. Eur. Wind Energy Conf. Exhib., pp. 1-10. Athens, Greece. ISBN 9781622764679.

Available at: http://scholar.google.com/scholar?hl=en\&btnG=Search\&q= intitle:Three-Dimensional+Corrections+of+Airfoil+Characteristics+ Based+on+Pressure+Distributions\#0\$ $\backslash$ delimiter"026E30F \$nhttp: //scholar.google.com/scholar?hl=en\&btnG=Search\&q=intitle: Three-dimensional+corrections+of+airfoi

Bamberger, K. and Carolus, T. (2012). Optimization of axial fans with highly swept blades with respect to losses and noise reduction. In: Fan 2012, pp. 1-12. Senlis, France.

Bamberger, K. and Carolus, T. (2014). Performance prediction of axial fans by cfdtrained meta-models. In: ASME Turbo expo 2014, pp. 1-10. Dusseldorf, Germany.

Beiler, M.G. and Carolus, T.H. (1999). Computation and Measurement of the Flow in Axial Flow Fans With Skewed Blades. J. Turbomach., vol. 121, no. 1, p. 59. ISSN 0889504X.

Bianchi, S., Corsini, A., Mazzucco, L., Monteleone, L. and Sheard, A.G. (2012). Stall Inception, Evolution and Control in a Low Speed Axial Fan With Variable Pitch in Motion. J. Eng. Gas Turbines Power, vol. 134, no. 4, p. 042602. ISSN 07424795 .

Bianchi, S., Corsini, A. and Sheard, A. (2014). A Critical Review of Passive Noise Control Techniques in Industrial Fans. J. Eng. Gas Turbines Power, vol. 136, no. 4, pp. 044001-1 - 044001-9. ISSN 0742-4795.

Available at: http://gasturbinespower.asmedigitalcollection.asme.org/ article.aspx?doi=10.1115/1.4025837 
Bredell, J., Kroger, D.G. and Thiart, G. (2006 June). Numerical investigation of fan performance in a forced draft air-cooled steam condenser. Appl. Therm. Eng., vol. 26, no. 8-9, pp. 846-852. ISSN 13594311.

Bruining, A. and Timmer, W.A. (1992). Airfoil characteristics of rotating wind turbine blades. J. Wind Eng. Ind. Aerodyn., vol. 39, pp. 35-39.

Bruneau, P.R.P. (1994). The design of an axial flow fan for a cooling tower application. Masters Thesis, University of Stellenbosch, Stellenbosch, R.S.A.

Butterfield, C.P. and Nelsen, E.N. (1990). Aerodynamic Testing of a Rotating Wind Turbine Blade. In: Proc. Sol. Denver, Colorado.

Corsini, A., Delibra, G., Rispoli, F., Sheard, A.G. and Volponi, D. (2014). Investigation on anti-stall ring aerodynamic performance in an axial flow fan. In: $A S M E$ Turbo expo 2014, pp. 1-22. Dusseldorf, Germany.

Corsini, A. and Rispoli, F. (2004 January). Using sweep to extend the stall-free operational range in axial fan rotors. Proc. Inst. Mech. Eng. Part A J. Power Energy, vol. 218, no. 3, pp. 129-139. ISSN 0957-6509.

Available at: http://pia.sagepub.com/lookup/doi/10.1243/ 095765004323049869

Corsini, A. and Rispoli, F. (2005 June). Flow analyses in a high-pressure axial ventilation fan with a non-linear eddy-viscosity closure. Int. J. Heat Fluid Flow, vol. 26, no. 3, pp. 349-361. ISSN 0142727X.

Available at: http://linkinghub.elsevier.com/retrieve/pii/ S0142727X04001572

Corsini, A., Rispoli, F. and Sheard, A.G. (2010). Shaping of Tip End-Plate to Control Leakage Vortex Swirl in Axial Flow Fans. J. Turbomach., vol. 132, no. 3, p. 031005. ISSN 0889504X.

Available at: http://link.aip.org/link/JOTUEI/v132/i3/p031005/s1\&Agg= doi

Downie, R., Thompson, M. and Wallis, R. (1993 May). An engineering approach to blade designs for low to medium pressure rise rotor-only axial fans. Exp. Therm. Fluid Sci., vol. 6, no. 4, pp. 376-401. ISSN 08941777.

Available at: http://linkinghub.elsevier.com/retrieve/pii/ $089417779390016 \mathrm{C}$

Eberlinc, M., Širok, B. and Hocevar, M. (2009). Experimental investigation of the interaction of two flows on the axial fan hollow blades by flow visualization and hot-wire anemometry. Exp. Therm. Fluid Sci., vol. 33, pp. 929-937.

Estevadeordal, J., Gogineni, S., Copenhaver, W., Bloch, G. and Brendel, M. (2000). Flow field in a low-speed axial fan : a DPIV investigation. Exp. Therm. Fluid Sci., vol. 23, pp. 11-21.

Fike, M., Bombek, G., Hriberserk, M. and Hribernik, A. (2014). Visualisation of rotating stall in an axial flow fan. Exp. Therm. Fluid Sci., vol. 53, pp. 269-276. 
Goldsschagg, H. (1993). Lessons learned from the world's largest forced draft direct air-cooled condenser. In: EPRI Meet. Washington, USA.

Govardhan, M., Kumar, O.G.K. and Sitaram, N. (2007). Computational study of the effect of sweep on the performance and flow field in an axial flow compressor rotor. Proc. Inst. Mech. Eng. Part A J. Power Energy, vol. 221, no. 3, pp. 315-329. ISSN 0957-6509.

Guedel, A., Robitu, M. and Chaulet, V. (2013). Energy Efficiency of an Axial Fan for Various Casing Configurations. J. Eng. Gas Turbines Power, vol. 135, no. 7, p. 074501. ISSN 0742-4795.

Available at: http://gasturbinespower.asmedigitalcollection.asme.org/ article.aspx?doi=10.1115/1.4023751

Hand, M.M., Simms, D.a., Fingersh, L.J., Jager, D.W., Cotrell, J.R., Schreck, S. and Larwood, S.M. (2001). Unsteady Aerodynamics Experiment Phase VI : Wind Tunnel Test Configurations and Available Data Campaigns. Tech. Rep. December, NREL, Colorado, USA.

Hansen, M., Sø rensen, J., Voutsinas, S., Sø rensen, N. and Madsen, H. (2006 June). State of the art in wind turbine aerodynamics and aeroelasticity. Prog. Aerosp. Sci., vol. 42, no. 4, pp. 285-330. ISSN 03760421.

Available at: http://linkinghub.elsevier.com/retrieve/pii/ S0376042106000649

Himmelskamp, H. (1947). Profile investigations on a rotating airscrew. PhD Thesis, University of Göttingen.

Hinze, J. (1975). Turbulence. McGraw-Hill Publishing Co., New York.

Horvath, C. and Vad, J. (2009). Broadband noise source model acoustical investigation on unskewed and skewed axial flow fan rotor cascades. In: 14th Int. Conf. fluid flow Technol. Budapest, Hungary.

Hotchkiss, P.J., Meyer, C.J. and Von Backström, T.W. (2006 February). Numerical investigation into the effect of cross-flow on the performance of axial flow fans in forced draught air-cooled heat exchangers. Appl. Therm. Eng., vol. 26, pp. 200-208. ISSN 13594311.

Hurault, J., Kouidri, S. and Bakir, F. (2012 July). Experimental investigations on the wall pressure measurement on the blade of axial flow fans. Exp. Therm. Fluid Sci., vol. 40, pp. 29-37. ISSN 08941777.

Available at: http://linkinghub.elsevier.com/retrieve/pii/ S0894177712000192

Hurault, J., Kouidri, S., Bakir, F. and Rey, R. (2010 June). Experimental and numerical study of the sweep effect on three-dimensional flow downstream of axial flow fans. Flow Meas. Instrum., vol. 21, no. 2, pp. 155-165. ISSN 09555986.

Ilikan, A.N. and Ayder, E. (2014). Influence of dihedral stacking on the performance of an axial fan. Eng. Appl. Comput. fluid Mech., vol. 8, no. 4, pp. 518-529. 
Ilikan, A.N. and Ayder, E. (2015). Influence of the Sweep Stacking on the Performance of an Axial Fan. J. Turbomach., vol. 137, no. 6.

Jin, G., Ouyang, H. and Du, Z. (2013). Experimental investigation of unsteady flow in axial skewed fans according to flow rates. Exp. Therm. Fluid Sci., vol. 48, pp. 81-96. ISSN 08941777.

Available at: http://linkinghub.elsevier.com/retrieve/pii/ S0894177713000484

Johansen, J. and Sorensen, N.N. (2004 October). Aerofoil characteristics from 3D CFD rotor computations. Wind Energy, vol. 7, no. 4, pp. 283-294. ISSN 1095-4244. Available at: http://doi.wiley.com/10.1002/we.127

Joubert, R. (2010). Influence of geometric and environmental parameters on aircooled steam condenser performance. Masters thesis, University of Stellenbosch, Stellenbosch, R.S.A.

Kergourlay, G., Kouidri, S., Rankin, G. and Rey, R. (2006 October). Experimental investigation of the 3D unsteady flow field downstream of axial fans. Flow Meas. Instrum., vol. 17, no. 5, pp. 303-314. ISSN 09555986.

Kim, J.-h., Hur, N. and Kim, W. (2012). Development of algorithm based on the coupling method with CFD and motor test results to predict performance and efficiency of a fuel cell air fan. Renew. Energy, vol. 42, pp. 157-162. ISSN 0960-1481. Available at: http://dx.doi.org/10.1016/j.renene.2011.08.029

Kröger, D.G. (2004). Air-cooled heat exchangers and cooling towers. PennWell Corporation, Tulsa, USA.

Lanzafame, R., Mauro, S. and Messina, M. (2013 April). Wind turbine CFD modeling using a correlation-based transitional model. Renew. Energy, vol. 52, pp. 31-39. ISSN 09601481.

Available at: http://linkinghub.elsevier.com/retrieve/pii/ S0960148112006441

Launder, B.E. and Spalding, D.B. (1974). The numerical computation of turbulent flows. Comput. Methods Appl. Mech. Eng., vol. 3, pp. 269-289.

Lee, G.H., Baek, J.H. and Myung, H.J. (2003). Structure of Tip Leakage Flow in a Forward-Swept Axial-Flow Fan. Flow, Turbul. Combust. (formerly Appl. Sci. Res., vol. 70, no. 1-4, pp. 241-265. ISSN 1386-6184.

Available at: http://www.springerlink.com/openurl.asp?id=doi:10.1023/B: APPL. $0000004936.88816 . e e$

Lewis, R. (1996). Turbomachinery performance analysis. Arnold Publishers, London, UK.

Lewis, R.I. and Hill, J.M. (1971). The influence of sweep and dihedral in turbomachinery blade rows. J. Mech. Eng. Sci., vol. 13, no. 4, pp. 266-285. ISSN 0022-2542. 
Li, Y., Liu, J., Ouyang, H. and Du, Z. (2008 June). Internal flow mechanism and experimental research of low pressure axial fan with forward-skewed blades. $J$. Hydrodyn., vol. 20, no. 3, pp. 299-305. ISSN 10016058.

Li, Y., Ouyang, H. and Du, Z. (2007 October). Experimental research on aerodynamic performance and exit flow field of low pressure axial flow fan with circumferential skewed blades. J. Hydrodyn. Ser. B, vol. 19, no. 5, pp. 579-586. ISSN 10016058 .

Li, Y. and Zhou, Z. (2008). Investigation and numerical simulation of inner-flow of an axial mineflow fan under low flow rate conditions. J. China Univ. Min. Technol., vol. 18, no. December 2007, pp. 0107-0111.

Lindenburg, C. (2003). Investigation into Rotor Blade Aerodynamics: Analysis of the stationary measurements on the UAE phase-VI rotor in the NASA-Ames wind tunnel. Tech. Rep. July, NOVEM.

Available at: http://www.ecn.nl/docs/library/report/2003/c03025.pdf

Liu, S.H., Huang, R.F. and Lin, C.A. (2010). Computational and experimental investigations of performance curve of an axial flow fan using downstream flow resistance method. Exp. Therm. Fluid Sci., vol. 34, no. 7, pp. 827-837. ISSN 0894-1777.

Available at: http://dx.doi.org/10.1016/j.expthermflusci.2010.01.011

Longhouse, R. (1977 July). Vortex shedding noise of low tip speed, axial flow fans. J. Sound Vib., vol. 53, no. 1, pp. 25-46. ISSN 0022460X.

Available at: http://linkinghub.elsevier.com/retrieve/pii/ $0022460 \times 7790092 \mathrm{X}$

Louw, F.G. (2011). Performance Trends of a Large Air-Cooled Steam Condenser during Windy Conditions. Masters thesis, University of Stellenbosch, Stellenbosch, R.S.A.

Louw, F.G., Bruneau, P.R.P., Von Backström, T.W. and Van der Spuy, S.J. (2012). The design of an axial flow fan for application in large air-cooled heat exchangers. In: Proceeding ASME Turbo expo 2012, pp. 1-15. ASME Turbo, Copenhagen ,Denmark.

Louw, F.G., Von Backström, T.W. and Van der Spuy, S.J. (2014a). Investigation of the flow field in the vicinity of an axial flow fan during low flow rates. In: Proc. ASME Turbo expo 2014. Dusseldorf, Germany.

Louw, F.G., Von Backström, T.W. and Van der Spuy, S.J. (2014b). Modelling of the flow field in the vicinity of an axial flow fan, focusing on low flow rates. In: Proc. SACAM 2014, January, pp. 1-10. Somerset West, RSA.

Louw, F.G., Von Backström, T.W. and Van der Spuy, S.J. (2015a). Experimental investigation of the blade surface pressure distribution in an axial flow fan for a range of flow rates. In: Proc. ASME Turbo Expo 2015 Turbine Tech. Conf. Expo. Montreal, Canada. 
Louw, F.G., Von Backström, T.W. and Van der Spuy, S.J. (2015b). Lift and drag characteristics of an air-cooled heat exchanger axial flow fan. J. Fluids Eng., vol. 137, no. 8, pp. 081101-1 - 081101-9.

Maeda, T. and Kawabuchi, H. (2005). Surface Pressure Measurement on a Rotating Blade of Field Horizontal Axis Wind Turbine in Yawed Condition . JSME Int. J., vol. 48 , no. 1, pp. $156-163$.

McGhee, R.J. and Beasley, W.D. (1976). Effects of thickness on the aerodynamic characteristics of an initial low-speed family of airfoils for general aviation applications. Tech. Rep., National aeronautics and space administration, Langley research center, Hampton, Virginia, USA.

McKenzie, A.B. (1997). Axial flow fans and compressors. Ashgate publishing company, Burlington, Vermont, U.S.A.

Menter, F., Kuntz, M. and Langtry, R. (2003). Ten years of industrial experience with the SST turbulence model. Turbul. Heat Mass Transf., vol. 4.

Available at: http://cfd.mace.manchester.ac.uk/flomania/pds_papers/ file_pds-1068134610Menter-SST-paper.pdf

Meyer, C.J. and Kröger, D.G. (1998). Plenum chamber flow losses in forced draught air-cooled heat exchangers. Appl. Therm. Eng., vol. 18, pp. 875-893.

Meyer, C.J. and Kröger, D.G. (2001 August). Numerical simulation of the flow field in the vicinity of an axial flow fan. Int. J. Numer. Methods Fluids, vol. 36, no. 8, pp. 947-969. ISSN 0271-2091.

Meyer, C.J. and Kröger, D.G. (2004). A Numerical Investigation of the Errors Associated with the Scaling of Axial Flow Fan Performance Characteristics. $R D$ Journal, SA Mech. Eng., vol. 20, no. 2, pp. 16-24.

Morris, S.C., Good, J.J. and Foss, J.F. (1998). Velocity measurements in the wake of an automotive cooling fan. Exp. Therm. Fluid Sci., vol. 17, pp. 100-106.

Owen, M.T.F. and Kröger, D.G. (2010 November). The effect of screens on air-cooled steam condenser performance under windy conditions. Appl. Therm. Eng., vol. 30, no. 16, pp. 2610-2615. ISSN 13594311.

Pelz, P.F. (2013). The Influence of Reynolds Number and Roughness on the Efficiency of Axial and Centrifugal FansA Physically Based Scaling Method. J. Eng. Gas Turbines Power, vol. 135, no. 5, p. 052601. ISSN 0742-4795.

Available at: http://gasturbinespower.asmedigitalcollection.asme.org/ article.aspx?doi=10.1115/1.4022991

Pericleous, K. and Patel, M. (1987). The modeling of tangential and axial agitators in chemical reactors. Physicochem. Hydrodyn., vol. 8, no. 2, pp. 105-123.

Ramakrishna, P.V. and Govardhan, M. (2009). Study of sweep and induced dihedral effects in subsonic axial flow compressor passages - Part I: Design considerations Changes in incidence, deflection, and streamline curvature. Int. J. Rotating Mach., vol. 2009, pp. 1-11. ISSN 1023621X. 
Ramakrishna, P.V. and Govardhan, M. (2011). On loading corrections and loss distributions in low-speed forward swept axial compressor rotors. Proc. Inst. Mech. Eng. Part A J. Power Energy, vol. 225, no. 1, pp. 120-130. ISSN 0957-6509.

Roehle, I., Schodl, R., Voigt, P. and Willert, C. (2000). Recent developments and applications of quantitative laser light sheet measuring techniques in turbomachinery components. Meas. Sci. Technol., vol. 11, pp. 1023-1035.

Salunkhe, P.B., Joseph, J. and Pradeep, A. (2011 September). Active feedback control of stall in an axial flow fan under dynamic inflow distortion. Exp. Therm. Fluid Sci., vol. 35, no. 6, pp. 1135-1142. ISSN 08941777.

Available at: http://linkinghub.elsevier.com/retrieve/pii/ S0894177711000586

Salunkhe, P.B. and Pradeep, A.M. (2009 January). Stall inception and its control in an axial flow fan under dynamic inflow distortion. Proc. Inst. Mech. Eng. Part A J. Power Energy, vol. 224, no. 3, pp. 383-398. ISSN 0957-6509.

Sarraf, C., Nouri, H., Ravelet, F. and Bakir, F. (2011 May). Experimental study of blade thickness effects on the overall and local performances of a Controlled Vortex Designed axial-flow fan. Exp. Therm. Fluid Sci., vol. 35, no. 4, pp. 684-693. ISSN 08941777 .

Schreck, S.J. (2007). Rotationally Augmented Flow Structures and Time Varying Loads on Turbine Blades Preprint. In: 45th AIAA Aerosp. Sci. Meet. Exhib. Wind Energy Symp., January. Reno, Nevada.

Seo, S.-J., Choi, S.-M. and Kim, K.-Y. (2008). Design optimization of a low-speed fan blade with sweep and lean. Proc. Inst. Mech. Eng. Part A J. Power Energy, vol. 222, no. 1, pp. 87-92. ISSN 0957-6509.

Sheard, A.G., Corsini, A. and Bianchi, S. (2011). Stall Warning in a Low-Speed Axial Fan by Visualization of Sound Signals. J. Eng. Gas Turbines Power, vol. 133, no. 4, p. 041601. ISSN 07424795.

Available at: http://link.aip.org/link/JETPEZ/v133/i4/p041601/s1\&Agg= doi

Shih, T.-H., Liou, A., Shabbir, A., Yang, Z. and Zhu, J. (1994). A new k-e eddy viscosity model for high Reynolds number turbulent flows - Model development and validation. Inst. Comput. Mech. Propuls. Cent. Model. Turbul. Transit.

Smith, L. and Yeh, H. (1963). Sweep and dihedral effects in axial-flow turbomachinery. J. basic Eng., pp. 401-415.

Stella, A., Guj, G. and Di Felice, F. (2000 January). Propeller wake flowfield analysis by means of LDV phase sampling techniques. Exp. Fluids, vol. 28, no. 1, pp. 1-10. ISSN 0723-4864.

Available at: http://www.springerlink. com/openurl.asp?genre=article\&id= doi: $10.1007 / \mathrm{s} 003480050001$ 
Stinnes, W.H. and Von Backström, T.W. (2002). Effect of cross-flow on the performance of air-cooled heat exchanger fans. Appl. Therm. Eng., vol. 22, pp. $1403-1415$.

Tangler, J.L. (2004 July). Insight into wind turbine stall and post-stall aerodynamics. Wind Energy, vol. 7, no. 3, pp. 247-260. ISSN 1095-4244.

Available at: http://doi.wiley.com/10.1002/we.122

Thiart, G.D. (1990). A numerical procedure for predicting the effects of distorted inflow conditions on the performance of axial flow fans. Ph.D thesis, University of Stellenbosch, Stellenbosch, R.S.A.

Thiart, G.D. and Von Backström, T.W. (1993 May). Numerical simulation of the flow field near an axial flow fan operating under distorted inflow conditions. $J$. Wind Eng. Ind. Aerodyn., vol. 45, no. 2, pp. 189-214. ISSN 01676105.

Ubaldi, M., Zunino, P. and Cattanei, A. (1994 August). Relative flow and turbulence measurements within and downstream of an axial flow rotor. Exp. Therm. Fluid Sci., vol. 9, no. 2, pp. 186-196. ISSN 08941777.

Available at: http://linkinghub.elsevier.com/retrieve/pii/ 0894177794901112

Vad, J. (2008 February). Aerodynamic effects of blade sweep and skew in low-speed axial flow rotors at the design flow rate: an overview. Proc. Inst. Mech. Eng. Part A J. Power Energy, vol. 222, no. 1, pp. 69-85. ISSN 0957-6509.

Available at: http://journals.pepublishing.com/openurl.asp?genre= article\&id=doi : 10.1243/09576509JPE471

Vad, J. and Bencze, F. (1998 December). Three-dimensional flow in axial flow fans of non-free vortex design. Int. J. Heat Fluid Flow, vol. 19, no. 6, pp. 601-607. ISSN 0142727X.

Vad, J., Bencze, F., Corsini, A. and Rispoli, F. (2001). Non-free vortex effects in axial flow rotor. Period. Polytech. Ser. Mech. Eng., vol. 45, no. 2, pp. 201-216.

Vad, J., Kwedikha, A.R.A., Horváth, C., Balczó, M., Lohász, M.M. and Régert, T. (2007 January). Aerodynamic effects of forward blade skew in axial flow rotors of controlled vortex design. Proc. Inst. Mech. Eng. Part A J. Power Energy, vol. 221, no. 7, pp. 1011-1023. ISSN 0957-6509.

Available at: http://journals.pepublishing.com/openurl.asp?genre= article\&id=doi : 10 .1243/09576509JPE420

Vad, J., Kwedikha, A.R.A. and Jaberg, H. (2006 January). Effects of blade sweep on the performance characteristics of axial flow turbomachinery rotors. Proc. Inst. Mech. Eng. Part A J. Power Energy, vol. 220, no. 7, pp. 737-749. ISSN 0957-6509.

Available at: http://journals.pepublishing.com/openurl.asp?genre= article\&id=doi:10.1243/09576509JPE249 
Van der Spuy, S.J., Le Roux, F.N., Von Backström, T.W. and Kroger, D.G. (2011). The simulation of an axial flow fan performance curve at low flow rates. In: ASME Turbo expo 2011, pp. 425-434. Vancouver, British Columbia, Canada.

Van Niekerk, C.G. (1958). Ducted fan design theory. J. Appl. Mech.

Van Rooyen, J.A. and Kröger, D.G. (2008). Performance Trends of an Air-Cooled Steam Condenser Under Windy Conditions. J. Eng. Gas Turbines Power, vol. 130, no. 2, pp. 023006-1 - 023006-7. ISSN 07424795.

Venter, S. and Kröger, D. (1992). The effect of tip clearance on the performance of an axial flow fan. Energy Convers. Manag., vol. 33, no. 2, pp. 89-97. ISSN 01968904 .

Venter, S.J. (1990). The effectiveness of axial flow fans in A-frame plenums. Ph.D Thesis, University of Stellenbosch, Stellenbosch, R.S.A.

Versteeg, H. and Malalasekera, W. (2007). An introduction to computational fluid dynamics. 2nd edn. Pearson Prentice Hall, Harlow, England.

Wadia, A.R., Szucs, P.N. and Crall, D.W. (1998). Inner Workings of Aerodynamic Sweep. J. Turbomach., vol. 120, no. 4, p. 671. ISSN 0889504X.

Wallis, R. (1983). Axial flow fans and ducts. John Wiley \& Sons Inc.

Walters, D.K. and Cokljat, D. (2008). A Three-Equation Eddy-Viscosity Model for Reynolds-Averaged NavierStokes Simulations of Transitional Flow. J. Fluids Eng., vol. 130, no. 12, p. 121401. ISSN 00982202.

Available at: http://fluidsengineering.asmedigitalcollection.asme.org/ article.aspx?articleid $=1478203$

Wernet, M.P. (2000 February). Development of digital particle imaging velocimetry for use in turbomachinery. Exp. Fluids, vol. 28, no. 2, pp. 97-115. ISSN 0723-4864. Available at: http://www.springerlink.com/openurl.asp?genre=article\&id= doi: $10.1007 / \mathrm{s} 003480050015$

Wilkinson, M.B. and Van der Spuy, S.J. (2015). The effect of fan tip configuration on air-cooled condenser axial flow fan performance. In: Fan2015, pp. 1-12. Lyon, France.

Wright, T. and Simmons, W.E. (1990). Blade Sweep for Low-Speed Axial Fans. J. Turbomach., vol. 112, no. 1, pp. 151-158.

Wu, Y., Jin, G., Ouyang, H. and Du, Z. (2010). Experimental Investigations on Tip Leakage Flow and Noise in Skewed Blades. In: 16th AIAA/CEAS Aeroacoustics Conf., pp. 1-14. Stockholm, Sweden.

Xu, C. and Amano, R.S. (2002 November). Unsteady Pressure Field Investigation of an Axial Fan Blade Unsteady Pressure Field Measurement. Int. J. Rotating Mach., vol. 8, no. 6, pp. 385-395. ISSN 1023-621X.

Available at: http://www.informaworld.com/openurl?genre=article\&doi=10 . 1080/10236210214327\&magic $=$ crossref | | D404A21C5BB053405B1A640AFFD44AE3 
Yang, B. and Gu, C.G. (2011). Research on the Multi-variable Optimization of Forward-skewed Blade. Int. J. turbo jet-engines, vol. 28, pp. 273-284.

Yang, B. and Gu, C.G. (2013). The Effects of Radially Distorted Incident Flow on Performance of Axial-Flow Fans With Forward-Skewed Blades. J. Turbomach., vol. 135, no. 1. ISSN 0889504X.

Ye, X., Li, P., Li, C. and Ding, X. (2015). Numerical investigation of blade tip grooving effect on performance and dynamics of an axial flow fan. Energy, vol. 82, pp. 556-569. ISSN 03605442.

Available at: http://linkinghub.elsevier.com/retrieve/pii/ S0360544215000894

Yoon, J. and Lee, S. (2004 October). Stereoscopic PIV measurements of flow behind an isolated low-speed axial-fan. Exp. Therm. Fluid Sci., vol. 28, no. 8, pp. 791-802. ISSN 08941777.

Available at: http://linkinghub.elsevier.com/retrieve/pii/ S0894177703001638

Yu, G., Shen, X., Zhu, X. and Du, Z. (2011 January). An insight into the separate flow and stall delay for HAWT. Renew. Energy, vol. 36, no. 1, pp. 69-76. ISSN 09601481 .

Available at:

http://linkinghub.elsevier.com/retrieve/pii/

S0960148110002557 


\section{Appendices}




\section{Appendix A}

\section{B2a-fan Specifications}

The B2a-fan used in the present study (depicted in figure A.1) is based on the B2-fan designed by Bruneau (1994) and also described by Louw et al. (2012). The basic dimensions and design parameters of the fan are given in section A.1. Hereafter, the specific aerofoil implemented and its Lift/Drag-characteristics are presented, as well as some comments on the blade shape in sections A.2 and A.3. Lastly some details are given on the construction of the fan used in the present study in section A.4.
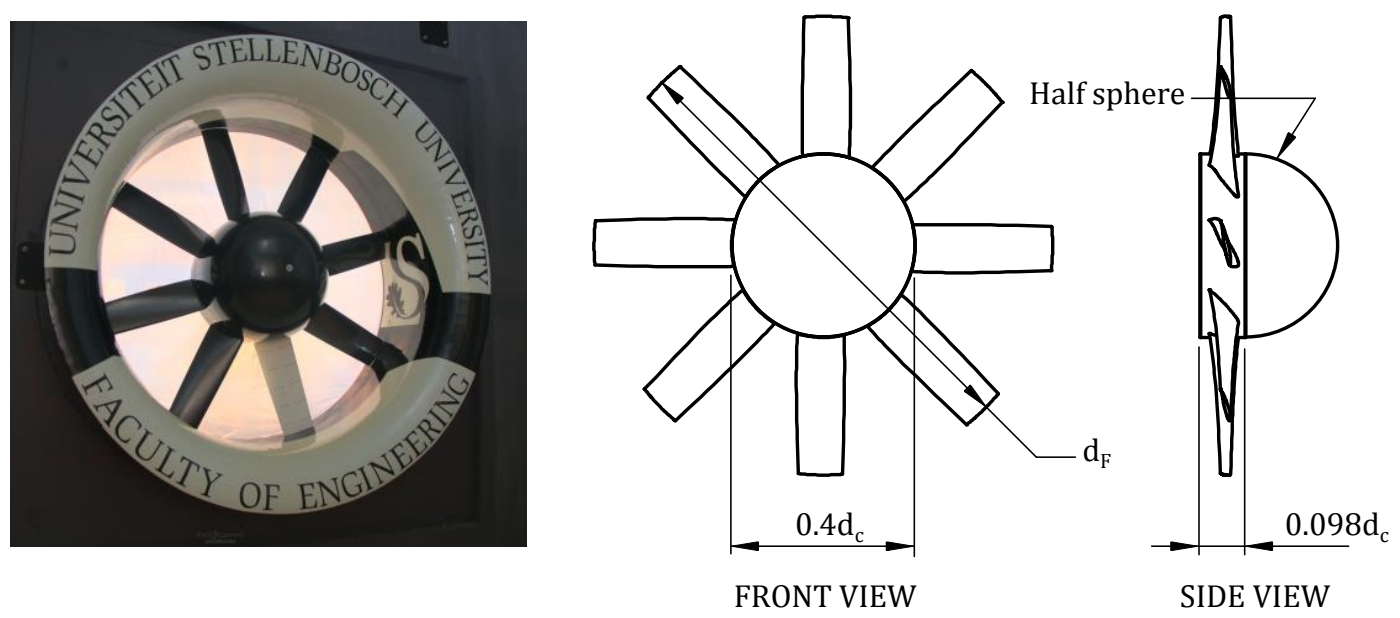

Figure A.1: Photo and schematic of the B2a-fan

\section{A.1 Design specifications}

Density:

Fan static pressure:

Volumetric flow rate:

$$
\begin{aligned}
& \rho_{a}=1.2 \mathrm{~kg} / \mathrm{m}^{3} \\
& \Delta p_{F s, D}=210 \mathrm{~Pa}\left(\Psi_{F s, D}=0.095\right) \\
& \dot{V}_{D}=16 \mathrm{~m}^{3} / \mathrm{s}\left(\phi_{D}=0.168\right)
\end{aligned}
$$


Fan static efficiency:

$$
\begin{aligned}
& \eta_{F s, D}=0.638 \\
& d_{c}=1.543 \mathrm{~m} \\
& x_{h}=0.4 \\
& c_{t}=3 \mathrm{~mm} \\
& n_{b}=8 \\
& N=750 \mathrm{rpm}(\omega=78.54 \mathrm{rad} / \mathrm{s}) \\
& z_{h}=150 \mathrm{~mm}
\end{aligned}
$$

Casing diameter:

Hub-tip ratio:

Tip clearance:

Number of blades:

Rotational speed:

Hub thickness:

Stagger angle (based on hub chord): $\quad \xi_{h}=59^{\circ}$

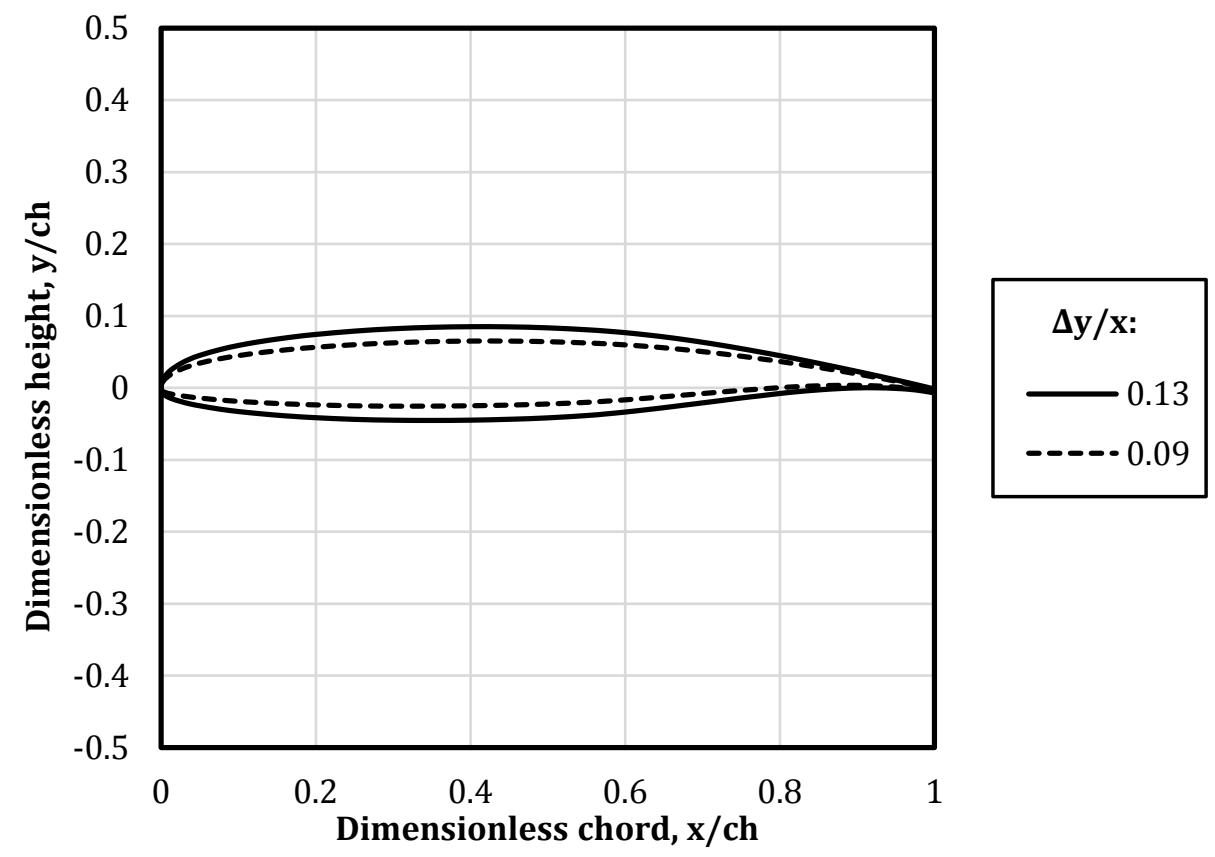

Figure A.2: NASA LS 0413 and 0409 aerofoil profiles (McGhee and Beasley, 1976)

\section{A.2 Aerofoil}

The NASA LS 0413 aerofoil (McGhee and Beasley, 1976) pictured in A.2 is used in the design of the B2a-fan blade. The aerofoil characteristics, shown in figure A.3, has a high lift to drag ratio (Bruneau, 1994) and a maximum thickness of $\Delta y / x=0.13$ at a dimensionless chord length of $c h=0.4$ from the leading edge. A spanwise linear distribution of aerofoil sections exists between the root and tip of the blade, enabling a lighter blade at higher span 
lengths. The blade therefore consists of a $\Delta y / x=0.13$ thickness foil at the root, tapering linearly off to a thickness of $\Delta y / x=0.09$ at the tip.

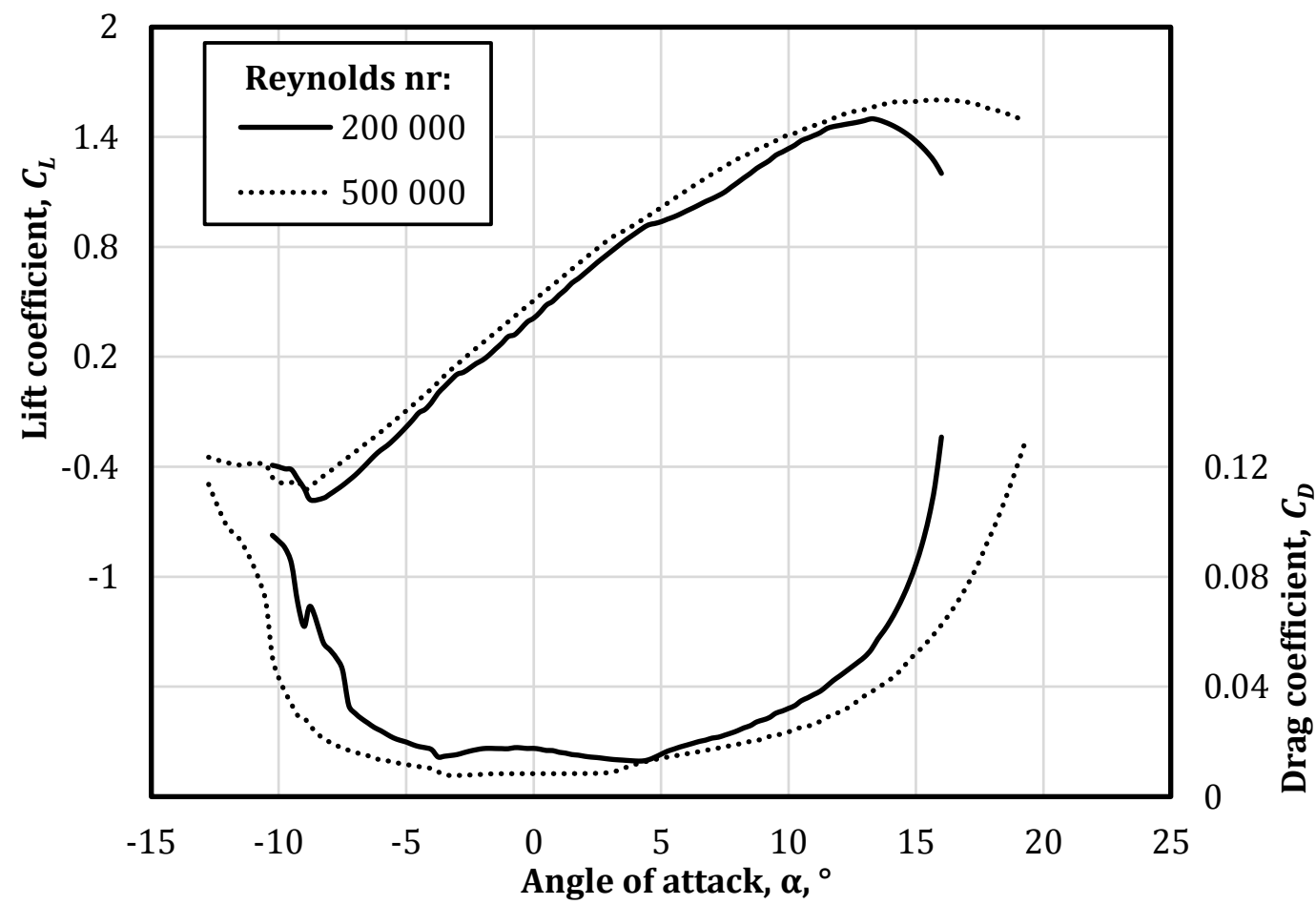

Figure A.3: Lift $\left(C_{L}\right)$ and Drag $\left(C_{D}\right)$ coefficients of the NASA LS 0413 aerofoil (McGhee and Beasley, 1976) profile versus angle of attack $(\alpha)$ as determined using XFOIL

\section{A.3 Blade shape}

The fan blade shape is a near free-vortex design between the hub and casing of the fan. The hub to casing ratio is chosen by means of an optimization process as discussed in Louw et al. (2012).

The advantage of a free-vortex designed fan is its relative simplicity to develop compared to controlled vortex designs, where other factors such as radial flow through the fan and variable outlet total pressure gradients should also be accounted for. However, a penalty of this advantage is the excessive blade twist that occurs, especially near the blade root. This has the potential of creating a small margin before stall occurs, especially since blade stall starts at the root and progresses outward towards the tip (Bruneau, 1994).

In the present case a free-vortex design is firstly established for the given design point values. Hereafter the stagger angles of the blade sections between midspan and the root were slightly decreased by a method discussed in Louw 


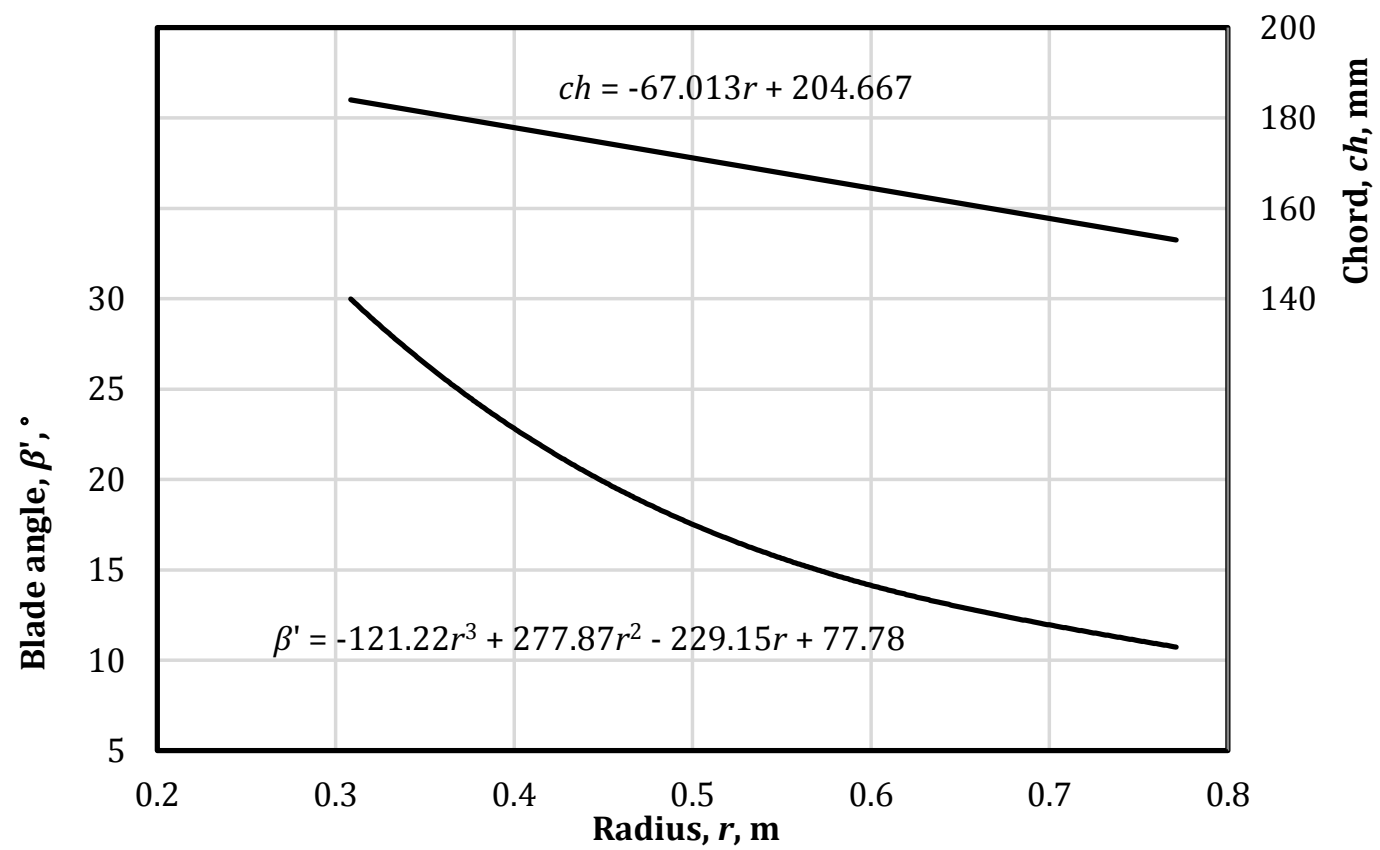

Figure A.4: B2a-fan spanwise chord and blade angle distribution (Louw et al., 2012)

et al. (2012). This effectively reduces the angle of attack at this blade section, creating a larger fan operating margin, before stall starts occurring. The spanwise blade chord length $(c h)$ and angle $\left(\beta^{\prime}\right)$ distribution are depicted in figure A.4, where:

$$
\beta^{\prime}=90^{\circ}-\xi \quad\left[{ }^{\circ}\right]
$$

and $\xi$ is the blade stagger angle.

\section{A.4 B2a-fan construction}

The construction of the B2a-fan comprises three assemblies, being the fan blades, hub and nose fairing. These are discussed in the following sections:

\section{A.4.1 Fan blade construction}

Due to the size of the fan it was decided to manufacture the fan blades from carbon fibre composite material. The blade manufacturing process is discussed with reference to figure A.5.

To maintain a high accuracy between repeated mouldings of the fan blades, an aluminium mould is cut by means of computer numerical control (CNC) milling. This mould consists of two parts, one being for the pressure side and another for the suction side of the blade (visible in figure A.5). 


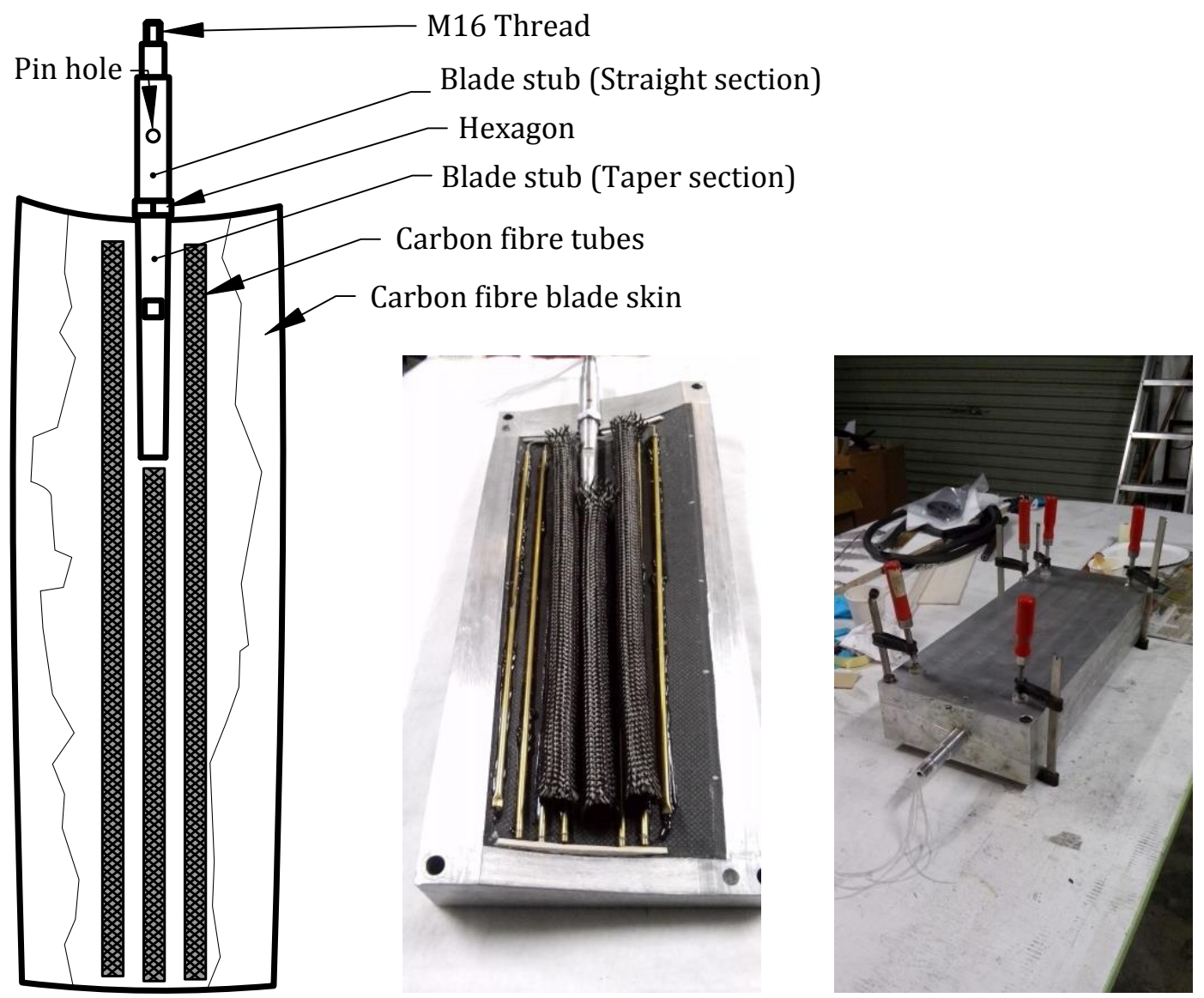

Figure A.5: B2a-fan blade construction

Each fan blade also has a CNC machined aluminium stub to connect the blade to the fan hub. The stub consists of a tapered section, located inside the blade and a straight section outside the blade. The sole purpose of the tapered section is to provide a bonding surface for the carbon fibre skins. The straight section consists of a hexagon crown at the blade root, a pin hole and a threaded part at the tip of which the purpose will be discussed shortly.

Before each moulding operation the moulds are waxed with a releasing agent, keeping the carbon fibre skins from sticking to the mould after curing has occurred. The layup consists of two layers of plain weaved carbon fibre sheets that are laminated in place under vacuum with epoxy resin.

After the first curing process has been concluded, the laminated skins are de-burred and cleaned, but not removed from their individual moulds, in preparation of the final joining between the suction and pressure side skins. Subsequently, the aluminium stub is put into place by locating the hexagon in a purpose-made position inside the mould and fixing it to this position with a pin through the pinholes of the stub and the mould. This orientation of the stub is important, since the hexagon forms part of a mechanism in the fan hub that is used for blade angle adjustment. All blades are therefore identical with regards to blade shape and the position of the stub. Additional epoxy 
resin is then spread over the taper part of the stub and sanded to remove the oxidized surface of the aluminium, creating a stronger bond. Three foam filled braided carbon fibre tubes are then impregnated with epoxy and positioned in the mould. Lastly, epoxy is spread around the edges of the blade surfaces after which the two parts of the mould is closed. The foam inside the braided carbon fibre tubes maintains an outward pressure causing a bonding between the tubes and the two laminated skins. These pressure and suction surfaces of the blade are held at the correct orientation to each other by means of four pins fitted through purpose-made pinholes at each corner of the mould. Subsequently a second round of curing takes place after which the blade is completed.
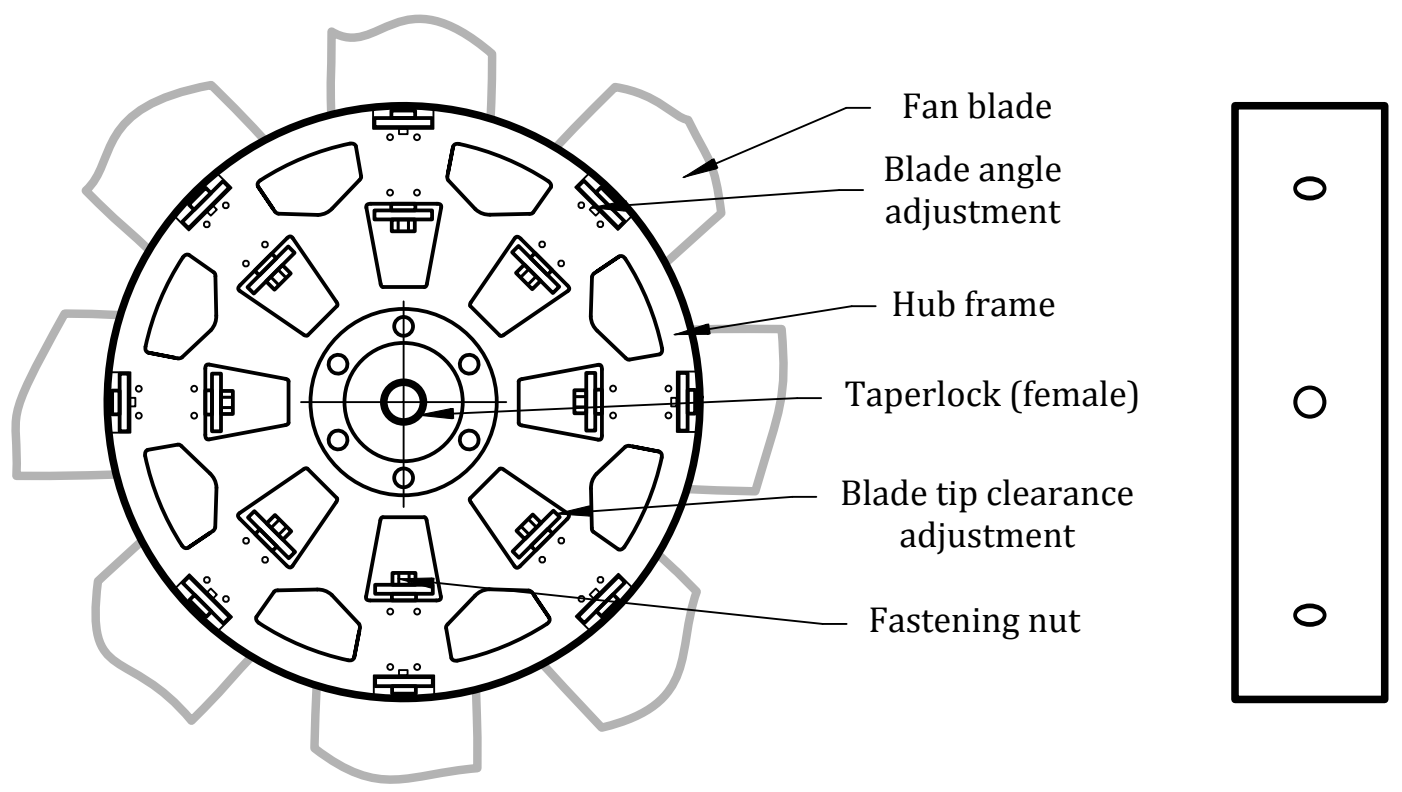

FRONT VIEW

SIDE VIEW

Figure A.6: B2a-fan hub construction

\section{A.4.2 Hub construction}

The fan hub depicted in figure A.6 (and also visible in figure 3.3b) is constructed (mainly from aluminium) to mount the eight B2a-fan blades. The hub conveniently has two mechanisms enabling the user to adjust the blade angle and tip clearance for investigation of the effect these two aspects would have on fan performance.

The blades are mounted through the holes on the side of the hub, where the blade stub is constrained within a sleeve. The male hexagonal part on the stub of the blade fits precisely into its female counterpart in the hub. This female part, forming part of the blade angle adjustment mechanism, keeps the blade at the set angle. A single M16 nut on the end of each blade's stub fixes 


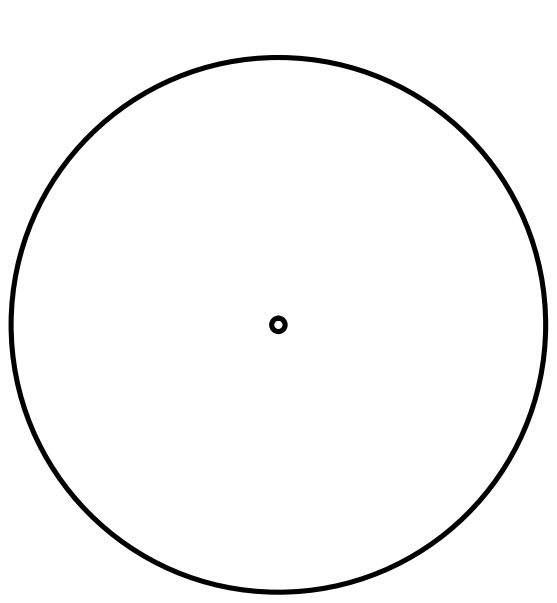

FRONT VIEW

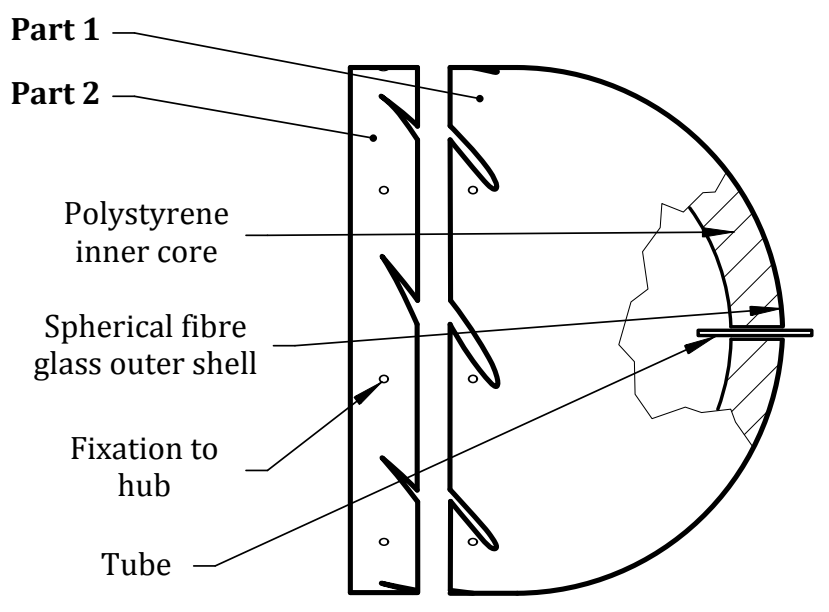

SIDE VIEW

Figure A.7: B2a-fan nose fairing construction

the blade in place and keeps it from dislodging in the radial direction. Lastly, a female taper lock fitting is mounted in the center of the hub, allowing it to be attached to the drive shaft in the test facility.

\section{A.4.3 Nose fairing construction}

The third part of the fan assembly is the spherical nose fairing. As can be observed in figure A.7, the fairing consists of two parts named part 1 and 2 . The reason for two parts is to simplify the regular dismounting of the nose fairing, necessary for the blade surface pressure measurements, discussed in section 3.2, without dismounting the entire fan. Part 2 of the fairing, fixed to the downstream end of the hub, ensures a continuous transition between the surfaces of part 1 and 2. These parts are each fastened to the hub by means of eight countersunk capscrews.

Part 1 is constructed by first cutting a dome shape of polystyrene and overlaying it with a glass fibre layup to provide structural strength. The surface is then filled, sanded, prepared and sprayed to provide a smooth surface finish. Part 2 is a band constructed solely of glass fibre and finished in the same fashion as part 1 . The $6 \mathrm{~mm}$ diameter tube, mounted on the axis-centre of part 2, is required for the blade surface pressure measurements that will be conducted. 


\section{Appendix B}

\section{Summarised literature}

All the literature obtained with regards to experimental measurements in applicable turbo-machinery is summarized in table B.1 and B.2. The numerical analyses found in literature are summarized in table B.3. If the information of the specific fans in the numerical investigations are not already given in table B.2, they are listed in table B.4.

The numbers in superscript next to the name(s) of certain authors in the first column of each table draw a link between different publications in which the same turbo-machine is used. E.g. all the authors with a number " 1 " in superscript next to their name(s) analysed the same machine. 


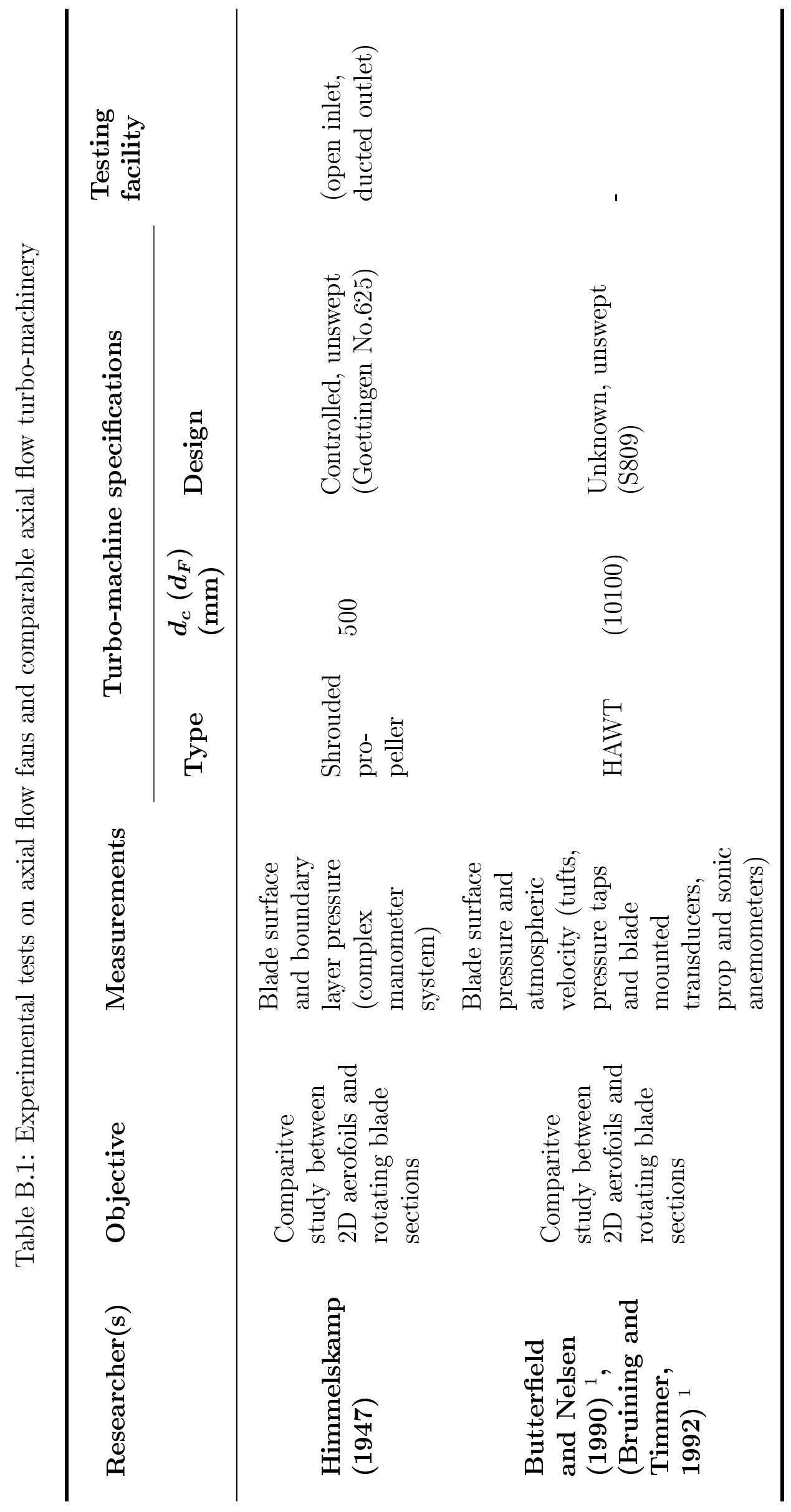




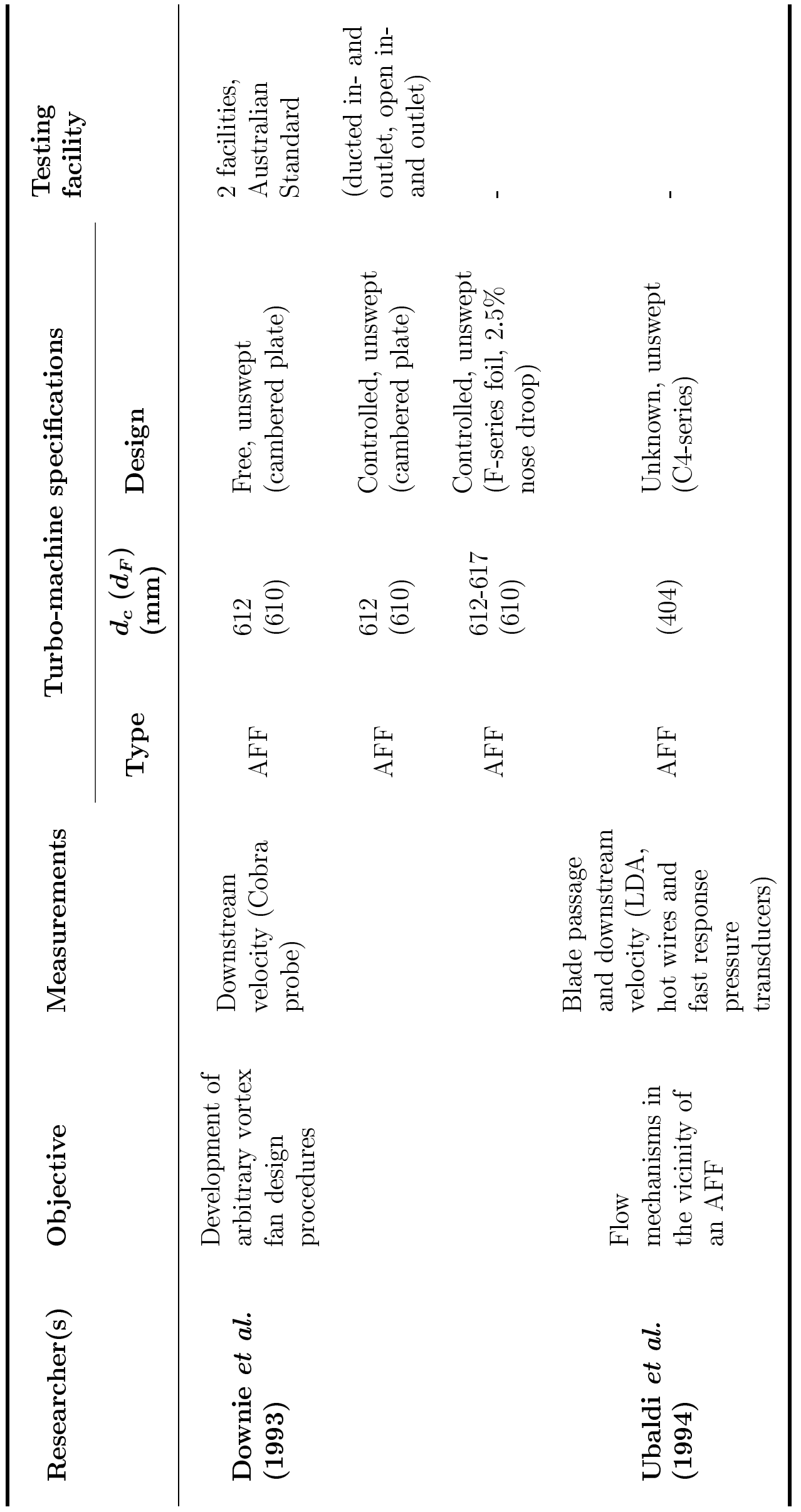




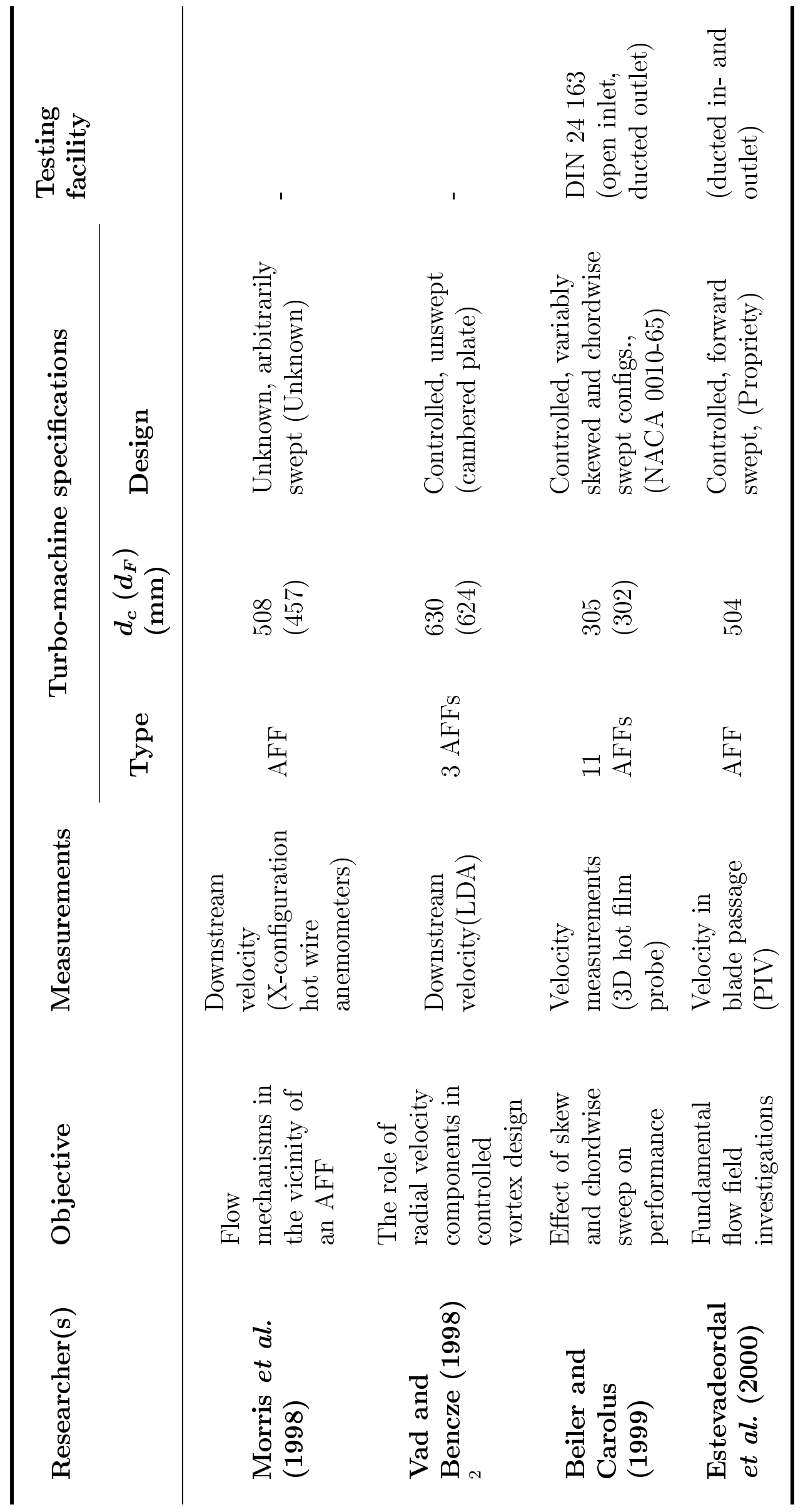




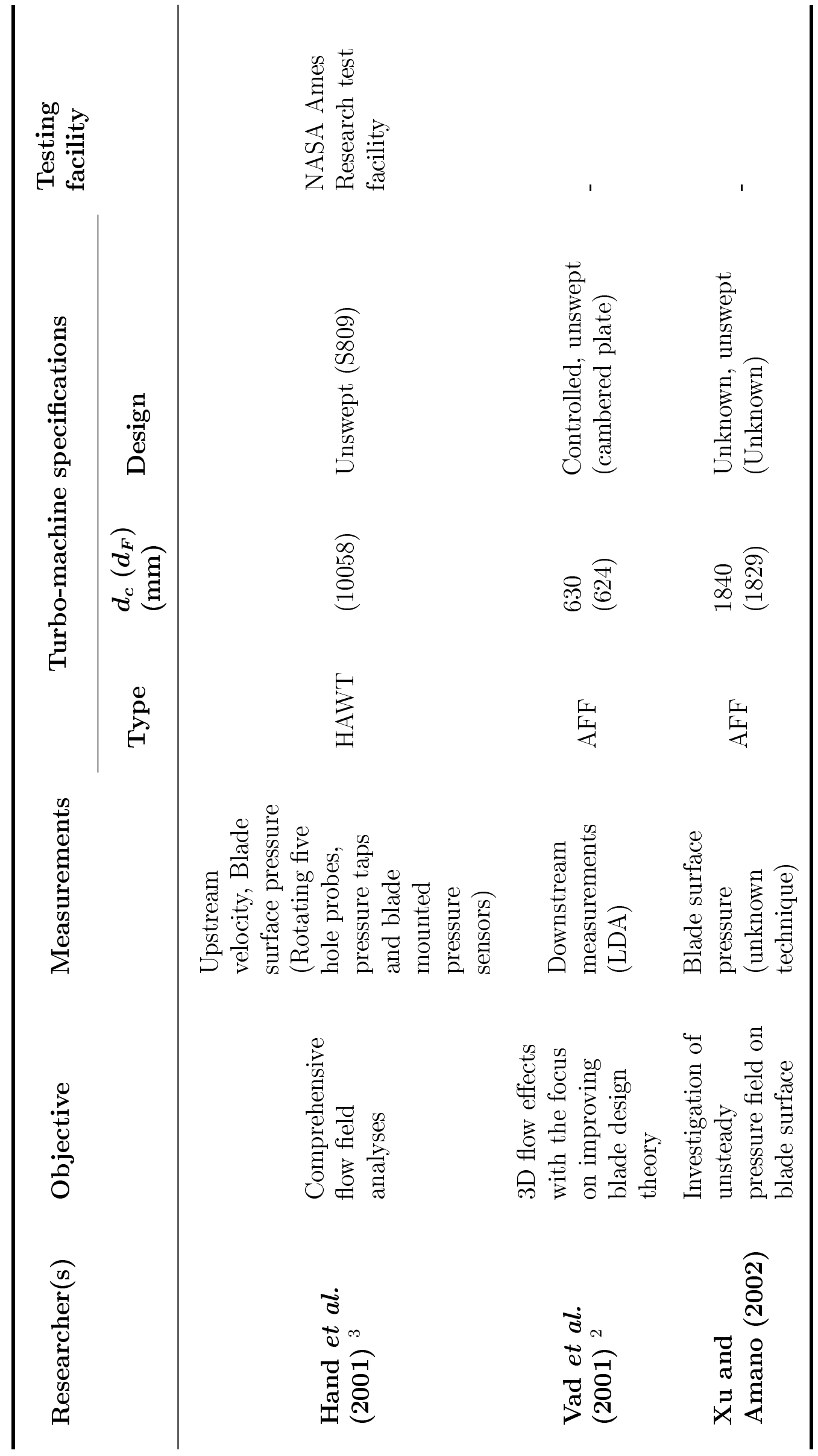




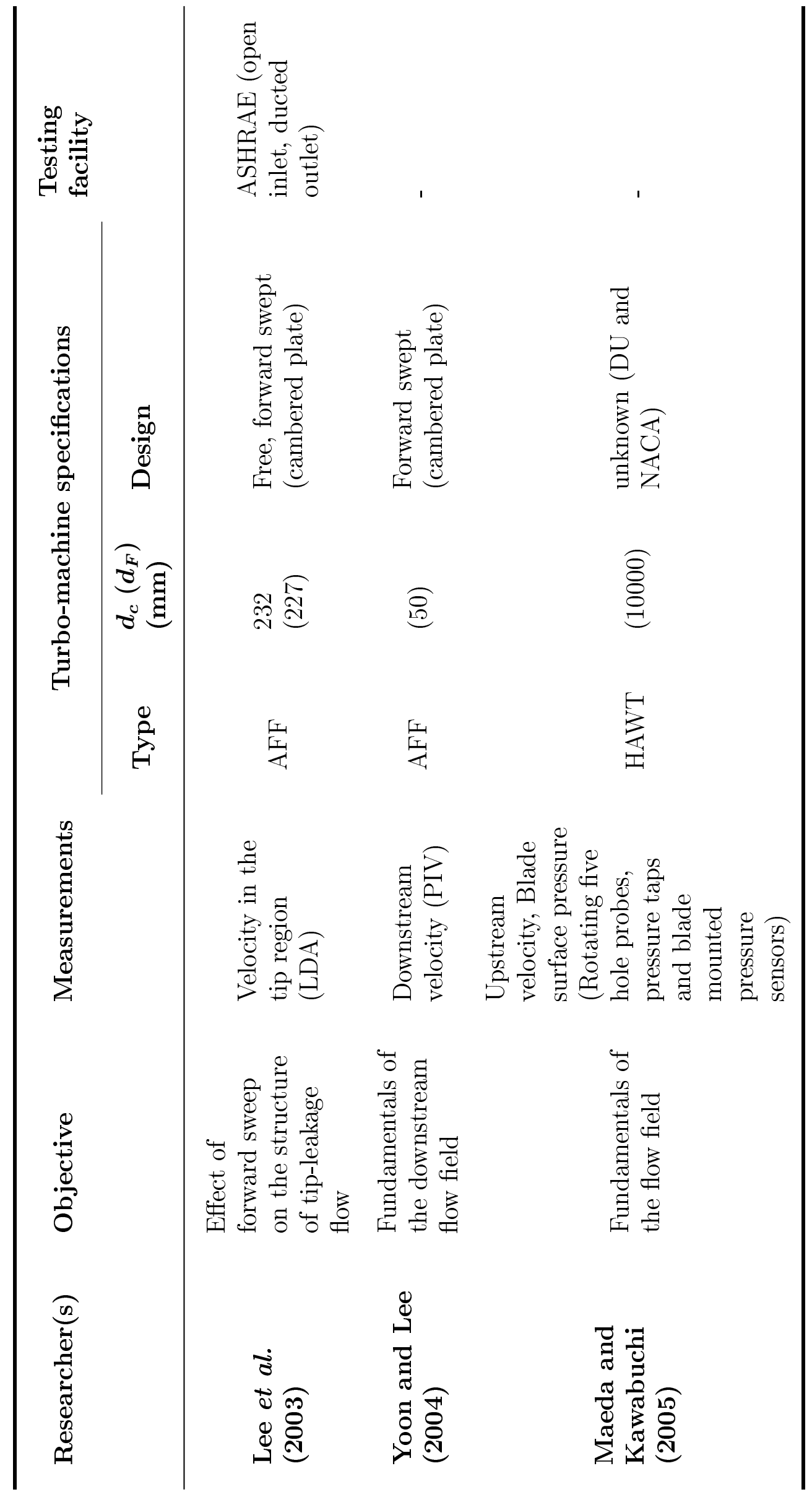




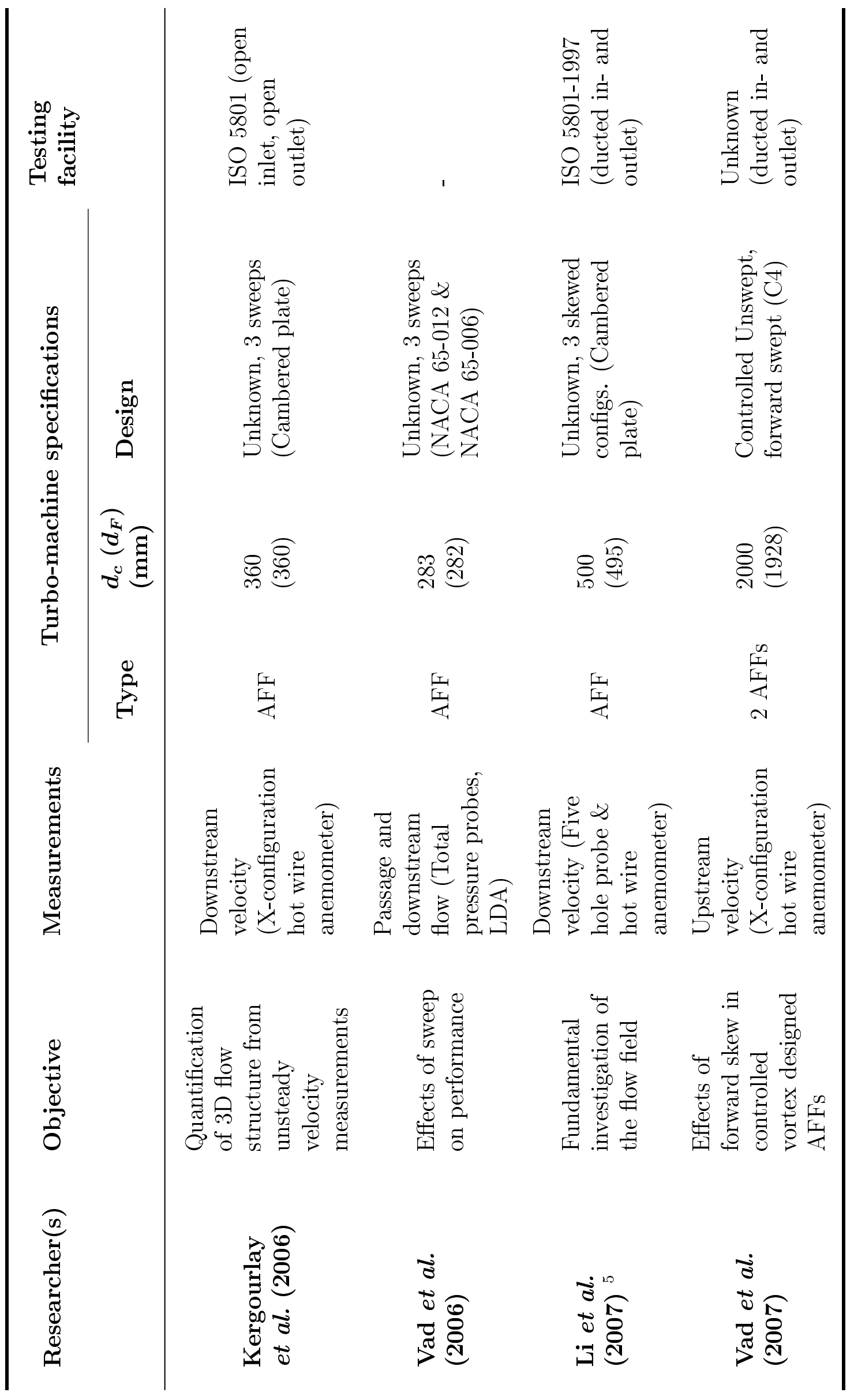


APPENDIX B. SUMMARISED LITERATURE

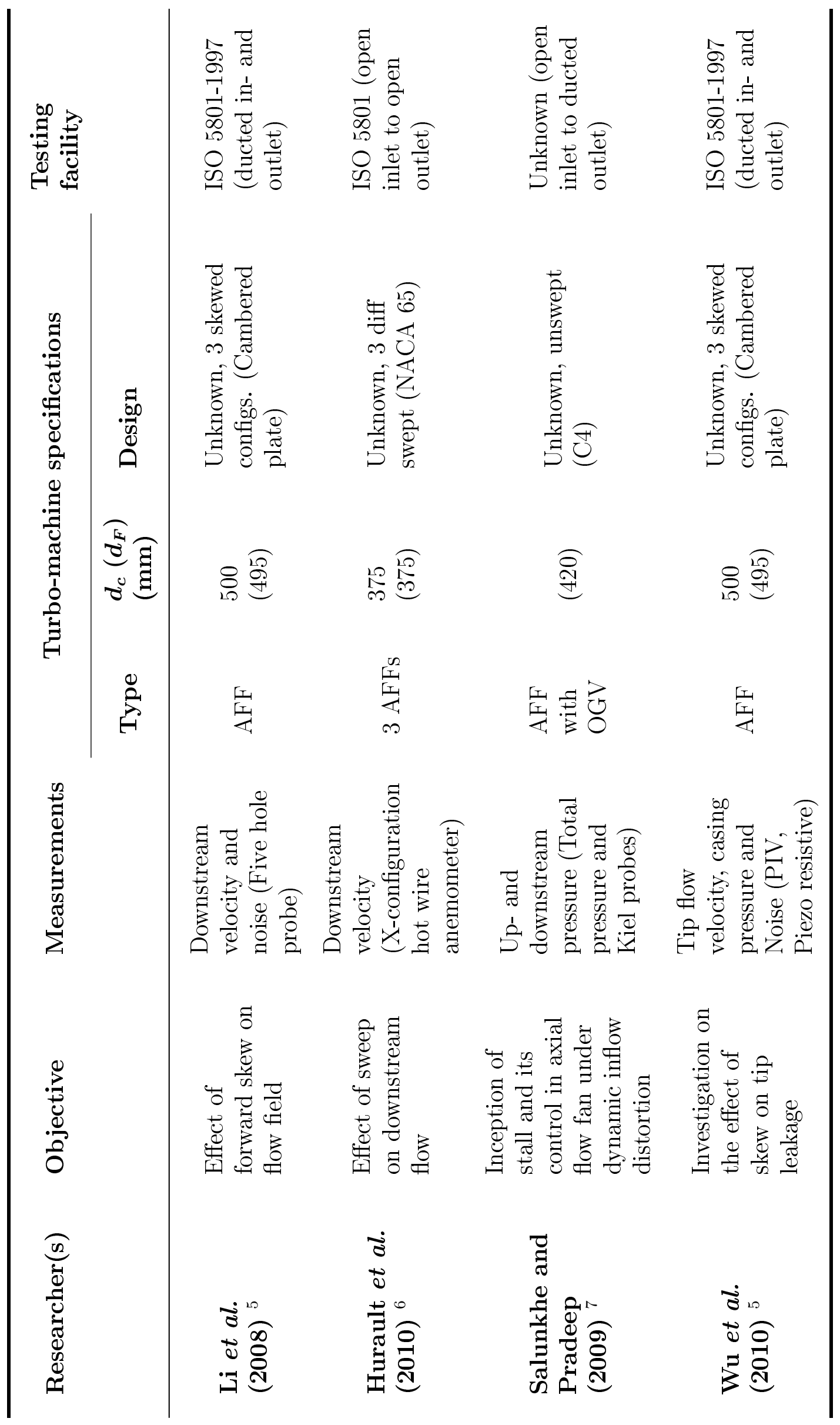




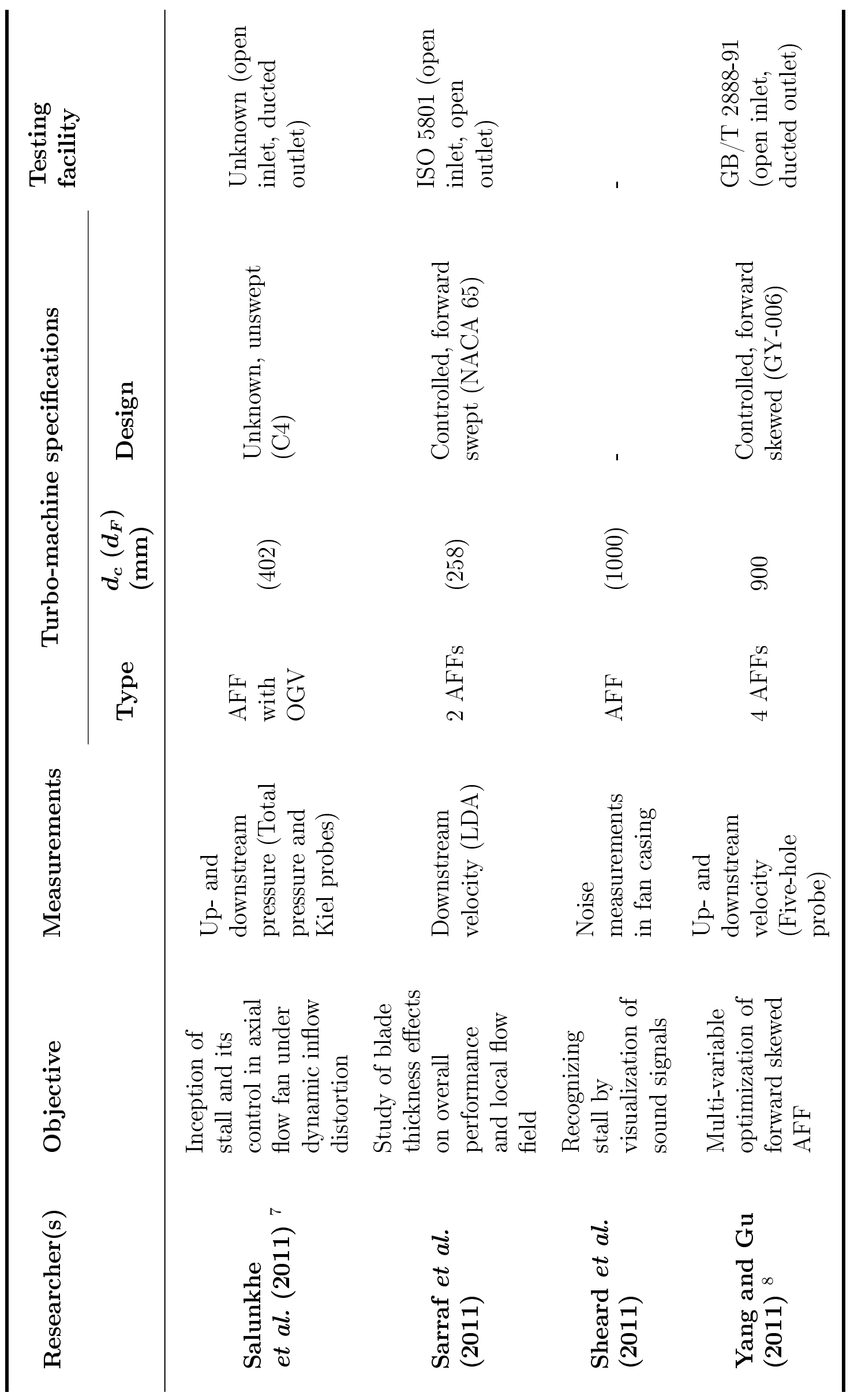


APPENDIX B. SUMMARISED LITERATURE

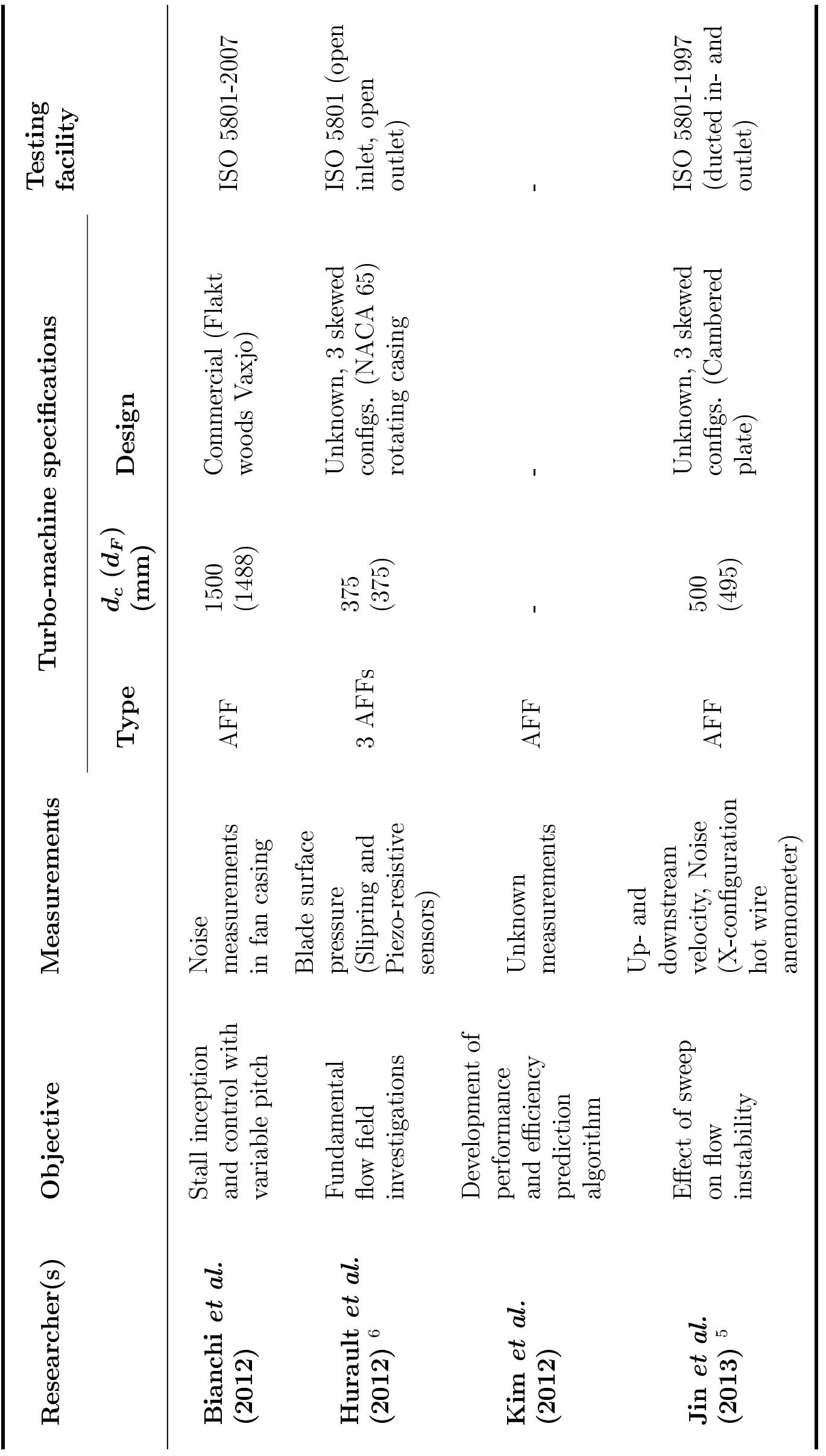




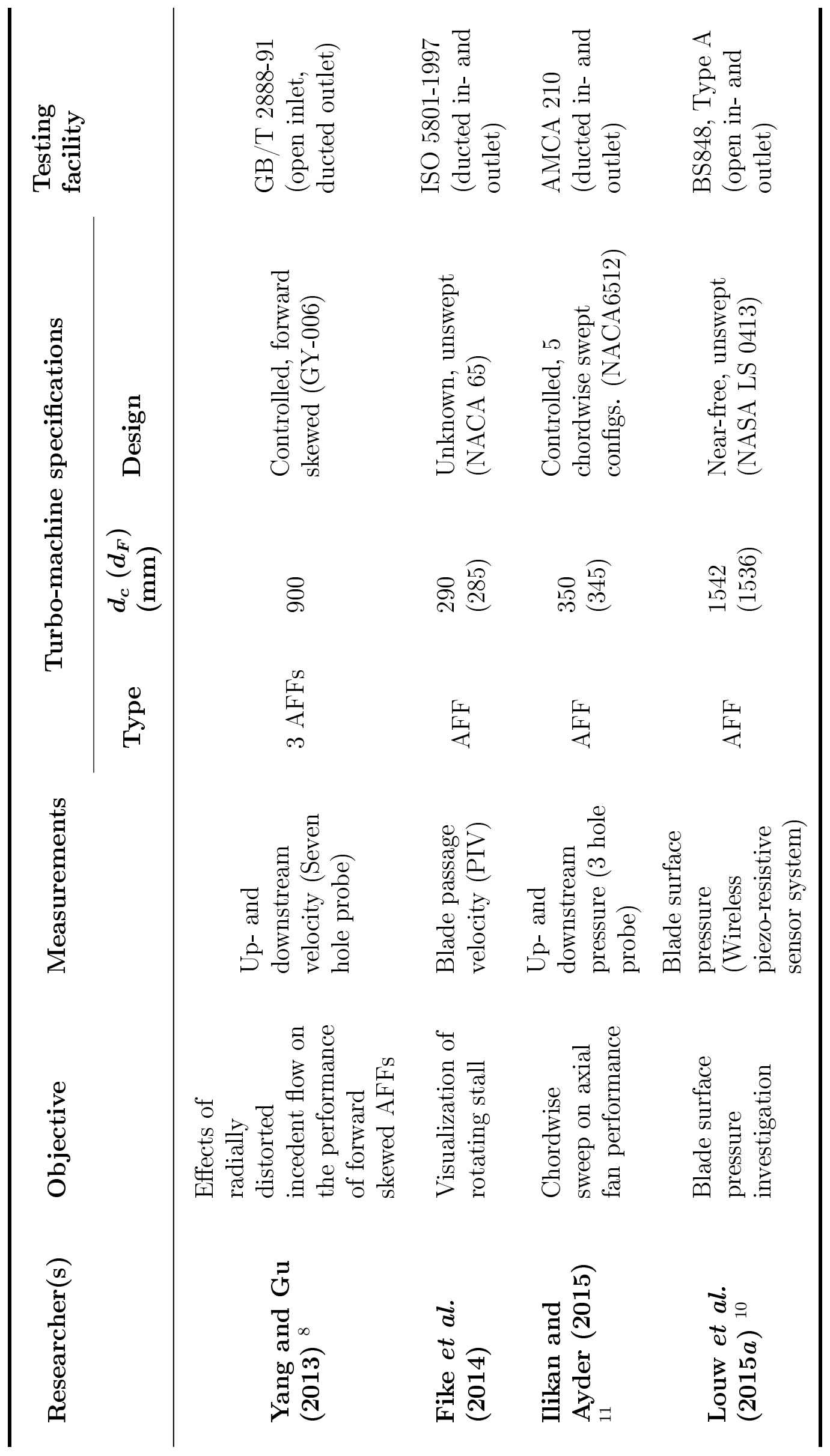


APPENDIX B. SUMMARISED LITERATURE

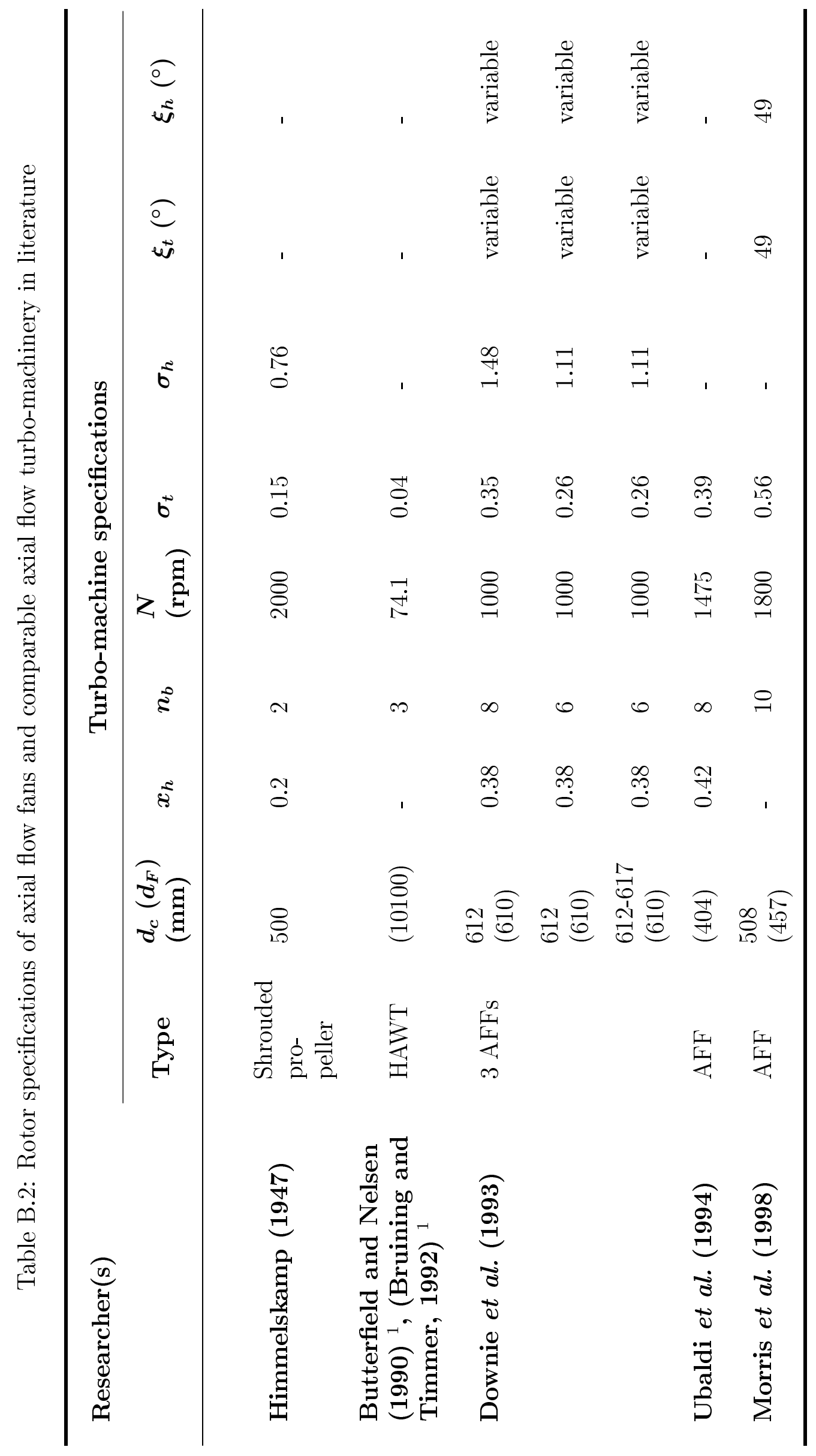




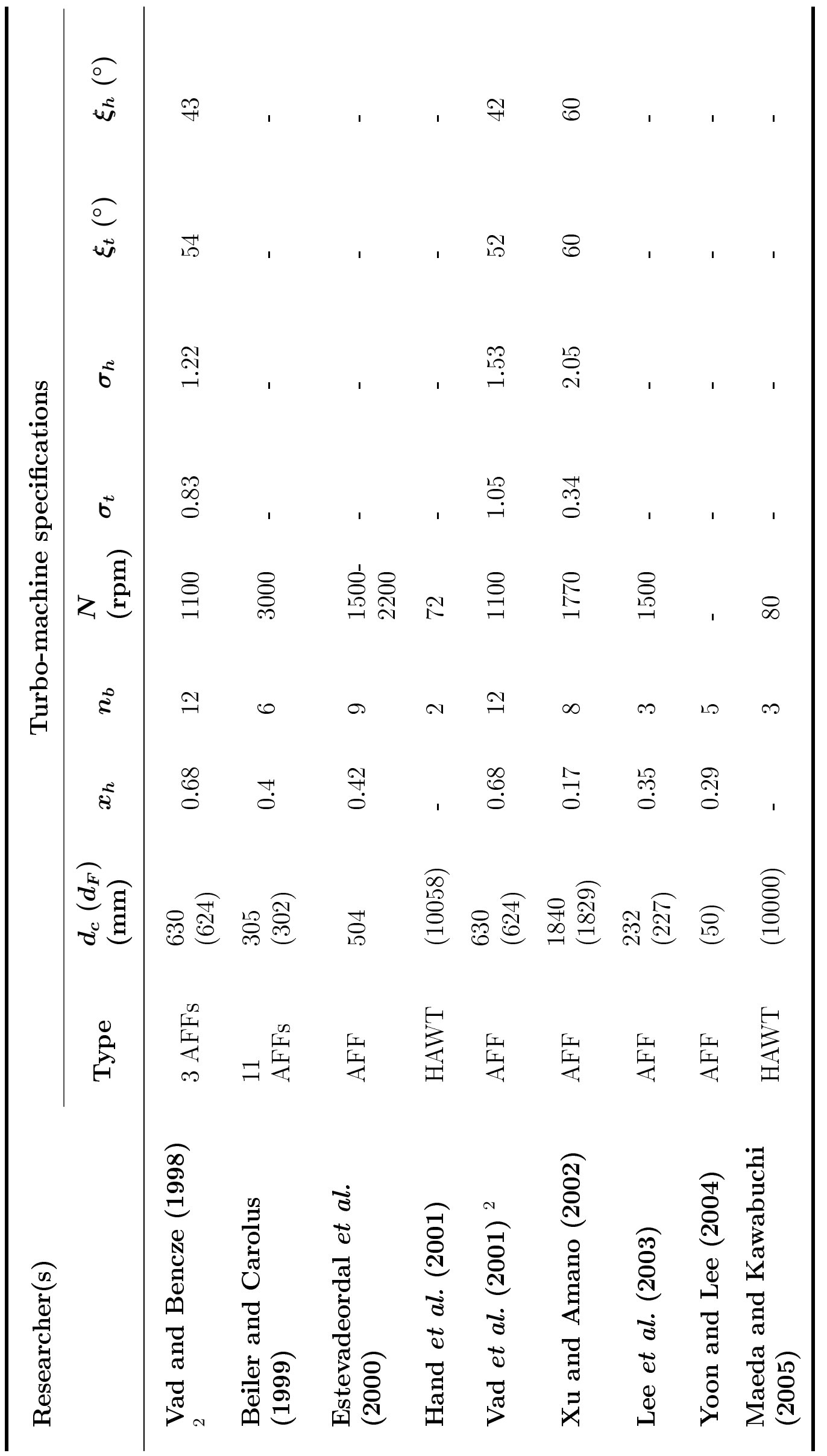




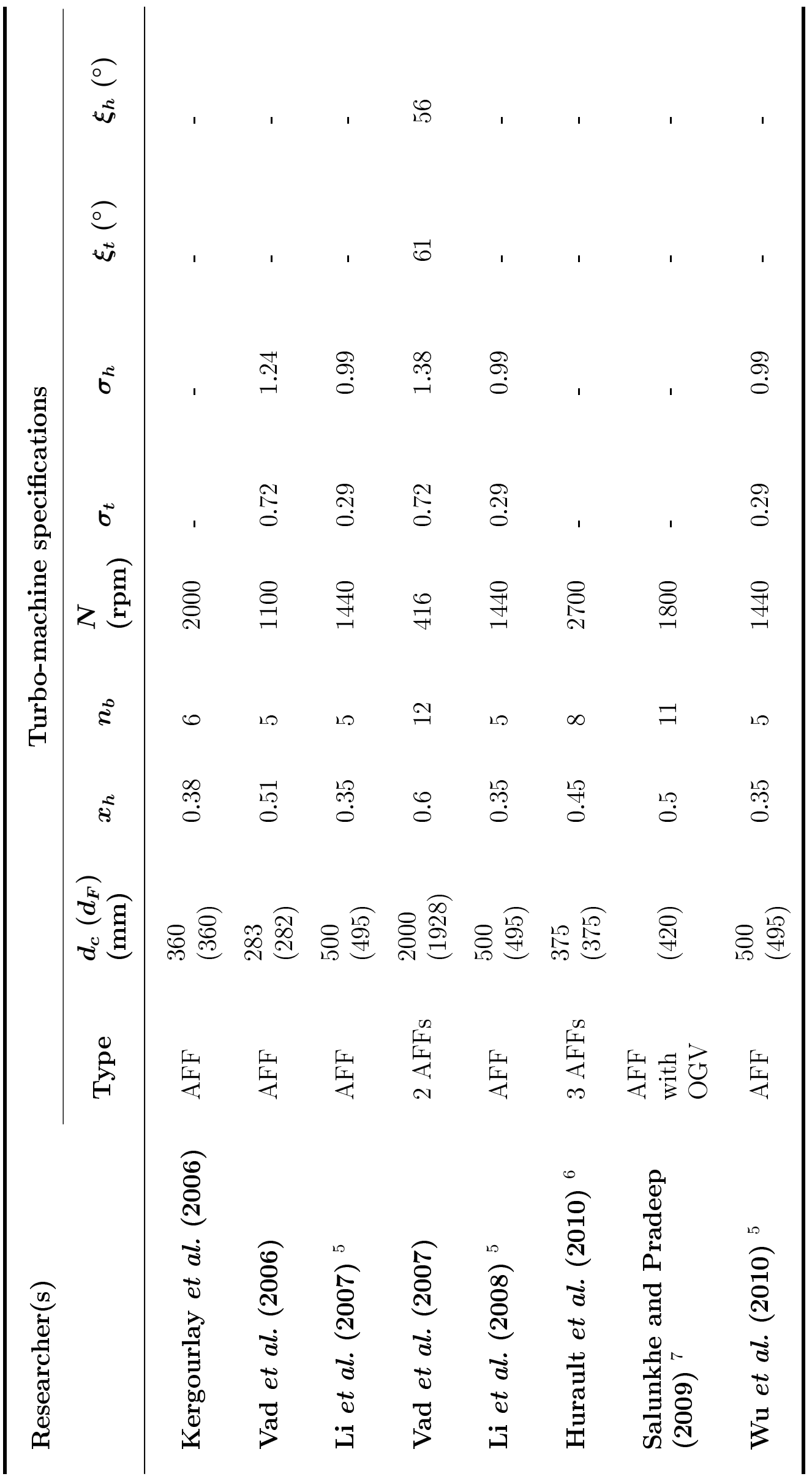




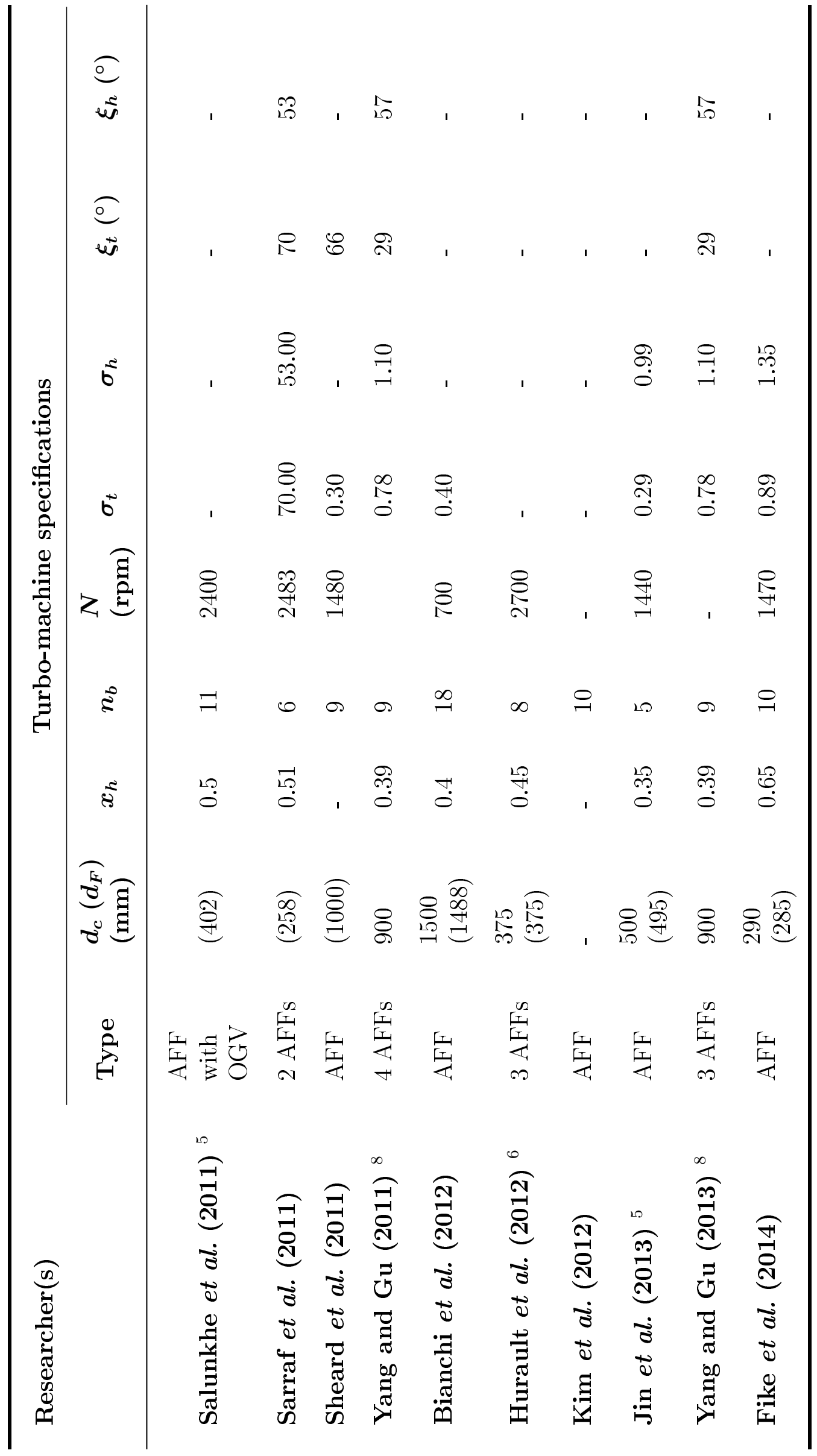


APPENDIX B. SUMMARISED LITERATURE

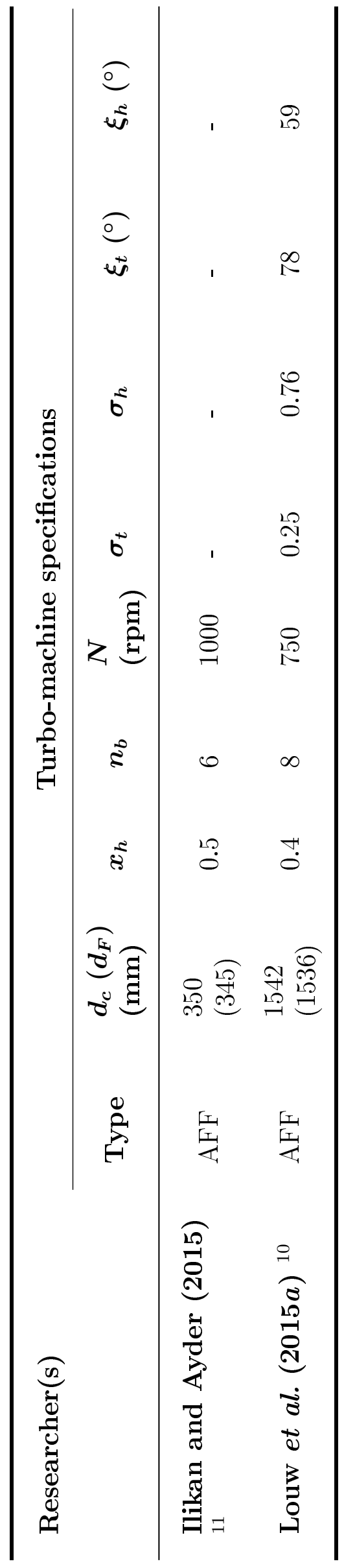




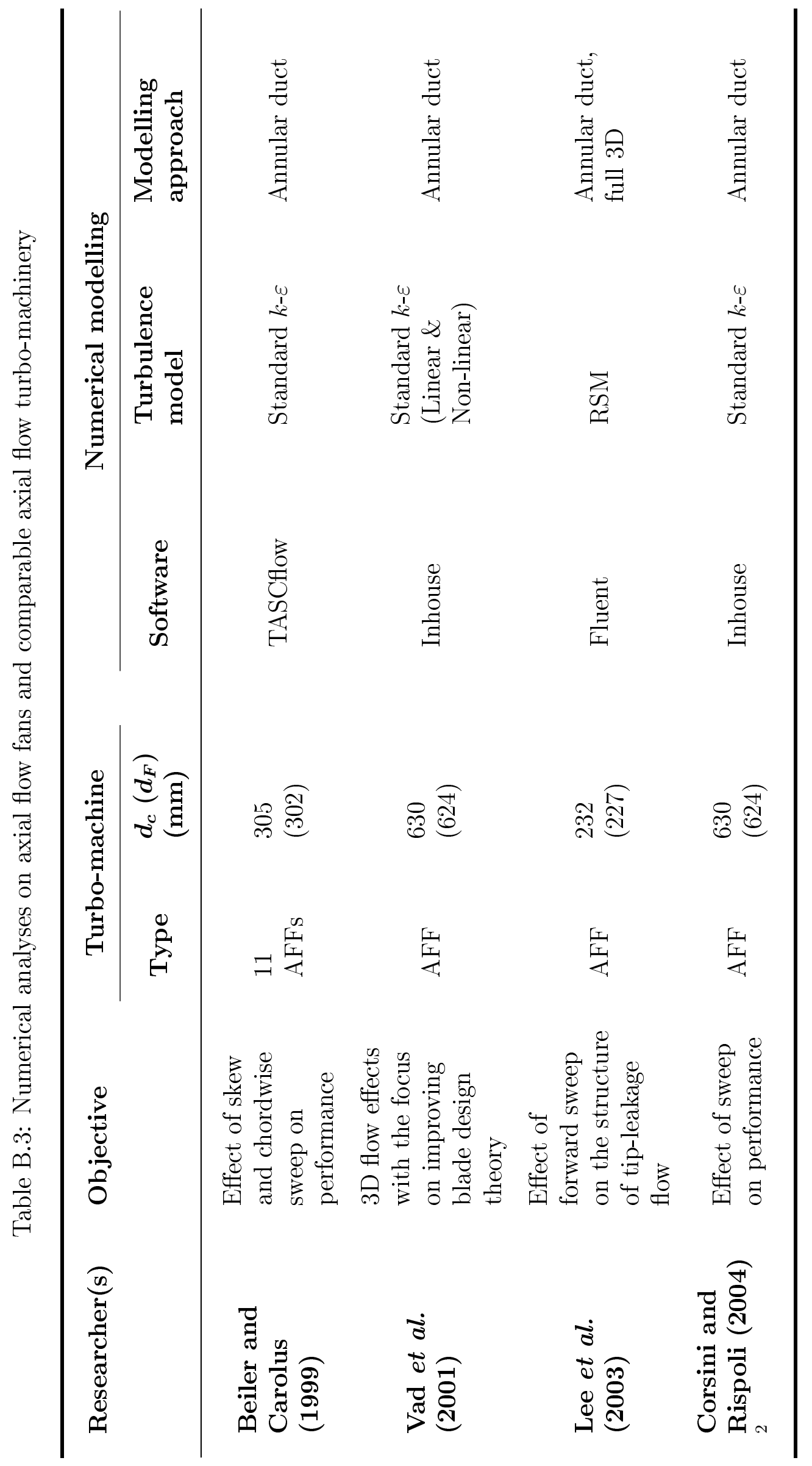


APPENDIX B. SUMMARISED LITERATURE

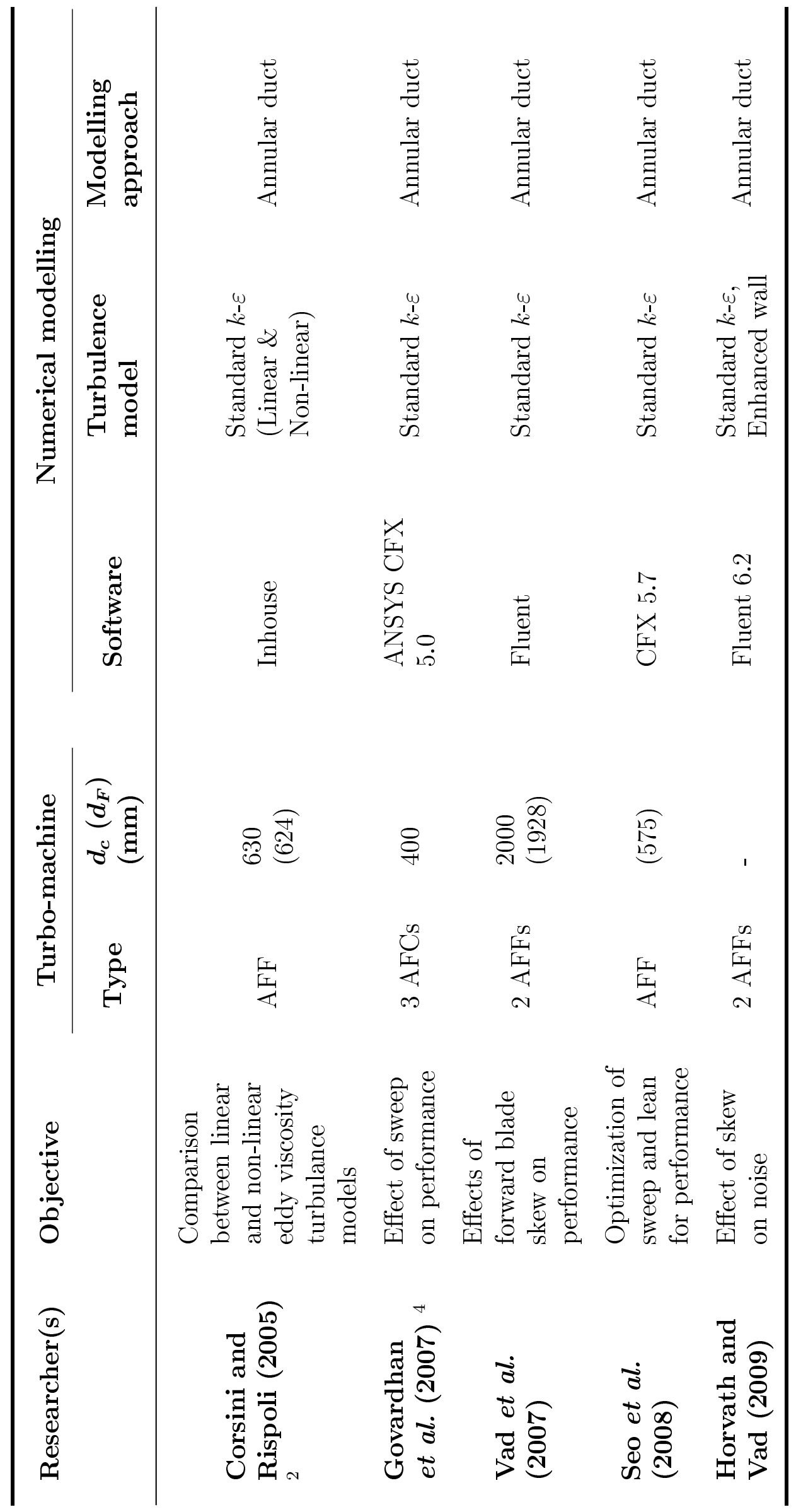


APPENDIX B. SUMMARISED LITERATURE

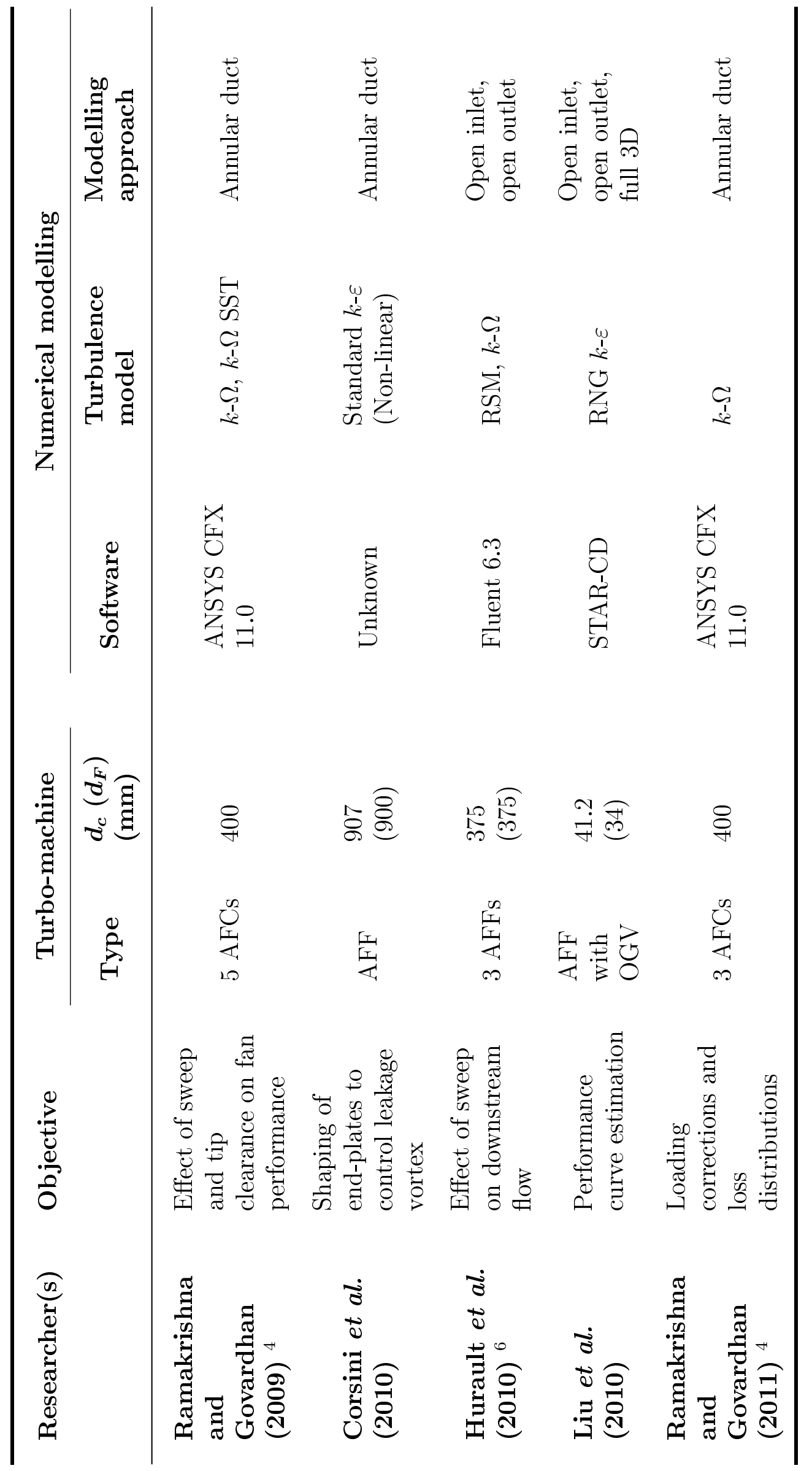




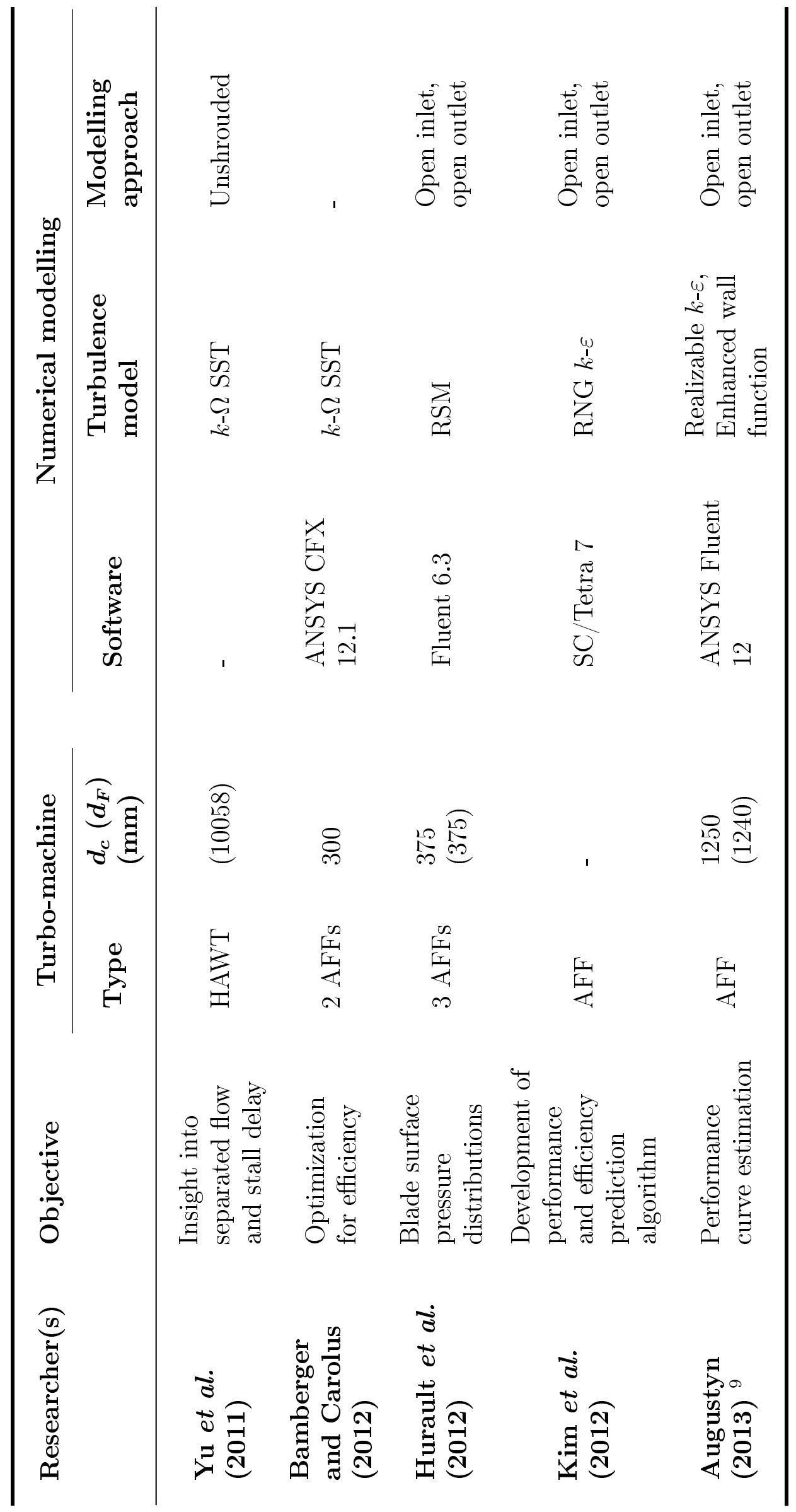




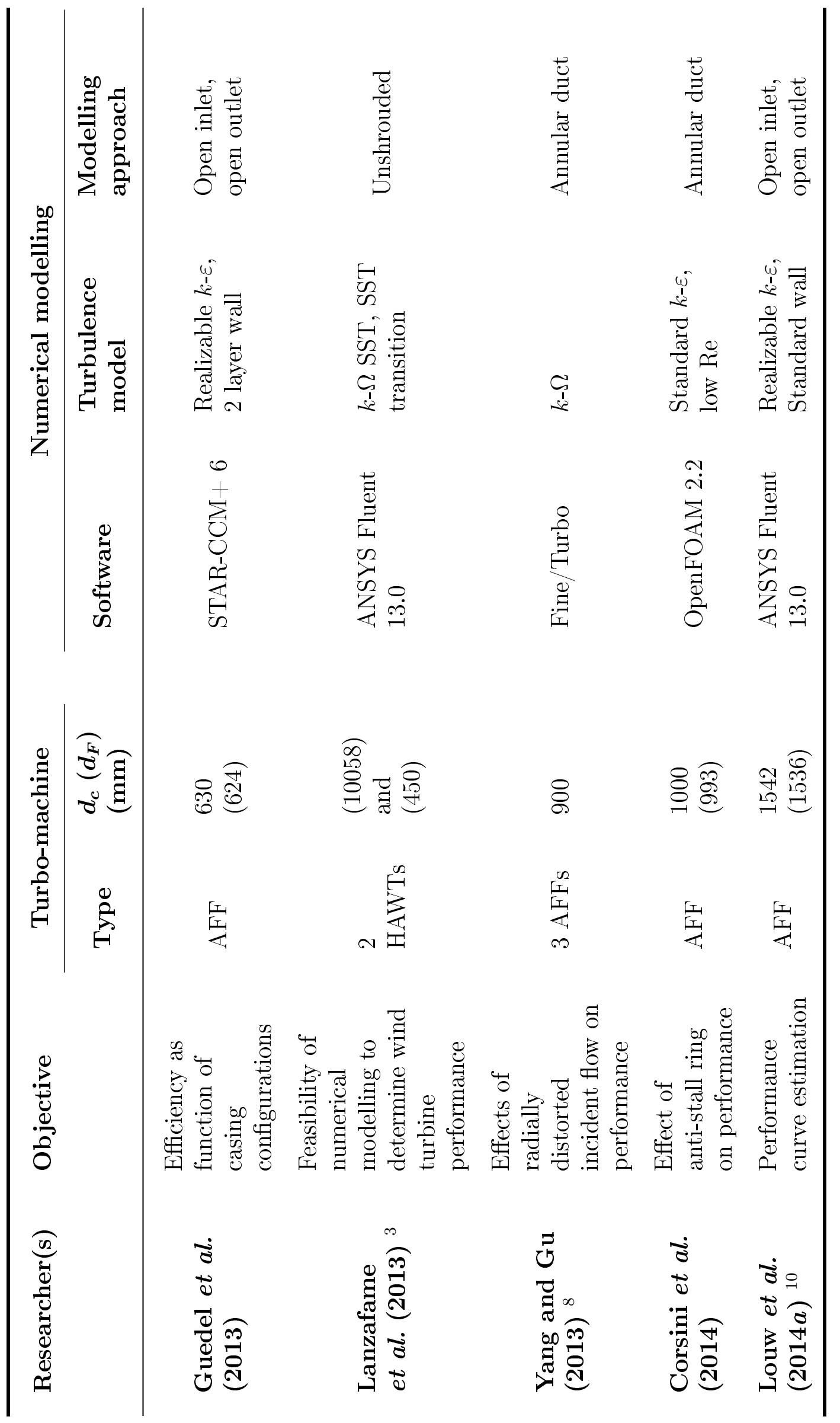




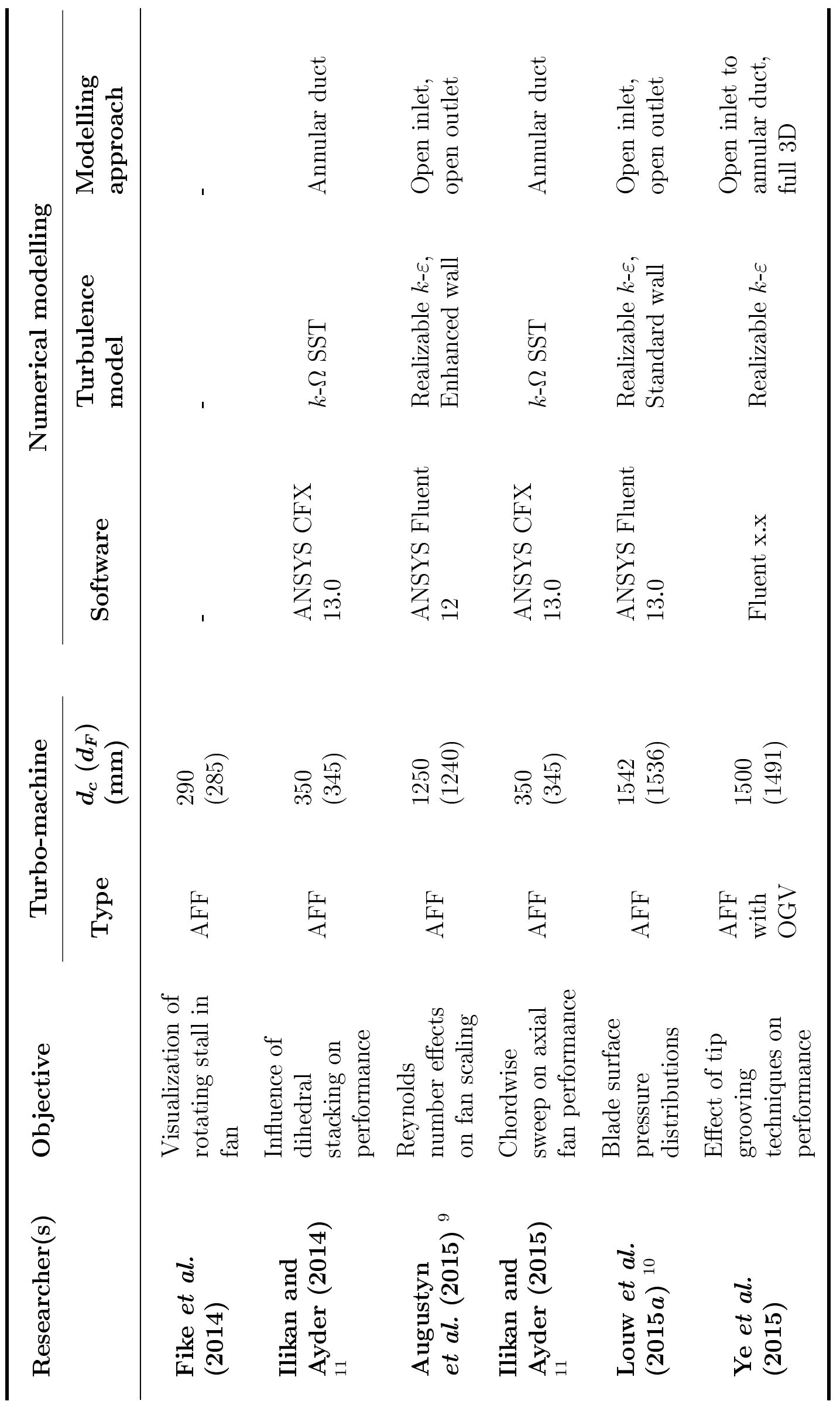




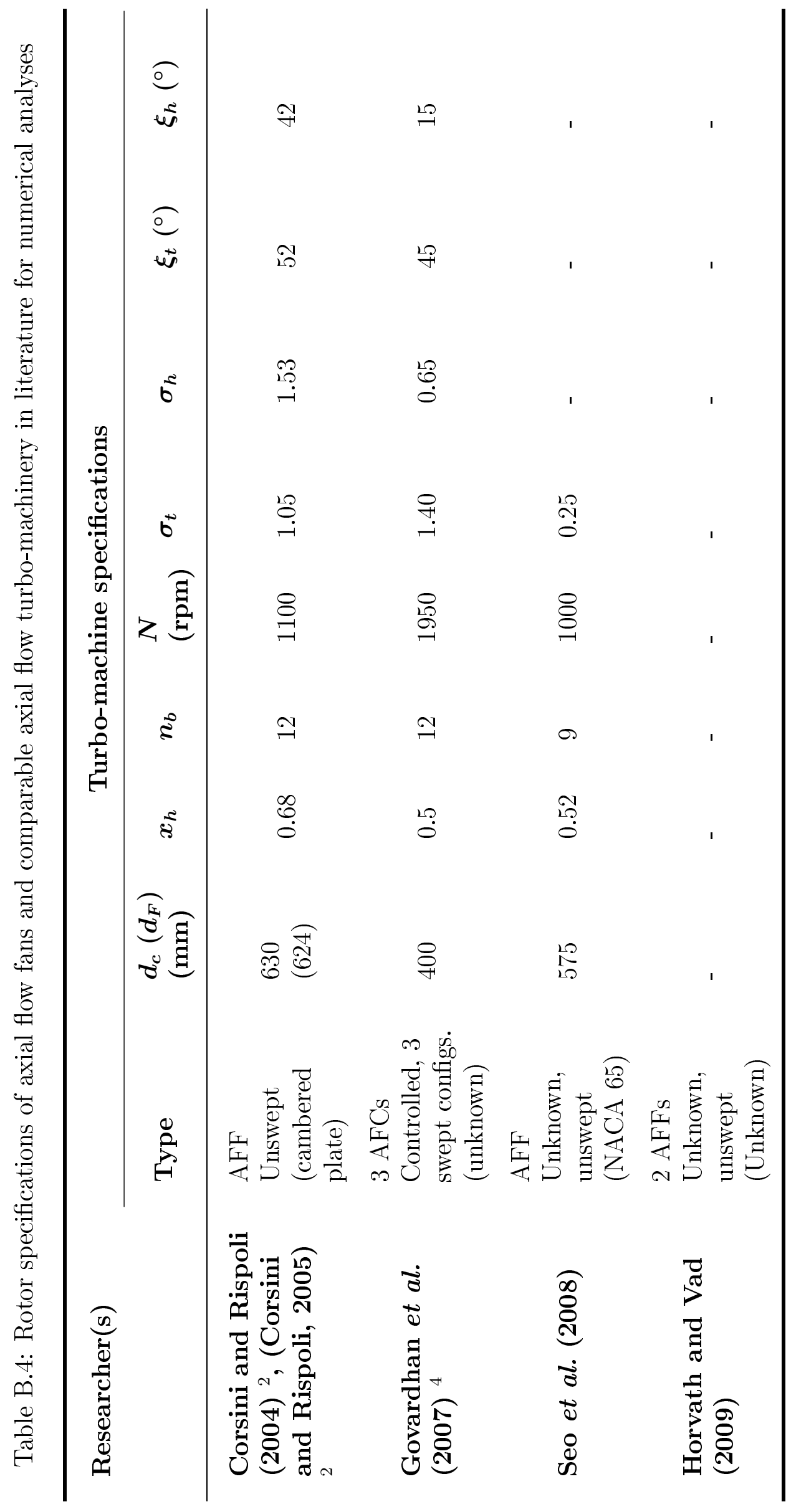


APPENDIX B. SUMMARISED LITERATURE

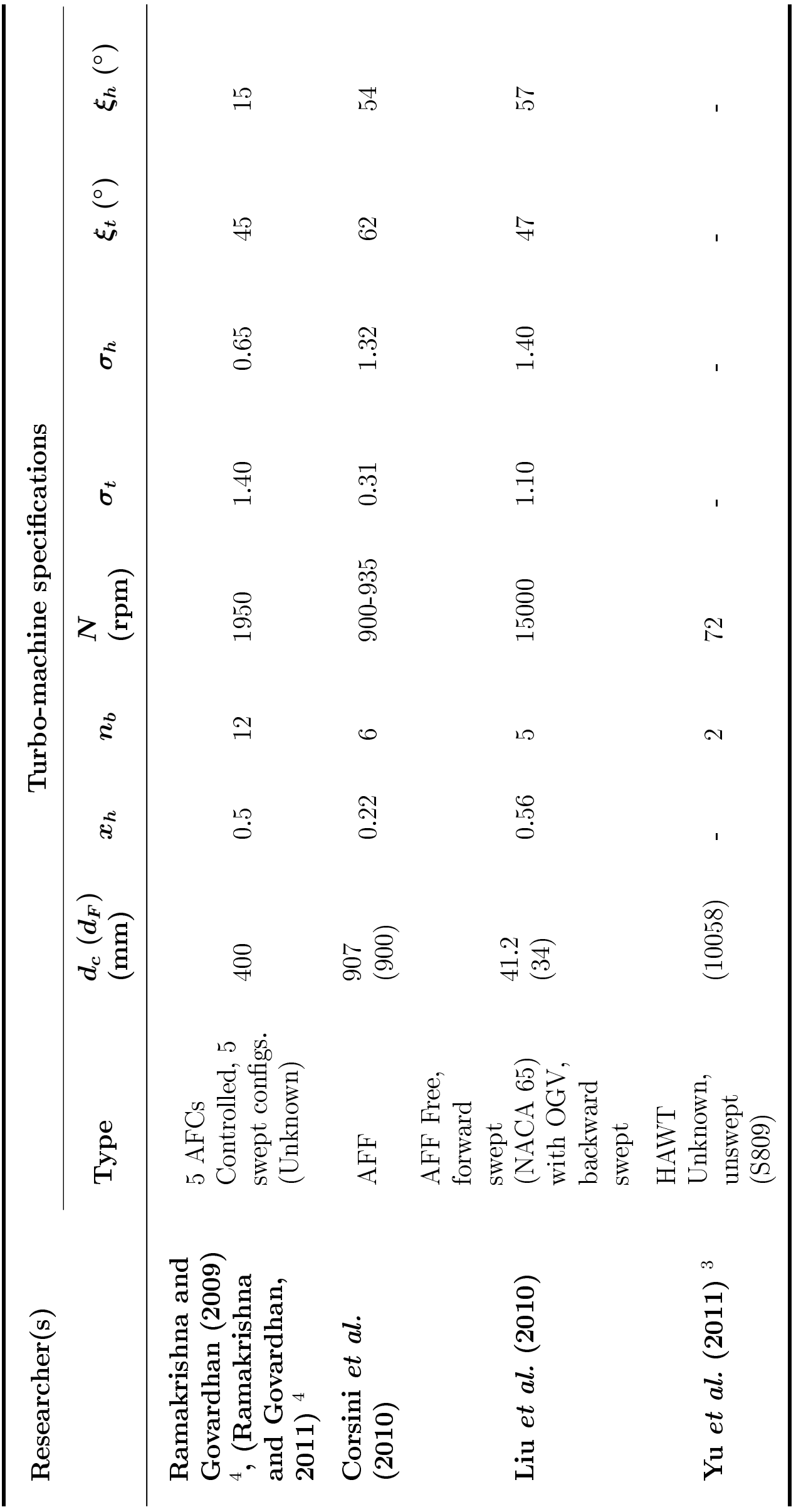




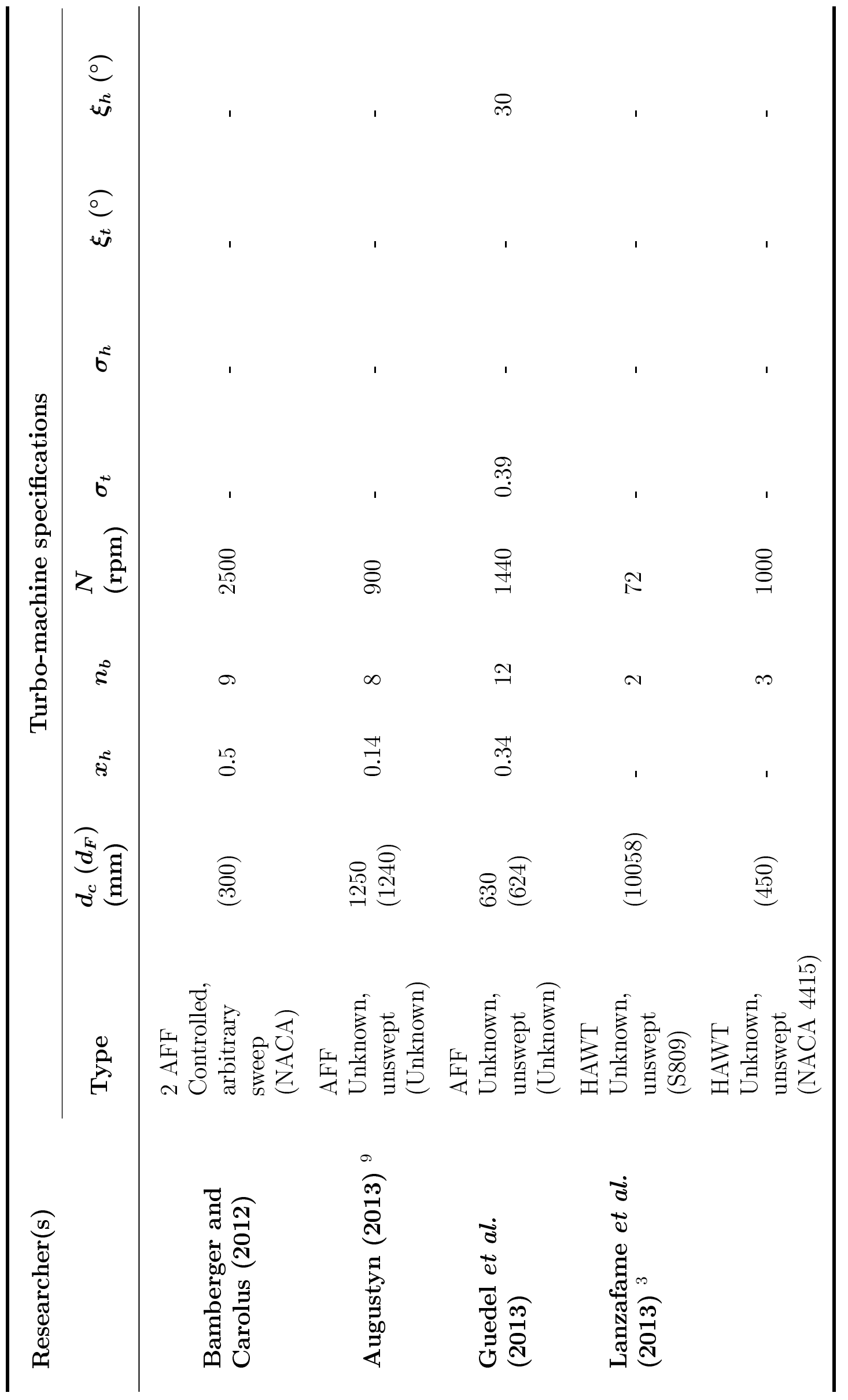




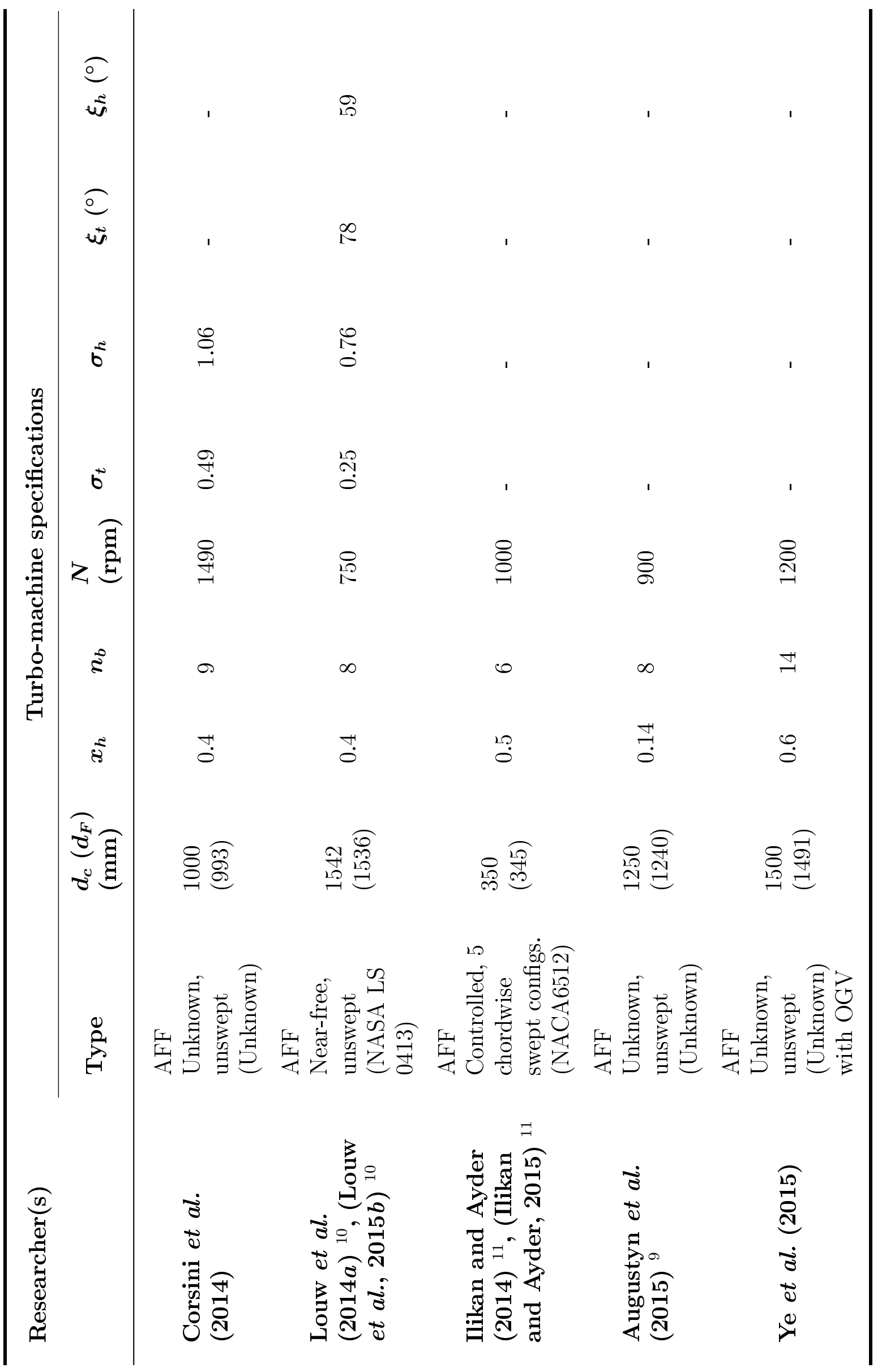




\section{Appendix C}

\section{BSPM error and repeatability analyses}

\section{C.1 Error analyses}

The accuracy of the measurement chain is governed by two measurement components, being the transducers and wireless modules. However, since the transducer is implemented differently than specified by the manufacturer (different excitation voltage), calibration of each transducer is conducted using a Betz micro-manometer, a calibration instrument which has an accuracy of $\pm 1 \mathrm{~Pa}$. The standard deviation (eq. C.2.1) for the measurement chain is determined to be $\sigma \leq 4.0 \mathrm{~Pa}(\varepsilon=0.003)$ on full-scale measurements using the results of four calibrations, giving an indication of the error of the BSPM apparatus alone. Therefore, the overall error of the BSPM apparatus can be given as $-5 \leq \Delta p_{\text {err }} \leq 5 \mathrm{~Pa}$.
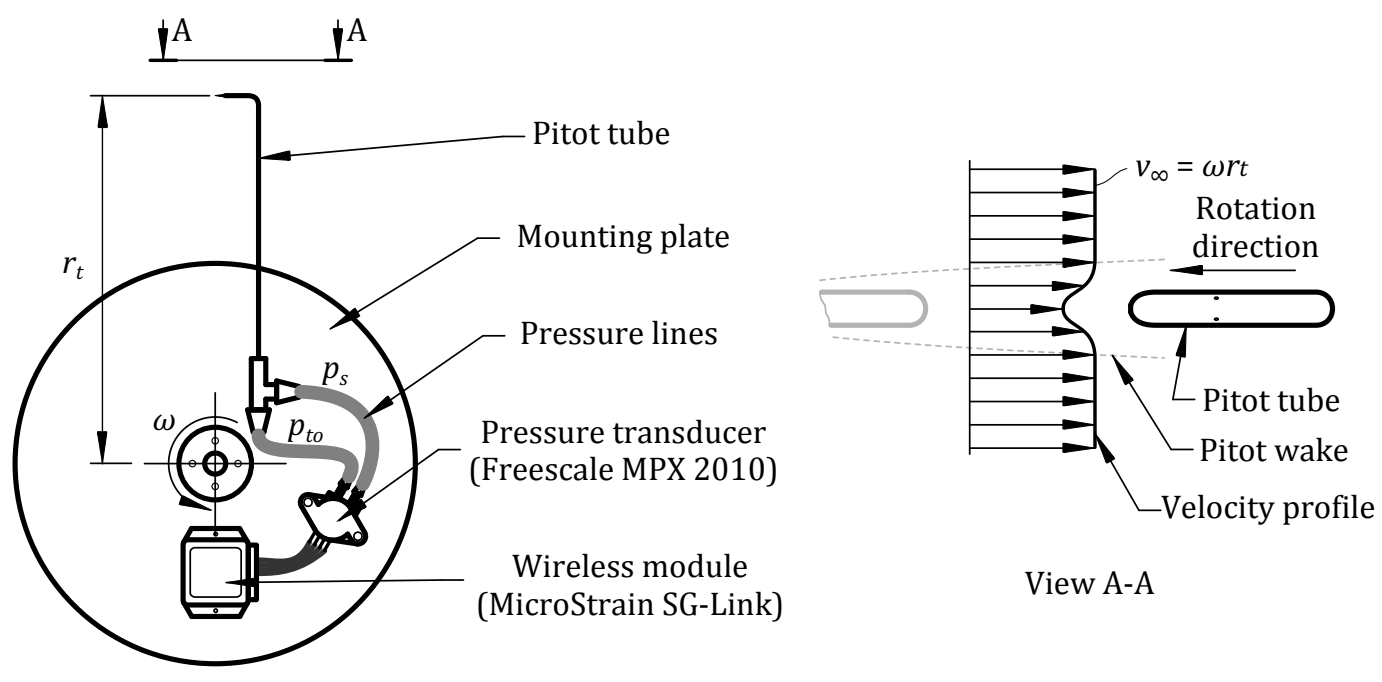

View A-A

Figure C.1: Pitot tube test apparatus 
A second verification is also conducted to test the pressure transducer functionality in a rotating environment. This is obtained by rotating a Pitot tube and measuring the difference between the stagnation and static pressures using a similar telemetry setup as described previously. For this purpose the apparatus in figure C.1 is constructed, consisting of a Pitot tube, one wireless module LORD MicroStrain SG-link and a single piezo-resistive pressure transducer Freescale MPX2010 (identical to those used for the BSPM analyses). The test apparatus was mounted on the shaft of the fan test facility and rotated as close as possible to the fan design speed $(N=750 \mathrm{rpm}, \omega=78.540 \mathrm{rad} / \mathrm{s})$. Subsequently, the differential pressure (dynamic pressure, $p_{d, \text { meas }}$ ) is measured and at the tip of Pitot tube.

The theoretical tangential speed at the radius of the Pitot tube tip $\left(v_{\infty}\right)$ can also be calculated, since the radius of the tip $\left(r_{t}\right)$ and the rotational speed $(\omega)$ are known.

$$
v_{\infty}=\omega r_{t} \quad[\mathrm{~m} / \mathrm{s}]
$$

Subsequently, the theoretical dynamic pressure $p_{d, t h e o r}$ at the tip of the Pitot tube can be obtained:

$$
p_{d, \text { theor }}=\frac{1}{2} \rho v_{\infty}^{2} \quad[\mathrm{~Pa}]
$$

A difference of $\Delta p_{\text {err }}=30.35 \mathrm{~Pa}(\varepsilon=0.07)$ is observed to exist between the theoretical and measured dynamic pressure $\left(p_{d}=400.22 \mathrm{~Pa}\right)$ results, while the measured values are always lower than the theoretical values. A reason for this might be due to the Pitot tube measuring a lower dynamic pressure, due to a lower velocity in its own wake (compared to the free stream, figure C.1), since it is rotating. It is therefore reasonable to assume that if the Pitot tube were to be infinitely thin, a measured dynamics pressure value $\left(p_{d, m e a s}\right)$ closer to the theoretical value would have been obtained and therefore $\Delta p_{\text {err }} \leq 30.35 \mathrm{~Pa}$ $(\varepsilon \leq 0.07)$.

\section{C.2 Repeatability analyses}

The standard and relative standard deviations ( $\sigma$ and $R S D$ ) are respectively given as:

$$
\sigma=\sqrt{\frac{1}{n} \sum_{i=1}^{n}\left(x_{i}-\mu\right)^{2}}
$$


where $n$ is the size of the population (data points in data set), $x_{i}$ the specific value of the data point and $\mu$ the arithmetic mean value of the population.

$$
R S D=\frac{\sigma}{\mu} \times 100
$$

Repeatability analyses of the blade surface pressure measurements are determined based on a statistical procedure. For this purpose five measurement runs are conducted for the suction side taps at blade spans of $s_{b}=0.1$ and 0.9 , respectively (Therefore ten runs in total). These spans are chosen, since they represent the lowest and highest pressures to be measured by the transducers on the suction side. Assuming a normal distribution of the measured pressure by each transducer, the standard deviation for each case is calculated, where a single case represents the measurement of one tap by one transducer at a specific volumetric flow rate. Since the measurements are repeated five times, each case has a population of five.

Through this procedure it was found that the standard deviations for each of the measurements ranged between a minimum of $\sigma=6.14 \mathrm{~Pa}$ and a maximum of $\sigma=35.19 \mathrm{~Pa}$. The relative standard deviations on all cases mostly ranged between 1 and $3 \%$ with only four cases giving errors of $4 \%$ and two giving $5 \%$. A maximum deviation of $5 \%$ from the arithmetic mean of the measured population occurred. 


\section{Appendix D}

\section{Experimental data analysis procedures}

\section{D.1 Fan characteristic measurement analysis}

The analysis procedure for calculating the fan performance characteristics as a function of flow rate follows. For a test run, a certain number of flow rates $\left(n_{V}\right)$ are measured consecutively. The time-averaged measured results obtained for a single flow rate are the plenum static pressure $\left(\Delta p_{s, p l e n, m}^{\prime}\right)$, facility inlet bellmouth pressure $\left(\Delta p_{s, n, m}^{\prime}\right)$, shaft torque $\left(T_{m}^{\prime}\right)$ and shaft rotational speed $\left(N_{m}^{\prime}\right)$. Zero load measurements $\left(\Delta p_{s, p l e n, m, z e r o 1}, \Delta p_{s, p l e n, m, z e r o 2}, \Delta p_{s, n, m, z e r o 1}\right.$, $\Delta p_{s, n, m, z e r o 2}, T_{m, z e r o 1}, T_{m, z e r o 2}, N_{m, z e r o 1}$ and $\left.N_{m, z e r o 2}\right)$ are made immediately before and after each test run to correct the data measurements made during the run. The temperature inside the plenum chamber $\left(T_{\text {plen } 1}\right.$ and $\left.T_{p l e n 2}\right)$ as well as the atmospheric pressure $\left(p_{a t m 1}\right.$ and $\left.p_{a t m 2}\right)$ are also measured immediately before and after a test run.

The measured values are firstly corrected for the zero offset by:

$$
\Phi=\Phi^{\prime}-\Phi_{z e r o}
$$

where $\Phi^{\prime}$ is a holding variable for the time-averaged variables $\Delta p_{s, p l e n, m}^{\prime}, \Delta p_{s, n, m}^{\prime}$, $T_{m}^{\prime}$ and $N_{m}^{\prime}$, $\Phi$ the corrected variable and $\Phi_{\text {zero }}$ calculated by linear interpolation between the first and last measurement for the specific flow rate as:

$$
\Phi_{z e r o}=\frac{i}{n_{s}}\left(\Phi_{z e r o 2}-\Phi_{z e r o 1}\right)+\Phi_{z e r o 1}
$$

In equation D.1.2, $i$ and $n_{s}$ refer to the specific flow rate number and the total number flow rates measured in the test run, respectively. Hence, the apostrophe is dropped for the corrected values $\left(\Delta p_{s, p l e n, m}, \Delta p_{s, n, m}, T_{m}, N_{m}\right.$, $T_{\text {plen }}$ and $\left.p_{a t m}\right)$.

The fan power can firstly be calculated as: 


$$
P_{F}=T_{m} \omega \quad[\mathrm{W}]
$$

where $\omega$ is the rotational speed in $\mathrm{rad} / \mathrm{s}$ :

$$
\omega=\frac{2 \pi N_{m}}{60} \quad[\mathrm{rad} / \mathrm{s}]
$$

Hereafter, the ambient density for the specific flow rate is calculated by:

$$
\rho_{a t m}=\frac{p_{a t m}}{R T_{a t m}} \quad\left[\mathrm{~kg} / \mathrm{m}^{3}\right]
$$

where $R$ is the specific gas constant for air and $T_{a t m}=T_{p l e n}$.

Subsequently the mass flow rate $(\dot{m})$ through the facility is obtained by:

$$
\dot{m}=K_{n} \frac{\pi d_{n}^{2}}{4} \sqrt{2 \rho_{a t m} \Delta p_{s, n, m}} \quad[\mathrm{~kg} / \mathrm{s}]
$$

where the test facility inlet loss coefficient $K_{n}=0.9082$ and the inlet diameter $d_{n}=1.008 \mathrm{~m}$.

The density in the plenum chamber is calculated as:

$$
\rho_{\text {plen }}=\frac{p_{a t m}-\Delta p_{s, p l e n, m}}{R T_{\text {plen }}} \quad\left[\mathrm{kg} / \mathrm{m}^{3}\right]
$$

Subsequently the dynamic pressure in the plenum chamber is calculated:

$$
p_{d, p l e n}=\frac{\dot{m}^{2}}{2 \rho_{\text {plen }} A_{\text {plen }}^{2}} \quad[\mathrm{~Pa}]
$$

where the area of the plenum, $A_{\text {plen }}=13.69 \mathrm{~m}^{2}$.

The total pressure inside the plenum chamber is obtained by:

$$
\Delta p_{t, p l e n}=\Delta p_{s, p l e n, m}+p_{d, p l e n} \quad[\mathrm{~Pa}]
$$

Subsequently the total pressure immediately downstream of the fan can be calculated by:

$$
\Delta p_{F t}=\Delta p_{t, p l e n}+p_{F d} \quad[\mathrm{~Pa}]
$$

where $p_{F d}$ is the dynamic pressure based on the fan annulus: 


$$
p_{F d}=\frac{\dot{m}^{2}}{2 \rho_{\text {plen }} A_{F}^{2}} \quad[\mathrm{~Pa}]
$$

and $A_{F}$ is the fan annulus:

$$
A_{F}=\frac{\pi\left(d_{c}^{2}-d_{h}^{2}\right)}{4} \quad\left[\mathrm{~m}^{2}\right]
$$

with $d_{c}$ and $d_{h}$ being the casing and hub diameters, respectively.

Furthermore, the volumetric flow rate through the facility can also be calculated by:

$$
\dot{V}=\frac{\dot{m}}{\rho_{\text {plen }}} \quad\left[\mathrm{m}^{3} / \mathrm{s}\right]
$$

Subsequently the fan total and static efficiencies, $\eta_{F} t$ and $\eta_{F} s$, can respectively be calculated by:

$$
\eta_{F t}=\frac{\Delta p_{F t} \dot{V}}{P_{F}}
$$

and

$$
\eta_{F s}=\frac{\Delta p_{F s} \dot{V}}{P_{F}}
$$

These calculated values for $\Delta p_{F t}, \Delta p_{F s}, \dot{V}$ and $P_{F}$ are all obtained for densities and rotational speeds close to the fan's design condition at the time of testing, but requires scaling to these exact conditions to make all results comparable using the turbo-machine scaling laws. As given in appendix A, these values for density and fan rotational speed are $\rho_{D}=1.2 \mathrm{~kg} / \mathrm{m}^{3}$ and $N_{D}=750 \mathrm{rpm}$. Subsequently the scaled values for $\Delta p_{F t}, \Delta p_{F s}, \dot{V}$ and $P_{F}$ are:

$$
\begin{aligned}
& \Delta p_{F t, \rho_{D}=1.2}=\Delta p_{F t}\left(\frac{d_{c}}{d_{c}}\right)^{2}\left(\frac{N_{D}}{N}\right)^{2}\left(\frac{\rho_{D}}{\rho}\right) \quad[\mathrm{Pa}] \\
& \Delta p_{F t, \rho_{D}=1.2}=\Delta p_{F t}\left(\frac{d_{c}}{d_{c}}\right)^{2}\left(\frac{N_{D}}{N}\right)^{2}\left(\frac{\rho_{D}}{\rho}\right) \quad[\mathrm{Pa}] \\
& \dot{V}_{\rho_{D}=1.2}=\dot{V}\left(\frac{d_{c}}{d_{c}}\right)^{3}\left(\frac{N_{D}}{N}\right) \quad\left[\mathrm{m}^{3} / \mathrm{s}\right]
\end{aligned}
$$


and

$$
P_{F, \rho_{D}=1.2}=P_{F}\left(\frac{d_{c}}{d_{c}}\right)^{5}\left(\frac{N_{D}}{N}\right)^{3}\left(\frac{\rho_{D}}{\rho}\right) \quad[\mathrm{W}]
$$

The efficiency values theoretically remain constant during scaling and hence $\eta_{F t, \rho_{D}=1.2}=\eta_{F t}$ and $\eta_{F t, \rho_{D}=1.2}=\eta_{F t}$.

Finally, the volumetric flow rate and pressure values are made dimensionless and presented as flow and pressure coefficients, respectively calculated by:

$$
\begin{aligned}
& \phi=c_{z, a v g} / u_{c} \\
& \Psi_{F s}=\frac{\Delta p_{F s, \rho_{D}=1.2}}{0.5 \rho u_{c}^{2}}
\end{aligned}
$$

and

$$
\Psi_{F t}=\frac{\Delta p_{F t, \rho_{D}=1.2}}{0.5 \rho u_{c}^{2}}
$$

where $u_{c}$ is the fan circumferential velocity at the casing and $c_{z, a v g}$ the average axial velocity through the fan, calculated by:

$$
c_{z, a v g}=\frac{\dot{V}_{\rho_{D}=1.2}}{A_{F}} \quad[\mathrm{~m} / \mathrm{s}]
$$

\section{D.2 BSPM analysis}

The analysis procedure to obtain the static pressure on the blade surfaces using the BSPM equipment follows. The series of brass and silicon tubes connecting the pressure taps on the blade surfaces to the pressure transducers is depicted in figure D.1a. The locations of these taps are at different radii compared to that of the transducer membranes (located on the axis of rotation). These differences in radii will lead to pressure differences between the taps and the transducers caused by centrifugal forces acting on the air constrained in the tubes. Considering a single pressure tap connected to one transducer, a derivation follows to obtain the static pressure at the tap from the value of pressure measured by the transducer.

Before the blade surface pressure can be determined, a relation is firstly derived in section D.2.1 to obtain the pressure gradient of air in the tubes as a function of radius. Secondly the calculation procedure is discussed in which the blade surface pressures at each pressure tap is calculated from the measured transducer data in section D.2.2. 


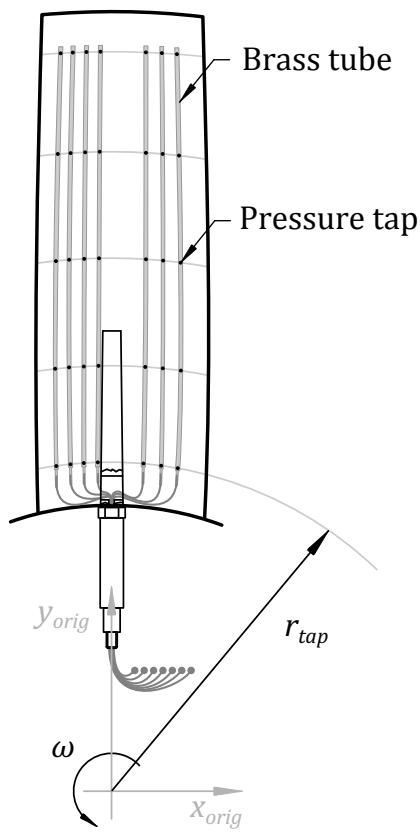

(a)

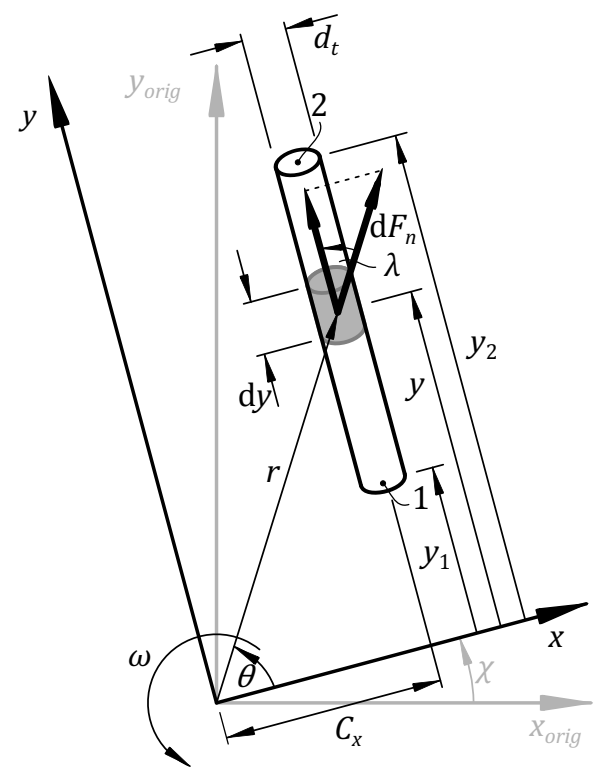

(b)

Figure D.1: (a) Details of the tube layout inside the fan blades and (b) a control volume of air constrained in a tube

\section{D.2.1 Derivation for centrifugal pressure distribution}

Consider a control volume of air constrained in an arbitrary angled tube element between the pressure tap and the pressure transducer as shown in figure D.1b. A Cartesian axis can be specified such that the $y$-direction is parallel to the tube element. The pressure due to centrifugal forces at height $y$ (the location of the control volume) can be given as:

$$
\mathrm{d} p=\frac{\mathrm{d} F_{n} \cos \lambda}{A} \quad[\mathrm{~Pa}]
$$

where

$$
\begin{aligned}
& A=\frac{\pi d_{t}}{4} \quad\left[\mathrm{~m}^{2}\right] \\
& \lambda=\frac{\pi}{2}-\theta \quad\left[{ }^{\circ}\right] \\
& \theta=\arctan \left(\frac{y}{C_{x}}\right) \quad\left[^{\circ}\right]
\end{aligned}
$$


The normal force, $\mathrm{d} F_{n}$, in equation D.2.1 can be defined as:

$$
\mathrm{d} F_{n}=a_{n} \mathrm{~d} m \quad[\mathrm{~N}]
$$

where

$$
\begin{aligned}
& \mathrm{d} m=\rho_{a} A \mathrm{~d} y \quad[\mathrm{~kg}] \\
& a_{n}=\omega^{2} r=\omega^{2} \sqrt{y^{2}+C_{x}^{2}} \quad\left[\mathrm{~m}^{2} / \mathrm{s}\right]
\end{aligned}
$$

Taking compressibility of the air inside the tube into account, the fluid density can be determined assuming the ideal gas law:

$$
\rho_{a}=\frac{p}{R T} \quad\left[\mathrm{~kg} / \mathrm{m}^{3}\right]
$$

where the gas constant of air, $R=287.08 \mathrm{~J} / \mathrm{kg} . \mathrm{K}$ and $T$ is the air temperature in $\mathrm{K}$. The mass of air is therefore given by:

$$
\mathrm{d} m=\frac{p A \mathrm{~d} y}{R T} \quad[\mathrm{~kg}]
$$

By substitution of the above into equation D.2.1, the differential pressure as a function of $y$ becomes:

$$
\mathrm{d} p(y)=\frac{p(y) \omega^{2} \sqrt{y^{2}+C_{x}^{2}} \cos \left[\frac{\pi}{2}-\arctan \left(\frac{y}{C_{x}}\right)\right] \mathrm{d} y}{R T} \quad[\mathrm{~Pa}]
$$

Integrating equation D.2.10 gives:

$$
\begin{aligned}
\int \frac{\mathrm{d} p(y)}{p(y)} & =\frac{\omega^{2}}{R T} \int \sqrt{y^{2}+C_{x}^{2}} \cos \left[\frac{\pi}{2}-\arctan \left(\frac{y}{C_{x}}\right)\right] \mathrm{d} y \\
\ln p(y) & =\frac{\omega^{2}}{R T} \int \sqrt{y^{2}+C_{x}^{2}} \frac{y}{\sqrt{y^{2}+C_{x}^{2}}} \mathrm{~d} y \\
& =\frac{\omega^{2} y^{2}}{2 R T}+C \quad[\mathrm{~Pa}]
\end{aligned}
$$

Although equation D.2.11 is in its final form, a relation for this pressure distribution as a function of radius is more beneficial. Substituting $y=\sqrt{r^{2}-C_{x}^{2}}$ into D.2.11 gives: 


$$
\begin{aligned}
\ln p(r) & =\frac{\omega^{2}\left(r^{2}-C_{x}^{2}\right)}{2 R T}+C \\
& =\frac{\omega^{2} r^{2}}{2 R T}-\frac{\omega^{2} C_{x}^{2}}{2 R T}+C \\
& =\frac{\omega^{2} r^{2}}{2 R T}+C_{1} \quad[\mathrm{~Pa}]
\end{aligned}
$$

Therefore, the centrifugal pressure distribution inside a rotating tube system is dependent only on the fluid temperature, rotational speed and radius.

\section{D.2.2 Measurement corrections for blade surface pressure}

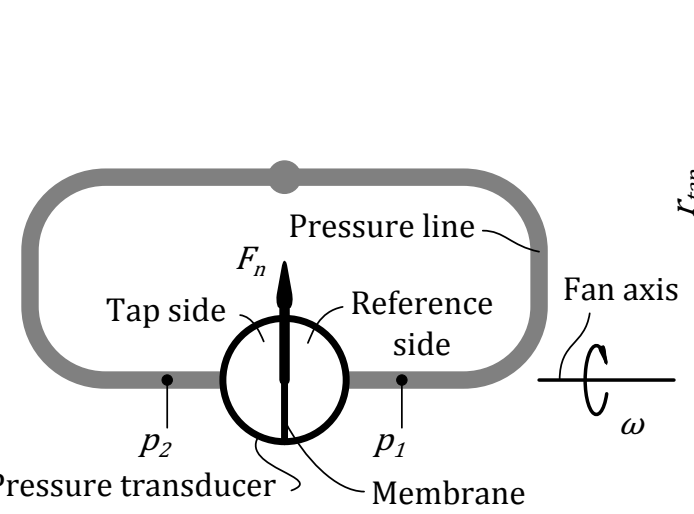

(a)

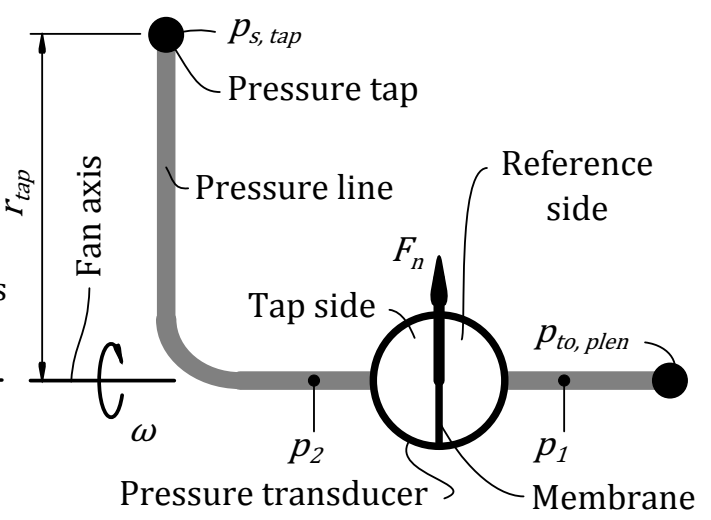

(b)

Figure D.2: Schematics of the pressure lines for (a) transducer membrane zero offset measurement and (b) blade surface pressure measurement

A line schematic of the tubes in the fan blade surface pressure measurement setup is shown in figures D.2a and b for two measuring configurations. As indicated in figure D.2b the tube connecting to the reference side of the pressure transducer is open to a location in the plenum chamber of the fan test facility to obtain a reference pressure (the total pressure in the plenum chamber). However, the tube on the tap side, leading to a pressure tap in the fan blade, is open to the blade surface environment at radius $r_{t a p}$ for static pressure measurement.

Due to centrifugal forces acting on the constrained air in the tubes, a correction is made to the measured pressure to ultimately obtain the static pressure at the applicable surface tap, $p_{s, t a p}$, positioned at radius $r_{\text {tap }}$. A derivation follows with reference to figures D.2a and b, explaining the pressure correction procedure. 
The pressure measured by the transducer is

$$
\Delta p_{m}=p_{2}-p_{1}+\Delta p_{\text {zero }} \quad[\mathrm{Pa}]
$$

where $\Delta p_{\text {zero }}$ is the initial pressure offset due to the combined effect of temperature and the centrifugal force, $F_{n}$, acting on the membrane of the transducer during rotation. This offset is obtained by temporarily connecting the reference side of the transducer to the tap side as shown in figure D.2a. Therefore the pressure on either side of the transducer membrane is equalized allowing the measured offset in pressure to be caused exclusively by the centrifugal force acting on the membrane. After the zero measurement is taken, the configuration is changed back to figure D.2b for blade surface pressure measurement.

The absolute pressure $p_{1}$ is given by

$$
p_{1}=p_{t o, p l e n}+p_{a t m} \quad[\mathrm{~Pa}]
$$

where $p_{t o, p l e n}$ is the total pressure in the plenum chamber, upstream of the fan and $p_{\text {atm }}$ the atmospheric pressure.

Furthermore, the absolute pressure $p_{2}$ is given by

$$
p_{2}=p_{s, t a p}+p_{a t m}-\Delta p_{n} \quad[\mathrm{~Pa}]
$$

where the $p_{s, t a p}$ is the static pressure at the blade tap and $\Delta p_{n}$ the difference in pressure due to the centrifugal forces acting on the air enclosed in the tubes between the fan axis and the tap radius, $r_{t a p}$. From equation D.2.12 it can be shown that $\Delta p_{n}$ is given as:

$$
\Delta p_{n}=p_{2}\left(\mathrm{e}^{\omega^{2} r_{\text {tap }}^{2} / 2 R T}-1\right) \quad[\mathrm{Pa}]
$$

Substituting D.2.16 into D.2.15 gives:

$$
\begin{aligned}
p_{2} & =p_{s, t a p}+p_{a t m}-p_{2}\left(\mathrm{e}^{\omega^{2} r_{\text {tap }}^{2} / 2 R T}-1\right) \\
& =\frac{p_{s, t a p}+p_{a t m}}{\mathrm{e}^{\omega^{2} r_{\text {tap }}^{2} / 2 R T}} \quad[\mathrm{~Pa}]
\end{aligned}
$$

By substituting D.2.17 and D.2.14 into D.2.13 the following relation is obtained for the measured pressure:

$$
\Delta p_{m}=\frac{p_{s, t a p}+p_{a t m}}{\mathrm{e}^{\omega^{2} r_{\text {tap }}^{2} / 2 R T}}-p_{t o, p l e n}-p_{a t m}+\Delta p_{\text {zero }} \quad[\mathrm{Pa}]
$$


Finally the static pressure at the relevant tap can be obtained by rearranging D.2.18:

$$
p_{s, t a p}=\mathrm{e}^{\omega^{2} r_{\text {tap }}^{2} / 2 R T}\left[\Delta p_{m}+p_{\text {to }, \text { plen }}+p_{\text {atm }}-\Delta p_{\text {zero }}\right]-p_{\text {atm }}
$$

The pressure values and volumetric flow rates are then made dimensionless and presented in the form of a pressure and flow coefficient, respectively:

$$
\begin{gathered}
\Psi_{F s}=\frac{p}{0.5 \rho u_{c}^{2}} \\
\phi=c_{z, \text { avg }} / u_{c}
\end{gathered}
$$

where $u_{c}$ is circumferential blade speed at the casing and $c_{z, \text { avg }}$ calculated by:

$$
c_{z, a v g}=\frac{\dot{V}}{A_{F}} \quad[\mathrm{~m} / \mathrm{s}]
$$

\section{D.2.3 Measured value interpolation}

Figure D.3a and b displays the measured blade surface pressure coefficients $\left(C_{p}\right)$ for a specific test run (pressure side, blade span $s_{b}=0.5$ ), representing the typical output of the measured data. These results are used to conduct a series of linear interpolations between data points allowing pressure values for other volumetric flow rates other than those measured, to be obtained. For example, to obtain a pressure value for a dimensionless chord length of $x / c h=0.3$ and a flow coefficient of $\phi=0.042,\left(\dot{V}=4 \mathrm{~m}^{3} / \mathrm{s}\right)$ the following holds true (considering the data points represented by the filled black circles in figure D.3):

$$
C_{p, \phi=0.042}=\frac{\phi-\phi_{1}}{\phi_{2}-\phi_{1}}\left(C_{p, \phi_{2}}-C_{p, \phi_{1}}\right)+C_{p, \phi_{1}}
$$

A linear interpolation is merited, considering the visible smooth gradient between consecutive data points as can be observed in fiqure D.3b. 


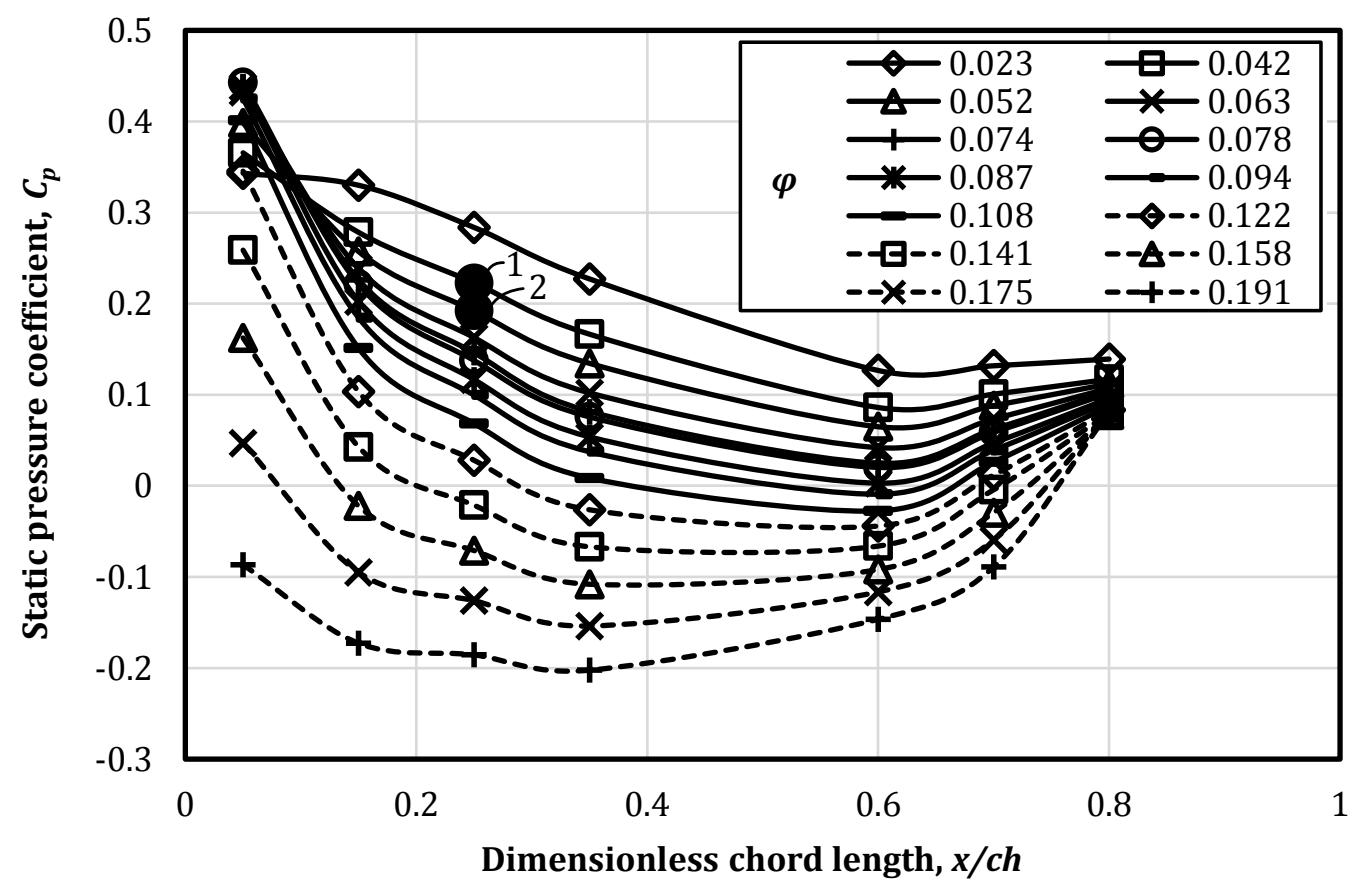

(a)

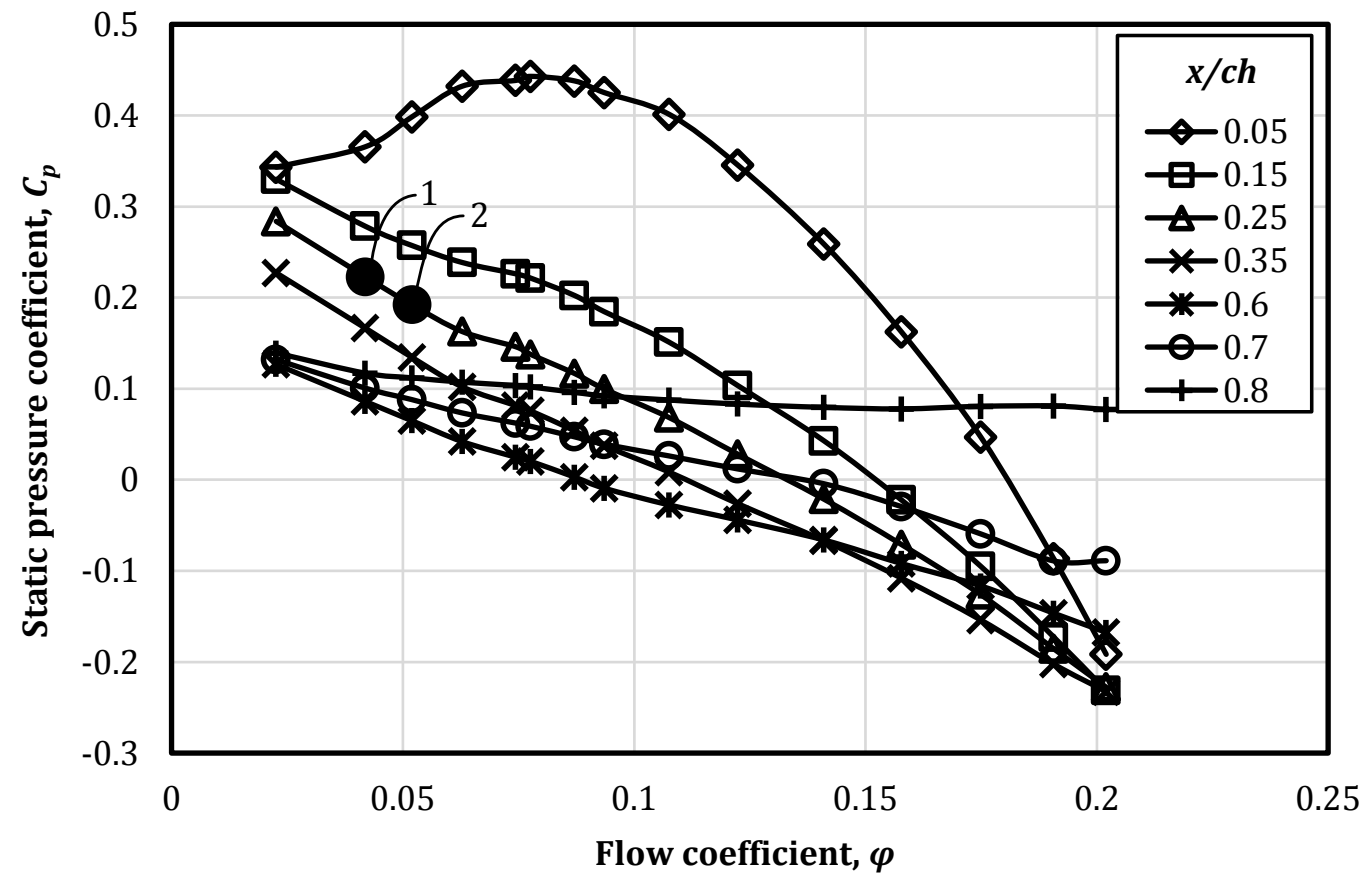

(b)

Figure D.3: Measured blade surface static pressures of the pressure side at a span of $s_{b}=0.5$ (a) as a function of dimensionless chord for a range of flow rates and (b) as a function of flow rate for each pressure tap. 


\section{Appendix E}

\section{P3DM sensitivity analyses}

Sensitivity analyses are conducted for the P3DM presented in chapter 4. A depiction of the computational domain is repeated in figure E.1.

As mentioned in chapter 4 the P3DM consists of three parts being the Inlet, Rotor and Outlet sub-domains. This configuration enables the separate grid construction of each sub-domain. A structured, hexagonal cell grid is implemented for the Rotor sub-domain (enclosing the blade surfaces) and constructed using the ANSYS CFX Turbogrid meshing tool. This type of grid presents a more accurate flow solution compared to other unstructured types, since the grid is generally aligned with the flow direction. Unstructured polyhedral grids are resorted to for the larger wedge shaped Inlet and Outlet sub-domains and constructed in ANSYS Design modeller and mesher in conjunction with Fluent. This allowed a dense population of cells to be placed near the Rotor sub-domain interfaces, with the cell population gradually de-

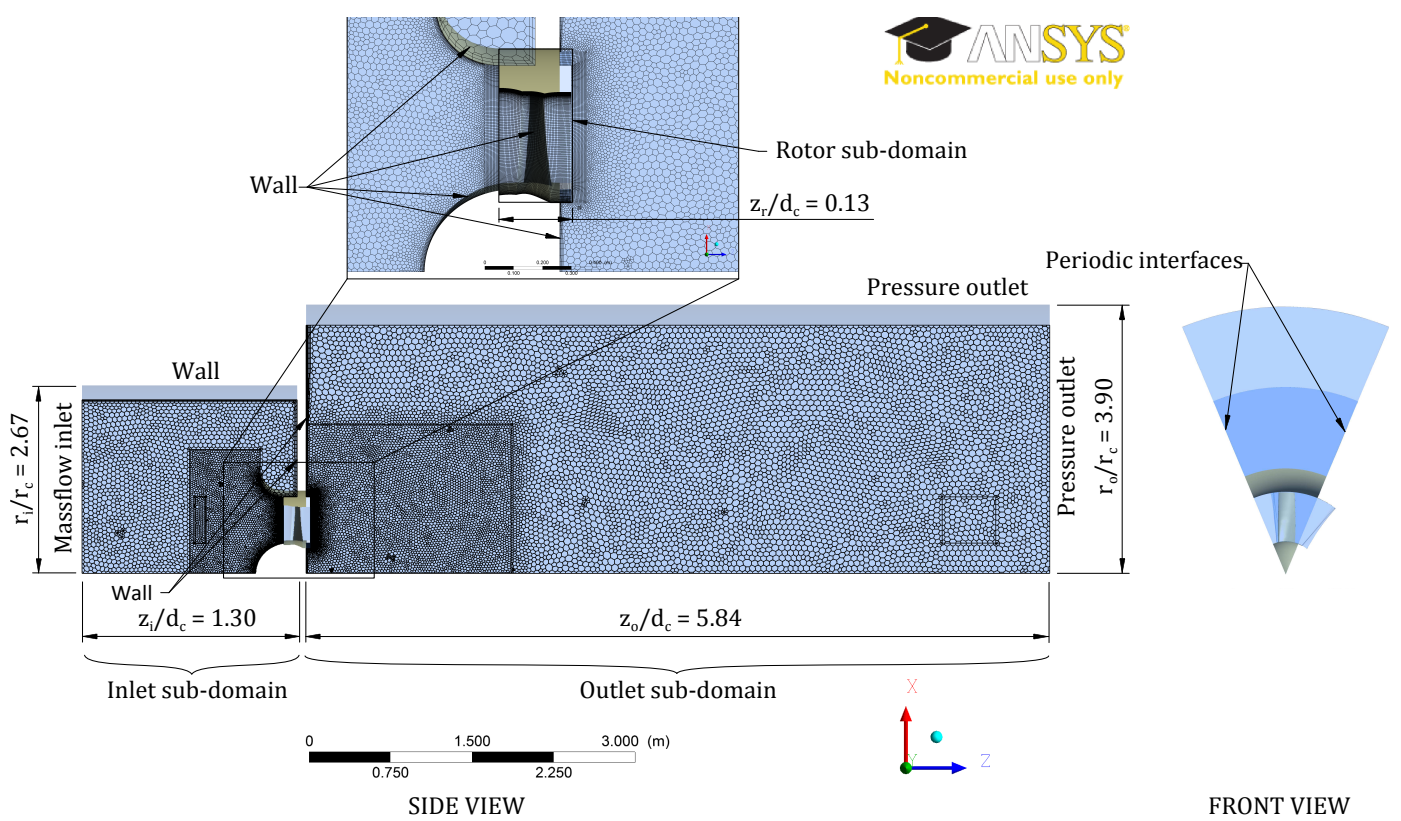

Figure E.1: Computational domain of the numerical fan model 
creasing in density towards the computational domain's outer extents where less resolution of the flow field is required.

This appendix presents the execution of the numerical sensitivity analyses. Firstly, the Rotor section alone is investigated to obtain the amount of cells that is required for a converged result (discussed in section E.1). Hereafter, an investigation is conducted to obtain the inlet and outlet boundary proximities from the fan blade model that would give converged results (presented in section E.2). Subsequently, grid resolution and time-step size analyses for the entire computational domain is conducted and chosen as discussed in sections E.3 and E.4, respectively. Lastly, the convergence of the time-dependent simulations is presented in E.5 and discussed.

\section{E.1 Rotor sub-domain grid sensitivity}

A grid sensitivity analysis for the Rotor sub-domain alone is conducted at the design flow rate of $\phi_{D}=0.168\left(\dot{V}_{D}=16 \mathrm{~m}^{3} / \mathrm{s}\right)$ by monitoring the fan static pressure difference between the inlet and outlet of the domain. The inlet to and outlet from the domain are modelled by Mass flow inlet and Pressure outlet boundaries respectively. It should also be mentioned that the tip-clearance is omitted for the Rotor sub-domain grid sensitivity analyses to reduce complexities in the flow and improve numerical convergence. Furthermore the Rotor sub-domain axial thickness is chosen as $z_{r} / d_{c}=0.13$.

Results for the grid sensitivity analysis can be seen in table E.1 comparing the fan static pressure difference across the fan to the number of cells in the domain. Observing these results it is evident that a small difference between a grid size of $1(10)^{6}$ and $2(10)^{6}$ cells exists. A Rotor sub-domain with a minimum of $1(10)^{6}$ cells is therefore used for further investigations.

\section{E.2 Boundary proximity}

The effect of boundary proximity is investigated in a stepwise manner for a flow rate of $\phi_{D}=0.168\left(\dot{V}_{D}=16 \mathrm{~m}^{3} / \mathrm{s}\right)$. Firstly the Outlet and Rotor sub-

Table E.1: Rotor sub-domain grid sensitivity

\begin{tabular}{cc}
\hline Cell count & $\boldsymbol{\Psi}_{\boldsymbol{F s}}\left(\boldsymbol{\Delta} \boldsymbol{p}_{\boldsymbol{F} \boldsymbol{s}}, \mathbf{P a}\right)$ \\
\hline $240(10)^{3}$ & $0.119(260.92)$ \\
$500(10)^{3}$ & $0.101(222.70)$ \\
$990(10)^{3}$ & $0.106(233.92)$ \\
$2000(10)^{3}$ & $0.106(233.46)$ \\
\hline
\end{tabular}




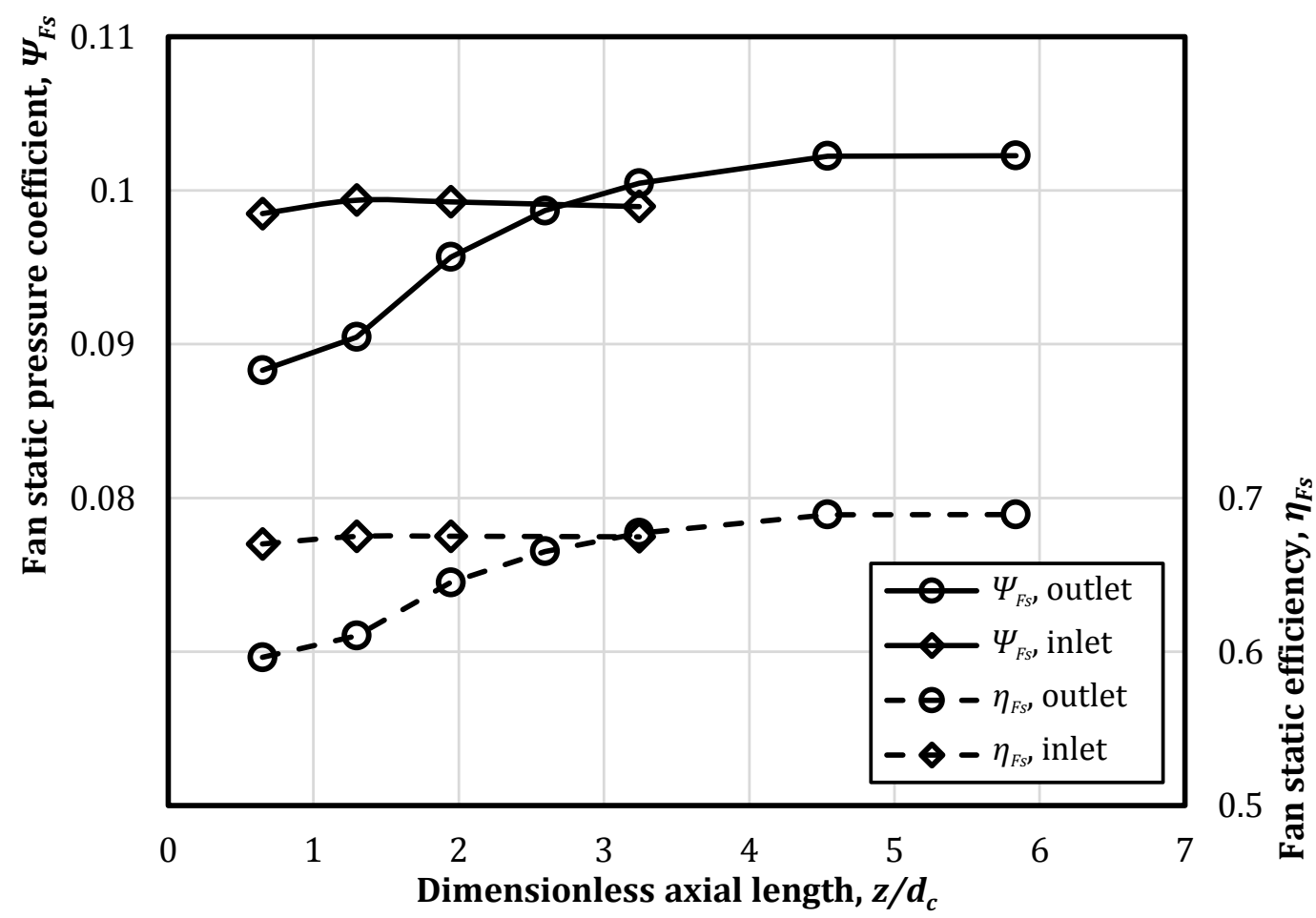

Figure E.2: Convergence of boundary proximity for Inlet and Outlet subdomains $\left(\phi_{D}=0.168\left(\dot{V}_{D}=16 \mathrm{~m}^{3} / \mathrm{s}\right)\right)$

domains are imported into Fluent and coupled together by the non-conformal interface already discussed. An appropriate axial outlet length of $z_{o} \leq 4.54 d_{c}$ is determined, by varying the distance between the outlet and the fan blade and monitoring the fan static pressure difference and fan static efficiency for each simulation as shown in figure E.2. The Inlet sub-domain is consequently also imported into Fluent and it is established that an inlet length of $z_{i} \leq 1.30 d_{c}$ provides converged fan static pressure difference and efficiency values, as indicated in figure E.2. A computational domain with inlet and outlet lengths of $z_{i} \leq 1.30 d_{c}$ and $z_{o} \leq 5.84 d_{c}$ is therefore used for the remaining analyses.

\section{E.3 Computational domain grid sensitivity}

A grid sensitivity analysis for the computational domain (shown in figure 9.6) is conducted at the design and lowest flow rates of $\phi_{D}=0.168\left(\dot{V}_{D}=16 \mathrm{~m}^{3} / \mathrm{s}\right)$ and $\phi=0.042\left(\dot{V}=4 \mathrm{~m}^{3} / \mathrm{s}\right)$ respectively. For this purpose two mesh densities are considered containing $2.5(10)^{6}$ and $5.5(10)^{6}$ cells respectively while conducting the analyses time-dependently. The time-steps for the individual flow rates are chosen in accordance with the conclusions made in section E.4. It should also be mentioned that the Enhanced wall treatment (a two layer model combined with other wall functions available in Fluent) was used for the finer computational grid $\left(5.5(10)^{6}\right.$ cells) since the size of the cells next to 
Table E.2: Computational domain grid sensitivity

\begin{tabular}{lccc}
\hline$\phi\left(\dot{\boldsymbol{V}}, \mathbf{m}^{3} / \mathrm{s}\right)$ & Cell count & $\boldsymbol{\Psi}_{\boldsymbol{F s}}\left(\boldsymbol{\Delta} \boldsymbol{p}_{\boldsymbol{F s}}, \mathrm{Pa}\right)$ & $\boldsymbol{\eta}_{\boldsymbol{F s}}$ \\
\hline $0.168(16)$ & $2.5(10)^{6}$ & $0.084(184.36)$ & 0.627 \\
& $5.5(10)^{6}$ & $0.084(184.63)$ & 0.617 \\
\hline $0.042(4)$ & $2.5(10)^{6}$ & $0.174(381.79)$ & 0.313 \\
& $5.5(10)^{6}$ & $0.175(385.02)$ & 0.307 \\
\hline
\end{tabular}

the blade surface was such that $1<y^{+}<5$ and therefore being below the recommended range of $\left(y^{+}>30\right)$ for utilization of the Standard wall function.

The results obtained for the mesh sensitivity analyses are shown in table E.2. It can be observed from the results for the design flow coefficient that no difference occurs between the fan static pressure coefficient if the value is rounded to the third decimal, whereas there is a one percent difference in static efficiency. The results for a flow coefficient of $\phi=0.042\left(\dot{V}=4 \mathrm{~m}^{3} / \mathrm{s}\right)$ similarly show small differences between the solutions of the two different grid densities.

Hence, a mesh density of $2.5(10)^{6}$ cells in the computational domain is sufficient for further analyses at flow rates close to the design point as well as lower flow rates.

\section{E.4 Time-step size}

Analyses are conducted to show time-step independence for the flow rates of $\phi_{D}=0.168\left(\dot{V}_{D}=16 \mathrm{~m}^{3} / \mathrm{s}\right)$ and $\phi=0.042\left(\dot{V}=4 \mathrm{~m}^{3} / \mathrm{s}\right)$ respectively. For this purpose the fan static pressure difference between the inlet and outlet of the domain, as well as the fan static efficiency are monitored. The results obtained for the different flow rates and time-steps are shown in table E.3. From the results for a flow coefficient of $\phi_{D}=0.168\left(\dot{V}_{D}=16 \mathrm{~m}^{3} / \mathrm{s}\right)$ it can be observed that no difference to the third decimal is evident for time-step values lower than $\Delta t<0.1(10)^{-3} \mathrm{~s}$. Therefore the time-step size is chosen as $\Delta t=$ $0.2(10)^{-3} \mathrm{~s}$ for the design flow rate. In the case of $\phi=0.042\left(\dot{V}=4 \mathrm{~m}^{3} / \mathrm{s}\right)$, no result is obtained for $\Delta t>0.1 \mathrm{~s}$ and the maximum difference between pressure coefficient and efficiency values are $0.1 \%$. Therefore a time-step value of $\Delta t=0.1 \mathrm{~s}$ proved to be sufficient to provide a converged result for low flow rates. 
Table E.3: Computational domain time-step independence analyses

\begin{tabular}{llcc}
\hline $\boldsymbol{\phi}\left(\dot{\boldsymbol{V}}, \mathbf{m}^{\mathbf{3}} / \mathbf{s}\right)$ & $\boldsymbol{\Delta} \boldsymbol{t},\left(\mathbf{1 0}^{-\mathbf{3}}\right) \mathbf{s}$ & $\boldsymbol{\Psi}_{\boldsymbol{F s} \boldsymbol{s}}\left(\boldsymbol{\Delta} \boldsymbol{p}_{\boldsymbol{F s}}, \mathbf{P a}\right)$ & $\boldsymbol{\eta}_{\boldsymbol{F s}}$ \\
\hline $0.168(16)$ & 0.5 & no result & no result \\
& 0.2 & 0.084 & 0.617 \\
& 0.1 & 0.084 & 0.618 \\
& 0.05 & 0.084 & 0.618 \\
& 0.025 & 0.084 & 0.618 \\
\hline \multirow{2}{*}{$0.042(4)$} & 0.2 & no result & no result \\
& 0.1 & 0.174 & 0.313 \\
& 0.05 & 0.173 & 0.313 \\
& 0.025 & 0.174 & 0.314 \\
\hline
\end{tabular}

\section{E.5 Simulation convergence}

The time-dependent numerical convergence of the fan static pressure and static efficiency is shown in figure E.3a-d for the design flow rate, $\phi_{D}=0.168\left(\dot{V}_{D}=\right.$ $\left.16 \mathrm{~m}^{3} / \mathrm{s}\right)$ and the low flow rates of $\phi=0.105,0.074,0.042\left(\dot{V}=10,7,4 \mathrm{~m}^{3} / \mathrm{s}\right)$. It can be observed that the convergence for the design flow rate is steady over time. However, the numerical results converge to a state where a periodic trend can be observed for the low flow rates. It is assumed that these simulations have converged when the moving average (based on the period lengths) of the static pressure coefficient and the static efficiency have reached a plato and the error between consecutive coefficient values are smaller than $10^{-4}$. The number of time-steps $\left(n_{t s}\right)$ in a single period is listed in table E.4 for each of the low flow rates. The numerical residuals also had to be less than the values specified in table 4.2 .

Table E.4: Time-steps in a single period of the converged numerical simulations at low flow rates

\begin{tabular}{llll}
\hline $\boldsymbol{\phi}\left(\dot{\boldsymbol{V}}, \mathbf{m}^{\mathbf{3}} / \mathbf{s}\right)$ & $0.105(10)$ & $0.074(7)$ & $0.042(4)$ \\
\hline $\boldsymbol{n}_{\boldsymbol{t s}}$ & 78 & 111 & 130 \\
\hline
\end{tabular}




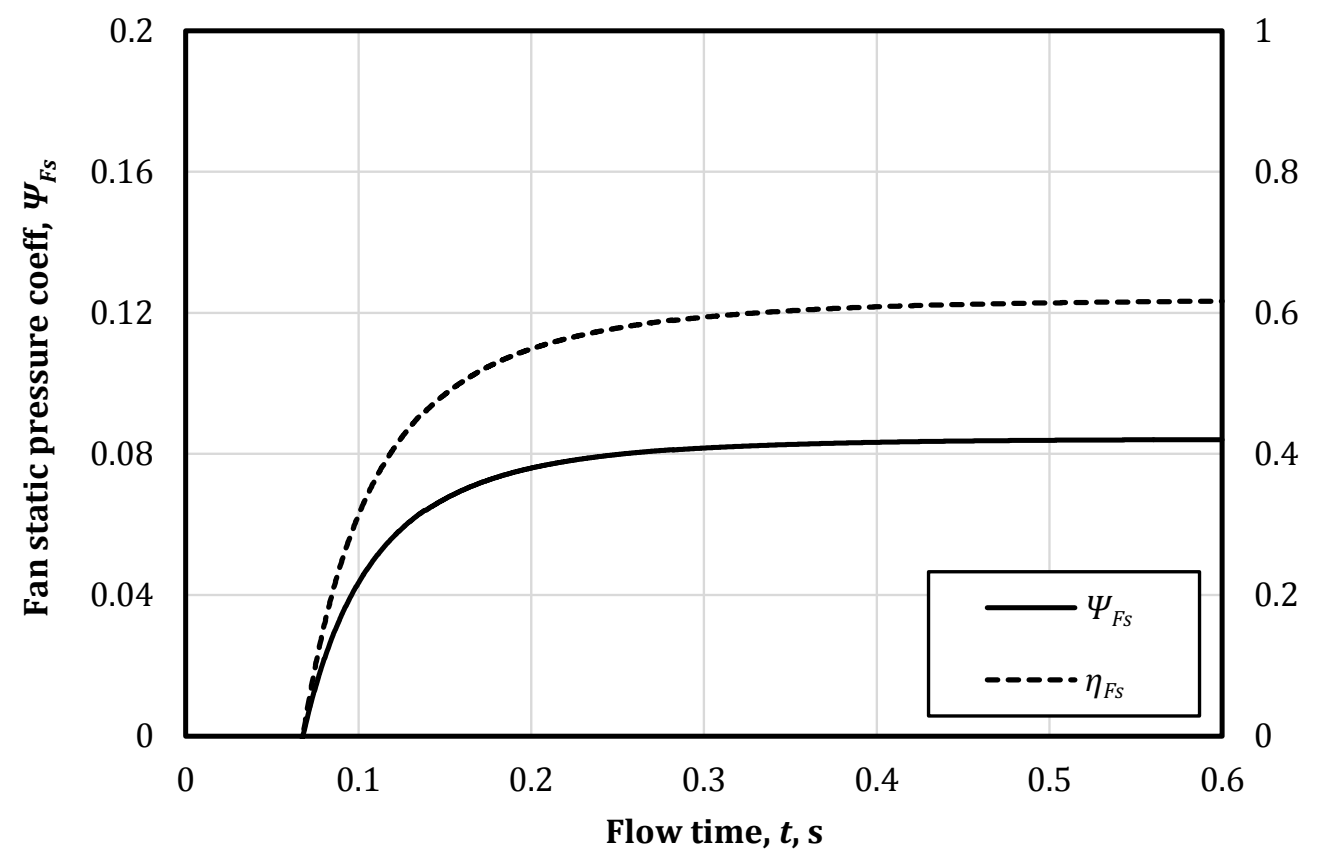

(a)

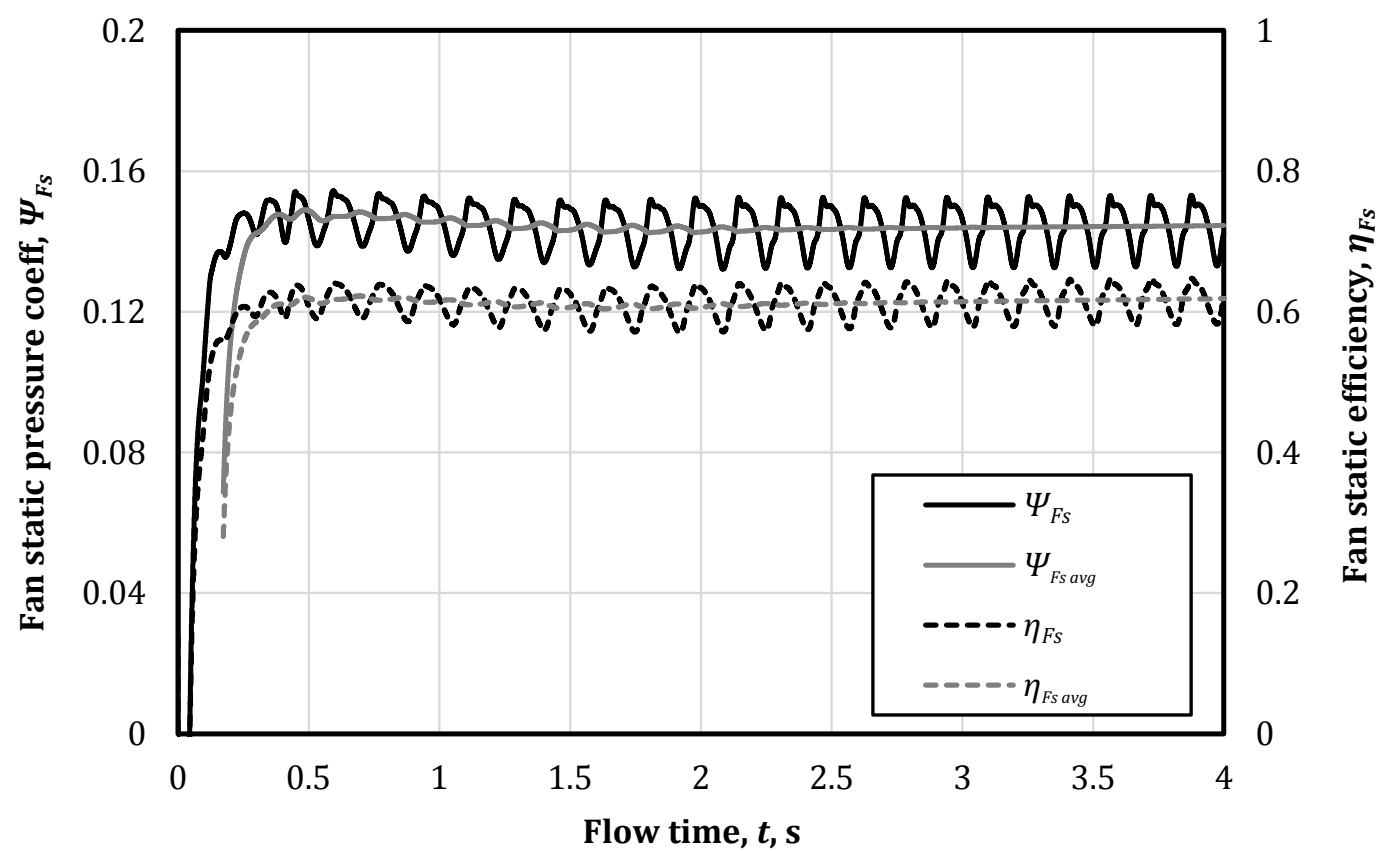

(b) 


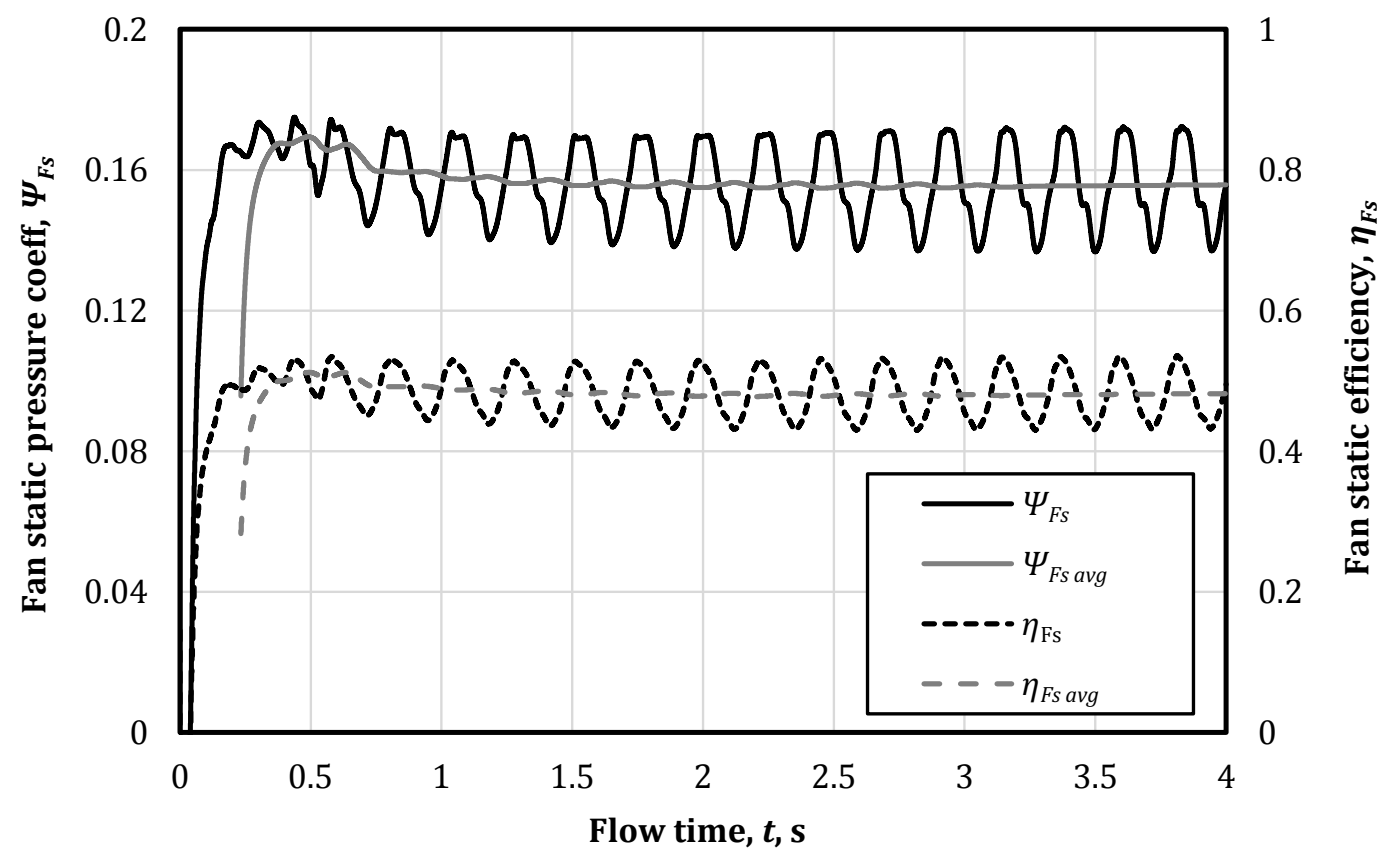

(c)

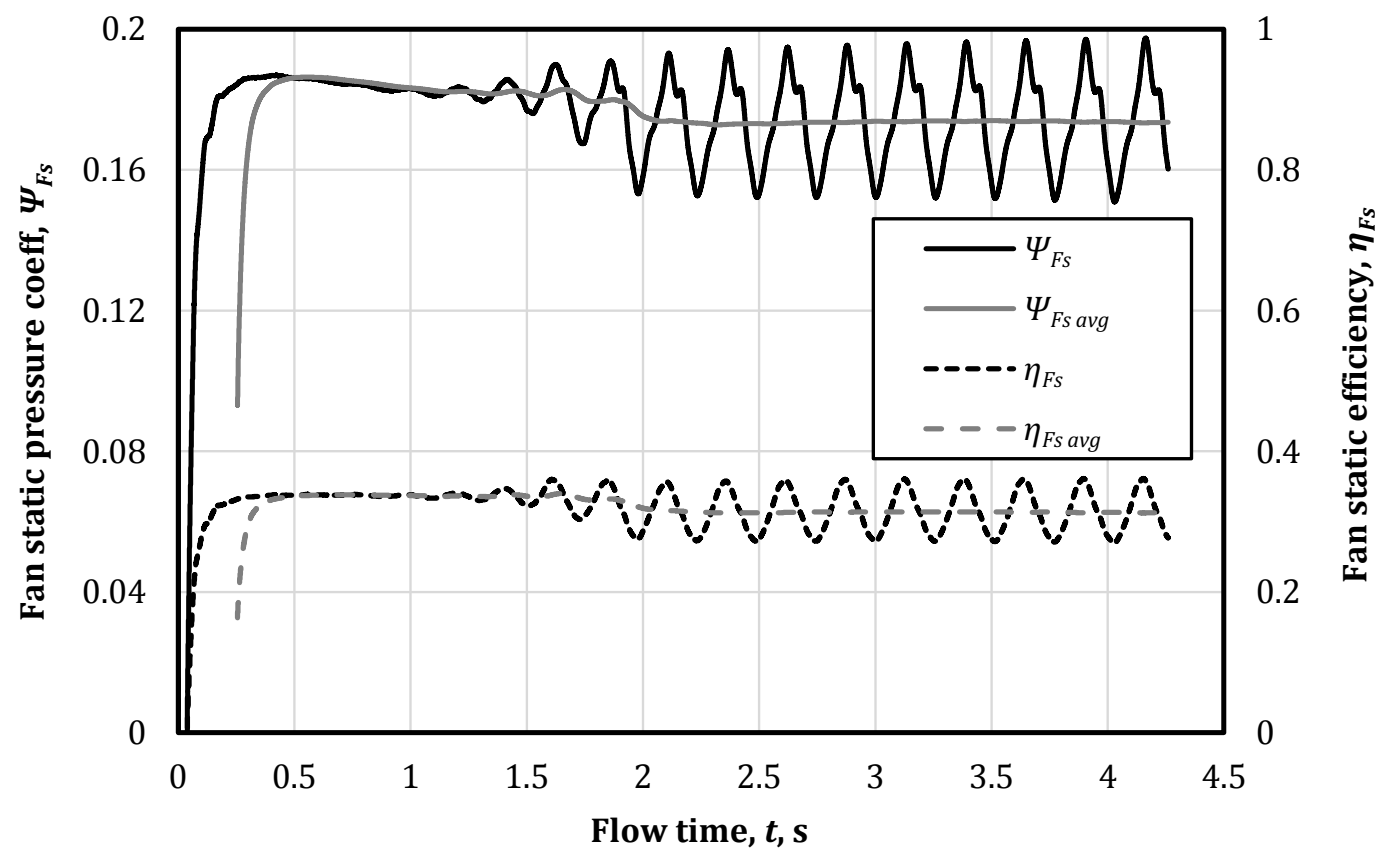

(d)

Figure E.3: Simulation convergence monitors of fan static pressure and static efficiency for (a) the design flow rate, $\phi_{D}=0.168\left(\dot{V}_{D}=16 \mathrm{~m}^{3} / \mathrm{s}\right)$ and the low flow rates of (b) $\phi=0.105\left(\dot{V}=10 \mathrm{~m}^{3} / \mathrm{s}\right)$, (c) $\phi=0.074\left(\dot{V}=7 \mathrm{~m}^{3} / \mathrm{s}\right)$ and (d) $\phi=0.042\left(\dot{V}=4 \mathrm{~m}^{3} / \mathrm{s}\right)$ 


\section{Appendix F}

\section{Turbulence modelling}

This appendix gives a brief overview of the fundamental governing flow equations in section F.1. Hereafter the Realizable $k-\varepsilon$ model (Shih et al., 1994) is presented in section F.2.

\section{F.1 Fundamental flow equations}

The fundamental fluid governing equations for a Newtonian incompressible fluid in Cartesian coordinates are given in tensor form as:

Continuity:

$$
\frac{\partial u_{i}}{\partial x_{i}}=0
$$

Momentum:

$$
\frac{\partial}{\partial t}\left(\rho u_{i}\right)+\frac{\partial}{\partial x_{j}}\left(\rho u_{i} u_{j}\right)=-\frac{\partial p}{\partial x_{i}}+\frac{\partial}{\partial x_{j}}\left(2 \mu s_{i j}\right)
$$

where the the strain rate tensor, $s_{i j}$ is defined as:

$$
s_{i j}=\frac{1}{2}\left(\frac{\partial u_{i}}{\partial x_{j}}+\frac{\partial u_{j}}{\partial x_{i}}\right)
$$

To obtain the Reynolds-averaged Navier Stokes (RANS) equations it is assumed that the velocity $\left(u_{i}\right.$, where $i$ is the Cartesian directions $x, y$ and $z$ ) and pressure components $(p)$ can be represented by the sum of the mean and fluctuating components:

$$
u_{i}=U_{i}+u_{i}^{\prime}, \quad p=P+p^{\prime}
$$

where the mean and fluctuating components conform to: 


$$
\begin{aligned}
& \overline{u_{i}}=U_{i}, \quad \bar{p}=P \\
& \overline{u_{i}^{\prime}}=0, \quad \overline{p_{i}^{\prime}}=0
\end{aligned}
$$

Substituting F.1.4 into F.1.1 and F.1.2 renders the RANS equations: Continuity:

$$
\frac{\partial \rho U_{i}}{\partial x_{i}}=0
$$

Momentum:

$$
\frac{\partial}{\partial t}\left(\rho U_{i}\right)+\frac{\partial}{\partial x_{i}}\left(\rho U_{i} U_{j}\right)=-\frac{\partial P}{\partial x_{i}}+\frac{\partial}{\partial x_{j}}\left(2 \mu S_{i j}\right)-\frac{\partial}{\partial x_{j}}\left(\rho \overline{u_{i}^{\prime} u_{j}^{\prime}}\right)
$$

where the mean strain rate tensor $\left(S_{i j}\right)$ is defined as:

$$
S_{i j}=\frac{1}{2}\left(\frac{\partial U_{i}}{\partial x_{j}}+\frac{\partial U_{j}}{\partial x_{i}}\right)
$$

In equation F.1.8 $\overline{u_{i}^{\prime} u_{j}^{\prime}}$ is known as the Reynolds stress tensor $\left(R_{i j}\right)$ which consists of nine components of which $\overline{u^{\prime} v^{\prime}}=\overline{v^{\prime} u^{\prime}}, \overline{u^{\prime} w^{\prime}}=\overline{w^{\prime} u^{\prime}}$ and $\overline{v^{\prime} w^{\prime}}=\overline{w^{\prime} v^{\prime}}$. Therefore six components are unknown being $\overline{u^{\prime} u^{\prime}}, \overline{v^{\prime} v^{\prime}}, \overline{w^{\prime} w^{\prime}}, \overline{u^{\prime} v^{\prime}}, \overline{u^{\prime} w^{\prime}}$ and $\overline{v^{\prime} w^{\prime}}$. Furthermore, the mean pressure $(P)$ is also unknown, producing a total of seven unknown variables. Since only the continuity and three momentum equations exist to solve these values, three unknown variables will still remain.

In order to obtain a closed set of fluid governing equations, the Reynolds stresses need to be modelled by means of a turbulence model. The Realizable $k-\varepsilon$ employs the Boussinesq hypothesis (Hinze, 1975) where the Reynolds stresses are approximated as:

$$
-\rho \overline{u_{i}^{\prime} u_{j}^{\prime}}=2 \mu_{t} S_{i j}-\frac{2}{3} k \delta_{i j}
$$

where $\delta_{i j}$ is the Kronecker delta which states that

$$
\begin{aligned}
& \delta_{i j}=0 \text { if } i \neq j \\
& \delta_{i j}=1 \text { if } i=j
\end{aligned}
$$

and $k$ is the turbulence kinetic energy defined as

$$
k=\frac{1}{2} \overline{u_{i}^{\prime} u_{i}^{\prime}}
$$


and $\mu_{t}$ is defined as the turbulent eddy viscosity, which is assumed to be an isotropic scalar quantity. Hence, the main goal in obtaining a flow solution using the Boussinesq approach to turbulence modelling is to obtain an approximation for turbulence viscosity $\left(\mu_{t}\right)$. This is obtained by means of the Realizable $k-\varepsilon$ model in the present study as discussed in the next section.

\section{F.2 Realizable $k$ - $\varepsilon$ turbulence model}

The transport equations for the turbulent kinetic energy $k$ and turbulent dissipation $\varepsilon$ rate in the Realizable $k-\varepsilon$ model are:

Turbulent kinetic energy:

$$
\begin{aligned}
\frac{\partial}{\partial t}(\rho k)+\frac{\partial}{\partial x_{j}}\left(\rho k U_{j}\right)= & \frac{\partial}{\partial x_{j}}\left[\left(\mu+\frac{\mu_{t}}{\sigma_{k}}\right) \frac{\partial k}{\partial x_{j}}\right]+G_{k}+G_{b} \\
& -\rho \varepsilon-Y_{M}+S_{k}
\end{aligned}
$$

Turbulent dissipation rate:

$$
\begin{aligned}
\frac{\partial}{\partial t}(\rho \varepsilon)+\frac{\partial}{\partial x_{j}}\left(\rho \varepsilon U_{j}\right)= & \frac{\partial}{\partial x_{j}}\left[\left(\mu+\frac{\mu_{t}}{\sigma_{\varepsilon}}\right) \frac{\partial \varepsilon}{\partial x_{j}}\right]+\rho G_{1} S_{\varepsilon}-\rho C_{2} \frac{\varepsilon^{2}}{k+\sqrt{\nu \varepsilon}} \\
& +C_{1 \varepsilon} \frac{\varepsilon}{k} C_{3 \varepsilon} G_{b}+S_{\varepsilon}
\end{aligned}
$$

where the value for $C_{1}$ is determined by

$$
C_{1}=\max \left[0.43, \frac{\eta}{\eta+5}\right], \quad \eta=S \frac{k}{\varepsilon}, \quad S=\sqrt{2 S_{i j} S_{i j}}
$$

In the turbulent kinetic energy transport equation F.2.1, $G_{k}$ represents the generation of turbulence kinetic energy due to the mean velocity gradients defined as

$$
G_{k}=\mu_{t} S^{2}
$$

where $S$ is defined in F.2.3. $G_{b}$ is the generation of turbulence kinetic energy due to buoyancy defined as

$$
G_{b}=\beta g_{i} \frac{\mu_{t}}{P r_{t}} \frac{\partial T}{\partial x_{i}}
$$

where the turbulent Prandtl number is $P r_{t}=0.85$ and $g_{i}$ is the gravitational component in the $i$-direction. The thermal expansion coefficient, $\beta$ is defined as

$$
\beta=-\frac{1}{\rho}\left(\frac{\partial \rho}{\partial T}\right)
$$


The effect of buoyancy on the turbulent dissipation rate transport equation F.2.2 is governed by $C_{3 \varepsilon}$, which is normally a constant value, but in Fluent it is calculated by

$$
C_{3 \varepsilon}=\tanh \left|\frac{v}{u}\right|
$$

The value $Y_{M}$ represents the effect of compressibility on turbulence, but for incompressible flow $Y_{M} \approx 0$

The remaining constants are $C_{1 \varepsilon}, C_{2}$ and the turbulent Prandtl numbers $\sigma_{k}$ and $\sigma_{\varepsilon}$ for $k$ and $\varepsilon$, respectively:

$$
C_{1 \varepsilon}=1.44, \quad C_{2}=1.9, \quad \sigma_{k}=1.0, \quad \sigma_{\varepsilon}=1.2
$$

In utilizing the Realizable $k-\varepsilon$ model, the turbulent viscosity is calculated in a similar fashion to the Standard $k-\varepsilon$ model by

$$
\mu_{t}=\rho C_{\mu} \frac{k^{2}}{\varepsilon}
$$

but $C_{\mu}$ is no longer a defined constant, but calculated through

$$
C_{\mu}=\frac{1}{A_{0}+A_{s} \frac{k U^{*}}{\varepsilon}}
$$

where

$$
\begin{aligned}
U^{*} & =\sqrt{S_{i j} S_{i j}+\tilde{\Omega}_{i j} \tilde{\Omega}_{i j}} \\
\tilde{\Omega}_{i j} & =\Omega_{i j}-2 \varepsilon_{i j k} \omega_{k} \\
\Omega_{i j} & =\overline{\Omega_{i j}}-\varepsilon_{i j k} \omega_{k}
\end{aligned}
$$

and the mean rate of rotation tensor $\left(\overline{\Omega_{i j}}\right)$ is defined as

$$
\overline{\Omega_{i j}}=\frac{1}{2}\left(\frac{\partial U_{i}}{\partial x_{j}}-\frac{\partial U_{j}}{\partial x_{i}}\right)
$$

viewed within a rotating reference frame with angular velocity $\omega_{k}$. Furthermore, the constants $A_{0}$ and $A_{s}$ are given as

$$
A_{0}=4.04, \quad A_{s}=\sqrt{6} \cos \phi
$$

where

$$
\phi=\frac{1}{3} \cos ^{-1}(\sqrt{6} W), \quad W=\frac{S_{i j} S_{j k} S_{k i}}{\tilde{S}^{3}}, \quad \tilde{S}=\sqrt{S_{i j} S_{i j}}
$$




\section{Appendix G}

\section{Data sampling techniques}

The techniques used to sample data from the numerical results are described in this appendix. For purposes of comparison to experimentally obtained values, the time-averaging of numerical results are required at low flow coefficients $\left(0.042 \leq \phi \leq 0.105\left(4 \leq \dot{V} \leq 10 \mathrm{~m}^{3} / \mathrm{s}\right)\right)$ for variables such as the fan static pressure and efficiency as well as blade surface pressures. The time-averaging of results are firstly discussed, whereafter the different data sampling routines used to extract specific data from the numerical results are presented in the subsequent sections.

\section{G.1 Time-averaging}

As mentioned in chapter 4, time-dependent numerical analyses are required for low flow coefficients through the fan $\left(0.042 \leq \phi \leq 0.105\left(4 \leq \dot{V} \leq 10 \mathrm{~m}^{3} / \mathrm{s}\right)\right)$. The convergence of these simulations, discussed in appendix E.5, displays a periodic trend. To be able to compare these numerical results to those obtained experimentally, an arithmetic mean is calculated for the applicable variable $\bar{\lambda}$ that is to be compared over one period of the converged simulation results as follow:

$$
\bar{\lambda}=\frac{\sum_{i=1}^{n_{t s}} \lambda_{i}}{n_{t s}}
$$

where $\lambda_{i}$ is the instantaneous value of a certain variable (e.g. fan static pressure $\left.\left(\Delta p_{F s}\right)\right)$ and $n_{t s}$ the number of time-steps in a single period of the converged simulation. The number of time-steps for a single period of each of the low flow rate simulations are listed in appendix E.5. 


\section{G.2 Fan performance characteristic data}

Step 1:

Set Fluent simulation to write out text files of surface monitors:

- Pressure on boundaries

- Fan torque moment

Step 2:

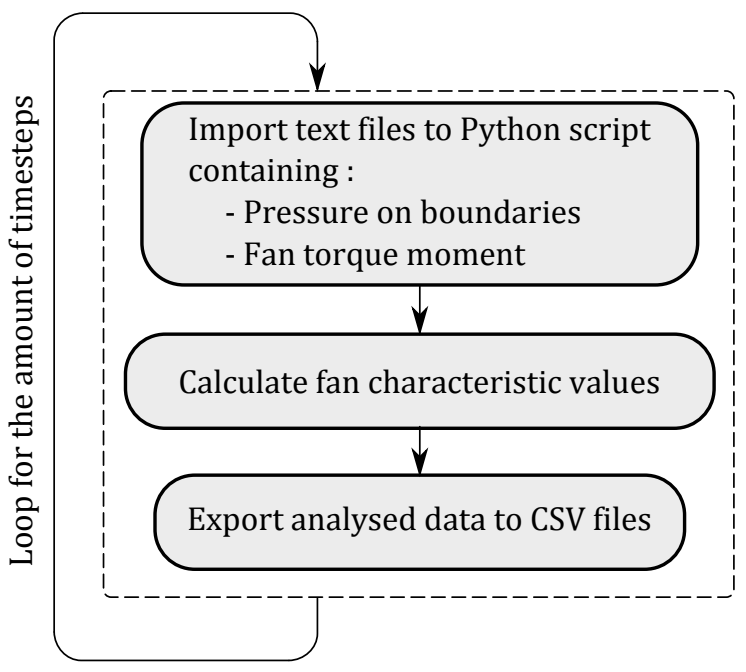

Step 3:

Calculate time average of variables

Figure G.1: Python routine for sampling and calculating fan performance characteristics 


\section{G.3 Blade pressure data}

Step 1:

Run batch script to export values from post processor (ANSYS CFD-Post) into CSV files:

- Blade surface pressures

Step 2:

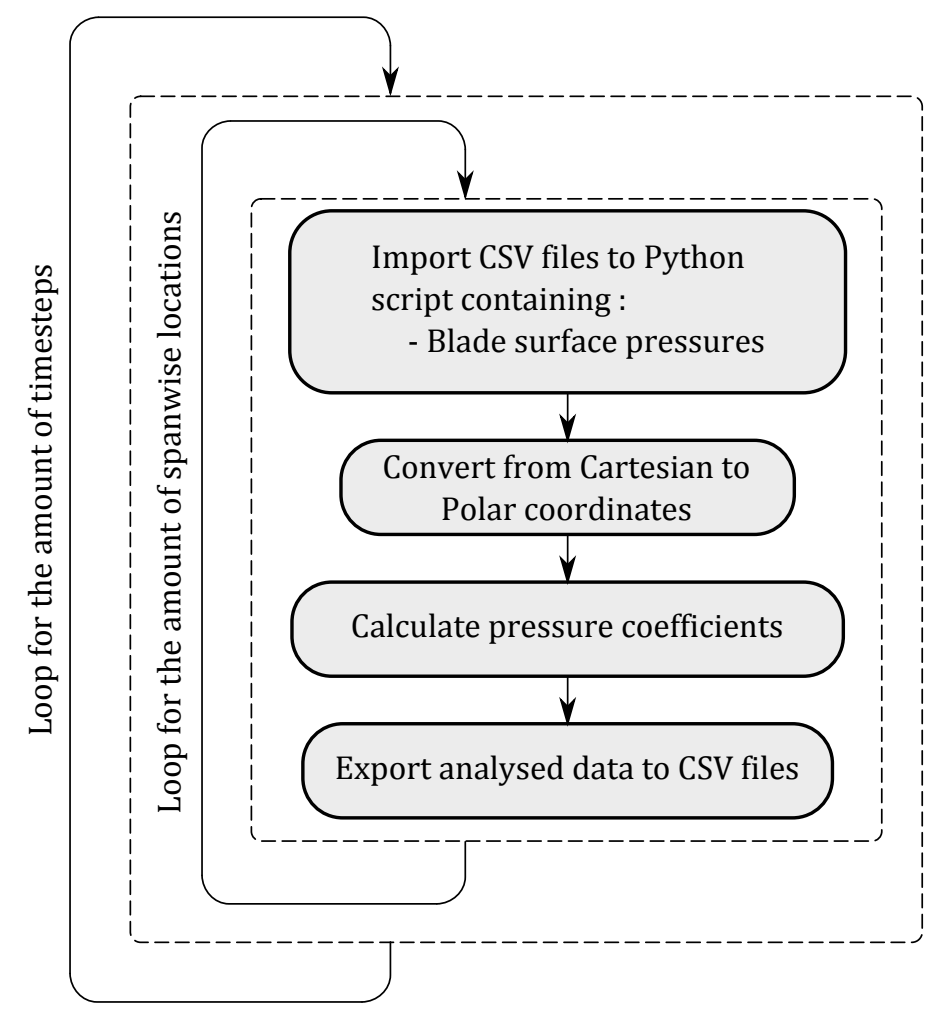

Step 3:

Calculate time average of variables

Figure G.2: Python routine for sampling and calculating blade surface pressure coefficients 


\section{G.4 Lift, drag and radial force data}

Step 1:

Run batch script to export values from post processor (ANSYS CFD-Post) into CSV files:

- Up- and downstream velocities

- Up- and downstream pressures

- Blade forces

Step 2:

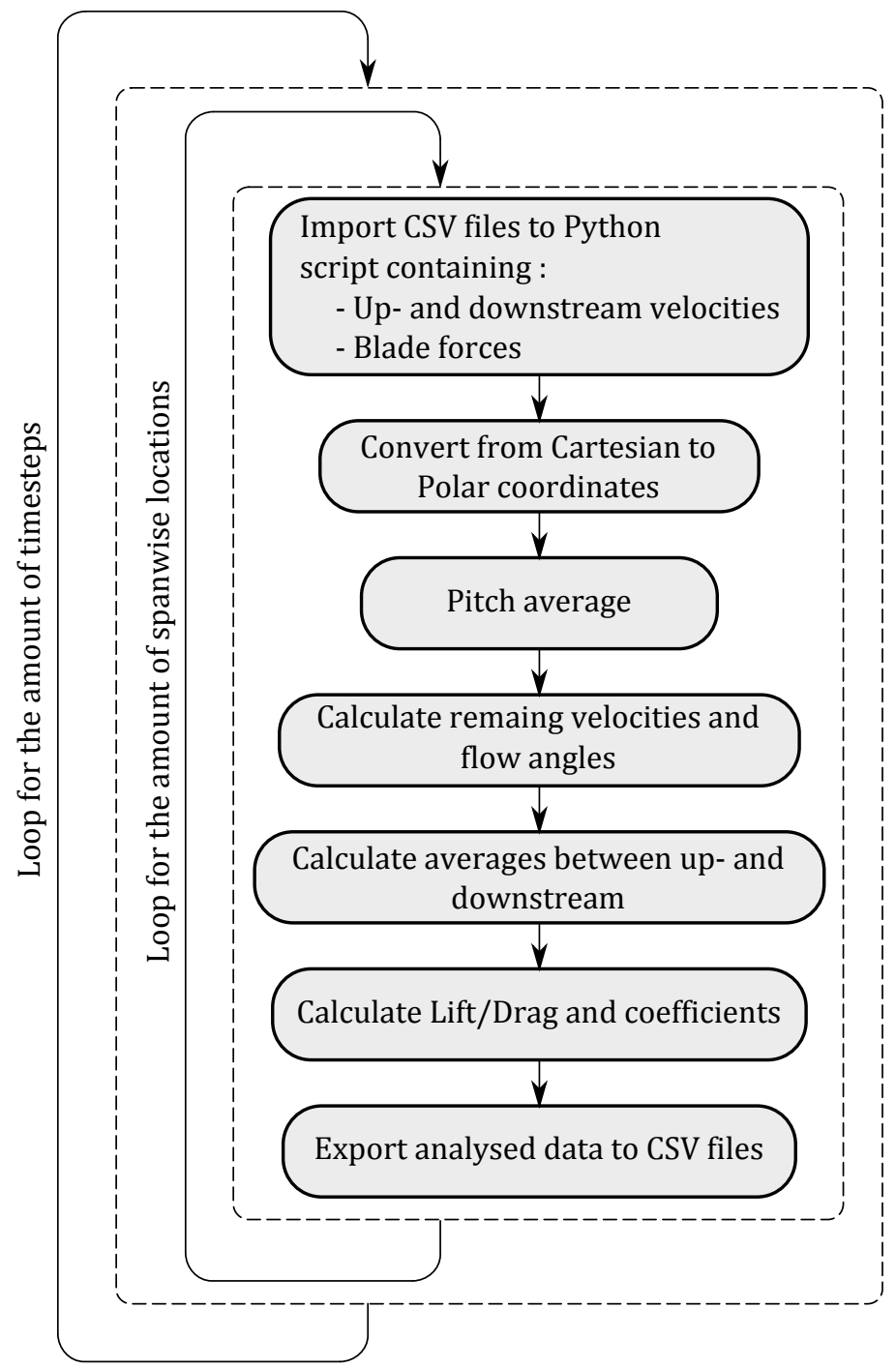

Step 3:

Calculate time average of variables

Figure G.3: Python routine for sampling and calculating blade sectional lift, drag and radial force coefficients 